\title{
United States Earthquakes, 1986
}

\section{U.S. GEOLOGICAL SURVEY BULLETIN 2089}

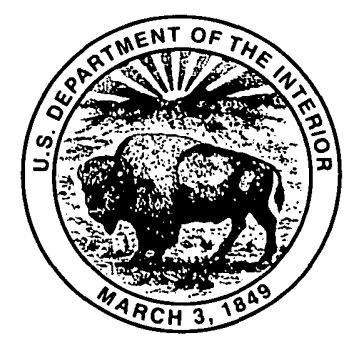




\section{AVAILABILITY OF BOOKS AND MAPS OF THE U.S. GEOLOGICAL SURVEY}

Instructions on ordering publications of the U.S. Geological Survey, along with prices of the last offerings, are given in the current-year issues of the monthly catalog "New Publications of the U.S. Geological Survey." Prices of available U.S. Geological Survey publications released prior to the current year are listed in the most recent annual "Price and Availability List." Publications that may be listed in various U.S. Geological Survey catalogs (see back inside cover) but not listed in the most recent annual "Price and Availability List" may no longer be available.

Reports released through the NTIS may be obtained by writing to the National Technical Information Service, U.S. Department of Commerce, Springfield, VA 22161; please include NTIS report number with inquiry

Order U.S. Geological Survey publications by mail or over the counter from the offices listed below.

BY MAIL

\section{Books}

Professional Papers, Bulletins, Water-Supply Papers, Techniques of Water-Resources Investigations, Circulars, publications of general interest (such as leaflets, pamphlets, booklets), single copies of Earthquakes \& Volcanoes, Preliminary Determination of Epicenters, and some miscellaneous reports, including some of the foregoing series that have gone out of print at the Superintendent of Documents, are obtainable by mail from

\section{U.S. Geological Survey, Map Distribution Box 25286, MS 306, Federal Center Denver, CO 80225}

Subscriptions to periodicals (Earthquakes \& Volcanoes and Preliminary Determination of Epicenters) can be obtained ONLY from the

\section{Superintendent of Documents Government Printing Office \\ Washington, DC 20402} Documents.)

(Check or money order must be payable to Superintendent of

\section{Maps}

For maps, address mail orders to

\section{U. S. Geological Survey, Map Distribution Box 25286, Bldg. 810, Federal Center Denver, CO 80225}

Residents of Alaska may order maps from

\author{
U.S. Geological Survey, Earth Science Information Center \\ 101 Twelfth Ave., Box 12 \\ Fairbanks, AK 99701
}

\section{OVER THE COUNTER}

\section{Books and Maps}

- Books-and maps of the U.S. Geological Survey are available over the counter at the following U.S. Geological Survey offices, all of which are authorized agents of the Superintendent of Documents.

- ANCHORAGE, Alaska-Rm. 101, 4230 University Dr.

- LAKEWOOD, Colorado-Federal Center, Bldg. 810

- MENLO PARK, California-Bldg. 3, Rm. 3128, 345 Middlefield Rd.

- RESTON, Virginia-USGS National Center, Rm. 1C402, 12201 Sunrise Valley Dr.

- SALT LAKE CITY, Utah-Federal Bldg., Rm. 8105, 125 South State St.

- SPOKANE, Washington-U.S. Post Office Bldg., Rm. 135, West 904 Riverside Ave.

- WASHINGTON, D.C.-Main Interior Bldg., Rm. 2650, 18th and C Sts., NW.

\section{Maps Only}

Maps may be purchased over the counter at the following U.S. Geological Survey offices:

- FAIRBANKS, Alaska-New Federal Bldg, 101 Twelfth Ave.

- ROLLA, Missouri-1400 Independence Rd.

- STENNIS SPACE CENTER, Mississippi-Bldg. 3101 


\section{United States Earthquakes, 1986}

By Carl. W. Stover and Lindie R. Brewer

\section{U.S. GEOLOGICAL SURVEY BULLETIN 2089}

A summary of data for earthquakes in the 50 States and Puerto Rico during 1986. Descriptions of individual earthquakes include hypocenters, magnitudes, intensities, and damages. The report also contains results from regional networks and data recorded by strong-motion seismographs

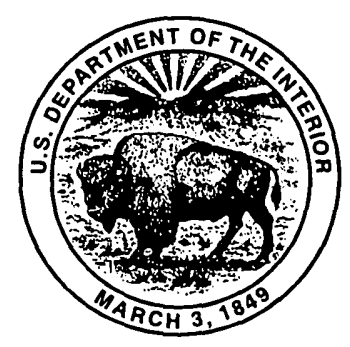




\title{
U.S. DEPARTMENT OF THE INTERIOR BRUCE BABBITT, Secretary
}

\author{
U.S. GEOLOGICAL SURVEY \\ Gordon P. Eaton, Director
}
For sale by U.S. Geological Survey, Map Distribution
Box 25286, MS 306, Federal Center
Denver, CO 80225

\begin{abstract}
Any use of trade, product, or firm names in this publication is for descriptive purposes only and does not imply endorsement by the U.S. Government
\end{abstract}

\section{Library of Congress Cataloging-in-Publication Data}

Stover, Carl W.

United States Earthquakes, 1986 / by Carl W. Stover and Lindie R. Brewer.

p. cm. - (U.S. Geological Survey bulletin ; 2089)

Includes bibliographical references.

Supt. of Docs. no.: I 19.3:2089

1. Earthquakes-United States-History-Chronology. I. Brewer, Lindie.

II. Title. III. Series.

QE75.B9 no. 2089

[QE535.2.U6]

$553.7 \mathrm{~s}-\mathrm{dc} 20$

[551.2'2'097309048] 


\section{Contributors}

Contributors to this publication are listed below according to the information furnished or service performed:

Hypocenters and magnitudes:

John H. Minsch, U.S. Geological Survey, Denver, Colo.

Robert Y. Koyanagi, Hawaiian Volcano Observatory, U.S. Geological Survey

Intensities:

Carl W. Stover, U.S. Geological Survey, Denver, Colo.

Robert Y. Koyanagi, Hawaiian Volcano Observatory, U.S. Geological Survey

Network operations (by institution)

University of California, Berkeley, Calif.

Hawaiian Volcano Observatory, U.S. Geological Survey, Hawaii.

Lamont-Doherty Geological Observatory, Columbia University, Palisades, N. Y.

Los Alamos National Laboratory, Los Alamos, N. Mex.

Montana Bureau of Mines and Geology, Butte, Mont.

New Mexico Institute of Mining and Technology, Socorro, N. Mex.

Oklahoma Geological Survey, Leonard, Okla.

Saint Louis University, St. Louis, Mo.

University of Nevada-Reno, Nev.

University of Washington, Seattle, Wash.

Virginia Polytechnic Institute and State University, Blacksburg, Va.

Weston Observatory, Boston College, Weston, Mass.

Strong-motion seismograph data:

Ronald L. Porcella and Josephine C. Switzer, U.S. Geological Survey, Menlo Park, Calif.

Editorial assistance and manuscript preparation:

Francis W. Baldwin, U.S. Geological Survey, Denver, Colo. 


\section{CONTENTS}

\section{Introduction 1}

Discussion of tables 1

Epicenter and isoseismal maps 1

Magnitude and intensity 3

Modified Mercalli Intensity scale of 19315

\section{Collaborators 8}

Earthquake descriptions 9

Alabama 9

Alaska 9

Arizona 21

Arkansas 21

California 21

Colorado 62

Connecticut 64

Delaware 64

District of Columbia 64

Georgia 64

Hawaii 66

Idaho 68

Illinois 69

Indiana 70

Kansas 70

Kentucky 70 .

Maine 71

Maryland 71

Massachusetts 71

Michigan 71

Missouri 71

Montana 72

Nevada 73

New Hampshire 75

New Jersey 76

New Mexico 76

New York 77

North Carolina 78

Ohio 78

Pennsylvania 94

Puerto Rico 94

Rhode Island 95

South Carolina 95

South Dakota 96

Tennessee 96

Texas 96

Utah 96

Vermont 97

Virginia 97 
Washington 98

West Virginia 100

Wisconsin 100

Wyoming 100

Summary of United States earthquakes for 1986103

Network operations 180

Eastern Aleutians seismicity, by J.J. Taber, M.A. Luckman, and S. Rosen 180

Northern and central California earthquakes, 1986, by Robert A. Uhrhammer 180

Seismicity and volcanic activity in Hawaii, 1986, by Robert Y. Koyanagi and Jennifer S. Nakata 182

Kansas and Nebraska earthquakes, 1986, by Choon Byong Park and Don W. Steeples 185

Mississippi Valley earthquakes, 1986, by W. Stauder, R. Herrmann, S. Neiers, S. Horton, C. Carr, C. Finn, and

\section{L. $\mathrm{Li} 188$}

Montana and adjacent area earthquakes, 1986, by Michael C. Stickney 189

Western Nevada and eastern California earthquakes, 1986, by Arturo Aburto 191

New England earthquakes, 1986, by James P. McCaffrey, S.J. 191

Socorro, New Mexico, area earthquakes, 1986, by Allan Sanford, Lawrence Jaksha, and Roderick Flores 192

Seismicity of New Mexico, 1986, by Allan Sanford, Lawrence Jaksha, and Dan Cash 195

Oklahoma earthquakes, 1986, by James. E. Lawson, Jr., and Kenneth V. Luza 196

Southeastern United States earthquakes, 1986, by M.S. Sibol, M.C. Chapman, and G.A. Bollinger 198

Washington earthquakes, 1986, by R.S. Ludwin, S.D. Malone, R.S. Crosson, and A.I. Qamar 199

Principal earthquakes of the World for 1986 (table 6) 203

Strong-motion accelerograph data, by Ronald L. Porcella and Josephine C. Switzer 205

Introduction 205

Accelerograph data (table 7) 206

References cited 238

\section{FIGURES}

1. Earthquake epicenters in the conterminous United States for 19862

2. Earthquake epicenters in Alaska for 19862

3. Earthquake epicenters in Hawaii for 19863

4. Epicenters in the conterminous United States for earthquakes with magnitudes $\geq 5.0$ in 19864

5. Epicenters in Alaska for earthquakes with magnitudes $\geq 5.0$ in 19864

6. Earthquakes in the conterminous United States that were felt or caused damage in 19866

7. Earthquakes in Alaska that were felt or caused damage in 19866

8. Earthquakes in Hawaii that were felt or caused damage in 19867

9. Isoseismal map for the central California earthquake of 26 January 1986, 192051.2 UTC 23

10. Isoseismal map for the Mount Lewis, California, earthquake of 31 March 1986, 115540.1 UTC 27

11. Isoseismal map for the North Palm Springs, California, earthquake of 8 July 1986, 092044.5 UTC 33

12. Photograph of Devers substation, Palm Springs, California, showing relatively tall porcelain-glass insulators on 10 - $f$ perches 34

13. Photograph of cracks in adobe walls of recently remodeled house in Whitewater Canyon, Calif. 35

14. Photograph of a fallen chimney in Whitewater Canyon 36

15. Isoseismal map for the earthquake of 13 July 1986, 134708.2 UTC off the coast of southern California 43

16. Isoseismal map for the Chalfant Valley, California, earthquake of 21 July 1986, 144226.5 UTC 48

17. Isoseismal map for the northern California, earthquake of 21 November 1986, 233301.7 UTC 60

18. Isoseismal map for the Georgia-Tennessee border earthquake of 11 July 1986, at 142614.8 UTC 65

19. Isoseismal map for the central New Hampshire earthquake of 25 October 1986, 17 16 38.4 UTC 77

20. Isoseismal map for the northeast Ohio earthquake of 31 January 1986, 164642.3 UTC 80 
21. Isoseismal map for the epicentral area of the northeast Ohio earthquake of 31 January 198682

22. Isoseismal map for the western Ohio earthquake of 12 July 1986, 081937.9 UTC 92

23. Seismicity located by the Shumagin seismic network from January 1 to December 30, 1986181

24. Cross section of Shumagin network seismicity along line $\mathbf{A}-\mathbf{A}^{\prime}$ in figure $23 \mathbf{1 8 1}$

25. University of California seismographic station network and northern and central California seismicity during 1986182

26. Seismograph stations for the island of Hawaii during 1986183

27. Map of the island of Hawaii showing locations of the active volcanoes Mauna Loa, Kilauea, and Loihi 183

28. Ground tilt, number of earthquakes, and lava volume associated with the eruption of Kilauea in 1986184

29. Earthquakes in Hawaii during 1986185

30. Locations of earthquakes of magnitude 2.5 or greater beneath the island of Hawaii region in 1986186

31. Locations of earthquakes of magnitude 1.5 or greater and standard errors of $2.0 \mathrm{~km}$ or less beneath Kilauea and Mauna Loa volcanoes in the southeastern region of the island of Hawaii for 1986186

32. Active seismograph stations in Kansas-Nebraska network during 1986187

33. Size-coded microearthquakes recorded by the Kansas Geological Survey during 1986 and major regional tectonic features that are apparently related to earthquake activity 188

34. Central Mississippi Valley earthquakes during 1986 within a $4^{\circ}$ by $5^{\circ}$ region centered at $36.5^{\circ} \mathrm{N}$. by $89.5^{\circ} \mathrm{W}$. 189

35. Central Mississippi Valley earthquakes during 1986 within a $1.5^{\circ}$ by $1.5^{\circ}$ area of the immediate New Madrid region 189

36. Permanent seismograph stations used to locate 1986 seismicity in Montana, parts of Idaho, and parts of Wyoming 190

37. Seismicity of Montana and adjacent regions during 1986191

38. Earthquakes in Montana and adjacent regions with magnitudes $\geq 3.0$ or reported felt 192

39. Earthquakes in western Nevada and eastern California during 1986 with magnitudes $\geq 4.0$ or reported felt 193

40. Northeastem United States earthquakes during 1986194

41. Seismicity of the Socorro, New Mexico, area for 1986195

42. Earthquakes in New Mexico during 1986 with magnitudes $\geq 1.5196$

43. Distribution of Oklahoma earthquakes for 1986197

44. Active seismographs in Oklahoma for 1986198

45. Southeastern United States earthquakes during 1986198

46. Southeastern United States Seismic Network (SEUSSN) stations operating at the end of 1986199

47. Distribution of focal depths for 1986 earthquakes (SEUSSN) 199

48. A plot of magnitude versus depth for 1986 earthquakes (SEUSSN) 199

49. Seismograph stations in Washington and Oregon operated by the University of Washington during 1986201

50. Felt earthquakes in Washington and northern Oregon during 1986, plus earthquakes with coda-length magnitudes greater than or equal to 2.7 which were not felt 201

\section{TABLES}

1. Summary of United States earthquakes for 1986103

2. Kansas and Nebraska earthquakes for 1986187

3. Oklahoma earthquakes for 1986197

4. Southeastern United States earthquakes for 1986200

5. Southeastern United States Seismic Network earthquake statistics for 1986200

6. Principal earthquakes of the World during 1986203

7. Summary of United States accelerograph records recovered during 1986206 


\title{
United States Earthquakes, 1986
}

\author{
ByCarl W. Stover and Lindie Brewer
}

\section{INTRODUCTION}

This publication describes all earthquakes that were reported felt in the United States and nearby territories in 1986. Its purpose is to provide a continuous history of U.S. earthquakes to be used in estimating areal seismic risk, for designing earthquake-resistant structures, and for answering inquiries from scientists, engineers, and the public.

The U.S. Geological Survey's National Earthquake Information Center (USGS/NEIC) collects intensity information primarily by mailing questionnaires, "Earthquake Report" forms, to postmasters and other public institutions (police departments and (or) fire departments) in the earthquake area. Completed questionnaires are returned to the USGS, where they are evaluated and intensities are assigned. For damaging earthquakes, the questionnaires are supplemented by USGS field investigations. The USGS/ NEIC publishes preliminary maximum intensity data for U. S. earthquakes in the Preliminary Determination of Epicenters, Monthly Listing (PDE) (for example, Irby and others, 1982). The latest and most complete information is published with maps, diagrams, and photographs in United States Earthquakes (now published as a USGS Bulletin) issued annually since 1928. Copies of issues prior to 1982 can be obtained from Open-File Services, Earth Science Information Center, U.S. Geological Survey, Box 25286, Mail Stop 517 Federal Center, Denver, CO 80225.

This current bulletin is composed of four major sections: (1) "Earthquake Descriptions," which includes a summary of macroseismic data reported for each earthquake and achronological list of earthquakes by State (table 1); (2) "Network Operations," which summarizes the results from local seismic networks; (3) "Miscellaneous Activities," which contains information on the principal earthquakes of the world (table 6); and (4) "Strong-Motion Seismograph Data" (table 7). The intensities and macroseismic data in "Earthquake Descriptions" are compiled from questionnaires returned to USGS/NEIC (see previous paragraph), newspaper articles, and reports prepared by other Federal government organizations, State institutions, local organizations, and individuals. Each description includes date, hypocenter, source of the hypocenter computation, magnitude, maximum intensity (Modified Mercalli), and (or) macroseismic effects reported from localities where the earthquake was felt.

\section{Discussion of Tables}

The earthquake parameters in tables 1 and 6 include date, origin time, hypocenter (epicenter and focal depth), and magnitude. Table 1 also lists the maximum observed Modified Mercalli (MM) intensity. The origin time and date are listed in Coordinated Universal Time (UTC). The epicenters are taken principally from those published in the USGS Preliminary Determination of Epicenters, Monthly Listings. These data have been updated and new data added from subsequent publications of universities or State agencies who operate seismic networks. The accuracy of the epicenters is that claimed by the institution supplying the hypocenter data and is not necessarily the accuracy indicated by the number of decimals listed. The epicenters located by the USGS/NEIC vary in degree of accuracy, but for most of the area of the United States the epicenters should be accurate to within two-tenths of a degree. See Preliminary Determination of Epicenters, Monthly Listing, for an explanation of the accuracy of USGS hypocenters. Depths are listed to the nearest kilometer.

Magnitudes listed in the tables 1 and 6 are either furnished by cooperating institutions or determined by the USGS. The computational sources are indicated by letter codes identified in headnotes to the tables.

\section{Epicenter and Isoseismal Maps}

Figures 1-3 are computer plots of all earthquake epicenters in the conterminous United States, Alaska, and Hawaii listed in table 1. Figures 4-5 show only those earthquakes whose computed magnitudes are 5.0 or larger. 


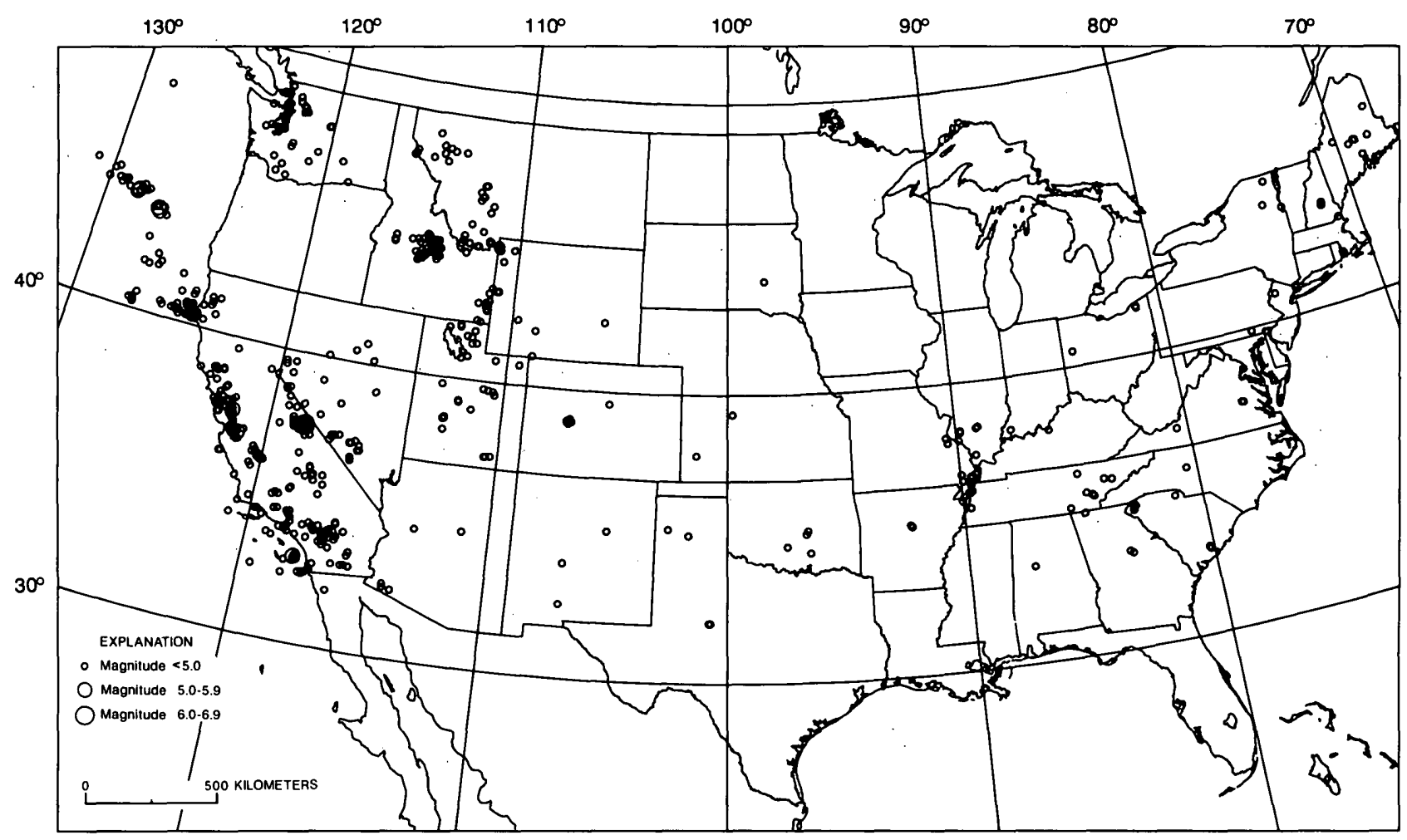

Figure 1. Earthquake epicenters in the conterminous United States for 1986 (from table 1).

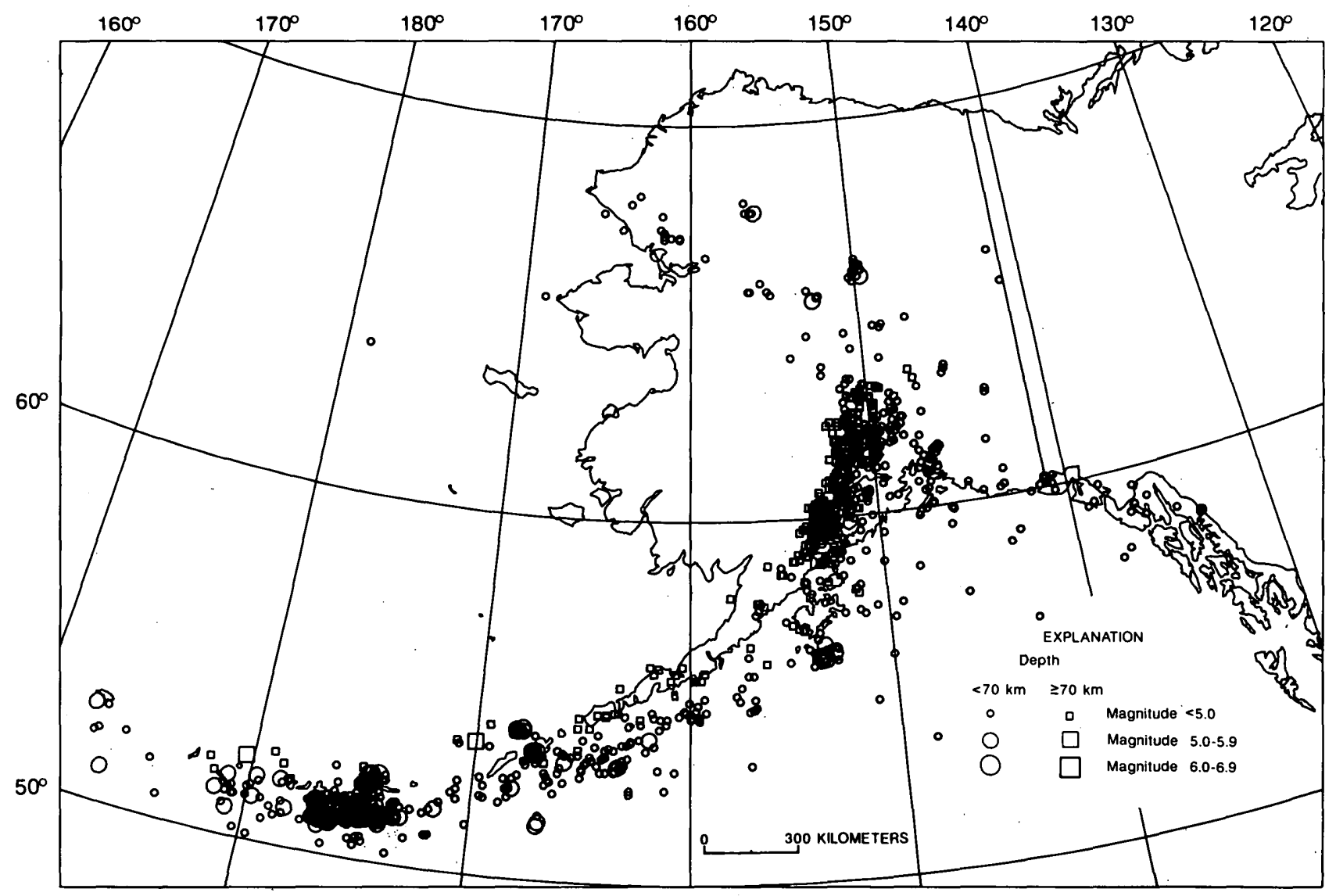

Figure 2. Earthquake epicenters in Alaska for 1986 (from table 1). 


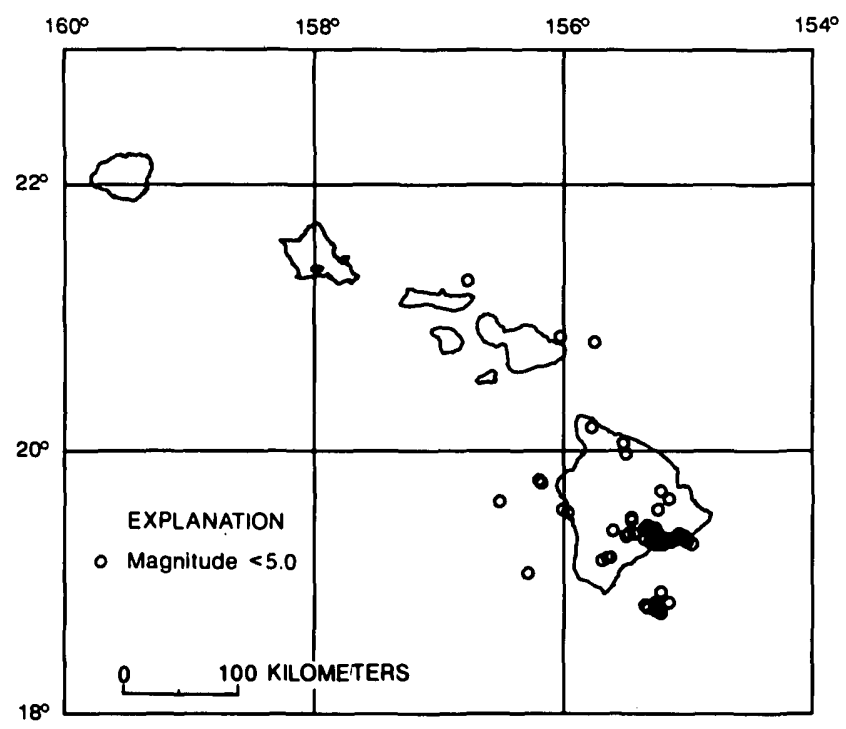

Figure 3. Earthquake epicenters in Hawaii for 1986 (from table 1).

Figures $6-8$ are maps showing the maximum intensity of earthquakes in the conterminous United States, Alaska, and Hawaii.

The USGS/NEIC coordinates the collection of all types of earthquake information; the special objective is to correlate instrumentally determined earthquake locations with noninstrumental locations indicated by intensity data. This correlation is achieved through regional investigations of earthquakes by local organizations and the USGS. Primary data are gathered by a mail canvass of the epicentral area using questionnaire cards. A field survey is usually made for damaging earthquakes. When returned and analyzed, this information is used to prepare isoseismal maps that show the areal pattern of intensity associated with individual earthquakes.

The selection of earthquakes for isoseismal maps (shown in the "Earthquake Descriptions") is governed largely by the size of the area affected. As a result, sharp, localized shocks of intensity VI, which are common in California, may not be represented by maps; more widely felt earthquakes of intensity V and VI, which are characteristic of the Eastern and Central States, commonly are illustrated because of the larger felt areas. Isoseismal contours are a generalization of intensity data and are extrapolated in regions that have reported few observations. The isoseismals do not account for each intensity observation because they are drawn to show the general patterns at a level of intensity or range of intensities.

\section{Magnitude and Intensity}

Magnitude, a measure of the size of an earthquake, is related to the energy release at the focus of an earthquake. Although the magnitude scale has neither maximum nor minimum values, the highest magnitude ever calculated was greater than 9.0 and the lowest magnitude ever calculated was about -3.0. On this logarithmic scale, a magnitude-6.0 shallow-focus earthquake represents elastic-wave energy about 30 times greater than that generated by a magnitude 5.0 earthquake, 900 times greater than that of a magnitude 4.0 shock, and so forth. Many factors enter into the determination of earthquake magnitude, including earthquake focal depth, frequency content of the sampled energy, and the earthquake radiation pattern. Magnitude values calculated by the USGS are based on the following five formulas:

Surface-wave magnitude

$$
M_{\mathrm{S}}=\log (A / T)+1.66 \log D+3.3 \text {, }
$$

as adopted by the International Association of Seismology and Physics of the Earth's Interior (IASPEI; Bath, 1966, p. 153 ), where $A$ is the maximum vertical surface-wave ground amplitude, in micrometers; $T$ is the period, in seconds, and $18 \leq T \leq 22$; and $D$ is the distance in geocentric degrees (station to epicenter), and $20^{\circ} \leq D \leq 160^{\circ}$. No depth correction is made for depth less than $50 \mathrm{~km}$, and no $M_{S}$ magnitudes are computed for depths greater than $50 \mathrm{~km}$.

Body-wave magnitude

$$
m_{\mathrm{b}}=\log (A / T)+Q(D, h),
$$

as defined by Gutenberg and Richter (1956), except that $T$, the period in seconds, is restricted to $0.1 \geq T \geq 3.0$, and $A$, the ground amplitude in micrometers, is not necessarily the maximum of the $P$-wave group. $Q$ is a function of distance $D$ and depth $\mathrm{h}$, where $D>5^{\circ}$.

Local magnitude

$$
M_{\mathrm{L}}=\log A-\log A_{\mathrm{o}}
$$

as defined by Richter (1958, p. 340), where $A$ is the maximum trace amplitude in millimeters, written by a WoodAnderson torsion seismometer, and $\log A_{0}$ is a standard value that is a function of distance, where distance $\leq 600 \mathrm{~km}$. Values of $M_{\mathrm{L}}$ are also calculated from other seismometers by conversion of recorded ground motion to the expected response of the torsion seismometer. $M_{\mathrm{L}}$ magnitudes are not listed for events with depths greater than $70 \mathrm{~km}$. 


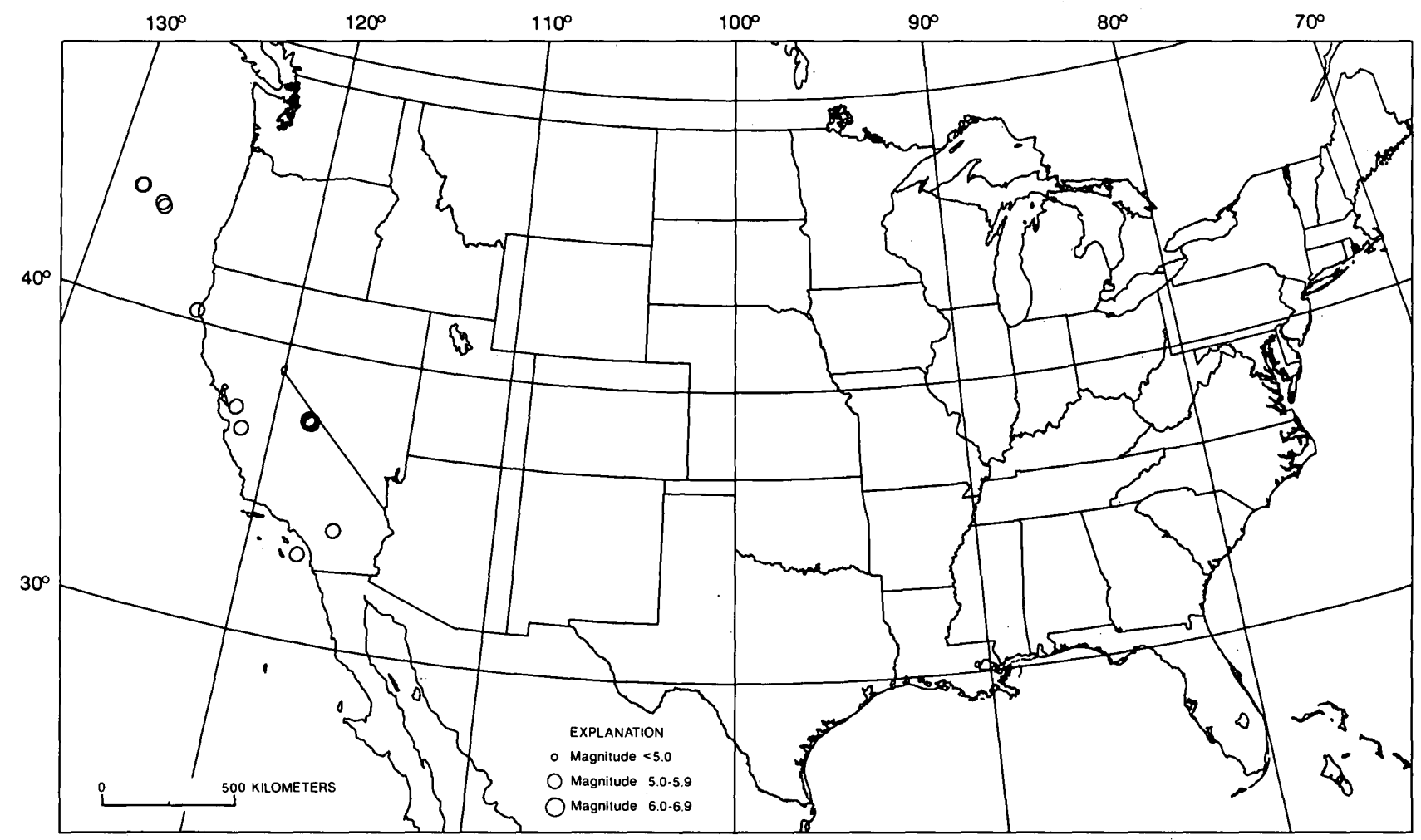

Figure 4. Epicenters in the conterminous United States for earthquakes with magnitudes greater than or equal to 5.0 in 1986.

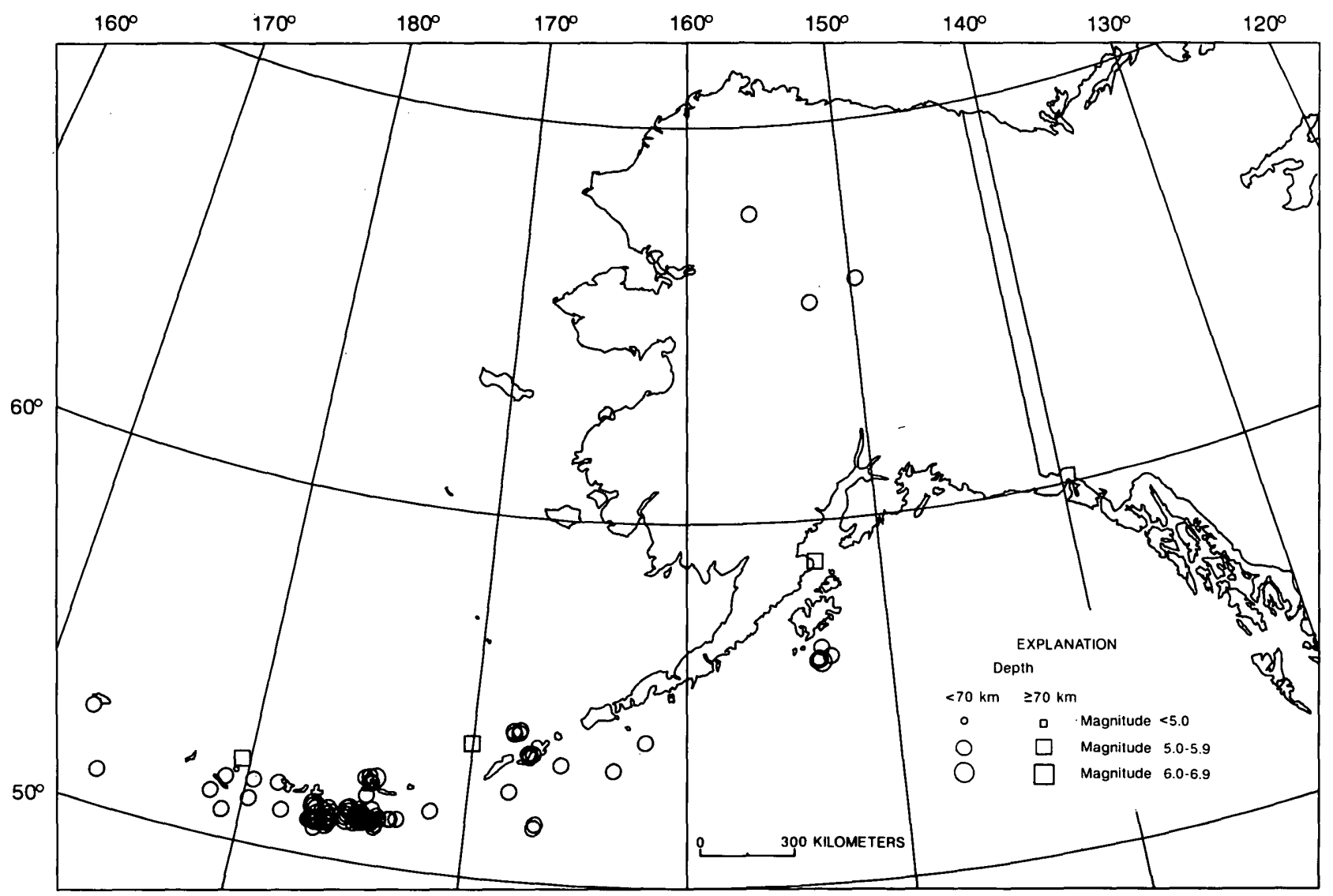

Figure 5. Epicenters in Alaska for earthquakes with magnitudes greater than or equal to 5.0 in 1986. 
Local magnitude

$$
\begin{gathered}
M_{\mathrm{n}}=3.75+0.90(\log D)+\log (A / T) \\
0.5^{\circ} \leq D \leq 4^{\circ}, \\
M_{\mathrm{n}}=3.30+1.66(\log D)+\log (A / T) \\
4^{\circ} \leq D \leq 30^{\circ}
\end{gathered}
$$

as proposed by Nuttli (1973) for North America east of the Rocky Mountains, where $A / T$ is expressed in micrometers per second, calculated from the vertical-component 1 -second $L g$ waves, and $D$ is the distance in geocentric degrees.

Moment magnitude

$$
M_{\mathrm{w}}=2 / 3 \log M_{\mathrm{o}}-10.7
$$

as defined by Hanks and Kanamori (1979), where $M_{\mathrm{o}}$ is the seismic moment in dyne-centimeters.

Other types of magnitudes computed by other organizations or universities are also listed in this publication and are defined in the following two paragraphs.

$M_{\mathrm{D}}$ designates duration or coda-length magnitude. $M_{\mathrm{D}}$ is usually computed from the difference, in seconds, between $P_{n}$ - or $P_{g}$-wave arrival time and the time the final coda amplitude decreases to the background-noise amplitude. Duration-magnitude scales are normally adjusted to agree with $M_{\mathrm{L}}$ or $M_{\mathrm{n}}$ estimates so that resulting magnitudes are compatible. Thus, the $M_{\mathrm{D}}$ formulas vary for different geographic regions and seismograph systems.

Some seismograph-network operators determine a magnitude formula for their specific network based on a comparison of their computed magnitudes with magnitudes published from other sources, such as from the USGS. These values are usually compared with $m_{\mathrm{b}}, M_{\mathrm{L}}$, or $M_{\mathrm{n}}$ magnitudes. In this bulletin these types of magnitudes are designated as $m_{\mathrm{x}}$ for body-wave magnitudes $\left(m_{\mathrm{b}}\right)$ and $M_{\mathrm{x}}$ for local magnitudes $\left(M_{\mathrm{L}}\right.$ or $\left.M_{\mathrm{n}}\right)$.

The seismic moment is tabulated for some earthquakes in the "Earthquake Descriptions" section. Moments contributed by the University of California, Berkeley, are computed according to the procedure described by Bolt and Herraiz (1983). Moments contributed by the U.S. Geological Survey, Golden, Colorado, are computed according to the procedure described by Sipkin (1982). Moments contributed by Harvard University are computed according to the procedure described by Dziewonski and others (1981).

Intensity, as applied to earthquakes, represents a quantity determined from the effects on people, manmade structures, and the Earth's surface (landslides, ground fissures, and such). Intensities are assigned according to the descriptions listed in the Modified Mercalli Intensity Scale of 1931 (Wood and Neumann, 1931). There are 12 discrete steps in the MM scale (see next section). An earthquake in a populated area will have different intensities at different localities, owing to the distance from the focus of the earthquake, type of focal mechanism, local geological conditions, structural design of buildings, and the earthquake magnitude.

The text of this bulletin gives the intensity at locations where an earthquake was reported felt and summaries of the strongest effects. Each earthquake is further characterized by its maximum intensity, which is given in the text and in table 1. The word "FELT" in the maximum intensity columns of table 1 indicates that only minimal or sketchy information was available. This designation does not imply that the earthquake was felt at a low-intensity level but indicates that the available data are not sufficient for assigning an intensity value.

Although the 1931 Modified Mercalli Intensity Scale is in many instances inadequate for present-day requirements, the scale has been the guide used by the USGS and will continue to be so until a new scale has been devised and has been accepted in the engineering and seismological communities.

\section{Modified Mercalli Intensity Scale of 1931}

Adapted from Sieberg's Mercalli-Cancani scale, modified and condensed.

I Not felt, or except rarely under especially favorable circumstances. Under certain conditions, at and outside the boundary of the area in which a great shock is felt: sometimes birds, animals, reported uneasy or disturbed; sometimes dizziness or nausea experienced; sometimes trees, structures, liquids, bodies of water, may sway - doors may swing very slowly.

II Felt indoors by few, especially on upper floors, or by sensitive, or nervous persons. Also, as in grade I, but often more noticeably: sometimes hanging objects may swing, especially when delicately suspended; sometimes trees, structures, liquids, bodies of water, may sway, doors may swing, very slowly; sometimes birds, animals, reported uneasy or disturbed; sometimes dizziness or nausea experienced.

III Felt indoors by several, motion usually rapid vibration. Sometimes not recognized to be an earthquake at first. Duration estimated in some cases. Vibration like 


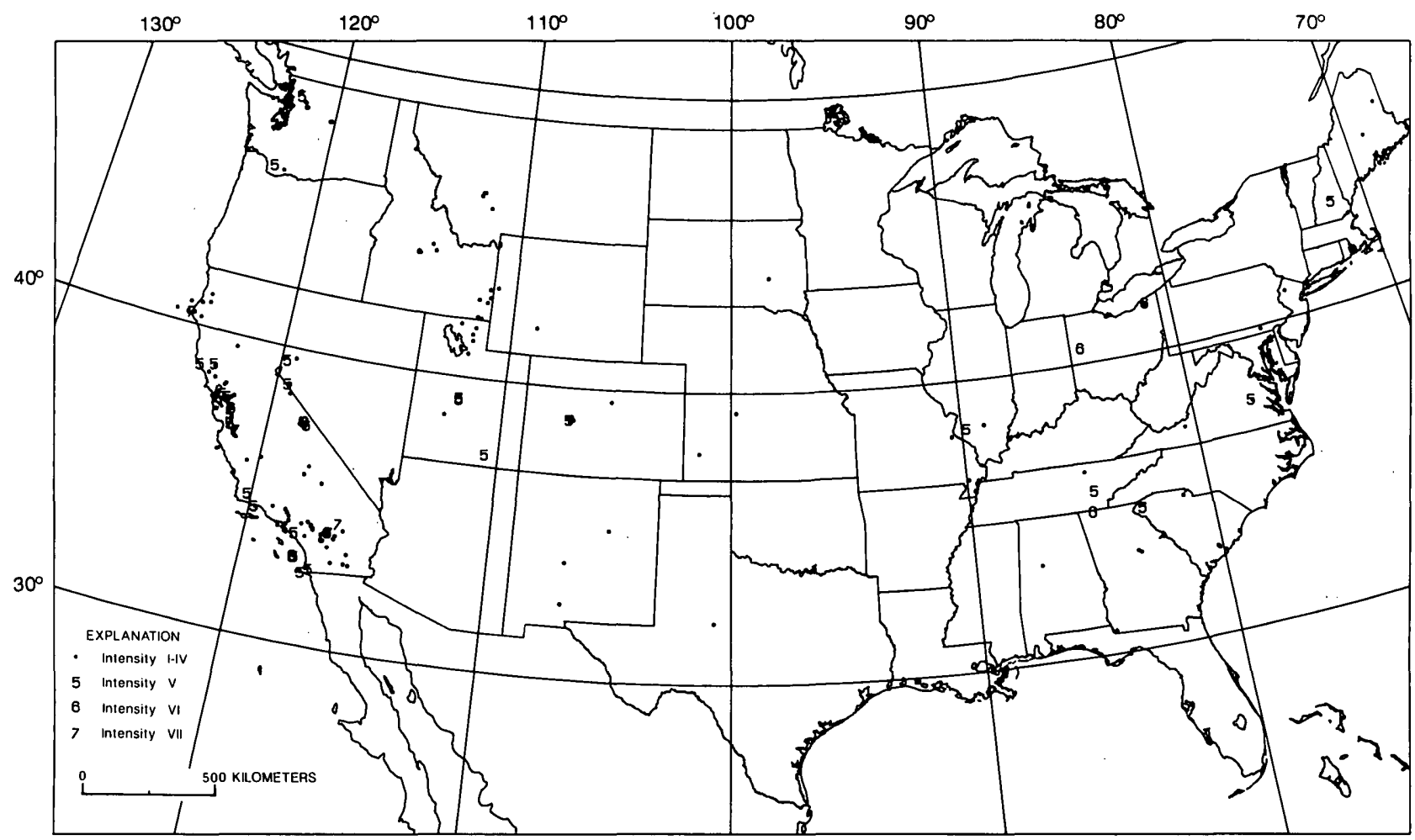

Figure 6. Earthquakes in the conterminous United States that were felt or caused damage in 1986. The maximum observed intensity for each earthquake is plotted at the epicenter.

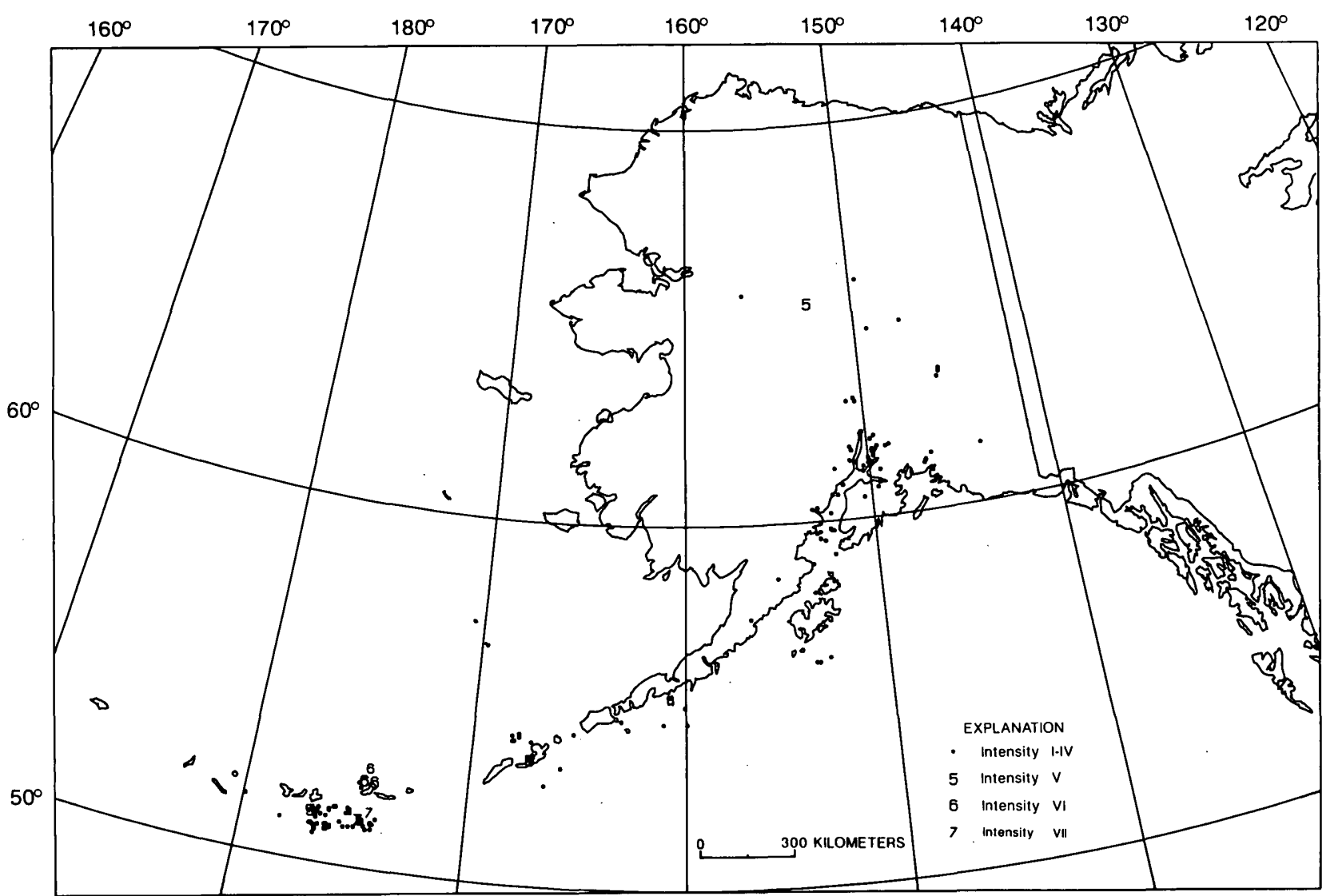

Figure 7. Earthquakes in Alaska that were felt or caused damage in 1986. The maximum observed intensity for each earthquake is plotted at the epicenter. 


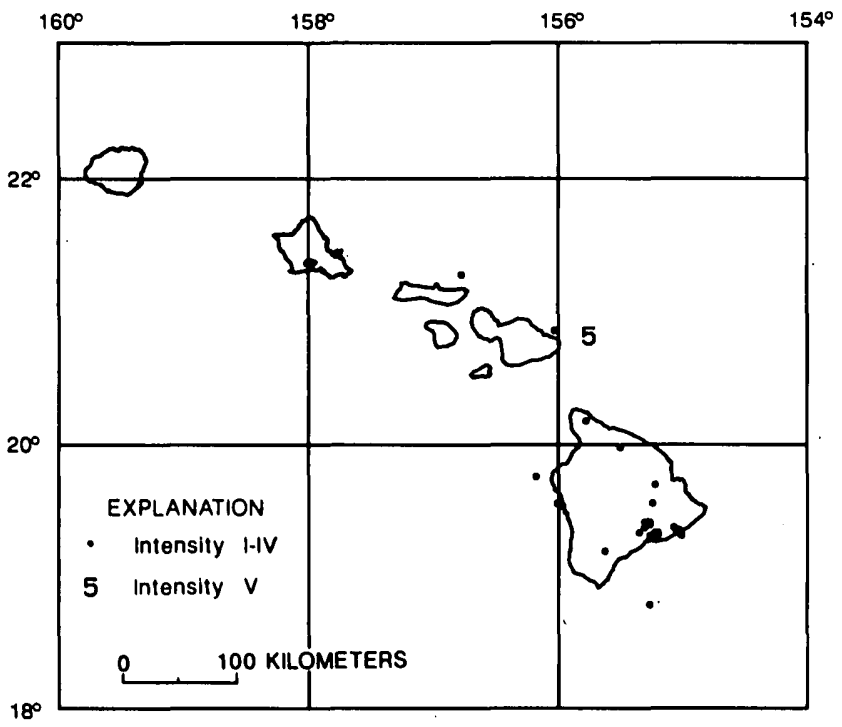

Figure 8. Earthquakes in Hawaii that were felt or caused damage in 1986. The maximum observed intensity for each earthquake is plotted at the epicenter.

that due to passing of light, or lightly loaded trucks, or heavy trucks some distance away. Hanging objects may swing slightly. Movements may be appreciable on upper levels of tall structures. Rocked standing motor cars slightly.

IV Felt indoors by many, outdoors by few. Awakened few, especially light sleepers. Frightened no one, unless apprehensive from previous experience. Vibration like that due to passing of heavy or heavily loaded trucks. Sensation like heavy body striking building, or falling of heavy objects inside. Rattling of dishes, windows, doors; glassware and crockery clink and clash. Creaking of walls, frame, especially in the upper range of this grade. Hanging objects swung, in numerous instances. Disturbed liquids in open vessels slightly. Rocked standing motor cars noticeably.

V Felt indoors by practically all, outdoors by many or most: outdoors direction estimated. Awakened many, or most. Frightened few-slight excitement, a few ran outdoors. Buildings trembled throughout. Broke dishes, glassware, to some extent. Cracked windows-in some cases, but not generally. Overturned vases, small or unstable objects, in many instances, with occasional fall. Hanging objects, doors, swing generally or considerably. Knocked pictures against walls, or swung them out of place. Opened, or closed, doors, shutters, abruptly. Pendulum clocks stopped, started, or ran fast, or slow. Moved small objects, furnishings, the latter to slight extent. Spilled liquids in small amounts from well filled open containers. Trees, bushes, shaken slightly.

VI Felt by all, indoors and outdoors. Frightened many, excitement general, some alarm, many ran outdoors. Awakened all. Persons made to move unsteadily.
Trees, bushes, shaken slightly to moderately. Liquid set in strong motion. Small bells rang-church, chapel, school, etc. Damage slight in poorly built buildings. Fall of plaster in small amount. Cracked plaster somewhat, especially fine cracks in chimneys in some instances. Broke dishes, glassware, in considerable quantity, also some windows. Fall of knickknacks, books, pictures. Overturned furniture in many instances. Moved furnishings of moderately heavy kind.

VII Frightened all. General alarm, all ran outdoors. Some, or many, found it difficult to stand. Noticed by persons driving motor cars. Trees and bushes shaken moderately to strongly. Waves on ponds, lakes, and running water. Water turbid from mud stirred up. Incaving to some extent of sand or gravel stream banks. Rang large church bells, etc. Suspended objects made to quiver. Damage negligible in buildings of good design and construction, slight to moderate in well-built ordinary buildings, considerable in poorly built or badly designed buildings, adobe houses, old walls (especially where laid up without mortar), spires, etc. Cracked chimneys to considerable extent, walls to some extent. Fall of plaster in considerable to large amount, also some stucco. Broke numerous windows, furniture to some extent. Shook down loosened brickwork and tiles. Broke weak chimneys at the roofline (sometimes damaging roofs). Fall of cornices from towers and high buildings. Dislodged bricks and stones. Overturned heavy furniture, with damage from breaking. Damage considerable to concrete irrigation ditches.

VIII Fright general. Alarm approaches panic. Disturbed persons driving motor cars. Trees shaken stronglybranches, trunks, broken off, especially palm trees. Ejected sand and mud in small amounts. Changes: temporary, permanent; in flow of springs and wells; dry wells renewed flow; in temperature of spring and well waters. Damage slight in structures (brick) built especially to withstand earthquakes. Considerable in ordinary substantial buildings, partial collapse: racked, tumbled down, wooden houses in some cases; threw out panel walls in frame structures, broke off decayed piling. Fall of walls. Cracked, broke, solid stone walls seriously. Wet ground to some extent, also ground on steep slopes. Twisting, fall, of chimneys, columns, monuments, also factory stacks, towers. Moved conspicuously, overturned, very heavy furniture.

IX Panic general. Cracked ground conspicuously. Damage considerable in (masonry) structures built especially to withstand earthquakes: threw out of plumb some wood-frame houses built especially to withstand earthquakes; great in substantial (masonry) buildings, some collapse in large part; or wholly shifted 
frame buildings off foundations, racked frames; serious to reservoirs; underground pipes sometimes broken.

$X$ Cracked ground, especially when loose and wet, up to widths of several inches; fissures up to a yard in width ran parallel to canal and stream banks. Landslides considerable from river banks and steep coasts. Shifted sand and mud horizontally on beaches and flat land. Changed level of water in wells. Threw water on banks of canals, lakes, rivers, etc. Damage serious to dams, dikes, embankments. Severe to well-built wooden structures and bridges, some destroyed. Developed dangerous cracks in excellent brick walls. Destroyed most masonry and frame structures, also their foundations. Bent railroad rails slightly. Tore apart, or crushed endwise, pipelines buried in earth. Open cracks and broad wavy folds in cement pavements and asphalt road surfaces.

XI Disturbances in ground many and widespread, varying with ground material. Broad fissures, earth slumps, and land slips in soft, wet ground. Ejected water in large amounts charged with sand and mud. Caused seawaves ("tidal" waves) of significant magnitude. Damage severe to wood-frame structures, especially near shock centers. Great to dams, dikes, embankments often for long distances. Few, if any (masonry) structures remained standing. Destroyed large well-built bridges by the wrecking of supporting piers, or pillars. Affected yielding wooden bridges less. Bent railroad rails greatly, and thrust them endwise. Put pipelines buried in earth completely out of service.

XII Damage total-Practically all works of construction damaged greatly or destroyed. Disturbances in ground great and varied, numerous shearing cracks. Landslides, falls of rock of significant character, slumping of river banks, etc., numerous and extensive. Wrenched loose, tore off, large rock masses. Fault slips in firm rock, with notable horizontal and vertical offset displacements. Water channels, surface and underground, disturbed and modified greatly. Dammed lakes, produced waterfalls, deflected rivers, etc. Waves seen on ground -surfaces (actually seen, probably, in some cases). Distorted lines of sight and level. Threw objects upward into the air.

\section{COLLABORATORS}

Active cooperation in earthquake investigations in the United States is provided by several seismological collaborators. The following served as collaborators to the USGS during 1986:
Alaska.-Staff of National Oceanic and Atmospheric Administration-Alaska Tsunami Warning Center, Palmer.

California (northern).--Robert A. Uhrhammer, University of California, Berkeley.

California (southern).-Clarence R. Allen and L.K. Hutton, California Institute of Technology, Pasadena.

Canada-Staff of Earth Physics Branch, Seismological Service of Canada, Ottawa.

Canada-Staff of Pacific Geoscience Centre, Sidney, British Columbia.

Connecticut.-Robert Miller, University of Connecticut, Groton.

Delaware.-Kenneth D. Woodruff, University of Delaware, Newark.

Florida and Georgia.-Leland T. Long, Georgia Institute of Technology, Atlanta.

Hawaii.-Robert Koyanagi, U.S. Geological Survey, Hawaiian Volcano Observatory, Hawaii National Park.

Indiana-Chi-Kin Lam, Department of Geology, Indiana University, Bloomington.

Kansas.-Don W. Steeples, Kansas Geological Survey, Lawrence.

Kentucky.-Ronald L. Street, University of Kentucky, Lexington.

Missouri, Illinois, Arkansas area-Robert B. Herrmann, Saint Louis University, Saint Louis.

Montana-Michael C. Stickney, Montana Bureau of Mines and Geology, Butte.

Nevada.-Arturo Aburto, University of Nevada, Reno.

New England.-James P. McCaffrey, S.J., Boston College, Weston, Mass.

New Mexico-Allan R. Sanford, New Mexico Institute of Mining and Technology, Socorro.

New York. - Lynn R. Sykes and J.J. Taber, LamontDoherty Geological Observatory, Palisades, N.Y.

Oklahoma._James E. Lawson, Jr., Oklahoma Geological Survey, Leonard.

Oregon.-Randy Jacobson, Oregon State University, Corvallis.

South Carolina.-Pradeep Talwani, University of South Carolina, Columbia, and Joyce Bagwell, Baptist College at Charleston, Charleston.

Tennessee.-Arch C. Johnston, Center for Earthquake Research and Information, Memphis State University, Memphis, Tenn.

Utah.-Ethan D. Brown, University of Utah, Salt Lake City.

Virginia.-G.A. Bollinger, Virginia Polytechnic Institute and State University, Blacksburg.

Washington.-Robert S. Crosson and Ruth S. Ludwin, University of Washington, Seattle.

Wyoming.-R.A. Hutchinson, National Park Service, Yellowstone National Park. 


\section{EARTHQUAKE DESCRIPTIONS}

All United States and Puerto Rico earthquakes that were reported felt in 1986 are listed in this section alphabetically by State and chronologically within each State. The origin time of each earthquake is given in Coordinated Universal Time (UTC). Time is expressed continuously from midnight to midnight, or 0 to 24 hours.

Sources of noninstrumental information (macroseismic data) in this publication include questionnaire canvasses conducted by the USGS, newspaper articles, bulletins of the Seismological Society of America, and special earthquake reports of other organizations. Instrumental data are provided by the USGS/NEIC, other government agencies, and universities that operate seismic networks.

Roman numerals in the earthquake descriptions refer to the Modified Mercalli Intensity Scale of 1931. Where more than one degree of earthquake intensity is reported from a town, the town is assigned the highest intensity reported. All earthquake questionnaires or press reports that do not contain enough detail from which to assign an intensity are listed as "Felt."

The following codes indicate sources for hypocenters, magnitudes, intensities, and (or) felt data:

(AK) Geophysical Institute, University of Alaska, College.

(BK) University of California, Berkeley.

(BU) Montana Bureau of Mines and Geology, Butte.

(DE) Delaware Geological Survey, Newark.

(EE) Engdahl, E. R., Billington, S., and Kisslinger, C., 1989, Journal of Geophysical Research, v. 94, no. B11, p. $15,481-15,498$.

(EN) Department of Energy, Washington, D.C.

(EP) Geophysics Division, Geological Survey of Canada, Ottawa, Ontario.

(GM) U.S. Geological Survey, Menlo Park, Calif.

(GP) U.S. Geological Survey, Pasadena, Calif.

(GS) U.S. Geological Survey, Golden, Colo.

(GT) Georgia Institute of Technology, Atlanta.

(HJ) Hauksson, Egill, and Jones, L.M., 1988, Seismological Society of America Bulletin, v. 78, no. 6, p. 1885-1906.

(HR) Harvard University, Cambridge, Mass.

(HV) Hawaiian Volcano Observatory, U.S. Geological Survey, Hawaii Volcanoes National Park.

(KS) Kansas Geological Survey, Lawrence.
(LD) Lamont-Doherty Geological Observatory, Palisades, N.Y.

(NI) Nicholson and others, 1988, Seismological Society of America Bulletin, v. 78, no. 1, p. 188-217.

(PG) Pacific Geoscience Centre, Sydney, B.C., Canada.

(PM) Alaska Palmer Observatory, National Oceanic and Atmospheric Administration, Palmer, Alaska.

(PS) California Institute of Technology, Pasadena.

(RN) University of Nevada, Reno.

(SC) University of South Carolina, Columbia.

(SL) St. Louis University, St. Louis, Mo.

(SZ) Schwartz, S.Y., and Christensen, D.H., 1988, Eastern Section, Seismological Society of America, Seismological Research Letters, v. 59, no. 2, p. 57-62.

(TC) Tennessee Earthquake Information Center, Memphis.

(TU) Oklahoma Geological Survey, Leonard.

(UU) University of Utah, Salt Lake City.

(VP) Virginia Polytechnic Institute and State University, Blacksburg.

(WA) University of Washington, Seattle.

(WO) Weston Observatory, Weston, Mass.

Depth: Normal. Depth was assumed to be $33 \mathrm{~km}$.

\section{ALABAMA}

7 May (GT) Central Alabama

Origin time: 022700.4

Epicenter: $33.335 N$., 87.347W.

Depth: $1 \mathrm{~km}$

Magnitude: $4.2 \mathrm{~m}_{\mathrm{b}}(\mathrm{GS}), 4.5 \mathrm{M}_{\mathrm{n}}(\mathrm{GS}), 4.0 \mathrm{M}_{\mathrm{n}}(T U)$

Felt: Tuscaloosa County. Mine collapse (GT).

\section{ALASKA}

14 January (GM) Southern Alaska

Origin time: 082013.9

Epicenter: 60.222N., 152.294W.

Depth: $81 \mathrm{~km}$

Magnitude: $3.7 \mathrm{M}_{\mathrm{L}}(\mathrm{PM})$

Intensity II: -Homer (PM).

\section{January (GM) Southern Alaska}

Origin time: 042953.2

Epicenter: 59.538N., 152.907W.

Depth: $91 \mathrm{~km}$ 
Magnitude: $3.7 \mathrm{M}_{\mathrm{L}}(\mathrm{PM})$

Intensity II: Homer (PM).

16 January (GM) Southern Alaska

Origin time: 143636.1

Epicenter: 61.489N., 146.537W.

Depth: $28 \mathrm{~km}$

Magnitude: $4.0 \mathrm{M}_{\mathrm{L}}(\mathrm{PM})$

Intensity IV: Valdez.

Felt: Anchorage (press report), Cordova (press report), Palmer (press report).

18 January (GM) Southern Alaska

Origin time: 043629.3

Epicenter: 61.542N., 150.996W.

Depth: $75 \mathrm{~km}$

Magnitude: $3.0 \mathrm{M}_{\mathrm{L}}(\mathrm{PM})$

Felt: Anchorage (PM).

19 January (GM) Southern Alaska

Origin time: 101147.1

Epicenter: 59.729N., 152.308W.

Depth: $63 \mathrm{~km}$

Magnitude: $4.2 \mathrm{M}_{\mathrm{L}}(\mathrm{PM})$

Felt: Homer (PM).

\section{January (GM) Southern Alaska}

Origin time: 231154.1

Epicenter: 61.869N., 148.738W.

Depth: $38 \mathrm{~km}$

Magnitude: $4.1 \mathrm{~m}_{\mathrm{b}}(\mathrm{GS}), 3.6 \mathrm{M}_{\mathrm{L}}(\mathrm{PM})$

Intensity II: Palmer (PM).

13 February (LD) Alaska Peninsula

Origin time: 084309.3

Epicenter: 54.655N., 159.998W.

Depth: $5 \mathrm{~km}$

Magnitude: $5.0 \mathrm{~m}_{\mathrm{b}}(\mathrm{GS}), 4.6 \mathrm{MS}_{\mathrm{S}}(\mathrm{GS}), 4.6 \mathrm{M}_{\mathrm{L}}(\mathrm{PM})$,

$$
4.5 \mathrm{~m}_{\mathrm{x}}(\mathrm{LD})
$$

Intensity IV: Sand Point.

Intensity III: False Pass.

Intensity II: King Cove.

14 February (GS) Central Alaska

Origin time: 124633.9

Epicenter: 63.069N., 150.835W.

Depth: $97 \mathrm{~km}$

Magnitude: None computed

Felt: Talkeetna (PM).

14 February (GS) Central Alaska

Origin time: 190129.5

Epicenter: 64.967N., 147.248W.
Depth: $10 \mathrm{~km}$

Magnitude: $3.2 \mathrm{M}_{\mathrm{L}}(\mathrm{PM})$

Felt: Fairbanks area (press report).

16 February (GM) Southern Alaska

Origin time: 213106.1

Epicenter: 61.495N., 150.711W.

Depth: $63 \mathrm{~km}$

Magnitude: $3.7 \mathrm{M}_{\mathrm{L}}(\mathrm{PM})$

Felt: Anchorage (PM).

24 February (GS) Central Alaska

Origin time: 185514.8

Epicenter: 63.075N., 150.383W.

Depth: $112 \mathrm{~km}$

Magnitude: $4.6 \mathrm{~m}_{\mathrm{b}}(\mathrm{GS})$

Intensity II: Gold Creek (PM).

28 February (GS) Alaska Peninsula

Origin time: 151224.1

Epicenter: 57.474N., 156.696W.

Depth: Normal

Magnitude: $4.1 \mathrm{~m}_{\mathrm{b}}(\mathrm{GS}), 4.1 \mathrm{M}_{\mathrm{L}}(\mathrm{PM})$

Felt: King Salmon (PM).

28 February (GS) Southern Alaska

Origin time: 170145.3

Epicenter: $60.345 \mathrm{~N} ., 152.966 \mathrm{~W}$.

Depth: $125 \mathrm{~km}$

Magnitude: $4.7 \mathrm{~m}_{\mathrm{b}}(\mathrm{GS})$

Intensity III: Homer (PM).

2 March (EE) Andreanof Islands, Aleutian Islands

Origin time: 204229.1

Epicenter: 51.373N., 176.714W.

Depth: $36 \mathrm{~km}$

Magnitude: $5.1 \mathrm{~m}_{b}(\mathrm{GS}), 4.4 \mathrm{MS}_{\mathrm{S}}(\mathrm{GS}), 5.2 \mathrm{M}_{\mathrm{L}}(\mathrm{PM})$

Felt: Adak Island (PM).

8 March (GS) Southern Alaska

Origin time: 053751.3

Epicenter: 60.673N., 151.843W.

Depth: $83 \mathrm{~km}$

Magnitude: $4.3 \mathrm{~m}_{\mathrm{b}}(\mathrm{GS})$

Felt: Anchorage (PM), Kenai (PM).

8 March (GM) Southern Alaska

Origin time: 173314.5

Epicenter: $61.038 \mathrm{~N} ., 146.566 \mathrm{~W}$.

Depth: $9 \mathrm{~km}$

Magnitude: $4.0 \mathrm{M}_{\mathrm{L}}(\mathrm{PM})$

Felt: Valdez (PM). 
9 March (GS) Fox Islands, Aleutian Islands

Origin time: 134928.2

Epicenter: $54.256 \mathrm{~N} ., 167.864 \mathrm{~W}$.

Depth: Normal

Magnitude: $5.2 \mathrm{~m}_{\mathrm{b}}(\mathrm{GS}), 5.5 \mathrm{MS}_{\mathrm{S}}(\mathrm{GS}), 5.5 \mathrm{M}_{\mathrm{S}}(\mathrm{BK})$,

Moment: $4.5 \times 10^{24}$ dyne-cm (HR)

Felt: Dutch Harbor (PM), Unalaska (PM).

12 March (GS) Central Alaska

Origin time: 024803.9

Epicenter: 64.882N., 149.197W.

Depth: Normal

Magnitude: $3.7 \mathrm{M}_{\mathrm{L}}(\mathrm{PM})$

Intensity III: Nenana (press report), Fairbanks (press report).

18 March (GS) Fox Islands, Aleutian Islands

Origin time: 161230.2

Epicenter: 54.028N., 168.070W.

Depth: Normal

Magnitude: $4.7 \mathrm{~m}_{\mathrm{b}}(\mathrm{GS}), 4.5 \mathrm{M}_{\mathrm{L}}(\mathrm{PM})$

Intensity III: Unalaska (PM).

20 March (GS) Fox Islands, Aleutian Islands

Origin time: 194008.8

Epicenter: 54.202N., 168.187W.

Depth: Normal

Magnitude: $4.8 \mathrm{~m}_{\mathrm{b}}(\mathrm{GS}), 4.3 \mathrm{M}_{\mathrm{S}}(\mathrm{GS})$, $5.0 \mathrm{M}_{\mathrm{L}}(\mathrm{PM})$

Felt: Unalaska (PM).

22 March (GM) Southern Alaska

Origin time: 053001.9

Epicenter: 60.348N., 153.297W.

Depth: $165 \mathrm{~km}$

Magnitude: $4.4 \mathrm{~m}_{\mathrm{b}}(\mathrm{GS})$

Felt: Homer (PM).

22 March (GM) Southern Alaska

Origin time: 214548.9

Epicenter: $61.217 \mathrm{~N}$., $150.382 \mathrm{~W}$.

Depth: $16 \mathrm{~km}$

Magnitude: $3.5 \mathrm{M}_{\mathrm{L}}(\mathrm{PM})$

Felt: Anchorage (PM).

30 March (GS) Andreanof Islands, Aleutian Islands

Origin time: 181329.3

Epicenter: 51.537N., 179.940W.

Depth: Normal

Magnitude: $4.8 \mathrm{~m}_{\mathrm{b}}(\mathrm{GS}), 3.9 \mathrm{M}_{\mathrm{L}}(\mathrm{PM})$

Felt: Adak Island (PM).

1 April (GM) Southern Alaska

Origin time: 202634.4

\section{ALASKA-Continued}

Epicenter: 61.558N., 149.984W.

Depth: $46 \mathrm{~km}$

Magnitude: $3.6 \mathrm{M}_{\mathrm{L}}(\mathrm{PM})$

Intensity II: Butte (PM), Palmer (PM).

1 April (GM) Southern Alaska

Origin time: 234651.4

Epicenter: $61.886 \mathrm{~N} ., 150.937 \mathrm{~W}$.

Depth: $72 \mathrm{~km}$

Magnitude: $3.9 \mathrm{~m}_{\mathrm{b}}(\mathrm{GS}), 4.0 \mathrm{M}_{\mathrm{L}}(\mathrm{PM})$

Intensity II: Anchorage (PM), Butte (PM), Palmer (PM).

3 April (GS) Southern Alaska

Origin time: 100236.8

Epicenter: 61.449N., 150.039W.

Depth: $45 \mathrm{~km}$

Magnitude: $3.0 \mathrm{M}_{\mathrm{L}}(\mathrm{PM})$

Felt: Anchorage (PM).

3 April (GS) Central Alaska

Origin time: 211147.7

Epicenter: 63.634N., 145.435W.

Depth: Normal

Magnitude: $3.7 \mathrm{M}_{\mathrm{L}}(\mathrm{PM})$

Felt: Richardson Highway, Mile 221 Marker (PM).

3 April (GS) Central Alaska

Origin time: 211449.4

Epicenter: 63.550N., 145.411W.

Depth: Normal

Magnitude: $2.8 \mathrm{M}_{\mathrm{L}}(\mathrm{PM})$

Felt: Richardson Highway, Mile 221 Marker (PM).

3 April (GS) Central Alaska

Origin time: 211655.0

Epicenter: 63.444N., 145.564W.

Depth: Normal

Magnitude: $3.2 \mathrm{M}_{\mathrm{L}}(\mathrm{PM})$

Felt: Richardson Highway, Mile 221 Marker (PM).

7 April (GS) Fox Islands, Aleutian Islands

Origin time: 170746.8

Epicenter: 54.058N., 167.343W.

Depth: Normal

Magnitude: $4.0 \mathrm{~m}_{\mathrm{b}}(\mathrm{GS}), 4.4 \mathrm{M}_{\mathrm{L}}(\mathrm{PM})$

Intensity II: Unalaska (PM).

8 April (GS) Fox Islands, Aleutian Islands

Origin time: 063648.0

Epicenter: 54.048 N., 168.156W.

Depth: Normal

Magnitude: $4.4 \mathrm{~m}_{\mathrm{b}}(\mathrm{GS}), 4.6 \mathrm{M}_{\mathrm{L}}(\mathrm{PM})$

Intensity III: Unalaska (PM). 


\section{ALASKA-Continued}

11 April (GS) Fox Islands, Aleutian Islands

Origin time: 172220.8

Epicenter: 54.164 N., 167.883W.

Depth: Normal

Magnitude: $5.3 \mathrm{~m}_{\mathrm{b}}(\mathrm{GS}), 5.9 \mathrm{MS}(\mathrm{GS}), 6.0 \mathrm{MS}_{\mathrm{S}}(\mathrm{BK})$

Moment: $1.1 \times 10^{25}$ (HR)

Intensity IV: Unalaska.

27 April (GM) Southern Alaska

Origin time: 105541.2

Epicenter: $59.723 \mathrm{~N} ., 152.918 \mathrm{~W}$.

Depth: $96 \mathrm{~km}$

Magnitude: $4.0 \mathrm{~m}_{\mathrm{b}}(\mathrm{GS}), 4.0 \mathrm{M}_{\mathrm{L}}(\mathrm{PM})$

Felt: Homer (PM).

28 April (GM) Southern Alaska

Origin time: 073256.1

Epicenter: 61.498N., 149.893W.

Depth: $59 \mathrm{~km}$

Magnitude: $3.5 \mathrm{M}_{\mathrm{L}}(\mathrm{PM})$

Intensity III: Anchorage (PM).

Intensity II: Palmer (PM).

2 May (GM) Southern Alaska

Origin time: 005157.7

Epicenter: 61.238N., 149.410W.

Depth: $35 \mathrm{~km}$

Magnitude: $2.1 \mathrm{M}_{\mathrm{L}}(\mathrm{PM})$

Felt: Anchorage (PM).

\section{May (GM) Southern Alaska}

Origin time: 101113.6

Epicenter: $61.426 \mathrm{~N} ., 149.859 \mathrm{~W}$.

Depth: $46 \mathrm{~km}$

Magnitude: $3.2 \mathrm{M}_{\mathrm{L}}(\mathrm{PM})$

Felt: Anchorage (PM).

7 May (EE) Andreanof Islands, Aleutian Islands

Origin time: 204333.3

Epicenter: 51.234N., 174.741W.

Depth: $25 \mathrm{~km}$

Magnitude: $6.1 \mathrm{~m}_{\mathrm{b}}(\mathrm{GS}), 6.0 \mathrm{M}_{\mathrm{S}}(\mathrm{GS}), 6.1 \mathrm{M}_{\mathrm{L}}(\mathrm{PM})$, $6.1 \mathrm{M}_{\mathrm{S}}(\mathrm{BK})$

Moment: $2.7 \times 10^{25}$ dyne-cm (HR)

Felt: Adak Island.

7 May (EE) Andreanof Islands, Aleutian Islands

Origin time: 224712.3

Epicenter: 51.325N., 174.751W.

Depth: $31 \mathrm{~km}$

Magnitude: $6.4 \mathrm{~m}_{\mathrm{b}}(\mathrm{GS}), 7.7 \mathrm{M}_{\mathrm{S}}(\mathrm{GS}), 7.9 \mathrm{M}_{\mathrm{S}}(\mathrm{BK})$

Moment: $1.0 \times 10^{28}$ dyne-cm (HR)

\section{ALASKA-Continued}

This earthquake is the largest to occur in Alaska since the February 4, 1965, Rat Islands earthquake. It caused moderate damage to structures on Adak Island and minor damage on Atka Island. The building damage consisted of cracked masonry and concrete walls, failure of partitions and suspended ceilings, spalling on concrete beams and concrete piers, and failure of piping (Glick and others, 1986).

Some houses experienced differential foundation settlements of several $\mathrm{cm}$, causing considerable damage to the structures (Glick and others, 1986).

Sand boils were noted in one localized area where an antenna array was located. One antenna foundation settled about $3 \mathrm{ft}$, and others were out of plumb. Soil liquefaction was noted in localized areas of back-filled soil. Lateral spreading cracks of about 8 to $20 \mathrm{~cm}$ and differential ground settlement occurred along a small wharf. Some slumping was observed on a side slope of the approach to a bridge. Rock falls occurred at two quarry sites, and snow avalanches were observed in steep mountain regions (Glick and others, 1986).

Horizontal peak ground accelerations between 0.20 $g$ and $0.25 g$ were recorded on the foundation slab near the AIMO hanger, and horizontal accelerations greater than $0.60 \mathrm{~g}$ were recorded at the bottom chord of a steel roof truss in the same area (Glick and others, 1986).

This earthquake also caused a tsunami that was recorded throughout the Pacific Ocean. In Alaska, Adak Island stations recorded wave heights of $175 \mathrm{~cm}$, Unalaska $20 \mathrm{~cm}$, and Sand Point $10 \mathrm{~cm}$. In Hawaii the heights ranged from 91 to $122 \mathrm{~cm}$ at Kapaa, Kauai, to $8 \mathrm{~cm}$ at Kailua-Kona, Hawaii. Wave heights were also measured in Japan, along the west coast of South America, and on many islands of the southern Pacific Ocean.

Intensity VII:

Adak Island-The AIMO hanger (two-story shop area) had many vertical and diagonal cracks in the masonry walls and in the stairwell area. The Navy Exchange (a precast-concrete structure) had permanent vertical offset, loose bolts, and bent connectors between adjacent precast roof panels. One concrete pier spalled and cracked near an expansion joint. Wood-frame housing units sustained minor interior wall cracking and differential settlement of as much as $13 \mathrm{~cm}$. File cabinets were overturned; windows were cracked; power was disrupted; and many items were displaced and overturned (Glick and others, 1986). 


\section{ALASKA-Continued}

Intensity VI:

Atka Island-Some parts of the airstrip "dropped" $30 \mathrm{~cm}$, and there were cracks in the strip. Landslides and slumping of road fill occurred. Standing and moving vehicles rocked moderately; plaster walls sustained hairline cracks; much merchandise was thrown off store shelves; small appliances overturned; a few windows cracked; many glassware items or dishes broke; many small objects overturned and fell; hanging pictures fell; buildings shook strongly; people had difficulty standing or walking; felt by and frightened everyone.

9 May (EE) Andreanof Islands, Aleutian Islands Origin time: 010531.2

Epicenter: 51.061N., 176.902W.

Depth: $23 \mathrm{~km}$

Magnitude: $5.5 \mathrm{~m}_{\mathrm{b}}(\mathrm{GS}), 5.6 \mathrm{M}_{\mathrm{S}}(\mathrm{GS}), 5.4 \mathrm{M}_{\mathrm{L}}(\mathrm{PM})$, $5.7 \mathrm{M}_{\mathrm{S}}(\mathrm{PS})$

Intensity III: Adak (PM).

9 May (EE) Andreanof Islands, Aleutian Islands

Origin time: 010810.5

Epicenter: $51.062 \mathrm{~N} ., 176.856 \mathrm{~W}$.

Depth: $18 \mathrm{~km}$

Magnitude: $5.6 \mathrm{~m}_{\mathrm{b}}(\mathrm{GS}), 5.5 \mathrm{MS}_{\mathrm{S}}(\mathrm{GS}), 5.5 \mathrm{M}_{\mathrm{L}}(\mathrm{PM})$

Intensity IV: Adak Island.

9 May (GS) Fox Islands, Aleutian Islands

Origin time: 182249.8

Epicenter: 54.324N., 165.372W.

Depth: $101 \mathrm{~km}$

Magnitude: $4.6 \mathrm{~m}_{\mathrm{b}}(\mathrm{GS})$

Felt: Cold Bay (PM), Sand Point (PM).

11 May (EE) Andreanof Islands, Aleutian Islands

Origin time: 104621.8

Epicenter: 50.970N., 176.095W.

Depth: $16 \mathrm{~km}$

Magnitude: $5.0 \mathrm{~m}_{\mathrm{b}}(\mathrm{GS}), 4.7 \mathrm{MS}_{\mathrm{S}}(\mathrm{GS}), 4.9 \mathrm{M}_{\mathrm{L}}(\mathrm{PM})$

Moment: $1.0 \times 10^{24}$ dyne-cm (HR)

Intensity II: Adak Island (PM).

11 May (EE) Andreanof Islands, Aleutian Islands

Origin time: 224847.2

Epicenter: 51.371N., 174.616W.

Depth: $29 \mathrm{~km}$

Magnitude: $5.5 \mathrm{~m}_{\mathrm{b}}(\mathrm{GS}), 5.2 \mathrm{M}_{\mathrm{S}}(\mathrm{GS}), 5.7 \mathrm{M}_{\mathrm{L}}(\mathrm{PM})$, $5.2 \mathrm{MS}(\mathrm{BK})$

Moment: $3.6 \times 10^{24}$ dyne-cm (HR)

Intensity IV: Adak Island (PM).

\section{ALASKA-Continued}

12 May (GS) Andreanof Islands, Aleutian Islands

Origin time: 201645.8

Epicenter: 52.700 N., $172.432 \mathrm{E}$.

Depth: Normal

Magnitude: $4.9 \mathrm{~m}_{\mathrm{b}}(\mathrm{GS}), 5.1 \mathrm{M}_{\mathrm{S}}(\mathrm{GS})$

Intensity III: Attu Island (PM).

15 May (EE) Andreanof Islands, Aleutian Islands

Origin time: 063837.9

Epicenter: 52.432N., 174.719W.

Depth: $15 \mathrm{~km}$

Magnitude: $5.7 \mathrm{~m}_{\mathrm{b}}(\mathrm{GS}), 6.4 \mathrm{M}_{\mathrm{S}}(\mathrm{GS}), 5.5 \mathrm{M}_{\mathrm{L}}(\mathrm{PM})$,

$$
6.4 \mathrm{M}_{\mathrm{S}}(\mathrm{BK})
$$

Moment: $4.4 \times 10^{25}$ dyne-cm (HR)

Intensity VI:

Atka Island-Underground pipes broke; there was slight damage to bridges; standing and moving vehicles rocked moderately; interior walls sustained hairline cracks; many items were shaken off store shelves; furniture was overturned; many glassware items or dishes broke; many small objects overturned and fell; hanging pictures fell; a few windows cracked; buildings shook strongly; people had difficulty standing and walking; felt by everyone.

Intensity II: False Pass (PM).

Felt: Adak Island.

15 May (LD) Alaska Peninsula

Origin time: 232113.2

Epicenter: 54.693N., 163.089W.

Depth: $3 \mathbf{~ k m}$

Magnitude: $4.5 \mathrm{~m}_{\mathrm{b}}(\mathrm{GS}), 4.4 \mathrm{~m}_{\mathrm{x}}(\mathrm{LD})$

Felt: Cold Bay (PM), False Pass (PM), Sand Point (PM).

17 May (GM) Southern Alaska

Origin time: 063114.2

Epicenter: 62.038N., 149.730W.

Depth: $51 \mathrm{~km}$

Magnitude: $2.6 \mathrm{M}_{\mathrm{L}}(\mathrm{PM})$

Felt: Anchorage (PM).

17 May (EE) Andreanof Islands, Aleutian Islands

Origin time: 162024.3

Epicenter: 52.443N., 174.271W.

Depth: $15 \mathrm{~km}$

Magnitude: $5.8 \mathrm{~m}_{\mathrm{b}}(\mathrm{GS}), 6.6 \mathrm{M}_{\mathrm{S}}(\mathrm{GS}), 6.5 \mathrm{MS}_{\mathrm{S}}(\mathrm{BK})$

Moment: $4.7 \times 10^{25}$ dyne-cm (GS)

Intensity VI:

Atka Island-Airstrip damaged and officially closed. Underground pipes broke; landslides occurred; standing vehicles rocked moderately; trees and bushes shook strongly; plaster walls cracked; furniture overturned; many items were shaken off store shelves; many glassware items or dishes broke; 


\section{ALASKA-Continued}

many small objects overturned and fell; a few windows cracked; hanging pictures fell; buildings shook strongly; people had difficulty standing or walking; felt by and frightened everyone.

Felt: Adak Island.

19 May (EE) Andreanof Islands, Aleutian Islands

Origin time: 023734.7

Epicenter: $52.359 \mathrm{~N} ., 174.955 \mathrm{~W}$.

Depth: $15 \mathrm{~km}$

Magnitude: $5.1 \mathrm{~m}_{\mathrm{b}}(\mathrm{GS}), 5.2 \mathrm{MS}_{\mathrm{S}}(\mathrm{GS}), 4.9 \mathrm{M}_{\mathrm{L}}(\mathrm{PM})$, $5.0 \mathrm{M}_{\mathrm{S}}(\mathrm{BK})$

Moment: $1.3 \times 10^{24}$ dyne-cm (HR)

Intensity II: Adak Island (PM), Atka Island (PM).

21 May (EE) Andreanof Islands, Aleutian Islands Origin time: 070515.7

Epicenter: 51.351N., 176.214W.

Depth: $33 \mathrm{~km}$

Magnitude: $4.6 \mathrm{~m}_{\mathrm{b}}(\mathrm{GS}), 4.4 \mathrm{M}_{\mathrm{L}}(\mathrm{PM})$

Intensity III: Adak Island (PM).

21 May (EE) Andreanof Islands, Aleutian Islands Origin time: 221220.9

Epicenter: 51.647N., 175.325W.

Depth: $46 \mathrm{~km}$

Magnitude: $4.8 \mathrm{~m}_{\mathrm{b}}(\mathrm{GS}), 4.6 \mathrm{M}_{\mathrm{L}}(\mathrm{PM})$

Intensity II: Adak Island (PM).

23 May (GS) Southern Alaska

Origin time: 231842.2

Epicenter: 58.906N., 153.377W.

Depth: $80 \mathrm{~km}$

Magnitude: $5.0 \mathrm{~m}_{b}(\mathrm{GS})$,

Moment: $5.5 \times 10^{23}$ dyne-cm (HR)

Intensity III: Homer (PM), Kodiak (PM).

Felt: Chiniak (press report).

29 May (GS) Southern Alaska

Origin time: 024011.2

Epicenter: 59.119N., 152.163W.

Depth: $61 \mathrm{~km}$

Magnitude: $4.5 \mathrm{~m}_{\mathrm{b}}(\mathrm{GS}), 4.2 \mathrm{M}_{\mathrm{L}}(\mathrm{PM})$

Felt: Homer (PM), Seldovia (PM).

29 May (EE) Andreanof Islands, Aleutian Islands

Origin time: 191846.3

Epicenter: $51.464 \mathrm{~N} ., 175.289 \mathrm{~W}$.

Depth: $35 \mathrm{~km}$

Magnitude: $4.9 \mathrm{~m}_{\mathrm{b}}(\mathrm{GS}), 4.7 \mathrm{M}_{\mathrm{L}}(\mathrm{PM})$

Felt: Adak Island (PM), Atka Island (PM).

3 June (EE) Andreanof Islands, Aleutian Islands Origin time: 230528.8

\section{ALASKA-Continued}

Epicenter: 51.256N., 174.631W.

Depth: $20 \mathrm{~km}$

Magnitude: $5.4 \mathrm{~m}_{\mathrm{b}}(\mathrm{GS}), 5.1 \mathrm{M}_{\mathrm{S}}(\mathrm{GS}), 5.8 \mathrm{M}_{\mathrm{L}}(\mathrm{PM})$, $5.1 \mathrm{M}_{\mathrm{S}}(\mathrm{BK})$

Intensity II: Adak Island (PM).

\section{June (GS) Central Alaska}

Origin time: 154820.8

Epicenter: $65.63 \mathrm{~N} ., 152.604 \mathrm{~W}$.

Depth: $10 \mathrm{~km}$

Magnitude: $5.2 \mathrm{~m}_{\mathrm{b}}(\mathrm{GS}), 4.7 \mathrm{M}_{\mathrm{S}}(\mathrm{GS}), 5.7 \mathrm{M}_{\mathrm{L}}(\mathrm{PM})$

Intensity V:

Tanana-A few items were shaken off store shelves; a few small objects overturned and fell; standing and moving vehicles rocked slightly; buildings shook moderately, felt by and frightened many.

Intensity IV: Galena Air Force Station, Hughes, Huslia, Indian Mountain Air Force Station, Lake Minchumina, Manley Hot Springs, Rampart.

Intensity III: Bettles, College, Fairbanks, Livingood (5 mi west), Minto, Ruby, Shungnak.

Felt: North Pole (press report).

4 June (EE) Andreanof Islands, Aleutian Islands Origin time: 192543.3

Epicenter: 51.287N., 174.580W.

Depth: $20 \mathrm{~km}$

Magnitude: $4.9 \mathrm{~m}_{\mathrm{b}}(\mathrm{GS}), 4.0 \mathrm{M}_{\mathrm{L}}(\mathrm{PM})$

Intensity II: Adak Island (PM).

5 June (GS) Andreanof Islands, Aleutian Islands Origin time: 142204.7

Epicenter: 51.093N., 174.341W.

Depth: Normal

Magnitude: $4.5 \mathrm{~m}_{\mathrm{b}}(\mathrm{GS}), 4.1 \mathrm{M}_{\mathrm{L}}(\mathrm{PM})$

Felt: Adak Island (PM).

5 June (EE) Andreanof Islands, Aleutian Islands Origin time: 152453.7

Epicenter: $51.138 \mathrm{~N}$., 174.177W.

Depth: $20 \mathrm{~km}$

Magnitude: $4.7 \mathrm{~m}_{\mathrm{b}}(\mathrm{GS}), 4.2 \mathrm{M}_{\mathrm{L}}(\mathrm{PM})$

Felt: Adak Island (PM).

5 June (EE) Andreanof Islands, Aleutian Islands Origin time: 173240.6

Epicenter: $51.094 \mathrm{~N} ., 175.350 \mathrm{~W}$.

Depth: $20 \mathrm{~km}$

Magnitude: $5.1 \mathrm{~m}_{\mathrm{b}}(\mathrm{GS}), 4.5 \mathrm{M}_{\mathrm{L}}(\mathrm{PM})$

Felt: Adak Island (PM).

5 June (EE) Andreanof Islands, Aleutian Islands Origin time: 202703.2

Epicenter: 51.296N., 174.210W. 


\section{ALASKA-Continued}

Depth: $22 \mathrm{~km}$

Magnitude: $5.4 \mathrm{~m}_{\mathrm{b}}(\mathrm{GS}), 4.8 \mathrm{MS}_{\mathrm{S}}(\mathrm{GS}), 4.5 \mathrm{M}_{\mathrm{L}}(\mathrm{PM})$, $4.8 \mathrm{Ms}(\mathrm{BK})$

Moment: $1.0 \times 10^{24}$ dyne-cm (HR)

Felt: Adak Island (PM).

15 June (GS) Andreanof Islands, Aleutian Islands Origin time: 022252.9

Epicenter: 51.396N., 174.760W.

Depth: Normal

Magnitude: $4.3 \mathrm{~m}_{\mathrm{b}}(\mathrm{GS}), 4.3 \mathrm{M}_{\mathrm{L}}(\mathrm{PM})$

Intensity III: Adak Island (PM).

16 June (GM) Southern Alaska

Origin time: 215402.0

Epicenter: 61.838N., 149.433W.

Depth: $43 \mathrm{~km}$

Magnitude: $3.8 \mathrm{M}_{\mathrm{L}}(\mathrm{PM})$

Intensity III: Hatcher Pass (PM).

Intensity II: Willow (PM).

18 June (EE) Andreanof Islands, Aleutian Islands

Origin time: 080516.4

Epicenter: 51.465N., 176.833W.

Depth: $41 \mathrm{~km}$

Magnitude: $5.8 \mathrm{~m}_{\mathrm{b}}(\mathrm{GS}), 6.3 \mathrm{M}_{\mathrm{S}}(\mathrm{GS}), 6.0 \mathrm{M}_{\mathrm{L}}(\mathrm{PM})$,

$$
6.4 \mathrm{Ms}(\mathrm{BK})
$$

Moment: $5.8 \times 10^{25}$ dyne-cm (HR)

Intensity IV: Adak Island (PM).

Intensity III: Atka (PM).

18 June (GS) Central Alaska

Origin time: 234304.4

Epicenter: 63.067N., 150.911W.

Depth: $131 \mathrm{~km}$

Magnitude: None computed

Felt: Talkeetna (PM).

19 June (GS) Southern Alaska

Origin time: 090909.2

Epicenter: 56.331N., 152.914W.

Depth: $17 \mathrm{~km}$

Magnitude: $6.0 \mathrm{~m}_{\mathrm{b}}(\mathrm{GS}), 6.3 \mathrm{M}_{\mathrm{S}}(\mathrm{GS}), 5.4 \mathrm{M}_{\mathrm{L}}(\mathrm{PM})$,

$$
6.4 \mathrm{Ms}(\mathrm{BK})
$$

Moment: $1.6 \times 10^{26}$ dyne-cm (HR)

Intensity IV: Kodiak.

19 June (LD) Alaska Peninsula

Origin time: 222838.8

Epicenter: 54.644N., 161.096W.

Depth: $15 \mathrm{~km}$

Magnitude: $4.3 \mathrm{~m}_{\mathrm{b}}(\mathrm{GS}), 3.4 \mathrm{~m}_{\mathrm{x}}(\mathrm{LD})$

Felt: False Pass (PM), King Cove (PM).
ALASKA-Continued

20 June (GM) Southern Alaska

Origin time: 073932.8

Epicenter: 60.676N., 152.107W.

Depth: $82 \mathrm{~km}$

Magnitude: $4.0 \mathrm{M}_{\mathrm{L}}(\mathrm{PM})$

Felt: Anchorage (PM), Homer (PM), Kenai (PM).

20 June (GM) Southern Alaska

Origin time: 221349.6

Epicenter: 62.248 N., $150.234 \mathrm{~W}$.

Depth: $10 \mathrm{~km}$

Magnitude: $3.8 \mathrm{M}_{\mathrm{L}}(\mathrm{PM})$

Felt: Talkeetna (PM).

21 June (GS) Southern Alaska

Origin time: 120730.2

Epicenter: 59.947N., 152.851W.

Depth: $101 \mathrm{~km}$

Magnitude: $4.9 \mathrm{~m}_{\mathrm{b}}(\mathrm{GS})$

Felt: Anchorage (PM), Eagle River (PM), Homer (PM), Kenai (PM).

22 June (EE) Andreanof Islands, Aleutian Islands

Origin time: 052852.4

Epicenter: 51.108 N., 175.170W.

Depth: $16 \mathrm{~km}$

Magnitude: $4.9 \mathrm{~m}_{\mathrm{b}}(\mathrm{GS}), 4.9 \mathrm{M}_{\mathrm{S}}(\mathrm{GS}), 4.3 \mathrm{M}_{\mathrm{L}}(\mathrm{PM})$

Moment: $1.3 \times 10^{24}$ dyne-cm (HR)

Felt: Adak Island (PM).

23 June (GM) Southern Alaska

Origin time: 024741.6

Epicenter: 61.740N., 149.765W.

Depth: $47 \mathrm{~km}$

Magnitude: $3.1 \mathrm{M}_{\mathrm{L}}(\mathrm{PM})$

Felt: Anchorage (PM), Eagle River (PM).

24 June (GM) Southern Alaska

Origin time: 093523.4

Epicenter: 58.529N., 155.219W.

Depth: $140 \mathrm{~km}$

Magnitude: None computed.

Felt: King Salmon Air Force Base (PM).

24 June (GS) Western Alaska

Origin time: 133825.6

Epicenter: 65.905N., 156.564W.

Depth: Normal

Magnitude: $3.6 \mathrm{M}_{\mathrm{L}}(\mathrm{PM})$

Felt: Kobuk (PM). 
24 June (GS) Central Alaska

Origin time: 204602.7

Epicenter: 66.133N., 149.639W.

Depth: $10 \mathrm{~km}$

Magnitude: $4.9 \mathrm{~m}_{\mathrm{b}}(\mathrm{GS}), 5.2 \mathrm{M}_{\mathrm{L}}(\mathrm{PM}), 5.9 \mathrm{M}_{\mathrm{S}}(\mathrm{BK})$

Intensity III: Fairbanks, Stevens Village.

Felt: Alyeska PLS Pump Station No. 6 (PM).

26 June (GM) Southern Alaska

Origin time: 135543.6

Epicenter: 59.730N., 152.182W.

Depth: $23 \mathrm{~km}$

Magnitude: $3.6 \mathrm{M}_{\mathrm{L}}(\mathrm{PM})$

Felt: Anchor Point (PM), Homer (PM).

26 June (GM) Southern Alaska

Origin time: 163419.0

Epicenter: 62.228N., 150.180W.

Depth: $12 \mathrm{~km}$

Magnitude: $3.3 \mathrm{M}_{\mathrm{L}}(\mathrm{PM})$

Felt: Talkeetna (PM).

28 June (LD) Alaska Peninsula

Origin time: 160119.7

Epicenter: $55.11 \mathrm{~N}$., $160.029 \mathrm{~W}$.

Depth: $52 \mathrm{~km}$

Magnitude: $4.3 \mathrm{~m}_{\mathrm{b}}(\mathrm{GS}), 4.4 \mathrm{~m}_{\mathrm{x}}(\mathrm{LD}), 4.3 \mathrm{M}_{\mathrm{L}}(\mathrm{PM})$

Felt: Sand Point (PM).

29 June (EE) Andreanof Islands, Aleutian Islands

Origin time: 043004.0

Epicenter: 52.539N., 174.792W.

Depth: $15 \mathrm{~km}$

Magnitude: $4.9 \mathrm{~m}_{\mathrm{b}}(\mathrm{GS}), 5.2 \mathrm{M}_{\mathrm{S}}(\mathrm{GS}), 4.6 \mathrm{M}_{\mathrm{L}}(\mathrm{PM})$, $5.3 \mathrm{M}_{\mathrm{S}}(\mathrm{BK})$

Moment: $1.5 \times 10^{24}$ dyne-cm (HR)

Felt: Adak Island (PM), Atka Island (PM).

29 June (GS) Andreanof Islands, Aleutian Islands

Origin time: 043210.8

Epicenter: $52.255 \mathrm{~N} ., 174.836 \mathrm{~W}$.

Depth: Normal

Magnitude: $4.7 \mathrm{~m}_{\mathrm{b}}(\mathrm{GS}), 4.3 \mathrm{M}_{\mathrm{L}}(\mathrm{PM})$

Felt: Adak Island (PM), Atka Island (PM).

30 June (EE) Andreanof Islands, Aleutian Islands

Origin time: 012130.0

Epicenter: 51.414N., 176.655W.

Depth: $39 \mathrm{~km}$

Magnitude: $4.8 \mathrm{~m}_{\mathrm{b}}(\mathrm{GS}), 4.1 \mathrm{M}_{\mathrm{S}}(\mathrm{GS}), 4.5 \mathrm{M}_{\mathrm{L}}(\mathrm{PM})$

Intensity III: Adak Island (PM).
30 June (EE) Andreanof Islands, Aleutian Islands

Origin time: 043908.8

Epicenter: 51.338N., 176.643W.

Depth: 33 km

Magnitude: $4.5 \mathrm{~m}_{\mathrm{b}}(\mathrm{GS})$

Intensity II: Adak Island (PM).

30 June (EE) Andreanof Islands, Aleutian Islands Origin time: 062347.1

Epicenter: 51.089N., 176.139W.

Depth: $21 \mathrm{~km}$

Magnitude: $5.1 \mathrm{~m}_{\mathrm{b}}(\mathrm{GS}), 4.7 \mathrm{MS}_{\mathrm{S}}(\mathrm{GS}), 4.9 \mathrm{M}_{\mathrm{L}}(\mathrm{PM})$

Moment: $1.3 \times 10^{24}$ dyne-cm (HR)

Intensity III: Adak Island (PM).

30 June (GS) Andreanof Islands, Aleutian Islands Origin time: 062801.9

Epicenter: 51.543N., 176.596W.

Depth: Normal

Magnitude: $4.2 \mathrm{~m}_{\mathrm{b}}(\mathrm{GS}), 4.3 \mathrm{M}_{\mathrm{L}}(\mathrm{PM})$

Intensity II: Adak Island (PM).

30 June (GS) Andreanof Islands, Aleutian Islands Origin time: 065509.4

Epicenter: 51.117N., 176.124W.

Depth: Normal

Magnitude: $4.2 \mathrm{~m}_{\mathrm{b}}(\mathrm{GS}), 4.7 \mathrm{M}_{\mathrm{L}}(\mathrm{PM})$

Intensity II: Adak Island (PM).

1 July (GM) Southern Alaska

Origin time: 192642.6

Epicenter: 61.597N., 149.704W.

Depth: $43 \mathrm{~km}$

Magnitude: $3.7 \mathrm{M}_{\mathrm{L}}(\mathrm{PM})$

Intensity III: Anchorage (PM), Eagle River (PM).

Intensity II: Palmer (PM).

3 July (EE) Andreanof Islands, Aleutian Islands Origin time: 173331.8

Epicenter: 51.204N., 175.589W.

Depth: $20 \mathrm{~km}$

Magnitude: $5.0 \mathrm{~m}_{\mathrm{b}}(\mathrm{GS}), 4.8 \mathrm{M}_{\mathrm{L}}(\mathrm{PM})$

Felt: Adak Island (PM), Atka Island (PM).

4 July (EE) Andreanof Islands, Aleutian Islands Origin time: 055851.9

Epicenter: 51.602N., 175.827W.

Depth: $42 \mathrm{~km}$

Magnitude: $4.9 \mathrm{~m}_{\mathrm{b}}(\mathrm{GS}), 4.7 \mathrm{M}_{\mathrm{L}}(\mathrm{PM})$

Felt: Adak Island (PM).

9 July (EE) Andreanof Islands, Aleutian Islands Origin time: 171024.6 
Epicenter: 51.545N., 176.083W.

Depth: $42 \mathrm{~km}$

Magnitude: $5.2 \mathrm{~m}_{\mathrm{b}}(\mathrm{GS}), 4.9 \mathrm{M}_{\mathrm{S}}(\mathrm{GS}), 4.9 \mathrm{M}_{\mathrm{L}}(\mathrm{PM})$, $4.9 \mathrm{Ms}(\mathrm{BK})$

Moment: $2.1 \times 10^{24}$ dyne-cm (HR)

Intensity IV: Adak Island (PM), Atka Island (PM).

9 July (EE) Andreanof Islands, Aleutian Islands

Origin time: 202452.7

Epicenter: 51.433 N., $176.815 W$.

Depth: $41 \mathrm{~km}$

Magnitude: $4.9 \mathrm{~m}_{\mathrm{b}}(\mathrm{GS}), 5.1 \mathrm{M}_{\mathrm{L}}(\mathrm{PM})$

Moment: $3.7 \times 10^{23}$ dyne-cm (HR)

Felt: Adak Island (PM), Atka Island (PM).

13 July (GM) Southern Alaska

Origin time: 035440.5

Epicenter: 62.254N., $150.228 \mathrm{~W}$.

Depth: $11 \mathrm{~km}$

Magnitude: $3.9 \mathrm{M}_{\mathrm{L}}(\mathrm{PM})$

Felt: Big Lake (PM), Palmer (PM), Talkeetna (PM).

13 July (GS) Southern Alaska

Origin time: 035802.5

Epicenter: 62.216 N., $150.286 \mathrm{~W}$.

Depth: $10 \mathrm{~km}$

Magnitude: $3.0 \mathrm{M}_{\mathrm{L}}(\mathrm{PM})$

Felt: Talkeetna (PM).

17 July (EE) Andreanof Islands, Aleutian Islands

Origin time: 183720.0

Epicenter: 51.180N., 174.494W.

Depth: $20 \mathrm{~km}$

Magnitude: $4.8 \mathrm{~m}_{b}(\mathrm{GS})$

Felt: Adak Island (PM).

19 July (GS) Fox Islands, Aleutian Islands

Origin time: 043155.9

Epicenter: 53.352N., 165.882W.

Depth: Normal

Magnitude: $5.5 \mathrm{~m}_{\mathrm{b}}(\mathrm{GS}), 5.1 \mathrm{M}_{\mathrm{S}}(\mathrm{GS}), 5.9 \mathrm{M}_{\mathrm{L}}(\mathrm{PM})$, $4.9 \mathrm{M}_{\mathrm{S}}(\mathrm{BK})$

Moment: $2.8 \times 10^{24}$ dyne-cm (HR)

Intensity IV: Akutan (press report), Unalaska (press report).

19 July (GS) Fox Islands, Aleutian Islands

Origin time: 050408.2

Epicenter: $53.339 \mathrm{~N} ., 165.859 \mathrm{~W}$.

Depth: Normal

Magnitude: $5.1 \mathrm{~m}_{\mathrm{b}}(\mathrm{GS}), 4.5 \mathrm{M}_{\mathrm{S}}(\mathrm{GS}), 5.6 \mathrm{M}_{\mathrm{L}}(\mathrm{PM})$

Moment: $6.9 \times 10^{23}$ dyne-cm (HR)

Intensity IV: Unalaska (press report).
19 July (GS) Fox Islands, Aleutian Islands

Origin time: 065317.8

Epicenter: 53.600N., 167.171W.

Depth: Normal

Magnitude: $5.5 \mathrm{~m}_{\mathrm{b}}(\mathrm{GS}), 5.7 \mathrm{MS}_{\mathrm{S}}(\mathrm{GS}), 5.8 \mathrm{M}_{\mathrm{L}}(\mathrm{PM})$, $5.6 \mathrm{Ms}(\mathrm{BK})$,

Moment: $1.0 \times 10^{25}$ dyne-cm (HR)

Intensity IV: Unalaska (press report).

Felt: Akutan (PM).

19 July (GS) Fox Islands, Aleutian Islands

Origin time: 113107.5

Epicenter: 53.617N., 167.408W.

Depth: Normal

Magnitude: $5.0 \mathrm{~m}_{\mathrm{b}}(\mathrm{GS}), 4.6 \mathrm{MS}_{\mathrm{S}}(\mathrm{GS}), 5.1 \mathrm{M}_{\mathrm{L}}(\mathrm{PM})$

Felt: Akutan (PM), Unalaska (PM).

19 July (GS) Fox Islands, Aleutian Islands

Origin time: 205209.6

Epicenter: 53.662N., 167.184W.

Depth: Normal

Magnitude: $4.9 \mathrm{~m}_{\mathrm{b}}(\mathrm{GS})$

Intensity III: Unalaska.

19 July (GS) Fox Islands, Aleutian Islands

Origin time: 223236.0

Epicenter: 53.521N., 167.301W.

Depth: Normal

Magnitude: $5.6 \mathrm{~m}_{\mathrm{b}}(\mathrm{GS}), 5.6 \mathrm{M}_{\mathrm{S}}(\mathrm{GS}), 5.6 \mathrm{MS}_{\mathrm{S}}(\mathrm{BK})$

Moment: $1.1 \times 10^{25}$ dyne-cm (HR)

Intensity $\mathrm{V}$ :

Akutan-Objects were knocked off walls and shelves; cars shook noticeably (press report).

Unalaska- Things were knocked off walls and shelves; cars shook noticeably; runway lights were knocked out at the Dutch Harbor-Unalaska airport (press report).

20 July (GS) Fox Islands, Aleutian Islands

Origin time: 015908.2

Epicenter: 53.530N., 167.344W.

Depth: Normal

Magnitude: $4.9 \mathrm{~m}_{\mathrm{b}}(\mathrm{GS}), 4.5 \mathrm{Ms}(\mathrm{GS}), 5.2 \mathrm{M}_{\mathrm{L}}(\mathrm{PM})$

Moment: $5.8 \times 10^{23}$ dyne-cm (HR)

Felt: Unalaska (PM), Cold Bay.

24 July (EE) Andreanof Islands, Aleutian Islands

Origin time: 004200.8

Epicenter: 51.012N., 176.639W.

Depth: $20 \mathrm{~km}$

Magnitude: $4.9 \mathrm{~m}_{\mathrm{b}}(\mathrm{GS}), 4.5 \mathrm{Ms}_{\mathrm{S}}(\mathrm{GS}), 5.3 \mathrm{M}_{\mathrm{L}}(\mathrm{PM})$

Felt: Adak Island (PM), Atka Island (PM).

24 July (EE) Andreanof Islands, Aleutian Islands

Origin time: 140330.0 
Epicenter: 51.492N., 175.173W.

Depth: $40 \mathrm{~km}$

Magnitude: $4.9 \mathrm{~m}_{\mathrm{b}}(\mathrm{GS}), 4.5 \mathrm{M}_{\mathrm{S}}(\mathrm{GS}), 4.3 \mathrm{M}_{\mathrm{L}}(\mathrm{PM})$

Felt: Adak Island (PM), Atka Island (PM).

25 July (EE) Andreanof Islands, Aleutian Islands

Origin time: 090132.6

Epicenter: 51.079N., 176.137W.

Depth: $21 \mathrm{~km}$

Magnitude: $5.3 \mathrm{~m}_{\mathrm{b}}(\mathrm{GS}), 5.6 \mathrm{MS}_{\mathrm{S}}(\mathrm{GS}), 5.3 \mathrm{M}_{\mathrm{L}}(\mathrm{PM})$, $5.5 \mathrm{Ms}(\mathrm{BK})$

Moment: $6.1 \times 10^{24}$ dyne-cm (HR)

Intensity IV: Adak Island (PM).

25 July (EE) Andreanof Islands, Aleutian Islands

Origin time: 090416.3

Epicenter: $51.056 \mathrm{~N} ., 175.996 \mathrm{~W}$.

Depth: $20 \mathrm{~km}$

Magnitude: $5.4 \mathrm{~m}_{\mathrm{b}}(\mathrm{GS}), 5.6 \mathrm{MS}_{\mathrm{S}}(\mathrm{GS})$

Felt: Adak Island (PM).

28 July (EE) Andreanof Islands, Aleutian Islands Origin time: 040650.5

Epicenter: 51.404N., 174.016W.

Depth: $21 \mathrm{~km}$

Magnitude: $5.4 \mathrm{~m}_{\mathrm{b}}(\mathrm{GS}), 4.8 \mathrm{M}_{\mathrm{S}}(\mathrm{GS}), 4.5 \mathrm{M}_{\mathrm{L}}(\mathrm{PM})$, 4.6 $\mathrm{MS}_{\mathrm{S}}(\mathrm{BK})$

Moment: $1.3 \times 10^{24}$ dyne-cm (HR)

Intensity III: Adak Island.

28 July (GS) Fox Islands, Aleutian Islands

Origin time: 050159.6

Epicenter: 52.862N., 166.590W.

Depth: Normal

Magnitude: $5.0 \mathrm{~m}_{\mathrm{b}}(\mathrm{GS}), 4.6 \mathrm{MS}(\mathrm{GS}), 4.7 \mathrm{M}_{\mathrm{L}}(\mathrm{PM})$

Felt: Unalaska (PM).

28 July (GM) Southern Alaska

Origin time: 143114.1

Epicenter: $60.577 \mathrm{~N} ., 150.386 \mathrm{~W}$.

Depth: $47 \mathrm{~km}$

Magnitude: $4.4 \mathrm{~m}_{\mathrm{b}}(\mathrm{GS}), 4.6 \mathrm{M}_{\mathrm{L}}(\mathrm{PM})$

Intensity IV: Anchorage (PM).

Felt: Homer(PM), Kenai (PM), Palmer (PM), Seward (PM).

28 July (EE) Andreanof Islands, Aleutian Islands

Origin time: 215716.6

Epicenter: 51.573N., 175.221W.

Depth: $42 \mathrm{~km}$

Magnitude: $5.4 \mathrm{~m}_{\mathrm{b}}(\mathrm{GS}), 4.9 \mathrm{MS}_{\mathrm{S}}(\mathrm{GS}), 4.9 \mathrm{M}_{\mathrm{L}}(\mathrm{PM})$

Moment: $1.9 \times 10^{24}$ dyne-cm (HR)

Intensity III: Adak Island.
31 July (GM) Southern Alaska

Origin time: 093314.3

Epicenter: $61.767 \mathrm{~N} ., 149.567 \mathrm{~W}$.

Depth: $41 \mathrm{~km}$

Magnitude: $3.2 \mathrm{M}_{\mathrm{L}}(\mathrm{PM})$

Felt: Anchorage (PM), Palmer (PM), Willows (PM).

31 July (GM) Southern Alaska

Origin time: 140434.5

Epicenter: $60.797 \mathrm{~N} ., 149.614 \mathrm{~W}$.

Depth: $45 \mathrm{~km}$

Magnitude: $3.7 \mathrm{M}_{\mathrm{L}}(\mathrm{PM})$

Felt: Anchorage (PM), Girdwood (PM).

1 August (GS) Fox Islands, Aleutian Islands

Origin time: 164306.4

Epicenter: 53.495N., 167.236W.

Depth: Normal

Magnitude: $4.6 \mathrm{~m}_{\mathrm{b}}(\mathrm{GS}), 4.5 \mathrm{M}_{\mathrm{L}}(\mathrm{PM}), 4.1 \mathrm{~m}_{\mathrm{x}}(\mathrm{LD})$

Felt: Unalaska (PM).

1 August (EE) Andreanof Islands, Aleutian Islands Origin time: 202459.3

Epicenter: 50.954N., 176.185W.

Depth: $20 \mathrm{~km}$

Magnitude: $4.8 \mathrm{~m}_{\mathrm{b}}(\mathrm{GS}), 4.9 \mathrm{MS}_{\mathrm{S}}(\mathrm{GS}), 4.9 \mathrm{M}_{\mathrm{L}}(\mathrm{PM})$

Felt: Adak Island (PM).

1 August (EE) Andreanof Islands, Aleutian Islands

Origin time: 210540.1

Epicenter: 51.262N., 174.224W.

Depth: $22 \mathrm{~km}$

Magnitude: $5.5 \mathrm{~m}_{\mathrm{b}}(\mathrm{GS}), 5.0 \mathrm{M}_{\mathrm{S}}(\mathrm{GS}), 4.6 \mathrm{M}_{\mathrm{L}}(\mathrm{PM})$

Moment: $1.5 \times 10^{24}$ dyne-cm (HR)

Intensity IV: Adak Island (PM).

3 August (EE) Andreanof Islands, Aleutian Islands Origin time: 023928.7

Epicenter: $51.244 \mathrm{~N} ., 174.125 \mathrm{~W}$.

Depth: $20 \mathrm{~km}$

Magnitude: $5.0 \mathrm{~m}_{\mathrm{b}}(\mathrm{GS}), 4.0 \mathrm{M}_{\mathrm{L}}(\mathrm{PM})$

Felt: Adak Island (PM).

3 August (EE) Andreanof Islands, Aleutian Islands Origin time: 132910.4

Epicenter: $51.026 \mathrm{~N} ., 176.749 \mathrm{~W}$.

Depth: $22 \mathrm{~km}$

Magnitude: $5.4 \mathrm{~m}_{\mathrm{b}}(\mathrm{GS}), 5.6 \mathrm{MS}_{\mathrm{S}}(\mathrm{GS}), 5.6 \mathrm{M}_{\mathrm{L}}(\mathrm{PM})$, $5.7 \mathrm{MS}_{\mathrm{S}}(\mathrm{BK})$

Moment: $8.1 \times 10^{24}$ dyne-cm (HR)

Intensity IV: Adak Island.

Felt: Atka (PM).

3 August (EE) Andreanof Islands, Aleutian Islands Origin time: 134454.2 
Epicenter: 50.808 N., $176.671 W$.

Depth: $20 \mathrm{~km}$

Magnitude: $4.7 \mathrm{~m}_{\mathrm{b}}(\mathrm{GS}), 5.1 \mathrm{M}_{\mathrm{L}}(\mathrm{PM})$

Felt: Adak Island (PM).

3 August (EE) Andreanof Islands, Aleutian Islands

Origin time: 200820.5

Epicenter: $50.918 \mathrm{~N} ., 176.638 \mathrm{~W}$.

Depth: $20 \mathrm{~km}$

Magnitude: $4.8 \mathrm{~m}_{\mathrm{b}}(\mathrm{GS}), 4.9 \mathrm{M}_{\mathrm{S}}(\mathrm{GS}), 4.2 \mathrm{M}_{\mathrm{L}}(\mathrm{PM})$

Felt: Adak Island (PM).

8 August (GS) Fox Islands, Aleutian Islands

Origin time: 043121.3

Epicenter: 53.594N., 167.320W.

Depth: Normal

Magnitude: $4.5 \mathrm{~m}_{\mathrm{b}}(\mathrm{GS}), 4.3 \mathrm{M}_{\mathrm{S}}(\mathrm{GS}), 5.0 \mathrm{M}_{\mathrm{L}}(\mathrm{PM})$

Felt: Unalaska (PM).

\section{August Central Alaska}

Origin time: 1100

Epicenter: Not located.

Depth: None computed.

Magnitude: $3.0 \mathrm{M}_{\mathrm{L}}(\mathrm{PM})$

Felt: Fairbanks (PM).

\section{August (GM) Southern Alaska}

Origin time: 232754.3

Epicenter: 61.352N., 150.333W.

Depth: $47 \mathrm{~km}$

Magnitude: $4.5 \mathrm{~m}_{\mathrm{b}}(\mathrm{GS}), 4.4 \mathrm{M}_{\mathrm{L}}(\mathrm{PM})$

Intensity III: Anchorage (PM), Palmer (PM), Willow (PM).

Intensity II: Talkeetna (PM).

3 September (EE) Andreanof Islands, Aleutian Islands

Origin time: 115106.4

Epicenter: $51.106 \mathrm{~N} ., 178.224 \mathrm{~W}$.

Depth: $30 \mathrm{~km}$

Magnitude: $5.0 \mathrm{~m}_{\mathrm{b}}(\mathrm{GS}), 5.0 \mathrm{M}_{\mathrm{L}}(\mathrm{PM})$

Intensity III: Adak Island.

12 September (GS) Southern Alaska

Origin time: 235715.6

Epicenter: 56.201N., 153.405W.

Depth: $31 \mathrm{~km}$

Magnitude: $6.1 \mathrm{~m}_{\mathrm{b}}(\mathrm{GS}), 6.3 \mathrm{M}_{\mathrm{S}}(\mathrm{GS}), 6.0 \mathrm{M}_{\mathrm{S}}(\mathrm{BK})$

Moment: $6.8 \times 10^{25}$ dyne-cm (GS)

Intensity IV: Larsen Bay.

13 September (GM) Southern Alaska

Origin time: 123040.7

Epicentcr: $61.245 \mathrm{~N} ., 146.939 \mathrm{~W}$.

Depth: $42 \mathrm{~km}$
Magnitude: $3.8 \mathrm{M}_{\mathrm{L}}(\mathrm{PM})$

Felt: Valdez (PM).

14 September (GM) Southern Alaska

Origin time: 113853.6

Epicenter: 61.703N., 149.682W.

Depth: $47 \mathrm{~km}$

Magnitude: $3.4 \mathrm{M}_{\mathrm{L}}(\mathrm{PM})$

Felt: Palmer (PM).

15 September (EE) Andreanof Islands, Aleutian Islands Origin time: 062938.6

Epicenter: 51.368N., 177.011W.

Depth: $35 \mathrm{~km}$

Magnitude: $4.9 \mathrm{~m}_{\mathrm{b}}(\mathrm{GS}), 4.0 \mathrm{M}_{\mathrm{S}}(\mathrm{GS}), 4.8 \mathrm{M}_{\mathrm{L}}(\mathrm{PM})$

Felt: Adak Island (PM).

15 September (GM) Southern Alaska

Origin time: 144822.1

Epicenter: 61.528N., 143.800W.

Depth: $52 \mathrm{~km}$

Magnitude: $4.5 \mathrm{~m}_{\mathrm{b}}(\mathrm{GS}), 4.7 \mathrm{M}_{\mathrm{L}}(\mathrm{PM}), 4.7 \mathrm{M}_{\mathrm{L}}(\mathrm{EP})$

Intensity IV: Chitina, Valdez.

Intensity III: Cordova (PM).

16 September (GS) Southern Alaska

Origin time: 205721.9

Epicenter: 56.222N., 153.600W.

Depth: Normal

Magnitude: $5.3 \mathrm{~m}_{\mathrm{b}}(\mathrm{GS}), 5.5 \mathrm{M}_{S}(\mathrm{GS}), 5.1 \mathrm{M}_{\mathrm{L}}(\mathrm{PM})$,

$$
5.1 \mathrm{M}_{\mathrm{S}}(\mathrm{BK})
$$

Moment: $7.7 \times 10^{24}$ dyne-cm (HR)

Intensity III: Kodiak.

18 September (GS) Southern Alaska

Origin time: 205605.8

Epicenter: 61.798N., 149.721W.

Depth: $57 \mathrm{~km}$

Magnitude: $4.6 \mathrm{~m}_{\mathrm{b}}(\mathrm{GS}), 4.6 \mathrm{M}_{\mathrm{L}}(\mathrm{PM})$

Intensity IV: Anchorage, Chugiak, Eagle River, Skwentna, Wasilla, Willow.

Intensity III: Anchorage International Airport, Moose Pass, Spenard, Sutton.

Intensity II: Whittier.

Felt: Girdwood (PM), King Mountain Lodge (PM), Mile 90

on Glenn Highway (PM), Palmer (PM).

28 September (GM) Southern Alaska

Origin time: 104148.8

Epicenter: 59.782N., 152.320W.

Depth: $61 \mathrm{~km}$

Magnitude: $4.1 \mathrm{M}_{\mathrm{L}}(\mathrm{PM})$

Intensity III: Homer (PM). 
29 September (EE) Andreanof Islands, Aleutian Islands Origin time: 122043.9

Epicenter: 51.118N., 174.957W:

Depth: $20 \mathrm{~km}$

Magnitude: $5.0 \mathrm{~m}_{\mathrm{b}}(\mathrm{GS}), 4.5 \mathrm{M}_{\mathrm{S}}(\mathrm{GS}), 5.2 \mathrm{M}_{\mathrm{L}}(\mathrm{PM})$

Intensity III: Adak Island (PM).

1 October (EE) Andreanof Islands, Aleutian Islands

Origin time: 155606.4

Epicenter: 51.623N., 175.901W.

Depth: $45 \mathrm{~km}$

Magnitude: $5.3 \mathrm{~m}_{\mathrm{b}}(\mathrm{GS}), 4.5 \mathrm{MS}_{\mathrm{S}}(\mathrm{GS}), 4.8 \mathrm{M}_{\mathrm{L}}(\mathrm{PM})$

Moment: $1.2 \times 10^{24}$ dyne-cm (HR)

Intensity III: Adak Island (PM).

5 October (EE) Andreanof Islands, Aleutian Islands

Origin time: 032557.7

Epicenter: 51.078N., 176.179W.

Depth: $20 \mathrm{~km}$

Magnitude: $4.9 \mathrm{~m}_{\mathrm{b}}(\mathrm{GS}), 4.5 \mathrm{M}_{\mathrm{L}}(\mathrm{PM})$

Intensity II: Adak Naval Air Station (PM).

6 October (EE) Andreanof Islands, Aleutian Islands

Origin time: 042148.9

Epicenter: 51.513N., 176.089W.

Depth: $48 \mathrm{~km}$

Magnitude: $5.1 \mathrm{~m}_{\mathrm{b}}(\mathrm{GS}), 4.2 \mathrm{MS}_{\mathrm{S}}(\mathrm{GS}), 4.9 \mathrm{M}_{\mathrm{L}}(\mathrm{PM})$

Moment: $7.3 \times 10^{23}$ dyne-cm (HR)

Intensity III: Adak Naval Air Station (PM).

\section{October (GM) Southern Alaska}

Origin time: 012106.1

Epicenter: 62.129N., 149.544W.

Depth: $58 \mathrm{~km}$

Magnitude: $4.5 \mathrm{~m}_{\mathrm{b}}(\mathrm{GS}), 4.3 \mathrm{M}_{\mathrm{L}}(\mathrm{PM})$

Intensity III: Anchorage (PM).

Intensity II: Talkeetna (PM).

15 October (GS) Southern Alaska

Origin time: 233551.2

Epicenter: 59.705N., 153.072W.

Depth: $121 \mathrm{~km}$

Magnitude: $4.6 \mathrm{~m}_{\mathrm{b}}(\mathrm{GS})$

Intensity III: Homer.

\section{October (GS) Central Alaska}

Origin time: 192210.1

Epicenter: 63.153N., 150.443W.

Depth: $119 \mathrm{~km}$

Magnitude: None computed

Felt: Cantwell (PM).
19 October (GM) Southern Alaska

Origin time: 215334.0

Epicenter: 59.509N., 152.682W.

Depth: $81 \mathrm{~km}$

Magnitude: $4.0 \mathrm{M}_{\mathrm{L}}(\mathrm{PM})$

Intensity II: Homer (PM).

22 October (GM) Southern Alaska

Origin time: 183134.1

Epicenter: 61.339N., 146.849W.

Depth: 38 km

Magnitude: $4.0 \mathrm{M}_{\mathrm{L}}(\mathrm{PM})$

Intensity III: Valdez (PM).

24 October (EE) Andreanof Islands, Aleutian Islands

Origin time: 110050.3

Epicenter: 51.384N., 176.750W.

Depth: $37 \mathrm{~km}$

Magnitude: $5.2 \mathrm{~m}_{\mathrm{b}}(\mathrm{GS}), 4.3 \mathrm{M}_{\mathrm{S}}(\mathrm{GS}), 4.7 \mathrm{M}_{\mathrm{L}}(\mathrm{PM})$

Moment: $7.1 \times 10^{23}$ dyne-cm (HR)

Intensity III: Adak Island (PM).

24 October (GM) Southern Alaska

Origin time: 143453.8

Epicenter: 60.946 N., $151.486 \mathrm{~W}$.

Depth: $69 \mathrm{~km}$

Magnitude: $3.2 \mathrm{M}_{\mathrm{L}}(\mathrm{PM})$

Felt: Eagle River (PM).

27 October (GM) Southern Alaska

Origin time: 193936.5

Epicenter: 60.928N., 149.464W.

Depth: $39 \mathrm{~km}$

Magnitude: $3.6 \mathrm{M}_{\mathrm{L}}(\mathrm{PM})$

Intensity IV: Anchorage.

Intensity III: Eagle River.

4 November (GM) Southern Alaska

Origin time: 061418.7

Epicenter: 61.341N., 151.900W.

Depth: $98 \mathrm{~km}$

Magnitude: $4.7 \mathrm{~m}_{\mathrm{b}}(\mathrm{GS})$

Intensity III: Hurricane (PM).

Intensity II: Anchorage (PM).

6 November (EE) Andreanof Islands, Aleutian Islands

Origin time: 182702.9

Epicenter: 51.242N., 176.631W.

Depth: $39 \mathrm{~km}$

Magnitude: $5.1 \mathrm{~m}_{\mathrm{b}}(\mathrm{GS}), 5.5 \mathrm{M}_{\mathrm{S}}(\mathrm{GS}), 5.2 \mathrm{M}_{\mathrm{L}}(\mathrm{PM})$, $5.6 \mathrm{Ms}(\mathrm{BK})$

Moment: $8.5 \times 10^{24}$ dyne-cm (HR)

Intensity IV: Adak Island. 
6 November (EE) Andreanof Islands, Aleutian Islands Origin time: 194540.5

Epicenter: 51.072N., 176.516W.

Depth: $20 \mathrm{~km}$

Magnitude: $4.8 \mathrm{~m}_{\mathrm{b}}(\mathrm{GS})$

Intensity IV: Adak Island.

19 November (GS) Andreanof Islands, Aleutian Islands

Origin time: 190011.4

Epicenter: $51.036 \mathrm{~N} ., 176.001 \mathrm{~W}$.

Depth: Normal

Magnitude: $4.5 \mathrm{M}_{\mathrm{L}}(\mathrm{PM})$

Intensity III: Adak Island.

26 November Central Alaska

Origin time: 0841

Epicenter: Not located.

Depth: None computed.

Magnitude: $3.5 \mathrm{M}_{\mathrm{L}}$ (AK)

Intensity IV: North Pole (press report).

Intensity III: Eielson Air Force Base.

Felt: Fairbanks (press report).

26 November (GM) Southern Alaska

Origin time: 210443.4

Epicenter: $61.774 \mathrm{~N} ., 150.887 \mathrm{~W}$.

Depth: $62 \mathrm{~km}$

Magnitude: $3.6 \mathrm{M}_{\mathrm{L}}(\mathrm{PM})$

Intensity III: Skwentna.

3 December (EE) Andreanof Islands, Aleutian Islands

Origin time: 050536.7

Epicenter: 51.352N., 176.465W.

Depth: $36 \mathrm{~km}$

Magnitude: $4.6 \mathrm{~m}_{\mathrm{b}}(\mathrm{GS})$

Intensity III: Adak Island.

16 December (EE) Andreanof Islands, Aleutian Islands

Origin time: 102724.4

Epicenter: $51.492 \mathrm{~N}$., $175.319 \mathrm{~W}$.

Depth: $35 \mathrm{~km}$

Magnitude: $5.1 \mathrm{~m}_{\mathrm{b}}(\mathrm{GS}), 4.4 \mathrm{M}_{\mathrm{S}}(\mathrm{GS}), 5.0 \mathrm{M}_{\mathrm{L}}(\mathrm{PM})$

Moment: $6.7 \times 10^{23}$ dyne-cm (HR)

Intensity IV: Adak Island.

19 December (EE) Andreanof Islands, Aleutian Islands

Origin time: 135013.3

Epicenter: 51.391N., 176.903W.

Depth: $37 \mathrm{~km}$

Magnitude: $5.3 \mathrm{~m}_{\mathrm{b}}(\mathrm{GS})$

Moment: $4.6 \times 10^{23}$ dyne-cm (HR)

Intensity V:

Adak Island-A few small objects overturned and fell; people had difficulty standing or walking; buildings shook strongly.

27 December (GM) Southern Alaska

Origin time: 033541.4

Epicenter: 61.828N., 148.954W.

Depth: $15 \mathrm{~km}$

Magnitude: $3.1 \mathrm{M}_{\mathrm{L}}(\mathrm{PM})$

Intensity II: Lazy Mountain (PM).

\section{ARIZONA}

23 June (PS) Baja California, Mexico

Origin time: 234608.5

See California listing.

8 July (GP) Southern California

Origin time: 092044.5

See California listing.

13 July (HJ) Off the coast of Southern California

Origin time: 134708.2

See California listing.

ARKANSAS

24 May (SL) Southeastern Missouri

Origin time: 124813.5

See Missouri listing.

\section{CALIFORNIA}

\section{January (BK) Central California}

Origin time: 051849.1

Epicenter: 37.262N., 121.665W.

Depth: $6 \mathrm{~km}$

Magnitude: $2.7 \mathrm{M}_{\mathrm{L}}(\mathrm{BK})$

Moment: $7.4 \times 10^{19}$ dyne-cm (BK)

Felt: Halls Valley area (BK).

Earthquake Descriptions 21 


\section{CALIFORNIA-Continued}

6 January (BK) Central California

Origin time: 195242.7

Epicenter: 37.010N., 121.483W.

Depth: $9 \mathrm{~km}$

Magnitude: $3.7 \mathrm{M}_{\mathrm{L}}(\mathrm{BK})$

Moment: $4.4 \times 10^{21}$ dyne-cm (BK)

Intensity III: Aptos, Morgan Hill.

Felt: Gilroy (press report), Hollister (press report).

\section{January (BK) Central California}

Origin time: 030754.9

Epicenter: 36.563N., 121.203W.

Depth: $7 \mathrm{~km}$

Magnitude: $3.4 \mathrm{M}_{\mathrm{L}}(\mathrm{BK})$

Moment: $1.8 \times 10^{20}$ dyne-cm (BK)

Felt: Bear Valley (press report), Gonzales (press report), Greenfield, Morgan Hill (press report), Soledad (press report).

14 January (BK) Central California

Origin time: 030936.3

Epicenter: 36.572N., 121.205W.

Depth: $7 \mathrm{~km}$

Magnitude: $5.0 \mathrm{~m}_{\mathrm{b}}(\mathrm{GS}), 4.8 \mathrm{M}_{\mathrm{L}}(\mathrm{BK})$

Moment: $9.1 \times 10^{22}$ dyne-cm (BK)

Intensity IV: Castroville, Chualar, Gonzales, Greenfield, Hollister, Pacific Grove, Paicines, Salinas, San Ardo, San Juan Bautista, Santa Cruz, Soledad.

Intensity III; Aromas, Coulterville, Davenport, Felton, Half Moon Bay, King City, La Selva Beach, Lockwood, Moss Landing, Redwood Estates, Seaside, Watsonville.

Felt: Placerville (press report).

14 January (PS) Southern California

Origin time: 131214.0

Epicenter: 33.914N., 116.697W.

Depth: $13 \mathrm{~km}$

Magnitude: $3.2 \mathrm{M}_{\mathrm{L}}(\mathrm{PS}), 3.4 \mathrm{M}_{\mathrm{L}}(\mathrm{GP})$

Felt: Palm Desert (PS), Palm Springs (PS).

16 January (BK) Central California

Origin time: 093847.4

Epicenter: 38.428N., 122.645W.

Depth: $5 \mathrm{~km}$

Magnitude: $2.5 \mathrm{M}_{\mathrm{L}}(\mathrm{BK})$

Intensity IV: Santa Rosa.

\section{January (BK) Central California}

Origin time: 200730.8

Epicenter: 38.543N., 122.995W.

Depth: $1 \mathrm{~km}$

Magnitude: $2.3 \mathrm{M}_{\mathrm{L}}(\mathrm{BK})$

Intensity IV: Guerneville (bottles reported to have been knocked from shelves in a liquor store).

\section{CALIFORNIA-Continued}

\section{January (BK) Central California}

Origin time: 192051.2

Epicenter: 36.810 N., 121.275W.

Depth: $7 \mathbf{~ k m}$

Magnitude: $5.3 \mathrm{~m}_{\mathrm{b}}(\mathrm{GS}), 5.3 \mathrm{MS}_{\mathrm{S}}(\mathrm{GS}), 5.5 \mathrm{M}_{\mathrm{L}}(\mathrm{BK})$

Moment: $2.0 \times 10^{24}$ dyne-cm (BK)

This earthquake was felt over a land area of about $36,000 \mathrm{~km}^{2}$ (fig. 9). Some of the effects listed below were taken from a survey by K.K. Harms, U.S. Geological Survey, Menlo Park, Calif., on 26 January 1986 supplemented by information from M.M. Clark, U.S. Geological Survey, Menlo Park, Calif., on 19 March 1986.

Intensity VII:

Paicines-At Almaden Vineyard's Cienaga Winery a 20,000-gallon vat of wine was moved $6.1 \mathrm{~m}$ off its foundation and shattered. Several other vats leaked, causing a total loss of 30,000 gallons of wine. The winery estimated damage and loss of wine at $\$ 800,000$ (from press reports). A few items were thrown from store shelves; hanging pictures fell; shaking was described as strong; felt by many people.

Intensity VI:

Hollister-Broken gas pipes were reported at a few homes and at one apartment building. A water line ruptured; a rockslide occurred on Airline Highway near Murphy Road east of Hollister; merchandise was shaken from shelves at Nob Hill and K\&S Markets breaking glass items; two people were slightly injured from glass cuts; and a tree fell onto an old house south of town (press reports). A few windows cracked; felt by many people.

Reynolds Martin Ranch (in Santa Ana Valley)Boulder-masonry walls at entrance to driveway were partly destroyed; many hanging pictures were shaken off wall hooks in house; pencils were ejected from glass container on table.

Santa Ana Valley-A chimney cracked; young girl was thrown to floor; plumbing for swimming pool was broken when its filter moved $20 \mathrm{~cm}$ off its mount. Items fell off shelves in homes; china fell in a cabinet; one house sustained some minor cracks. A water heater moved off its mount in one home (press report).

Tres Pinos-Tres Pinos Inn had vertical structural cracks in the front part of building and a cracked window. At the 19 th Hole Bar, two chimneys fell; kitchen stove shifted; bottles fell off shelves and broke; and customers ran out into the street. At the post office a 10-lb scale was knocked off the safe and landed about $0.9 \mathrm{~m}$ away. One residential chimney cracked; plaster and dry wall sustained large cracks; many items were thrown off store shelves; many glassware items broke; many small 


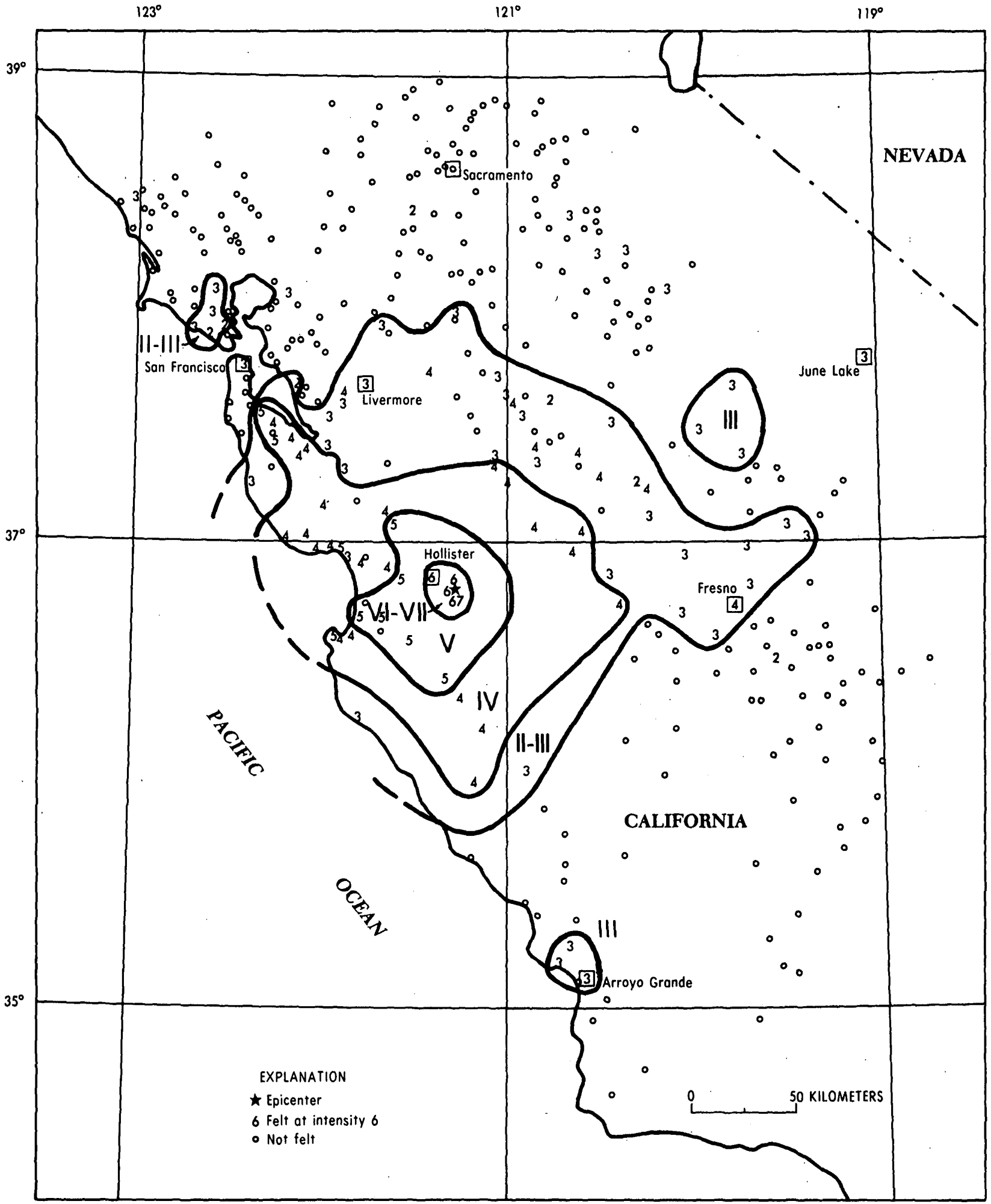

Figure 9. Isoseismal map for the central California earthquake of 26 January, 1986, 192051.2 UTC. Roman numerals represent Modified Mercalli intensities between isoseismals; Arabic numerals represent intensities at specific sites; dashed contour lines are inferred isoseismals, and small boxes show locations of towns and cities whose names are plotted. 


\section{CALIFORNIA-Continued}

objects overturned and fell; buildings shook strongly; people had difficulty standing; felt by everyone.

Intensity $\mathrm{V}$ :

Aptos-A few items were thrown from store shelves; a few small objects overturned and fell; shaking was described as strong; felt by and frightened several people.

Chualar-Plaster walls sustained hairline cracks; a few items were thrown off store shelves; a few small objects overturned and fell; buildings shook strongly; windows, doors, and dishes rattled loudly; trees and bushes shook moderately; standing vehicles rocked moderately; felt by everyone.

Marina-A few small objects overturned and fell; hanging pictures were out of place; shaking was described as strong; felt by everyone.

Pacific Grove-Standing and moving vehicles rocked moderately; trees and bushes shook moderately; windows, doors, and dishes rattled loudly; buildings shook moderately; the vibration was described as strong; felt by and frightened many people.

Redwood City-A few small objects fell; hanging pictures fell; water splashed onto sides of swimming pools; felt by many people.

Salinas-Trees and bushes shook moderately; standing vehicles rocked slightly; a few small objects overturned and fell; hanging pictures swung; buildings shook moderately; shaking was described as moderate with some difficulty in standing; felt by many people.

San Juan Bautista (press report)-Standing and moving vehicles rocked slightly; trees and bushes shook slightly; hanging pictures swung; shaking was described as moderate; felt by everyone.

San Martin-A few items were thrown off store shelves; standing vehicles rocked slightly; trees and bushes shook slightly; pictures swung out of place; windows, doors, and dishes rattled loudly; shaking was described as moderate with some difficulty in standing; felt by everyone.

San Mateo-Standing and moving vehicles rocked slightly; trees and bushes shook slightly; a few items were thrown off store shelves; a few glassware items broke; a few small objects overturned and fell; hanging objects swung violently; shaking was described as strong; felt by and frightened many people.

Silva Ranch-Items fell off shelves; water sloshed out of the toilet; there were some minor cracks in the house.

Soledad-Standing vehicles rocked slightly; trees and bushes shook slightly; a few small objects overturned and fell; hanging pictures swung; buildings shook slightly; felt by and frightened many people.

Intensity IV: Aromas, Campbell, Capitola, Ceres, Davenport, East Santa Cruz, Felton, Fresno, Greenfield, Gustine, Hilmar, Jolon, King City, LeGrand, Los Altos,

\section{CALIFORNIA-Continued}

Los Banos, Mendota, Merced, Moffett Field Naval Air Station, Monterey, Monterey Bay Academy, Morgan Hill, Mount Hermon, Mountain View, Newman, Palo Alto, Pleasanton, Redwood Estates, San Carlos, Santa Clara, Santa Cruz, Santa Rita Park, Seaside, Soquel, South Dos Palos, Stanford University (press report), Tracy, Watsonville, Winton.

Intensity III: Ahwahnee, Alviso, Arnold, Arroyo Grande, Auberry, Avila Beach, Big Sur, Bolinas, Brentwood, Chowchilla, Clovis, Coyote, Crows Landing, El Portal, Fairfax, Firebaugh, Fremont, Friant, June Lake, Kerman, Keyes, La Selva Beach, Lathrop, Livermore, Long Barn, Madera, Mariposa, Modesto, Mountain Ranch, Novato, Pescadero, Pine Grove, Port Costa, Raisin, San Luis Obispo (press report), Salida, San Ardo, San Francisco (press report), San Francisco International Airport, San Jose, Stevinson, Stockton (press report), Sunol, Tollhouse, Villa Grande.

Intensity II: Clarksburg, Dos Palos, Kingsburg, Larkspur, Planada, San Lorenzo, Stinson Beach, Waterford.

Felt: Gilroy (press report).

\section{January (BK) Central California}

Origin time: 174707.5

Epicenter: $37.645 \mathrm{~N} ., 121.837 \mathrm{~W}$.

Depth: $4 \mathrm{~km}$

Magnitude: $2.9 \mathrm{M}_{\mathrm{L}}(\mathrm{BK})$

Moment: $6.6 \times 10^{20}$ dyne-cm (BK)

Felt: Livermore (BK), Pleasanton (BK).

2 February (BK) Northern California

Origin time: 193122.8

Epicenter: 40.802N., 124.063W.

Depth: $25 \mathrm{~km}$

Magnitude: $3.2 \mathrm{M}_{\mathrm{L}}(\mathrm{BK})$

Moment: $1.6 \times 10^{20}$ dyne-cm (BK)

Felt: Eureka (BK).

15 February (BK) Northern California

Origin time: 222701.0

Epicenter: 39.630N., 122.072W.

Depth: $22 \mathrm{~km}$

Magnitude: $3.1 \mathrm{M}_{\mathrm{L}}(\mathrm{BK})$

Moment: $1.0 \times 10^{22}$ dyne-cm (BK)

Intensity III: Durham.

Intensity II: Artois.

Felt: Chico (BK).

17 February (PS) Southern California

Origin time: 021233.5

Epicenter: $34.116 \mathrm{~N} ., 116.030 \mathrm{~W}$.

Depth: $11 \mathrm{~km}$ 
Magnitude: $4.0 \mathrm{M}_{\mathrm{L}}(\mathrm{PS}), 3.8 \mathrm{M}_{\mathrm{L}}(\mathrm{GP})$

Felt: Palm Springs (PS).

\section{February (PS) Southern California}

Origin time: 105838.6

Epicenter: 32.966N., 115.552W.

Depth: $8 \mathrm{~km}$

Magnitude: $3.3 \mathrm{M}_{\mathrm{L}}(\mathrm{PS}), 3.4 \mathrm{M}_{\mathrm{L}}(\mathrm{GP})$

Intensity IV: Brawley (press report).

18 February (PS) Southern California

Origin time: 012529.0

Epicenter: 32.962N., 115.543W.

Depth: $5 \mathrm{~km}$

Magnitude: $3.0 \mathrm{M}_{\mathrm{L}}$ (PS)

Felt: Brawley (press report).

18 February (PS) Southern California

Origin time: 012629.0

Epicenter: 32.957N., 115.554W.

Depth: $5 \mathrm{~km}$

Magnitude: $3.0 \mathrm{M}_{\mathrm{L}}(\mathrm{PS}), 3.1 \mathrm{M}_{\mathrm{L}}(\mathrm{GP})$

Felt: Brawley (press report).

19 February (GP) Southern California Origin time: 004724.5

Epicenter: 32.485 N., 117.567 W.

Depth: $6 \mathrm{~km}$

Magnitude: $3.9 \mathrm{M}_{\mathrm{L}}(\mathrm{PS}), 3.8 \mathrm{M}_{\mathrm{L}}(\mathrm{GP})$

Intensity III: San Diego (press report).

19 February (BK) Central California

Origin time: 030109.1

Epicenter: 36.827N., 121.277W.

Depth: 9 km

Magnitude: $3.1 \mathrm{M}_{\mathrm{L}}(\mathrm{BK})$

Moment: $1.2 \times 10^{21}$ dyne-cm (BK)

Felt: Hollister (press report).

19 February (BK) Central California

Origin time: 234907.7

Epicenter: 36.848N., 121.297W.

Depth: $9 \mathrm{~km}$

Magnitude: $3.1 \mathrm{M}_{\mathrm{L}}(\mathrm{BK})$,

Moment: $1.7 \times 10^{21}$ dyne-cm (BK)

Felt: Hollister (press report).

3 March (PS) Southern California

Origin time: 131820.3

Epicenter: 33.746 N., $117.525 W$.

Depth: $6 \mathrm{~km}$

Magnitude: $3.2 \mathrm{M}_{\mathrm{L}}(\mathrm{PS}), 3.3 \mathrm{M}_{\mathrm{L}}(\mathrm{GP})$

Intensity IV: Corona.
Intensity III: Canyon Lake, El Toro, Rio, Riverside, Silverado.

Felt: Colton (press report).

3 March (BK) Central California

Origin time: 144520.0

Epicenter: $36.835 \mathrm{~N} ., 121.268 \mathrm{~W}$.

Depth: $6 \mathrm{~km}$

Magnitude: $2.7 \mathrm{M}_{\mathrm{L}}$ (BK)

Felt: Hollister (BK).

9 March (BK) Central California

Origin time: 012813.0

Epicenter: 37.672N., 122.498W.

Depth: $7 \mathrm{~km}$

Magnitude: $3.0 \mathrm{M}_{\mathrm{L}}(\mathrm{BK})$

Moment: $4.8 \times 10^{20}$ dyne-cm (BK)

Felt: Daly City (BK), San Francisco area (BK).

9 March (PS) Southern California

Origin time: 224142.6

Epicenter: 34.113N., 117.769W.

Depth: $5 \mathrm{~km}$

Magnitude: $3.3 \mathrm{M}_{\mathrm{L}}(\mathrm{PS}), 3.5 \mathrm{M}_{\mathrm{L}}(\mathrm{GP})$

Intensity IV: Azusa, Claremont, La Verne, Mount Baldy, Pomona.

Intensity III: Cucamonga, Glendora, Guasti, Ontario, Upland.

10 March (GP) Southern California

Origin time: 153316.0

Epicenter: 34.403 N., $119.813 \mathrm{~W}$.

Depth: $24 \mathrm{~km}$

Magnitude: $4.4 \mathrm{~m}_{\mathrm{b}}(\mathrm{GS}), 4.1 \mathrm{M}_{\mathrm{L}}(\mathrm{PS}), 4.0 \mathrm{M}_{\mathrm{L}}(\mathrm{GP})$,

Intensity $\mathrm{V}$ : $4.4 \mathrm{M}_{\mathrm{L}}(\mathrm{BK})$

Goleta-A few windows cracked; a few glassware items or dishes broke; a few small objects overturned and fell; interior walls sustained hairline cracks; a foundation cracked; trees and bushes shook slightly; standing vehicles rocked slightly; felt by everyone.

Santa Barbara-People ran out into the streets; gas leaks were reported; it was felt on offshore drilling platforms; standing and moving vehicles rocked slightly; trees and bushes shook slightly; felt by most people.

Intensiy IV: Los Olivos, Santa Ynez, Solvang, Summerland, Ventura.

Intensity II: Oxnard.

16 March (PS) Southern California

Origin time: 014545.3

Epicenter: 34.150N., 117.313W.

Depth: $5 \mathrm{~km}$ 
Magnitude: $2.9 \mathrm{M}_{\mathrm{L}}(\mathrm{PS}), 3.0 \mathrm{M}_{\mathrm{L}}(\mathrm{GP})$

Felt: San Bernardino (press report).

19 March (GS) Central California

Origin time: 092739.2

Epicenter: 37.468 N., $118.611 W$.

Depth: 5 km

Magnitude: $3.4 \mathrm{M}_{\mathrm{L}}(\mathrm{PS}), 3.4 \mathrm{M}_{\mathrm{L}}(\mathrm{BK})$

Intensity III: Miramonte.

Intensity II: Auberry.

20 March (PS) Southern California

Origin time: 064940.3

Epicenter: 33.794N., 118.310W.

Depth: $10 \mathrm{~km}$

Magnitude: $3.2 \mathrm{M}_{\mathrm{L}}(\mathrm{PS}), 3.3 \mathrm{M}_{\mathrm{L}}(\mathrm{GP})$

Felt: Carson (press report), Downey (press report), Gardena (press report), Long Beach (press report), San Pedro (press report), Torrance (PS).

\section{March (BK) Central California}

Origin time: 045801.4

Epicenter: $38.845 N ., 122.887 \mathrm{~W}$.

Depth: $2 \mathrm{~km}$

Magnitude: $3.7 \mathrm{~m}_{\mathrm{b}}(\mathrm{GS}), 3.7 \mathrm{M}_{\mathrm{L}}(\mathrm{BK})$

Intensity V:

Cobb-A few small objects overturned; buildings shook strongly; windows, doors, and dishes rattled loudly; shaking was described as strong; felt by and frightened many people.

Intensity IV: Jenner, Loch Lomond.

Intensity III: Boyes Hot Springs, Freestone.

\section{March (PS) Southern California}

Origin time: 051440.0

Epicenter: $33.785 \mathrm{~N} ., 118.305 \mathrm{~W}$.

Depth: $8 \mathrm{~km}$

Magnitude: $2.8 \mathrm{M}_{\mathrm{L}}(\mathrm{PS})$

Intensity IV: Palos Verdes Peninsula, Paramount, Torrance, Wilmington.

Intensity III: Gardena, Lomita.

Felt: Carson (press report), Compton (press report), Long Beach (press report).

\author{
29 March (BK) Central California \\ Origin time: 162404.2 \\ Epicenter: 37.872N., 122.201W. \\ Depth: $9 \mathrm{~km}$ \\ Magnitude: $4.1 \mathrm{M}_{\mathrm{L}}(\mathrm{BK})$ \\ Moment: $2.5 \times 10^{22}$ dyne-cm (BK)
}

Intensity V:

Alameda Naval Air Station-Plaster cracked; small objects moved; windows and doors rattled.

Concord-A few windows cracked; a few items were shaken off store shelves; a few glassware items or dishes broke; a few small objects overturned or fell.

Kensington-Exterior stucco walls cracked and some stucco fell; shaking was described as strong; felt by and frightened many people.

Martinez-A few glassware items or dishes broke; a few small objects overturned and fell; felt by and frightened many people.

Pleasant Hill-A few windows cracked; a few items were shaken off store shelves; a few glassware items or dishes broke; a few small objects overturned and fell; a bottom hinge on the entrance door of the post office was broken; felt by many people.

Intensity IV: Alameda, Albany, Berkeley, Berkeley (Grizzly Peak area-press report), Canyon, Danville, Diablo, El Cerrito (a plate-glass store window cracked), Hayward, Hercules, Lafayette, Moraga, Martinez, Oakland, Pacheco, Pinole, Port Costa, Richmond, Rodeo, Ross, San Pablo, South Berkeley.

Intensity III: San Leandro, Vallejo.

Intensity II: Oakland International Airport.

Felt: Mill Valley (press report), San Francisco (press report).

\section{March (BK) Central California}

Origin time: $115540: 1$

Epicenter: 37.488N., 121.693W.

Depth: $8 \mathrm{~km}$

Magnitude: $5.5 \mathrm{~m}_{\mathrm{b}}(\mathrm{GS}), 5.5 \mathrm{M}_{S}(\mathrm{GS}), 5.7 \mathrm{M}_{\mathrm{L}}(\mathrm{BK})$

Moment: $2.6 \times 10^{24}$ dyne-cm (BK)

This earthquake, called the Mount Lewis earthquake, caused minor injuries to six people (two in Fremont and four in San Jose) and light damage in Fremont, Mount Hamilton, Newark, and San Jose. It was felt over a land area of about $39,500 \mathrm{~km}^{2}$ (fig. 10). No surface rupture was reported by geologists in the field (Bolt and Uhrhammer, 1986).

The Mount Lewis earthquake was preceded by two $\mathrm{M}_{\mathrm{L}} 2.6$ foreshocks, the first on March 24 at 01:54 UTC and the second on March 31 at 04:05 UTC, and was followed by 22 aftershocks with magnitudes $\geq M_{L} 2.5$ (Bolt and Uhrhammer, 1986).

Thiel and Arnold (1986) reported a peak acceleration of $0.31 \mathrm{~g}$ in the north-south direction from the strongmotion records for the roof of the Santa Clara County Administration building. Damage to the building was estimated at between $\$ 45,000$ and $\$ 100,000$; most of the damage was to the elevator. The other damage occurred on 


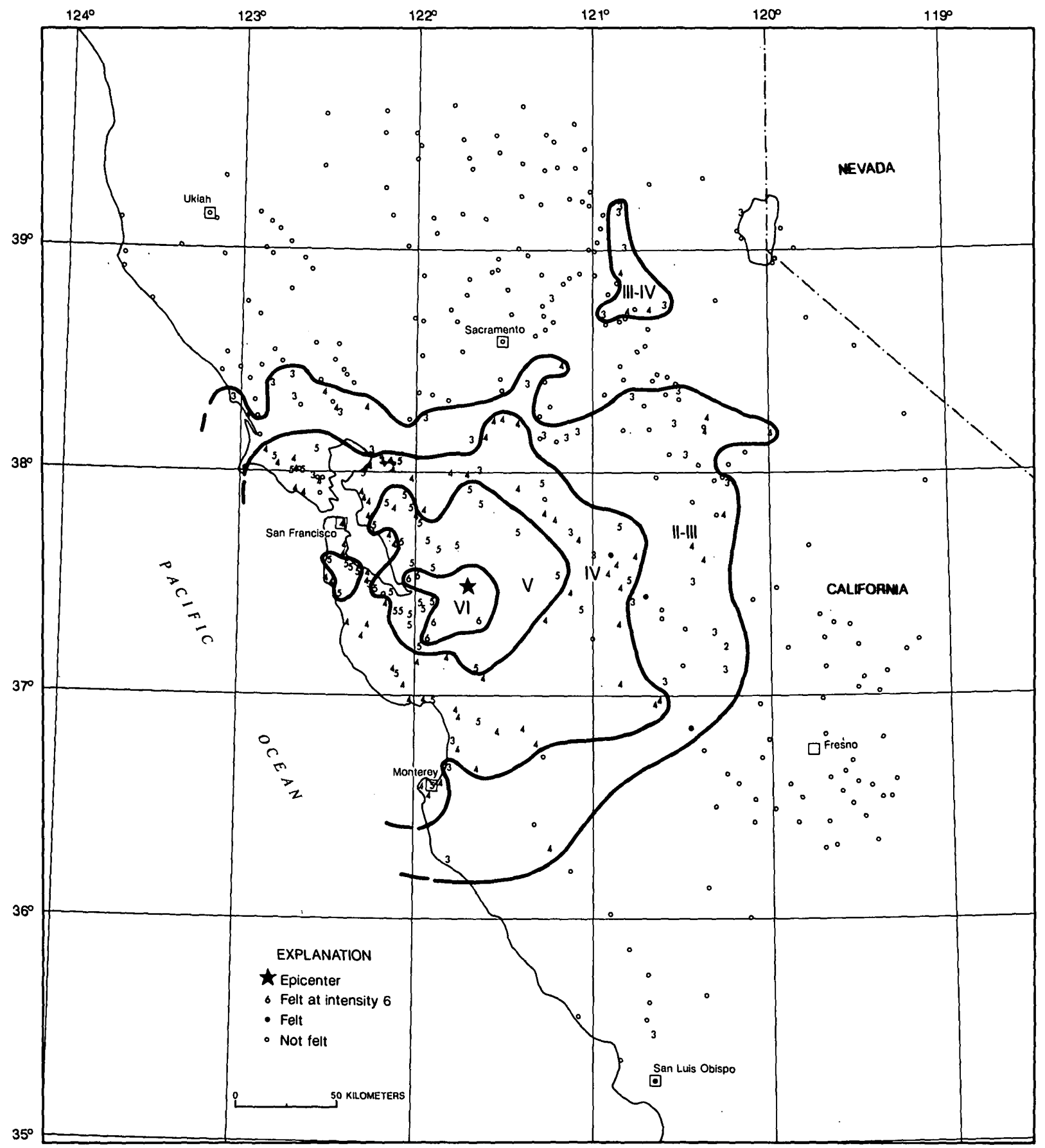

Figure 10. Isoseismal map for the Mount Lewis, California, earthquake of 31 March, 1986, 115540.1 UTC. Roman numerals represent Modified Mercalli intensities between isoseismals; Arabic numerals represent intensities at specific sites; dashed contour lines are inferred isoseismals, and small boxes show locations of towns and cities whose names are plotted. 


\section{CALIFORNIA-Continued}

the upper stories of the building, from the 7th through the 12th floors, where cabinets and bookcases were thrown down, glass panels broken, and file cabinets tipped over. Other damage was to overhead power lines (15 burned; 15 had fuses burned out, causing some disruption; and 14 shorted) (Schiff, 1986).

\section{Intensity VI:}

Fremont-A water line broke at a Safeway warehouse; many bottles broke at Abe's Liquors; at Lucky Food Center many jars and bottles broke; 14 ceiling tiles fell; and a 3-month-old store wall cracked; at SyQuest Technology a half-dozen wooden structural beams were cracked (press reports). A few windows cracked; many small objects overturned and fell; buildings shook strongly; felt by and frightened many people.

Mount Hamilton-Exterior reinforced-concrete walls cracked; many glassware items or dishes broke; many small objects overturned and fell; hanging pictures fell; buildings shook strongly; standing vehicles rocked moderately; trees and bushes shook moderately. At Lick Observatory several existing cracks in a building were widened; some fittings in the hydraulic system of the telescope were cracked and required repair (press report).

Newark-Ceiling tiles fell in the Walgreen drug store; picture frames, clocks, and ceramic gift items fell to the floor and broke in Scribbles and Giggles store; merchandise fell off store shelves in Alpha Beta Market and the floor had a small crack (press report).

San Jose-Chimneys cracked; foundations cracked; tombstones fell; interior walls sustained hairline cracks; small appliances overturned; many glassware items or dishes broke; many items were shaken off store shelves; buildings shook strongly; people had difficulty standing or walking; felt by many people. The Santa Clara Administration building had damage to two elevators (one guide rail was bent; one brake failed; and several motor generator sets moved); many file cabinets and bookcases were thrown over on the upper floors (Thiel and Amold, 1986). Twelve power lines burned, causing power failure (Schiff, 1986).

San Jose (Cambrian Park)-Some windows were broken out; a few items were shaken off store shelves; a few glassware items or dishes broke; a few small objects overturned and fell; felt by and frightened many people.

The most common effects at the places listed below were that: shaking was described as moderate; buildings shook moderately; a few small objects overturned and fell;

\section{CALIFORNIA-Continued}

pictures swung with some left out of place; windows, doors, and dishes rattled; felt by many people.

Intensity $V$ :

Agnew-A few windows cracked; a few glassware items or dishes broke; a few small appliances overturned; a few items were shaken off store shelves; many small objects overturned and fell; trees and bushes shook moderately.

Alameda-A few items were shaken off store shelves; windows, doors, and dishes rattled loudly.

Alamo-Dry wall sustained hairline cracks; windows, doors, and dishes rattled loudly.

Alviso-A few windows cracked; a few glassware items or dishes broke; people had difficulty standing.

Aptos-A few items were shaken off store shelves; hanging pictures fell; small appliances overturned; a few glassware items or dishes broke; many small objects overturned and fell; trees and bushes shook moderately.

Aromas-Interior walls sustained hairline cracks.

Benicia-Plaster walls sustained hairline cracks.

Brentwood-Interior walls sustained hairline cracks; trees and bushes shook slightly.

Brookdale.

Burlingame-A few items fell from shelves; racked an armoire (press report).

Byron-Buildings shook strongly; windows, doors, and dishes rattled loudly.

Canyon-People had difficulty standing; trees and bushes shook slightly.

Crows Landing-Small appliances overturned.

Cupertino-A few windows cracked.

Denair-A few glassware items or dishes broke.

Dublin-People had difficulty standing; interior walls sustained hairline cracks; mortar was loosened on a fireplace.

East Palo Alto-A few glassware items or dishes broke; windows, doors, and dishes rattled loudly; shaking was described as strong.

Half Moon Bay-A few items were shaken off store shelves; a few glassware items or dishes broke; buildings shook strongly.

Lagunitas-A few windows cracked; a few glassware items or dishes broke.

Livermore-A water heater was knocked off its supports; minor rockslides occurred nearby (press report).

Los Altos-Dry wall sustained hairline cracks.

Los Gatos-Plaster walls sustained hairline cracks; moving vehicles rocked slightly; trees and bushes shook slightly.

Millbrae-A few small appliances overturned.

Milpitas-There were hairline cracks in a wall of one of the minimum security buildings of the Elmwood Rehabilitation Center (press report). A few glassware items or 


\section{CALIFORNIA-Continued}

dishes broke; a foundation cracked; buildings shook strongly.

Monterey-A few windows cracked; a few glassware items or dishes broke.

Morgan Hill-Dry wall sustained hairline cracks.

Mountain View-A few items were shaken off store shelves; standing and moving vehicles rocked slightly; trees and bushes shook slightly; small appliances overturned.

Novato-Standing and moving vehicles rocked slightly; trees and bushes shook slightly.

Oakdale-Plaster walls sustained hairline cracks.

Pacifica-A few items shook off of shelves in the Central Market (press report).

Pinole-Interior walls sustained hairline cracks; there were cracks in the street; trees and bushes shook slightly.

Pleasanton-Interior walls sustained hairline cracks; standing vehicles rocked moderately; trees and bushes shook moderately; hanging objects swung violently.

Point Reyes Station.

Redwood City-Plaster walls sustained hairline cracks; a few items fell from shelves; standing vehicles rocked slightly; trees and bushes shook slightly; felt by everyone.

San Jose (Blossom Hill)-A few items were shaken off store shelves; standing vehicles rocked slightly; trees and bushes shook slightly.

San Mateo (press report)-A few items shook off of shelves (press report).

San Pablo-A few windows cracked; a few items were shaken off store shelves; interior walls sustained hairline cracks; a few glassware items or dishes broke.

Stockton (press report)-Pictures were knocked off walls; water sloshed in a swimming pool; a few items were shaken off store shelves (press report).

Sunnyvale-Interior walls sustained hairline cracks.

Sunol-Hanging pictures fell.

Tracy-A few glassware items or dishes broke; windows, doors, and dishes rattled loudly.

Union City-Many items were shaken off store shelves; a few small appliances overturned; a few glassware items or dishes broke; buildings shook strongly.

Walnut Creek-A few items were shaken off store shelves. Westley-A few items were shaken off store shelves.

Woodacre-Plaster walls sustained hairline cracks; windows, doors, and dishes rattled loudly; shaking was described as strong.

Intensity IV: Albany, Antioch, Arnold, Belmont, Berkeley, Big Oak Flat, Bolinas, Boulder Creek, Brisbane, Camino, Campbell, Capitola, Carmel, Castroville, Concord, Danville, Diablo, Dillon Beach, Dos Palos, El Cerrito, El Granada, Felton, Forest Knolls, Foster City (press report),

\section{CALIFORNIA-Continued}

Freedom, Georgetown, Glen Ellen, Greenfield, Hathaway Pines, Hayward, Hercules, Hickman, Hollister, Holt, Hughson, Inverness, Isleton, Keyes, La Grange, La Honda, Lathrop, Loma Mar, Los Banos, Manteca, Martinez, Millbrae, Moffett Field, Montara, Moraga, Moss Beach, Mount Hermon, Napa, New Almaden, Nicasio, Oakland, Oakley, Olema, Patterson, Pebble Beach, Pinecrest, Placerville, Port Costa, Redwood Estates, Rheem Valley, Rodeo, Ross, Ryde, Salida, Salinas, San Bruno, San Carlos, San Francisco International Airport, San Francisco, San Gregorio, San Juan Bautista, San Leandro, San Lorenzo, San Martin, Santa Clara, Santa Cruz, Seaside, Slough House, Sonoma, South Dos Palos, Stevinson, Stinson Beach, Thornton, Tres Pinos, Turlock, Walnut Grove, Waterford, Watsonville.

Intensity III: Alamo, Atascadero, Bethel Island, Big Sur, Bodega Bay, Chowchilla, Clements, Crockett, Delhi, Dutch Flat, Elk Grove, Fairfax, Foresthill, Gold Run, Jackson, Lockeford, Marina, Modesto, Moss Landing, Mountain Ranch, Planada, Pollock Pines, Rescue, Rio Vista, Ripon, Rocklin, Rohnert Park, Santa Rita Park, Santa Rosa, Sebastopol, Snelling, Sonoma, South San Francisco, Tahoe City, Travis Air Force Base, Tuolumne, Vallecito, Vallejo, Vineburg, Wilseyville.

Intensity II: Le Grand.

Felt: Ballico, Empire, Firebaugh, San Luis Obispo (press report).

\section{April (PS) Southern California}

Origin time: 003050.6

Epicenter: 33.975 N., 117.248W.

Depth: $13 \mathrm{~km}$

Magnitude: $2.6 \mathrm{M}_{\mathrm{L}}(\mathrm{PS})$

Felt: Riverside (PS).

\section{April (PS) Southern California}

Origin time: 065040.4

Epicenter: 33.730N., 118.010W.

Depth: $14 \mathrm{~km}$

Magnitude: $3.6 \mathrm{M}_{\mathrm{L}}(\mathrm{PS}), 3.9 \mathrm{M}_{\mathrm{L}}(\mathrm{GP})$

Intensity V:

Huntington Beach-Plaster walls sustained hairline cracks; a few small objects overturned and fell; a few items were shaken off store shelves; buildings shook moderately; felt by many people.

Long Beach-Plaster walls sustained hairline cracks; a few small objects overturned and fell; a few glassware items or dishes broke; a few windows cracked; buildings shook strongly; felt by many people.

Santa Ana-A few small objects overturned and fell; buildings shook moderately; shaking was described as strong; felt by many people.

Intensity IV: Buena Park, Cerritos, Costa Mesa, Cypress, Fountain Valley, Fullerton, Garden Grove, La Mirada, Los 
Alamitos, Paramount, Seal Beach, South Gate, Stanton, Sunset Beach, Torrance, Upland, Walnut, Westminster, Whittier, Yorba Linda.

Intensity III: Anaheim, Artesia, La Mirada, Norwalk, Palos Verdes, Santa Ana.

Intensity II: Glendora.

Felt: Orange (press report).

\section{April (PS) Southern California}

Origin time: 172149.5

Epicenter: $33.336 \mathrm{~N} ., 115.709 \mathrm{~W}$.

Depth: $3 \mathrm{~km}$

Magnitude: $3.8 \mathrm{M}_{\mathrm{L}}(\mathrm{PS}), 3.7 \mathrm{M}_{\mathrm{L}}(\mathrm{GP})$

Intensity IV: Bombay Beach (press report).

Intensity III: Niland.

\section{April (BK) Central California}

Origin time: 092556.7

Epicenter: 36.677 N., 121.347W.

Depth: $4 \mathrm{~km}$

Magnitude: $3.6 \mathrm{M}_{\mathrm{L}}(\mathrm{BK})$

Moment: $3.6 \times 10^{21}$ dyne-cm (BK)

Intensity II: San Juan Bautista (press report).

\section{April (BK) Central California}

Origin time: 110021.7

Epicenter: $38.230 \mathrm{~N} ., 122.178 \mathrm{~W}$.

Depth: $3 \mathrm{~km}$

Magnitude: $2.3 \mathrm{M}_{\mathrm{L}}(\mathrm{BK})$

Felt: Suisun Bay area (BK).

20 April (PS) Southern California

Origin time: 124549.2

Epicenter: $34.224 \mathrm{~N} ., 117.469 \mathrm{~W}$.

Depth: $12 \mathrm{~km}$

Magnitude: $2.7 \mathrm{M}_{\mathrm{L}}(\mathrm{PS})$

Felt: San Bernardino (PS).

21 April (PS) Southern California

Origin time: 091217.1

Epicenter: 34.378N., 119.768W.

Depth: $11 \mathrm{~km}$

Magnitude: $3.0 \mathrm{M}_{\mathrm{L}}(\mathrm{PS})$

Felt: Goleta (PS), Santa Barbara (PS).

\section{April (BK) Central California}

Origin time: 163506.0

Epicenter: $37.428 \mathrm{~N} ., 121.800 \mathrm{~W}$.

Depth: $2 \mathrm{~km}$

Magnitude: $2.2 \mathrm{M}_{\mathrm{L}}(\mathrm{BK})$

Felt: Milpitas (BK), San Jose (BK).
28 April (BK) Central California

Origin time: 173347.8

Epicenter: 37.478N., 121.693W.

Depth: $7 \mathrm{~km}$

Magnitude: $3.5 \mathrm{M}_{\mathrm{L}}(\mathrm{BK})$

Moment: $3.9 \times 10^{21}$ dyne-cm (BK)

Felt: Fremont (press report), Pleasanton (press report).

28 April (BK) Central California

Origin time: 221840.6

Epicenter: $36.815 \mathrm{~N} ., 121.258 \mathrm{~W}$.

Depth: $8 \mathrm{~km}$

Magnitude: $3.6 \mathrm{M}_{\mathrm{L}}(\mathrm{BK})$

Moment: $3.2 \times 10^{21}$ dyne-cm (BK)

Intensity IV: Paicines, Tres Pinos.

30 April (BK) Northern California

Origin time: 223730.4

Epicenter: $40.760 \mathrm{~N} ., 124.560 \mathrm{~W}$.

Depth: $19 \mathrm{~km}$

Magnitude: $3.5 \mathrm{~m}_{\mathrm{b}}(\mathrm{GS}), 3.9 \mathrm{M}_{\mathrm{L}}(\mathrm{BK})$

Moment: $1.3 \times 10^{21}$ dyne-cm (BK)

Intensity III: Samoa.

Felt: Eureka (telephone report).

2 May (PS) Southern California

Origin time: 081907.9

Epicenter: $33.677 \mathrm{~N} ., 116.826 \mathrm{~W}$.

Depth: $12 \mathrm{~km}$

Magnitude: $2.6 \mathrm{M}_{\mathrm{L}}$ (PS)

Felt: Epicentral area (PM).

10 May (BK) Central California

Origin time: 223013.1

Epicenter: 37.348 N., $122.288 \mathrm{~W}$.

Depth: $13 \mathrm{~km}$

Magnitude: $2.7 \mathrm{M}_{\mathrm{L}}(\mathrm{BK})$

Moment: $1.2 \times 10^{20}$ dyne-cm (BK)

Felt: Redwood City (BK).

12 May (BK) Central California

Origin time: 091709.3

Epicenter: 37.462N., 121.693W.

Depth: $6 \mathrm{~km}$

Magnitude: $3.1 \mathrm{M}_{\mathrm{L}}(\mathrm{BK})$

Moment: $7.2 \times 10^{20}(\mathrm{BK})$

Felt: San Jose (BK), Sunnyvale (BK).

12 May (BK) Central California

Origin time: 230019.7

Epicenter: 36.848 N., 121.297W.

Depth: $6 \mathrm{~km}$

Magnitude: $3.2 \mathrm{M}_{\mathrm{L}}(\mathrm{BK}), 3.5 \mathrm{M}_{\mathrm{L}}(\mathrm{GP})$ 


\section{CALIFORNIA-Continued}

Moment: $1.8 \times 10^{21}$ dyne-cm (BK)

Felt: San Benito County (BK).

\section{May (PS) Southern California}

Origin time: 115540.3

Epicenter: 33.793N., 118.312W.

Depth: $10 \mathrm{~km}$

Magnitude: $2.7 \mathrm{M}_{\mathrm{L}}(\mathrm{PS})$

Felt: Carson (PS).

13 May (PS) Southern California

Origin time: 163545.0

Epicenter: 33.791N., 118.302W.

Depth: $8 \mathrm{~km}$

Magnitude: $2.7 \mathrm{M}_{\mathrm{L}}(\mathrm{PS})$

Felt: Epicentral area (PS).

\section{May (BK) Central California}

Origin time: 003009.6

Epicenter: 37.363N., 122.262W.

Depth: $14 \mathrm{~km}$

Magnitude: $3.2 \mathrm{M}_{\mathrm{L}}(\mathrm{BK})$

Moment: $7.8 \times 10^{20}$ dyne-cm (BK)

Felt: Monterey (press report), Palo Alto (BK), Redwood City (BK).

15 May (BK) Central California

Origin time: 083202.1

Epicenter: 37.477N., 121.695W.

Depth: $7 \mathrm{~km}$

Magnitude: $3.3 \mathrm{M}_{\mathrm{L}}(\mathrm{BK})$

Moment: $9.5 \times 10^{20}$ dyne-cm (BK)

Felt: Fremont (BK), San Jose (BK).

19 May (PS) Southern California

Origin time: 041253.3

Epicenter: 33.892N., 118.387W.

Depth: $10 \mathrm{~km}$

Magnitude: $3.0 \mathrm{M}_{\mathrm{L}}(\mathrm{PS}), 3.1 \mathrm{M}_{\mathrm{L}}(\mathrm{GP})$

Felt: Torrance (PS).

20 May (PS) Southern California

Origin time: 071140.2

Epicenter: 33.940N., 118.668W.

Depth: $6 \mathrm{~km}$

Magnitude: $2.8 \mathrm{M}_{\mathrm{L}}(\mathrm{PS})$

Felt: Santa Monica (PS).

23 May (PS) Central California

Origin time: 114155.1

Epicenter: 35.806N., 118.019W.

Depth: $10 \mathrm{~km}$

\section{CALIFORNIA-Continued}

Magnitude: $3.6 \mathrm{~m}_{\mathrm{b}}(\mathrm{GS}), 4.1 \mathrm{M}_{\mathrm{L}}(\mathrm{PS}), 3.9 \mathrm{M}_{\mathrm{L}}(\mathrm{GP})$, 4.0 $\mathrm{M}_{\mathrm{L}}(\mathrm{BK})$

Intensity IV: Wofford Heights.

Intensity III: Bakersfield, Ducor, Edison, Lake Isabella, Tehachapi.

Intensity II: Onyx.

25 May (BK) Central California

Origin time: 095103.1

Epicenter: 38.810N., 122.798W.

Depth: $4 \mathrm{~km}$

Magnitude: $3.2 \mathrm{M}_{\mathrm{L}}(\mathrm{BK})$

Moment: $5.4 \times 10^{21}$ dyne-cm (BK)

Felt: Lake Berryessa (BK).

31 May (BK) Central California

Origin time: 084756.1

Epicenter: 36.618N., 121.255W.

Depth: $4 \mathrm{~km}$

Magnitude: $4.6 \mathrm{~m}_{\mathrm{b}}(\mathrm{GS}), 3.7 \mathrm{M}_{\mathrm{S}}(\mathrm{GS}), 4.7 \mathrm{M}_{\mathrm{L}}(\mathrm{BK})$

Moment: $8.0 \times 10^{22}$ dyne-cm (BK)

Intensity IV: Hollister (3 mi south), Paicines, Salinas, Tres Pinos.

Intensity III: Big Sur, Carmel, Castroville, Freedom, Moss Landing, Seaside, Watsonville.

3 June (PS) Southern California

Origin time: 141449.3

Epicenter: 33.788N., 116.344W.

Depth: $11 \mathrm{~km}$

Magnitude: $3.7 \mathrm{M}_{\mathrm{L}}(\mathrm{PS}), 3.7 \mathrm{M}_{\mathrm{L}}(\mathrm{GP})$

Intensity IV: Palm Desert (press report).

Felt: Palm Springs (press report).

9 June (BK) Central California

Origin time: 044805.8

Epicenter: 37.960N., 121.668W.

Depth: $1 \mathrm{~km}$

Magnitude: $2.1 \mathrm{M}_{\mathrm{L}}(\mathrm{BK})$

Felt: Brentwood area (BK).

11 June (BK) Central California

Origin time: 130301.8

Epicenter: 36.945N., 121.618W.

Depth: $6 \mathrm{~km}$

Magnitude: $2.7 \mathrm{M}_{\mathrm{L}}(\mathrm{BK})$

Felt: Gilroy (BK).

11 June (BK) Central California

Origin time: 150859.5

Epicenter: $36.620 \mathrm{~N} ., 121.277 \mathrm{~W}$.

Depth: $7 \mathrm{~km}$

Magnitude: $3.5 \mathrm{M}_{\mathrm{L}}(\mathrm{BK}), 3.5 \mathrm{M}_{\mathrm{L}}(\mathrm{PS})$ 


\section{CALIFORNIA-Continued}

Moment: $6.2 \times 10^{21}(\mathrm{BK})$

Felt: Hollister (BK), Salinas (BK).

13 June (GP) Central California

Origin time: 132515.4

Epicenter: $36.060 \mathrm{~N} ., 119.938 \mathrm{~W}$.

Depth: $6 \mathrm{~km}$

Magnitude: $3.6 \mathrm{M}_{\mathrm{L}}(\mathrm{GP}), 3.9 \mathrm{M}_{\mathrm{L}}(\mathrm{PS})$

Intensity II: Orosi.

18 June (PS) Southern California

Origin time: 141326.4

Epicenter: 33.935N., 116.742W.

Depth: $17 \mathrm{~km}$

Magnitude: $3.5 \mathrm{M}_{\mathrm{L}}(\mathrm{PS}), 3.4 \mathrm{M}_{\mathrm{L}}(\mathrm{GP})$

Felt: Palm Springs (PS).

23 June (PS) Baja California, Mexico

Origin time: 234608.5

Epicenter: $32.174 \mathrm{~N} ., 115.160 \mathrm{~W}$.

Depth: $6 \mathrm{~km}$

Magnitude: $4.4 \mathrm{~m}_{\mathrm{b}}(\mathrm{GS}), 4.4 \mathrm{M}_{\mathrm{L}}(\mathrm{PS})$

Intensity IV: Arizona-San Luis.

Intensity II: California-Bard.

Felt: Arizona-Yuma (telephone report).

26 June (PS) Southern California

Origin time: 053947.8

Epicenter: $33.869 \mathrm{~N} ., 118.450 \mathrm{~W}$.

Depth: $7 \mathrm{~km}$

Magnitude: $3.2 \mathrm{M}_{\mathrm{L}}(\mathrm{PS}), 3.4 \mathrm{M}_{\mathrm{L}}(\mathrm{GP})$

Intensity IV: Compton, Manhattan Beach, Palos Verdes Estates, Santa Monica, Torrance, Venice.

Intensity III: Redondo Beach.

Felt: Hermosa Beach (press report), Lennox (press report), Long Beach (PS), Los Angeles (press report).

\section{June (BK) Central California}

Origin time: 040643.4

Epicenter: $37.745 N$ N., 121.982W.

Depth: $4 \mathrm{~km}$

Magnitude: $2.4 \mathrm{M}_{\mathrm{L}}(\mathrm{BK})$

Felt: Danville (BK), San Ramon (BK).

30 June (BK) Central California

Origin time: 110005.9

Epicenter: 37.842N., 121.763W.

Depth: $11 \mathrm{~km}$

Magnitude: $3.3 \mathrm{M}_{\mathrm{L}}(\mathrm{BK})$

Moment: $1.7 \times 10^{21}$ dyne-cm (BK)

Felt: Alamo (BK), Berkeley (BK), Danville (BK), Richmond (BK).

\section{CALIFORNIA-Continued}

2 July Central California

Origin time 0947

Epicenter: Not located.

Depth: None computed.

Magnitude 1.6 $\mathrm{M}_{\mathrm{L}}(\mathrm{BK})$

Intensity IV: Willits.

7 July (GP) Southern California

Origin time: 091322.7

Epicenter: 34.149N., 117.744W.

Depth: 5 km

Magnitude: $3.0 \mathrm{M}_{\mathrm{L}}(\mathrm{PS})$

Felt: Fontana (PS).

8 July (BK) Central California

Origin time: 004023.4

Epicenter: $36.083 \mathrm{~N} ., 121.827 \mathrm{~W}$.

Depth: 19 km

Magnitude: $4.4 \mathrm{~m}_{\mathrm{b}}(\mathrm{GS}), 4.4 \mathrm{M}_{\mathrm{L}}(\mathrm{BK}), 3.6 \mathrm{M}_{\mathrm{L}}(\mathrm{PS})$

Moment: $2.6 \times 10^{22}$ dyne-cm (BK)

Intensity IV: Big Sur.

Intensity III: Monterey.

Intensity II: Lockwood.

8 July (GP) Southern California

Origin time: 092044.5

Epicenter: $33.998 \mathrm{~N} ., 116.606 \mathrm{~W}$.

Depth: $12 \mathrm{~km}$

Magnitude: $5.8 \mathrm{~m}_{\mathrm{b}}(\mathrm{GS}), 6.0 \mathrm{MS}_{\mathrm{S}}(\mathrm{GS}), 5.9 \mathrm{M}_{\mathrm{L}}(\mathrm{PS})$,

$$
5.6 \mathrm{M}_{\mathrm{L}}(\mathrm{GP})
$$

Moment: $2.3 \times 10^{25}$ dyne-cm (GS)

This earthquake, known as the North Palm Springs, Calif., earthquake, caused minor injuries to 40 people and an estimated loss of $\$ 6$ million to structures and their contents (press reports). It was felt over an area of about $130,500 \mathrm{~km}^{2}$ of Arizona, California, and Nevada (fig. 11). Most of the damage occurred in Riverside County where 4 homes and 16 businesses were destroyed; 102 homes (many of the damaged homes were mobile homes) and 117 businesses were damaged (Earthquake Engineering Research Institute, 1986). There was also major damage to a highway bridge on Interstate 10 and to the Southern California Edison Devers Substation (fig. 12) near the intersection of Interstate 10 and State Highway 62. Minor landslides temporarily closed State Highways 62, 74, and 243. Jones and others (1986) suggested that this earthquake probably occurred on the Banning fault.

The most serious damage was located in the northern end of Coachella Valley and in the Whitewater Canyon area. The Whitewater bridge over Interstate 10 (about $9 \mathrm{mi}$ northwest of Palm Springs) was displaced laterally, leaving a 6-in. gap between the deck and the abutment. In the 


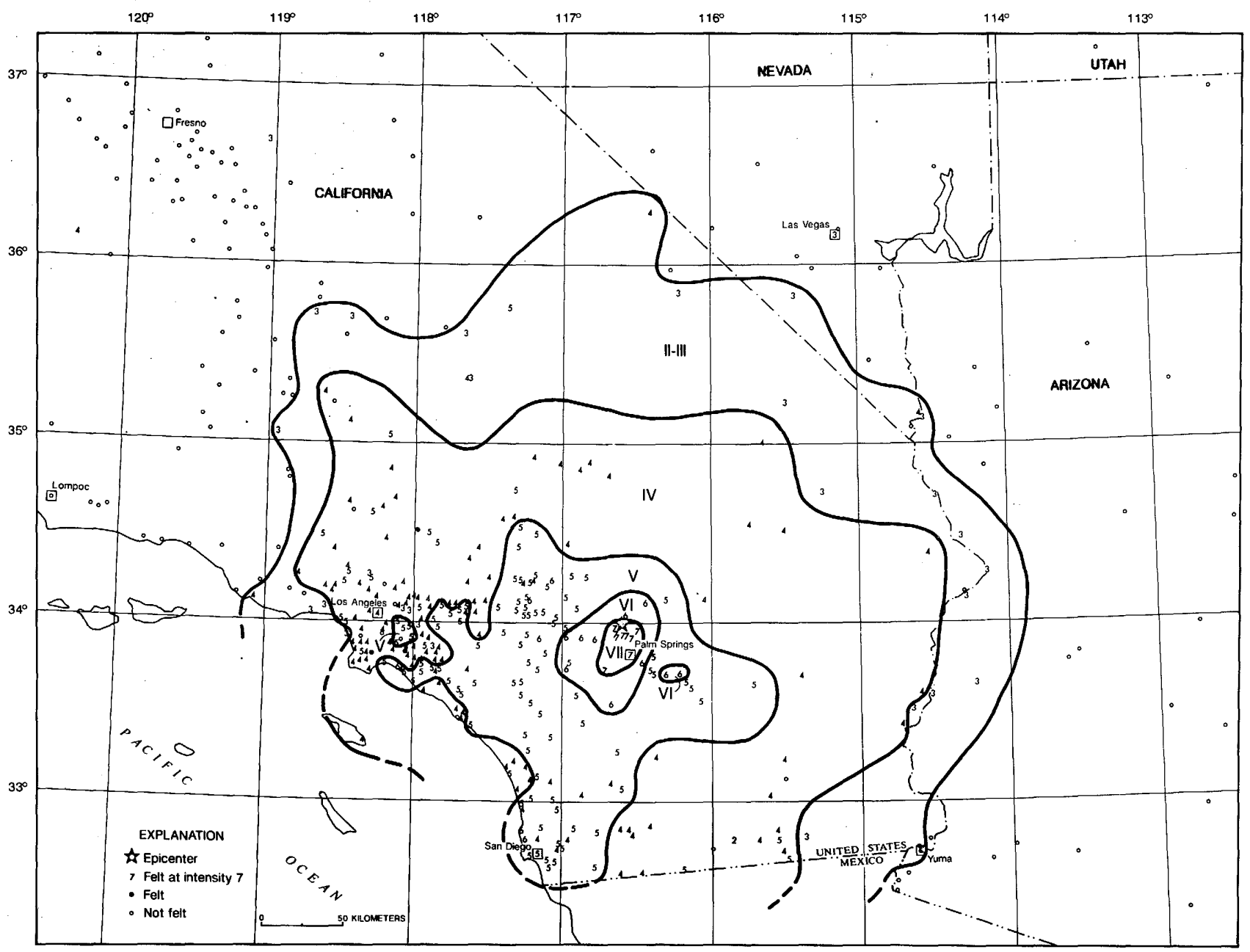

Figure 11. Isoseismal map for the North Palm Springs, California, earthquake of 8 July, 1986, 092044.5 UTC. Roman numerals represent Modified Mercalli intensities between isoseismals; Arabic numerals represent intensities at specific sites; dashed contour lines are inferred isoseismals, and small boxes show locations of towns and cities whose names are plotted.

\section{CALIFORNIA-Continued}

Whitewater Canyon area, three homes were destroyed when walls were severely cracked, with some partial collapse, and chimneys fell (figs. 13 and 14). In Coachella Valley, near the intersection of Interstate 10 and State Highway 62, the Southern CaliforniaEdison substation was severely damaged when many ceramic columns were broken and when transformers sheared retaining bolts and moved as much as $10 \mathrm{in}$. (Earthquake Engineering Research Institute, 1986; Borchardt and Manson, 1986).

En echelon fractures formed along the Banning fault for about $9 \mathrm{~km}$ on both sides of State Highway 62 north of Palm Springs; the fractures had a tiny $(<1 \mathrm{~mm})$ right-lateral offset (Sharp and others, 1986).

\section{CALIFORNIA-Continued}

The accelerations recorded on the four accelerographs nearest the epicenter were $0.44 \mathrm{~g}$ vertical and $0.66 \mathrm{~g}$ horizontal at the Whitewater Trout Farm $5 \mathrm{~km}$ northwest; $0.78 \mathrm{~g}$ vertical and $0.70 \mathrm{~g}$ horizontal at North Palm Springs $9 \mathrm{~km}$ southeast; $0.59 \mathrm{~g}$ vertical and $0.33 \mathrm{~g}$ horizontal at Desert Hot Springs $10 \mathrm{~km}$ east; and $0.35 \mathrm{~g}$ vertical and $0.23 \mathrm{~g}$ horizontal at Morongo Valley $10 \mathrm{~km}$ north (from Earthquake Engineering Research Institute, 1986; Borchardt and Manson, 1986).

This earthquake also caused failure of the electrical power, failure of the telephone systems, breaks in water and gas lines, and failure of two Metropolitan Water District pumping stations. The power failure affected 


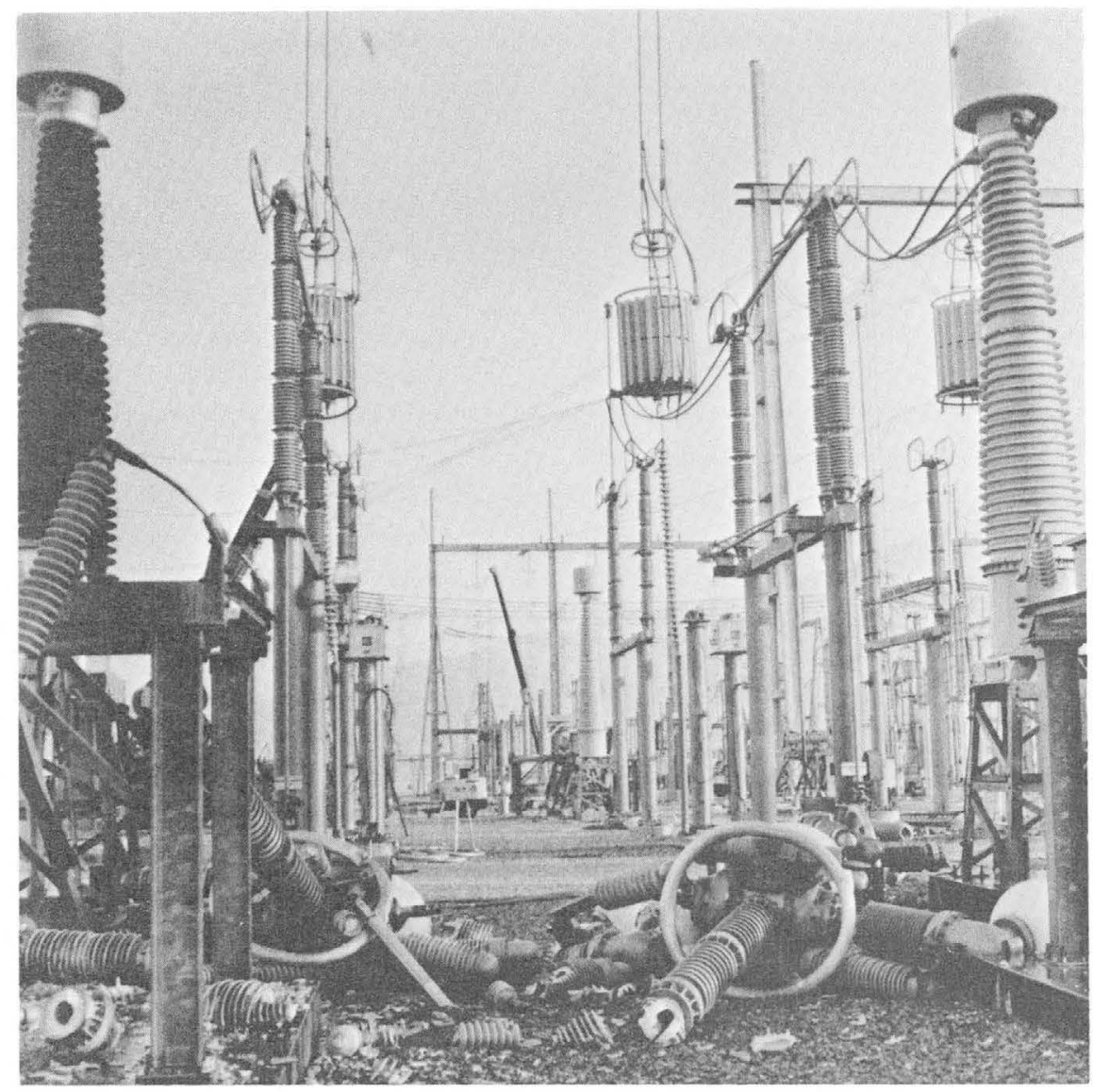

Figure 12. Devers electrical substation, Palm Springs, showing relatively tall porcelainglass insulators on 10-ft perches. Insulators built in 1982 were destroyed by the July 8, 1986, earthquake (Borchardt and Manson, 1986).

\section{CALIFORNIA-Continued}

100,000 people for several hours. Telephone failure was minimal; most problems were due to overloading the system. Most water- and gas-line failures occurred in private residences. The pumping-station failures on the Colorado River Aqueduct caused 977 million gallons of water to be dumped into the desert.

\section{Intensity VII:}

\section{California-}

Desert Hot Springs-Many plate-glass windows were broken in businesses; water lines were broken in many places; two condominiums at Mission Lake Country Club sustained large cracks in walls and broken water lines that flooded some units. Ceiling panels fell in many commercial buildings; many items shook off of shelves in stores; books were shaken off shelves in the library and some shelves were knocked over. At Von's

\section{CALIFORNIA-Continued}

Market a 5-in. water main was broken; a ceiling beam separated from the roof over the meat department; ceiling tiles fell; and much of the merchandise was thrown onto the floor. At City Hall, filing cabinets were tipped over (information from press and fire-department reports).

Devers substation (Southern California Edison) $2 \mathrm{mi}$ northwest of North Palm Springs.- This substation is about 200 meters north of the Banning fault, which displayed minor cracks just south of the substation. Most damage was concentrated in ceramic columns in transformer lightning arrestors, bus taps, disconnect switches, and circuit breakers. Heaviest damage was in the $500-\mathrm{kV}$ switchyard (fig. 12). About 75 percent of the ceramic members were destroyed. One $650,000-1 b$ transformer moved $10 \mathrm{in}$., shearing the four 1 -in. 


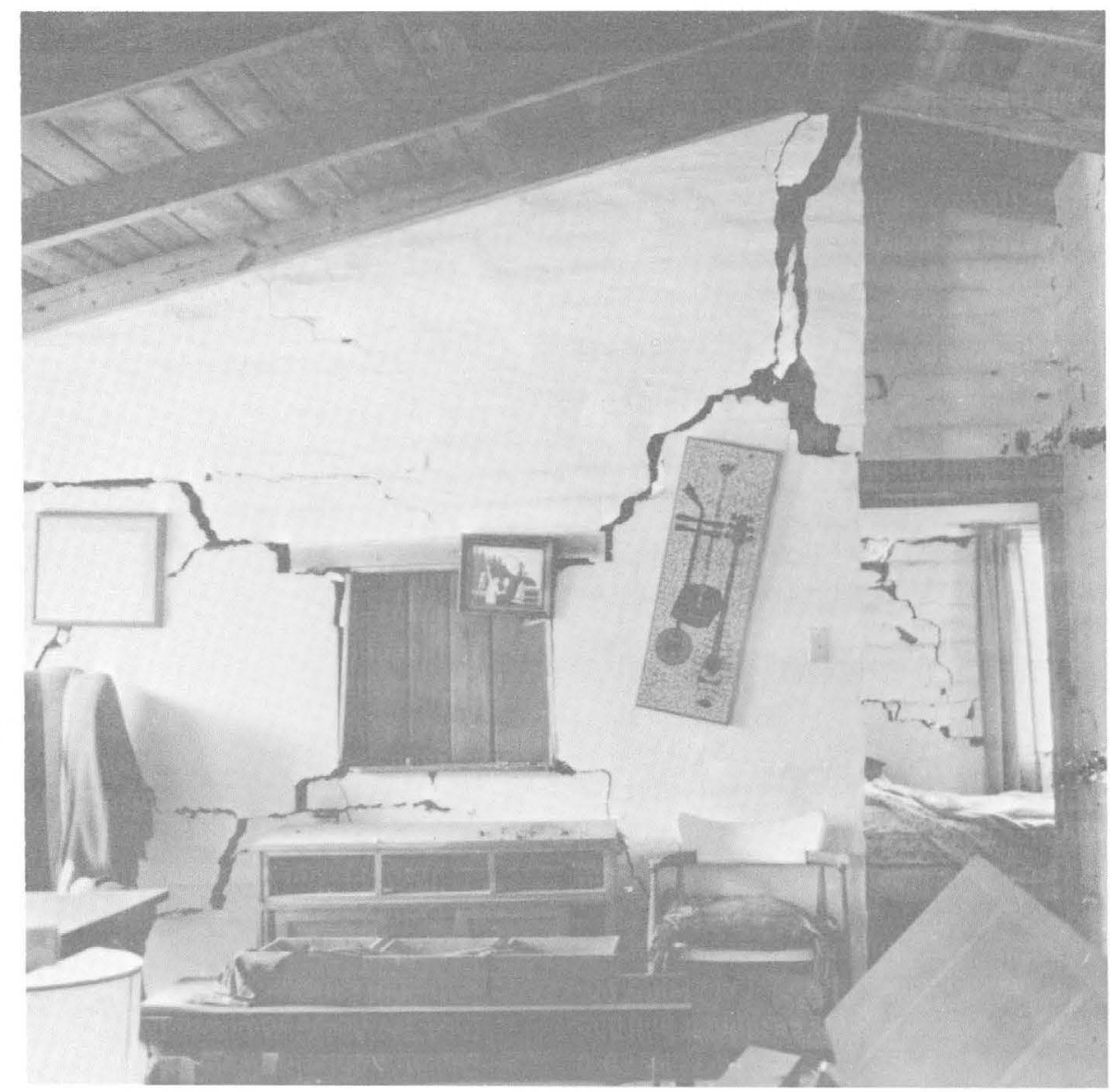

Figure 13. Cracks in adobe walls of the recently remodeled McKenzie house in Whitewater Canyon, Caliifornia (Borchardt and Manson, 1986).

\section{CALIFORNIA-Continued}

retaining bolts. Another moved enough to bend the anchoring clamps. In the control building relays were tripped; batteries moved in their racks; ceiling tiles fell; and several storage cabinets fell (from Earthquake Engineering Research Instiute, 1986; Borchardt and Manson, 1986).

Idyllwild-Chimneys were broken at the roof line; one house shifted on its foundation; a back-porch roof fell, causing extensive damage; and a bedroom wall cracked. Many windows broke; small appliances were overturned; many small objects overturned and fell; many items were shaken off store shelves. The elementary school had minor cracking of interior walls in the kitchen and toilet rooms; an unanchored heater moved 1 in.; ceiling acoustical tiles fell; and book shelves pulled away from the wall; books fell to the floor (partially from Earthquake Engineering Research Institute, 1986, press report).

\section{CALIFORNIA-Continued}

McKenzie House-The house is about $4.5 \mathrm{mi}$ north of White Water Post Office in Whitewater Canyon. Built about 1946, the house is one story and L-shaped with 12-in. unreinforced adobe walls and tile roof. Damage consisted of major diagonal cracking throughout the south part of the building and eastern wall and the southeast corner separating from the building (fig. 13). The adobe chimney collapsed completely (fig. 14). The house was virtually destroyed (Earthquake Engineering Research Institute, 1986).

North Palm Springs-Bridges were damaged; underground pipes broke; stone or brick fences broke; foundations cracked; trees and bushes shook strongly; small landslides occurred; heavy furniture or appliances overturned; many windows were broken out; many items were shaken off store shelves; many glassware items or dishes broke; many small objects overturned 


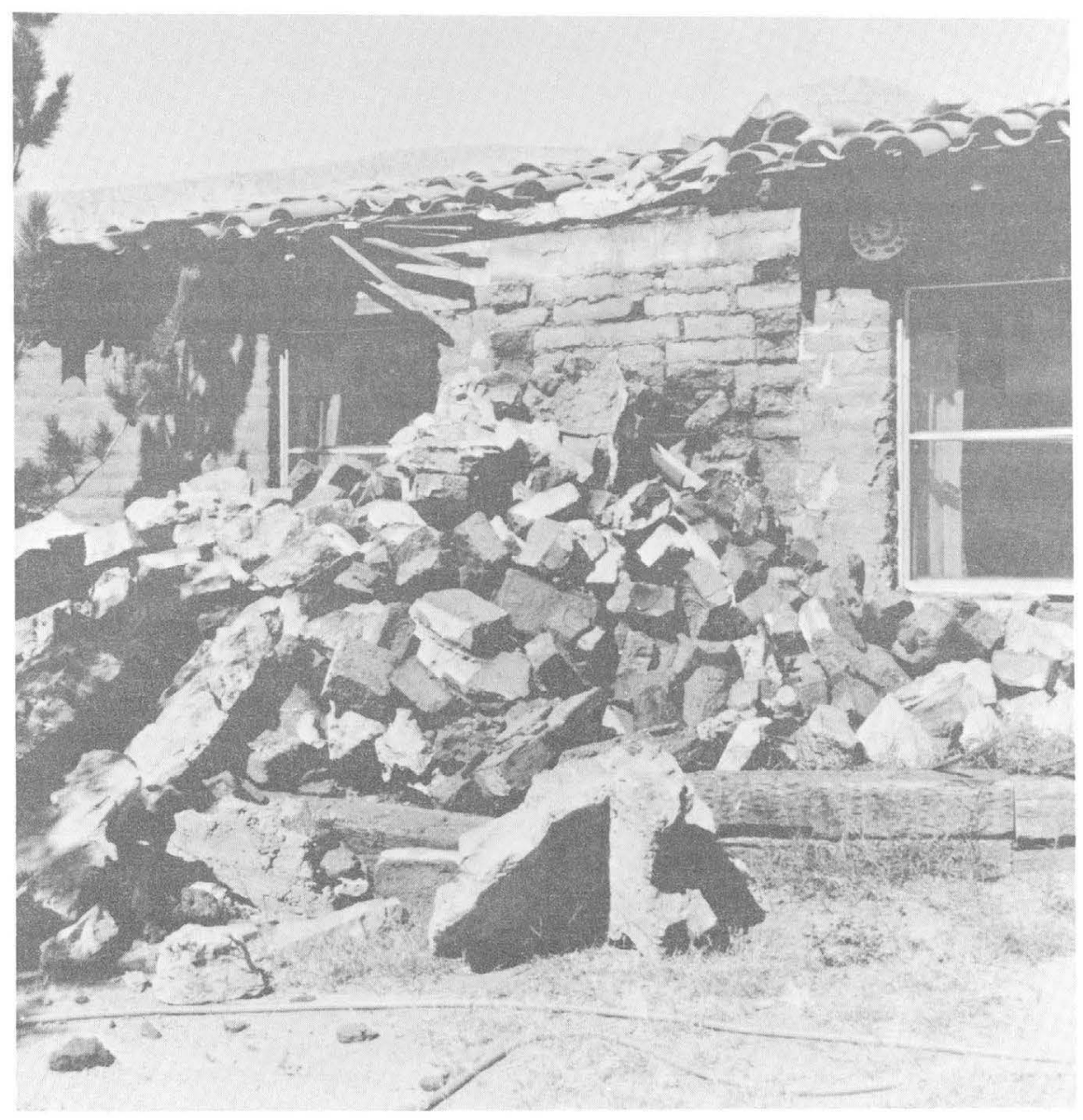

Figure 14. A fallen chimney in Whitewater Canyon, Caliifornia (Borchardt and Manson, 1986).

\section{CALIFORNIA-Continued}

and fell; hanging pictures fell; buildings shook strongly; felt by everyone.

Painted Hills (about 10 mi northwest of Palm Springs)-A house on Painted Hills Road sustained cracked walls and a cracked concrete foundation; a pot-bellied stove was moved $1 \mathrm{ft}$, breaking the chimney pipe; a dresser moved across the room until it hit the bed; every window broke or cracked; everything was shaken into the middle of the room; and the garage was totally destroyed. Another house on Estrellito Road was moved 2-3 in. from its original position; the hot-water heater was torn from the water lines and moved about $2 \mathrm{ft}$; a heavy storage shed on the patio was moved $1 \mathrm{ft}$ across the concrete slab. Other homes had cracks in walls, broken dishes, smashed potted plants, toppled furniture, movement of heavy appliances across the floor, chimneys fallen, and drawers opened with contents dumped onto the floor. At one home an automobile (Cadillac) in

\section{CALIFORNIA-Continued}

a carport was moved several feet (press report). Twenty-four homes reported damage (Riverside County damage survey).

Palm Springs-There were many reports of minor damage throughout the city. Some of the most common types of damage were many instances of fallen acoustical ceiling tile, many broken plate-glass windows in downtown businesses, many cracked interior and exterior walls in both homes and businesses, floors being covered with items thrown from shelves in both homes and businesses, and fallen overhead light fixtures.

Some of the more serious effects in Palm Springs are listed in detail below:

Desert Spa Hotel-There was substantial glass breakage on the upper floors and cracked stucco on the end walls both inside and outside. 


\section{CALIFORNIA-Continued}

Maxim de Paris Hotel-There were some hairline cracks at floor-level joints; glass falling out of panels in two atrium elevators shut down service; and a large chunk of concrete fell from a third-story beam in the lobby.

Desert Inn Fashion Plaza-Plaster fell and numerous wall cracks occurred throughout the plaza.

Professional Building at 1301 North Palm Canyon Drive-An underground parking structure had most of the columns cracked.

Ramada Inn-There were some cracked columns in the parking structure, and the east-side facia was cracked.

671 North Riverside Drive-Veneer separated about 1 in. from the front of the building and was cracked throughout the entire width; there were numerous cracks in the west wall of the building; and a concrete block wall on the west side was cracked.

Bank of America-The north wall was cracked and a front panel pulled away from the building.

167 North Indian Avenue-Interior plaster walls cracked and the exterior wall sustained small cracks.

Security Pacific National Bank at 756 North Palm Canyon Drive-There were cracks around the beams at the front entry, cracks at the rear of the building, and cracks and splitting at the southwest corner.

Stroke Activity Center at 1776 North Palm Canyon Drive-There were three cracks in the wall that extends from the roof line to the ground.

Old Lucky's Center at 2500 North Palm Canyon DriveThere was severe cracking on the east end of the building; a wall at the rear of the building sustained severe cracking that extended upward into the mezzanine; and a wall and exterior column cracked near the receiving entrance.

Dansks at 2550 North Palm Canyon Drive-An interior east wall sustained cracking; a concrete slab in the hallway of the receiving area cracked; and there were exterior cracks between windows.

White Water (at the mouth of Whitewater Canyon)-The main building of the Bridgehaven alcohol and drug abuse recovery house collapsed; it was constructed of partly mortared stone. Walls were severely cracked; the kitchen was destroyed; and the building was uninhabitable (press report).

White Water overpass (near the White Water Post Office)-There was structural damage due to excessive movement at the south abutment. A lateral displacement left a 6-in. gap between a deck and an abutment. The displacement broke lateral restrainers and snapped restraining cables. Pounding damage was visible at joints. Concrete posts shattered, exposing the steel reinforcing rods.

\section{CALIFORNIA-Continued}

Intensity VI:

The most common effects at the places listed below were that: buildings shook moderately to strongly; small objects overturned and fell; glassware or dishes broke; many items were shaken off store shelves; standing and moving vehicles rocked slightly to moderately; shaking was described as strong; felt by everyone.

California-

Anza-A few windows cracked; interior walls sustained hairline cracks; trees and bushes shook moderately; hanging pictures fell; people had difficulty standing and walking.

Banning-A wall cracked at City Hall; a ceiling cracked in one home; a side porch separated from one house; a grocery store sustained minor roof and ceiling damage; plaster fell from a ceiling; windows broke (from press report).

Beaumont-Walls cracked at Valley View Hospital; a house shifted on its piers; plate-glass windows broke; plaster fell inside the church tower of San Gorgonio Parish (from press report).

Cabazon-Interior walls cracked; exterior stone wall cracked; a foundation cracked; hanging pictures fell.

Cathedral City-Fire Station 34 had minor damage. A fire caused by a gas leak damaged the Cathedral City Mirror and Glass store (press report). People had difficulty standing or walking; small appliances overturned.

Green Valley Lake-Bricks fell from chimneys; interior walls sustained hairline cracks; small landslides occurred.

Hemet-Walls cracked in the auditorium of OurLady of the Valley school.

Indian Wells-Brick fences and streets cracked.

Indio-Walls cracked from the floor to the ceiling and a large decorative metal eagle fell off the front of the First Trust Bank (press report).

La Jolla-Windows broke (press report).

Moreno Valley-Chimneys cracked; interior walls sustained hairline cracks; a few windows cracked.

Morongo Valley-Chimneys cracked; concrete bridges sustained slight damage; small landslides occurred; elevated water tanks cracked; interior walls sustained hairline cracks; hanging pictures fell; furniture overturned; some windows were broken out.

Pacific Beach-Windows broke (press report).

Patton-Chimneys cracked; interior walls sustained hairline cracks; light furniture and (or) appliances overturned. 


\section{CALIFORNIA - Continued}

Seal Beach-Exterior concrete walls cracked.

Yucca Valley-At Hi-Desert Concrete Products plant, one of four legs of a 45-ft-high tower, used to mix concrete materials, collapsed causing the tower to fall, and plate-glass windows at Yucca Valley Ford dealership broke (press reports); chimneys cracked; a foundation cracked; sidewalks cracked; interior walls sustained hairline cracks; some windows were broken out; hanging pictures fell.

Intensity V:

The most common effects at the places listed below were that: a few small objects overturned and fell; buildings shook moderately to strongly; earthquake was felt by many people or all; shaking was described as moderate to strong.

\section{California-}

Aguanga-A few items were shaken off store shelves.

Alpine-Plaster walls sustained hairline cracks.

Angelus Oaks-Plaster walls sustained hairline cracks; a few items were shaken off store shelves; a few windows cracked; a few glassware items or dishes broke; small landslides occurred; trees and bushes shook slightly; standing vehicles rocked slightly.

Apple Valley-A few glassware items or dishes broke; hanging pictures swung out of place; trees and bushes shook slightly; standing vehicles rocked slightly.

Baldwin Park-A few windows cracked; a few glassware items or dishes broke; plaster walls sustained hairline cracks; trees and bushes shook slightly; standing vehicles were rocked slightly; hanging pictures swung out of place.

Bellflower-A few windows cracked; a few items were shaken off store shelves; a few glassware items or dishes broke; hanging pictures swung out of place; trees and bushes shook moderately; standing and moving vehicles rocked slightly.

Big Bear City-A few items were shaken off store shelves; a few glassware items or dishes broke; hanging pictures swung out of place.

Bonsall-A few items were shaken off store shelves; a few glassware items or dishes broke; plaster walls sustained hairline cracks; trees and bushes shook slightly; standing vehicles were rocked slightly.

Bryn Mawr-People had difficulty standing and walking. Calexico-Trees and bushes shook slightly.

Calimesa-Plaster walls sustained hairline cracks; hanging pictures swung out of place; people had difficulty standing and walking. Items were shaken off store shelves, breaking glass jars. An outdoor sign fell into the street at $\mathrm{C}$ and $\mathrm{H}$ Liquor (press report).

\section{CALIFORNIA-Continued}

Canyon Lake-A few windows broke; streets cracked; hanging pictures swung out of place.

Carlsbad-A few items were shaken off store shelves.

Castaic-There was report of displaced tombstones.

Cedarpines Park.

Cherry Valley-Knickknacks shaken from shelves (press report).

Chino-People had difficulty standing and walking.

Chiriaco Summit-People had difficulty standing and walking.

Chula Vista-Plaster walls sustained hairline cracks; hanging pictures fell. There were minor cosmetic cracks in the County building (press report).

Coachella-A few windows cracked; a few items were shaken off store shelves; trees and bushes shook slightly; a few glassware items or dishes broke; small appliances were overturned.

Colton-Plaster walls sustained hairline cracks; a few glassware items or dishes broke; people had difficulty standing and walking; hanging pictures swung out of place.

Corona-A few glassware items or dishes broke; a few items were shaken off store shelves; people had difficulty standing and walking.

Crestline-A few windows cracked.

Del Rosa.

Diamond Bar-A few glassware items or dishes broke; a few items were shaken off store shelves; hanging pictures swung out of place.

Dulzura-Interior walls sustained hairline cracks.

East Irvine-A few windows cracked; a few glassware items or dishes broke; people had difficulty standing or walking.

El Centro-A few items were shaken off store shelves.

El Toro-Plaster walls sustained hairline cracks; trees and bushes shook slightly.

Fallbrook-Interior walls sustained hairline cracks; a few items were shaken off store shelves; a few windows cracked; a few glassware items or dishes broke.

Fawnskin-A few items were shaken off store shelves; hanging pictures swung out of place.

Fontana-A few windows broke; a few items were shaken off store shelves; a few glassware items or dishes broke; interior walls sustained hairline cracks; trees and bushes shook slightly.

Forest Falls-A few windows cracked; a few items were shaken off store shelves; a few glassware items or dishes broke; interior walls sustained hairline cracks; large landslides occurred; hanging pictures swung out of place.

Fountain Valley.

Fullerton.

Garden Grove-A few items were shaken off store shelves. 
Helendale-Trees and bushes shook slightly.

Hesperia-A few windows cracked; trees and bushes shook moderately.

Highland-A few windows cracked; a few items were shaken off store shelves; trees and bushes shook slightly.

Huntington Beach-A few windows cracked; a few items were shaken off store shelves; a few glassware items or dishes broke.

Jacumba-One wooden porch floor cracked.

Joshua Tree-Hanging pictures fell; a few glassware items or dishes broke; many items were shaken off store shelves.

Julian-Plaster walls sustained hairline cracks; trees and bushes shook moderately.

Lake Elsinore-A few windows cracked; a few items were shaken off store shelves; a few glassware items or dishes broke; hanging pictures swung out of place.

La Mesa-Trees and bushes shook slightly.

La Mirada-Plaster walls sustained hairline cracks; hanging pictures swung out of place.

Lakeside-A few windows cracked; a few items were shaken off store shelves; plaster walls sustained hairline cracks.

Lakeview.

Leona Valley-A few small appliances overturned; trees and bushes shook slightly.

Loma Linda.

Long Beach-People had difficulty standing and walking; a pendulum and tubular bells of a grandfather clock clashed against each other and the glass case.

Los Nietos-A few windows cracked; a few glassware items or dishes broke; plaster walls sustained hairline cracks; trees and bushes shook slightly.

March Air Force Base.

Mecca.

Mentone-A few windows cracked; a few interior walls sustained hairline cracks; hanging pictures swung out of place.

Miramar Naval Air Station-A few windows cracked; a few items were shaken off store shelves; interior walls sustained hairline cracks; a few glassware items or dishes broke; trees and bushes shook slightly.

Mission Viejo-A few windows cracked; a few items were shaken off store shelves; a few glassware items or dishes broke; interior walls sustained hairline cracks; trees and bushes shook slightly.

Mojave-A few glassware items or dishes broke.

Montclair-A few glassware items or dishes broke; trees and bushes shook slightly.
Montebello-Interior walls sustained hairline cracks; trees and bushes shook slightly.

Murrieta-A few glassware items or dishes broke; a few items were shaken off store shelves; hanging pictures fell; people had difficulty standing or walking; trees and bushes shook strongly.

National City-A few items were shaken off store shelves; hanging pictures fell.

North Shore-Trees and bushes shook slightly.

Norton Air Force Base-People had difficulty standing and walking.

Pala-A few items were shaken off store shelves.

Palm Desert-There were small cracks in City Hall buildings (press report).

Palomar Mountain-A few items were shaken off store shelves; hanging pictures swung out of place.

Pearblossom-A few glassware items or dishes broke.

Perris-A 2,000-lb safe moved 2 in. at the post office; a few small parcels were knocked to the floor. People had difficulty standing or walking; a painting fell; and water beds shook strongly (press reports).

Pomona-A few glassware items or dishes broke.

Poway-Trees and bushes shook moderately.

Rancho Bernardo-A few dishes broke; a grandfather clock stopped (press report).

Rancho Mirage-A few windows cracked; a few items were shaken off store shelves; a few glassware items or dishes broke; hanging pictures swung out of place; trees and bushes shook slightly. There were fine cracks in some bridges (press report).

Rancho Santa Fe-Trees and bushes shook slightly.

Redlands-Plaster walls sustained hairline cracks; hanging pictures swung out of place; water splashed over the sides of a swimming pool.

Rimforest-A few glassware items or dishes broke; a few items were shaken off store shelves; hanging pictures swung out of place.

Riverside-A few items were shaken off store shelves; plaster walls sustained hairline cracks; hanging pictures swung out of place.

Romoland-A few windows cracked; a few items were shaken off store shelves; hanging pictures swung out of place; trees and bushes shook slightly.

Running Springs-A few windows cracked; many items were shaken off store shelves; a few glassware items or dishes broke; hanging pictures swung out of place and some fell; one report of bricks fallen from chimneys.

San Bernardino-People had difficulty standing and walking. One report of broken dishes; a painting fell off a wall (press report). 


\section{CALIFORNIA-Continued}

San Clemente-A few items were shaken off store shelves; hanging pictures fell; people had difficulty standing and walking; there was a report of broken underground pipes.

San Diego-A few glassware items or dishes broke; small landslides occurred; small appliances overturned; windows cracked.

San Diego (North Island Naval Air Station)-Small appliances and (or) furniture moved.

San Diego (Paradise Hills)-Aisles in Victory Foods Grocery were littered with groceries, cans, and broken bottles (press report).

San Dimas-A few windows cracked; a few items were shaken off store shelves; a few glassware items or dishes broke; hanging pictures fell.

San Fernando-Trees and bushes shook moderately; standing and moving vehicles rocked moderately.

San Jacinto-People had difficulty standing or walking.

San Marcos.

Santa Ana-A few windows cracked; a few items were shaken off store shelves; a few glassware items or dishes broke; trees and bushes shook slightly.

Santa Monica-A few merchandise items were shaken off store shelves.

Sepulveda-Interior walls sustained hairline cracks; a few items were shaken off store shelves; a few glassware items and dishes broke; trees and bushes shook moderately.

Solana Beach.

Spring Valley-Interior walls sustained hairline cracks; hanging pictures swung out of place; people had difficulty standing and walking.

Sunnymead.

Sunset Beach-A few glassware items or dishes broke.

Temecula- A few windows cracked; a few items were shaken off store shelves; a few glassware items or dishes broke; plaster walls sustained hairline cracks; hanging pictures swung out of place; trees and bushes shook slightly.

Thermal-A few items were shaken off store shelves; interior walls sustained hairline cracks; hanging pictures swung out of place; trees and bushes shook slightly.

Thousand Palms-A few windows cracked; a few items were shaken off store shelves; a few glassware items or dishes broke; hanging pictures fell; interior walls sustained hairline cracks; trees and bushes shook strongly.

Torrance-A few windows cracked; trees and bushes shook moderately.

Trabuco Canyon-A few windows cracked; a few items were shaken from store shelves; a few glassware items or dishes broke; trees and bushes shook slightly.

\section{CALIFORNIA-Continued}

Trona-A few windows cracked; a few items were shaken off store shelves; trees and bushes shook slightly.

Tujunga-Plaster walls sustained hairline cracks; trees and bushes shook slightly.

Tustin-A few windows cracked; a few items were shaken off store shelves; a few glassware items or dishes broke; people had difficulty standing and walking; trees and bushes shook slightly.

Upland-A few windows cracked; a few glassware items or dishes broke; hanging pictures swung out of place.

Valyermo-Interior walls sustained hairline cracks; hanging pictures swung out of place.

Venice-A few windows cracked; many items were shaken off store shelves; a few glassware items or dishes broke; hanging pictures swung out of place; trees and bushes shook moderately.

Victorville.

Walnut-People had difficulty standing and walking.

Warner Springs.

Whittier-A few windows cracked; a few items were shaken off store shelves; a few glassware items or dishes broke; plaster walls sustained hairline cracks; trees and bushes shook slightly.

Wildomar-Trees and bushes shook slightly.

Yucaipa-A few glassware items or dishes broke; plaster walls sustained hairline cracks; hanging pictures swung out of place.

Intensity IV:

Arizona-Mohave Valley.

California-Acton, Adelanto, Alta Loma, Altadena, Amboy, Anaheim (one report of a cracked driveway, press report), Arcadia, Arrowhead Highlands, Atwood, Avalon, Azusa, Barstow, Blue Jay, Blythe, Borrego Springs, Brea, Buena Park (press report), Burbank, Cadiz, Caliente, Campo, Canoga Park, Canyon Country, Capistrano Beach, Cerritos, Claremont, Coalinga, Covina, Crest Park, Culver City (press report), Cypress, Daggett, Death Valley Junction, Del Mar, Descanso, Desert Center, Duarte, El Cajon, El Modena, El Toro Marine Corps Air Station, Escondido, Etiwanda, Gardena, Glendale, Guasti, Guatay, Heber, Hermosa Beach, Hinkley, Imperial, Inglewood (press report), Irvine (press report), Kelso, La Habra, La Puente, La Verne, Laguna Niguel, Lake Hughes, Lake San Marcos, Lakewood, Lancaster, Lawndale, Lemon Grove, Leucadia, Lomita, Los Angeles, Lucerne Valley, Lytle Creek, Manhattan Beach, Maywood, Monrovia, Mount Baldy, Mount Laguna, Mount Wilson, Newberry Springs, Newport Beach, Niland, Norco, Oceanside, Olive, Ontario, Orange, Oro Grande, Palo Verde, Paramount, Phelan, Pine Valley, PointMugu Pacific Missle Test Center, Potrero, Quartz Hill, Ramona, Rancho Palos Verdes (press report), Randsburg, Redondo Beach, 


\section{CALIFORNIA-Continued}

Reseda, Rolling Hills Estates, Rosamond, San Diego (Clairemont), San Gabriel, San Juan Capistrano, San Luis Rey, San Pedro (press report), Santa Ysabel, Seeley, Silverado, Simi Valley, South Gate, Stanton, Sun Valley, Surfside, Sylmar, Tarzana, Tecate, Tehachapi, Temple City, Twentynine Palms, Twin Peaks, Vista, Westminster, Westmorland, Wrightwood, Yermo.

Nevada - Laughlin.

Intensity III:

Arizona-Bullhead City, Ehrenberg, Lake Havasu City, Parker, Quartzsite, Topock.

California-Bell (press report), Castle Air Force Base, Cima, El Monte, Essex, Glennville, Hacienda Heights, Holtville, Johannesburg, Malibu, Mettler, Miramonte, Parker Dam, Placentia, Playa Del Rey, Ridgecrest, Ripley, Rosemead, Sunland, Tecopa, Topanga, Wofford Heights. Nevada-Henderson, Jean, Las Vegas (press report).

Intensity II:

California-Plaster City.

Nevada-Pioche.

Felt:

Arizona-Yuma.

California-Carson (press report), El Segundo, La Palma (press report), Littlerock, Stanton (press report).

9 July (GP) Southern California

Origin time: 001232.1

Epicenter: 33.987 N., $116.569 \mathrm{~W}$.

Depth: $9 \mathrm{~km}$

Magnitude: $4.2 \mathrm{~m}_{\mathrm{b}}(\mathrm{GS}), 4.2 \mathrm{M}_{\mathrm{L}}(\mathrm{PS}), 4.2 \mathrm{M}_{\mathrm{L}}(\mathrm{BK})$, $4.4 \mathrm{M}_{\mathrm{L}}(\mathrm{GP})$

Felt: Palm Spring area (PS), San Diego (PS).

9 July (RN) Central California

Origin time: 125359.7

Epicenter: 37.565N., 118.435W.

Depth: $4 \mathrm{~km}$

Magnitude: $3.4 \mathrm{MD}_{\mathrm{D}}(\mathrm{RN}), 3.0 \mathrm{M}_{\mathrm{L}}(\mathrm{PS})$

Felt: Chalfant (press report).

10 July (PS) Southern California

Origin time: 120250.9

Epicenter: 33.962N., 116.593W.

Depth: $12 \mathrm{~km}$

Magnitude: $3.4 \mathrm{M}_{\mathrm{L}}(\mathrm{PS})$

Intensity V:

North Palm Springs-A few small objects overturned and fell; a few glassware items or dishes broke; a few merchandise items were shaken off store shelves; standing vehicles rocked slightly.

Palm Desert-A few small objects fell; a few items were shaken off store shelves; hanging pictures swung out of
CALIFORNIA-Continued

place; trees and bushes shook moderately; standing vehicles were rocked moderately; felt by many people.

Palm Springs-A few small objects overturned and fell; interior walls sustained hairline cracks; trees and bushes shook slightly; standing vehicles rocked slightly; awakened many people.

White Water-People had difficulty standing and walking; awakened people.

Intensity IV: Cabazon, Desert Hot Springs.

Intensity III: Idyllwild.

\section{July (PS) Southern California}

Origin time: 074814.0

Epicenter: $33.997 \mathrm{~N} ., 116.572 \mathrm{~W}$.

Depth: $12 \mathrm{~km}$

Magnitude: $3.1 \mathrm{M}_{\mathrm{L}}(\mathrm{PS})$

Intensity III: Palm Springs (press report).

11 July (PS) Southern California

Origin time: 085128.7

Epicenter: $33.967 \mathrm{~N} ., 116.575 \mathrm{~W}$.

Depth: $7 \mathrm{~km}$

Magnitude: $3.3 \mathrm{M}_{\mathrm{L}}$ (PS)

Intensity III: Palm Springs (press report).

11 July (PS) Southern California

Origin time: 151330.6

Epicenter: $34.020 \mathrm{~N} ., 116.653 \mathrm{~W}$.

Depth: $12 \mathrm{~km}$

Magnitude: $3.2 \mathrm{M}_{\mathrm{L}}$ (PS)

Intensity III: Palm Springs (press report).

11 July (PS) Southern California

Origin time: 212852.5

Epicenter: 34.298 N., 118.292W.

Depth: $8 \mathrm{~km}$

Magnitude: $3.0 \mathrm{M}_{\mathrm{L}}(\mathrm{PS})$

Intensity IV: Pacoima, Sierra Madre.

Intensity III: Canyon Country.

Felt: Burbank (press report), Glendale (press report), Palm Springs (press report).

12 July (PS) Southern California

Origin time: 054527.5

Epicenter: 33.986N., 116.652W.

Depth: $7 \mathrm{~km}$

Magnitude: $4.0 \mathrm{M}_{\mathrm{L}}$ (PS)

Intensity III: Palm Spring (press report).

13 July (PS) Southern California

Origin time: 014138.2

Epicenter: 33.951N., 116.613W.

Depth: $12 \mathrm{~km}$

Magnitude: $3.7 \mathrm{M}_{\mathrm{L}}(\mathrm{PS})$

Felt: Palm Springs (press report). 
13 July (HJ) Off the coast of Southern California

Origin time: 134708.2

Epicenter: 32.978N., 117.858W.

Depth: $9 \mathrm{~km}$

Magnitude: $5.6 \mathrm{~m}_{\mathrm{b}}(\mathrm{GS}), 5.8 \mathrm{Ms}_{\mathrm{S}}(\mathrm{GS}), 5.4 \mathrm{M}_{\mathrm{L}}(\mathrm{PS})$, $5.3 \mathrm{M}_{\mathrm{L}}(\mathrm{HJ})$

Moment: $6.5 \times 10^{24}$ dyne-cm (HR)

This earthquake was felt over a land area of about $48,500 \mathrm{~km}^{2}$ of Arizona and California (fig. 15). It caused an estimated $\$ 700,000$ damage in San Diego County and injured one man when piles of books fell on him (press report). Ninety-nine aftershocks of magnitude $\geq 3.0$ occurred during the period 13 July 1986 and 30 April 1987 (Hauksson and Jones, 1988).

Intensity VI:

California-

Chula Vista-Plate-glass windows broke (press report); a few glassware items or dishes broke; interior walls sustained hairline cracks; many small objects fell; shaking was described as strong (press report).

Escondido-Plate-glass windows broke or cracked in four businesses; one chimney fell; the ceiling of a tire dealership collapsed (press report); a few items were shaken off store shelves; plaster walls sustained hairline cracks; a few small objects overturned and fell; felt by and awakened many people.

Imperial Beach-Two roof-support beams at Bayside Elementary School cracked, and Oneonta Elementary School reported minor damage (press report).

Lindbergh Field-Walls were cracked in both airline-ticket terminals; some windows were broken out (press report). A few items were shaken off store shelves; a few glassware items and dishes broke; a few small objects overturned and fell; underground pipes broke; felt by and awakened all.

National City-Display windows in stores were broken (press report).

Oceanside-Some windows were broken out; a few items were shaken off store shelves; a few small objects overturned and fell; plaster walls sustained hairline cracks; standing and moving vehicles rocked slightly; felt by everyone.

San Clemente-The top portion of an old (1920's) chimney fell, and a few residential walls were cracked (press report).

San Diego-The upper portion of a chimney collapsed; a 500-lb statue on a roof shook loose and fell onto a car at the Lincoln Hotel; County Administration Center had a little fallen plaster; in the Golden Hill area, several parked cars were damaged when a chimney collapsed onto them (press report).
Spring Valley-Windows broke; a roof partially collapsed; a small landslide temporarily blocked Wildcat Canyon Road (press report). Interior walls sustained hairline cracks; shaking was described as strong; felt by everyone.

Intensity V:

The most common effects at the places listed below were that: a few small objects overturned and fell; buildings shook moderately; hanging pictures swung, leaving many out of place; the earthquake was felt by and awakened many people.

California-

Anaheim-A few small objects fell; there was a report of broken underground pipes; shaking was described as strong.

Artesia-A few windows cracked; interior walls sustained hairline cracks; a foundation cracked; hanging pictures fell; a few glassware items or dishes broke; shaking was described as strong.

Bellflower-A few items were shaken off store shelves; light furnishings overturned; many small objects overturned and fell; a few windows cracked; a few glassware items or dishes broke.

Bonita-Shaking was described as strong; buildings shook moderately.

Carlsbad-A few items were shaken off store shelves; a foundation cracked; plaster walls sustained hairline cracks; a few glassware items or dishes broke; many small objects overturned and fell.

Colton-Shaking was described as moderate.

Downey-A few windows cracked; interior dry wall was cracked; a few items were shaken off store shelves; a few glassware items or dishes broke.

East Irvine-Plaster walls sustained hairline cracks; standing vehicles rocked slightly; shaking was described as moderate.

El Cajon-A few windows cracked; interior walls sustained hairline cracks; a few items were shaken off store shelves; hanging pictures fell; buildings shook strongly.

El Segundo-A few windows cracked, a few glassware items or dishes broke; shaking was described as strong.

Encinitas-Everyone ran outside of a restaurant because of the strong shaking (press report).

Fallbrook-There was a report of broken windows; shaking was described as moderate.

Fullerton-Water splashed onto sides of swimming pools; shaking was described as strong; felt by and awakened everyone.

Huntington Park-A few items were shaken off store shelves; a few glassware items and dishes broke; standing vehicles were rocked slightly. 


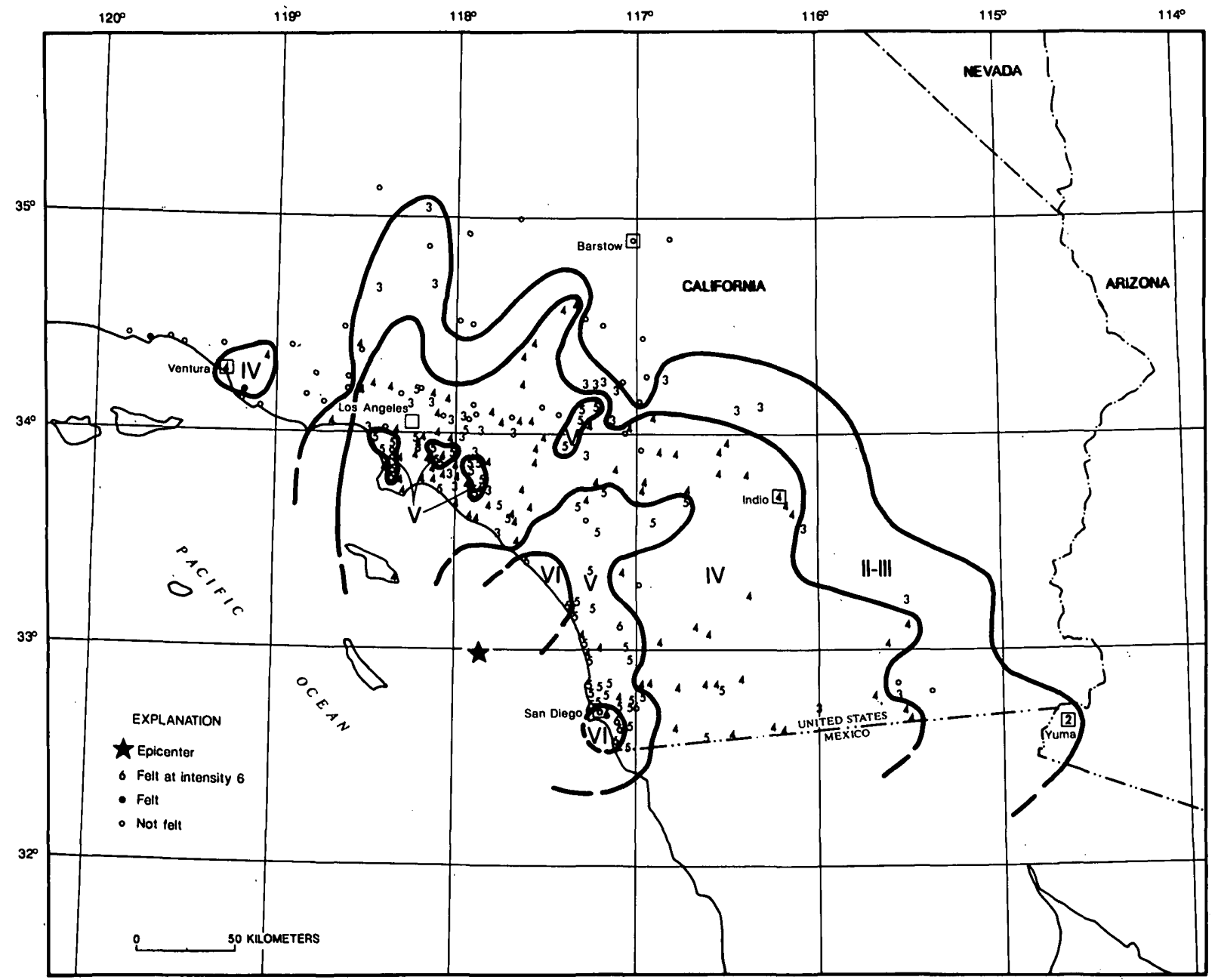

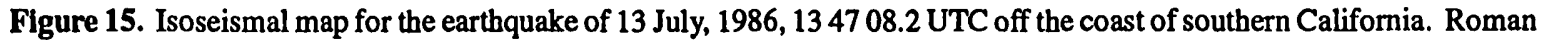
numerals represent Modified Mercalli intensities between isoseismals; Arabic numerals represent intensities at specific sites; dashed contour lines are inferred isoseismals, and small boxes show locations of towns and cities whose names are plotted.

\section{CALIFORNIA-Continued}

Inglewood-A few items were shaken off store shelves; standing and moving vehicles were rocked slightly; felt by everyone.

Laguna Hills-Standing vehicles were rocked slightly; shaking was described as moderate; felt by and awakened everyone.

La Jolla-There was $\$ 500-\$ 600$ damage to merchandise shaken off grocery-store shelves. Interior walls sustained minor cracks (press reports).

Lake Elsinore-A few windows cracked; a few items were shaken off store shelves; interior walls sustained hairline cracks; a few glassware items or dishes broke; standing and moving vehicles were rocked slightly.

La Mesa-People had difficulty standing and walking; shaking was described as strong.

\section{CALIFORNIA-Continued}

La Mirada-Interior walls sustained hairline cracks; a few windows cracked; a few items were shaken off store shelves; a few glassware items or dishes broke; shaking was described as moderate.

La Puente-A few windows cracked; plaster walls sustained hairline cracks; a few glassware items or dishes broke; trees and bushes were strongly shaken; standing and moving vehicles rocked slightly.

Lemon Grove-A few glassware items or dishes broke; a few items were shaken off store shelves; interior dry wall cracked; standing vehicles rocked moderately.

Miramar Naval Air Station area-Plaster walls sustained hairline cracks; many items were shaken off store shelves; shaking was described as moderate. 


\section{CALIFORNIA-Continued}

Montrose-A few merchandise items were shaken off store shelves.

Mountain Center-It was felt by and awakened everyone.

Murrieta-A few windows cracked; a few glassware items or dishes broke; a few items were shaken off store shelves; standing vehicles rocked slightly.

Ocean Beach-A lot of liquor containers were shaken off shelves and broke; merchandise was shaken off every shelf in the store (press report).

Orange-Plaster walls sustained hairline cracks; standing vehicles rocked moderately; shaking was described as strong.

Palomar Mountain-Small landslides occurred along road cuts; many small objects overturned and fell; buildings shook strongly.

Patton-A few windows cracked; interior walls sustained hairline cracks; a cracked chimney was reported; a few glassware items or dishes broke; standing and moving vehicles were rocked slightly.

Pine Valley-Shaking was described as moderate.

Placentia-A few glassware items or dishes broke.

Potrero-Trees and bushes shookmoderately; standing and moving vehicles were rocked moderately; felt by everyone.

Poway-Plaster walls sustained hairline cracks; a foundation cracked; sidewalks were cracked; there was a report of broken underground pipes; a few items were shaken off store shelves; standing vehicles rocked slightly.

Rancho Bernardo-A few windows cracked; a few glassware items or dishes broke; a few items were shaken off store shelves; plaster walls sustained hairline cracks.

Riverside-A few glassware items or dishes broke.

Romoland-Sidewalks cracked; plaster walls sustained hairline cracks; standing and moving vehicles rocked slightly; shaking was described as moderate.

San Bernardino-Streets and sidewalks were cracked; a few items were shaken off store shelves; trees and bushes shook slightly; shaking was described as moderate.

San Diego (City Heights)-A few windows cracked; a few glassware items or dishes broke; a few items were shaken off store shelves; interior walls sustained hairline cracks; standing and moving vehicles rocked slightly.

San Diego (Clairemont)-A few glassware items or dishes broke; a few items were shaken off store shelves; there was a report of windows broken out; standing vehicles were rocked slightly; shaking was described as strong.

San Diego (Del Mar)-Items were shaken off store shelves (press report).

\section{CALIFORNIA-Continued}

San Diego (Hillcrest)-A few windows cracked; a few glassware items or dishes broke; many items were shaken off store shelves; shaking was described as moderate.

San Diego (Ocean Beach)-Interior dry wall cracked; standing vehicles rocked slightly.

San Diego (Old Town)-Interior walls sustained hairline cracks.

San Diego (University City)-A few glassware items or dishes broke; interior walls sustained hairline cracks; a few items were shaken off store shelves; standing and moving vehicles shook slightly; shaking was described as strong.

San Luis Rey-Plaster walls sustained hairline cracks; trees and bushes shook moderately; buildings shook strongly.

San Ysidro-A few windows cracked; a few glassware items or dishes broke; a few items were shaken off store shelves; standing vehicles rocked slightly; interior wall was cracked.

Santa Ana-Plaster walls sustained hairline cracks.

Seal Beach-A few items were shaken off store shelves; standing and moving vehicles rocked slightly.

Torrance-Trees and bushes shook slightly; shaking was described as moderate.

Venice-A few glassware items or dishes broke; a few items were shaken off store shelves; interior walls sustained hairline cracks.

Vista-A few items were shaken off store shelves; felt and awakened everyone.

Walteria-Interior walls sustained hairline cracks; a few items were shaken off store shelves; standing and moving vehicles rocked slightly; felt by and awakened everyone.

Intensity IV:

California-Adelanto, Alpine, Altadena, Atwood, Avalon, Balboa Island, Banning, Borrego Springs, Boulevard, Buena Park, Cabazon, Calexico, Calipatria, Campo, Canyon Country, Canyon Lake, Coachella, Compton, Corona, Descanso, Desert Hot Springs, Dulzura, El Toro, Forest Falls, Garden Grove, Gardena, Grantville, Guasti, Guatay, Hawaiian Gardens, Heber, Hemet, Huntington Beach, Idyllwild, Indio, Irvine, Jacumba, Julian, Lakeside, Lakeview, Lakewood, Leucadia, Loma Linda, Lomita, Long Beach, Los Alamitos, Los Angeles, Malibu (press report), Manhattan Beach, Maywood, Mira Loma, Mission Viejo, Montebello, Mount Baldy, Mount Laguna, Newport Beach (press report), Norco, North Island Naval Air Station, North Palm Springs, Norwalk, Ontario, Oro Grande, Pala, Palm Springs, Paramount, Perris, Phelan, Pomona, Ramona, Rancho Santa Fe, Redondo Beach, Reseda, San Dimas, San Gabriel, San Jacinto, San Juan Capistrano, San Pedro, Santa Paula, Santa Ysabel, Santee, Seeley, Sepulveda, Sierra Madre, 


\section{CALIFORNIA-Continued}

Silverado, Solana Beach, South Gate, Stanton, Sun Valley, Tecate, Thermal, Thousand Palms, Trabuco Canyon, Ventura, Westmorland, Whittier, Willow Brook, Wrightwood, Yorba Linda, Yucaipa.

Intensity III:

California-Baldwin Park, Big Bear City, Brea, Chino, Crestline, Cypress, El Centro, El Monte, Glendale, Hacienda Heights, Joshua Tree, Laguna Beach, Lake Arrowhead, Lake Hughes, Lancaster, Los Angeles, March Air Force Base, Mecca, Mentone, Mojave, Niland, Ocotillo, Pasadena, Running Springs, Santa Monica, Tustin, Twin Peaks, Walnut, Westminster, Yucca Valley.

Nevada-Las Vegas (press report).

Felt:

Arizona-Yuma (press report).

California-Oxnard (press report), Santa Barbara (press report).

\section{July (HJ) Off the coast of Southern California}

Origin time: 124701.1

Epicenter: 32.972N., 117.805W.

Depth: $3 \mathrm{~km}$

Magnitude: $3.8 \mathrm{M}_{\mathrm{L}}(\mathrm{PS}), 3.7 \mathrm{M}_{\mathrm{L}}(\mathrm{HJ})$

Intensity $\mathrm{V}$ :

Bonsall-Plaster walls sustained hairline cracks; a few glassware items or dishes broke; a few small objects overturned and fell; standing vehicles rocked slightly; buildings shook moderately; felt by and awakened many people.

ElCajon-A few items were shaken off store shelves; a few small objects overturned and fell; hanging pictures swung out of place; felt by many people.

Escondido-Interior walls sustained hairline cracks; a few glassware items or dishes broke; a few small objects overturned and fell; trees and bushes shook moderately; standing and moving vehicles rocked slightly; hanging pictures swung out of place; felt by and awakened many people.

Laguna Hills-People had difficulty standing and walking; shaking was described as moderate.

Poway-Plaster walls sustained hairline cracks; many items were shaken off store shelves; a few glassware items or dishes broke; many small objects fell; hanging pictures swung out of place; trees and bushes shook slightly; standing and moving vehicles rocked slightly; buildings shook strongly; felt by everyone and awakened many people.

San Diego (Lindbergh Field)-A few windows cracked; a few glassware items or dishes broke; interior walls sustained hairline cracks; a few small objects overturned and fell; trees and bushes shook slightly; standing vehicles rocked slightly.

\section{CALIFORNIA-Continued}

San Diego (South Park)-A few windows cracked; a few small objects overturned and fell; hanging pictures swung out of place; felt by many people.

San Marcos-Dry wall sustained hairline cracks; a few small objects overturned and fell; hanging pictures swung out of place; buildings shook moderately; felt by many people.

Vista-Dry wall was cracked; a foundation cracked; exterior walls were reported cracked; shaking was described as strong.

Intensity IV: Lemon Grove, Spring Valley, Valley Center. Intensity III: Fallbrook, Huntington Beach.

Intensity II: Carlsbad.

16 July (BK) Central California

Origin time: 162749.9

Epicenter: 37.747N., 121.972W.

Depth: $4 \mathrm{~km}$

Magnitude: $2.4 \mathrm{M}_{\mathrm{L}}(\mathrm{BK})$

Felt: San Ramon (BK).

16 July (BK) Central California

Origin time: 205903.8

Epicenter: 37.297N., 121.662W.

Depth: $6 \mathrm{~km}$

Magnitude: $3.0 \mathrm{M}_{\mathrm{L}}(\mathrm{BK})$

Felt: Eastern San Jose (BK), San Felipe Valley (press report).

17 July (GP) Southern California

Origin time: 203515.0

Epicenter: 33.989N., 116.649W.

Depth: $6 \mathrm{~km}$

Magnitude: $4.4 \mathrm{~m}_{\mathrm{b}}(\mathrm{GS}), 4.6 \mathrm{M}_{\mathrm{L}}(\mathrm{PS}), 4.6 \mathrm{M}_{\mathrm{L}}(\mathrm{BK})$,

$$
4.0 \mathrm{M}_{\mathrm{L}}(\mathrm{GP})
$$

This earthquake is an aftershock of the July 8 North Palm Springs earthquake.

Intensity VI :

California-

White Water-Chimneys broke at the roof line; tombstones fell; foundation cracked; interior walls cracked; there was slight damage to concrete bridges; there were broken underground pipes. Rockslides occurred on White Water Road.

Intensity V:

California-

Angelus Oaks-A few items were shaken off store shelves; trees and bushes shook slightly; standing vehicles rocked slightly; felt by many people.

Banning-There were hairline cracks in the floor of the basement and a wall of the County building (press report). 


\section{CALIFORNIA-Continued}

Coachella-A few small objects overturned and fell; people had difficulty standing and walking; felt by many people.

Highland-A few items were shaken off store shelves; a few glassware items or dishes broke; a few small objects fell; many small objects overturned; hanging pictures fell; standing and moving vehicles rocked slightly; buildings shook strongly.

Landers-A few small objects overturned; buildings shook moderately; felt by most people.

Yorba Linda-A few small objects overturned and fcll; felt by many people.

Intensity IV:

California-Altadena, Anza, Cabazon, Cathedral City, Colton, Desert Hot Springs, Fawnskin, Forest Falls, Huntington Beach, Imperial, Indio, Joshua Tree, La Verne, Mead Valley, Morongo Valley, Mountain Center, Norton Air Force Base, Ontario, Palm Springs, Palomar Mountain, Riverside, San Pedro, Solana Beach, Torrance.

Intensity III:

California-Acton, Baker, Beaumont, Calexico, Calimesa, Carlsbad, Julian, La Quinta, Lemon Grove, Long Beach, Mecca, Midway City, Nuevo, Oro Grande, Palmdale, Palm Desert, Pico Rivera, Ramona, Rosemead, San Bernardino, San Diego (Lindbergh Field), Seeley, Spring Valley, Thermal, Vista, Westminster, Whittier, Winchester.

Nevada-Las Vegas.

Intensity II:

California - Crestline, Phelan, Westmorland.

Felt:

California-Alpine, Octillo, Pasadena (PS).

17 July (GP) Southern California

Origin time: 215445.2

Epicenter: 33.991N., 116.641W.

Depth: 7 km

Magnitude: $4.1 \mathrm{~m}_{\mathrm{b}}(\mathrm{GS}), 4.4 \mathrm{M}_{\mathrm{L}}(\mathrm{PS})$

This earthquake is an aftershock of the July 8 North Palm Springs earthquake.

Intensity IV: Palm Springs area (press report).

Intensity III: Yucaipa.

18 July (PS) Central California

Origin time: 155834.1

Epicenter: 37.576N., 118.441W.

Depth: 6 km

Magnitude: $3.1 \mathrm{M}_{\mathrm{L}}(\mathrm{PS}), 3.2 \mathrm{M}_{\mathrm{D}}(\mathrm{RN})$

Felt: Chalfant Valley (RN).

18 July (BK) Central California

Origin time: 160008.5

Epicenter: 37.570 N., $118.443 W$.
CALIFORNIA-Continued

Depth: $4 \mathrm{~km}$

Magnitude: $3.9 \mathrm{M}_{\mathrm{L}}(\mathrm{BK}), 4.0 \mathrm{M}_{\mathrm{L}}(\mathrm{PS}), 3.6 \mathrm{M}_{\mathrm{L}}(\mathrm{RN})$

Felt: Chalfant Valley (press report).

18 July (GP) Central California

Origin time: 170036.8

Epicenter: $36.093 \mathrm{~N} ., 117.849 \mathrm{~W}$.

Depth: $2 \mathrm{~km}$

Magnitude: $3.8 \mathrm{M}_{\mathrm{L}}(\mathrm{PS}), 3.8 \mathrm{M}_{\mathrm{L}}(\mathrm{BK}), 3.8 \mathrm{M}_{\mathrm{L}}(\mathrm{RN})$, 3.7 $\mathrm{M}_{\mathrm{L}}(\mathrm{GP}), 3.7 \mathrm{M}_{\mathrm{L}}(\mathrm{GP})$

Intensity IV: CampNelson, Cerro Gordo mine (7.5 mi northeast of Keeler), Keeler.

Intensity III: China Lake, Ridgecrest, Trona.

Felt: Wofford Heights.

18 July (PS) Southern California

Origin time: 195801.8

Epicenter: 33.967N., 116.569W.

Depth: $7 \mathrm{~km}$

Magnitude: $3.2 \mathrm{M}_{\mathrm{L}}(\mathrm{PS})$

This earthquake is an aftershock of the July 8 North Palm Springs earthquake.

Intensity IV: Angelus Oaks, Calimesa, Colton, Desert Hot Springs, Forest Falls, Indio, Joshua Tree, North Palm Springs, Palm Springs.

Intensity III: Aguanga, Idyllwild, Patton, Thousand Palms. Intensity II: Loma Linda.

Felt: Perris, Winchester.

\section{July (GM) Owens Valley area}

Origin time: 142945.5

Epicenter: 37.580N., 118.450W.

Depth: $8 \mathrm{~km}$

Magnitude: $5.6 \mathrm{~m}_{\mathrm{b}}(\mathrm{GS}), 5.6 \mathrm{M}_{\mathrm{S}}(\mathrm{GS}), 5.9 \mathrm{M}_{\mathrm{L}}(\mathrm{BK})$, $5.9 \mathrm{M}_{\mathrm{L}}(\mathrm{PS}), 5.6 \mathrm{M}_{\mathrm{L}}(\mathrm{RN})$

Moment: $1.4 \times 10^{24}$ dyne-cm (BK),

This earthquake was felt from Modesto to Bakersfield and eastward into Nevada (press report). No extensive questionnaire canvass of this earthquake was done; therefore information is available only for towns near the hypocenter. This earthquake was a foreshock to the July 21, 144226.5 UTC earthquake.

Intensity V:

California-

Chalfant-Merchandise was knocked off shelves in the grocery store and minor damage to contents occurred in some homes (mostly mobile homes) (press report).

Benton-Hanging pictures fell; trees and bushes were moderately shaken; standing and moving vehicles rocked 


\section{CALIFORNIA-Continued}

moderately; people had difficulty standing and walking; shaking was described as strong; felt by everyone.

Big Pine-Many merchandise items were shaken off store shelves; a few glassware items or dishes broke; a few small objects overturned and fell; hanging pictures fell; trees and bushes shook slightly; standing and moving vehicles rocked slightly; shaking was described as strong; felt by many people.

Bishop-A few windows were cracked; a few merchandise items were shaken off store shelves; a few small objects overturned and fell; shaking was described as moderate; felt by everyone. A few dirt roads were closed by Nevadaboulders rolling onto the roadway (press report).

Dyer-Many merchandise items were shaken off store shelves; a few small objects overturned and fell; hanging pictures fell; people had difficulty standing and walking; buildings shook strongly; landslides occurred in the mountains; felt by everyone.

\section{July (BK) Owens Valley area}

Origin time: 183654.1

Epicenter: 37.533N., 118.460W.

Depth: $5 \mathrm{~km}$

Magnitude: $3.8 \mathrm{M}_{\mathrm{L}}(\mathrm{BK}), 3.7 \mathrm{M}_{\mathrm{L}}(\mathrm{PS})$

Intensity IV: Bishop.

Felt: Big Pine.

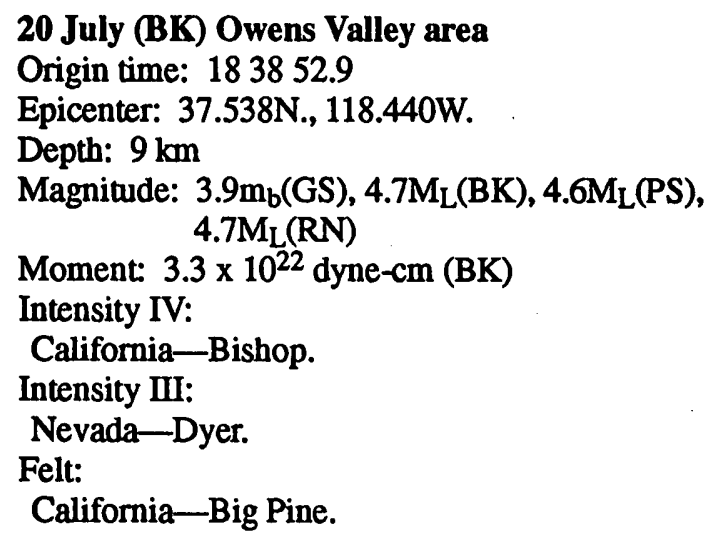

\section{July (BK) Owens Valley area}

Origin time: 144226.5

Epicenter: $37.537 \mathrm{~N} ., 118.450 \mathrm{~W}$.

Depth: 9 km

Magnitude: $6.0 \mathrm{~m}_{\mathrm{b}}(\mathrm{GS}), 6.2 \mathrm{Ms}(\mathrm{GS}), 6.4 \mathrm{M}_{\mathrm{L}}(\mathrm{BK})$, $5.9 \mathrm{M}_{\mathrm{L}}(\mathrm{PS}), 6.6 \mathrm{M}_{\mathrm{L}}(\mathrm{RN})$

Moment: $3.5 \times 10^{25}$ dyne-cm (BK),

$$
2.8 \times 10^{25} \text { dyne-cm (GS) }
$$

This earthquake, named the Chalfant Valley, California, earthquake, caused minor damage in the Bishop

\section{CALIFORNIA-Continued}

area and moderate damage in Chalfant (about $15 \mathrm{~km}$ north of Bishop). Two people in Chalfant were hurt by falling objects. Most of the serious damage in Chalfant was to mobile homes being shaken off their supports, either jack stands or concrete-block piers, with resulting damage to the interior contents, and broken water and sewer lines. The building damage in Bishop consisted of a few cracked chimneys, a few cracked exterior walls, and broken windows. Total damage was estimated at $\$ 2.7$ million. The earthquake was felt over a contiguous area of about $255,000 \mathrm{~km}^{2}$ of California and Nevada (fig. 16). It was also felt in tall buildings as far away as Salt Lake City, Utah.

The Chalfant Valley earthquake was preceded (on 20 July, 142945.5 UTC) by a foreshock of $\mathrm{M}_{\mathrm{L}}=5.9(\mathrm{BK})$ and followed (on 31 July, 072240.2 UTC) by an aftershock of $\mathrm{M}_{\mathrm{L}}=5.8(\mathrm{BK})$. Cockerham and Corbett (1987) located 4,114 earthquakes occurring between 1 July and 30 September 1986 that outline an area $24 \mathrm{~km}$ long north-south by $6-8 \mathrm{~km}$ wide east-west, surrounding the epicenter of the main shock.

Surface ground fracturing occurred on the White Mountains frontal fault zone for a discontinuous length of $13.2 \mathrm{~km}$, according to Lienkaemper and others (1987) or a length of $15.5 \mathrm{~km}$, according to DePolo and Ramelli (1987). Numerous small landslides occurred in the epicentral area, temporarily blocking some secondary roads (Brewer, 1989). Spectacular rock falls occurred in Chidago Canyon (near the epicenter) and in canyons of the White Mountains (east of the epicentral area) (Smith, 1987). The peak accelerations recorded for this earthquake were $0.46 \mathrm{~g}$ horizontal and $0.35 \mathrm{~g}$ vertical at Zack Brothers Ranch about $14 \mathrm{~km}$ north of Chalfant and the epicenter (Earthquake Engineering Research Institute, 1986).

Intensity VI:

California-

Bishop-The brick facade cracked on the front of First Sierra Bank on Main Street. A plate-glass window of the Western Auto store, also on Main Street, broke. Walls crackedat Sears Roebuck Co., Joseph's Bi-Rite Market, and City Hall; windows broke or cracked in other businesses; ceiling tile fell in a few places; and plaster fell from the third-floor ceiling of the Masonic Temple. Many items were shaken off store shelves-especially grocery-store shelves. Streets cracked; water flow was disturbed in wells; buildings shook strongly; people had difficulty standing and walking; felt by everyone.

Chalfant-Most damage was to mobile homes; single family fixed structures were relatively undamaged (Earthquake Engineering Research Institute, 1986). The mobile homes (single and double width) were mounted on jack stands or concrete-block piers 2-3 ft long and $2 \mathrm{ft}$ 


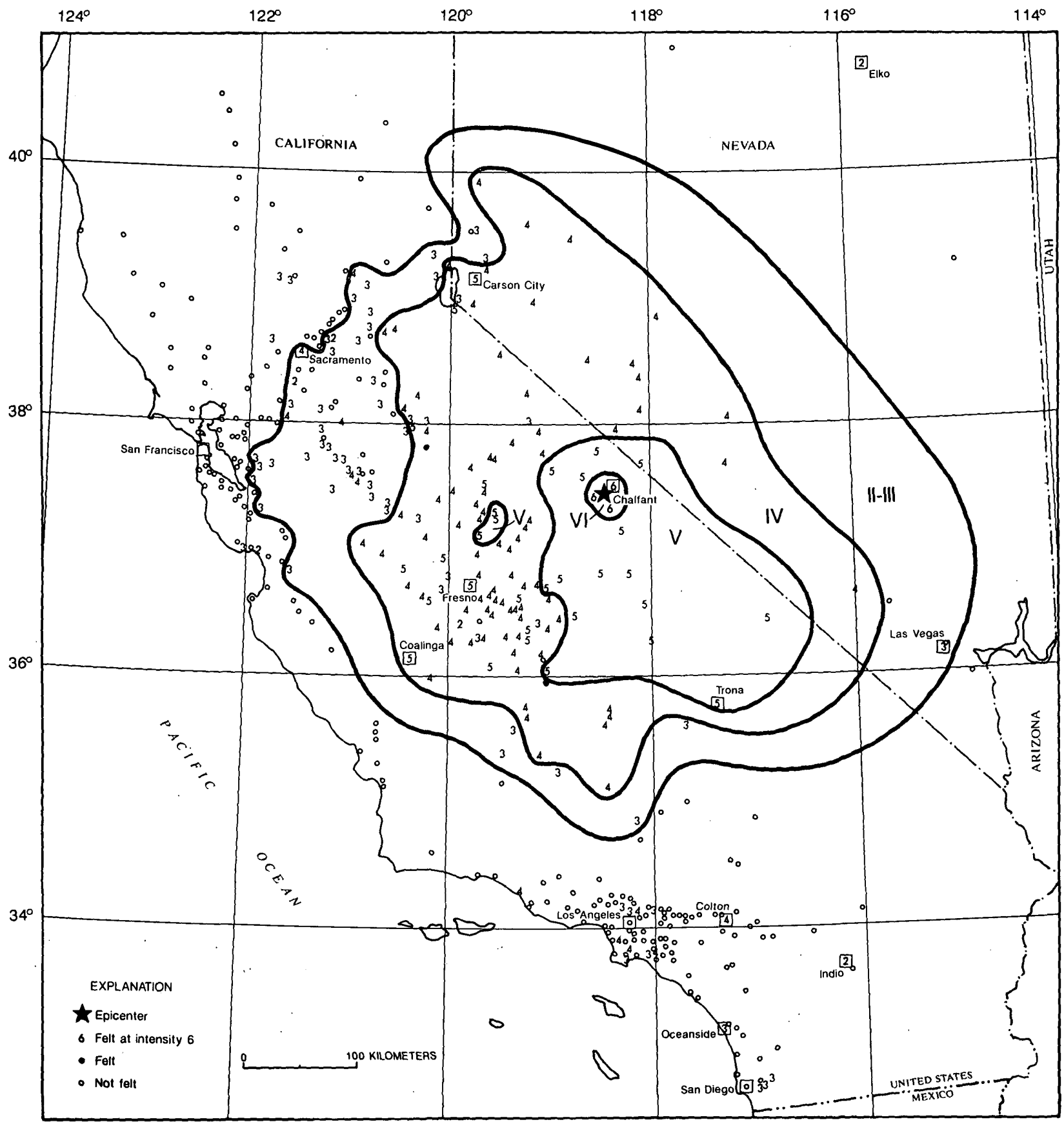

Figure 16. Isoseismal map for the Chalfant Valley, California, earthquake of 21 July, 1986, 144226.5 UTC. Roman numerals represent Modified Mercalli intensities between isoseismals; Arabic numerals represent intensities at specific sites, and small boxes show locations of towns and cities whose names are plotted.

\section{CALIFORNIA-Continued}

high. The shaking moved the mobile homes as much as 18 in. laterally (Earthquake Engineering Research Institute, 1986). This lateral movement broke water and sewer connections and virtually destroyed one

\section{CALIFORNIA-Continued}

double-width mobile home when jacks penetrated the floor. The press reported 53 of the 72 mobile homes in Chalfant were displaced and damaged. Damage to other types of homes (other than mobile homes) consisted 


\section{CALIFORNIA-Continued}

of a few bricks off chimneys, broken windows, and cracked stucco. At Chalfant Mercantile store, much of its merchandise was knocked off shelves, breaking all glass jars or bottles. Across U.S. Highway 6, west of Chalfant, interior walls in a dome-shaped house sustained many cracks (some of the above information is from press reports).

Control Gorge power plant-A connection wire to a transformer was loosened, shutting off power; three bushing seals cracked and had to be replaced. An 8-ft-diameter penstock connects the three Gorge hydroelectric plants. This pipe is supported on concrete footings, some of which cracked and spalled and had anchor bolts pulled (from Earthquake Engineering Research Institute, 1986).

Intensity V:

The most common effects at the places listed below were that: a few small objects overturned and fell; pictures swung, leaving some out of place; buildings shook slightly to moderately; windows rattled; shaking was described as moderate to strong; the earthquake was felt by most people.

\section{California-}

Bass Lake-A few items were shaken off store shelves; trees and bushes shook slightly.

Benton-A few windows broke; hanging pictures fell; buildings shook strongly; trees and bushes shook strongly; standing vehicles rocked moderately; people had difficulty standing or walking.

Big Pine-A few items were shaken off store shelves.

Coalinga-A few items were shaken off store shelves; trees and bushes shook slightly.

Corcoran-A few items were shaken off store shelves; trees and bushes shook slightly; standing vehicles rocked slightly.

Crowley Lake-A few items were shaken off store shelves; a few glassware items or dishes broke; buildings shook strongly; people had difficulty standing or walking; trees and bushes shook moderately; standing vehicles rocked moderately.

Death Valley-Trees and bushes shook slightly; underground pipes were out of service.

Farmersville-A few windows cracked; a few items were shaken off store shelves; a few glassware items or dishes broke; people had difficulty standing and walking; interior walls sustained hairline cracks; trees and

\section{CALIFORNIA-Continued}

bushes shook moderately; standing and moving vehicles rocked slightly.

Firebaugh-A few windows cracked; a few glassware items or dishes broke; trees and bushes shook slightly; standing vehicles rocked slightly.

Fresno-A few windows cracked; a few glassware items or dishes broke; a few items were shaken off store shelves; trees and bushes shook slightly; and standing vehicles rocked slightly.

Hume-Plaster walls sustained hairline cracks; buildings shook strongly; trees and bushes shook slightly.

Independence-A few windows cracked; a few glassware items or dishes broke; interior walls sustained hairline cracks; trees and bushes shook moderately; standing and moving vehicles rocked moderately.

Ivanhoe.

Kings Canyon National Park-Many items were shaken off store shelves; people had difficulty standing and walking; dry wall sustained hairline cracks; trees and bushes shook moderately; small landslides occurred.

Lone Pine-A few windows cracked; buildings shook strongly; dry wall sustained hairline cracks; trees and bushes shook slightly.

Madera-A few items were shaken off store shelves; plaster walls sustained hairline cracks; trees and bushes shook slightly; standing vehicles rocked slightly.

Mammoth Lakes-Trees and bushes shook moderately.

Miramonte-Trees and bushes shook moderately; standing vehicles rocked moderately.

North Fork-Plaster walls sustained hairline cracks; buildings shook strongly.

O'Neals-A moving car was shaken strongly; trees and bushes shook slightly.

Olancha.

Orange Cove-People had difficulty standing or walking.

Porterville-Plaster walls sustained hairline cracks; trees and bushes shook slightly; standing vehicles rocked slightly.

San Joaquin-A few windows cracked; a few glassware items or dishes broke; a few items were shaken off store shelves; dry wall sustained hairline cracks; sidewalks were reported cracked; trees and bushes shook slightly; standing vehicles rocked slightly.

Sequoia National Park-Trees and bushes shook slightly.

Sonora-Interior walls sustained hairline cracks.

South Lake Tahoe-A few glassware items or dishes broke; trees and bushes shook slightly; standing vehicles rocked slightly.

Toms Place-People had difficulty standing and walking.

Trona-Trees and bushes shook slightly; standing vehicles rocked slightly. 


\section{CALIFORNIA-Continued}

Wawona-A few items were shaken off store shelves; trees and bushes shook slightly.

Nevada-

Carson City-A few windows cracked.

Dyer.

Intensity IV:

California-Ahwahnee, Arnold, Auberry, Avenal, Badger, Bakersfield, Bethel Island, Big Creek, Bridgeport, Camino, Carson, Caruthers, Catheys Valley, Cedar Ridge, Ceres, Chowchilla, Clovis, Coarsegold, Coleville, Colton, Cutler, Del Rey, Delano, Dinuba, Dos Palos, Dunlap, East Fresno, El Portal, Fish Camp, Five Points, Fowler, Friant, Goshen, Hanford, Hawthorne, June Lake, Kaweah, Kernville, Keyes, Kings Beach, Kingsburg, Lake Isabella, Lakeshore, Lee Vining, Lemoncove, Lemoore, Lemoore Naval Air Station, Linden, Lindsay, Los Banos, Mariposa, McFarland, Mendota, Merced, Murphys, Oakhurst, Orosi, Parlier, Pasadena, Piedra, Pollock Pines, Prather, Raymond, Reedley, Sacramento, Selma, Shaver Lake, Squaw Valley, Stanton, Sultana, Sanger (press report), Tehachapi, Tipton, Tollhouse, Tranquillity, Tulare, Tuolumne, Tuolumne Meadows, Ventura, Visalia, Wofford Heights, Yettem, Yosemite Lodge, Yosemite National Park.

Nevada - Babbitt, Candelaria (press report), Fallon, Fernley, Gardnerville, Goldfield, Gabbs (press report), Hawthorne, Incline Village, Luning, Mercury, Mina, Minden, Montgomery Pass (press report), Tonopah, Yerington.

Intensity III:

California-Arcadia, Armona, Atwater, Biola, Burbank, Buttonwillow, Citrus Heights, Columbia, Delhi, Denair, Dublin (press report), Empire, Fremont, Georgetown, Glendale, Iowa Hill, Isleton, Jackson, Jamestown, Kelsey, Kerman, Lamont, Lathrop, Lemon Grove, Livermore, Lodi, Los Alamitos, Lundy Canyon (press report), Manteca, Mather Air Force Base, Meadow Vista, Modesto, Oceanside, Patterson, Planada, Pleasanton, Ridgecrest, Ripon, Rosamond, Salida, San Andreas, San Jose, San Juan Bautista, San Pedro, Santa Cruz, Santee, Shingle Springs, Spring Valley, Stockton, Sutter (press report), Tahoe City, Tracy, Truckee, Twain Harte, Wasco, Winton, Woodlake, Woodland, Yuba City.

Nevada-Las Vegas, Sparks, Virginia City, Zephyr Cove. Intensity II:

California-Aptos, Courtland, Folsom, Indio, Riverdale. Nevada-Elk.

Felt:

California-Groveland, Terra Bella.

21 July (BK) Owens Valley area

Origin time: 145110.1

Epicenter: 37.570N., 118.525W.

Depth: $1 \mathrm{~km}$

\section{CALIFORNIA-Continued}

Magnitude: $5.1 \mathrm{~m}_{\mathrm{b}}(\mathrm{GS}), 5.7 \mathrm{M}_{\mathrm{L}}(\mathrm{BK}), 5.4 \mathrm{M}_{\mathrm{L}}(\mathrm{PS})$, $5.8 \mathrm{M}_{\mathrm{L}}(\mathrm{RN})$

Moment: $4.3 \times 10^{23}$ dyne-cm (BK)

Intensity V:

California-

Benton-Many small objects overturned; hanging pictures fell; trees and bushes shook strongly; people had difficulty standing; felt by everyone.

Big Pine-Many items were shaken off store shelves; a few glassware items or dishes broke; hanging pictures fell; a few small objects overturned and fell; people had difficulty standing or walking; shaking was described as strong; trees and bushes shook moderately; standing and moving vehicles rocked moderately; felt by everyone.

Bishop-A few windows cracked; a few glassware items or dishes broke; many items were shaken off store shelves; a few small objects overturned and fell; trees and bushes shook slightly; standing vehicles rocked slightly; feltby everyone.

Nevada-

Dyer-People had difficulty standing, felt by everyone.

21 July (HJ) Off the coast of Southern California

Origin time: 182930.8

Epicenter: $33.018 \mathrm{~N} ., 117.802 \mathrm{~W}$.

Depth: $8 \mathrm{~km}$

Magnitude: $3.8 \mathrm{M}_{\mathrm{L}}(\mathrm{PS}), 3.8 \mathrm{M}_{\mathrm{L}}(\mathrm{HJ})$

Felt: Hawthorne (PS), Laguna Beach (PS), Long Beach (PS).

\section{July (BK) Central California}

Origin time: 220717.0

Epicenter: $37.483 \mathrm{~N} ., 118.367 \mathrm{~W}$.

Depth: $6 \mathrm{~km}$

Magnitude: $5.5 \mathrm{~m}_{\mathrm{b}}(\mathrm{GS}), 5.0 \mathrm{M}_{\mathrm{S}}(\mathrm{GS}), 5.6 \mathrm{M}_{\mathrm{L}}(\mathrm{BK})$,

$$
5.4 \mathrm{M}_{\mathrm{L}}(\mathrm{PS}), 5.8 \mathrm{M}_{\mathrm{L}}(\mathrm{RN})
$$

Moment: $4.2 \times 10^{23}$ dyne-cm (BK)

Felt: Bishop (press report).

22 July (BK) Owens Valley area

Origin time: 133359.8

Epicenter: $37.517 \mathrm{~N} ., 118.477 \mathrm{~W}$.

Depth: $9 \mathrm{~km}$

Magnitude: $4.2 \mathrm{~m}_{\mathrm{b}}(\mathrm{GS}), 4.7 \mathrm{M}_{\mathrm{L}}(\mathrm{BK}), 4.6 \mathrm{M}_{\mathrm{L}}(\mathrm{RN})$, 5.0 $\mathrm{M}_{\mathrm{L}}(\mathrm{PS})$

Moment: $4.9 \times 10^{22}$ dyne-cm (BK)

Intensity IV: Bishop (press report).

23 July (HJ) Off the coast of Southern California

Origin time: 025758.2

Epicenter: 32.998N., 117.788W.

Depth: 4 km 


\section{CALIFORNIA-Continued}

Magnitude: $3.5 \mathrm{M}_{\mathrm{L}}(\mathrm{PS}), 3.5 \mathrm{M}_{\mathrm{L}}(\mathrm{HJ})$

Felt: Epicentral area (PS).

23 July (PS) Southern California

Origin time: 063834.2

Epicenter: 34.103N., 117.314W.

Depth: $15 \mathrm{~km}$

Magnitude: $2.7 \mathrm{M}_{\mathrm{L}}(\mathrm{PS})$

Felt: San Bernardino (PS).

23 July (HJ) Off the coast of Southern California

Origin time: 102901.6

Epicenter: 33.013N., 117.817W.

Depth: $6 \mathrm{~km}$

Magnitude: $3.4 \mathrm{M}_{\mathrm{L}}(\mathrm{PS}), 3.4 \mathrm{M}_{\mathrm{L}}(\mathrm{HJ})$

Felt: Epicentral area (PS).

24 July (PS) Southern California

Origin time: 015823.1

Epicenter: 33.970N., 116.556W.

Depth: 9 km

Magnitude: $3.3 \mathrm{M}_{\mathrm{L}}(\mathrm{PS})$

Felt: Palm Springs area (PS).

25 July (PS) Southern California

Origin time: 054403.7

Epicenter: $34.424 \mathrm{~N} ., 118.407 \mathrm{~W}$.

Depth: $11 \mathrm{~km}$

Magnitude: $3.0 \mathrm{M}_{\mathrm{L}}(\mathrm{PS})$

Intensity III: Canyon Country.

Intensity II: Leona Valley.

Felt: San Fernando (press report).

25 July (PS) Southern California

Origin time: 113909.7

Epicenter: 33.997N., 116.571W.

Depth: $8 \mathrm{~km}$

Magnitude: $2.8 \mathrm{M}_{\mathrm{L}}(\mathrm{PS})$

Felt: Epicentral area (PS).

26 July (PS) Southern California

Origin time: 054031.5

Epicenter: 34.001N., 116.574W.

Depth: $10 \mathrm{~km}$

Magnitude: $2.9 \mathrm{M}_{\mathrm{L}}(\mathrm{PS})$

Felt: Long Beach (PS), South Bay Beaches area (PS).

28 July (HJ) Off the coast of Southern California

Origin time: 025445.7

Epicenter: 32.953N., 117.812W.

Depth: $5 \mathrm{~km}$

Magnitude: $3.6 \mathrm{M}_{\mathrm{L}}(\mathrm{PS}), 3.6 \mathrm{M}_{\mathrm{L}}(\mathrm{HJ})$

Felt: Epicentral area (PS).

\section{CALIFORNIA-Continued}

29 July (PS) Southern California

Origin time: 064350.3

Epicenter: 33.965N., 116.590W.

Depth: $7 \mathrm{~km}$

Magnitude: $3.2 \mathrm{M}_{\mathrm{L}}(\mathrm{PS})$

Felt: Epicentral area (PS).

29 July (BK) Central California

Origin time: 071158.8

Epicenter: $37.543 N$., 118.445W.

Depth: $8 \mathrm{~km}$

Magnitude: $4.2 \mathrm{M}_{\mathrm{L}}(\mathrm{BK}), 3.6 \mathrm{M}_{\mathrm{L}}(\mathrm{PS}), 4.3 \mathrm{M}_{\mathrm{L}}(\mathrm{RN})$

Moment: $1.4 \times 10^{22}$ (BK)

Felt: Crowley Lake (press report).

29 July (HJ) Off the coast of Southern California

Origin time: 081741.4

Epicenter: 32.945N., 117.828W.

Depth: $6 \mathrm{~km}$

Magnitude: $3.9 \mathrm{~m}_{\mathrm{b}}(\mathrm{GS}), 4.3 \mathrm{M}_{\mathrm{L}}(\mathrm{PS}), 4.3 \mathrm{M}_{\mathrm{L}}(\mathrm{HJ})$

Felt in Orange, Riverside, and San Diego Counties (press report).

Intensity V:

Oceanside-A few items were shaken off store shelves; a few small objects fell; awakened few people.

Rancho Bernardo-A few windows cracked; buildings shook moderately; felt by and awakened many people.

San Diego-A few windows cracked; interior walls sustained hairline cracks; a few glassware items or dishes broke; a few small objects overturned and fell; trees and bushes shook slightly; standing and moving vehicles rocked slightly; felt by many people.

San Diego (Golden Hill)-A few items were shaken off store shelves; hanging pictures fell; a few small objects overturned and fell.

Intensity IV: Capistrano Beach, Laguna Beach (press report), Poway, San Clemente (press report), San Diego (Lindberg Field), San Diego (Ocean Beach), San Diego (Paradise Hills), Vista.

Intensity III: Encinitas, Escondido, Lemon Grove, Leucadia, San Clemente, San Luis Rey, San Marcos, Santee, San Ysidro, Yucaipa.

Felt: El Cajon (press report), Lakeside (press report).

29 July (BK) Owens Valley area

Origin time: 095757.2

Epicenter: 37.595 N., 118.477 W.

Depth: $7 \mathrm{~km}$

Magnitude: $3.7 \mathrm{~m}_{\mathrm{b}}(\mathrm{GS}), 4.6 \mathrm{M}_{\mathrm{L}}(\mathrm{BK}), 4.6 \mathrm{M}_{\mathrm{L}}(\mathrm{PS})$,

$$
\text { 4.7 } \mathrm{M}_{\mathrm{L}}(\mathrm{RN})
$$

Moment: $3.8 \times 10^{22}$ dyne-cm (BK)

Intensity IV: Bishop.

29 July (HJ) Off the coast of Southern California

Origin time: 112222.4 


\section{CALIFORNIA-Continued}

Epicenter: 32.957N., 117.815W.

Depth: $5 \mathrm{~km}$

Magnitude: $3.5 \mathrm{M}_{\mathrm{L}}(\mathrm{PS}), 3.5 \mathrm{M}_{\mathrm{L}}(\mathrm{HJ})$

Felt: Epicentral area (press report).

29 July (PS) Southern California

Origin time: 120319.5

Epicenter: $33.966 \mathrm{~N} ., 116.568 \mathrm{~W}$.

Depth: $7 \mathrm{~km}$

Magnitude: $3.0 \mathrm{M}_{\mathrm{L}}(\mathrm{PS})$

Felt: Epicentral area (PS).

30 July (PS) Southern California

Origin time: 011401.1

Epicenter: $34.000 \mathrm{~N} ., 118.378 \mathrm{~W}$.

Depth: $4 \mathrm{~km}$

Magnitude: $2.8 \mathrm{M}_{\mathrm{L}}(\mathrm{PS})$

Felt: Culver City (PS), West Hollywood (PS).

30 July (BK) Owens Valley area

Origin time: 064152.9

Epicenter: 37.582N., 118.468W.

Depth: $7 \mathrm{~km}$

Magnitude: $4.1 \mathrm{~m}_{\mathrm{b}}(\mathrm{GS}), 4.8 \mathrm{M}_{\mathrm{L}}(\mathrm{BK}), 4.8 \mathrm{M}_{\mathrm{L}}(\mathrm{PS})$

Moment: $5.1 \times 10^{22}$ dyne-cm (BK)

Intensity IV: Benton, Bishop.

Felt: Toms Place.

30 July (HJ) Off the coast of Southern California

Origin time: 225113.0

Epicenter: 33.012N., 117.782W.

Depth: $7 \mathrm{~km}$

Magnitude: $3.9 \mathrm{M}_{\mathrm{L}}(\mathrm{PS}), 3.9 \mathrm{M}_{\mathrm{L}}(\mathrm{HJ})$

Felt: Epicentral area (PS).

\section{July (BK) Owens Valley area}

Origin time: 072240.2

Epicenter: 37.463N., 118.367W.

Depth: 7 km

Magnitude: $5.5 \mathrm{~m}_{\mathrm{b}}(\mathrm{GS}), 5.2 \mathrm{M}_{\mathrm{S}}(\mathrm{GS}), 5.8 \mathrm{M}_{\mathrm{L}}(\mathrm{BK})$,

$$
5.9 \mathrm{M}_{\mathrm{L}}(\mathrm{PS}), 5.5 \mathrm{M}_{\mathrm{L}}(\mathrm{RN})
$$

Moment: $1.6 \times 10^{24}$ dyne-cm (BK)

This earthquake was an aftershock of the 21 July 14

4226.5 UTC earthquake.

Intensity VI:

California-

Bishop-A plate-glass window broke at the Safeway store; a few other windows broke; light fixtures fell at the U.S. National Weather Service office; people ran outside; items were shaken off store shelves (press report).

Intensity V:

California-

\section{CALIFORNIA-Continued}

Big Pine-A few items were shaken off store shelves; shaking was described as strong; felt by and awakened many people.

Sequoia National Park-Dry wall sustained hairline cracks; a few items were shaken off store shelves; a few glassware items or dishes broke; a few small objects overturned and fell; buildings shook strongly; felt by and awakened many people.

Sonora-Plaster walls sustained hairline cracks; trees and bushes shook slightly; hanging pictures swung out of place; felt by many people.

Nevada-

Dyer-Many items were shaken off store shelves; hanging pictures swung out of place; felt by everyone; awakened many people.

Intensity IV:

California-Armona, Auberry, Bass Lake, Benton, Big Creek, Bridgeport, Camp Nelson, China Lake, Grant Grove, Hume, Independence, June Lake, Kaweah, Kerman, Lindsay, Lone Pine, Mammoth Lakes, Miramonte, Orange Cove, Orosi, Ridgecrest, Tollhouse, Traver, Wawona.

Nevada-Goldfield.

Intensity III:

California-Ahwahnee, Badger, Biola, Coarsegold, Dinuba, El Portal, Firebaugh, Fish Camp, Fresno, Friant, Hanford, Kernville, Kinsburg, Mendota, North Fork, Oakhurst, O'Neals, Paso Robles, Piedra, Prather, Ripon, Riverdale, Shaver Lake, South Lake Tahoe, Tulare, Winton, Wofford Heights, Woodlake.

Nevada-Mina, Tonopah.

Intensity II:

California-Tipton.

Felt:

California-Bakersfield (press report), Chowchilla, Coalinga, Farmersville, Toms Place, Yosemite Lodge.

31 July (PS) Southern California

Origin time: 075142.9

Epicenter: $33.966 \mathrm{~N} ., 116.574 \mathrm{~W}$.

Depth: $7 \mathrm{~km}$

Magnitude: $3.3 \mathrm{M}_{\mathrm{L}}(\mathrm{PS})$

Felt: Palm Springs (PS).

31 July (PS) Southern California

Origin time: 191645.9

Epicenter: 33.995N., 118.368W.

Depth: $4 \mathrm{~km}$

Magnitude: $2.8 \mathrm{M}_{\mathrm{L}}(\mathrm{PS})$

Felt: Culver City (press report), West Hollywood (PS).

1 August (BK) Owens Valley area

Origin time: 142716.4

Epicenter: 37.501N., 118.398W. 
Depth: $6 \mathrm{~km}$

Magnitude: $4.2 \mathrm{~m}_{\mathrm{b}}(\mathrm{GS}), 4.8 \mathrm{M}_{\mathrm{L}}(\mathrm{BK}), 3.4 \mathrm{M} \mathrm{M}_{\mathrm{L}}(\mathrm{PS})$, $4.1 \mathrm{M}_{\mathrm{L}}(\mathrm{RN})$

Felt: Bishop (press report).

1 August (BK) Owens Valley area

Origin time: 142819.6

Epicenter: 37.468N., 118.448W.

Depth: 14 km

Magnitude: $4.9 \mathrm{~m}_{\mathrm{b}}(\mathrm{GS}), 5.1 \mathrm{M}_{\mathrm{L}}(\mathrm{BK}), 5.2 \mathrm{M}_{\mathrm{L}}(\mathrm{RN})$

Moment: $2.1 \times 10^{23}$ dyne-cm (BK)

Felt: Bishop (press report).

\section{August (PS) Southern California}

Origin time: 113657.8

Epicenter: 34.036N., 116.696W.

Depth: $11 \mathrm{~km}$

Magnitude: $3.4 \mathrm{M}_{\mathrm{L}}(\mathrm{PS}), 3.5 \mathrm{M}_{\mathrm{L}}(\mathrm{PS})$

Felt: Palm Springs area (press report).

\section{August (BK) Central California}

Origin time: 103305.3

Epicenter: 37.615 N., 118.455W.

Depth: $7 \mathrm{~km}$

Magnitude: $3.6 \mathrm{~m}_{\mathrm{b}}(\mathrm{GS}), 4.3 \mathrm{M}_{\mathrm{L}}(\mathrm{BK}), 4.0 \mathrm{M}_{\mathrm{L}}(\mathrm{RN})$

Moment: $2.3 \times 10^{22}$ dyne-cm (BK)

Felt: Chalfant (PS).

\section{August (BK) Central California}

Origin time: 034141.9

Epicenter: 37.432N., 121.773W.

Depth: $8 \mathrm{~km}$

Magnitude: $3.4 \mathrm{M}_{\mathrm{L}}(\mathrm{BK})$

Moment: $1.7 \times 10^{21}$ dyne-cm (BK)

Intensity IV: San Jose.

Intensity III: Santa Clara.

\section{August (BK) Northern California}

Origin time: 173135.3

Epicenter: 40.862N., 123.725W.

Depth: $42 \mathrm{~km}$

Magnitude: $3.8 \mathrm{M}_{\mathrm{L}}(\mathrm{BK})$

Felt: Eureka (BK).

9 August (PS) Off the coast of Southern California Origin time: 005229.3

Epicenter: $32.500 \mathrm{~N} ., 117.398 \mathrm{~W}$.

Depth: $6 \mathrm{~km}$

Magnitude: $3.4 \mathrm{M}_{\mathrm{L}}(\mathrm{PS})$

Intensity III: Spring Valley.

Intensity II: San Diego.

Felt: Imperial Beach (PS), Pacific Beach (PS), Point Loma (PS).
14 August (BK) Northern California

Origin time: 005040.8

Epicenter: $40.377 \mathrm{~N} ., 124.328 \mathrm{~W}$.

Depth: $18 \mathrm{~km}$

Magnitude: $3.7 \mathrm{M}_{\mathrm{L}}(\mathrm{BK})$

Moment: $3.6 \times 10^{21}$ dyne-cm (BK)

Intensity III: Rio Dell, Scotia (BK).

15 August (HJ) Off the coast of Southern California

Origin time: 184516.1

Epicenter: 32.948N., 117.797W.

Depth: $6 \mathrm{~km}$

Magnitude: $3.7 \mathrm{M}_{\mathrm{L}}(\mathrm{PS}), 3.8 \mathrm{M}_{\mathrm{L}}(\mathrm{GS})$

Felt: Epicentral area (PS).

\section{August (BK) Central California}

Origin time: 070216.8

Epicenter: 37.122N., 121.563W.

Depth: 6 km

Magnitude: $3.3 \mathrm{M}_{\mathrm{L}}(\mathrm{BK}), 3.1 \mathrm{M}_{\mathrm{L}}(\mathrm{PS})$

Intensity IV: Morgan Hill (press report).

Felt: Gilroy (BK).

20 August (BK) Central California

Origin time: 095021.0

Epicenter: 37.120N., 121.562W.

Depth: $6 \mathrm{~km}$

Magnitude: $2.8 \mathrm{M}_{\mathrm{L}}(\mathrm{BK}), 2.7 \mathrm{M}_{\mathrm{L}}(\mathrm{PS})$

Intensity III: Morgan Hill (press report).

Felt: Gilroy (BK).

20 August (BK) Central California

Origin time: 095705.0

Epicenter: $37.118 \mathrm{~N} ., 121.565 \mathrm{~W}$.

Depth: $7 \mathrm{~km}$

Magnitude: $3.3 \mathrm{M}_{\mathrm{L}}(\mathrm{BK}), 3.0 \mathrm{M}_{\mathrm{L}}(\mathrm{PS})$

Intensity III: Morgan Hill (press report).

23 August (BK) Central California

Origin time: 030130.3

Epicenter: 37.493N., 118.383W.

Depth: $5 \mathrm{~km}$

Magnitude: $3.9 \mathrm{M}_{\mathrm{L}}(\mathrm{BK}), 4.0 \mathrm{M}_{\mathrm{L}}(\mathrm{RN})$

Intensity III: Bishop.

25 August (BK) Central Califormia

Origin time: 114751.2

Epicenter: 37.747 N., 121.968W.

Depth: $4 \mathrm{~km}$

Magnitude: $2.6 \mathrm{M}_{\mathrm{L}}(\mathrm{BK})$

Felt: Danville (BK), San Ramon (BK).

28 August (PS) Off the coast of Southern California Origin time: 101416.3 


\section{CALIFORNIA-Continued}

Epicenter: $32.491 \mathrm{~N} ., 117.420 \mathrm{~W}$.

Depth: $6 \mathrm{~km}$

Magnitude: $3.6 \mathrm{M}_{\mathrm{L}}(\mathrm{PS})$

Intensity V:

San Diego (Encanto)-A few windows cracked ; a few glassware items or dishes broke; a few small objects overturned and fell; felt by and awakened many people.

Solana Beach-A few windows cracked; a few glassware items or dishes broke; a few small objects overturned and fell; awakened a few people.

Intensity IV: San Diego (Lindbergh Fields), San Diego

(Sierra Mesa).

Intensity III: San Diego (Mission Gorge area), San Diego

(Ocean Beach), San Marcos, Spring Valley.

\section{August (PS) Southern California}

Origin time: 163214.6

Epicenter: 33.919N., 116.273W.

Depth: $8 \mathrm{~km}$

Magnitude: $3.2 \mathrm{M}_{\mathrm{L}}(\mathrm{PS})$

Felt: Palm Springs (press report).

\section{August (BK) Central California}

Origin time: 064442.5

Epicenter: 35.888N., 120.475W.

Depth: $5 \mathrm{~km}$

Magnitude: $3.2 \mathrm{M}_{\mathrm{L}}(\mathrm{BK}), 3.6 \mathrm{M}_{\mathrm{L}}(\mathrm{PS})$

Intensity III: Paso Robles, San Ardo.

Felt: Parkfield (BK).

\section{August (PS) Southern California}

Origin time: 074654.5

Epicenter: 33.956 N., 116.599W.

Depth: $8 \mathrm{~km}$

Magnitude: $3.7 \mathrm{M}_{\mathrm{L}}(\mathrm{PS})$

Intensity V:

Desert Hot Springs- A few items were shaken off store shelves; shaking was described as strong; felt by many people.

Hemet-A few items were shaken off store shelves; interior walls sustained hairline cracks; a few glassware items or dishes broke; small objects overturned and fell; buildings shook strongly; felt by everyone.

Intensity IV: Cathedral City, Palm Springs, Yucca Valley.

Intensity III: Beaumont, Cabazon, Forest Falls, Indio, North

Palm Springs.

2 September (BK) Northern California

Origin time: 135956.1

Epicenter: 41.128 N., 123.755W.

Depth: $12 \mathrm{~km}$

Magnitude: $3.4 \mathrm{M}_{\mathrm{L}}(\mathrm{BK})$

\section{CALIFORNIA-Continued}

Moment: $8.4 \times 10^{20}$ dyne-cm (BK)

Felt: Epicentral area (BK).

3 September (BK) Central California

Origin time: 043114.8

Epicenter: 37.295 N., $121.667 \mathrm{~W}$.

Depth: $10 \mathrm{~km}$

Magnitude: $4.0 \mathrm{M}_{\mathrm{L}}(\mathrm{BK}), 3.6 \mathrm{M}_{\mathrm{L}}(\mathrm{PS})$

The press reported that this earthquake was felt from Monterey County to Marin County.

Intensity $\mathrm{V}$ :

Soquel-A few glassware items or dishes broke; a few small objects overturned and fell; buildings shookmoderately; standing and moving vehicles rocked slightly; felt by everyone.

Intensity IV: Brookdale, Fremont, Loma Mar, Mount Hamilton, Mount Hermon, New Almaden, Redwood Estates, San Jose, Santa Cruz.

Intensity III: Aptos, Felton, Gilroy, Ladera, Morgan Hill.

Felt: San Francisco (press report).

\section{September (BK) Central California}

Origin time: 162244.8

Epicenter: 36.838N., 121.395W.

Depth: $7 \mathrm{~km}$

Magnitude: $2.4 \mathrm{M}_{\mathrm{L}}(\mathrm{BK}), 2.5 \mathrm{M}_{\mathrm{L}}(\mathrm{PS})$

Felt: Hollister (BK).

9 September (PS) Southern California

Origin time: 162250.6

Epicenter: $33.966 \mathrm{~N} ., 116.567 \mathrm{~W}$.

Depth: $6 \mathrm{~km}$

Magnitude: $3.5 \mathrm{M}_{\mathrm{L}}(\mathrm{PS})$

Intensity IV: Cabazon, Desert Hot Springs, Morongo Valley, Mountain Center, North Palm Springs.

Intensity III: Joshua Tree, La Quinta, Palm Springs.

Intensity II: Thousand Palms.

Felt: Desert Hot Springs (PS).

10 September (PS) Southern California

Origin time: 15.5152 .4

Epicenter: 33.958N., 116.669W.

Depth: 9 km

Magnitude: $3.0 \mathrm{M}_{\mathrm{L}}(\mathrm{PS})$

Intensity III: Palm Springs (press report).

16 September (RN) Owens Valley area

Origin time: 131425.8

Epicenter: 37.615N., 118.430W.

Depth: $8 \mathrm{~km}$

Magnitude: $3.5 \mathrm{M}_{\mathrm{L}}(\mathrm{BK}), 3.8 \mathrm{M}_{\mathrm{L}}(\mathrm{RN})$

Intensity IV: Chalfant (press report).

Intensity III: Benton. 
18 September (RN) Owens Valley area

Origin time: 075947.7

Epicenter: 37.622N., 118.435W.

Depth: $8 \mathrm{~km}$

Magnitude: $4.0 \mathrm{M}_{\mathrm{L}}(\mathrm{BK}), 4.2 \mathrm{M}_{\mathrm{L}}(\mathrm{PS})$

Felt: Bishop (PS).

18 September (PS) Southern California

Origin time: 090510.9

Epicenter: $33.700 \mathrm{~N} ., 116.746 \mathrm{~W}$.

Depth: $17 \mathrm{~km}$

Magnitude: $2.7 \mathrm{M}_{\mathrm{L}}$ (PS)

Felt: Hemet (PS).

\section{September (BK) Central California}

Origin time: 144115.1

Epicenter: $37.365 N$., 121.742W.

Depth: $6 \mathrm{~km}$

Magnitude: $3.2 \mathrm{M}_{\mathrm{L}}(\mathrm{BK}), 2.7 \mathrm{M}_{\mathrm{L}}(\mathrm{PS})$,

Moment: $3.4 \times 10^{20}$ dyne-cm (BK)

Intensity II: Cupertino, Mount Hamilton.

23 September (BK) Central California

Origin time: 192756.7

Epicenter: 36.073 N., $121.792 W$.

Depth: $18 \mathrm{~km}$

Magnitude: $3.7 \mathrm{M}_{\mathrm{L}}(\mathrm{BK})$

Moment: $1.8 \times 10^{21}$ dyne-cm (BK)

Intensity II: Big Sur.

Felt: King City (BK).

24 September (PS) Southern California

Origin time: 104630.1

Epicenter: $34.540 \mathrm{~N} ., 119.036 \mathrm{~W}$.

Depth: $18 \mathrm{~km}$

Magnitude: $3.4 \mathrm{M}_{\mathrm{L}}$ (PS)

Felt: Ventura (PS).

26 September (PS) Off the coast of Southern California Origin time: 003524.1

Epicenter: $32.685 \mathrm{~N} ., 117.152 \mathrm{~W}$.

Depth: $8 \mathrm{~km}$

Magnitude: $2.8 \mathrm{M}_{\mathrm{L}}(\mathrm{PS})$

This earthquake was felt in scattered areas of San Diego County (press report).

Felt: San Diego (PS).

28 September (PS) Southern California

Origin time: 070626.9

Epicenter: $34.010 \mathrm{~N} ., 116.577 \mathrm{~W}$.

Depth: $10 \mathrm{~km}$
Magnitude: $3.2 \mathrm{M}_{\mathrm{L}}(\mathrm{PS})$

Intensity IV: Morongo Valley.

29 September (PS) Southern California

Origin time: 140026.6

Epicenter: 34.018N., 117.239W.

Depth: $16 \mathrm{~km}$

Magnitude: $2.9 \mathrm{M}_{\mathrm{L}}(\mathrm{PS})$

Felt: San Bernardino (PS).

30 September (HJ) Off the coast of Southern California Origin time: 095211.2

Epicenter: 33.008N., 117.777W.

Depth: $0 \mathrm{~km}$

Magnitude: $3.9 \mathrm{M}_{\mathrm{L}}(\mathrm{PS}), 3.9 \mathrm{M}_{\mathrm{L}}(\mathrm{HJ})$

Intensity IV: Lakeside, Pala, San Diego.

Intensity III: Avalon, El Toro, Escondido, Laguna Hills, Mission Viejo, San Luis Rey, San Marcos, Vista.

Intensity II: Spring Valley.

1 October (HJ) Off the coast of Southern California

Origin time: 201218.3

Epicenter: 32.973 N., 117.840W.

Depth: $8 \mathrm{~km}$

Magnitude: $4.0 \mathrm{M}_{\mathrm{L}}(\mathrm{PS}), 4.0 \mathrm{M}_{\mathrm{L}}(\mathrm{HJ})$

Felt: Oceanside (press report), San Diego (press report), southern Orange County (press report).

2 October (PS) Southern California

Origin time: 045542.0

Epicenter: $34.033 \mathrm{~N} ., 116.639 \mathrm{~W}$.

Depth: $11 \mathrm{~km}$

Magnitude: $2.9 \mathrm{M}_{\mathrm{L}}(\mathrm{PS})$

Felt: Palm Springs (PS).

2 October (HJ) Southern California

Origin time: 152328.6

Epicenter: 33.010N., 117.753W.

Depth: $0 \mathrm{~km}$

Magnitude: $3.4 \mathrm{M}_{\mathrm{L}}(\mathrm{PS}), 3.4 \mathrm{M}_{\mathrm{L}}(\mathrm{HJ})$

Intensity III: Oceanside (press report).

9 October (RN) Owens Valley area

Origin time: 053725.0

Epicenter: 37.348N., 118.370W.

Depth: 15 km

Magnitude: $4.4 \mathrm{M}_{\mathrm{L}}(\mathrm{BK}), 4.3 \mathrm{M}_{\mathrm{L}}(\mathrm{PS}), 3.8 \mathrm{M}_{\mathrm{L}}(\mathrm{RN})$

Moment: $1.7 \times 10^{22}$ dyne-cm (BK)

Intensity III: Bishop (press report), Lone Pine.

10 October (PS) Southern California

Origin time: 152302.6

Epicenter: 33.948N., 116.786W.

Depth: $2 \mathrm{~km}$ 


\section{CALIFORNIA-Continued}

Magnitude: $3.5 \mathrm{M}_{\mathrm{L}}$ (PS)

Intensity IV: Angelus Oaks.

Intensity III: Anza, Desert Hot Springs, Forest Falls, North Palm Springs, Palm Springs, Rancho Mirage, Thousand Palms.

\section{October (BK) Central California}

Origin time: 051736.4

Epicenter: $37.827 \mathrm{~N} ., 121.960 \mathrm{~W}$.

Depth: $6 \mathrm{~km}$

Magnitude: $4.2 \mathrm{M}_{\mathrm{L}}(\mathrm{BK})$

Moment: $8.3 \times 10^{21}$ dyne-cm (BK)

Intensity V:

Canyon-Hanging pictures fell; many books overturned; buildings shook strongly; felt by many people.

Intensity IV: Concord, Danville, Dublin, Lafayette, Oakland, Pleasanton, Pleasant Hill (press report), San Ramon (press report).

Intensity III: Clayton, Concord (press report), Hayward (press report), Martinez (press report), Richmond, Sunol, Union City, Walnut Creek (press report).

Felt: Berkeley (press report), Livermore (press report), San Francisco (press report).

\section{October (BK) Central California}

Origin time: 063938.2

Epicenter: $37.827 \mathrm{~N} ., 121.952 \mathrm{~W}$.

Depth: $5 \mathrm{~km}$

Magnitude: $3.2 \mathrm{M}_{\mathrm{L}}(\mathrm{BK})$

Moment: $1.9 \times 10^{21}$ dyne-cm (BK)

Felt: Danville (press report).

\section{October (BK) Central California}

Origin time: 064301.9

Epicenter: 38.718 N., $123.500 \mathrm{~W}$.

Depth: $11 \mathrm{~km}$

Magnitude: $4.2 \mathrm{~m}_{\mathrm{b}}(\mathrm{GS}), 4.0 \mathrm{M}_{\mathrm{L}}(\mathrm{BK})$

Moment: $1.6 \times 10^{22}$ dyne-cm (BK)

Intensity $\mathrm{V}$ :

Sea Ranch-A few items were shaken off store shelves; a few glassware items or dishes broke; a few small objects overturned and fell; hanging pictures swung out of place; felt by everyone.

Intensity IV: Annapolis, Gualala, Point Arena.

Intensity III: Manchester, Ville Grande.

\section{October (PS) Southern California}

Origin time: 022847.8

Epicenter: 33.953 N., $116.572 W$.

Depth: $9 \mathrm{~km}$

Magnitude: $4.3 \mathrm{~m}_{\mathrm{b}}(\mathrm{GS}), 4.9 \mathrm{M}_{\mathrm{L}}(\mathrm{PS}), 4.5 \mathrm{M}_{\mathrm{L}}(\mathrm{BK})$,

$$
\text { 4.7 } \mathrm{M}_{\mathrm{L}}(\mathrm{GP})
$$

\section{CALIFORNIA-Continued}

Intensity V:

Desert Hot Springs-A few small objects overturned; buildings shook strongly; trees and bushes shook slightly; felt by many people.

La Quinta-A few glassware items or dishes broke; a few small objects overturned and fell; trees and bushes shook slightly; standing vehicles rocked slightly; felt by many people.

Palm Springs-A few small objects overturned; trees and bushes shook moderately; standing and moving vehicles rocked moderately; felt by everyone.

Rancho Mirage-A few small objects overturned and fell; trees and bushes shook slightly; standing vehicles rocked slightly; felt by many people.

Thousand Palms-Plaster walls sustained hairline cracks; a few small objects fell; trees and bushes shook slightly; standing vehicles rocked slightly; felt by everyone.

Intensity IV: Beaumont, Coachella, Forest Falls, Hemet, Indio, Joshua Tree, North Palm Springs, Palm Desert, Palm Springs, San Jacinto, Thermal, Twentynine Palms, White Water, Yucca Valley.

Intensity III: Anza, Banning, Fontana, Morongo Valley, Pala, Landers.

Felt: Cathedral City.

15 October (PS) Southern California

Origin time: 054618.8

Epicenter: $34.165 \mathrm{~N} ., 118.274 \mathrm{~W}$.

Depth: $3 \mathrm{~km}$

Magnitude: $2.5 \mathrm{M}_{\mathrm{L}}(\mathrm{PS})$

Felt: Burbank (PS), Glendale (PS).

16 October (BK) Northern California

Origin time: 061640.7

Epicenter: 40.327N., 123.927W.

Depth: $6 \mathrm{~km}$

Magnitude: $2.4 \mathrm{M}_{\mathrm{L}}(\mathrm{BK})$

Intensity III: Rio Dell.

Felt: Scotia (BK).

16 October (PS) Southern California

Origin time: 101617.0

Epicenter: 33.977N., 116.565W.

Depth: $8 \mathrm{~km}$

Magnitude: $2.9 \mathrm{M}_{\mathrm{L}}(\mathrm{PS})$

Felt: Palm Springs (PS).

16 October (BK) Central California

Origin time: 112037.6

Epicenter: 37.755 N., $122.138 \mathrm{~W}$.

Depth: $7 \mathrm{~km}$

Magnitude: $2.2 \mathrm{M}_{\mathrm{L}}(\mathrm{BK})$

Felt: San Leandro (BK). 


\section{CALIFORNIA-Continued}

19 October (PS) Southern California

Origin time: 145026.1

Epicenter: $34.015 N$., 116.687W.

Depth: $8 \mathrm{~km}$

Magnitude: $2.9 \mathrm{M}_{\mathrm{L}}(\mathrm{PS})$

Felt: Epicentral area (PS).

\section{October (BK) Central California}

Origin time: 131458.6

Epicenter: 36.760N., 121.367W.

Depth: $11 \mathrm{~km}$

Magnitude: $3.3 \mathrm{M}_{\mathrm{L}}(\mathrm{BK})$,

Moment: $7.8 \times 10^{20}$ dyne-cm (BK)

Felt: Hollister (BK), Santa Cruz (BK).

\section{October (BK) Central California}

Origin time: 020645.5

Epicenter: $37.175 \mathrm{~N} ., 121.583 \mathrm{~W}$.

Depth: $8 \mathrm{~km}$

Magnitude: $3.6 \mathrm{M}_{\mathrm{L}}(\mathrm{BK})$

Moment: $5.8 \times 10^{21}$ dyne-cm (BK)

Intensity II: Morgan Hill.

Felt: Near Anderson Reservoir (BK).

\section{October (PS) Southern California}

Origin time: 023815.3

Epicenter: 32.615N., 117.152W.

Depth: $15 \mathrm{~km}$

Magnitude: $3.9 \mathrm{~m}_{\mathrm{b}}(\mathrm{GS}), 4.1 \mathrm{M}_{\mathrm{L}}(\mathrm{PS}), 4.4 \mathrm{M}_{\mathrm{L}}(\mathrm{BK})$

This earthquake was felt with a Modified Mercalli intensity $\mathrm{V}$ at Tijuana, Mexico, and Modified Mercalli intensity IV at Tecate, Mexico.

\section{Intensity V:}

Chula Vista-A few items were shaken off store shelves; a few glassware items or dishes broke; a few small objects overturned and fell; standing and moving vehicles rocked slightly; people had difficulty standing; felt by many people.

Coronado-Stucco walls sustained hairline cracks; buildings shook moderately; felt by many people.

Dulzura-A few small objects overturned and fell; shaking was described as strong; felt by many people.

El Cajon-A few small objects overturned; trees and bushes shook slightly; shaking was described as strong; felt by many people.

Encinitas-A few windows cracked; standing and moving vehicles rocked slightly; shaking was described as strong; felt by everyone.

Imperial Beach-A few glassware items or dishes broke; a few small objects overturned and fell; shaking was described as moderate; trees and bushes shook slightly;

\section{CALIFORNIA-Continued}

felt by several people. The press reported a broken water line.

San Diego-A few windows cracked; a few glassware items or dishes broke; a few small objects overturned and fell; hanging pictures swung out of place; shaking was described as moderate; felt by many people.

San Diego (Clairemont)-A few items were shaken off store shelves; a few glassware items or dishes broke; a few small objects overturned and fell; shaking was described as moderate; felt by everyone.

San Diego (Encanto)-A few items were shaken off store shelves; a few windows cracked; a few glassware items or dishes broke; a few small objects overturned and fell; standing and moving vehicles rocked slightly; trees and bushes shook slightly; shaking was described as moderate; felt by many people.

San Diego(Ocean Beach)-A few small objects overturned and fell; hanging pictures swung out of place; standing vehicles rocked slightly; trees and bushes shook slightly; shaking was described as strong; felt by many people.

Spring Valley-A few small objects overturned and fell; buildings shook strongly; trees and bushes shook slightly; standing vehicles rocked slightly; felt by many people.

Intensity IV: Bonita, Campo, Descanso, Julian, La Jolla, Lakeside, La Mesa, Lemon Grove, Murrieta, National City, North Island Naval Air Station, Palomar Mountain, Pine Valley, Poway, Potrero, Rancho Penasquitos, Ramona, San Diego (City Heights), San Diego (College Heights), San Diego (Logan Heights), San Diego (Serra Mesa), San Diego (Shelter Island-press report), San Diego (University City), San Luis Rey, Santee, Santa Ysabel:

Intensity III: Alhambra, Boulevard, Guatay, Indio, Jacumba, Miramar Naval Air Station, Mount Laguna, Oceanside, Pala, Pauma Valley, Perris, San Diego (Mission Beach), Vista.

Intensity II: Leucadia.

30 October (BK) Central California

Origin time: 181208.3

Epicenter: 36.827N., 121.578W.

Depth: $4 \mathrm{~km}$

Magnitude: $3.1 \mathrm{M}_{\mathrm{L}}(\mathrm{BK})$

Intensity IV: San Martin (press report).

Intensity III: Aptos.

\section{October (BK) California-Nevada border area}

Origin time: 035728.9

Epicenter: 38.420N., 119.323W.

Depth: 1 km

Magnitude: $4.6 \mathrm{M}_{\mathrm{L}}(\mathrm{BK}), 4.4 \mathrm{M}_{\mathrm{L}}(\mathrm{RN})$

Moment: $4.7 \times 10^{22}$ dyne-cm (BK) 


\section{CALIFORNIA-Continued}

Intensity IV:

California-Bridgeport, Topaz.

Nevada-Schurz, Wellington.

Intensity III:

California-Angels Camp, Avery, Bear Valley, Kyburz, Pine Grove.

Felt: Seven miles north of Lee Vining, Coleville (BK), South Lake Tahoe (BK), Walker (BK).

\section{October (PS) Central California}

Origin time: 142705.2

Epicenter: 35.578 N., $117.178 W$.

Depth: $6 \mathrm{~km}$

Magnitude: $3.8 \mathrm{M}_{\mathrm{L}}(\mathrm{PS})$

Intensity III: China Lake, Ridgecrest.

31 October (BK) Central California

Origin time: 184614.2

Epicenter: 36.947 N., 121.572W.

Depth: $7 \mathrm{~km}$

Magnitude: $3.5 \mathrm{M}_{\mathrm{L}}(\mathrm{BK})$

Moment: $7.6 \times 10^{21}$ dyne-cm (BK)

Intensity $\mathrm{V}$ :

Aromas-A few small objects overturned and fell; trees and bushes shook slightly; felt by many people.

Intensity IV: Gavilan College (press report).

Intensity III: Morgan Hill, Moss Landing, Santa Cruz.

Intensity II: San Juan Bautista.

Felt: Gilroy (press report), Hollister (BK), Oakland (BK), Salinas (BK), San Jose (BK), Watsonville (BK).

\section{November (BK) Central California}

Origin time: 145057.4

Epicenter: 37.347 N., $121.730 \mathrm{~W}$.

Depth: $6 \mathrm{~km}$

Magnitude: $3.4 \mathrm{M}_{\mathrm{L}}(\mathrm{BK})$

Moment: $2.1 \times 10^{21}$ dyne-cm (BK)

Intensity IV: Tres Pinos.

Intensity III: San Jose, Los Gatos.

Felt: Santa Cruz.

1 November (BK) Western Nevada

Origin time: 192338.3

See Nevada listing.

\section{November (BK) Central California}

Origin time: 034614.4

Epicenter: 37.630N., 122.483W.

Depth: $10 \mathrm{~km}$

Magnitude: $3.0 \mathrm{M}_{\mathrm{L}}(\mathrm{BK})$

Moment: $4.1 \times 10^{20}$ dyne-cm (BK)

Felt: San Francisco (BK).

\section{CALIFORNIA-Continued}

3 November (PS) Southern California

Origin time: 184040.4

Epicenter: 33.689N., 116.813W.

Depth: $18 \mathrm{~km}$

Magnitude: $2.9 \mathrm{M}_{\mathrm{L}}(\mathrm{PS})$

Intensity III: Palm Springs (press report).

3 November (GP) Southern California

Origin time: 210401.4

Epicenter: 33.874N., 116.859W.

Depth: $11 \mathrm{~km}$

Magnitude: $3.1 \mathrm{M}_{\mathrm{L}}(\mathrm{PS})$

Intensity III: Palm Springs (press report).

6 November (PS) Southern California

Origin time: 091958.3

Epicenter: 34.735 N., $120.147 \mathrm{~W}$.

Depth: 0 km

Magnitude: $4.0 \mathrm{~m}_{\mathrm{b}}(\mathrm{GS}), 4.0 \mathrm{M}_{\mathrm{L}}(\mathrm{PS})$

Intensity V:

Los Alamos-A few glassware items or dishes broke; a few small objects overturned and fell; hanging pictures fell; felt by many people.

Los Olivos-People had difficulty standing and walking; buildings shook strongly; felt by many people.

Intensity IV: Santa Ynez, Solvang.

Intensity III: Buellton.

Intensity II: Casmalia.

Felt: Lompoc (PS), Santa Maria (press report).

12 November (PS) Southern California

Origin time: 232647.9

Epicenter: $34.528 \mathrm{~N} ., 118.488 \mathrm{~W}$.

Depth: $2 \mathrm{~km}$

Magnitude: $2.7 \mathrm{M}_{\mathrm{L}}(\mathrm{PS})$

Felt: Epicentral area (PS).

13 November (PS) Southern California

Origin time: 051228.0

Epicenter: 33.959N., 116.734W.

Depth: $10 \mathrm{~km}$

Magnitude: $3.2 \mathrm{M}_{\mathrm{L}}(\mathrm{PS})$

Intensity IV: Palm Springs.

Intensity III: North Palm Springs.

13 November (RN) Owens Valley area

Origin time: 165538.3

Epicenter: $37.479 \mathrm{~N} ., 118.510 \mathrm{~W}$.

Depth: $3 \mathrm{~km}$

Magnitude: $3.9 \mathrm{M}_{\mathrm{L}}(\mathrm{BK}), 3.6 \mathrm{M}_{\mathrm{L}}(\mathrm{PS}), 4.1 \mathrm{M}_{\mathrm{L}}(\mathrm{RN})$

Felt: Bishop.

14 November (BK) Central California

Origin time: 042620.4 
Epicenter: 38.528N., 122.977W.

Depth: $2 \mathrm{~km}$

Magnitude: $2.6 \mathrm{M}_{\mathrm{L}}(\mathrm{BK})$

Felt: Forestville to Guerneville (BK).

19 November (BK) Off the coast of Northern California

Origin time: 161906.0

Epicenter: $40.375 \mathrm{~N} ., 125.060 \mathrm{~W}$.

Depth: $8 \mathrm{~km}$

Magnitude: $4.2 \mathrm{~m}_{\mathrm{b}}(\mathrm{GS}), 3.9 \mathrm{M}_{\mathrm{L}}(\mathrm{BK})$

Moment: $3.4 \times 10^{21}$ dyne-cm (BK)

Intensity IV: Ferndale.

Intensity II: Rio Dell.

\section{November (BK) Northern California}

Origin time: 233301.7

Epicenter: 40.372 N., $124.443 W$.

Depth: $15 \mathrm{~km}$

Magnitude: $5.3 \mathrm{~m}_{\mathrm{b}}(\mathrm{GS}), 5.1 \mathrm{MS}_{\mathrm{S}}(\mathrm{GS}), 5.1 \mathrm{M}_{\mathrm{L}}(\mathrm{BK})$

Moment: $6.1 \times 10^{23}$ dyne-cm (BK)

This earthquake was felt over about $12,000 \mathrm{~km}^{2}$ of northern California (fig. 17). Two people were injured.

Intensity VII:

Petrolia-Shelves in the Petrolia General Store almost emptied from shaking that broke most glass bottles and jars. An old teacherage at Mattole Union Elementary School was knocked off its cinder-block foundation. A 64-year-old man was injured when thrown to the ground and a wood pile fell on him. A home was reported to have sustained split walls. A water well ceased to pump water (from press reports). Chimneys were cracked and twisted; small appliances were overturned; many glassware items or dishes broke; a few windows cracked; hanging pictures fell; buildings shook strongly; small landslides occurred; felt by everyone.

Intensity VI:

Carlotta-Chimneys cracked; a few items were shaken off store shelves; many glassware items or dishes broke; many small objects overturned and fell; hanging pictures fell; trees and bushes shook strongly; standing vehicles rocked moderately; moving vehicles rocked slightly; buildings were shaken strongly; felt by everyone.

Ferndale-Two plate-glass windows broke; a few items shaken off of shelves (press report).

Fortuna-Many windows broke out; many items were shaken off store shelves; many glassware items or dishes broke; many small objects overturned and fell; ceiling tiles fell in the post office; standing vehicles rocked moderately; trees and bushes shook moderately; buildings shook strongly; felt by everyone.

Honeydew-A chimney was thrown down; one person was injured by a falling bookcase; a pressure cooker was almost thrown off a stove. A few items were shaken off store shelves; a few windows cracked; many glassware items or dishes broke; many small objects overturned and fell; hanging pictures fell; trees and bushes shook strongly; standing vehicles rocked moderately; buildings shook strongly; people had difficulty standing; felt by many people.

Hydesville-Chimneys were cracked; a few items were shaken off store shelves; a few glassware items or dishes broke; a few small objects overturned and fell; standing and moving vehicles rocked slightly; shaking was described as strong; felt by everyone.

Rio Dell-Some windows were broken out; interior plaster walls sustained cracks; many items were shaken off store shelves; small appliances overturned; some glassware items or dishes broke; some small objects overturned and fell; trees and bushes shook moderately; standing and moving vehicles rocked moderately; felt by everyone.

Scotia-Windows broke (press report). Many items were shaken off store shelves; small appliances overturned; a few glassware items and dishes broke; a few small objects overturned and fell; shaking was described as strong; felt by many people.

Intensity V:

Alderpoint-A few small objects overturned and fell; buildings shook moderately; trees and bushes shook slightly; standing vehicles rocked slightly; felt by many people.

Arcata-A few small objects overturned and fell; shaking was described as moderate; pictures swung out of place; felt by many people.

Blocksburg-A few items were shaken off store shelves; trees and bushes shook slightly; standing vehicles rocked slightly; shaking was described as moderate; felt by many people.

Cutten-A few items were shaken off store shelves; $a$ few glassware items or dishes broke; a few small objects overturned and fell; shaking was described as moderate; felt by many people.

Fields Landing-People had difficulty standing and walking; hanging pictures swung out of place; trees and bushes shook moderately; standing vehicles rocked slightly; buildings shook strongly; felt by everyone.

Loleta-A few items were shaken off store shelves; a few glassware items or dishes broke; many small objects fell; buildings shook strongly; standing vehicles rocked moderately; felt by everyone. 


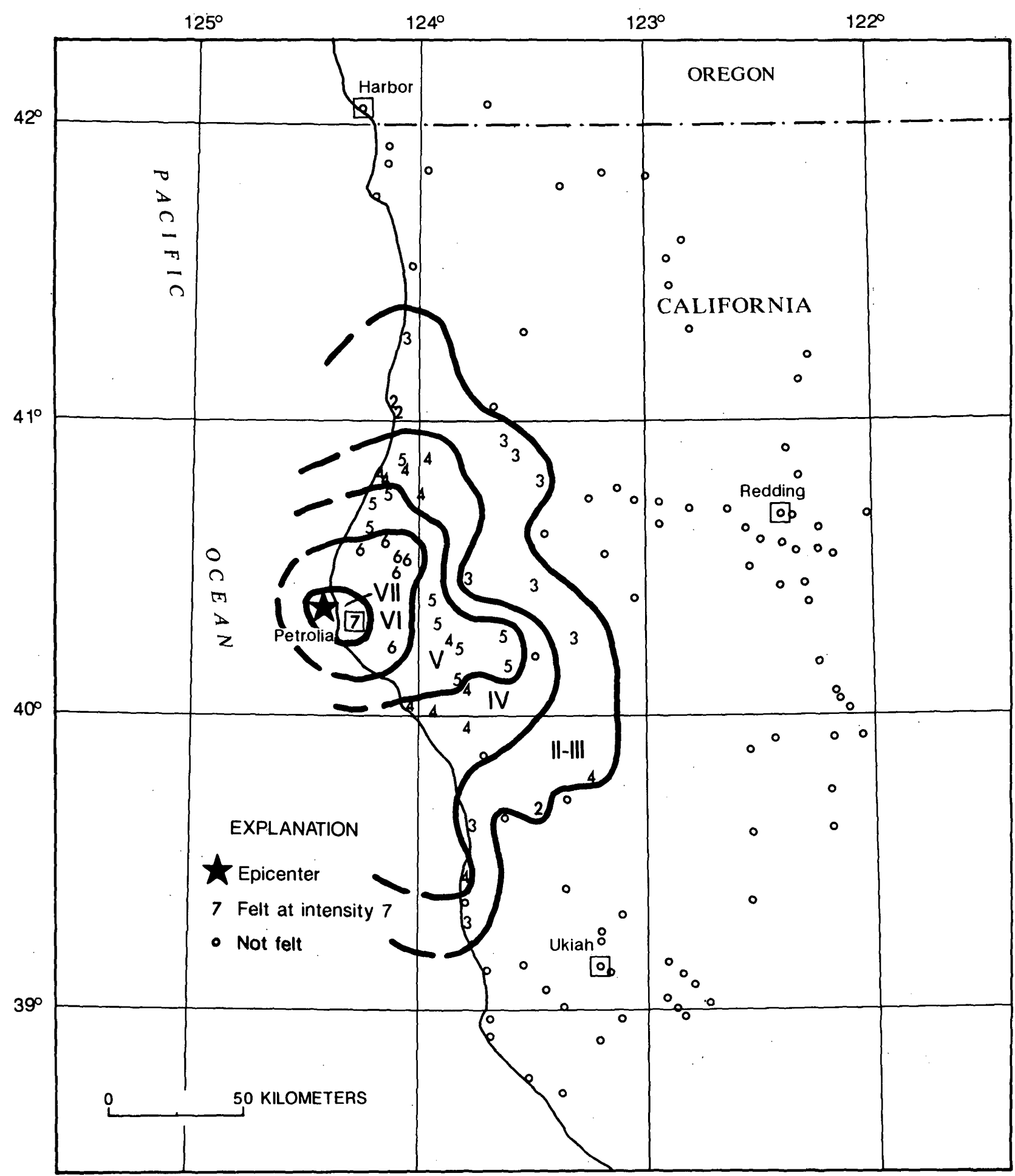

Figure 17. Isoseismal map for the northern California earthquake of 21 November, 1986, 233301.7 UTC. Roman numerals represent Modified Mercalli intensities between isoseismals; Arabic numerals represent intensities at specific sites; dashed contour lines are inferred isoseismals, and small boxes show locations of towns and cities whose names are plotted. 


\section{CALIFORNIA-Continued}

Miranda-A few small objects overturned and fell; people had difficulty standing and walking; shaking was described as strong; trees and bushes shook moderately; standing vehicles rocked moderately; felt by many people.

Phillipsburg-A few items were shaken off store shelves; a few small objects fell; many small objects overturned; hanging pictures fell; buildings shook strongly; standing vehicles rocked slightly; felt by many people.

Redcrest-A few items were shaken off store shelves; a few small objects fell; standing and moving vehicles rocked slightly; buildings shook moderately; felt by everyone.

Redway-A few merchandise were shaken off store shelves; a few small objects overturned and fell; hanging pictures swung out of place; buildings shook moderately; felt by everyone.

Weott-A few glassware items or dishes broke; a few small objects overturned and fell; hanging pictures swung out of place; trees and bushes shook moderately; standing vehicles rocked moderately; moving vehicles rocked slightly; buildings shook moderately; felt by everyone.

Intensity IV: Bayside, Blue Lake, Covelo, Eureka, Fort

Bragg (press report), Garberville, Kneeland, Korbel, Myers Flat, Piercy, Samoa, Shelter Cove, Whitethom.

Intensity III: Bridgeville, Burnt Ranch, Mad River, Mendocino, Orick, Ruth, Salyer, Westport, Willow Creek.

Intensity II: Laytonville, Trinidad, Westhaven.

\section{November (BK) Northern California}

Origin time: 233418.0

Epicenter: $40.367 \mathrm{~N} ., 124.450 \mathrm{~W}$.

Depth: $15 \mathrm{~km}$

Magnitude: $5.1 \mathrm{~m}_{\mathrm{b}}(\mathrm{GS}), 5.1 \mathrm{M}_{\mathrm{L}}(\mathrm{BK})$

Moment: $4.6 \times 10^{23}$ dyne-cm (BK)

This earthquake was felt over about the same area and with the same intensity as the 21 November 233301.7 UTC earthquake. The shaking effects could not be differentiated from the first earthquake; therefore, all the intensity values were assigned to the first earthquake.

\section{November (BK) Northern California}

Origin time: 054106.3

Epicenter: $40.360 \mathrm{~N} ., 124.528 \mathrm{~W}$.

Depth: $20 \mathrm{~km}$

Magnitude: $4.0 \mathrm{~m}_{\mathrm{b}}(\mathrm{GS}), 4.0 \mathrm{M}_{\mathrm{L}}(\mathrm{BK})$

Moment: $2.9 \times 10^{22}$ dyne-cm (BK)

Intensity III: Rio Dell.

Felt: Fortuna (press report).

\section{December (PS) Southern California}

Origin time: 105519.6

Epicenter: 33.713 N., 116.836W.

Depth: $16 \mathrm{~km}$

\section{CALIFORNIA-Continued}

Magnitude: $2.6 \mathrm{M}_{\mathrm{L}}(\mathrm{PS})$

Felt: Epicentral area (PS).

7 December (BK) Central California

Origin time: 123308.8

Epicenter: 35.352N., 120.980W.

Depth: $6 \mathrm{~km}$

Magnitude: $3.3 \mathrm{M}_{\mathrm{L}}(\mathrm{BK}), 3.3 \mathrm{M}_{\mathrm{L}}(\mathrm{PS})$

Intensity IV: Morro Bay (press report).

Intensity III: Chorro Valley (press report), Los Osos (press report).

Felt: Cayucos (BK), San Luis Obispo (BK).

\section{December (BK) Central California}

Origin time: 141805.3

Epicenter: 37.568N., 121.665W.

Depth: $4 \mathrm{~km}$

Magnitude: $4.1 \mathrm{M}_{\mathrm{L}}(\mathrm{BK})$

Moment: $2.6 \times 10^{22}$ dyne-cm (BK)

Intensity IV: Fremont (press report), Livermore, San Jose (Cambrian Park).

Intensity III: Aromas, Banta, Port Costa, San Jose, San Pablo, San Ramon.

Intensity II: Oakley, San Leandro.

Felt: Santa Rosa (press report).

\section{December (BK) Central California}

Origin time: 014706.5

Epicenter: 38.298 N., $122.153 W$.

Depth: $7 \mathrm{~km}$

Magnitude: $2.9 \mathrm{M}_{\mathrm{L}}(\mathrm{BK})$,

Moment: $2.5 \times 10^{21}$ dyne-cm (BK)

Intensity III: Fairfield (press report)

Felt: Vacaville (BK).

20 December (BK) Central California

Origin time: 021941.8

Epicenter: $37.453 \mathrm{~N} ., 121.802 \mathrm{~W}$.

Depth: $6 \mathrm{~km}$

Magnitude: $3.6 \mathrm{M}_{\mathrm{L}}(\mathrm{BK}), 3.2 \mathrm{M}_{\mathrm{L}}(\mathrm{PS})$

Moment: $6.9 \times 10^{21}$ dyne-cm (BK)

Intensity IV: Fremont (press report).

Intensity III: Milpitas, San Jose, Santa Clara (Agnew).

Felt: Monta Vista.

20 December (BK) Northern California

Origin time: 194532.9

Epicenter: $40.363 \mathrm{~N} ., 124.590 \mathrm{~W}$.

Depth: $13 \mathrm{~km}$

Magnitude: $3.7 \mathrm{M}_{\mathrm{L}}(\mathrm{BK})$

Moment: $8.7 \times 10^{21}$ dyne-cm (BK)

Intensity III: Rio Dell. 


\section{CALIFORNIA-Continued}

25 December (BK) Northern California

Origin time: 022515.7

Epicenter: 40.313N., 124.565W.

Depth: $22 \mathrm{~km}$

Magnitude: $3.4 \mathrm{M}_{\mathrm{L}}(\mathrm{BK})$

Moment: $1.2 \times 10^{21}$ dyne-cm (BK)

Intensity III: Honeydew.

25 December (BK) Central California

Origin time: 132828.1

Epicenter: 37.745N., 122.570W.

Depth: $9 \mathrm{~km}$

Magnitude: $2.7 \mathrm{M}_{\mathrm{L}}(\mathrm{BK})$

Moment: $3.6 \times 10^{20}$ dyne-cm (BK)

Felt: San Francisco (BK).

25 December (PS) Southern California

Origin time: 173522.9

Epicenter: 32.984 N., 116.286W.

Depth: 8 km

Magnitude: $3.4 \mathrm{M}_{\mathrm{L}}(\mathrm{PS})$

Intensity III: Dulzura, Jamul.

Intensity II: Calipatria, San Diego (Ocean Beach).

26 December $(\mathrm{RN})$ Owens Valley area

Origin time: 095627.4

Epicenter: $37.572 \mathrm{~N} ., 118.402 \mathrm{~W}$.

Depth: $5 \mathrm{~km}$

Magnitude: $4.0 \mathrm{M}_{\mathrm{L}}(\mathrm{BK})$, 4.0M $(\mathrm{RN}), 3.5 \mathrm{M}_{\mathrm{L}}(\mathrm{PS})$

Felt: Bishop (RN), Chalfant Valley (RN).

27 December (PS) Southern California

Origin time: 191303.9

Epicenter: $33.506 \mathrm{~N} ., 116.551 \mathrm{~W}$.

Depth: $12 \mathrm{~km}$

Magnitude: $3.2 \mathrm{M}_{\mathrm{L}}(\mathrm{PS})$

Felt: Palm Springs (PS).

29 December (BK) Central California

Origin time: 152804.9

Epicenter: 37.458 N., $121.800 \mathrm{~W}$.

Depth: $6 \mathrm{~km}$

Magnitude: $3.8 \mathrm{~m}_{\mathrm{b}}(\mathrm{GS}), 4.0 \mathrm{M}_{\mathrm{L}}(\mathrm{BK})$

Intensity IV: Boulder Creek, Burlingame, Campbell, Cupertino, Fremont, Milpitas, Moffett Field, Mountain View, Pleasanton (press report), Redwood City, San Bruno, San Carlos, San Jose, San Jose (Cambrian Park), San Lorenzo, Santa Clara.

Intensity III: Belmont, Bolinas, Brisbane, Byron, Dublin, Forest Knolls, French Camp, Hayward (press report), Livermore, Loma Mar, Los Altos, Moss Landing, New Almaden, Palo Alto, Port Costa, Santa Cruz, Scotts Valley, Stinson Beach, Stockton, Sunol, Sunnyvale.

\section{CALIFORNIA-Continued}

Intensity II: Half Moon Bay.

Felt: Monterey (press report), Salinas (press report), San Rafael (press report).

29 December (PS) Southern California

Origin time: 160514.0

Epicenter: $33.020 \mathrm{~N} ., 115.769 \mathrm{~W}$.

Depth: $4 \mathrm{~km}$

Magnitude: $3.4 \mathrm{M}_{\mathrm{L}}(\mathrm{PS})$

Intensity IV: Calipatria.

Intensity III: Imperial.

Felt: Brawley (PS).

30 December (BK) Northern California

Origin time: 062906.6

Epicenter: 40.380N., 124.273W.

Depth: $18 \mathrm{~km}$

Magnitude: $3.2 \mathrm{M}_{\mathrm{L}}(\mathrm{BK})$

Moment: $1.4 \times 10^{21}$ dyne-cm (BK)

Intensity III: Rio Dell.

\section{COLORADO}

11 April (GS) Western Colorado

Origin time: 061714.7

Epicenter: 38.982N., 106.940W.

Depth: $5 \mathrm{~km}$

Magnitude: $2.9 \mathrm{M}_{\mathrm{L}}$ (GS)

Intensity III: Basalt, Snowmass Village.

9 May (GS) Western Colorado

Origin time: 215526.7

Epicenter: 38.887N., 106.884W.

Depth: $5 \mathrm{~km}$

Magnitude: $2.7 \mathrm{M}_{\mathrm{L}}(\mathrm{GS})$

Intensity II: Snowmass Village.

13 August (GS) Western Colorado

Origin time: 024255.6

Epicenter: 38.814N., 106.996W.

Depth: 5 km

Magnitude: $2.6 \mathrm{M}_{\mathrm{L}}(\mathrm{GS})$

Felt: Crested Butte, Meridian Park (4.5 mi north of Crested Butte).

\section{August (GS) Western Colorado}

Origin time: 121343.9

Epicenter: 38.879N., 107.039W.

Depth: 5 km

Magnitude: $2.4 \mathrm{M}_{\mathrm{L}}(\mathrm{GS})$

Felt: Crested Butte, Meridian Park (4.5 mi north of Crested Butte). 


\section{COLORADO-Continued}

\section{August (GS) Western Colorado}

Origin time: 173925.9

Epicenter: 38.908N., 107.082W.

Depth: $5 \mathrm{~km}$

Magnitude: 2.6M $(\mathrm{GS})$

Felt: Crested Butte, Meridian Park (4.5 mi north of Crested Butte).

\section{August (GS) Western Colorado}

Origin time: 221028.3

Epicenter: 38.897N., 107.076W.

Depth: $5 \mathrm{~km}$

Magnitude: $2.4 \mathrm{M}_{\mathrm{L}}(\mathrm{GS})$

Felt: Crested Butte, Meridian Park (4.5 mi north of Crested Butte).

\author{
18 August (GS) Western Colorado \\ Origin time: 011515.0 \\ Epicenter: 38.914N., 107.087W. \\ Depth: $5 \mathrm{~km}$ \\ Magnitude: $3.0 \mathrm{M}_{\mathrm{L}}(\mathrm{GS})$ \\ Intensity III: Aspen, Carbondale. \\ Felt: Crested Butte, Meridian Park (4.5 mi north of \\ Crested Butte), Snowmass Village (press report).
}

\section{August (GS) Western Colorado}

Origin time: 044340.1

Epicenter: 38.892N., 107.077W.

Depth: 5 km

Magnitude: $2.3 \mathrm{M}_{\mathrm{L}}(\mathrm{GS})$

Felt: Crested Butte, Meridian Park (4.5 mi north of Crested Butte).

\section{August (GS) Western Colorado \\ Origin time: 202132.9 \\ Epicenter: 38.892N., 107.068W. \\ Depth: $5 \mathrm{~km}$ \\ Magnitude: $2.7 \mathrm{M}_{\mathrm{L}}(\mathrm{GS})$ \\ Felt: Crested Butte, Meridian Park (4.5 mi north of Crested Butte).}

\section{August (GS) Western Colorado}

Origin time: 141131.6

Epicenter: 38.903N., 107.063W.

Depth: $5 \mathrm{~km}$

Magnitude: $1.9 \mathrm{M}_{\mathrm{L}}(\mathrm{GS})$

Felt: Crested Butte, Meridian Park (4.5 mi north of Crested Butte).

\section{August (GS) Western Colorado}

Origin time: 051303.0

Epicenter: 38.905N., 107.095W.

Depth: $5 \mathrm{~km}$

\section{COLORADO-Continued}

Magnitude: $2.4 \mathrm{M}_{\mathrm{L}}(\mathrm{GS})$

Felt: Crested Butte.

24 August (GS) Western Colorado

Origin time: 035917.5

Epicenter: 38.967N., 107.141W.

Depth: $5 \mathrm{~km}$

Magnitude: $2.1 \mathrm{M}_{\mathrm{L}}(\mathrm{GS})$

Felt: Crested Butte.

26 August (GS) Western Colorado

Origin time: 020602.6

Epicenter: 38.900N., 107.041W.

Depth: $5 \mathrm{~km}$

Magnitude: $3.1 \mathrm{M}_{\mathrm{L}}(\mathrm{GS})$

Intensity IV: Meridian Park (4.5 mi north of Crested Butte).

Intensity III: Carbondale, Crested Butte.

Intensity II: Mount Crested Butte.

\section{September (GS) Western Colorado}

Origin time: 062050.9

Epicenter: 38.912N., 107.090W.

Depth: $5 \mathrm{~km}$

Magnitude: $3.5 \mathrm{M}_{\mathrm{L}}(\mathrm{GS})$

Intensity V:

Crested Butte-A few glassware items or dishes broke; a few small objects overturned and fell; shaking was described as strong; felt by and awakened many people.

Intensity III: Aspen.

Intensity II: Gunnison.

18 September (GS) Western Colorado

Origin time: 045321.6

Epicenter: 38.937N., 107.116W.

Depth: $5 \mathbf{~ k m}$

Magnitude: $3.2 \mathrm{M}_{\mathrm{L}}(\mathrm{GS})$

Felt: Crested Butte.

18 September (GS) Western Colorado

Origin time: 092638.1

Epicenter: 38.925N., 107.086W.

Depth: $5 \mathrm{~km}$

Magnitude: $3.4 \mathrm{M}_{\mathrm{L}}(\mathrm{GS})$

Intensity III: Aspen, Snowmass Village.

21 September (GS) Central Colorado

Origin time: 092046.6

Epicenter: $39.597 \mathrm{~N} ., 105.285 \mathrm{~W}$.

Depth: $5 \mathrm{~km}$

Magnitude: $2.5 \mathrm{M}_{\mathrm{L}}(\mathrm{GS})$

Felt: Conifer, Tiny Town.

7 October (GS) Western Colorado

Origin time: 123503.2 
Epicenter: $38.947 \mathrm{~N} ., 107.090 \mathrm{~W}$.

Depth: $5 \mathbf{~ k m}$

Magnitude: $1.8 \mathrm{M}_{\mathrm{L}}(\mathrm{GS})$

Felt: Crested Butte.

\section{CONNECTICUT}

\section{October (GS) Central New Hampshire \\ Origin time: 171638.4}

See New Hampshire listing.

\section{DELAWARE}

\section{January (NI) Northeastern Ohio \\ Origin time: 164642.3}

See Ohio listing.

\section{DISTRICT OF COLUMBIA}

\section{January (NI) Northeastern Ohio}

Origin time: 164642.3

See Ohio listing.

\section{GEORGIA}

\section{February (TC) Northwestern South Carolina}

Origin time: 113545.3

See South Carolina listing.

64 United States Earthquakes, 1986

\section{February (GT) Central Georgia}

Origin time: 041257.9

Epicenter: 33.296N., 83.245W.

Depth: $1 \mathrm{~km}$

Magnitude: $1.7 \mathrm{M}_{\mathrm{D}}(\mathrm{GT}), 2.4 \mathrm{M}_{\mathrm{D}}(\mathrm{TC})$

Intensity IV: Lake Sinclair area (press report).

13 March (GT) Central Georgia

Origin time: 022931.0

Epicenter: $33.35 \Subset$ N., 83.394W.

Depth: $1 \mathrm{~km}$

Magnitude: $2.2 \mathrm{M}_{\mathrm{D}}(\mathrm{GT}), 2.5 \mathrm{M}_{\mathrm{D}}(\mathrm{TC})$

Intensity IV: Epicentral area near Lake Sinclair (press report), Milledgeville.

Intensity III: Eatonton, Haddock.

11 July (TC) Georgia-Tennessee Border

Origin time: 142614.8

Epicenter: $34.937 \mathrm{~N} ., 84.987 \mathrm{~W}$.

Depth: $13 \mathrm{~km}$

Magnitude: $3.7 \mathrm{~m}_{\mathrm{b}}(\mathrm{GS}), 3.8 \mathrm{M}_{\mathrm{n}}(\mathrm{GS}), 3.8 \mathrm{MD}(\mathrm{GT})$

This earthquake was felt over a contiguous area of about $13,800 \mathrm{~km}^{2}$ of Georgia, North Carolina, and Tennessee (fig. 18).

Intensity VI:

Georgia-

Cohutta-A house foundation cracked (press report); chimneys cracked; exterior brick walls cracked; hanging pictures fell; buildings shook moderately; felt by everyone.

Intensity V:

Georgia-

Dalton-A few windows cracked; a few glassware items or dishes broke; a few small objects overturned and fell; trees and bushes shook slightly; hanging pictures swung out of place; shaking was described as moderate.

Tunnel Hill-A few windows cracked; standing vehicles rocked slightly; buildings shook moderately; felt by many people.

Tennessee-

Chattanooga-A few small objects overturned and fell; a few glassware items or dishes broke; hanging pictures fell; felt by many people.

Turtletown-A few glassware items or dishes broke; a few small objects overturned and fell; interior dry wall was cracked; trees and bushes shook slightly; shaking was described as moderate.

Intensity IV:

Georgia-Blue Ridge, Crandall, Dawsonville, Eton, Ringgold, Rocky Face, Tennga, Varnell.

Tennessee-Apison, Benton, Charleston, Collegedale, East Ridge, Ocoee, Ooltewah. 


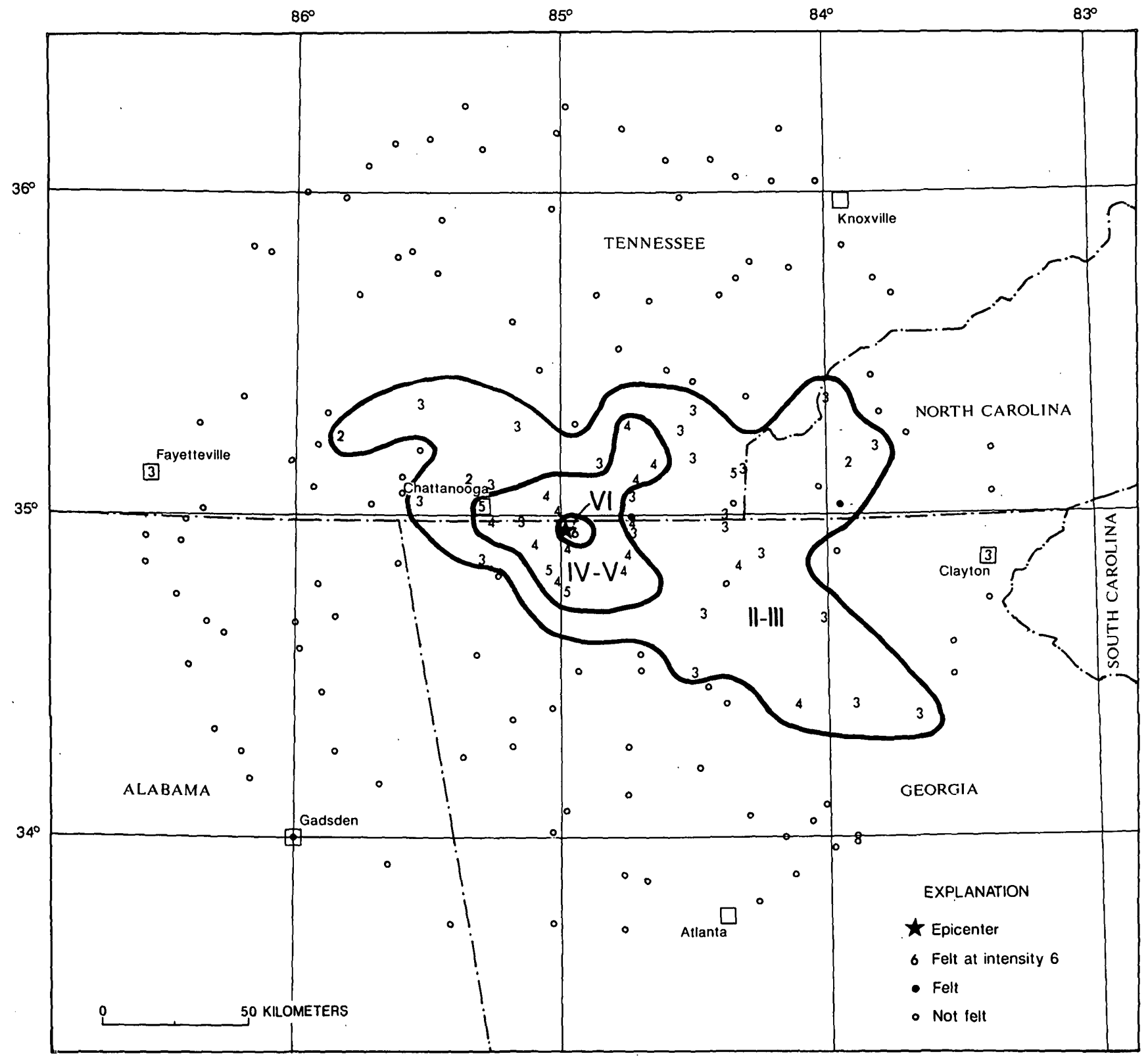

Figure 18. Isoseismal map for the Georgia-Tennessee-border earthquake of $11 \mathrm{July,} \mathrm{1986,} \mathrm{at} 142614.8$ UTC. Roman numerals represent Modified Mercalli intensities between isoseismals; Arabic numerals represent intensities at specific sites, and small boxes show locations of towns and cities whose names are plotted.

\section{GEORGIA-Continued}

Intensity III:

Georgia-Chickamauga, Cisco, Clayton, East Ellijay, Ellijay, Epworth, Graysville, Lula, McCaysville, Morganton, Murrayville, Suches, Talking Rock.

North Carolina-Andrews.

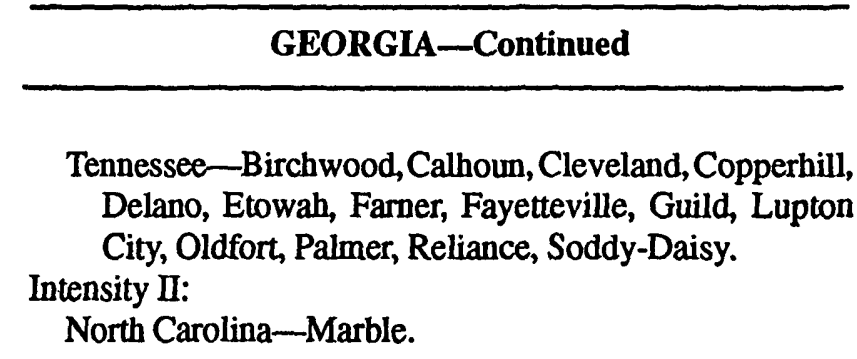

Earthquake Descriptions 65 


\section{GEORGIA-Continued}

Tennessee-East Chattanooga, Monteagle, Signal Mountain.

Felt:

North Carolina-Brasstown.

Tennessee - Conasauga, Knoxville (press report).

\section{HAWAII}

17 January (HV) Hawaii Island

Origin time: 234831.6

Epicenter: $19.359 \mathrm{~N} ., 155.063 \mathrm{~W}$.

Depth: $10 \mathrm{~km}$

Magnitude: $3.6 \mathrm{M}_{\mathrm{L}}(\mathrm{HV})$

Intensity II: Hilo.

23 January (HV) Hawaii Island

Origin time: 223557.9

Epicenter: 19.340N., 155.199W.

Depth: $8 \mathrm{~km}$

Magnitude: $3.7 \mathrm{M}_{\mathrm{L}}(\mathrm{HV})$

Intensity III: Hilo, Keaau.

Intensity II: Volcano.

27 January (HV) Hawaii Island

Origin time: 233628.1

Epicenter: 19.315N., 155.228W.

Depth: $6 \mathrm{~km}$

Magnitude: $4.0 \mathrm{M}_{\mathrm{L}}(\mathrm{HV})$

Intensity III: Hilo, Volcano.

Intensity II: Hawaiian Beaches, Pahala.

3 February (HV) Hawaii Island

Origin time: 210122.2

Epicenter: 19.353N., 155.021W.

Depth: $7 \mathrm{~km}$

Magnitude: $3.7 \mathrm{M}_{\mathrm{L}}(\mathrm{HV})$

Intensity II: Hilo.

4 February (HV) Hawaii Island

Origin time: 205633.0

Epicenter: 19.553N., 155.234W.

Depth: $25 \mathrm{~km}$

Magnitude: $3.3 \mathrm{M}_{\mathrm{L}}(\mathrm{HV})$

Intensity II: Hilo, Papaikou, Pepeekeo, Puna areas, Volcano.

20 February (HV) Hawaii Island

Origin time: 071747.7

Epicenter: 19.320N., 155.191W.

Depth: $1 \mathrm{~km}$

Magnitude: $3.5 \mathrm{M}_{\mathrm{L}}(\mathrm{HV})$

Intensity III: Volcano.

\section{HAWAII-Continued}

28 February (HV) Hawaii Island

Origin time: 072114.3

Epicenter: 19.317N., 155.192W.

Depth: $1 \mathrm{~km}$

Magnitude: $3.7 \mathrm{M}_{\mathrm{L}}(\mathrm{HV})$

Intensity III: Mountain View.

Intensity II: Papaikou.

1 March (HV) Hawaii Island

Origin time: 211034.2

Epicenter: 19.410N., 155.292W.

Depth: $16 \mathrm{~km}$

Magnitude: $3.3 \mathrm{M}_{\mathrm{L}}(\mathrm{HV})$

Intensity II: Volcano.

12 March (HV) Hawaii Island

Origin time: 222934.6

Epicenter: 19.302N., 155.219W.

Depth: $11 \mathrm{~km}$

Magnitude: $3.8 \mathrm{M}_{\mathrm{L}}(\mathrm{HV})$

Intensity III: Hilo.

Intensity II: Puna areas, Volcano.

16 March (HV) Hawaii Island

Origin time: 205834.8

Epicenter: $19.768 \mathrm{~N} ., 156.175 \mathrm{~W}$.

Depth: $42 \mathrm{~km}$

Magnitude: $4.2 \mathrm{M}_{\mathrm{L}}(\mathrm{HV})$

Intensity II: Hilo.

30 March (HV) Hawaii Island

Origin time: 194803.1

Epicenter: $19.327 \mathrm{~N} ., 155.038 \mathrm{~W}$.

Depth: $5 \mathrm{~km}$

Magnitude: $3.9 \mathrm{M}_{\mathrm{L}}(\mathrm{HV})$

Intensity III: Hilo.

7 April (HV) Hawaii Island

Origin time: 083749.7

Epicenter: 19.199N., 155.620W.

Depth: $11 \mathrm{~km}$

Magnitude: $4.2 \mathrm{~m}_{\mathrm{b}}(\mathrm{GS}), 4.3 \mathrm{M}_{\mathrm{L}}(\mathrm{HV})$

Intensity IV: Discovery Harbour, Hawaiian Ocean View Estates, Waiohinu.

Intensity III: Ahualoa, Captain Cook, Hilo, Papaikou, Volcano, Volcano Golf Course.

23 April (HV) Hawaii Island

Origin time: 044351.3

Epicenter: 19.305 N., 155.271W.

Depth: $31 \mathrm{~km}$

Magnitude: $4.5 \mathrm{M}_{\mathrm{L}}(\mathrm{HV})$

Intensity IV: Hilo, Mountain View, Pahala, Volcano. 
Intensity III: Honokaa, Kohala area, Papaikou. Intensity II: Discovery Harbour, Makiki (Oahu Island), Aiea (Oahu Island).

\section{April (HV) Hawaii Island}

Origin time: 114951.9

Epicenter: 19.309 N., $155.263 \mathrm{~W}$.

Depth: $31 \mathrm{~km}$

Magnitude: $3.0 \mathrm{M}_{\mathrm{L}}(\mathrm{HV})$

Intensity III: Hilo.

26 April (GS) Maui Island area

Origin time: 171946.5

Epicenter: 20.811N., 155.749W.

Depth: Normal

Magnitude: $5.1 \mathrm{~m}_{\mathrm{b}}(\mathrm{GS}), 4.9 \mathrm{M}_{\mathrm{L}}(\mathrm{HV})$

Intensity V:

Molokai Island-

Kalaupapa-A few windows cracked; interior walls sustained hairline cracks; buildings shook strongly. Intensity IV:

Hawaii Island-Ahualoa, Hawi, Honokaa, Kamuela, Mountain View, Paauhau, Pahala.

Kauai Island-Waimea.

Lanai Island-Lanai City.

Maui Island-Hana, Kihei, Kula, Pukalani, Wailuku.

Molokai Island-Maunaloa.

Oahu Island-Aina Haina, Honolulu, Kaneohe.

Intensity III:

Hawaii Island-Glenwood, Hilo, Kailua, Kapaau, Kealakekua, Papaaloa, Pepeekeo, Volcano.

Maui Island-Haleakala, Lualapuu, Paia, Weilea (press report).

Oahu Island-Ewa, Hawaii Kai (press report), Kailua (press report), Makiki (press report), Mililani, WaialaeKahala, Waialua, Waimanalo, Waipio (press report).

Intensity II:

Oahu Island-Waianae.

Kauai Island-Hanalei (press report), Kilauea (press report).

8 May (HV) Hawaii Island

Origin time: 014526.3

Epicenter: $19.292 \mathrm{~N} ., 155.235 \mathrm{~W}$.

Depth: $10 \mathrm{~km}$

Magnitude: $3.2 \mathrm{M}_{\mathrm{L}}(\mathrm{HV})$

Intensity II: Papaikou.

13 May (HV) Hawaii Island

Origin time: 170839.1

Epicenter: $19.412 \mathrm{~N} ., 155.270 \mathrm{~W}$.

Depth: $17 \mathrm{~km}$

Magnitude: $3.4 \mathrm{M}_{\mathrm{L}}(\mathrm{HV})$

Intensity II: Glenwood, Mountain View, Volcano.
21 May (HV) Hawaii Island

Origin time: 183716.6

Epicenter: 19.379N., 155.302W.

Depth: $30 \mathrm{~km}$

Magnitude: $3.1 \mathrm{M}_{\mathrm{L}}(\mathrm{HV})$

Intensity II: Ahualoa.

27 May (HV) Hawaii Island

Origin time: 110218.1

Epicenter: 19.399N., 155.259W.

Depth: 4 km

Magnitude: $3.0 \mathrm{M}_{\mathrm{L}}(\mathrm{HV})$

Intensity III: Hawaii Volcanoes National Park.

8 June (HV) Hawaii Island

Origin time: 213309.7

Epicenter: $19.321 \mathrm{~N} ., 155.009 \mathrm{~W}$.

Depth: $10 \mathrm{~km}$

Magnitude: $3.2 \mathrm{M}_{\mathrm{L}}(\mathrm{HV})$

Intensity II: Kalapana.

25 June (HV) Hawaii Island

Origin time: 121359.7

Epicenter: 19.419N., 155.312W.

Depth: $4 \mathrm{~km}$

Magnitude: $3.2 \mathrm{M}_{\mathrm{L}}(\mathrm{HV})$

Intensity III: Namakani, Paio, Volcano.

\section{July (HV) Hawaii Island}

Origin time: 122809.1

Epicenter: $19.552 \mathrm{~N} ., 155.999 \mathrm{~W}$.

Depth: $20 \mathrm{~km}$

Magnitude: $4.2 \mathrm{~m}_{\mathrm{b}}(\mathrm{GS}), 4.2 \mathrm{M}_{\mathrm{L}}(\mathrm{HV})$

Intensity IV: Captain Cook, Holualoa, Honalo, Kealakekua, Napoopoo, Pahala.

Intensity III: Discovery Harbour, Seaview, Volcano.

\section{July (HV) Hawaii Island}

Origin time: 124525.4

Epicenter: 19.537 N., $155.970 \mathrm{~W}$.

Depth: $11 \mathrm{~km}$

Magnitude: $3.1 \mathrm{M}_{\mathrm{L}}(\mathrm{HV})$

Intensity II: Kaawaloa.

11 August (HV) Hawaii Island

Origin time: 031612.2

Epicenter: 19.372N., 155.079W.

Depth: $10 \mathrm{~km}$

Magnitude: $3.7 \mathrm{M}_{\mathrm{L}}(\mathrm{HV})$

Intensity II: Hilo, Pu'u O’o

8 September (HV) Molokai Island area

Origin time: 141621.0

Epicenter: 21.284N., 156.782W. 
Depth: $1 \mathrm{~km}$

Magnitude: $3.3 \mathrm{M}_{\mathrm{L}}(\mathrm{HV})$

Intensity II: Kalaupapa.

19 September (HV) Hawaii Island

Origin time: 144442.5

Epicenter: 19.333 N., $155.349 W$.

Depth: $31 \mathrm{~km}$

Magnitude: $4.2 \mathrm{M}_{\mathrm{L}}(\mathrm{HV})$

Intensity IV: Hawaiian Ocean View Estates, Homestead, Kiolaka'a, Pahala.

Intensity III: Discovery Harbour, Hilo.

Intensity II: Honomu, Papaikou.

\section{September (HV) Hawaii Island}

Origin time: 093033.6

Epicenter: 18.795 N., $155.268 \mathrm{~W}$.

Depth: $12 \mathrm{~km}$

Magnitude: $3.7 \mathrm{M}_{\mathrm{L}}(\mathrm{HV})$

Intensity IV: Pahala.

\section{September (HV) Hawaii Island}

Origin time: 171602.0

Epicenter: 19.979N., 155.502W.

Depth: $37 \mathrm{~km}$

Magnitude: $3.9 \mathrm{M}_{\mathrm{L}}(\mathrm{HV})$

Intensity IV: Ahualoa, Mauna Kea Observatory (Summit). Intensity III: Hilo, Honokaa.

Intensity II: Hawaiian Volcano Observatory, Volcano.

\section{October (HV) Hawaii Island}

Origin time: 080216.0

Epicenter: 19.702N., 155.224W.

Depth: $36 \mathrm{~km}$

Magnitude: $3.7 \mathrm{M}_{\mathrm{L}}(\mathrm{HV})$

Intensity IV: Hilo.

Intensity III: Glenwood, Hale Pohaku-Mauna Kea, Kurtistown.

Intensity II: Hawaiian Volcano Observatory, Honomu, Keahou Bay-Kona, Kohala area, Papaikou, Pepeekeo, Volcano.

15 November (HV) Hawaii Island

Origin time: 205854.3

Epicenter: 19.344N., 155.218W.

Depth: $8 \mathrm{~km}$

Magnitude: $4.0 \mathrm{M}_{\mathrm{L}}(\mathrm{HV})$

Intensity III: Volcano.

Intensity II: Hamakua area, Hilo.

18 November (HV) Hawaii Island

Origin time: 024022.6

Epicenter: 20.189N., 155.774W.
Depth: $37 \mathrm{~km}$

Magnitude: $3.7 \mathrm{M}_{\mathrm{L}}(\mathrm{HV})$

Intensity III: Ahualoa.

6 December (HV) Hawaii Island

Origin time: 221040.6

Epicenter: 19.360N., 155.038W.

Depth: $1 \mathrm{~km}$

Magnitude: $3.8 \mathrm{M}_{\mathrm{L}}(\mathrm{HV})$

Intensity IV: Wahaula.

Intensity III: Kalapana, Hilo.

Intensity II: Hamakua area.

7 December (HV) Maui Island

Origin time: 024530.1

Epicenter: 20.864N., 156.036W.

Depth: $38 \mathrm{~km}$

Magnitude: $4.2 \mathrm{M}_{\mathrm{L}}(\mathrm{HV})$

Intensity IV: Kamuela-Hawaii Island, eastern Maui Island.

11 December (HV) Hawaii Island

Origin time: 004742.9

Epicenter: 19.335N., 155.036W.

Depth: $4 \mathrm{~km}$

Magnitude: $3.2 \mathrm{M}_{\mathrm{L}}(\mathrm{HV})$

Intensity III: Wahaula.

\section{DAHO}

\section{January (GS) Central Idaho \\ Origin time: 054501.5 \\ Epicenter: 44.153N., 113.946W.}

Depth: $5 \mathrm{~km}$

Magnitude: $4.0 \mathrm{M}_{\mathrm{L}}(\mathrm{GS}), 4.3 \mathrm{M}_{\mathrm{L}}(\mathrm{BU})$

Intensity IV: Chilly (five earthquakes were felt-pressreport).

Intensity III: Challis.

17 February (GS) Southeastern Idaho

Origin time: 085338.6

Epicenter: 42.596N., 111.301W.

Depth: $5 \mathrm{~km}$

Magnitude: $3.0 \mathrm{M}_{\mathrm{L}}(\mathrm{GS})$

Intensity III: Montpelier, Georgetown.

24 February (GS) Southeastern Idaho

Origin time: 031333.0

Epicenter: 43.081N., 111.224W.

Depth: $5 \mathrm{~km}$

Magnitude: $2.8 \mathrm{M}_{\mathrm{L}}(\mathrm{GS})$ 


\section{IDAHO-Continued}

Intensity III:

Wyoming-Alpine.

Intensity II:

Idaho-Wayan.

Wyoming-Thayne.

12 March (BU) Northern Idaho

Origin time: 163256.7

Epicenter: 47.460N., 115.802W.

Depth: $0 \mathrm{~km}$

Magnitude: $2.6 \mathrm{M}_{\mathrm{L}}(\mathrm{BU}), 2.0 \mathrm{M}_{\mathrm{L}}(\mathrm{GS})$

Rockburst in the Lucky Friday Mine near Mullan, Idaho. One person was killed and two were injured.

Felt: Lucky Friday mine.

7 April (GS) Central Idaho

Origin time: 140725.8

Epicenter: 44.337 N., $114.177 \mathrm{~W}$.

Depth: $5 \mathrm{~km}$

Magnitude: $4.1 \mathrm{M}_{\mathrm{L}}(\mathrm{GS}), 4.5 \mathrm{M}_{\mathrm{L}}(\mathrm{BU})$

Intensity III: Thompson Creek (7 mi from Clayton).

21 June (GS) Idaho-Wyoming border area

Origin time: 203053.5

Epicenter: 42.793N., 111.153W.

Depth: $5 \mathrm{~km}$

Magnitude: $3.5 \mathrm{M}_{\mathrm{L}}(\mathrm{GS})$

Intensity III:

Wyoming-Auburn.

29 August (UU) Southeastern Idaho

Origin time: 093734.7

Epicenter: 42.096N., 111.650W.

Depth: $1 \mathrm{~km}$

Magnitude: $2.4 \mathrm{M}_{\mathrm{L}}(\mathrm{UU})$

Intensity III: Preston.

3 September (GS) Central Idaho

Origin time: 185349.1

Epicenter: 44.039N., 114.764W.

Depth: $5 \mathrm{~km}$

Magnitude: $3.9 \mathrm{M}_{\mathrm{L}}(\mathrm{GS}), 4.2 \mathrm{M}_{\mathrm{L}}(\mathrm{BU})$

Intensity III: Clayton.

24 September (GS) Central Idaho

Origin time: 153226.7

Epicenter: 44.003N., 114.755W.

Depth: $5 \mathrm{~km}$

Magnitude: $3.7 \mathrm{M}_{\mathrm{L}}(\mathrm{GS}), 3.9 \mathrm{M}_{\mathrm{L}}(\mathrm{BU})$

Felt: Clayton.
IDAHO-Continued

26 September (GS) Central Idaho

Origin time: 212808.5

Epicenter: $44.016 \mathrm{~N} ., 114.750 \mathrm{~W}$.

Depth: $5 \mathrm{~km}$

Magnitude: $4.3 \mathrm{M}_{\mathrm{L}}(\mathrm{GS}), 4.4 \mathrm{M}_{\mathrm{L}}(\mathrm{BU})$

Intensity IV: Clayton.

Intensity III: Stanley.

26 September (GS) Central Idaho

Origin time: 224857.9

Epicenter: 44.043N., 114.756W.

Depth: $5 \mathrm{~km}$

Magnitude: $4.6 \mathrm{~m}_{\mathrm{b}}(\mathrm{GS}), 4.5 \mathrm{M}_{\mathrm{L}}(\mathrm{GS}), 4.6 \mathrm{M}_{\mathrm{L}}(\mathrm{BU})$

Intensity IV: Clayton.

14 October (GS) Central Idaho

Origin time: 121753.3

Epicenter: 44.023N., 114.674W.

Depth: $5 \mathrm{~km}$

Magnitude: $3.9 \mathrm{M}_{\mathrm{L}}(\mathrm{GS}), 4.1 \mathrm{M}_{\mathrm{L}}(\mathrm{BU})$

Intensity IV: Clayton.

18 October (GS) Idaho-Utah border area

Origin time: 212129.0

Epicenter: 42.014N., 111.448W.

Depth: $7 \mathrm{~km}$

Magnitude: $3.5 \mathrm{M}_{\mathrm{L}}(\mathrm{UU})$

Intensity IV:

Idaho- Saint Charles.

Intensity III:

Idaho-Fish Haven.

Utah-Garden City, Laketown.

15 November (GS) southeastern Idaho

Origin time: 090013.2

Epicenter: 42.706 N., $111.667 \mathrm{~W}$.

Depth: 5 km

Magnitude: $3.3 \mathrm{M}_{\mathrm{L}}(\mathrm{GS})$

Intensity IV: Soda Springs.

17 November (GS) Western Wyoming

Origin time: 083413.3

See Wyoming list.

\section{ILLINOIS}

31 January (NI) Northeastern Ohio

Origin time: 164642.3

See Ohio listing. 
26 August (SL) Southern Illinois

Origin time: 164124.8

Epicenter: 38.320 N., $89.790 \mathrm{~W}$.

Depth: $5 \mathrm{~km}$

Magnitude: $3.7 \mathrm{M}_{\mathrm{n}}(\mathrm{GS}), 3.6 \mathrm{M}_{\mathrm{n}}(\mathrm{SL})$

Intensity V:

Illinois-

Belleville-A few small objects overturned and fell; buildings shook moderately; felt by several people.

Lenzburg-A few small objects overturned and fell; plaster walls sustained hairline cracks; standing vehicles rocked slightly; hanging pictures swung out of place; felt by everyone.

Marissa-A few windows cracked; a few glassware items or dishes broke; a few small objects overturned and fell; standing and moving vehicles rocked slightly; hanging pictures swung out of place; felt by many people.

Intensity IV:

Illinois-Alhambra, Baldwin, Coulterville, Hecker, Lebanon, Modoc, New Athens, New Memphis, Prairie du Rocher, Smithton, Summerfield, Troy.

Intensity III:

Illinois-Evansville, Carterville, Freeburg, Highland (press report), Mascoutah, Nashville, New Baden, Oakdale, Okawville, Red Bud, Saint Jacob, Saint Libory, Tilden, Trenton, Venedy.

Missouri-Pevely, Richmond Heights, Valles Mines.

Intensity II:

Illinois-DuBois, Fieldon, Menard.

Missouri-Florissant, Hillsboro.

Felt:

Missouri-South Saint Louis (press report).

29 October (SL) Southern Illinois

Origin time: 050341.3

Epicenter: 38.440N., 89.040W.

Depth: $5 \mathrm{~km}$

Magnitude: $2.7 \mathrm{M}_{\mathrm{n}}(\mathrm{GS}), 3.0 \mathrm{M}_{\mathrm{n}}(\mathrm{SL})$

Intensity III: Salem.

Felt: Northern Jefferson County (press report).

30 December (SL) Southeastern Missouri

Origin time: 071519.1

See Missouri listing.
31 January (NI) Northeastern Ohio

Origin time: 164642.3

See Ohio listing.

12 July (GS) Western Ohio

Origin time: 081937.9

See Ohio listing.

\section{KANSAS}

\section{June (GS) Northern Kansas}

Origin time: 040405.2

Epicenter: 39.344N., 99.781W.

Depth: $5 \mathrm{~km}$

Magnitude: $3.0 \mathrm{M}_{\mathrm{n}}(\mathrm{GS}), 3.0 \mathrm{M}_{\mathrm{D}}(\mathrm{KS})$

Intensity IV: Nicodemus (press report).

Intensity III: Bogue, Damar, Hill City, Lenora, New Almelo, Penokee, Stockton.

20 October (GS) Southwestern Kansas

Origin time: 043249.0

Epicenter: 37.918N., 101.372W.

Depth: $5 \mathrm{~km}$

Magnitude: $3.0 \mathrm{M}_{\mathrm{n}}(\mathrm{GS}), 2.9 \mathrm{M}_{\mathrm{n}}(\mathrm{TU}), 3.0 \mathrm{M}_{\mathrm{D}}(\mathrm{KS})$

Intensity IV: Lakin.

Intensity III: Friend.

\section{KENTUCKY}

31 January (NI) Northeastern Ohio

Origin time: 164642.3

See Ohio listing.

12 July (GS) Western Ohio

Origin time: 081937.9

See Ohio listing.

30 December (SL) Southeastern Missouri

Origin time: 071519.1

See Missouri listing. 


\section{MAINE}

\section{June (WO) Central Maine}

Origin time: 024015.7

Epicenter: 45.203N., 69.177W.

Depth: $2 \mathrm{~km}$

Magnitude: $2.5 \mathrm{M}_{\mathrm{n}}(\mathrm{WO}), 2.5 \mathrm{M} \mathrm{D}(\mathrm{WO})$

Felt: Dover-Foxcroft (WO).

\section{July (WO) Eastern Maine}

Origin time: 203248.4

Epicenter: 46.170N., 68.198W.

Depth: $9 \mathrm{~km}$

Magnitude: $3.4 \mathrm{M}_{\mathrm{n}}(\mathrm{WO}), 3.5 \mathrm{M} \mathrm{D}(\mathrm{WO})$

Felt: Houlton (WO), Island Falls (WO), Ludlow (WO), Sherman (WO), Smyrna Mills (WO).

25 October (GS) Central New Hampshire

Origin time: 171638.4

See New Hampshire listing.

\section{MARYLAND}

31 January (NI) Northeastern Ohio

Origin time: 164642.3

See Ohio listing.

\section{MASSACHUSETTS}

\section{April (WO) Northeastern Massachusetts}

Origin time: 042142.7

Epicenter: $42.847 \mathrm{~N} ., 70.982 \mathrm{~W}$.

Depth: $5 \mathrm{~km}$

Magnitude: $2.6 \mathrm{M}_{\mathrm{D}}(\mathrm{WO})$

Intensity III: Amesbury (press report), Groveland (press report), Merrimac (press report), West Newbury (press report).

\section{October (GS) Central New Hampshire}

Origin time: 171638.4

See New Hampshire listing.

\section{MICHIGAN}

31 January (NI) Northeastern Ohio

Origin time: 164642.3

See Ohio listing.

12 July (GS) Western Ohio

Origin time: 081937.9

See Ohio listing.

\section{MISSOURI}

24 May (SL) Southeastern Missouri

Origin time: 124813.5

Epicenter: $36.580 \mathrm{~N} ., 89.880 \mathrm{~W}$.

Depth: $10 \mathrm{~km}$

Magnitude: $3.4 \mathrm{M}_{\mathrm{n}}(\mathrm{SL}), 3.4 \mathrm{M}_{\mathrm{n}}(\mathrm{GS}), 3.1 \mathrm{M}_{\mathrm{D}}(\mathrm{TC})$,

Intensity IV:

$$
3.4 \mathrm{M}_{\mathrm{n}}(\mathrm{TU})
$$

Missouri-Bernie, Caruthersville, Malden, Parma, Portageville, Risco.

Intensity III:

Arkansas-Pollard.

Missouri-Brosley, Campbell, Canalou, Catron, Dudley,

Fisk, Kewanee, New Madrid, Steele, Tallapoosa.

Tennessee-Tiptonville.

Intensity II:

Missouri-Graybridge.

Felt:

Missouri-Conran, East Prairie (SL), Sikeston (SL).

26 August (SL) Southern Illinois

Origin time: 164124.8

See Illinois listing.

24 October (SL) Southeastern Missouri

Origin time: 055745.8

Epicenter: 36.170 N., $89.660 \mathrm{~W}$.

Depth: $9 \mathrm{~km}$

Magnitude: $2.9 \mathrm{M}_{\mathrm{n}}(\mathrm{SL}), 2.6 \mathrm{M}_{\mathrm{D}}(\mathrm{TC})$

Intensity IV: Caruthersville (SL).

6 November (SL) Missouri

Origin time: 192147.2

Epicenter: 38.110N., 90.420W.

Depth: 9 km

Magnitude: $2.7 \mathrm{M}_{\mathrm{n}}(\mathrm{SL})$

Intensity III: Crystal City (SL). 
30 December (SL) Southeastern Missouri

Origin time: 071519.1

Epicenter: $36.420 \mathrm{~N}$., $89.580 \mathrm{~W}$.

Depth: $14 \mathrm{~km}$

Magnitude: $3.5 \mathrm{M}_{\mathrm{n}}(\mathrm{GS}), 3.4 \mathrm{M}_{\mathrm{n}}(\mathrm{SL})$

Intensity IV:

Missouri-Portageville.

Intensity III:

Illinois-Tamms.

Missouri-Blodgett, Dexter, Gideon, Harviell, Hayti, Sikeston.

Tennessee-Finley, Tigrett.

Intensity II:

Kentucky-Clinton.

Missouri-Tallapoosa.

Tennessee-Tiptonville.

Felt:

Missouri-Point Pleasant (SL).

\section{MONTANA}

8 January (UU) Yellowstone National Park

Origin time: 073225.7

See Wyoming listing.

8 January (UU) Yellowstone National Park Origin time: 110815.7

See Wyoming listing.

8 January (UU) Yellowstone National Park

Origin time: 133628.5

See Wyoming listing.

14 January (UU) Yellowstone National Park Origin time: 015037.8

See Wyoming listing.

14 January (UU) Yellowstone National Park Origin time: 164629.9

See Wyoming listing.

15 January (UU) Yellowstone National Park Origin time: 211018.0

See Wyoming listing.
2 March (UU) Yellowstone National Park

Origin time: 105525.4

See Wyoming listing.

2 March (UU) Yellowstone National Park

Origin time: 125936.5

See Wyoming listing.

18 April (UU) Yellowstone National Park

Origin time: 141755.6

See Wyoming listing.

24 August (BU) Western Montana

Origin time: 180425.5

Epicenter: 45.802N., 111.594W.

Depth: $13 \mathrm{~km}$

Magnitude: $3.9 \mathrm{M}_{\mathrm{L}}(\mathrm{GS}), 3.9 \mathrm{MD}(\mathrm{BU})$

Intensity IV: Willow Creek.

Intensity III: Manhattan, Three Forks.

2 October Yellowstone National Park

Origin time: 0645

See Wyoming listing.

18 October (BU) Southwestern Montana

Origin time: 142046.9

Epicenter: 46.304N., 112.060W.

Depth: $3 \mathrm{~km}$

Magnitude: $3.1 \mathrm{M}_{\mathrm{L}}(\mathrm{BU}), 3.2 \mathrm{M}_{\mathrm{L}}(\mathrm{GS})$

Intensity IV: Jefferson City.

Felt: Boulder (BU).

18 October (BU) Southwestern Montana Origin time: 185538.7

Epicenter: 46.293N., 112.026W.

Depth: $0 \mathrm{~km}$

Magnitude: $3.4 \mathrm{M}_{\mathrm{L}}(\mathrm{BU}), 3.5 \mathrm{M}_{\mathrm{L}}(\mathrm{GS})$

Intensity IV: Jefferson City.

Felt: Boulder (BU).

19 October (BU) Southwestern Montana Origin time: 100143.4

Epicenter: 46.292N., 112.025W.

Depth: $0 \mathrm{~km}$

Magnitude: $3.3 \mathrm{M}_{\mathrm{L}}(\mathrm{BU}), 3.3 \mathrm{M}_{\mathrm{L}}(\mathrm{GS})$

Intensity IV: Jefferson City.

Felt: Boulder (BU).

24 October (BU) Southwestern Montana

Origin time: 045447.7 
Epicenter: $46.305 \mathrm{~N} ., 112.046 \mathrm{~W}$.

Depth: $3 \mathrm{~km}$

Magnitude: $3.2 \mathrm{M}_{\mathrm{L}}(\mathrm{BU}), 3.5 \mathrm{M}_{\mathrm{L}}(\mathrm{GS})$

Felt: Boulder (BU).

\section{November (BU) Southwestern Montana}

Origin time: 120635.3

Epicenter: 46.205 N., $112.112 \mathrm{~W}$.

Depth: $10 \mathrm{~km}$

Magnitude: $3.2 \mathrm{M}_{\mathrm{L}}(\mathrm{BU}), 3.3 \mathrm{M}_{\mathrm{L}}(\mathrm{GS})$

Felt: Boulder (BU).

15 November (UU) Yellowstone National Park

Origin time: 005657.0

See Wyoming listing.

24 November (UU) Yellowstone National Park

Origin time: 021058.7

See Wyoming listing.

24 November (UU) Yellowstone National Park

Origin time: 063150.4

See Wyoming listing.

25 November (UU) Yellowstone National Park

Origin time: 204525.4

See Wyoming listing.

\section{NEVADA}

12 January (RN) Western Nevada

Origin time: 040743.3

Epicenter: $39.626 \mathrm{~N} ., 119.380 \mathrm{~W}$.

Depth: None computed.

Magnitude: $3.1 \mathrm{M}_{\mathrm{D}}(\mathrm{RN})$

Felt: Fallon (RN), near Gooseberry mine (RN), Painted Rock (RN).

22 March (EN) Southern Nevada

Origin time: 161500.076

Epicenter: $37.083 \mathrm{~N} ., 116.066 \mathrm{~W}$.

Depth: $0 \mathrm{~km}$

Magnitude: $5.1 \mathrm{~m}_{\mathrm{b}}(\mathrm{GS}), 5.1 \mathrm{M}_{\mathrm{L}}(\mathrm{BK})$
Nevada Test Site explosion "GLENCOE" at $37^{\circ} 04^{\prime} 58.81^{\prime \prime} \mathrm{N}$., $116^{\circ} 03^{\prime} 57.81^{\prime \prime} \mathrm{W}$., surface elevation 1,260 m., depth of burial $600 \mathrm{~m}$.

10 April (EN) Southern Nevada

Origin time: 140830.095

Epicenter: 37.218 N., $116.183 \mathrm{~W}$.

Depth: $0 \mathrm{~km}$

Magnitude: $4.9 \mathrm{~m}_{\mathrm{b}}(\mathrm{GS}), 4.8 \mathrm{M}_{\mathrm{L}}(\mathrm{BK})$

Nevada Test Site explosion "MIGHTY OAK" at $37^{\circ} 13^{\prime} 05.97^{\prime \prime} \mathrm{N}$., $116^{\circ} 10^{\prime} 59.20^{\prime \prime} \mathrm{W}$., surface elevation 2,111 $\mathrm{m}$., depth of burial $400 \mathrm{~m}$, tunnel shot.

22 April (EN) Southern Nevada

Origin time: 143000.086

Epicenter: 37.264 N., $116.440 \mathrm{~W}$.

Depth: $0 \mathrm{~km}$

Magnitude: $5.3 \mathrm{~m}_{\mathrm{b}}(\mathrm{GS}), 4.2 \mathrm{M}_{S}(\mathrm{GS}), 5.4 \mathrm{M}_{\mathrm{L}}(\mathrm{BK})$

Nevada Test Site explosion "JEFFERSON" at $37^{\circ} 15^{\prime} 50.82^{\prime \prime} \mathrm{N}$., $116^{\circ} 26^{\prime} 24.73^{\prime \prime} \mathrm{W}$., surface elevation 1,982 m., depth of burial $600 \mathrm{~m}$.

21 May (EN) Southern Nevada

Origin time: 135900.083

Epicenter: 37.125 N., $116.060 \mathrm{~W}$.

Depth: $0 \mathrm{~km}$

Magnitude: $4.1 \mathrm{M}_{\mathrm{L}}(\mathrm{BK})$

Nevada Test Site explosion "PANAMINT" at $37^{\circ} 07^{\prime} 30.12^{\prime \prime} \mathrm{W}$., $116^{\circ} 03^{\prime} 37.40^{\prime \prime}$ surface elevation $1,286 \mathrm{~m}$, depth of burial $500 \mathrm{~m}$.

5 June (EN) Southern Nevada

Origin time: 150400.064

Epicenter: 37.098 N., 116.016W.

Depth: $0 \mathrm{~km}$

Magnitude: $5.3 \mathrm{~m}_{\mathrm{b}}(\mathrm{GS}), 4.2 \mathrm{M}_{\mathrm{S}}(\mathrm{GS}), 5.2 \mathrm{M}_{\mathrm{L}}(\mathrm{BK})$

Nevada Test Site explosion "TAJO" at $37^{\circ} 05^{\prime} 53.84^{\prime \prime} \mathrm{N}$., $116^{\circ} 00^{\prime} 55.84^{\prime \prime} \mathrm{W}$., surface elevation 1,314 m., depth of burial $500 \mathrm{~m}$.

15 June (RN) Western Nevada

Origin time: 140051.3

Epicenter: 38.766 N., $119.402 W$.

Depth: $5 \mathrm{~km}$

Magnitude: $3.2 \mathrm{M}_{\mathrm{D}}(\mathrm{RN})$

Intensity IV: Smith (RN), Wellington (RN).

25 June (EN) Southern Nevada

Origin time: 202745.1

Epicenter: $37.265 \mathrm{~N} ., 116.499 \mathrm{~W}$.

Depth: $0 \mathrm{~km}$

Magnitude: $5.5 \mathrm{~m}_{\mathrm{b}}(\mathrm{GS}), 4.2 \mathrm{M}_{\mathrm{S}}(\mathrm{GS}), 5.4 \mathrm{M}_{\mathrm{L}}(\mathrm{BK})$ 
Nevada Test Site explosion "DARWIN" at $37^{\circ} 15^{\prime} 52.51^{\prime \prime} \mathrm{N}$., $116^{\circ} 29^{\prime} 57.51^{\prime \prime} \mathrm{W}$., surface elevation 1,876 m., depth of burial $500 \mathrm{~m}$.

\section{June (RN) Western Nevada}

Origin time: 020629.6

Epicenter: $39.518 \mathrm{~N} ., 119.761 \mathrm{~W}$.

Depth: None computed.

Magnitude: $3.7 \mathrm{M}_{\mathrm{L}}(\mathrm{BK}), 3.9 \mathrm{M}_{\mathrm{L}}(\mathrm{RN})$

Intensity V:

Reno-A few glassware items or dishes broke; a few small objects overturned and fell; buildings shook strongly; felt by many people.

Sparks-A few small objects fell; felt by and frightened many people.

Intensity III: Fernley.

Felt: Sun Valley.

8 July (PS) Southern California

Origin time: 092044.5

See California listing.

13 July $(\mathrm{HJ})$ Off the coast of Southern California

Origin time: 134708.2

See California listing.

17 July (GP) Southern California

Origin time: 203515.0

See California listing.

17 July (EN) Southern Nevada

Origin time: 210000.055

Epicenter: 37.279N., 116.356W.

Depth: $0 \mathrm{~km}$

Magnitude: $5.7 \mathrm{~m}_{\mathrm{b}}(\mathrm{GS}), 5.6 \mathrm{M}_{\mathrm{L}}(\mathrm{BK})$

Nevada Test Site explosion "CYBAR" at $37^{\circ} 16^{\prime} 43.22^{\prime \prime} \mathrm{N}$., $116^{\circ} 21^{\prime} 20.19^{\prime}$ W., surface elevation 2,044 $\mathrm{m}$, depth of burial $600 \mathrm{~m}$.

20 July (GM) Owens Valley area

Origin time: 142945.5

See California listing.

20 July (BK) Owens Valley area

Origin time: 183852.9

See California listing.

\section{July (BK) Owens Valley area}

Origin time: 144226.5

See California listing.

21 July (BK) Owens Valley area

Origin time: 145110.1

See California listing.

24 July (EN) Southern Nevada

Origin time: 150500.086

Epicenter: 37.143 N., 116.071W.

Depth: $0 \mathrm{~km}$

Magnitude: $4.4 \mathrm{~m}_{\mathrm{b}}(\mathrm{GS}), 4.5 \mathrm{M}_{\mathrm{L}}(\mathrm{BK})$

Nevada Test Site explosion "CORNUCOPIA" at $37^{\circ} 08^{\prime} 33.89^{\prime \prime} \mathrm{N}$., $116^{\circ} 04^{\prime} 16.02^{\prime \prime} \mathrm{W}$., surface elevation 1,314 $\mathrm{m}$., depth of burial $400 \mathrm{~m}$.

31 July (BK) Owens Valley area

Origin time: 072240.2

See California listing.

\section{August (RN) Western Nevada}

Origin time: 140610.4

Epicenter: 39.521N., 119.753W.

Depth: None computed.

Magnitude: $2.7 \mathrm{M}_{\mathrm{L}}(\mathrm{GS}), 2.5 \mathrm{M}_{\mathrm{D}}(\mathrm{RN})$

Felt: Sparks (press report), Reno (RN).

\section{August (RN) Western Nevada}

Origin time: 170636.9

Epicenter: $39.638 \mathrm{~N}$., $119.803 \mathrm{~W}$.

Depth: $5 \mathbf{~ k m}$

Magnitude: $2.8 \mathrm{M}_{\mathrm{L}}(\mathrm{GS}), 2.9 \mathrm{M}_{\mathrm{L}}(\mathrm{BK}), 3.2 \mathrm{M}_{\mathrm{L}}(\mathrm{RN})$

Intensity III: Sparks.

Felt: Reno (press report).

25 August (RN) Western Nevada

Origin time: 175742.1

Epicenter: 39.638N., 119.803W.

Depth: $5 \mathrm{~km}$

Magnitude: $2.5 \mathrm{M}_{\mathrm{L}}(\mathrm{GS}), 3.2 \mathrm{M}_{\mathrm{L}}(\mathrm{RN})$

Felt: Reno (press report), Sparks (press report).

11 September (EN) Southern Nevada

Origin time: 145700.107

Epicenter: 37.069N., 116.050W.

Depth: $0 \mathrm{~km}$

Magnitude: $3.2 \mathrm{M}_{\mathrm{L}}(\mathrm{GS})$

Nevada Test Site explosion "ALEMAN" at $37^{\circ} 04^{\prime} 08.68^{\prime \prime} \mathrm{N}$., $116^{\circ} 02^{\prime} 58.89^{\prime \prime}$ W., surface elevation 1,244 m., depth of burial $500 \mathrm{~m}$. 


\section{NEVADA-Continued}

30 September (EN) Southern Nevada

Origin time: 223000.102

Epicenter: 37.300N., 116.307W.

Depth: 0 km

Magnitude: $5.5 \mathrm{~m}_{b}(\mathrm{GS}), 4.5 \mathrm{MS}_{\mathrm{S}}(\mathrm{GS}), 5.3 \mathrm{M}_{\mathrm{L}}(\mathrm{BK})$

Nevada Test Site explosion "LABQUARK" at $37^{\circ} 18^{\prime} 00.29^{\prime} \mathrm{N}$., $116^{\circ} 18^{\prime} 26.74^{\prime \prime} \mathrm{W}$., surface elevation 2,127 m., depth of burial $600 \mathrm{~m}$.

16 October (EN) Southern Nevada

Origin time: 192500.089

Epicenter: 37.220N., 116.462W.

Depth: $0 \mathrm{~km}$

Magnitude: $5.6 \mathrm{~m}_{\mathrm{b}}(\mathrm{GS}), 5.4 \mathrm{M}_{\mathrm{L}}(\mathrm{BK})$

Nevada Test Site explosion "BELMONT" at $37^{\circ} 13^{\prime} 12.77^{\prime \prime} \mathrm{N}$., $116^{\circ} 27^{\prime} 41.89^{\prime \prime} \mathrm{W}$., surface elevation 1,898 m., depth of burial $600 \mathrm{~m}$.

\section{October (BK) California-Nevada border area}

Origin time: 035728.9

See California listing.

\section{November (BK) Western Nevada}

Origin time: 192338.3

Epicenter: $38.712 \mathrm{~N} ., 119.540 \mathrm{~W}$.

Depth: $17 \mathrm{~km}$

Magnitude: $4.6 \mathrm{M}_{\mathrm{L}}(\mathrm{BK}), 4.3 \mathrm{M}_{\mathrm{L}}(\mathrm{RN})$

Moment: $4.2 \times 10^{22}$ dyne-cm (BK)

Intensity $\mathrm{V}$ :

California-

Topaz- A few small objects fell; shaking was described as moderate; felt by many people.

White Pines-People had difficulty standing; shaking was described as strong.

Intensity IV:

California- Avery, Pioneer, Twain Harte.

Nevada - Wellington.

Intensity III:

California - Coleville, Pine Grove, Standard.

Nevada-Schurz, Smith.

Intensity II:

California_ Arnold, Bridgeport.

14 November (EN) Southern Nevada

Origin time: 160000.066

Epicenter: 37.100N., 116.048W.

Depth: $0 \mathrm{~km}$

Magnitude: $5.8 \mathrm{~m}_{b}(\mathrm{GS}), 4.5 \mathrm{MS}_{\mathrm{S}}(\mathrm{GS}), 5.5 \mathrm{M}_{\mathrm{L}}(\mathrm{BK})$

\section{NEVADA-Continued}

Nevada Test Site explosion "GASCON" at $37^{\circ} 06^{\prime} 01.54^{\prime \prime} \mathrm{W} ., 116^{\circ} 02^{\prime} 53.05^{\prime \prime} \mathrm{W}$., surface elevation 1,263 $\mathrm{m}$., depth of burial $600 \mathrm{~m}$.

Intensity III: Las Vegas (press report).

14 November (GS) Southern Nevada

Origin time: 200238.7

Epicenter: 37.081N., 116.014W.

Depth: 0 km

Magnitude: $4.0 \mathrm{~m}_{\mathrm{b}}(\mathrm{GS})$

Nevada Test Site collapse from explosion "GASCON".

13 December (EN) Southern Nevada

Origin time: 175005.093

Epicenter: 37.263N., 116.412W.

Depth: $0 \mathrm{~km}$

Magnitude: $5.5 \mathrm{~m}_{\mathrm{b}}(\mathrm{GS}), 5.4 \mathrm{M}_{\mathrm{L}}(\mathrm{BK})$

Nevada Test Site explosion "BODIE" at $37^{\circ} 15^{\prime} 46.64^{\prime \prime} \mathrm{N}$., $116^{\circ} 24^{\prime} 42.06^{\prime \prime} \mathrm{W}$., surface elevation 2,018 $\mathrm{m}$., depth of burial $600 \mathrm{~m}$.

\section{NEW HAMPSHIRE}

\section{January (WO) Central New Hampshire}

Origin time: 143357.5

Epicenter: $43.500 \mathrm{~N}$., $71.568 \mathrm{~W}$.

Depth: $5 \mathrm{~km}$

Magnitude: $2.6 \mathrm{M}_{\mathrm{L}}(\mathrm{WO}), 2.5 \mathrm{M}_{\mathrm{D}}(\mathrm{WO})$

Intensity III: Gaza.

\section{January (NI) Northeastern Ohio}

Origin time: 164642.3

See Ohio listing.

25 October (GS) Central New Hampshire

Origin time: 171638.4

Epicenter: $43.399 \mathrm{~N}$., $71.590 \mathrm{~W}$.

Depth: $5 \mathrm{~km}$

Magnitude: $3.9 \mathrm{M}_{\mathrm{n}}(\mathrm{GS})$

This earthquake was felt over a contiguous area of about $9,500 \mathrm{~km}^{2}$ of Maine, Massachusetts, and New Hampshire (fig. 19).

Intensity V:

New Hampshire- 


\section{NEW HAMPSHIRE-Continued}

Boscawen-A few small objects fell; kitchen cabinet doors with magnetic catches opened; shaking was described as strong; felt by everyone.

Canterbury-A few items were shaken off store shelves; a few small objects overturned; underground pipes broke; buildings were shaken moderately; felt by many people.

Chesterfield-A few small objects overturned and fell; felt by several people.

Henniker-A few small objects overturned and fell; shaking was described as moderate; felt by many people.

Jaffrey-A few small objects overturned and fell; felt by many people.

Lakeport-A few small objects overturned and fell; felt by several people.

Walpole-A few windows cracked; a few glassware items or dishes broke; a few small objects overturned and fell; felt by several people.

Intensity IV:

Massachusetts-East Templeton, Gardner, Woburn (press report).

New Hampshire-Andover, Auburn, Barnstead, Belmont, Brookline (press report), Center Barnstead, Concord, Contoocook, Elkins, Epsom, Franklin, Gilmanton, Greenfield, Hill, Hillsboro, Keene, Loudon, Milford, New Durham, New Hampton, New Ipswich, Northfield (press report), Peterborough, Pittsfield, Rindge, Sanbornton, South Newbury, Strafford, Tilton, Warner, Washington, Westmoreland, Winchester.

Vermont-Hartland.

Intensity III:

Maine-Scarborough.

Massachusetts-Ashburnham, Ashby, Athol, Baldwinville, Billerica (press report), Boxford, East Longmeadow, Fitchburg, Framingham (press report), Greenfield (press report), Holliston (press report), Lancaster, Lawrence (press report), Lowell (press report), Natick, Shrewsbury (press report), Townsend, Ware, Wilbraham, Winchendon, Worcester (press report).

New Hampshire-Amherst, Antrim, Ashland, Bennington, Bradford, East Derry, Fitzwilliam, Goshen, Hancock, Harrisville, Laconia, Marlborough, Marlow, Munsonville, North Sutton, Potter Place, Salisbury, South Sutton, Troy, Wilmot Flat.

Intensity II:

Connecticut-Torrington (press report).

Massachusetts-Holden, West Springfield.

Maine-Brunswick.

New Hampshire-Dublin, Ossipee.

Rhode Island-Greenville.

\section{NEW HAMPSHIRE-Continued}

Felt:

Maine-North Waterboro.

New Hampshire-Greenville (press report), Temple (press report).

\section{NEW JERSEY}

\section{January (NI) Northeastern Ohio}

Origin time: 164642.3

See Ohio listing.

23 November (LD) New Jersey

Origin time: 212938.8

Epicenter: $40.956 \mathrm{~N}$., $74.820 \mathrm{~W}$.

Depth: $7 \mathrm{~km}$

Magnitude: $2.8 \mathrm{M}_{\mathrm{D}}(\mathrm{LD})$

Intensity IV: Allamuchy, Byram, Lake Tranquility area.

Intensity III: Waterloo Village.

\section{NEW MEXICO}

17 April (GS) Southern New Mexico

Origin time: 210430.3

Epicenter: 32.587N., 106.912W.

Depth: $5 \mathrm{~km}$

Magnitude: $2.7 \mathrm{M}_{\mathrm{D}}(\mathrm{GS})$

Felt: Leasburg.

\section{April (GS) Central New Mexico}

Origin time: 130016.0

Epicenter: 34.009N., 106.821W.

Depth: $5 \mathrm{~km}$

Magnitude: $2.6 \mathrm{M}_{\mathrm{D}}(\mathrm{GS})$

Felt: Luis Lopez.

27 August (GS) Central New Mexico

Origin time: 180656.3

Epicenter: 35.160N., 105.094W.

Depth: $5 \mathrm{~km}$

Magnitude: $3.2 \mathrm{M}_{\mathrm{L}}(\mathrm{GS}), 3.0 \mathrm{M}_{\mathrm{L}}$ (TU)

Felt: Anton Chico, Las Vegas. 


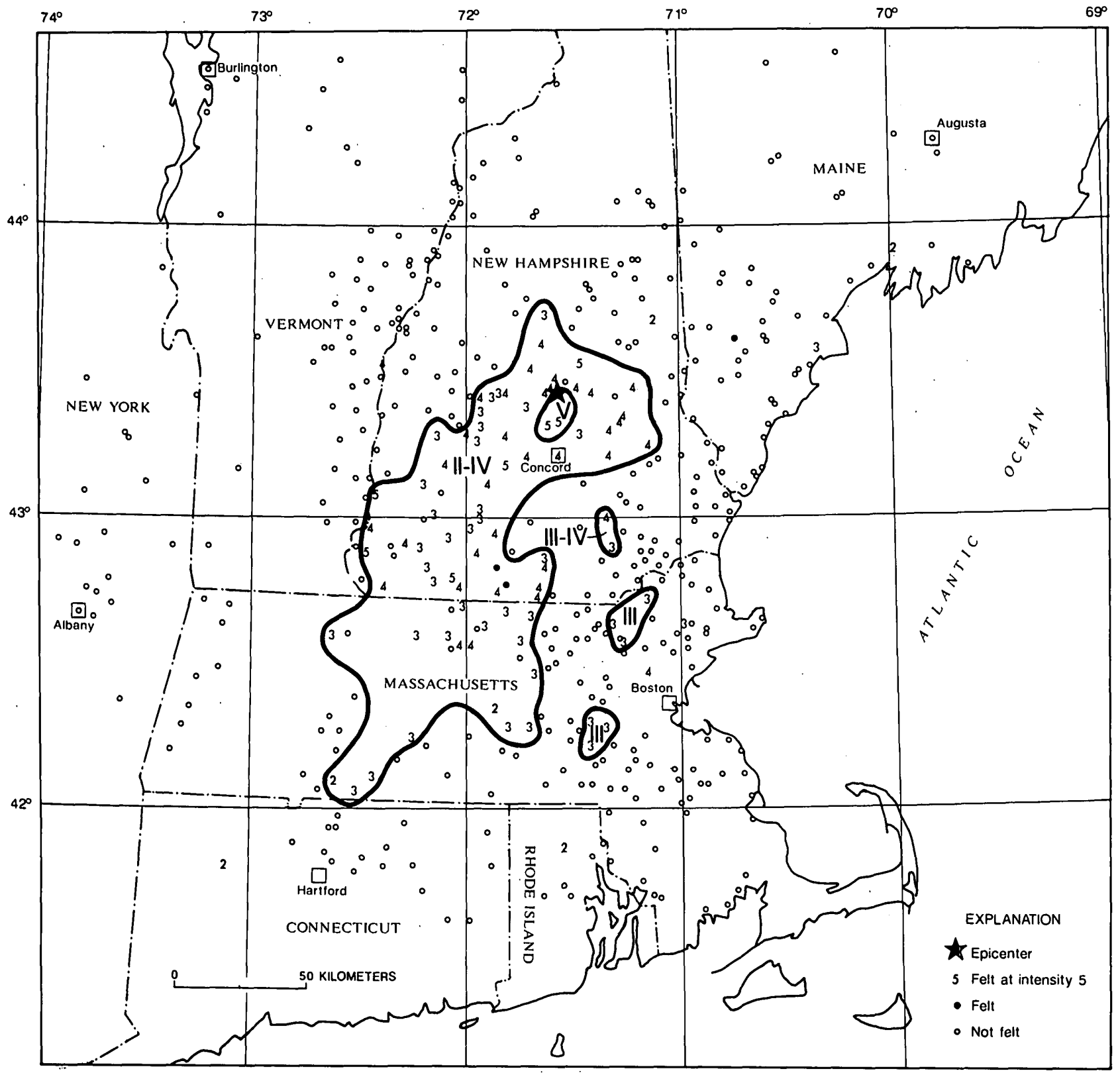

Figure 19. Isoseismal map for the central New Hampshire earthquake of 25 October, 1986, 171638.4 UTC. Roman numerals represent Modified Mercalli intensities between isoseismals; Arabic numerals represent intensities at specific sites, and small boxes show locations of towns and cities whose names are plotted.

\section{NEW YORK}

\section{January (LD) Southeastern New York}

Origin time: 033556.2

Epicenter: 40.996 N., $73.833 W$.

Depth: $6 \mathrm{~km}$

Magnitude: $2.5 \mathrm{M}_{\mathrm{n}}(\mathrm{LD})$

\section{NEW YORK-Continued}

Felt throughout southern Westchester County (press report).

Intensity IV: Ardsley (press report), Dobbs Ferry (press report), Hastings-On-Hudson, Tuckahoe, White Plains, Yonkers. 
NEW YORK-Continued

Intensity III: Eastchester, Irvington, Mamaroneck, New Rochelle, Scarsdale, Tarrytown.

\section{January (NI) Northeastern Ohio}

Origin time: 164642.3

See Ohio listing.

\section{April (LD) Southeastern New York}

Origin time: 072823.7

Epicenter: 40.980N., 73.834W.

Depth: $6 \mathrm{~km}$

Magnitude: $2.7 \mathrm{M}_{\mathrm{D}}(\mathrm{LD})$

Intensity IV: Ardsley (press report), Eastchester (press report), Harrison (press report), Mount Vernon, Rye, Scarsdale, Tuckahoe (press report), Yonkers.

Intensity III: Ardsley-On-Hudson, Bronxville (press report), Dobbs Ferry (press report), Elmsford (press report), Greenburgh (press report), Hastings-On-Hudson (press report), Irvington (press report), Mamaronek, New Rochelle (press report), Pelham, White Plains (press report).

\section{August (LD) Southwestern Quebec, Canada}

Origin time: 045518.4

Epicenter: 45.131N., 74.246W.

Depth: $24 \mathrm{~km}$

Magnitude: $3.4 \mathrm{M}_{\mathrm{D}}(\mathrm{LD}), 3.3 \mathrm{M}_{\mathrm{n}}(\mathrm{EP})$

Felt:

New York-Hogansburg, Malone, Massena, Moira, Potsdam, Trout River, Westville (all from press reports).

20 December (LD) Southeastern New York

Origin time: 131531.0

Epicenter: 40.999N., 73.831W.

Depth: $5 \mathrm{~km}$

Magnitude: $1.9 \mathrm{M}_{\mathrm{D}}(\mathrm{LD})$

Felt: Ardsley (press report).

\section{NORTH CAROLINA}

13 February (TC) Northwestern South Carolina Origin time: 113545.3

See South Carolina listing.

11 July (TC) Georgia-Tennessee border Origin time: 142614.8

See Georgia listing.

\section{OHIO}

31 January (NI) Northeastern Ohio

Origin time: 164642.3

Epicenter: 41.650N., 81.162W.

Depth: $2 \mathrm{~km}$

Magnitude: $5.0 \mathrm{~m}_{\mathrm{b}}(\mathrm{GS}), 4.9 \mathrm{M}_{\mathrm{n}}(\mathrm{SL}), 5.3 \mathrm{M}_{\mathrm{n}}(\mathrm{EP})$

Moment: $3.4 \times 10^{23}$ dyne-cm (GS)

This earthquake injured 17 people and produced minor damage in the epicentral area. The maximum intensity is assigned a MM VI. However, a few isolated reports of damage indicated a possible intensity of MM VII. Also, well water changed in level or was muddied, indicating a possible intensity of MM VII. Because of the few instances of marginal MM VII effects, the overall intensity is rated as a high MM VI.

The injuries resulted from falls during the evacuation of buildings, cuts from flying broken glass, bruises from falling objects, and exposure due to extended periods in the cold weather while buildings were being examined for damage. The damage to homes and commercial buildings consisted mostly of cracked chimneys, brick walls, and plastered walls; falling of suspended ceiling tiles; and cracked and broken windows. Other types of damage consisted primarily of broken glassware, either from glass items thrown off store shelves or chinaware that broke in china cabinets.

The earthquake affected more than a dozen water wells in Lake and George Counties. The effects varied from sediment in the water to variations of water flow (from too much to not enough), and in one place, water appeared where there had been none. There was one report of an old artesian water well that suddenly started filling an old water trough with water. In Leroy Township, a small pond formed from the flow of a new artesian well.

The earthquake was felt over a contiguous area of approximately $305,000 \mathrm{~km}^{2}$ (fig. 20) in all or parts of eight states and Ontario, Canada (Stover, 1986). These states were Illinois, Indiana, Kentucky, Michigan, Ohio, New York, Pennsylvania, and West Virginia. Isolated felt reports were received from Delaware, Maryland, New Jersey, Virginia, and Wisconsin as well as Washington, D.C. (District of Columbia). These isolated reports generally originated from people on the upper floors of multistory buildings.

Thirteen aftershocks were detected, following the main shock, until April 15, 1986, and 13 more were detected from April 15, 1986, to April 15, 1987. Magnitudes ranged from about 0.5 to 2.5 for the first 13 and about 1.0 for the second 13 (Nicholson and others, 1988). 


\section{OHIO-Continued}

The peak ground acceleration at the Perry Nuclear Power Station (about $10 \mathrm{mi}$ north of the epicenter) was $0.18 \mathrm{~g}$ in the north-south direction and $0.10 \mathrm{~g}$ in the eastwest direction (Monroe and Stevenson, 1986; Wesson and Nicholson, 1986).

The intensities in the United States (figs. 20 and 21) were evaluated from data collected by the U.S. Geological Survey/National Earthquake Information Center, supplemented by a canvass of the epicentral region by Weston Geophysical Corp., Westboro, Mass. and by numerous press reports. The intensity data in Canada were furnished by R.J. Wetmiller, Geophysics Division, Geological Survey of Canada, Ottawa, supplemented by press reports.

\section{Intensity VI:}

United States-

Ohio-

Bainbridge Center (press report)-At Kenston Intermediate School a chimney shifted, and several walls of each building were cracked. Ceiling tile fell at Bainbridge Town hall (press report).

Bowling Green (press reports)-

At Wood County Office building, marble cracked in the front entrance; damage occurred to the Law Library ceiling; plaster cracked and fell in the Sheriff's entry to the parking garage; tiles in front of several parking garage spaces were damaged; ceramic wall tiles cracked in four restrooms on the first, second, and fifth floors (cracks about $10 \mathrm{ft}$ long); there was a 0.5 -inch-wide crack between the floor and the wall of the main restrooms on the first floor; some mortar joints split in the stairwell on the first, second, and fifth floors.

Wood County Courthouse-Hairline cracks opened on all three of its floors; cracks occurred in the ceiling of the Probate Court and above the door in another courtroom, and cracks opened in the walls and ceiling of the prosecutor's office.

Chardon.

Geneva.

Grand River.

Huntsburg.

Kirtland-Chimneys, basement floors, and cement-block basement walls were cracked, several pieces of plaster fell from the walls and ceiling of the Kirtland Temple Visitor Center, a suspended ceiling fell on the third floor of Lakeland Community College, and a mirror broke and lamps were knocked over.

Leroy-Several chimneys and one fireplace cracked. At Leroy Elementary School walls cracked; chimneys cracked; bricks fell off one chimney; four windows broke; and light bulbs broke.

\section{OHIO-Continued}

Madison-At Madison High School cement-block walls cracked; ceiling tiles fell; and 1,300 students were dismissed because of a chemical spill. Effects at other locations in Madison included: cracked chimneys with fallen brick, cracks in exterior brick walls, hairline cracks in interior walls, cracked windows, broken glassware, a few small objects overturned and fallen, pictures fallen; felt by and frightened many people.

Mentor-Walls cracked at Center Street Elementary School, Garfield Elementary School, and Memorial Junior High School. At Heinen's Supermarket several ceiling tiles fell and much merchandise was thrown off shelves. Ceiling tiles fell at the Great Lakes Mall. One window broke and six cracked at St. Bede's Catholic Church. The press reported 15 buildings damaged. Much merchandise was shaken off store shelves, and there were some gas leaks at homes and businesses. Shaking was described as strong; felt by everyone.

Metals Park (west of Newburg)-Stairs of the American Society of Metals building cracked. Other effects in Metals Park were cracked chimneys, small sidewalk cracks, and hairline plaster cracks. Shaking was described as strong; a few glassware items broke; a few small objects overturned and fell; felt by everyone.

Middlefield-Chimneys cracked; a reinforced concrete wall cracked; plaster walls sustained hairline cracks; windows cracked; there was a report of visible waves on the ground; well water was muddied; a few items were thrown from store shelves; pictures fell; a few small objects overturned and fell; felt by many people to all.

Painesville-At Lake Erie College, 19 windows cracked or broke, and a large crack opened in the stairwell of the Commons (dining facility). The walls and ceilings of Thomas W. Harvey High School cracked, and a 2.5-ton machine was moved 3/16 in. at a sheet-metal shop. Tiles fell from the ceiling at Lake East Hospital. Other damage in Painesville included damaged chimneys, cracked plaster, cracked basement walls, and a cracked fireplace front.

Perry-At one home a garage ceiling cracked, the crack extending part way down the wall; baseboards separated from the wall in several rooms; bathroom walls separated from the ceiling, leaving a 3/16-in. crack; dishes broke in a china closet; windows broke; and a basement floor cracked.

Perry Nuclear Power Plant-There were hairline floor cracks, hairline wall cracks, and several minor leaks in nonsafety pipes.

Thompson-There were cracks in a gymnasium wall of the Lodgemont Elementary and Junior High School. Other damage in Thompson included: cracks in walls and basement floors, cracked chimneys, broken glassware, cracked windows, muddied well water, a few objects 
Figure 20. Isoseismal map for the northeast Ohio earthquake of 31 January, 1986, 164642.3 UTC. Roman numerals represent Modified Mercalli intensities between isoseismals; Arabic numerals represent intensities at specific sites; dashed contour lines are inferred isoseismals, and small boxes show locations of towns and cities whose names are plotted.

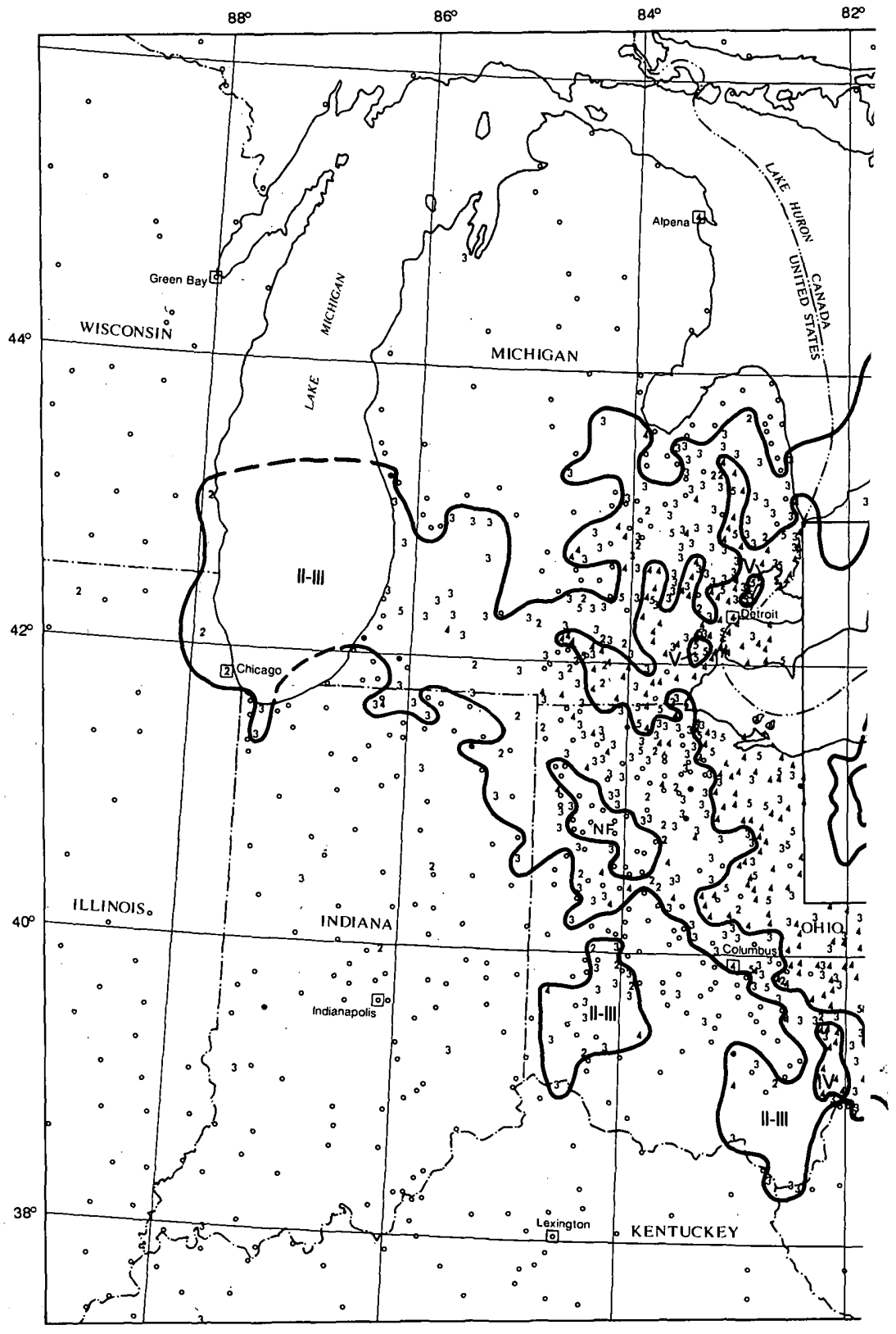

\section{OHIO_Continued}

overturned and fallen, a few items were shaken off store shelves; shaking was described as strong, felt by everyone.

Warren-Warren Western Reserve High School had some cracked walls (press report).

\section{OHIO-Continued}

Willoughby-Some windows broke; plaster-board walls cracked; a cement-block foundation cracked; a few glassware iterms broke; much merchandise was thrown off store shelves; streets were cracked; hanging pictures fell; shaking described as moderate; felt by everyone. 


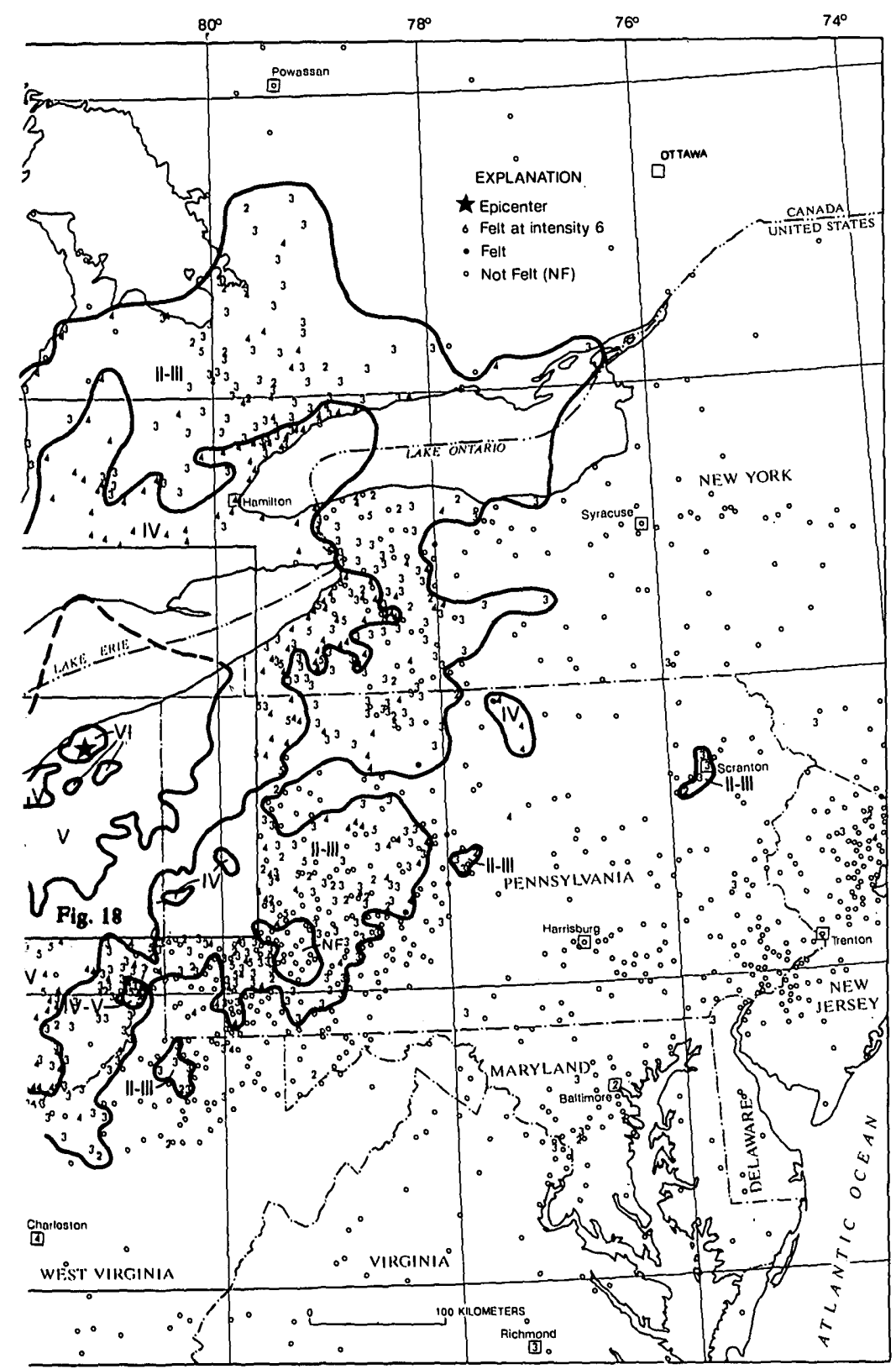

OHIO-Continued

Windsor-Interior and exterior brick walls were damaged; water level in wells changed; many items were shaken off store shelves; light furniture overturned; a few windows cracked; a few small objects overturned and fell; shaking was described as strong; felt by many people.

\section{OHIO-Continued}

\section{Pennsylvania-}

Albion-Chimneys, foundations, exterior brick walls, and interior plaster walls cracked; a few windows cracked; a few glassware items broke; a few small objects overturned and fell; buildings shook moderately; felt by and frightened many people. 


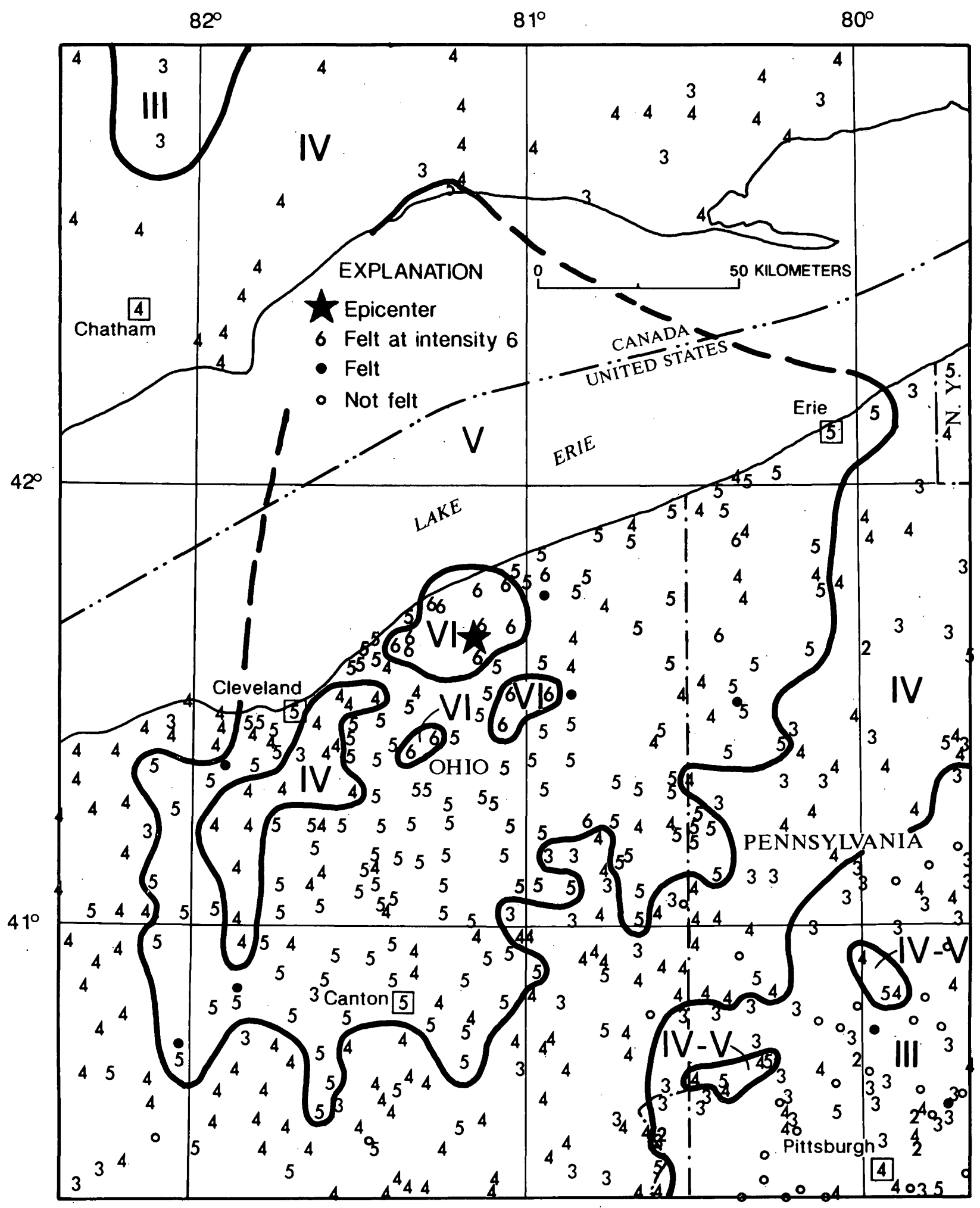

Figure 21. Isoseismal map for the epicentral area of the northeast Ohio earthquake of 31 January, 1986, 1646 42.3 UTC. Roman numerals represent Modified Mercalli intensities between isoseismals; Arabic numerals represent intensities at specific sites; dashed lines are inferred isoseismals, and boxes represent towns and cities whose names are plotted. 


\section{OHIO-Continued}

Linesville-Chimneys, foundations, and windows cracked; a few glassware items broke; a few items were shaken off store shelves; a few small objects overturned and fell; hanging pictures fell; shaking was described as strong, felt by everyone.

Intensity V:

The most common effects at the places listed below were that a few small objects overturned and fell; a few glassware items and dishes broke; trees and bushes shook slightly; hanging pictures swung, leaving some out of place; buildings shook slightly to moderately; windows, doors, and dishes rattled; standing and moving vehicles shook slightly; the shaking was described as moderate to strong; felt by many people.

\section{Canada- \\ Ontario- \\ Creemore. \\ Leamington. \\ Port Stanley. \\ United States_- \\ Michigan- \\ Almont-A few items were shaken off store shelves. \\ Bangor-A few windows cracked. \\ Burnside-A few bottles broke and ceiling tiles sustained minor damage at Dick's Place at the intersection of State Highways M-90 and M-53 (press report). \\ Carleton-A few items were shaken off store shelves. \\ Clawson-A few windows cracked. \\ Detroit Metro Airport-A few items were shaken off store shelves; a few windows cracked; interior walls sus- tained hairline cracks.}

East Detroit.

Fair Haven.

Flat Rock-A few merchandise items were shaken off store shelves.

Grand Blanc-A few merchandise items were shaken off store shelves.

Gregory.

Hamburg-A few windows cracked.

Lincoln Park-A few windows cracked.

Mount Clemens-A refrigerator moved 15 in.; a chair with a woman sitting in it moved on a kitchen floor (press report).

New Boston-A few windows cracked; interior walls sustained hairline cracks.

Rives Junction.

\section{OHIO-Continued}

Roseville-An above-ground, backyard swimming pool ruptured, causing all the water to drain out (press report).

Saint Clair Shores-Concrete cell walls of the city jail cracked; water splashed out of a bathtub (press report).

Smiths Creek- Hanging pictures fell; a few items were shaken off store shelves.

Somerset Center-A few items were shaken off store shelves.

New York-

Collins Center.

Conewango Valley-Ripples were observed (from east to west) in the snow.

Dewittville-Interior walls sustained hairline cracks.

Farnham-A few windows cracked; hanging pictures fell; a few items were shaken off store shelves; plaster-board walls sustained hairline cracks; there was a report of a cracked chimney.

Forestville-A few windows cracked.

Gasport.

Grand Island-A few windows cracked; interior walls sustained hairline cracks.

Maple Springs-A few items were shaken off store shelves.

Ripley-A few items were shaken off store shelves; interior walls sustained hairline cracks.

Ohio-

Akron-A few items were shaken off store shelves; interior walls sustained hairline cracks.

Akron (North Hill)-A few items were shaken off store shelves; interior walls sustained hairline cracks.

Alliance-A few windows cracked; a few items were shaken off store shelves; interior walls sustained hairline cracks; stone fences cracked.

Alliance (Mount Union)-A few windows cracked.

Andover-Interior walls sustained cracks.

Ashtabula-A few windows cracked; a few items were shaken off store shelves; interior walls sustained hairline cracks.

Attica-A few merchandise items were shaken off store shelves.

Atwater-Standing vehicles rocked moderately.

Aurora-A few windows cracked; interior walls sustained hairline cracks; standing vehicles rocked moderately; there was a report of broken underground pipes and cracked sidewalks.

Austinburg.

Baltic-A few items shook off store shelves.

Bartlett.

Bath-There was a report of cracked chimneys.

Bay Village-A few windows cracked; many small objects overturned and fell. 


\section{OHIO-Continued}

Beach City-There was a report of bricks falling from chimneys.

Beachwood-A few items were shaken off store shelves.

Bedford-Windows cracked; it was felt in a moving vehicle; everyone ran out of the newspaper office to find out the cause of the shaking; coffee splashed out of cups; windows rattled (press report).

Berea.

Boardman-A few windows cracked; a few items were shaken off store shelves; light furniture overturned.

Brady Lake-A few items were shaken off store shelves.

Brewster-There was a report of broken underground pipes.

Brice-A few items were thrown from store shelves; plaster walls sustained hairline cracks.

Bristolville-The Post Office and safe inside swayed "like jello" from east to west.

Brooklyn-A few windows cracked; many small objects overturned and fell; many merchandise items fell off store shelves.

Burghill-There was a report of cracked chimneys.

Burton-Many items were shaken off store shelves.

Cambridge-There was minor damage to water pipes and plumbing; a mobile home was shaken off its supports; buildings shook (press report).

Canal Fulton-A few windows cracked; a few items were shaken off store shelves.

Canton-A 2-in.-wide crack formed in the street in front of 2135 Sandwich Avenue; the couch moved; chairs jumped up and down; two jars fell from store shelves and broke (press report).

Carroll-Existing cracks in a stairwell at Malvern Elementary School widened, and the stairwell shifted about 0.75 in. at the bottom (press report).

Castalia-A few windows cracked; interior walls cracked.

ChagrinFalls-Cans, bottles of beer, bottles of wine, jars of jelly, and jars of mustard fell off store shelves; people ran out of buildings (press report). A few windows cracked; interior walls sustained hairline cracks.

Chesterland-A few windows cracked; a few items were shaken off store shelves.

Cleveland-In Severance Hall, doors and chandeliers swung; panels over the Cleveland Orchestra moved; and the audience and orchestra left the hall. There was a small crack in a wall of the State Office building. The Standard Oil building and the Terminal Tower shook strongly (press report). A few items were shaken off store shelves; buildings shook strongly.

Cleveland (Noble)-Interior walls damaged.

Cleveland (Midpark).

\section{OHIO-Continued}

Columbia Station-Interior walls sustained hairline cracks.

Conneaut-A few windows cracked; a few items were shaken off store shelves; interior walls sustained hairline cracks.

Cortland.

Cuyahoga Falls-Interior walls sustained hairline cracks; a foundation cracked.

Dalton.

Damascus-Many small objects overturned and fell.

Dellroy-Many small objects overturned and fell; a few items were shaken off store shelves; a street cracked.

Diamond-Plaster walls sustained hairline cracks.

Dorset-Interior walls sustained hairline cracks; a foundation cracked.

Dundee-A few windows cracked; plaster walls sustained hairline cracks.

East Canton-A few windows cracked; a few items were shaken off store shelves.

East Claridon-A few windows cracked; light furniture overturned; plaster walls sustained hairline cracks.

Eastlake-Glass objects fell and broke; a lamp broke; a few dishes and glasses fell out of cupboards; video tapes fell; everyone left a building.

Ellet.

Elyria-A few items were shaken off store shelves; plaster walls sustained hairline cracks.

Euclid-Hanging objects swung violently; pictures fell.

Fairport Harbor-Two stress fractures developed in the 50-year-old high-school building (press report). A few windows cracked; standing and moving vehicles rocked moderately; knickknacks overturned.

Fowler-Well-water levels changed and water in wells was muddied.

Garrettsville.

Geneva-On-The-Lake-Plaster walls sustained hairline cracks; items fell from window sills; cabinet doors opened and some of the contents fell out onto the floor; knickknacks shook off of shelves; a garage foundation cracked.

Genoa.

Girard-A few windows cracked; a few items were shaken off store shelves; interior walls sustained hairline cracks.

Grafton.

Grand Rapids-A few windows cracked.

Greensburg.

Hambden-Ceiling tiles fell; furniture moved; a few instances of fallen plaster (press report).

Hartford-A few items were shaken off store shelves.

Hartsgrove-People ran outside; walls moved at least 2 in.

Hinckley-A few windows cracked; a few items were shaken off store shelves. 


\section{OHIO-Continued}

Hiram-A few windows cracked; interior walls sustained hairline cracks; a foundation cracked.

Homeworth-A few windows cracked; a few items were shaken off store shelves; dry wall sustained hairline cracks.

Howland Corners-A 500-lb wood stove moved (press report).

Hudson.

Kent-Knickknacks fell, and people ran outside at Kent State University. Elsewhere, a few windows cracked.

Killbuck-Light furniture overturned.

Kingsville-A few items were shaken off store shelves; a foundation cracked.

Kinsman.

Lake Milton-A few windows cracked; a few items were shaken off store shelves.

Lakewood.

Lindsey-A few windows cracked.

Lisbon-A few windows cracked; a few items were shaken off store shelves; interior walls sustained hairline cracks.

Lodi.

Loudonville.

Lyndhurst.

Mayfield.

Malta-Trees and bushes shook moderately.

Malvern-The inside stairway at the sewer plant cracked (press report).

Mansfield-A few windows cracked; a few items were shaken off store shelves.

Mantua-A few merchandise items were shaken off store shelves.

Marshallville.

Massillon-Potted plants on a window moved; fire trucks moved back and forth; and a water heater leaked (press report). A few windows cracked; a few items were shaken off store shelves; interior walls sustained hairline cracks.

Masury-A few items were shaken off store shelves.

Maximo-A few items were shaken off store shelves; dry wall sustained hairline cracks; water flow in wells was disturbed.

McDonald-A few windows cracked; a few items were shaken off store shelves; interior walls sustained hairline cracks.

Mentor-On-The-Lake-Ceiling tiles fell in two department stores located in the Great Lakes Mall. Ceiling tiles also fell in the Heinen Supermarket at 8850 Mentor Avenue. Some small items fell off shelves at Gray's Drug Store. A false ceiling fell in the science classroom of the junior high school; walls of two elementary schools cracked.

\section{OHIO-Continued}

St. Bede's Catholic Church had one broken window and six cracked windows (press report).

Mesopotamia-Plaster walls sustained hairline cracks; a few merchandise items shook off store shelves.

Metamora.

Middleburg Heights-One window and an interior wall cracked. Buildings shook strongly.

Milan-A few windows cracked.

Mingo Junction-A few windows cracked; a few items were shaken off store shelves; interior walls sustained hairline cracks; some light furniture overturned.

Mogadore-Small items fell off shelves (press report).

Montville-Plaster walls cracked; small objects fell.

Mount Gilead-A few items were shaken off store shelves.

Munroe Falls-A few items were shaken off store shelves.

Munson-Plaster cracked in a few instances; small objects overturned.

Neffs.

New Philadelphia-A few windows cracked; a few items were shaken off store shelves.

Newbury-Many items were shaken off store shelves.

Newcomerstown-Interior wall board sustained hairline cracks.

Newton Falls-Plaster walls sustained hairline cracks; a few items were thrown from store shelves.

Nimisila-There was a report of a cracked chimney.

North Bloomfield-Plaster walls sustained hairline cracks.

North Canton-A few windows cracked; a few items were shaken off store shelves.

North Jackson-A few windows cracked; a few items were shaken off store shelves; interior walls sustained hairline cracks; a foundation cracked.

North Madison-Dishes and other objects fell out of cupboards and off shelves; pictures fell off walls; a basement foundation cracked.

Northfield-A few items were shaken off store shelves.

Norton.

Nova.

Novelty-Plaster walls sustained hairline cracks; well water was muddied.

Oak Harbor-Interior walls sustained hairline cracks.

Orrville-A few windows cracked.

Parkman-Trees and bushes shook moderately.

Parma-Interior walls sustained hairline cracks; a few items were shaken off store shelves; hanging pictures fell.

Peninsula.

Pepper Pike.

Pierpont-A few windows cracked; a few items were shaken off store shelves; interior walls sustained hairline 


\section{OHIO_Continued}

cracks; and there was an unconfirmed report of cracked chimneys.

Port Washington-A few windows cracked; interior walls sustained hairline cracks.

Randolph-A few windows cracked; dry wall sustained hairline cracks.

Ravenna-There was a report of broken underground pipes.

Richfield-Interior walls sustained hairline cracks; a few windows cracked; a few items were shaken off store shelves; small landslides occurred in an area of road fill; there was an unconfirmed report of cracked chimneys.

Richmond Heights-People had difficulty in standing and walking.

Rittman-Interior walls sustained hairline cracks; a few windows cracked; a few items were shaken off store shelves.

Robertsville-Interior walls sustained hairline cracks.

Rocky River-Buildings shook strongly; trees and bushesshook strongly; standing and moving vehicles shook moderately; felt by everyone.

Rootstown-On a farm, a full tank moved across a floor. People had difficulty standing.

Sandyville.

Sardis-A few windows cracked.

Sebring-A few windows cracked; a few items were shaken off store shelves; interior walls sustained hairline cracks.

Shaker Heights-Trees and bushes shook moderately; standing and moving vehicles rocked moderately.

Shalersville-A few knickknacks fell; an anchored mobile home moved (press report).

Sharon Center.

Shelby.

Shreve-A few windows cracked.

Solon-People had difficulty standing and walking; a few windows cracked; buildings shook strongly; small landslides occurred; felt by everyone.

Southington-Crystal fell off a kitchen chandelier (press report). A few windows cracked; a few items were shaken off store shelves; interior walls sustained hairline cracks.

Sparta-A few items were shaken off store shelves; a few light furniture pieces overturned.

Spencer-A few windows cracked; a few merchandise items were shaken off store shelves.

Streetsboro-People ran out of city offices thinking the building was going to fall (press report).

Swanton-A few windows cracked; a few merchandise items were shaken off store shelves.

Sylvania.

\section{OHIO-Continued}

Tallmadge-A few windows cracked; trees and bushes shook moderately.

Toronto-A few items were shaken off store shelves.

Twinsburg-A few windows cracked; a few merchandise items were shaken off store shelves.

Uniontown.

Unionville-A few windows cracked; a few items were shaken off store shelves; plaster walls sustained hairline cracks.

Wadsworth.

Waldo.

Warrensville Heights-Walls cracked; a few items fell off store shelves.

Washingtonville-Pictures were knocked off a wall in one home (press report).

Wayland-A large earthen dam sustained slight damage; people had difficulty standing.

West Farmington-Interior walls sustained hairline cracks.

West Salem.

Westfield Center-Interior walls sustained hairline cracks; cracks in an exterior brick wall were reported.

Wickliffe-Well water muddied; trees and bushes shook moderately. People ran out of their homes (press report); people had difficulty standing.

Willard-The press reported cracks in the walls of Central Elementary School and cracks in the walls and floor tile at the junior high school. Plaster walls sustained hairline cracks; standing vehicles rocked moderately.

Willowick-Buildings shook strongly; damage of an unknown type was reported by the postmaster; felt by everyone.

Windham-A few windows cracked; a few items were shaken off store shelves; many small objects overturned and fell.

Wooster-A few items were shaken off store shelves.

Youngstown (West Side)-A few windows cracked; interior walls sustained hairline cracks.

Youngstown (South Side)-A foundation cracked.

Youngstown (Fosterville)-Hanging pictures fell.

Youngstown-Stress cracks opened in the walls of the Department of Human Services building on West Federal Street (press report). A few windows cracked; a few items were shaken off store shelves; interior walls sustained hairline cracks; a foundation cracked.

Pennsylvania-

Atlantic.

Avonmore-A few windows cracked; a few items were shaken off store shelves; some light furniture overturned.

Charleroi-Many items were shaken off store shelves; some light furniture overturned.

Conneaut Lake-A few windows cracked. 


\section{OHIO-Continued}

Corry-A few windows cracked; plaster walls sustained hairline cracks; foundations cracked.

Dayton-A few windows cracked; hanging pictures fell; plaster walls sustained hairline cracks.

East Springfield.

Edinboro-Interior. walls sustained hairline cracks.

Erie-Interior walls sustained hairline cracks.

Fairview-Hanging pictures fell; trees and bushes shook moderately.

Farrell-Plaster walls sustained hairline cracks; a few items were shaken off store shelves.

Girard-Plaster walls sustained hairline cracks; a few items shook from store shelves.

Greenville-A few windows cracked; a few items were shaken off store shelves; interior walls sustained hairline cracks; foundations cracked; there was a report of broken underground pipes.

Hadley-A few merchandise items were shaken from store shelves.

Harborcreek-A few items were shaken off store shelves.

Hartstown-Plaster walls sustained hairline cracks; a few items shook from store shelves.

Hermitage.

Industry.

Ingomar-Dry wall sustained hairline cracks.

Lyndora-A few windows cracked; a few items were shaken off store shelves.

Meadville-Interior walls sustained hairline cracks; a few windows cracked; a few items shook from store shelves; light furniture and small appliances overturned.

Monongahela.

North Springfield.

Pittsfield-A few windows cracked.

Pulaski-Dry wall sustained hairline cracks.

Reno-Interior walls sustained hairline cracks; a foundation cracked; a few windows cracked; a few items were shaken off store shelves.

Rochester.

Rockton.

Sharon-An $8 \times 20-\mathrm{ft}$ wood and metal marquee, on the front of an empty store on West State Street, fell onto the sidewalk when the support cables snapped. Two windows were reportedly broken (press reports). A few windows cracked; interior walls sustained hairline cracks; a foundation cracked.

Sharpsville-A few windows cracked; a few items were shaken off store shelves; plaster walls sustained hairline cracks.

Smethport-A few windows cracked; dry wall sustained hairline cracks.

\section{OHIO-Continued}

Tionesta-Hanging pictures fell.

Titusville-A few windows cracked.

Venango-A few windows cracked; a few merchandise items were shaken off store shelves.

Wampum-A few windows cracked; a few items were shaken off store shelves; interior walls sustained hairline cracks.

West Newton-A few items were shaken off store shelves. Wilmerding-Many small objects overturned and fell.

Yatesboro-Some windows were reportedly broken.

West Virginia-

Benwood-A few items were shaken off store shelves.

Glen Dale-A few windows cracked.

Washington-Interior walls sustained hairline cracks.

Wellsburg.

Intensity IV:

Canada-

Ontario-Amherstburg, Ancaster, Aurora, Aylmer, Baden, Barrie, Belle River, Belleville, Blenheim, Bolton, Bracebridge, Brampton, Brantford, Brooklin, Burford (press report), Burlington, Chatham, Clarkson, Clinton, Coburg, Coldwater, Cottam, Courtland, Courtright, Cultus (press report), Delhi, Don Mills, Downsville, Dresden, Dublin, Erieau, Essex, Etobicoke, Exeter, Fort Erie, Georgetown, Glanworth, Grand Valley, Grimsby, Guilds, Hagersville, Hamilton, Hanover, Harrow, Highgate, Highland Creek, Ingersoll, Kincardine, Kingsville, La Salle, Listowel, London, Malton, McGregor, Mississauga, Mitchell, Mount Pleasant (press report), Niagara-On-The-Lake, Oakville, Oro Station, Oshawa, Owen Sound, Pefferlaw, Pickering, Port Darlington, Port Dover, Port Elgin, Port Rowan, Richmond Hill, Ridgetown, Rockwood, Rosemont, Saint Catherines (press report), Saint Mary's, Saint Thomas, Sarnia, Scarborough, Sebringville, Simcoe, South Woodslee, Strathroy, Streetsville, Tavistock, Thamesford, Thornbury, Thorndale, Tillsonburg, Toronto, Union, Wallaceburg, Wardsville, Wartburg, Waterford, Waterloo, Welland, West Hill, Willowdale, Windsor, Woodstock, Wroxeter, Zephyr.

United States-

Indiana-Mishawaka.

Michigan-Adrian, Algonac, Alpena, Ann Arbor, Bancroft, Bay City (press report), Birmingham, Blissfield, Brighton, Brooklyn, Brown City, Chesterfield (press report), Clarklake (press report), Clarkston, Columbiaville, Concord, Corunna, Dearborn, Deerfield, Detroit, Dexter, Dryden, East Lansing, Fowlerville, Frontier, Gibralter (press report), Goodrich, Grosse Ile, Harsens Island, Hartland, Hazel Park, Horton, Hudson, Ida, Imlay City (press report), Inkster, Kingston, Lake Orion, Lakeland, Lansing (press report), Leonard, Manitou Beach, Marine City, Marlette, Milan, Monroe, Morenci, New Haven, Newport 
(press report), North Lakeport (press report), Novi, Onsted, Pearl Beach, Petersburg, Port Huron, Portage, Reading, Richmond, Riga, Rockwood (press report), Romeo, Romulus, Royal Oak, Saline, South Rockwood, Southfield, Spring Arbor, Sterling Heights, Taylor, Tecumseh, Trenton, Union Lake, Warren, Waterford, Wayne (press report), Weston, Willis, Wixom, Wyandotte.

New Jersey-Cedar Grove.

New York-Angola, Arcade, Attica, Bemus Point, Blasdell (press report), Brant, Brockport, Cattaraugus, Cherry Creek, Colden, Collins, Cuba, Derby, East Aurora, Ellicottville, Ellington, Findley Lake, Fredonia, Holland, Irving, Java Center, Kennedy, Mayville, Newfane, Nunda, Otto, Portageville, Salamanca, Sandusky, Sherman, Silver Creek, South Dayton, Springville, Stockton, Stow, Wyoming, Yorkshire.

Ohio-Adamsville, Akron (Maple Valley), Albany, Amherst, Amsterdam, Archbold (press report), Ashland, Ashtabula (west end), Athens (press report), Avon, Avon Lake, Baltimore, Barberton, Barton, Bay Village (press report), Bellevue, Beloit, Bergholz, Berkey, Berlin, Berlin Heights, Bethesda, Birmingham, Bloomingdale, Bloomville, Bolivar, Bowerston, Brecksville, Bridgeport, Brilliant, Brinkhaven, Brook Park (press report), Brookfield, Brownsville, Brunswick, Buckeye Lake, Bucyrus (press report), Burbank, Butler, Byesville, Caledonia, Canfield, Cardington (press report), Carrollton, Centerburg, Charm, Chatfield, Chesterville, Clay Center, Cleveland (beachland), Cleveland Heights, Cleveland (Puritas Parks), Cleveland (Willow), Cleveland (Newburg), Clyde, Columbiana, Columbus, Conesville, Copley, Crestline, Crooksville, Croton, Cumberland, Curtice, Defiance (press report), Delaware, Dennison, Derwent, Dillonvale, Dover, Doylestown, Dresden, Dunbridge, Duncan Falls, East Cleveland, East Fultonham, East Palestine, East Rochester, East Sparta, Eaton (press report), Edison, Elkton, Elmore, Fairlawn, Fairview Park, Farmdale, Fleming, Frazeysburg, Fredericksburg, Fredericktown, Fremont, Fulton, Galena, Galion, Garfield Heights, Gates Mills, Gibsonburg, Glouster, Gnadenhutten, Granville, Graytown, Green Springs, Greenford, Greenwich, Hammondsville, Hanoverton, Hartville, Haskins, Hayesville, Hebron, Holloway, Holmesville, Homerville, Hopewell, Hubbard, Huron, Independence (press report), Jacksontown, Jacksonville, Jacobsburg, Jefferson, Jeromesville, Junction City, Kelleys Island, Kensington, Kenton (press report), Kidron, Kimbolton, Kipton, Kunkle, Lafferty, Lakemore, Lakeside-Marblehead, Lakeview, Lakeville, Langsville, Leetonia, Limaville, Lorain, Louisville (press report), Lucas, Lyndhurst, Macedonia, Macksburg, Magnetic Springs, Magnolia, Maple Heights, Marengo, Marietta, Martel, Martins Ferry, Mayfield Heights (press report), McConnelsville, Mechanicstown, Medina (press report), Melmore, Middlebranch, Midvale, Millersburg, Millfield, Mineral City, Mineral Ridge, Minerva, Monroeville, Mount Eaton, Mount Hope, Moxahala, Murray City, Nankin, Napoleon, Nashville, Navarre, Nelsonville, New Lexington, New London, New Springfield, New Washington, Newark (press report), Niles, North Baltimore (press report), North Benton, North Georgetown, North Industry, North Kingsville, North Olmsted, North Royalton, Norwalk (press report), Oberlin, Old Fort, Old Washington, Orangeville, Ostrander, Paris, Paulding, Perrysville, Petersburg, Philo, Piedmont, Pleasant City, Pleasant Grove (press report), Pleasantville, Plymouth, Polk, Pomeroy, Port Clinton, Prospect, Put-In-Bay, Republic, Richwood, Rock Creek, Rockbridge, Rome, Saint Louisville, Salem (press report), Savannah, Scio, Senecaville, Seville, Shauck, Sherrodsville, Shiloh, Sidney, Springfield (press report), Sterling, Steubenville, Stone Creek, Stow, Stratton, Strongsville, Struthers, Sugarcreek, Sullivan, Summitville, Sunbury, Sycamore, Thornville, Tiffin, Tiro, Toledo (a couple of items fell from store shelves), Toledo Express Airport, Trimble, Tuppers Plains, Tuscarawas, Uhrichsville, University Heights, Upper Sandusky, Utica, Valley City, Vermilion, Vickery, Vienna, Vincent, Wakeman, Walhonding, Walnut Creek, Wapakoneta, Warsaw, Waterford, Waverly, Wellington, West Akron, West Mansfield, West Point, Westlake (press report), Whipple, Williamsfield, Willoughby Heights, Wilmington (press report), Youngstown (east side), Zanesville (press report), Zoar, Zoarville.

Pennsylvania-Barnesboro, Beaver, Bellwood, Bessemer, Bridgeville, Brookville (press report), Bruin, Butler, Cambridge Springs, Carlton, Carnegie, Center Hill (press report), Centerville, Clarks Mills, Clearfield (press report), Coalport (press report), Conneautville, Cowanesque (press report), Cranesville, Curwensville (press report), Dickerson Run, Du Bois, East Brady, East Vandergrift, Edinburg, Elderton, Eldred, Ellwood City, Enon Valley, Falls Creek (press report), Fenelton, Freeport, Georgetown, Grand Valley, Greensboro, Grove City, Harmony, Hilliards, Hillsville, Homestead, Irvine, Irvona (press report), Jackson Center, Jamestown, Jerome, Johnsonburg, Kennerdell, Kylertown (press report), Lake City, Leechburg, Leetsdale, Lock Haven (press report), Mahoningtown (press report), Mather, McGrann, McKeesport, Mercer, Mill Village, Morris (press report), Mount Jewett, New Bedford, New Castle, New Galilee, Oakmont, Oil City, Osceola Mills, Pittsburgh, Pleasantville, Polk, Pricedale, Reynoldsville (press report), Rogersville, Rural Ridge, Saegertown, Sandy Lake, Seneca, Shippingport, Slickville, South Heights, Spring Creek, Springboro, Sugargrove, Sutersville, Swede Hill (press 


\section{OHIO-Continued}

report), Tidioute, Tiona, Union City, Villa Maria, Waterford, Wellsboro, West Springfield, Westford, Wilcox, Youngsville.

West Virginia-Charleston (Yeager Airport), Morgantown, Petroleum, Weirton (press report), Williamstown.

Intensity III:

Canada-

Ontario-Agincourt, Ajax, Alliston, Arthur, Bayfield, Baysville, Beaverton, Beeton, Bethany, Blackstock, Borden, Bradford, Bramalea, Brougham, Brunner, Caledon East, Cambridge, Clark Point, Clarksburg, Columbus, Downsview, East York, Elmvale, Everett, Fesserton, Fingal, Gads Hill, Gamebridge, Goderich, Gravenhurst, Guelph, Hastings, Huntsville, Inglewood, Islington, Jarvis, Kingston (press report), Kitchener, Kleinburg, La Salette, Lambton Generating Station, Langton, Leaksdale, Maidstone, Mansfield, Maple, Markham, Milton, Mount Hope, Newmarket, North York, Oil Springs, Orangeville, Orillia, Orono, Orton, Penetanguishene, Peterborough, Port Burwell, Port Carling, Port Hope, Rexdale, Rostock, Shelburne, Southampton, Stayner, Stouffville, Stratford, Stroud, Sutton, Thedford, Thornhill, Tottenham, Unionville, Uxbridge, Wasaga Beach, Washago, Weston, Whitby, Wingham, Woodbridge, Wyoming.

United States-

Delaware-Newark.

Illinois-Galesburg.

Indiana-Berne, Crown Point, Elkhart, Evansville, Fort Wayne, Gary, Goshen, Greensburg, Lafayette, South Bend, Sullivan, Warsaw.

Kentucky-Ashland, Madisonville.

Maryland-College Park, Sykesville.

Michigan-Ada, Allenton, Anchorville, Armada, Atlas, Avoca, Belleville, Bloomfield Hills, Britton, Burt, Camden, Capac, Caro, Cass City, Cement City, Center Line, Clinton, Coldwater (press report), Croswell, Davisburg, Davison, Dearborn Heights, Decker, Deford, Drayton Plains, Durand, Edwardsburg, Fairgrove, Flint, Fostoria, Fraser, Galesburg, Garden City, Genesee, Gilford, Grand Haven (press report), Grand Rapids, Grandville, Hadley (press report), Hastings, Highland, Hillsdale, Holland, Holly, Howell, Ithaca, Jerome, Kalamazoo, Keego Harbor, Laingsburg, Lakeville, Lambertville, Lapeer, Lexington, Litchfield, Lowell, Marshall, Marysville, Melvin, Metamora, Michigan Center, Midland, Milford, Munith, New Hudson, North Branch, North Street, Norvell, Okemos, Onondaga, Ortonville, Otter Lake, Oxford, Palmyra, Parma, Paw Paw, Pinckney, Pittsford, Plainwell, Pleasant Lake, Pontiac, Port Sanilac, Saginaw (press report), SaintClair, Sandusky, Shaftsburg, Shields (press report), South Haven Highlands (press report), South Lyon, Sturgis, Sunfield, Tekonsha, Temperance, TraverseCity, Troy,

\section{OHIO-Continued}

Utica, Vicksburg, Waldron, Walled Lake, Washington, Whitmore Lake, Whittaker, Yale.

New Jersey-Rockaway.

New York-Albion, Alden, Alfred, Alfred Station, Allegany, Ashville, Athol Springs, Batavia, Belfast, Belmont, Boston, Brocton, Buffalo, Canaseraga, Cassadaga, Celoron, Ceres, Chaffee, Chautauqua, Cheektowaga, Clarence Center, Corfu, Cowlesville, Crittenden, Dale, Dalton, Dayton, Depew, East Amherst, East Otto, East Randolph, Elba, Falconer, Farmersville Station, Fillmore, Frewsburg, Gerry, Gowanda, Greenhurst, Jamestown, Java Village, Johnson City, Kenmore, Kill Buck, Leon, Lewiston, Lily Dale, Little Genesee, Little Valley, Machias, Marilla, Monticello, Niagara Falls, North Boston, North Java, Penn Yan, Portland, Randolph, Rochester, Sanborn, Scio, Sinclairville, Sodus, Strykersville, Swormville, Tonawanda, Varysburg, Versailles, Warsaw, Wayland, West Valley, Westfield, Youngstown.

Ohio-Adena, Amesville, Anna (press report), Apple Creek, Augusta, Austintown, Bannock, Barlow, Barnesville, Bascom, Beallsville, Bellaire, Belle Valley, Belpre, Beverly, Bladensburg, Blue Rock, Buchtel, Burgoon, Cadiz, Campbell, Chandlersville, Chesapeake, Chester, Chesterhill, Christiansburg, Cincinnati, Clarington, Collins, Corning, Coshocton, Danville, Dayton, Deerfield, Dexter City, East Liberty, East Liverpool (press report), East Springfield, Ellsworth, Empire, Enon, Fairborn, Findlay, Flat Rock, Flushing, Fort Seneca, Franklin (press report), Fresno, Glandorf, Gratiot, Green Camp, Greentown, Grover Hill, Guysville, Hamilton, Hamler, Harbor View, Harrisville, Hilliard, Holgate, Holland, Homer, Howard, Iberia, Irondale, Jerusalem, Jewell, Jewett, Johnstown, Kettlersville, Lagrange, Lancaster, Lansing, Lewis Center, Liberty Center, Lima, Lithopolis, Little Hocking, Lloydsville (press report), Logan, Londonderry, Lordstown (press report), Lore City, Lowell, Lower Salem, Lyons, Martin, Marysville, Maumee, Maynard, McClure, McCutchenville, Miamisburg, Millbury, Miller City, Monclova, Morral, Morristown, Morrow (press report), Mount Blanchard, Mount Cory, Mount Perry, Mount Pleasant, Mount Vernon, Mount Victory, Nashport, Neapolis, Negley, Nevada, New Athens, New Bremen, New Concord, New Holland, New Riegel, New Straitsville, Newport, Ney, North Fairfield, North Lawrence, North Lima, North Robinson, Okolona, Ontario (press report), Ottawa, Pataskala, Pemberville, Pettisville, Pickerington, Piney Fork, Portsmouth, Powell, Powhatan Point, Quaker City, Racine, Rawson, Reedsville, Reno, Reynoldsburg, Richmond, Ridgeway, Rocky Ridge, Roseville, Russells Point, Rutland, Scott, Sedalia, Seven Hills, Sheffield Lake, Stewart, Strasburg, Stryker, Sulphur Springs, Summerfield, Tiltonsville, Trinway, Unionville Center, Van Wert (press report), Vandalia, 


\section{OHIO-Continued}

Vanlue, Venedocia, Versailles, Walbridge, Waterville, Wauseon, Wayne, West Lafayette, West Unity, Westerville, Weston, Wharton, White Cottage, Williston, Winona, Woodville, Zenia (press report).

Pennsylvania-Allenport, Allentown, Anita, Apollo, Austin, Avoca, Baden, Bakerstown, Bear Lake, Beaver Falls, Belle Vernon, Bigler, Black Lick, Bolivar, Brackenridge, Bradford, Brisbin, Brockport, Buena Vista, Byrnedale, Callensburg, Callery, Cassandra, Cheswick, Clairton, Clarendon, Clark, Clarks Summit, Columbus, Commodore, Confluence, Coudersport, Coulters, Cranberry, Creekside, Cuddy, Darlington, Distant, Dravosburg, Duke Center, Dunlevy, Duquesne, Dysart, East Pittsburgh, East Smethport, Elmora, Emeigh, Finleyville, Flinton, Fombell, Ford City, Forestville, Foxburg, Fredonia, Freedom, Gibsonia, Glasgow, Glenshaw, Glenwillard, Grampian, Grapeville, Harrisville, Hawk Run, Herminie, Hickory, Home, Hookstown, Hydetown, Indian Head, Jacobs Creek, Jefferson, Johnstown, Julian, Kane (press report), Kittanning, Koppel, Larimer, Lecontes Mills (press report), Lewis Run, Ludlow, Manorville, McConnellstown, McKean, Mercersburg, Midland, Mill Run, Millsboro, Nanty Glo, New Bethlehem, New Brighton, New Kensington, New Stanton, New Wilmington, North Apollo, North East, North Versailles, North Washington, Parker, Penn Run, Perryopolis, Petrolia, Point Marion, Port Allegany, Republic, Rew, Rices Landing, Rixford, Robinson, Rockwood, Roscoe, Rossiter, Rouseville, Russell, Saint Petersburg, Sarver, Scranton (press report), Seward, Shawville, Sheakleyville, Sheffield, Shinglehouse, Sipesville, Slippery Rock, Smithton, Somerset, South Fork, Spangler, Spartansburg, State College, Stoneboro, Stoystown, Sturgeon, Summerville, Tarrs, Taylorstown, Townville, Turtlepoint, University Park, Utica, Volant, Warren, Washington, Wattsburg, Webster, West Middlesex, West Sunbury, Wildwood, Windber, Woodland (press report), Wrights Corners (press report), Yukon.

Virginia-Falls Church, Richmond.

West Virginia-Beech Bottom, Belleville, Belmont, Bigbend, Burton, Cairo, Charleston, Chester, Colliers, Elizabeth, Follansbee, Grafton, Gypsy, Harrisville, Huntington, Mannington, Mason, Mineralwells, Moundsville, Newell, Osage, Paden City, Palestine, Parkersburg, Proctor, Rachel, Saint Marys, Sistersville, Smithfield, Waverly, Wheeling, Windsor Heights.

Intensity II:

Canada-

Ontario-Angus, Duntroon, Glen Williams, Midland, Rosseau, Schomberg, Seagrave, Sharon, Waubaushene.

United States-

Illinois-Chicago, Des Plaines, Rockford.

Indiana-Angola, Carmel, Garrett, Marion.

Kentucky-Glasgow.
OHIO-Continued

Maryland-Baltimore, Brentwood, Swanton.

Michigan-Albion, Byron, Clayton, Clifford, Flushing, Leslie, Livonia, Manchester, Memphis, Napoleon, North Adams, Owosso, Silverwood, Three Rivers, Ubly.

New Jersey-Pompton Lakes.

New York-Barker, Bliss, Castile, Hilton, Knowlesville, Olcott, Olean (press report), West Falls.

Ohio-Arlington, Belle Center, Belmont, Bloomdale, Dun-

kirk, Edon, Freeport, Kilbourne, Long Bottom, Mason,

McComb, New Carlisle, Rushsylvania, Saint Marys,

Sarahsville, Shawnee, Thurston, Tontogany, Troy, Wellston, Yellow Springs.

Pennsylvania-Ashville, Bellefonte, Bulger, Dixonville, Elco, Everson, Genesee, Greensburg, Guys Mills, Heilwood, Hyde Park, Indianola, Mars, Sagamore, Saint Benedict, Templeton, Venus, Verona, West Elizabeth, Westover, Wilkes-Barre (press report), Worthington.

Wisconsin-Milwaukee (press report).

West Virginia - Grantsville, Lumberport, New Manchester, Weston.

Felt:

Delaware-New Castle.

Indiana-Brazil, Syracuse.

Michigan-Benton Harber (press report), Dowagiac (press report), Harrison (press report), Muskegon (press report), New Baltimore (press report), Snover (press report),

Ohio-Carey, Chillicothe (press report), Clarksfield (press report), Coolville (press report), Devola (press report), Fostoria (press report), Harmer (press report), Harpersfield, Olmstead Falls (press report), Orwell, Rayland (press report), Smithville (press report), Springville (press report), Williamstown (press report).

Pennsylvania-Adamsville, Altoona (press report), Clymer, Emporium (press report), Graham (press report), Oak Grove (press report), Renfrew, Springdale, Westfield (press report).

7 February (GS) Northeastern Ohio

Origin time: 183622.3

Epicenter: 41.645N., 81.157W.

Depth: $6 \mathrm{~km}$

Magnitude: $2.5 \mathrm{M}_{\mathrm{n}}(\mathrm{GS})$

Intensity IV: Concord (press report), Leroy (press report), Thompson (press report).

Intensity III: Painesville (press report).

Felt: Chardon (press report), Hambden (press report), Kirtland (press report), Mentor (press report).

12 July (GS) Western Ohio

Origin time: 081937.9

Epicenter: $40.537 \mathrm{~N} ., 84.371 \mathrm{~W}$.

Depth: $10 \mathrm{~km}$

Magnitude: $4.5 \mathrm{~m}_{\mathrm{b}}(\mathrm{GS}), 4.6 \mathrm{M}_{\mathrm{n}}(\mathrm{SL}), 4.9 \mathrm{M}_{\mathrm{n}}(\mathrm{EP})$

Moment: $4.5 \times 10^{22}$ dyne-cm (SZ) 


\section{OHIO-Continued}

This earthquake was felt over a contiguous area of about $85,500 \mathrm{~km}^{2}$ (fig. 22) of Indiana, Kentucky, Michigan and Ohio. It was also felt in a few places in West Virginia.

Intensity VI:

Ohio-

Anna-A fireplace cracked; chimneys cracked; interior walls sustained hairline cracks; a few small objects overturned and fell; hanging pictures swung out of place; shaking was described as strong; felt by and awakened many people.

Minster-Bricks fell from chimneys; plaster walls sustained hairline cracks; a few small objects overturned; hanging pictures fell; shaking was described as strong; felt by and awakened many people.

New Bremen-Chimneys cracked; a few glassware items or dishes broke; a few windows cracked; a few small objects overturned and fell; buildings shook moderately.

Saint Marys-Walls cracked at Joint Township District Memorial Hospital, and a few bricks fell from the top of the chimney at the Goodyear Tire and Rubber Co. plant (press report). A few items were shaken off store shelves; a few small objects overturned and fell; hanging pictures swung out of place; buildings shook moderately; felt by and awakened many people.

Intensity $\mathrm{V}$ :

The most common effects at the places listed below were that a few small objects overturned and fell; buildings shook slightly; windows, doors, and dishes rattled slightly; felt by many people.

Kentucky-

Botkins-Some windows broke; hanging pictures fell; a few items were shaken off store shelves; a few glassware items or dishes broke; a few small objects overturned and fell; felt by and awakened many people.

Mount Olivet-Tombstones were displaced; trees and bushes shook slightly; awakened several people.

Silver Grove-Interior walls cracked.

\section{Ohio-}

Batavia-Plaster walls sustained hairline cracks; trees and bushes shook moderately; standing and moving vehicles were rocked moderately; hanging pictures swung out of place.

Bloomdale.

Botkins-Some windows broke; hanging pictures fell; a few items were shaken off store shelves; a few glassware items or dishes broke; a few small objects overturned and fell; felt by and awakened many people.

Broadway-Awakened many people.

Cherry Fork.

Chillicothe-A few glassware items or dishes broke; hanging pictures swung out of place.
OHIO-Continued

Croton-Many small objects overturned; hanging pictures swung out of place; awakened many people.

Danville-A few glassware items or dishes broke; standing vehicles rocked slightly.

Fort Jennings.

Gomer-Awakened many people.

Grelton-Hanging pictures fell.

Harrod-A few merchandise items were shaken off store shelves; a few glassware items or dishes broke; hanging pictures fell; awakened many people.

Houston-Hanging pictures swung out of place; buildings shook moderately, awakened many people.

Kettlersville-A few items were shaken off store shelves; hanging pictures swung out of place; awakened many people.

Lafayette-A few items were shaken off store shelves; trees and bushes shook slightly; awakened several people.

Lakeview-Hanging pictures swung out of place; awakened many people.

Lima-A window cracked, and bricks fell off an old building (press report).

Manchester-Interior walls sustained hairline cracks.

Maplewood-People had difficulty standing and walking; buildings shook moderately; standing and moving vehicles rocked slightly.

Maria Stein-Plaster walls sustained hairline cracks.

McComb-Awakened several people.

Mendon-Interior walls sustained hairline cracks; hanging pictures swung out of place; buildings shook moderately; awakened many people.

Montezuma-A few glassware items or dishes broke; hanging pictures swung out of place; trees and bushes shook slightly; awakened many people.

Mount Sterling-Awakened several people.

Napoleon-A few items were shaken off store shelves; a few glassware items or dishes broke; shaking was described as moderate.

New Knoxville-People had difficulty standing and walking; awakened many people.

New London.

Newtonsville-A few windows cracked; a few glassware items or dishes broke; plaster walls sustained hairline cracks; awakened several people.

Owensville-A few items were shaken off store shelves; hanging pictures swung out of place; awakened many people.

Pickerington-A few glassware items or dishes broke; trees and bushes shook slightly; standing and moving vehicles rocked slightly.

Quincy-Plaster walls sustained hairline cracks; awakened many people.

Richwood-Hanging pictures swung out of place; awakened several people. 


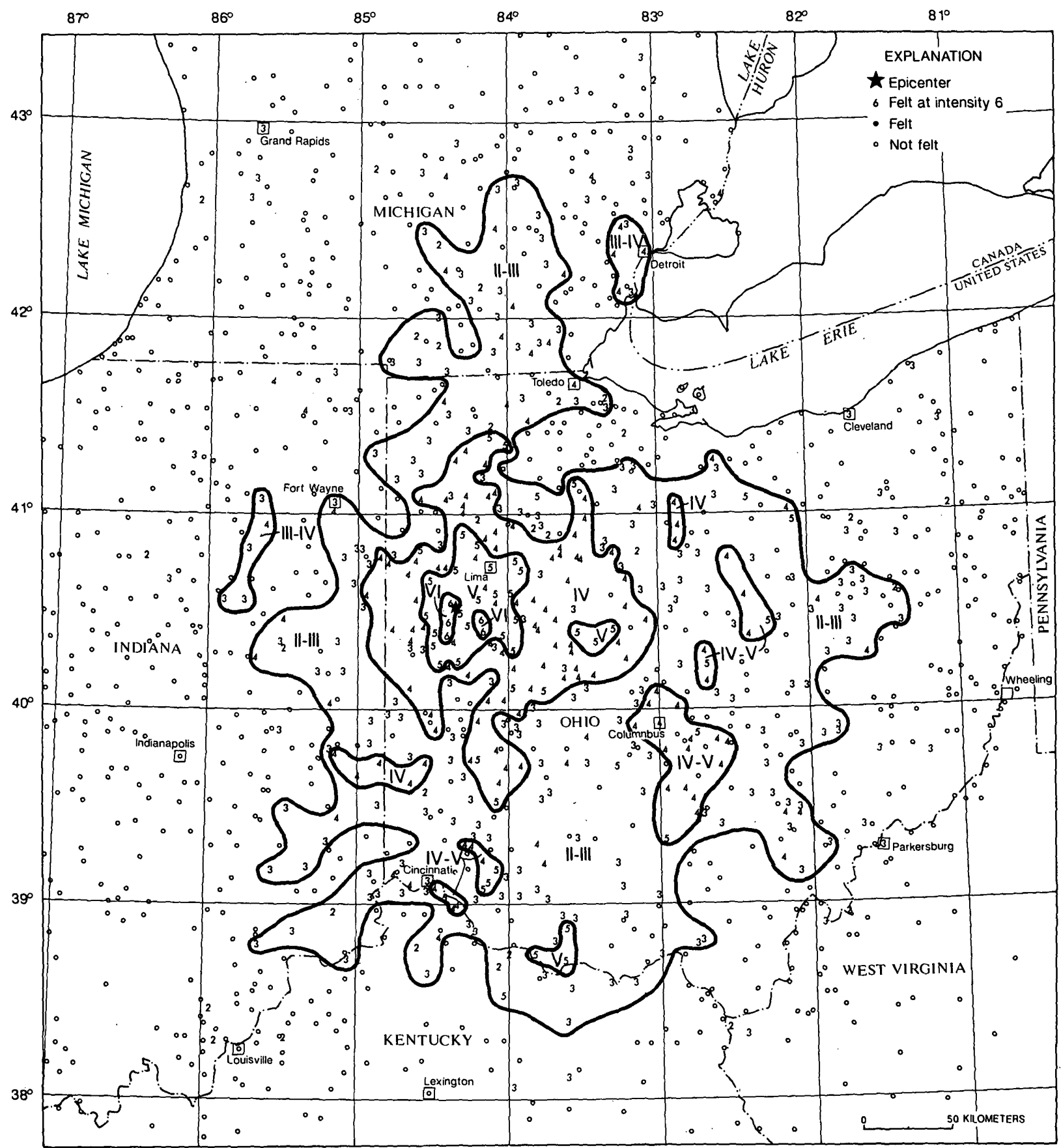

Figure 22. Isoseismal map for the western Ohio earthquake of 12 July 1986, 08 1937.9 UTC. Roman numerals represent Modified Mercalli intensities between isoseismals; Arabic numerals represent intensities at specific sites, and small boxes show locations of towns and cities whose names are plotted.

\section{OHIO-Continued}

Ripley-A few windows cracked; a few items were shaken off store shelves; a few glassware items or dishes broke; awakened several people.

\section{OHIO-Continued}

Roosevelt-A few items were shaken off store shelves; a few windows cracked; a few glassware items or dishes broke; hanging pictures swung out of place. 


\section{OHIO-Continued}

Saint Paris-Awakened several.

Spencerville-A few items were shaken off store shelves; a few glassware items or dishes broke; hanging pictures fell; awakened everyone.

Uniopolis-A few windows cracked; a few items were shaken off store shelves; a few glassware items or dishes broke; hanging pictures swung out of place; awakened many people.

Urbana-A few windows cracked; a few glassware items or dishes broke; awakened several people.

Van Wert-Hanging pictures fell; shaking was described as moderate.

Versailles-Interior walls sustained hairline cracks; a few windows cracked; a few glassware items or dishes broke; a few items were shaken off store shelves; hanging pictures swung out of place; awakened several people.

Wapakoneta-A few items were shaken off store shelves; a few glassware items or dishes broke; interior walls sustained hairline cracks; awakened many people.

Waynesfield-Hanging pictures swung out of place; buildings shook moderately; awakened many people.

Waynesville-A few windows cracked; a few glassware items or dishes broke; trees and bushes shook slightly; standing and moving vehicles rocked slightly.

West Mansfield-A few items were shaken off store shelves; dry wall cracked; awakened many people.

West Salem-Plaster walls were cracked; shaking was described as moderate; awakened a few people.

Yorkshire-A few windows cracked; a few items were shaken off store shelves; a few glassware items or dishes broke; plaster walls sustained hairline cracks; trees and bushes shook slightly; standing and moving vehicles rocked slightly; awakened many people.

Intensity IV:

Indiana-Alexandria, Bippus, Boston, Brownsville, Geneva, Losantville, Metamora, Milton, Pleasant Mills, Shipshewana, Upland, Waynedale, Wolcottville.

Kentucky-Alexandria, Newport.

Michigan-Adrian, Algonac (press report), Blissfield, Clarkston, Clinton, Dearborn Heights, Detroit (press report), East Lansing (press report), Gregory, Jackson, Petersburg, Redford (press report), Southfield (press report), Woodhaven (press report).

Ohio-Adelphi, Albany, Alpha, Attica, Baltimore, Bellbrook, Belle Center, Bellefontaine, Buchtel, Burkettsville, Butler, Cable, Cairo, Camden, Canal Winchester, Carthagena, Casstown, Celina, Centerburg, Christiansburg, Circleville, Cloverdale, Columbus (press report), Columbus Grove, Continental, Cridersville, Defiance, Delta, Dola, Dunkirk, Dupont, East Liberty, Elgin, Elida, Findlay, Forest, Fort Loramie, Fort Recovery, Gahanna (press report), Galloway, Gambier, Glandorf, Goshen, Green

\section{OHIO-Continued}

Camp, Greenville, Homerville, Huber Heights, Huntsville, Jacksonville, Jewell, Johnstown, Kenton, Kirby, Lancaster, Latty, Laura, Lithopolis, Lockbourne, London, Lynchburg, Magnetic Springs, Maineville, Mansfield (press report), Martinsville, Mason, Melmore, Melrose, Mid City, Middleburg, Milan, Milford Center, Miller City, Millersburg, Morning View, Morral, Mount Blanchard, Mount Saint Joseph, Neapolis, New Hampshire, New Washington, Newcomerstown, North Bend, North Hampton, North Lewisburg, Ohio City, Osgood, Ostrander, Ottoville, Pandora, Patterson, Pemberton, Piqua, Pitsburg, Rockford, Rosewood, Roundhead, Rushsylvania, Russia, Saint Henry, Saint Johns, Sidney, South Solon, Stoutsville, Sulphur Springs, Tarlton, Thurston, Tipp City, Toledo, Upper Arlington (press report), Vanlue, Vaughnsville, Venedocia, Verona, Waldo, West Alexandria, West Carrollton, West Liberty, West Unity, Westville, Williamstown, Willshire, Woodstock, Worthington, Wright Patterson Air Force Base, Xenia, Zanesfield.

Intensity III:

Indiana-Ashley, Aurora, Bath, Bourbon, Bryant, Cambridge City, Canaan, Converse, Craigville, Deputy, Earlham, Farmland, Fort Wayne (press report), Fountain City, Guilford, Kennard, Lagro, Linn Grove, Milroy, Monroe, Napoleon, Oldenburg, Orland, Preble, Redkey, Selma, South Whitley, Spencerville, Summitville, Sweetser, Walton, Westport.

Kentucky-California, Covington (press report), Garrison, Ghent, Hebron, Mount Sterling, Petersburg, Salt Lick, South Portsmouth, Tollesboro, Vanceburg, Wallingford, Williamstown.

Michigan-Ann Arbor (press report), Brighton, Britton, Brooklyn, Deerfield, Dorr, Dundee, Fowlerville, Frontier, Grand Rapids, Grosse Ile, Hopkins, Jerome, Laingsburg, Lansing, Leonard, Manchester, Marlette, Michigan Center, Oak Grove, Onondaga, Otisville, Palmyra, Reading, Royal Oak, Sand Creek, Tipton, Union Lake, Waldron, Washington, Whitmore Lake.

Ohio-Aberdeen, Akron (press report), Alexandria, Amelia, Antwerp, Athens, Bainbridge, Baltic, Barlow, Bascom, Beaverdam, Belle Valley, Benton Ridge, Bettsville, Big Prairie, Bloomville, Blue Rock, Bowersville, Brinkhaven, Caledonia, Cardington, Cecil, Cedarville, Chandlersville, Charm, Cincinnati (press report), Clayton, Cleveland (press report), Commercial Point, Coshocton, Covington, Crestline, Decatur, Donnelsville, Dresden, Dublin, Dundee, Eaton, Enon, Fayetteville, Fletcher, Forest Park, Frazeysburg, Fresno, Gilboa, Glenford, Glouster, Granville, Harpster, Harrisburg, Hilliard, Holgate, Hollansburg, Iberia, Irwin, Junction City, Killbuck, Kings Mills, Lakeside-Marblehead, Latham, Laurelville, Lebanon, Leesburg, Lewisville, Logan, Marathon, Marion, Mark Center, Marshallville, Martin, 


\section{OHIO-Continued}

McGuffey, Mechanicsburg, Miamisburg, Miamitown, Middle Point, Middlefield, Midvale, Mifflin (press report), Mingo, Monroe, Morrow, Moscow, Mount Eaton, Mount Gilead (press report), Mount Hope, Mount Orab, Mount Vernon (press report), Mowrystown, Murray City, New Bavaria, New Carlisle, New Lebanon, New Madison, New Straitsville, New Weston, Ney, North Canton, North Fairfield, North Robinson, North Star, Northridge, Norwalk (press report), Oakwood, Old Fort, Ottawa, Painesville, Palestine, Peebles, Philo, Piketon, Port Jefferson, Port William, Rawson, Ridgeville Corners, Ridgeway, Roseville, Russells Point, Sardinia, Scioto Furnace, Seaman, Sedalia, Seven Mile, Shauck, South Bloomingville; South Lebanon, South Webster, Springboro, Springfield, Stewart, Stryker, Sunbury, Swanton, Sycamore, Tremont City, Trimble, Upper Sandusky, Walbridge, West Jefferson, Wharton, Williamsburg, Williamsport, Wilmot, Winchester, Wren.

West Virginia-Lavalette, Letart, Morgantown, Parkersburg (press report).

Intensity II:

Indiana-Borden, Friendship, Gaston, Jeffersonville, Logansport.

Kentucky-Augusta, Brooksville, Crestwood, Crittenden, Pikeville.

Michigan-Brown City, Fennville, Hillsdale, Mulliken, Norvell, Pittsford, Rives Junction.

Ohio-Deerfield, Doylestown, Jenera, Kalida, Liberty Center, Lindsey, Maumee, Mineral City, Mount Cory,Nevada, West Elkton, Williston.

West Virginia-Kenova, Ravenswood.

\section{PENNSYLVANIA}

31 January (NI) Northeastern Ohio

Origin time: 164642.3

See Ohio listing.

2 May (GS) Southeastern Pennsylvania

Origin time: 135352.6

Epicenter: $39.925 N$., $76.293 W$.

Depth: $5 \mathrm{~km}$

Magnitude: $2.5 \mathrm{M}_{\mathrm{D}}(\mathrm{DE})$

Intensity IV: Conestoga.

Intensity III: Craley, Millersville (press report), Pequea, Refton, Washington Boro, West Willow.

Intensity II: Brogue, Holtwood, New Providence.

Felt: Marticville (press report), Rawlinsville (press report), Safe Harbor (press report).

\section{PUERTO RICO}

13 February (GS) Northeast of Puerto Rico

Origin time: 130055.2

Epicenter: 19.193N., 65.286W.

Depth: Normal.

Magnitude: $4.4 \mathrm{~m}_{\mathrm{b}}(\mathrm{GS})$

Felt: San Juan area.

18 February (GS) Southern Puerto Rico

Origin time: 025844.5

Epicenter: 17.918N., 66.474W.

Depth: $21 \mathrm{~km}$

Magnitude: $4.7 \mathrm{~m}_{\mathrm{b}}(\mathrm{GS})$

Intensity V:

Barranquitas-A few items were shaken off store shelves; felt by many people.

Cayey-A few items were shaken off store shelves; a few glassware items or dishes broke; a few small objects overturned, standing and moving vehicles rocked slightly; felt by many people.

Cidra-A few windows cracked; a few glassware items or dishes broke; a few items were shaken off store shelves; a few small objects overturned and fell; standing and moving vehicles rocked slightly; buildings shook strongly; felt by many people.

Coama-A few windows cracked; a few items were shaken off store shelves; a few glassware items or dishes broke; a few small objects overturned and fell; interior walls sustained hairline cracks; standing and moving vehicles rocked slightly; shaking was described as strong; felt by many people.

Comerio-Standing vehicles rocked moderately; a few items were shaken off store shelves; a few small objects overturned; shaking was described as strong; felt by everyone.

Humacao-A few small objects overturned and fell; felt by many people.

Maricao-Plaster walls sustained hairline cracks.

San Lorenzo-Interior walls sustained hairline cracks; a few small objects overturned and fell.

San Isabel-Light furniture or small appliances overturned; a few small objects overturned and fell; buildings shook strongly; people had difficulty standing or walking; standing vehicles rocked slightly; felt by many people.

Villalba-Hanging pictures fell; small objects overturned and fell; felt by many people.

Intensity IV: Adjuntas, Aibonito, Aguirre, Angeles, Arecibo, Caguas, Ceiba, Ciales, Corozal, Coto Laurel, Fajardo, Gurabo, LaPlata, Loiza, Moca, Ponce, Puerto Real, San Sebastian, Utuado, Yabucoa.

Intensity III: Aguas Buenas, Isabela, Lajas, Luquillo, Morovis, Naguabo, Yauco.

Intensity II: Lares, San Juan. 


\section{PUERTO RICO-Continued}

Felt: Bayamon (press report), Caguas (press report), Canovanas, Guaynabo (press report), Ponce (press report).

5 June (GS) Southwestern Puerto Rico

Origin time: 183058.2

Epicenter: 18.075N., 66.860W.

Depth: 19 km

Magnitude: $4.1 \mathrm{~m}_{\mathrm{b}}(\mathrm{GS})$

This earthquake was felt over most of Puerto Rico, strongly in Ponce (press report).

Felt: Caguas (press report), Mayaguez (press report), Ponce (press report).

31 August (GS) Virgin Islands

Origin time: 232756.4

Epicenter: 18.337N., 65.076W.

Depth: Normal

Magnitude: None computed.

Felt: Aguas Buenas, Bayamon.

\section{RHODE ISLAND}

25 October (GS) Central New Hampshire

Origin time: 171638.4

See New Hampshire listing.

\section{SOUTH CAROLINA}

\section{February (TC) Northwestern South Carolina}

Origin time: 113545.3

Epicenter: 34.793N., 82.907W.

Depth: $5 \mathrm{~km}$

Magnitude: $3.5 \mathrm{M}_{\mathrm{n}}(\mathrm{GS}), 3.5 \mathrm{M}_{\mathrm{D}}(\mathrm{TC})$

Intensity V:

South Carolina-

Central-A few glassware items or dishes broke; a few small objects overturned and fell; felt by many people.

Long Creek-A few windows cracked; a few glassware items or dishes broke; a few small objects overturned and fell; people had difficulty standing; felt by many people.

Walhalla-A few items were shaken off store shelves; a few glassware items or dishes broke; a few small objects overturned and fell; trees and bushes shook slightly; standing and moving vehicles rocked slightly; felt by many people.
SOUTH CAROLINA-Continued

Westminster-A few windows cracked; a few items were shaken off store shelves; a few glassware items or dishes broke; a few small objects overturned and fell; people had difficulty standing; felt by everyone.

Intensity IV:

Georgia-Bowersville, Lavonia, Martin, Toccoa (press report).

South Carolina-Fair Play, Liberty, Newry, Richland, Salem, Six Mile, Seneca, Tamassee, West Union.

Intensity III:

Georgia - Canon, Franklin Springs, Rabun Gap.

North Carolina-Cedar Mountain, Hendersonville,

Highlands, Sylva.

South Carolina-Mountain Rest, Williamston.

Intensity II:

Georgia-Athens.

Felt:

South Carolina-Pickens, Townville.

South Carolina-Greenville (press report), Oconee Nuclear Power Station (press report).

\section{March (GS) Charleston area}

Origin time: 234915.3

Epicenter: 32.968N., 80.169W.

Depth: $6 \mathrm{~km}$

Magnitude: $2.2 \mathrm{M}_{\mathrm{D}}(\mathrm{GS})$

Intensity III: Summerville.

17 September (GS) Charleston area

Origin time: 093349.4

Epicenter: $32.928 \mathrm{~N}$., $80.152 \mathrm{~W}$.

Depth: $8 \mathrm{~km}$

Magnitude: $2.6 \mathrm{MD}(\mathrm{GS})$

Intensity IV: Summerville.

Intensity III: Middleton Place (press report).

18 October (TC) North Carolina border area

Origin time: 083138.8

Epicenter: $34.946 \mathrm{~N} ., 81.172 \mathrm{~W}$.

Depth: 23 km

Magnitude: $2.9 \mathrm{M}_{\mathrm{D}}(\mathrm{TC})$

Felt: Rock Hill (TC).

11 December (TC) Northwestern South Carolina

Origin time: 140550.2

Epicenter: $34.889 \mathrm{~N} ., 82.887 \mathrm{~W}$.

Depth: $9 \mathrm{~km}$

Magnitude: $2.9 \mathrm{MD}(\mathrm{TC}), 2.0 \mathrm{M}_{\mathrm{D}}(\mathrm{GT})$

Felt: Salem (TC).

11 December (TC) Northwestern South Carolina

Origin time: 140711.5

Epicenter: $34.898 \mathrm{~N} ., 82.880 \mathrm{~W}$.

Depth: $9 \mathrm{~km}$ 
Magnitude: $3.0 \mathrm{M}_{\mathrm{D}}(\mathrm{TC}), 2.6 \mathrm{M}_{\mathrm{D}}(\mathrm{SC})$

Felt: Salem (TC).

30 December (SL) Southeastern Missouri

Origin time: 071519.1

See Missouri listing.

\section{SOUTH DAKOTA}

25 May (GS) Southeastern South Dakota

Origin time: 071322.1

Epicenter: 43.937N., 98.289W.

Depth: $5 \mathrm{~km}$

Magnitude: $3.4 \mathrm{M}_{\mathrm{n}}(\mathrm{GS}), 3.6 \mathrm{M}_{\mathrm{n}}(\mathrm{TU})$

Intensity IV: Letcher, Mount Vernon, Plankinton, Stickney.

Intensity III: Woonsocket.

\section{TENNESSEE}

7 January (TC) Eastern Tennessee

Origin time: 012643.3

Epicenter: $35.609 \mathrm{~N}$., 84.762W.

Depth: $22 \mathrm{~km}$

Magnitude: $3.2 \mathrm{M}_{\mathrm{D}}(\mathrm{TC}), 3.1 \mathrm{M}_{\mathrm{D}}(\mathrm{GT})$

This earthquake was felt in parts of Rhea County (press report).

Intensity V:

Watts Bar area-Glass broke and windows rattled (press report).

Intensity IV: Decatur (TC).

\section{May (SL) Southeastern Missouri}

Origin 124813.5

See Missouri listing.

11 July (TC) Georgia-Tennessee border

Origin time: 142614.8

See Georgia listing.

19 August (TC) Northern Tennessee

Origin time: 205126.0

Epicenter: $36.291 \mathrm{~N} ., .85 .020 \mathrm{~W}$.

Depth: $30 \mathrm{~km}$

Magnitude: $2.9 \mathrm{M}_{\mathrm{D}}(\mathrm{TC}), 2.9 \mathrm{M}_{\mathrm{D}}(\mathrm{GT})$

Felt: Jamestown (TC).

\section{UTAH}

30 January (GS) West Texas

Origin time: 222637.0

Epicenter: 32.066N., 100.693W.

Depth: $5 \mathrm{~km}$

Magnitude: $3.3 \mathrm{M}_{\mathrm{n}}(\mathrm{GS}), 3.1 \mathrm{M}_{\mathrm{n}}(\mathrm{TU})$

Intensity III: Robert Lee, Silver.

\section{January (UU) Northern Utah}

Origin time: 123204.6

Epicenter: $41.715 N ., 111.665 \mathrm{~W}$.

Depth: $7 \mathrm{~km}$

Magnitude: $3.3 \mathrm{M}_{\mathrm{L}}(\mathrm{UU})$

Intensity IV: Logan (River Heights).

Intensity II: Riverside.

\section{March (UU) Central Utah}

Origin time: 224023.4

Epicenter: $39.234 \mathrm{~N} ., 112.062 \mathrm{~W}$.

Depth: $1 \mathrm{~km}$

Magnitude: $4.7 \mathrm{~m}_{\mathrm{b}}(\mathrm{GS}), 4.4 \mathrm{M}_{\mathrm{L}}(\mathrm{UU})$

Intensity V:

Scipio-A few items were shaken off store shelves; a few small objects overturned and fell; trees and bushes shook slightly; people had difficulty standing or walking; windows, doors, and dishes rattled loudly; felt by many people.

Intensity IV: Axtell, Fayette, Gunnison, Redmond.

Intensity III: Centerfield, Ephraim, Fountain Green, Mountain Home, Oak City, Salina.

25 March (UU) Central Utah

Origin time: 025301.3

Epicenter: $39.225 \mathrm{~N} ., 112.013 \mathrm{~W}$.

Depth: $1 \mathrm{~km}$

Magnitude: $4.5 \mathrm{~m}_{\mathrm{b}}(\mathrm{GS}), 3.9 \mathrm{M}_{\mathrm{L}}(\mathrm{UU})$

Intensity $\mathrm{V}$ :

Scipio-A few items were shaken off store shelves; a few small objects overturned and fell; trees and bushes 
UTAH-Continued

shook slightly; shaking was described as strong; windows, doors, and dishes rattled loudly; felt by many people.

Redmond-A few small objects overturned and fell; a brick fell from one chimney; standing vehicles rocked slightly; trees and bushes shook slightly; felt by many people. Intensity IV: Axtell, Fayette, Gunnison, Salina. Intensity III: Centerfield, Ephraim, Oak City. Intensity II: Aurora.

\section{June (UU) Northeastern Utah}

Origin time: 080541.7

Epicenter: $41.266 \mathrm{~N} ., 111.684 \mathrm{~W}$.

Depth: $10 \mathrm{~km}$

Magnitude: $3.6 \mathrm{M}_{\mathrm{L}}(\mathrm{UU})$

Felt: Epicentral area (UU).

\section{August (GS) Southern Utah}

Origin time: 132633.3

Epicenter: 37.420 N., $110.574 \mathrm{~W}$.

Depth: $5 \mathrm{~km}$

Magnitude: $4.0 \mathrm{M}_{\mathrm{L}}(\mathrm{UU}), 3.8 \mathrm{M}_{\mathrm{L}}(\mathrm{GS})$

Intensity V:

Oljeto-Dry wall cracked; a few small objects fell; standing vehicles rocked slightly; buildings shook moderately; felt by many people.

Intensity III: Monument Valley area.

19 September (UU) Northeastern Utah

Origin time: 104128.2

Epicenter: 41.466 N., $111.702 W$.

Depth: $7 \mathrm{~km}$

Magnitude: $3.4 \mathrm{M}_{\mathrm{L}}(\mathrm{UU})$

Intensity III: Hyrum, Millville, Paradise, Wellsville, Willard.

\section{October (UU) Northern Utah}

Origin time: 115146.7

Epicenter: 40.818N., 111.821W.

Depth: $5 \mathrm{~km}$

Magnitude: $2.7 \mathrm{M}_{\mathrm{D}}(\mathrm{UU})$

Intensity III: Salt Lake Valley (press report).

\section{October (UU) Central Utah}

Origin time: 154733.4

Epicenter: 38.631N., 112.558W.

Depth: $0 \mathrm{~km}$

Magnitude: $3.3 \mathrm{M}_{\mathrm{L}}$ (UU)

Intensity III: Elsinore.

18 October (GS) Idaho-Utah border area

Origin time: 212128.7

See Idaho listing.

\section{UTAH-Continued}

29 October (UU) Northern Utah

Origin time: 221314.5

Epicenter: 41.821N., 112.318W.

Depth: $5 \mathrm{~km}$

Magnitude: $3.6 \mathrm{M}_{\mathrm{L}}(\mathrm{UU})$

Intensity IV: Garland, Howell.

Intensity III: Plymouth, Portage, Riverside, Snowville, Tremonton (press report).

\section{October (UU) Northern Utah}

Origin time: 115828.2

Epicenter: $41.823 \mathrm{~N} ., 112.316 \mathrm{~W}$.

Depth: $4 \mathrm{~km}$

Magnitude: $3.5 \mathrm{M}_{\mathrm{L}}$ (UU)

Intensity IV: Howell (plaster walls sustained hairline cracks).

Intensity III: Garland, Portage.

Intensity II: Plymouth, Riverside.

Felt: Tremonton (press report).

13 November Northern Utah

Origin time: 2328

Epicenter: Not located.

Depth: None computed.

Magnitude: $2.6 \mathrm{MD}(\mathrm{UU})$

Felt: Salt Lake Valley (press report).

31 December (UU) Utah

Origin time: 112156.5

Epicenter: 41.822N., 112.316W.

Depth: $5 \mathrm{~km}$

Magnitude: $3.3 \mathrm{M}_{\mathrm{L}}(\mathrm{UU})$

Intensity IV: Howell.

Intensity III: Garland, Ogden, Plymouth, Portage, Riverside, Willard.

\section{VERMONT}

25 October (GS) Central New Hampshire

Origin time: 171638.4

See New Hampshire listing.

\section{VIRGINIA}

31 January (NI) Northeastern Ohio

Origin time: 164642.3

See Ohio listing. 
VIRGINIA-Continued

26 March (VP) Western Virginia

Origin time: 163623.9

Epicenter: $37.245 \mathrm{~N} ., 80.494 \mathrm{~W}$.

Depth: $12 \mathrm{~km}$

Magnitude: $2.9 \mathrm{M}_{\mathrm{D}}(\mathrm{VP}), 3.0 \mathrm{M}(\mathrm{TC})$

Maximum intensity IV effects occurred in the epicentral area along the Giles-Montgomery County line and in the northern part of Blacksburg (Virginia Polytechnic Institute and State University, 1986). Felt in southern Giles County and northern Montgomery County.

Intensity IV: Blacksburg.

Intensity III: Eggleston, Newport.

\section{December (VP) Eastern Virginia}

Origin time: 094421.1

Epicenter: 37.580N., 77.458W.

Depth: $1 \mathrm{~km}$

Magnitude: $1.5 \mathrm{M}_{\mathrm{D}}(\mathrm{VP})$

Intensity IV: Richmond.

10 December (VP) Eastern Virginia

Origin time: 113006.1

Epicenter: 37.584N., 77.468W.

Depth: $1 \mathrm{~km}$

Magnitude: $2.2 \mathrm{M}_{\mathrm{D}}(\mathrm{VP})$

At least 11 earthquakes were felt in Richmond between December 1 and 28, 1986 (Davison and Bode', 1987). Most of them were felt at an intensity III-IV level in the central part of the city. The larger earthquakes that were located were felt throughout the city. A report on this series was published by Virginia Polytechnic Institute and State University, Virginia Tech. Seismological Observatory (VTSO), VTSO Special Study Series 87-1, 11 February 1987.

Intensity V:

Richmond (near the epicenter)-A few small objects fell; pendulum clocks stopped; felt by people driving cars on Interstate 95 ; felt by many people.

Intensity IV: Richmond (north side), Richmond (south side).

24 December (VP) Eastern Virginia

Origin time: 175838.2

Epicenter: 37.583N., 77.458W.

Depth: $1 \mathrm{~km}$

Magnitude: $1.5 \mathrm{M}_{\mathrm{D}}(\mathrm{VP})$

Intensity IV: Richmond.

\section{WASHINGTON}

10 February (WA) Northwestern Washington

Origin time: 171207.4

Epicenter: $48.395 \mathrm{~N} ., 121.955 \mathrm{~W}$.

Depth: $4 \mathrm{~km}$

Magnitude: $3.1 \mathrm{M}_{\mathrm{D}}(\mathrm{WA}), 2.5 \mathrm{M}_{\mathrm{L}}(\mathrm{EP})$

Intensity IV: Day Creek (press report).

10 February (WA) Northwestern Washington

Origin time: 180508.0

Epicenter: 48.397N., 121.941W.

Depth: 0 km

Magnitude: $3.7 \mathrm{M}_{\mathrm{L}}(\mathrm{GS}), 3.9 \mathrm{MD}(\mathrm{WA})$

Intensity V:

Day Creek-Pans fell off the wall in a home (press report).

Lyman-A few small objects overturned and fell; a building shook strongly; shaking was described as strong; felt by many people.

Sedro-Wooley-A few dishes or glassware items broke; a few small objects overturned and fell; standing vehicles rocked slightly; windows, doors, and dishes rattled loudly; walls creaked loudly; felt by many people.

Intensity IV: Cape Hom (press report), Cedargrove (press report), Clearlake, Concrete, Rockport (press report).

Intensity III: Darlington, Hamilton, Marblemount, Silvana, Stanwood.

Felt: Day Lake (WA), Mount Vernon (press report).

11 March (WA) Puget Sound, Washington

Origin time: 072321.0

Epicenter: 47.335N., 122.488W.

Depth: $7 \mathrm{~km}$

Magnitude: $2.9 \mathrm{M}_{\mathrm{D}}(\mathrm{WA}), 2.3 \mathrm{M}_{\mathrm{L}}(\mathrm{EP})$

Intensity III: Dockton, Lakebay.

Intensity II: Tacoma.

11 March (WA) Southwestern Washington

Origin time: 104810.4

Epicenter: 45.941N., 122.411W.

Depth: $15 \mathrm{~km}$

Magnitude: $3.1 \mathrm{M}_{\mathrm{D}}(\mathrm{WA})$

Intensity V:

Ariel-A few large cracks opened in plaster walls; buildings shook moderately; windows, doors, and dishes rattled loudly; shaking was described as strong.

Intensity IV: Cougar, Vancouver.

Intensity III: Amboy, LaCenter.

21 March (PG) Eastern British Columbia

Origin time: 235619.0

Epicenter: $53.24 \mathrm{~N} ., 122.00 \mathrm{~W}$.

Depth: $10 \mathrm{~km}$

Magnitude: $5.4 \mathrm{~m}_{\mathrm{b}}(\mathrm{GS}) ; 5.2 \mathrm{M}_{\mathrm{S}}(\mathrm{GS}), 5.4 \mathrm{M}_{\mathrm{n}}(\mathrm{PG})$

Maximum intensity was VI in the epicentral area (PG). 
WASHINGTON-Continued

Intensity III:

Washington-Mount Vernon.

27 March (WA) Northwestern Washington

Origin time: 121012.8

Epicenter: $48.265 \mathrm{~N} ., 121.732 \mathrm{~W}$.

Depth: $2 \mathrm{~km}$

Magnitude: $2.8 \mathrm{M}_{\mathrm{L}}(\mathrm{GS}), 2.9 \mathrm{M}_{\mathrm{L}}(\mathrm{WA})$

Intensity IV: Fortson.

Intensity III: Darrington (press report).

28 March (WA) Northwestern Washington

Origin time: 034834.7

Epicenter: 48.256 N., $121.736 \mathrm{~W}$.

Depth: $2 \mathrm{~km}$

Magnitude: $3.1 \mathrm{M}_{\mathrm{L}}(\mathrm{GS}), 3.1 \mathrm{M}_{\mathrm{L}}(\mathrm{WA})$

Intensity IV: Darrington (press report).

28 March (WA) Northwestern Washington

Origin time: 041246.7

Epicenter: 48.260N., 121.734W.

Depth: $2 \mathrm{~km}$

Magnitude: $3.6 \mathrm{M}_{\mathrm{L}}(\mathrm{GS}), 3.6 \mathrm{M}_{\mathrm{L}}(\mathrm{WA})$

Intensity $\mathrm{V}$ :

Arlington-Some things were knocked off walls and shelves (WA).

Intensity IV: Carnation Farms, Concrete, Darrington.

Intensity II: Index.

Felt: Duvall (WA).

28 March (WA) Northwestern Washington

Origin time: 054055.3

Epicenter: 48.254N., 121.740W.

Depth: $3 \mathrm{~km}$

Magnitude: $2.4 \mathrm{M}_{\mathrm{D}}(\mathrm{WA})$

Felt: Darrington (WA).

28 March (WA) Washington

Origin time: 121114.9

Epicenter: 48.258N., 121.732W.

Depth: $2 \mathrm{~km}$

Magnitude: $2.1 \mathrm{MD}(\mathrm{WA})$

Intensity III: Darrington (WA), Whitehorse (WA).

29 March (WA) Northwestern Washington

Origin time: 130924.0

Epicenter: 48.258 N., $121.732 W$.

Depth: 2 km

Magnitude: $3.1 \mathrm{M}_{\mathrm{L}}(\mathrm{GS}), 3.3 \mathrm{M}_{\mathrm{L}}(\mathrm{WA})$

Felt: Darrington (press report), Whitehorse (WA).

31 March (WA) Northwestern Washington

Origin time: 071127.2

\section{WASHINGTON_Continued}

Epicenter: 48.260N., 121.736W.

Depth: 2 km

Magnitude: $2.3 \mathrm{MD}(\mathrm{WA})$

Felt: Power plant near Darrington (WA).

8 April (WA) Central Washington

Origin time: 105735.6

Epicenter: 47.770N., 120.230W.

Depth: 14 km

Magnitude: $2.9 \mathrm{M}_{\mathrm{L}}(\mathrm{GS}), 3.3 \mathrm{M} \mathrm{D}(\mathrm{WA})$

Felt: Staymen Flats area south of Chelan (press report).

20 April (WA) Northwestern Washington

Origin time: 164033.1

Epicenter: 48.840N., 122.526W.

Depth: $18 \mathrm{~km}$

Magnitude: $2.8 \mathrm{M}_{\mathrm{L}}(\mathrm{GS}), 3.0 \mathrm{M}_{\mathrm{D}}(\mathrm{WA})$

Intensity III: Lynden.

Intensity II: Bellingham, Deming, Nugents Comer, Sumas.

\section{June (WA) Puget Sound, Washington}

Origin time: 061242.3

Epicenter: 47.776N., 120.168W.

Depth: $9 \mathrm{~km}$

Magnitude: $2.7 \mathrm{M}$ (WA), $2.4 \mathrm{M}_{\mathrm{L}}(\mathrm{GS})$

Intensity III: Chelan, Chelan Falls, Entiat.

Intensity II: Ardenvois.

16 June (PG) Vancouver Island region

Origin time: 155437.0

Epicenter: 49.431N., 127.017W.

Depth: $35 \mathrm{~km}$

Magnitude: $4.9 \mathrm{~m}_{\mathrm{b}}(\mathrm{GS}), 5.0 \mathrm{M}_{\mathrm{s}}(\mathrm{GS}), 5.2 \mathrm{M}_{\mathrm{L}}(\mathrm{PG})$

Felt: Northwest Washington (press report).

8 July (WA) Puget Sound, Washington

Origin time: 051632.4

Epicenter: 48.264N., 122.512W.

Depth: 63 km

Magnitude: $3.5 \mathrm{M}_{\mathrm{D}}(\mathrm{WA}), 3.4 \mathrm{M}_{\mathrm{L}}(\mathrm{EP})$

Intensity IV: Freeland, Greenbank (press report), Lyman, Oak Harbor, Silvana.

Intensity III: Burlington, Coupeville, Hamilton, LaConner, Marysville, Mount Vernon, Mukilteo, Shaw Island.

Intensity II: Lake Stevens.

Felt: Alger, Concrete (press report), Greenbank Beach (press report), Ledgewood Beach (press report).

28 August (WA) Southern Washington

Origin time: 043413.5

Epicenter: 45.835N., 121.923W.

Depth: $9 \mathrm{~km}$

Magnitude: $2.7 \mathrm{M}(\mathrm{WA})$

Felt: Near Carson Valley (WA). 
16 September (WA) Northwestern Washington

Origin time: 231949.5

Epicenter: 48.221N., 121.643W.

Depth: $2 \mathrm{~km}$

Magnitude: $1.6 \mathrm{M}_{\mathrm{D}}(\mathrm{WA})$

Felt: Near Darrington (WA).

16 September (WA) Northwestern Washington

Origin time: 233857.8

Epicenter: $48.065 \mathrm{~N}$., $121.523 \mathrm{~W}$.

Depth: $6 \mathrm{~km}$

Magnitude: $2.8 \mathrm{M}_{\mathrm{D}}(\mathrm{WA}), 3.0 \mathrm{M}_{\mathrm{L}}(\mathrm{EP})$

Felt: Near Darrington (WA).

16 September (WA) Northwestern Washington

Origin time: 234937.1

Epicenter: $48.067 \mathrm{~N} ., 121.542 \mathrm{~W}$.

Depth: $8 \mathrm{~km}$

Magnitude: $2.4 \mathrm{M}_{\mathrm{D}}(\mathrm{WA}), 2.4 \mathrm{M}_{\mathrm{L}}(\mathrm{EP})$

Felt: Near Darrington (WA).

26 September (WA) Northwestern Washington

Origin time: 233454.7

Epicenter: $48.552 \mathrm{~N}$., $121.989 \mathrm{~W}$.

Depth: $0 \mathrm{~km}$

Magnitude: $2.4 \mathrm{M}(\mathrm{WA})$

Felt: Hamilton (press report), Lyman (press report), SedroWoolley (press report).

29 September (WA) Northwestern Washington

Origin time: 193706.9

Epicenter: 48.551N., 121.983W.

Depth: $0 \mathrm{~km}$

Magnitude: $2.2 \mathrm{MD}(\mathrm{WA})$

Felt: Epicentral area (WA).

7 November (EP) Northwestern Washington

Origin time: 1035.54 .0

Epicenter: 48.120N., 123.317W.

Depth: $38 \mathrm{~km}$

Magnitude: $3.9 \mathrm{M}_{\mathrm{L}}(\mathrm{EP})$

Felt: James Bay area of Victoria, B.C., Canada (EP).

\section{WEST VIRGINIA}

\section{January (NI) Northeastern Ohio}

Origin time: 164642.3

See Ohio listing.
12 July (GS) Western Ohio

Origin time: 081937.9

See Ohio listing.

\section{WISCONSIN}

31 January (NI) Northeastern Ohio

Origin time: 164642.3

See Ohio listing.

\section{WYOMING}

2 January (UU) Yellowstone National Park

Origin time: 155340.9

Epicenter: 44.620N., 110.997W.

Depth: $6 \mathrm{~km}$

Magnitude: $3.0 \mathrm{M}_{\mathrm{L}}(\mathrm{GS}), 3.4 \mathrm{M}_{\mathrm{L}}(\mathrm{BU})$

Intensity II: Madison Junction.

\section{January (UU) Yellowstone National Park}

Origin time: 073225.7

Epicenter: 44.620N., 111.003W.

Depth: $8 \mathrm{~km}$

Magnitude: $2.8 \mathrm{M}_{\mathrm{L}}(\mathrm{GS}), 3.2 \mathrm{M}_{\mathrm{L}}(\mathrm{BU})$

Intensity II:

Montana-West Yellowstone.

8 January (UU) Yellowstone National Park

Origin time: 110815.7

Epicenter: 44.621N., 110.998W.

Depth: 7 km

Magnitude: $3.0 \mathrm{M}_{\mathrm{L}}(\mathrm{GS}), 3.3 \mathrm{M}_{\mathrm{L}}(\mathrm{BU})$

Intensity III:

Montana-West Yellowstone.

8 January (UU) Yellowstone National Park

Origin time: 133628.5

Epicenter: 44.635N., 110.999W.

Depth: $7 \mathrm{~km}$

Magnitude: $2.9 \mathrm{M}_{\mathrm{L}}(\mathrm{GS}), 3.4 \mathrm{M}_{\mathrm{L}}(\mathrm{BU})$

Intensity III:

Montana-West Yellowstone.

14 January (UU) Yellowstone National Park

Origin time: 015037.8 
Epicenter: 44.637N., 111.002W.

Depth: 6 km

Magnitude: $2.9 \mathrm{M}_{\mathrm{L}}(\mathrm{GS}), 3.2 \mathrm{M}_{\mathrm{L}}(\mathrm{BU})$

Intensity II:

Montana-West Yellowstone.

14 January (UU) Yellowstone National Park

Origin time: 164629.9

Epicenter: 44.659N., 111.018W.

Depth: $7 \mathrm{~km}$

Magnitude: $3.2 \mathrm{M}_{\mathrm{L}}(\mathrm{GS}), 3.5 \mathrm{M}_{\mathrm{L}}(\mathrm{BU})$

Intensity II:

Montana-West Yellowstone.

15 January (UU) Yellowstone National Park

Origin time: 211018.0

Epicenter: 44.633N., 111.002W.

Depth: $7 \mathrm{~km}$

Magnitude: $2.8 \mathrm{M}_{\mathrm{L}}(\mathrm{GS}), 3.1 \mathrm{M}_{\mathrm{L}}(\mathrm{BU})$

Intensity III:

Montana- West Yellowstone.

16 January (UU) Yellowstone National Park

Origin time: 102947.7

Epicenter: 44.621N., 111.001W.

Depth: $6 \mathrm{~km}$

Magnitude: $3.4 \mathrm{M}_{\mathrm{L}}(\mathrm{GS}), 3.7 \mathrm{M}_{\mathrm{L}}(\mathrm{BU})$

Intensity III: Madison Junction.

1 February (UU) Yellowstone National Park

Origin time: 060954.8

Epicenter: 44.626 N., $110.996 \mathrm{~W}$.

Depth: $7 \mathbf{~ k m}$

Magnitude: $3.1 \mathrm{M}_{\mathrm{L}}(\mathrm{BU}), 2.8 \mathrm{M}_{\mathrm{L}}(\mathrm{GS})$

Intensity III: Madison Junction.

5 February (UU) Yellowstone National Park Origin time: 142619.3

Epicenter: 44.644N., 111.016W.

Depth: $4 \mathrm{~km}$

Magnitude: $3.4 \mathrm{M}_{\mathrm{L}}(\mathrm{GS}), 3.9 \mathrm{M}_{\mathrm{L}}(\mathrm{BU})$

Felt: Epicentral area (BU).

5 February (UU) Yellowstone National Park Origin time: 143701.7

Epicenter: 44.640N., 111.014W.

Depth: $3 \mathrm{~km}$

Magnitude: $3.3 \mathrm{M}_{\mathrm{L}}(\mathrm{BU}), 2.9 \mathrm{M}_{\mathrm{L}}(\mathrm{GS})$

Felt: Epicentral area (BU).

11 February (UU) Yellowstone National Park

Origin time: 222655.6

Epicenter: 44.647N., 111.016W.
Depth: $7 \mathrm{~km}$

Magnitude: $4.2 \mathrm{M}_{\mathrm{L}}(\mathrm{GS}), 4.3 \mathrm{M}_{\mathrm{L}}(\mathrm{BU})$

Felt: Epicentral area (BU).

24 February (GS) Southeastern Idaho

Origin time: 031333.0

See Idaho listing.

2 March (UU) Yellowstone National Park

Origin time: 105525.4

Epicenter: 44.652N., 111.018W.

Depth: $7 \mathrm{~km}$

Magnitude: $3.2 \mathrm{M}_{\mathrm{L}}(\mathrm{GS}), 3.6 \mathrm{M}_{\mathrm{L}}(\mathrm{BU})$

Intensity II:

Montana-West Yellowstone.

2 March (UU) Yellowstone National Park

Origin time: 125936.5

Epicenter: 44.653N., 111.022W.

Depth: $7 \mathrm{~km}$

Magnitude: $3.0 \mathrm{M}_{\mathrm{L}}(\mathrm{GS}), 3.4 \mathrm{M}_{\mathrm{L}}(\mathrm{BU})$

Intensity II:

Montana-West Yellowstone.

12 April (UU) Yellowstone National Park

Origin time: 230547.8

Epicenter: 44.644N., 111.006W.

Depth: 8 km

Magnitude: $3.0 \mathrm{M}_{\mathrm{L}}(\mathrm{GS}), 3.3 \mathrm{M}_{\mathrm{L}}(\mathrm{BU})$

Intensity II: Epicentral area (BU).

18 April (UU) Yellowstone National Park

Origin time: 141755.6

Epicenter: 44.642N., 111.004W.

Depth: $6 \mathrm{~km}$

Magnitude: $3.2 \mathrm{M}_{\mathrm{L}}(\mathrm{GS}), 3.6 \mathrm{M}_{\mathrm{L}}(\mathrm{BU})$

Intensity II:

Montana-West Yellowstone.

Wyoming-Old Faithful.

21 June (GS) Idaho-Wyoming border area

Origin time: 203053.5

See Idaho listing.

5 July (UU) Yellowstone National Park

Origin time: 021805.6

Epicenter: 44.666 N., $111.024 W$.

Depth: $9 \mathrm{~km}$

Magnitude: $3.6 \mathrm{M}_{\mathrm{L}}(\mathrm{GS}), 4.0 \mathrm{M}_{\mathrm{L}}(\mathrm{BU})$

Intensity II: Epicentral area (BU). 


\section{WYOMING-Continued}

2 October Yellowstone National Park area

Origin time: 0645

Epicenter: Not located.

Depth: None computed.

Magnitude: None computed.

Intensity III:

Montana-West Yellowstone.

Wyoming-Old Faithful.

3 November (GS) Southwestern Wyoming

Origin time: 002345.0

Epicenter: 41.922N., 108.896W.

Depth: $5 \mathbf{~ k m}$

Magnitude: $3.3 \mathrm{M}_{\mathrm{L}}(\mathrm{GS})$

Possible mine explosion.

Intensity IV:

Wyoming-Superior.

Intensity III:

Wyoming-Farson.

15 November (UU) Yellowstone National Park

Origin time: 005657.0

Epicenter: $44.67 \mathrm{~N} ., 111.030 \mathrm{~W}$.

Depth: 7 km

Magnitude: $3.4 \mathrm{M}_{\mathrm{L}}(\mathrm{GS}), 3.4 \mathrm{M}_{\mathrm{L}}(\mathrm{BU})$

Intensity IV:

Montana-West Yellowstone.

Intensity III:

Wyoming-Madison Junction.

17 November (GS) Western Wyoming

Origin time: 083413.3

Epicenter: $43.156 \mathrm{~N} ., 110.812 \mathrm{~W}$.

Depth: $5 \mathrm{~km}$

Magnitude: $3.9 \mathrm{M}_{\mathrm{L}}(\mathrm{GS}), 4.2 \mathrm{M}_{\mathrm{L}}(\mathrm{BU})$

\section{WYOMING-Continued}

Intensity III:

Idaho-Driggs, Palisades.

Wyoming-Alpine.

24 November (UU) Yellowstone National Park Origin time: 021058.7

Epicenter: 44.674N., 111.021W.

Depth: 7 km

Magnitude: $2.6 \mathrm{M}_{\mathrm{L}}(\mathrm{BU}), 2.7 \mathrm{M}_{\mathrm{D}}(\mathrm{BU})$

Intensity II:

Montana-West Yellowstone.

24 November. (UU) Yellowstone National Park Origin time: 063150.4

Epicenter: 44.670N., 111.029W.

Depth: $7 \mathrm{~km}$

Magnitude: $3.2 \mathrm{M}_{\mathrm{L}}(\mathrm{GS}), 3.4 \mathrm{M}_{\mathrm{L}}(\mathrm{BU})$

Intensity IV:

Wyoming-Madison Junction.

Intensity III:

Montana-West Yellowstone.

Intensity II:

Wyoming-Old Faithful.

25 November (UU) Yellowstone National Park

Origin time: 204525.4

Epicenter: 44.671N., 111.027W.

Depth: $7 \mathrm{~km}$

Magnitude: $3.5 \mathrm{M}_{\mathrm{L}}(\mathrm{GS}), 3.4 \mathrm{M}_{\mathrm{L}}(\mathrm{BU})$

Intensity IV:

Montana-West Yellowstone.

Intensity III:

Wyoming-Madison Junction.

Felt:

Montana-Bozeman (press report). 
Table 1. Summary of United States earthquakes for 1986

[The following codes are used to indicate sources of hypocenters and (or) magnitudes and local times:

BK University of California, Berkeley

BU Montana Bureau of Mines and Geology, Butte

DE Delaware Geological Survey, Newark

EE Engdahl, E. R., Billington, S., and Kisslinger, C., 1989, Journal of Geophysical Research, v. 94, no. B11, p. $15,481-15,498$

EN Department of Energy, Washington, D.C.

EP Geophysics Division, Geological Survey of Canada, Ottawa, Ontario

GM U.S. Geological Survey, Menlo Park, Calif.

GP U.S. Geological Survey, Pasadena, Calif.

GS U.S. Geological Survey, Golden, Colo.

GT Georgia Institute of Technology, Atlanta

HJ Hauksson, Egill, and Jones, L.M., 1988, Seismological Society of America Bulletin, v. 78, no. 6, p. 1885-1906

HV Hawaiian Volcano Observatory, U.S. Geological Survey, Hawaii National Park

LD Lamont-Doherty Geological Observatory, Palisades, N.Y.

NI Nicholson and others, 1988, Seismological Society of America Bulletin, v. 78, no, 1, p. 188-217

PM Alaska Palmer Observatory, National Oceanic and Atmospheric Administration, Palmer, Alaska
PS California Institute of Technology, Pasadena

RN University of Nevada, Reno

SC University of South Carolina, Columbia

SL St. Louis University, St. Louis, Mo.

TC Tennessee Earthquake Information Center, Memphis

TU University of Oklahoma, Leonard

UU University of Utah, Salt Lake City

VP Virginia Polytechnic Institute and State University, Blacksburg

WA University of Washington, Seattle

WO Weston Observatory, Weston, Mass.

AST Alaska Standard Time

CST Central Standard Time

EST Eastern Standard Time

HST Hawaii Standard Time

MST Mountain Standard Time

PST Pacific Standard Time

UTC Coordinated Universal Time

FELT Not enough data available to assign an intensity

- Information not available; hr, hours; min, minutes; sec, seconds; $k m$, kilometers; $m_{b}, M_{s}, M_{L}$, and $m_{x}$ magnitudes, see p. 2-3; Max., maximum]

\begin{tabular}{|c|c|c|c|c|c|c|c|c|c|c|c|c|c|}
\hline \multirow[t]{2}{*}{ Date } & \multirow{2}{*}{$\begin{array}{l}\text { Origin time } \\
\text { (UTC) } \\
\text { hr min sec }\end{array}$} & \multirow{2}{*}{$\begin{array}{l}\text { Latitude } \\
\text { ( ) }\end{array}$} & \multirow{2}{*}{$\begin{array}{l}\text { Longitude } \\
\text { (9) }\end{array}$} & \multirow{2}{*}{$\begin{array}{l}\text { Depth } \\
(\mathrm{km})\end{array}$} & \multirow{2}{*}{$\begin{array}{l}\text { Hypo- } \\
\text { center } \\
\text { Source }\end{array}$} & \multicolumn{3}{|c|}{ Magnitude } & \multirow{2}{*}{$\begin{array}{l}\text { Max. } \\
\text { inten- } \\
\text { sity }\end{array}$} & \multicolumn{4}{|c|}{ Local time } \\
\hline & & & & & & $\mathbf{m}_{\mathbf{b}}$ & $\mathrm{M}_{s}$ & Local & & Date & & hr & zone \\
\hline MAY & 022700.4 & $33.335 \mathrm{~N}$. & $87.347 w$. & 1 & GT & 4.2 & - & 4.5Mn(GS) & FELT & MAY & 6 & $20: 2$ & $7 \mathrm{CST}$ \\
\hline
\end{tabular}

ALASKA

\begin{tabular}{|c|c|c|c|c|c|c|c|c|c|c|c|c|c|}
\hline JAN. & 1 & 163341.5 & $60.717 \mathrm{~N}$ & $147.680 \mathrm{~W}$. & 20 & GM & - & - & - & - & JAN. & 1 & 07:33 AST \\
\hline JAN. & 1 & 185937.9 & $60.285 \mathrm{~N}$ & $151.531 \mathrm{~W}$ & 59 & GM & - & - & - & - & JAN. & 1 & 09:59 AST \\
\hline JAN. & 2 & 052340.0 & $66.039 \mathrm{~N}$ & $150.132 \mathrm{~W}$ & 10 & GS & - & - & $3.4 \mathrm{M}_{\mathrm{L}}(\mathrm{PM})$ & & JAN. & 1 & $20: 23$ AST \\
\hline JAN. & 3 & 095759.2 & $53.391 \mathrm{~N}$ & $164.857 \mathrm{~W}$ & 33 & LD & - & - & $3.1 \mathrm{~m}_{\mathrm{X}}(\mathrm{LD})$ & & JAN. & 3 & 00:57 AST \\
\hline JAN. & 3 & 113455.4 & $60.072 \mathrm{~N}$ & 153.328W. & 124 & GM & - & - & - & & JAN. & 3 & 02:34 AST \\
\hline JAN. & 5 & 043434.3 & $57.748 \mathrm{~N}$. & 153.194W. & 62 & GM & - & - & - & & JAN. & 4 & 19:34 AST \\
\hline JAN. & 5 & 194133.0 & $64.330 \mathrm{~N}$ & $150.678 \mathrm{~W}$ & 33 & GS & - & - & $3.9 \mathrm{M}_{\mathrm{L}}(\mathrm{PM})$ & & JAN. & 5 & $10: 41$ AST \\
\hline JAN. & 5 & 230214.9 & $53.580 \mathrm{~N}$. & $166.011 \mathrm{~W}$ & 20 & LD & - & - & $3.5 \mathrm{~m}_{\mathrm{X}}(\mathrm{LD})$ & & JAN. & 5 & $14: 02$ AST \\
\hline JAN. & 6 & 055340.0 & $61.705 \mathrm{~N}$ & $150.706 \mathrm{~W}$. & 71 & GM & - & - & $3.6 \mathrm{M}_{\mathrm{L}}(\mathrm{PM})$ & & JAN. & 5 & $20: 53$ AST \\
\hline JAN. & 6 & 085416.0 & $60.166 \mathrm{~N}$ & $153.072 \mathrm{~W}$. & 122 & GM & - & - & - & & JAN. & 5 & 23:54 AST \\
\hline JAN. & 6 & 160913.2 & $54.697 \mathrm{~N}$ & $159.965 \mathrm{~W}$. & 28 & LD & 4.6 & - & $3.9 \mathrm{~m}_{\mathrm{X}}(\mathrm{LD})$ & & JAN. & 6 & 07:09 AST \\
\hline JAN. & 6 & 163807.8 & $62.380 \mathrm{~N}$ & $152.449 \mathrm{~W}$ & 153 & GM & - & - & - & & JAN. & 6 & 07:38 AST \\
\hline JAN. & 7 & 170513.0 & $52.574 \mathrm{~N}$ & $168.379 \mathrm{~W}$ & 33 & GS & 4.0 & - & - & & JAN. & 7 & 08:05 AST \\
\hline JAN. & 7 & 224141.8 & $54.644 \mathrm{~N}$. & $159.560 \mathrm{~W}$ & 29 & LD & - & - & $3.1 \mathrm{~m}_{\mathrm{X}}(\mathrm{LD})$ & 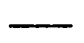 & JAN. & 7 & $13: 41$ AST \\
\hline JAN. & 8 & 223406.5 & $59.746 \mathrm{~N}$ & $152.828 \mathrm{~W}$ & 76 & GM & 4.3 & - & $3.9 \mathrm{M}_{\mathrm{L}}(\mathrm{PM})$ & & JAN. & 8 & 13:34 AST \\
\hline JAN. & 9 & 092618.3 & $54.353 \mathrm{~N}$ & $161.468 \mathrm{~W}$. & 5 & LD & - & - & $3.1 \mathrm{~m}_{\mathrm{X}}(\mathrm{LD})$ & 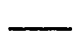 & JAN. & 9 & 00:26 AST \\
\hline JAN. & 9 & 092812.7 & $62.497 \mathrm{~N}$. & $151.275 \mathrm{~W}$ & 97 & GM & - & - & - & - & JAN. & 9 & $00: 28$ AST \\
\hline JAN. & 10 & 101938.3 & $61.382 \mathrm{~N}$ & $150.658 \mathrm{~W}$ & 60 & GM & - & - & - & - & JAN. & 10 & 01:19 AST \\
\hline
\end{tabular}


Table 1. Summary of United States earthquakes for 1986 Continued

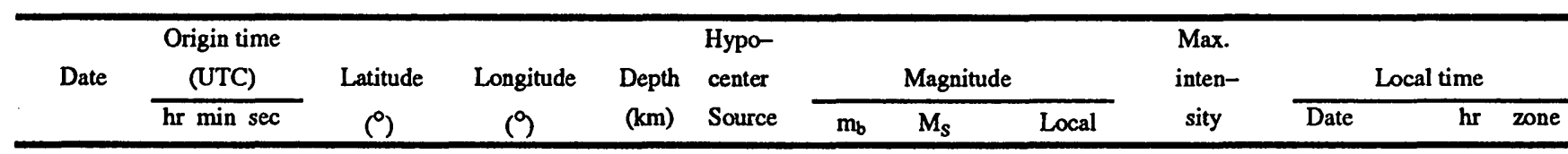

ALASKA-Continued

JAN. $11 \quad 022315.9$

JAN. $11 \quad 080225.5$

JAN. $11 \quad 095623.3$

JAN. $12 \quad 040026.7$

JAN. $13 \quad 025957.0$

JAN. $13 \quad 081259.1$

JAN. $13 \quad 210728.5$

$\begin{array}{lll}\text { JAN. } & 14 \quad 074840.9\end{array}$

JAN. $14 \quad 082013.9$

JAN. $14 \quad 181258.1$

JAN. $14 \quad 194106.6$

JAN. $15 \quad 010833.3$

JAN. $\quad 15 \quad 012102.3$

JAN. $15 \quad 042953.2$

JAN. $15 \quad 062805.0$

JAN. $15 \quad 072539.6$

JAN. $16 \quad 140314.0$

JAN. $16 \quad 143636.1$ JAN. $16 \quad 154346.4$

JAN. $17 \quad 010315.3$

JAN. $17 \quad 055905.3$

JAN. $17 \quad 154555.1$

JAN. $\quad 18 \quad 002628.0$

JAN. $18 \quad 011010.1$

JAN. $18 \quad 015900.9$

JAN. $18 \quad 043629.3$

JAN. $18 \quad 050535.5$

JAN. $18 \quad 102431.9$

JAN. $\quad 19 \quad 041512.8$

JAN. 19081857.9

JAN. $19 \quad 092433.9$

JAN. $19 \quad 101147.1$

JAN. $20 \quad 162923.6$

JAN. $21 \quad 151241.6$

JAN. $21 \quad 183123.2$

JAN. $22 \quad 131147.3$

JAN. $22 \quad 150949.1$
$54.606 \mathrm{~N} .159 .584 \mathrm{~W}$.

$60.108 \mathrm{~N} .153 .405 \mathrm{~W}$.

$59.405 \mathrm{~N} .151 .949 \mathrm{~W}$.

$68.323 \mathrm{~N} .163 .258 \mathrm{~W}$.

$60.784 \mathrm{~N} .152 .401 \mathrm{~W}$.

$62.352 \mathrm{~N} .151 .168 \mathrm{~W}$.

$67.308 \mathrm{~N}$. 161.240W.

$58.258 \mathrm{~N} . \quad 152.980 \mathrm{~W}$

$60.222 \mathrm{~N} .152 .294 \mathrm{~W}$.

$60.710 \mathrm{~N} .143 .155 \mathrm{~W}$.

$52.631 \mathrm{~N} .175 .023 \mathrm{~W}$.

$66.505 \mathrm{~N} .149 .889 \mathrm{~W}$

$66.200 \mathrm{~N} .150 .105 \mathrm{~W} .10$ GS

$59.538 \mathrm{~N} .152 .907 \mathrm{~W} . \quad 91 \quad \mathrm{GM}$

66.312N. 149.712W. 10 GS

66.574N. 149.876W. 10 GS

$55.500 \mathrm{~N}$. $157.496 \mathrm{~W} .34$ LD

61.489N. 146.537W. 28 GM 61.689 N. $150.762 \mathrm{~W} .63$

$61.494 \mathrm{~N}$. 146.518W. 30

$67.874 \mathrm{~N} .156 .247 \mathrm{~W} . \quad 33$

66.441N. 149.621W. 10 GS

$60.068 \mathrm{~N} .141 .368 \mathrm{~W}$.

$60.291 \mathrm{~N} .153 .043 \mathrm{~W}$.

$51.387 \mathrm{~N} .173 .055 \mathrm{~W}$.

$61.542 \mathrm{~N} .150 .996 \mathrm{~W}$.

$61.545 \mathrm{~N} .151 .023 \mathrm{~W}$.

$7 \mathrm{GM}$

128

16

75

75

60.154N. $153.289 \mathrm{~W}, \quad 137$

$61.053 \mathrm{~N} .152 .205 \mathrm{~W} .107$

$54.458 \mathrm{~N} .162 .412 \mathrm{~W}$.

$61.710 \mathrm{~N} .149 .565 \mathrm{~W}$.

59.729 N. $152.308 \mathrm{~W}$.

3 LD

$40 \mathrm{GM}$

$63 \mathrm{GM}$

57.983N. $153.853 \mathrm{~W} . \quad 34 \quad \mathrm{GM}$

$61.526 \mathrm{~N} .152 .403 \mathrm{~W} .130 \mathrm{GM}$

61.277 N. $151.471 \mathrm{~W} .78 \quad$ GM

$59.830 \mathrm{~N}$. 150.905W. $26 \quad \mathrm{GM}$

$62.869 \mathrm{~N} .148 .347 \mathrm{~W} . \quad 59 \mathrm{GM}$

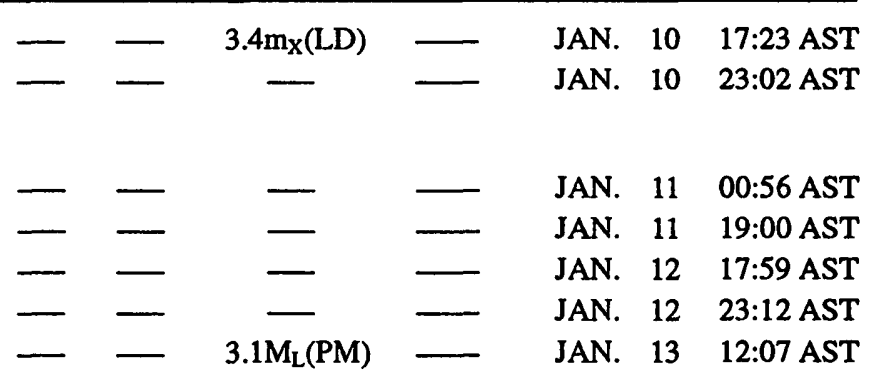

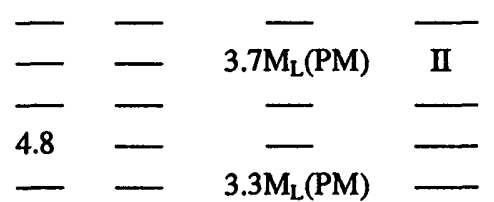

JAN. $13 \quad 22: 48$ AST

JAN. 13 23:20 AST

JAN. 14 09:12 AST

JAN. 14 09:41 HST

JAN. 14 16:08 AST

JAN. 14 16:21 AST

JAN. 14 19:29 AST

JAN. $14 \quad 21: 28$ AST

JAN. 14 22:25 AST

JAN. 16 05:03 AST

JAN. $16 \quad$ 05:36 AST JAN. 16 06:43 AST JAN. 16 16:03 AST JAN. 16 20:59 AST JAN. 17 06:45 AST

JAN. $17 \quad$ 15:26 AST JAN. 17 16:10 AST JAN. 17 15:59 HST JAN. 17 19:36 AST JAN. 17 20:05 AST

JAN. $18 \quad 01: 24$ AST JAN. 18 19:15 AST JAN. 18 23:18 AST JAN. 19 00:24 AST JAN. 19 01:11 AST

JAN. $20 \quad$ 07:29 AST JAN. 21 06:12 AST JAN. $21 \quad 09: 31$ AST JAN. 22 04:11 AST JAN. 22 06:09 AST 
Table 1. Summary of United States earthquakes for 1986-Continued

\begin{tabular}{|c|c|c|c|c|c|c|c|c|c|c|c|c|}
\hline \multirow[t]{2}{*}{ Date } & \multirow{2}{*}{$\begin{array}{l}\text { Origin time } \\
\text { (UTC) }\end{array}$} & \multirow{2}{*}{$\begin{array}{c}\text { Latitude } \\
\left({ }^{\circ}\right)\end{array}$} & \multirow{2}{*}{$\begin{array}{l}\text { Longitude } \\
\text { (9) }\end{array}$} & \multirow{2}{*}{$\begin{array}{c}\text { Depth } \\
(\mathrm{km})\end{array}$} & \multirow{2}{*}{$\begin{array}{l}\text { Hypo- } \\
\text { center } \\
\text { Source }\end{array}$} & \multicolumn{3}{|c|}{ Magnitude } & \multirow{2}{*}{$\begin{array}{l}\text { Max. } \\
\text { inten- } \\
\text { sity }\end{array}$} & \multicolumn{3}{|c|}{ Local time } \\
\hline & & & & & & $\mathrm{m}_{\mathrm{b}}$ & $\mathbf{M}_{\mathbf{S}}$ & Local & & Date & $\mathrm{hr}$ & zone \\
\hline
\end{tabular}

ALASKA-Continued

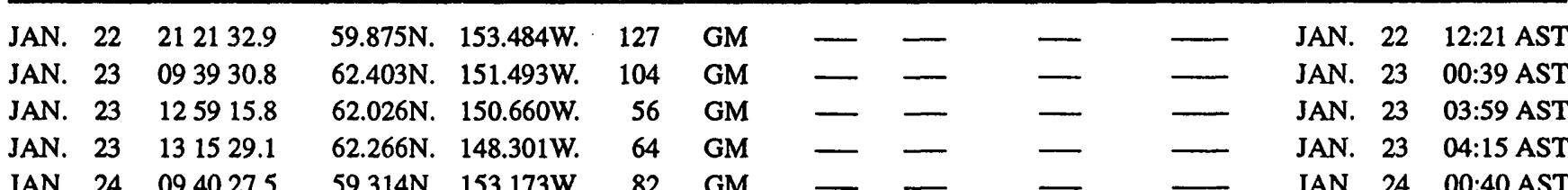

JAN. $24 \quad 094027.5$

59.314N. $153.173 \mathrm{~W} . \quad 82 \quad \mathrm{GM}$

JAN. $25 \quad 103258.0$

JAN. $25 \quad 110355.5$

JAN. $25 \quad 143541.7$

JAN. $25 \quad 150421.1$

JAN. $26 \quad 231154.1$

JAN. $27 \quad 034943.1$ JAN. $27 \quad 050434.8$ JAN. $27 \quad 112959.1$ JAN. $28 \quad 071229.0$ JAN. $28 \quad 183755.2$

JAN. $29 \quad 101901.9$ JAN. $29 \quad 143915.7$

JAN. $29 \quad 203913.5$

JAN. $30 \quad 103720.9$

FEB. $1 \quad 041121.4$

FEB. $\quad 2 \quad 203945.1$

FEB. 3170308.4

FEB. 4075226.2

FEB. $4 \quad 122738.3$

FEB. $4 \quad 195743.3$

FEB. $5 \quad 071507.6$ FEB. $5 \quad 101856.4$ FEB. $5 \quad 154534.1$ FEB. $6 \quad 084959.5$ FEB. $6 \quad 151458.3$

FEB. $\quad 7 \quad 065759.8$

FEB. $\quad 7 \quad 193735.2$

FEB. $8 \quad 045704.5$

FEB. $\quad 8 \quad 093900.2$

FEB. $8 \quad 132223.4$

FEB. $8 \quad 135423.0$ FEB. $8 \quad 235005.0$ FEB. 9121355.9
$59.870 \mathrm{~N} .146 .066 \mathrm{~W} . \quad 40 \quad \mathrm{GM}$ $59.917 \mathrm{~N} .146 .091 \mathrm{~W} .27$ GM $59.931 N$. $146.081 \mathrm{~W} .25 \mathrm{GM}$ $59.907 \mathrm{~N} .146 .144 \mathrm{~W} . \quad 33 \mathrm{GM}$ 61.869 N. $148.738 \mathrm{~W} .38 \mathrm{GM}$

50.701N. 176.062E. 33 GS 59.684N. $153.014 \mathrm{~W} . \quad 82 \quad \mathrm{GM}$ $57.966 \mathrm{~N}$. $157.980 \mathrm{~W} .146$ GS $61.428 \mathrm{~N}$. 151.553W. $88 \quad \mathrm{GM}$ $59.589 \mathrm{~N} . \quad 153.612 \mathrm{~W} .117 \quad \mathrm{GM}$

52.631N. 162.820W. 5 LD 61.213N. 147.426W. 17 GM $66.157 \mathrm{~N}$. $149.760 \mathrm{~W} .10$ GS $53.666 \mathrm{~N}$. 166.254W. 43 LD $66.244 \mathrm{~N}$. $149.962 \mathrm{~W} .10$ GS

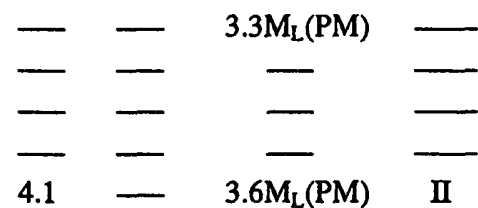

JAN. 25 JAN. 25 JAN. 25 JAN. 25 JAN. 26

01:32 AST 02:03 AST 05:35 AST 06:04 AST 14:11 AST

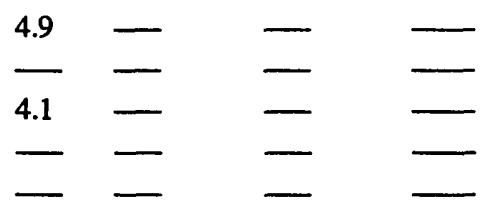

JAN. 26

JAN. 26 20:04 AST JAN. 27 02:29 AST JAN. $27 \quad 22: 12$ AST JAN. $28 \quad 09: 37$ AST

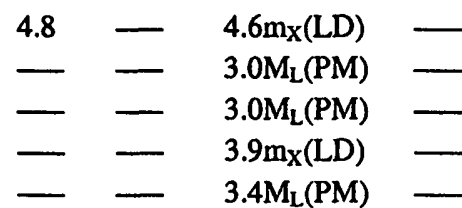

JAN. $29 \quad 01: 19$ AST JAN. 29 05:39 AST JAN. 29 11:39 AST JAN. $30 \quad 01: 37$ AST JAN. $31 \quad$ 19:11 AST

$51.424 \mathrm{~N}$. 179.289E. 33 GS 59.007N. 153.964W. 105 GM $62.385 \mathrm{~N} .150 .912 \mathrm{~W} .76 \mathrm{GM}$ $60.512 \mathrm{~N} .151 .816 \mathrm{~W} .73 \mathrm{GM}$ 57.470 N. $151.436 \mathrm{~W} .56 \quad \mathrm{GM}$

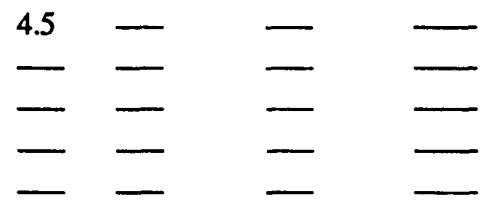

FEB. 2 10:39 HST FEB. 3 08:03 AST FEB. 3 22:52 AST FEB. 4 03:27 AST FEB. 4 10:57 AST

67.883N. $156.503 \mathrm{~W} .10$ GS $61.664 \mathrm{~N} .150 .693 \mathrm{~W} . \quad 59 \quad \mathrm{GM}$ $67.901 \mathrm{~N} .156 .160 \mathrm{~W} .33$ GS $68.152 \mathrm{~N} .156 .689 \mathrm{~W} .33$ GS $67.898 \mathrm{~N} .155 .994 \mathrm{~W} . \quad 33$ GS

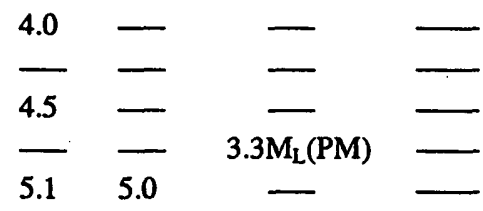

FEB. 4 22:15 AST FEB. 5 01:18 AST FEB. 5 06:45 AST FEB. 5 23:49 AST FEB. 6 06:14 AST

52.459N. $166.284 \mathrm{~W} . \quad 11 \quad$ LD $60.296 \mathrm{~N}$. 153.498W. 172 PS 54.941N. 156.779W. 22 LD $52.015 \mathrm{~N}$. $172.814 \mathrm{E} . \quad 33$ GS

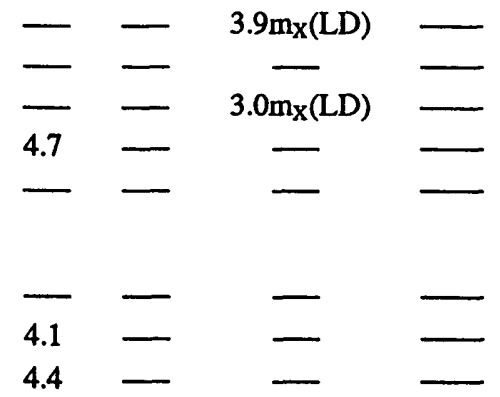

FEB. $6 \quad 21: 57$ AST FEB. 7 10:37 AST FEB. 7 19:57 AST FEB. 7 23:39 HST FEB. 8 04:22 AST

FEB. 8 04:54 AST FEB. 8 13:50 HST FEB. 9 03:13 AST 
Table 1. Summary of United States earthquakes for 1986-Continued

\begin{tabular}{|c|c|c|c|c|c|c|c|c|c|c|c|c|}
\hline \multirow[t]{2}{*}{ Date } & \multirow{2}{*}{$\begin{array}{l}\text { Origin time } \\
\text { (UTC) } \\
\text { hr min sec }\end{array}$} & \multirow{2}{*}{$\begin{array}{l}\text { Latitude } \\
\text { (०) }\end{array}$} & \multirow{2}{*}{$\begin{array}{c}\text { Longitude } \\
\text { (9) }\end{array}$} & \multirow{2}{*}{$\begin{array}{l}\text { Depth } \\
\text { (km) }\end{array}$} & \multirow{2}{*}{$\begin{array}{l}\text { Hypo- } \\
\text { center } \\
\text { Source }\end{array}$} & \multicolumn{3}{|c|}{ Magnitude } & \multirow{2}{*}{$\begin{array}{l}\text { Max. } \\
\text { inten- } \\
\text { sity }\end{array}$} & \multicolumn{3}{|c|}{ Local time } \\
\hline & & & & & & $m_{b}$ & $\mathrm{M}_{\mathrm{S}}$ & Local & & Date & hr & zone \\
\hline
\end{tabular}

ALASKA-Continued

\begin{tabular}{|c|}
\hline FEB $\quad 9 \quad 2101589$ \\
\hline
\end{tabular}

FEB. $10 \quad 044934.8 \quad 62.127 \mathrm{~N}$. $150.870 \mathrm{~W} . \quad 72 \quad \mathrm{GM} \quad-\quad-\quad-\quad-\quad-\quad$ FEB. 9 19:49 AST

FEB. $10050211.9 \quad 59.659 \mathrm{~N} .152 .528 \mathrm{~W} . \quad 67 \mathrm{GM}-\quad-\quad-\quad-\quad-\quad$ FEB. 9 20:02 AST FEB. $10 \quad 123829.4 \quad 60.266 \mathrm{~N}$. $152.802 \mathrm{~W} . \quad 115 \quad \mathrm{GM} \quad-\quad-\quad-\quad-\quad-\quad-\quad$ FEB. 10 03:38 AST FEB. $10132940.1 \quad 50.188 \mathrm{~N}$. $179.648 \mathrm{~W} . \quad 33$ GS $4.2 \quad-\quad-\quad-\quad-\quad$ FEB. 10 03:29 HST FEB. $12234431.253 .493 \mathrm{~N}$. $165.580 \mathrm{~W} .16$ LD $\quad-\quad-3.5 \mathrm{~m}_{\mathrm{X}}(\mathrm{LD}) \quad-\quad$ FEB. 12 14:44 AST FEB. $13055330.0 \quad 60.218 \mathrm{~N}$. $153.207 \mathrm{~W} .129 \quad \mathrm{GM} \quad-\quad-\quad-\quad-\quad-\quad$ FEB. 12 20:53 AST

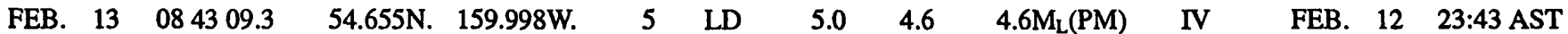
FEB. $13164626.5 \quad 53.743 \mathrm{~N}$. $163.598 \mathrm{~W}$. 33 GS $4.4 \quad-\quad-\quad-\quad-\quad$ FEB. 13 07:46 AST $\begin{array}{llllllllllllll}\text { FEB. } & 14 & 124633.9 & 63.069 \mathrm{~N} . & 150.835 \mathrm{~W} . & 97 & \text { GS } & - & - & - & \text { FELT } & \text { FEB. } 14 & \text { 03:46 AST }\end{array}$ FEB. $14190129.5 \quad 64.967 \mathrm{~N}$. $147.248 \mathrm{~W} .10 \quad 10$ GS $\quad-\quad-\quad 3.2 \mathrm{M}_{\mathrm{L}}$ (PM) $\quad$ FELT $\quad$ FEB. 14 10:01 AST FEB. $15 \quad 135152.6 \quad 62.693 \mathrm{~N}$. $151.002 \mathrm{~W} . \quad 78 \quad \mathrm{GM} \quad-\quad-\quad-\quad-\quad-\quad$ FEB. 15 04:51 AST

FEB. $\quad 15 \quad 151608.8 \quad 60.110 \mathrm{~N} . \quad 152.877 \mathrm{~W} . \quad 107 \quad$ GM FEB. $16 \quad 072648.6$ FEB. $\quad 16 \quad 085152.8$ FEB. $16 \quad 213106.1$ FEB. $18 \quad 145311.3$

FEB. $20 \quad 035623.7$ FEB. $20 \quad 040904.7$ FEB. $21 \quad 001417.8$ FEB. $21 \quad 183048.9$ FEB. $22 \quad 181739.3$

FEB. $\quad 23 \quad 042743.9$ FEB. $23 \quad 153012.5$ FEB. $23 \quad 170612.5$ FEB. $24 \quad 074413.8$ FEB. $24 \quad 185514.8$

FEB. $24 \quad 193938.6$ FEB. $24 \quad 195925.4$ FEB. $25 \quad 170202.6$ FEB. $25 \quad 211834.3$ FEB. $27 \quad 060018.9$

FEB. $28 \quad 063349.2$ FEB. $28 \quad 151224.1$ FEB. $28 \quad 151528.9$ FEB. $28 \quad 170145.3$ FEB. $28 \quad 170152.0$

\section{$54.664 \mathrm{~N}$. $159.936 \mathrm{~W}$. $30 \quad$ LD}

$59.768 \mathrm{~N} .152 .295 \mathrm{~W} . \quad 54 \quad \mathrm{GM}$

$61.495 \mathrm{~N} .150 .711 \mathrm{~W} . \quad 63 \quad \mathrm{GM}$

$58.780 \mathrm{~N}$. $143.348 \mathrm{~W} . \quad 30 \quad \mathrm{GM}$

$60.463 \mathrm{~N} . \quad 152.918 \mathrm{~W} . \quad 145$ $52.925 \mathrm{~N} . \quad 167.982 \mathrm{~W} . \quad 33$ $56.128 \mathrm{~N}$. $155.109 \mathrm{~W} .33$ $59.998 \mathrm{~N} .153 .179 \mathrm{~W} .121$ 51.437N. 175.169 W. 33

$52.819 \mathrm{~N} .168 .261 \mathrm{~W}$. $60.307 \mathrm{~N} .141 .069 \mathrm{~W}$. $63.404 \mathrm{~N} .150 .077 \mathrm{~W}$. $66.225 \mathrm{~N} .150 .007 \mathrm{~W}$. $63.075 \mathrm{~N} .150 .383 \mathrm{~W}$.
62.071N. $149.771 \mathrm{~W} .55 \mathrm{GM}$ 59.497N. $152.718 \mathrm{~W} . \quad 70 \quad \mathrm{GM}$ 66.332N. 149.935W. 10 GS 59.949N. 153.509W. 141 GM 62.401N. 150.635W. 73 GM

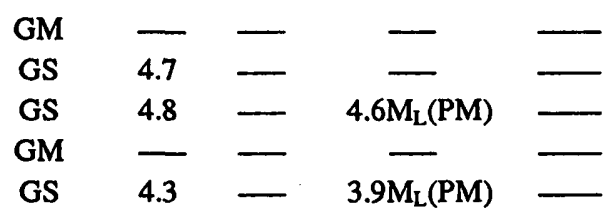

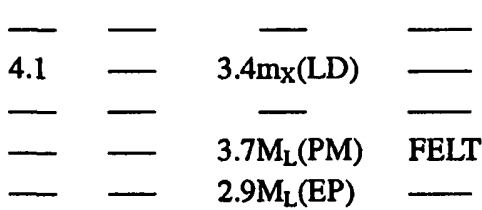

FEB, 15

FEB. $15 \quad 22: 26$ AST

FEB. 15 23:51 AST

FEB. $16 \quad$ 12:31 AST

FEB. $18 \quad 05: 53$ AST

FEB. $19 \quad$ 18:56 AST FEB. 19 19:09 AST FEB. $20 \quad 15: 14$ AST FEB. $21 \quad 09: 30$ AST FEB. 22 08:17 HST

FEB. $22 \quad$ 19:27 AST FEB. 23 06:30 AST FEB. 23 08:06 AST FEB. $23 \quad 22: 44$ AST FEB. 24 09:55 AST
FEB. $24 \quad 10: 39$ AST FEB. $24 \quad 10: 59$ AST FEB. 25 08:02 AST FEB. $25 \quad 12: 18$ AST FEB. 26 21:00 AST

FEB. $27 \quad 21: 33$ AST FEB. 28 06:12 AST FEB. $28 \quad 06: 15$ AST FEB. 28 08:01 AST FEB. 28 07:01 HST 
Table 1. Summary of United States earthquakes for 1986-Continued

\begin{tabular}{|c|c|c|c|c|c|c|c|c|c|c|c|c|}
\hline \multirow{3}{*}{ Date } & \multirow{3}{*}{$\begin{array}{l}\text { Origin time } \\
\text { (UTC) } \\
\text { hr min sec }\end{array}$} & \multirow{3}{*}{$\begin{array}{l}\text { Latitude } \\
\text { () }\end{array}$} & \multirow{3}{*}{$\begin{array}{c}\text { Longitude } \\
\text { (9) }\end{array}$} & \multirow{3}{*}{$\begin{array}{l}\text { Depth } \\
(\mathrm{km})\end{array}$} & \multirow{3}{*}{$\begin{array}{l}\text { Hypo- } \\
\text { center } \\
\text { Source }\end{array}$} & \multirow{2}{*}{\multicolumn{3}{|c|}{ Magnitude }} & \multirow{3}{*}{$\begin{array}{l}\text { Max. } \\
\text { inten- } \\
\text { sity }\end{array}$} & \multirow{2}{*}{\multicolumn{3}{|c|}{ Local time }} \\
\hline & & & & & & & & & & & & \\
\hline & & & & & & $\mathbf{m}_{\mathbf{b}}$ & $\overline{M_{S}}$ & Local & & Date & hr & zone \\
\hline
\end{tabular}

ALASKA-Continued

$\begin{array}{llllllllll}\text { FEB. } 28 & 223837.5 & 61.083 N & 152.173 W & 109 & \text { GM } & \text { FEB. } 28 \quad 13.38 \text { AST }\end{array}$

MAR. 1085820.5

MAR. 2045554.2

MAR. $2 \quad 054012.1$

MAR. $2 \quad 142846.5$

MAR. 2192318.3

MAR. $2 \quad 204229.1$

MAR. $3 \quad 060602.7$

MAR. $3 \quad 084929.3$

MAR. $3 \quad 193240.8$

MAR. $4 \quad 082943.6$

MAR. $4 \quad 084714.6$

MAR. $4 \quad 085033.8$

MAR. $4 \quad 091226.5$

MAR. $4 \quad 103025.3$

MAR. $4 \quad 125244.0$

MAR. $4 \quad 153533.0$

MAR. $5 \quad 010504.1$

MAR. $5 \quad 184204.0$

MAR. $5 \quad 184616.3$

$\begin{array}{llr}\text { MAR. } \quad 5 \quad 21 & 1034.8\end{array}$

MAR. $6 \quad 033901.2$

MAR. $6 \quad 034136.3$

MAR. $6 \quad 040818.8$

MAR. $6 \quad 101600.2$

MAR. $6 \quad 191414.4$

MAR. $6 \quad 205002.9$

MAR. $7 \quad 000811.1$

MAR. $7 \quad 165559.4$

MAR. $7 \quad 182146.5$

MAR. $8 \quad 025812.0$

MAR. $8 \quad 053751.3$

MAR. $8 \quad 173314.5$

MAR. 9015524.9

MAR. 9024104.0

MAR. 9025608.4

MAR. $9 \quad 115005.9$

MAR. $9 \quad 132741.8$

$58.819 \mathrm{~N} .153 .176 \mathrm{~W} .96$

$54.779 \mathrm{~N}$. 163.423W. 104

50.789 N. $179.176 \mathrm{E}$. 33

59.817 N. $147.855 \mathrm{~W} .34$

$\begin{array}{lll}\text { GM } & - & - \\ \text { GD } & - & - \\ \text { GM } & 5.4 & 5.3\end{array}$

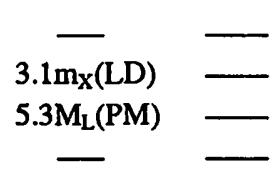

$61.556 \mathrm{~N} .149 .982 \mathrm{~W}$.

$51.373 \mathrm{~N}$. 176.714W.

$60.752 \mathrm{~N} .151 .743 \mathrm{~W}$.

$57.348 \mathrm{~N} .151 .240 \mathrm{~W} . \quad 37$

60.434 N. $153.119 \mathrm{~W} .136$

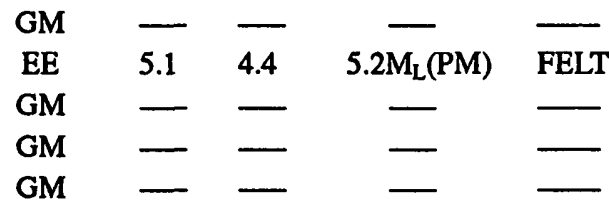

$51.252 \mathrm{~N} . \quad 178.524 \mathrm{E}$.

$51.553 \mathrm{~N} .166 .943 \mathrm{~W} . \quad 33$

$51.817 \mathrm{~N} .166 .952 \mathrm{~W} . \quad 33$

$60.832 \mathrm{~N}$. 152.378W. 100

51.649 N. $166.883 \mathrm{~W} . \quad 33$

GS
GS
GS
GM
GS

$\begin{array}{ll}4.5 & - \\ 5.6 & 4.6 \\ 4.8 & - \\ 4.9 & -\end{array}$

$5.1 \mathrm{M}_{\mathrm{L}}(\mathrm{PM})$

$5.6 \mathrm{M}_{\mathrm{L}}(\mathrm{PM})$

$-$

$59.954 \mathrm{~N} .153 .356 \mathrm{~W} .131$

$59.597 \mathrm{~N} . \quad 152.787 \mathrm{~W} . \quad 99$

58.702 N. $149.988 W .22$

$61.929 \mathrm{~N} .151 .203 \mathrm{~W} .79$

61.139 N. 152.084W. 97

GM

GM

GM

GM

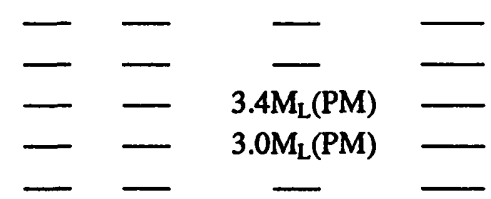

$59.922 \mathrm{~N} . \quad 152.837 \mathrm{~W}$.

$56.354 \mathrm{~N} .153 .694 \mathrm{~W}$.

$56.315 \mathrm{~N} .153 .460 \mathrm{~W}$.

$56.286 \mathrm{~N} .153 .524 \mathrm{~W}$.

$67.455 \mathrm{~N}$. 164.299W.

87 GM

33

33

33

33

33

$56.357 \mathrm{~N} . \quad 153.846 \mathrm{~W} . \quad 33$

$58.884 \mathrm{~N} .154 .734 \mathrm{~W} . \quad 130$

$61.746 \mathrm{~N} . \quad 151.037 \mathrm{~W} . \quad 69$

58.292 N. $154.078 \mathrm{~W} .72$

55.708 N. $160.992 \mathrm{~W} .147$
GS

GM

GM

GM

LD \begin{tabular}{ll}
-4.3 & - \\
5.1 & 4.8 \\
5.3 & 5.0 \\
\hline
\end{tabular}

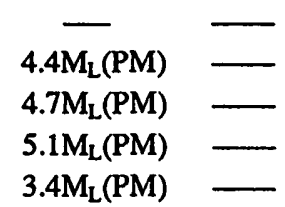

4

- -

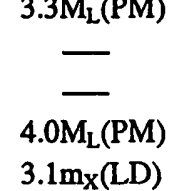

$59.698 \mathrm{~N} .153 .108 \mathrm{~W}$.

$60.673 \mathrm{~N} .151 .843 \mathrm{~W}$.

$61.038 \mathrm{~N} .146 .566 \mathrm{~W}$.

$52.220 \mathrm{~N}$. 169.428W.

$59.901 \mathrm{~N} .153 .107 \mathrm{~W}$.
GM

GS

GM

GS

GM

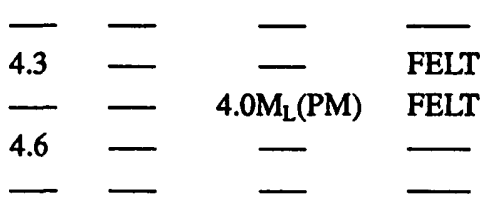

FEB. $28 \quad 13: 38$ AST

FEB. 28 23:58 AST

MAR. 1 19:55 AST

MAR. 1 19:40 HST

MAR. 2 05:28 AST

MAR. 2 10:23 AST MAR. 2 10:42 HST MAR. 2 21:06 AST MAR. 2 23:49 AST MAR. $3 \quad 10: 32$ AST

MAR. $3 \quad 22: 29$ HST MAR. 3 23:47 AST MAR. 3 23:50 AST MAR. 4 00:12 AST MAR. 4 01:30 AST

MAR. 4 03:52 AST MAR. 4 06:35 AST MAR. 4 16:05 AST MAR. 5 09:42 AST MAR. 5 09:46 AST

MAR. $5 \quad$ 12:10 AST MAR. 5 18:39 AST MAR. 5 18:41 AST MAR. 5 19:08 AST MAR. 6 01:16 AST

MAR. $6 \quad$ 10:14 AST MAR. $6 \quad 11: 50$ AST MAR. $6 \quad$ 15:08 AST MAR. 7 07:55 AST MAR. 7 09:21 AST

MAR. 7 17:58 AST MAR. 7 20:37 AST MAR. 8 08:33 AST MAR. 8 16:55 AST MAR. 8 17:41 AST

MAR. 8 17:56 AST MAR. 9 02:50 AST

MAR. 9 04:27 AST $\begin{array}{llrr}65.098 \mathrm{~N} & 153.066 \mathrm{~W} & 130 & \mathrm{LD}\end{array}$ 54.088 N. $168.127 \mathrm{~W} . \quad 33$ GS $\square_{4.4}-$

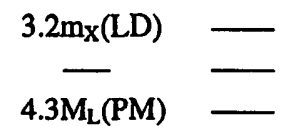


Table 1. Summary of United States earthquakes for 1986-Continued

\begin{tabular}{|c|c|c|c|c|c|c|c|c|c|c|c|c|}
\hline \multirow[t]{2}{*}{ Date } & \multirow{2}{*}{$\begin{array}{l}\text { Origin time } \\
\text { (UTC) } \\
\text { hr min sec }\end{array}$} & \multirow{2}{*}{$\begin{array}{c}\text { Latitude } \\
()^{\circ}\end{array}$} & \multirow{2}{*}{$\begin{array}{l}\text { Longitude } \\
(9)\end{array}$} & \multirow{2}{*}{$\begin{array}{c}\text { Depth } \\
(\mathrm{km})\end{array}$} & \multirow{2}{*}{$\begin{array}{l}\text { Hypo- } \\
\text { center } \\
\text { Source }\end{array}$} & \multicolumn{3}{|c|}{ Magnitude } & \multirow{2}{*}{$\begin{array}{l}\text { Max. } \\
\text { inten- } \\
\text { sity }\end{array}$} & \multicolumn{3}{|c|}{ Local time } \\
\hline & & & & & & $\mathbf{m}_{\mathbf{b}}$ & $\mathrm{M}_{\mathbf{S}}$ & Local & & Date & $\mathrm{hr}$ & zone \\
\hline
\end{tabular}

\section{ALASKA-Continued}

\begin{tabular}{llllllllllllll}
\hline MAR. & 9 & 134928.2 & $54.256 \mathrm{~N}$. & $167.864 \mathrm{~W}$. & 33 & GS & 5.2 & 5.5 & - & FELT & MAR. & 9 & $04: 49$ AST \\
MAR. & 9 & 140900.9 & $54.225 \mathrm{~N}$. & $168.160 \mathrm{~W}$ & 33 & GS & 4.6 & - & $4.3 \mathrm{M}_{\mathrm{L}}(\mathrm{PM})$ & - & MAR. & 9 & $05: 09$ AST
\end{tabular}

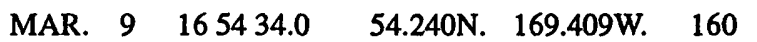

MAR. 10.030539 .0

MAR. $10 \quad 085603.7$

MAR. 11053846.5

MAR. $11 \quad 152021.6$

MAR. $11 \quad 172116.6$

MAR. $11 \quad 222443.5$

MAR. $11 \quad 231828.4$

MAR. $12 \quad 024803.9$

MAR. $12 \quad 040942.1$

MAR. $12 \quad 131501.2$

MAR. $12 \quad 231721.8$

MAR. $13 \quad 131638.6$

MAR. $13 \quad 132512.7$

MAR. $13 \quad 185528.8$

MAR. 13

MAR. 14

MAR. 14

MAR. 14

MAR. 14

213410.7

032027.9

094312.6

112709.6

122050.9

MAR, 14

MAR. 15

MAR. 15

MAR. 15

MAR. 15

173637.8 023412.8

071507.6

073429.8

204807.5

MAR. $17 \quad 122933.7$

MAR. $17 \quad 135439.5$

MAR. $18 \quad 071157.5$

MAR. $18 \quad 154850.2$

MAR. $18 \quad 161230.2$

MAR. $18 \quad 184501.1$ MAR. $18 \quad 232516.6$ MAR. 19092908.9 MAR. $19 \quad 182850.3$ MAR. $19 \quad 212058.4$

$54.101 \mathrm{~N} .168 .379 \mathrm{~W} . \quad 33$

60.184 N. 153.101W. 145

63.934 N. 152.483W. 33

$54.841 \mathrm{~N} .160 .220 \mathrm{~W} . \quad 35$

$54.257 \mathrm{~N} .168 .112 \mathrm{~W}$.

59.545 N. $152.762 \mathrm{~W} .77$

64.882 N. $149.197 \mathrm{~W} . \quad 33$

$54.112 \mathrm{~N} .168 .340 \mathrm{~W} . \quad 33$

61.685N. 151.072W. 75 $54.038 \mathrm{~N} .162 .421 \mathrm{~W} .4$ 51.649 N. $172.648 \mathrm{~W} . \quad 33$ 58.999 N. 153.930W. 122 59.987N. 153.025W. 111

$67.515 \mathrm{~N} .161 .887 \mathrm{~W} . \quad 33$ $67.425 \mathrm{~N} . \quad 161.738 \mathrm{~W} . \quad 33$ $61.710 \mathrm{~N} .151 .452 \mathrm{~W} .80$ $52.579 \mathrm{~N}$. 179.129W. 219 60.355N. 152.046W. 76

$60.177 \mathrm{~N} . \quad 151.147 \mathrm{~W} . \quad 65$ 62.613 N. $151.232 \mathrm{~W} . \quad 87$ 54.027N. 168.305W. 33 $59.052 \mathrm{~N} .150 .871 \mathrm{~W} . \quad 62$ $67.350 \mathrm{~N} .161 .696 \mathrm{~W} .33$

60.299 N. $140.678 \mathrm{~W} .4$ 61.693 N. 151.834W. 103 $56.351 \mathrm{~N} .157 .171 \mathrm{~W} .66$ 66.788 N. $159.157 \mathrm{~W} . \quad 33$ 54.028 N. 168.070W. 33
$54.086 \mathrm{~N} .168 .075 \mathrm{~W} . \quad 33$ 56.974 N. $154.295 \mathrm{~W} .86$ 63.205N. 150.693W. 143 $52.168 \mathrm{~N} .174 .060 \mathrm{E} . \quad 33$ 59.814 N. $152.502 \mathrm{~W}$. 87

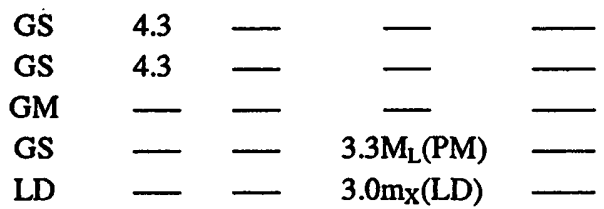

GS
GM
GM
GS
GS

GM
LD
GM
GM
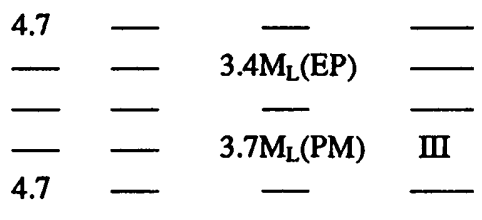

MAR.

07:54 AST

MAR. 9 18:05 AST

MAR. 9 23:56 AST

MAR. $10 \quad$ 20:38 AST

MAR. 11 06:20 AST

MAR. $11 \quad 08: 21$ AST MAR. 11 13:24 AST MAR. $11 \quad$ 14:18 AST MAR. 11 17:48 AST MAR. $11 \quad$ 19:09 AST

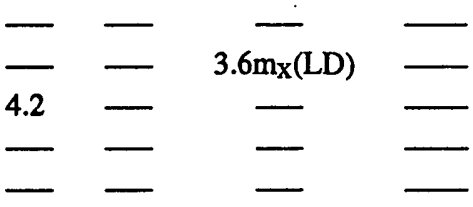

MAR. 12 04:15 AST MAR. $12 \quad$ 14:17 AST MAR. 13 03:16 HST MAR. 13 04:25 AST MAR. 13 09:55 AST

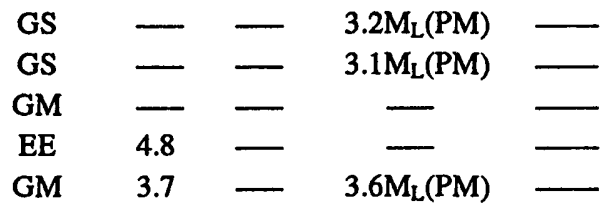

MAR. $13 \quad 12: 34$ AST MAR. 13 18:20 AST MAR. 14 00:43 AST MAR. 14 01:27 HST MAR. 14 03:20 AST

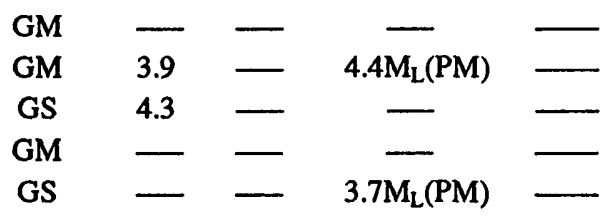

MAR. 14 08:36 AST MAR. 14 17:34 AST MAR. 14 22:15 AST MAR. 14 22:34 AST MAR. $15 \quad$ 11:48 AST

MAR. 17 03:29 AST MAR. 17 04:54 AST MAR. 17 22:11 AST MAR. 18 06:48 AST MAR. $18 \quad 07: 12$ AST

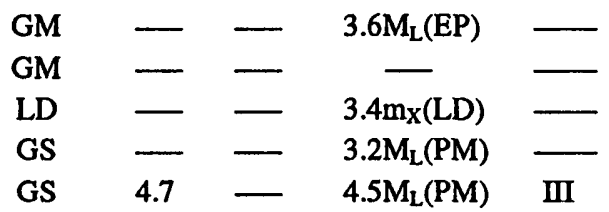

$\begin{array}{ccccc}\text { GS } & 4.6 & - & 4.3 \mathrm{M}_{\mathrm{L}}(\mathrm{PM}) & - \\ \mathrm{GM} & 4.7 & - & - & - \\ \mathrm{GS} & - & - & - & - \\ \mathrm{GS} & 4.8 & - & - & - \\ \mathrm{GM} & - & - & - & -\end{array}$
MAR. $18 \quad 09: 45$ AST MAR. 18 14:25 AST MAR. 19 00:29 AST MAR. 19 08:28 HST MAR. 19 12:20 AST 
Table 1. Summary of United States earthquakes for 1986-Continued

\begin{tabular}{|c|c|c|c|c|c|c|c|c|c|c|c|c|}
\hline \multirow[t]{2}{*}{ Date } & \multirow[t]{2}{*}{$\begin{array}{l}\text { Origin time } \\
\text { (UTC) }\end{array}$} & \multirow{2}{*}{$\begin{array}{c}\text { Latitude } \\
\left(^{\circ}\right)\end{array}$} & \multirow{2}{*}{$\begin{array}{c}\text { Longitude } \\
\text { (9) }\end{array}$} & \multirow{2}{*}{$\begin{array}{l}\text { Depth } \\
(\mathrm{km})\end{array}$} & \multirow{2}{*}{$\begin{array}{l}\text { Hypo- } \\
\text { center } \\
\text { Source }\end{array}$} & \multicolumn{3}{|c|}{ Magnitude } & $\begin{array}{l}\text { Max. } \\
\text { inten- }\end{array}$ & \multicolumn{3}{|c|}{ Local time } \\
\hline & & & & & & $\mathrm{m}_{\mathrm{b}}$ & $\mathrm{M}_{\mathrm{s}}$ & Local & sity & Date & $\mathrm{hr}$ & zone \\
\hline
\end{tabular}

ALASKA-Continued

\begin{tabular}{|c|c|c|c|c|c|c|c|c|c|c|c|}
\hline MAR. 20 & 034735.5 & $62.793 \mathrm{~N}$. & 149.782W. & 59 & GM & - & - & - & & MAR. 19 & 18:47 AST \\
\hline MAR. 20 & 161735.5 & $60.118 \mathrm{~N}$ & 153.281W. & 143 & GM & - & - & - & & MAR. 20 & 07:17 AST \\
\hline MAR. 20 & 194008.8 & $54.202 \mathrm{~N}$. & $168.187 \mathrm{~W}$. & 33 & GS & 4.8 & 4.3 & $5.0 \mathrm{M}_{\mathrm{L}}(\mathrm{PM})$ & FELT & MAR. 20 & 10:40 AST \\
\hline MAR. 21 & 195737.7 & $61.512 \mathrm{~N}$ & $146.759 \mathrm{~W}$. & 28 & GM & - & - & - & - & MAR. 21 & $10: 57$ AST \\
\hline MAR. 22 & 053001.9 & $60.348 \mathrm{~N}$ & 153.297W. & 165 & GM & 4.4 & - & - & FELT & MAR. 21 & 20:30 AST \\
\hline
\end{tabular}

MAR. $22 \quad 214548.9$

MAR. $24 \quad 171128.5$

MAR. $24 \quad 232001.4$

MAR. 25054939.8

MAR. $25 \quad 114010.9$

MAR. $25 \quad 212252.5$

MAR. $26 \quad 053053.6$

MAR. 26060016.0

MAR. $27 \quad 224236.6$

MAR. $28 \quad 214048.9$

MAR. $29 \quad 041430.3$

MAR. 29072852.6

MAR. $29 \quad 211507.0$

MAR. $30 \quad 081757.2$

MAR. $30 \quad 181329.3$

MAR. $31 \quad 041413.3$

MAR. 31042334.6

MAR. $31 \quad 093158.9$

APR. 1060129.8

APR. $1 \quad 070619.4$

APR. $\quad 1 \quad 140825.7$ APR. $1 \quad 202634.4$ APR. $\quad 1 \quad 234651.4$ APR. $2 \quad 133707.1$ APR. 2205907.5

APR. $\quad 2 \quad 225740.9$ APR. $3 \quad 0211 \quad 10.6$ APR. $3 \quad 024325.9$ APR. $3 \quad 025711.1$ APR. $3 \quad 034350.5$

APR. $\quad 3 \quad 100236.8$ APR. $3 \quad 122027.9$ APR. $3 \quad 211147.7$
$61.217 \mathrm{~N} .150 .382 \mathrm{~W} . \quad 16$ $62.388 \mathrm{~N} .151 .396 \mathrm{~W} .88$ $59.544 \mathrm{~N} .152 .242 \mathrm{~W}$. 65 $62.517 \mathrm{~N} .151 .178 \mathrm{~W} .90$ 62.796 N. 150.498 W. 90

52.279 N. $178.632 \mathrm{~W} . \quad 250$ $54.152 \mathrm{~N} .168 .232 \mathrm{~W} . \quad 33$ $59.956 \mathrm{~N} . \quad 152.222 \mathrm{~W} . \quad 62$ $53.780 \mathrm{~N}$. $148.483 \mathrm{~W} . \quad 33$ $57.923 \mathrm{~N} .153 .976 \mathrm{~W} .105$
GM

GM

GM

GM

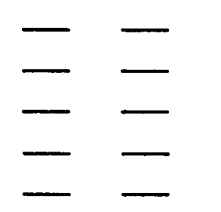

3.5 $\mathrm{M}_{\mathrm{L}}(\mathrm{PM})$

$3.5 \mathrm{M}_{\mathrm{L}}(\mathrm{PM})$

FELT

$-$

$\overline{3.6 \mathrm{M}_{\mathrm{L}}(\mathrm{PM})}$

$\longrightarrow$
MAR. 22

MAR. 24

MAR. 24

MAR. 24

MAR. 25

MAR. $25 \quad 11: 22$ HST

MAR. $25 \quad 20: 30$ AST

MAR. 25 21:00 AST

MAR. 27 13:42 AST

MAR. $28 \quad 12: 40$ AST

61.791N. $150.407 \mathrm{~W} . \quad 65$ $59.961 \mathrm{~N} .150 .333 \mathrm{~W} . \quad 55$ $59.103 \mathrm{~N} .152 .486 \mathrm{~W} . \quad 83$ $60.599 \mathrm{~N} . \quad 151.855 \mathrm{~W} . \quad 67$ $51.537 \mathrm{~N} .179 .940 \mathrm{~W} . \quad 33$

$58.307 \mathrm{~N} . \quad 154.185 \mathrm{~W} . \quad 74$ $59.143 \mathrm{~N} . \quad 152.572 \mathrm{~W} . \quad 64$ 61.751N. 151.954W. 111 56.901N. 153.553W. 33 $53516 \mathrm{~N} .164 .136 \mathrm{~W} . \quad 42$

61.741N. $150.912 \mathrm{~W} .65$ $61.558 \mathrm{~N} .149 .984 \mathrm{~W} . \quad 46$ $61.886 \mathrm{~N} . \quad 150.937 \mathrm{~W} . \quad 72$ $58.075 \mathrm{~N} .154 .085 \mathrm{~W} .64$ $59.996 \mathrm{~N} . \quad 152.998 \mathrm{~W} . \quad 114$

59.879 N. $153.396 \mathrm{~W} . \quad 130$ $60.110 \mathrm{~N} .153 .215 \mathrm{~W} .130$ $56.410 \mathrm{~N} .153 .436 \mathrm{~W} . \quad 33$ $56.257 \mathrm{~N} . \quad 153.473 \mathrm{~W} . \quad 33$ $51.525 \mathrm{~N}$. 173.603W. 33

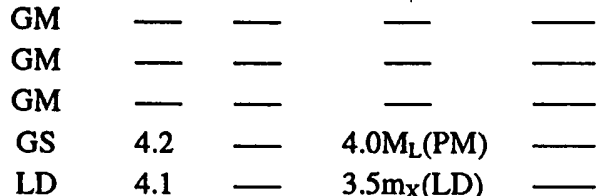

GM

GM

GM

GS
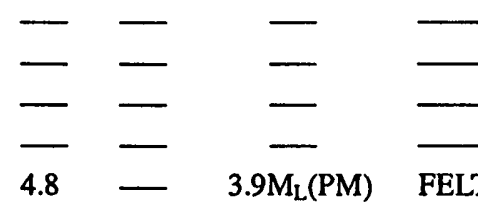
$3.9 \mathrm{M}_{\mathrm{L}}(\mathrm{PM})$

FELT
MAR. $28 \quad$ 19:14 AST MAR. $28 \quad 22: 28$ AST MAR. $29 \quad 12: 15$ AST MAR. $29 \quad 23: 17$ AST MAR. $30 \quad 08: 13$ HST

MAR. $30 \quad$ 19:14 AST MAR. $30 \quad$ 19:23 AST MAR. $31 \quad 00: 31$ AST MAR. 31 21:01 AST MAR. 31 22:06 AST

APR. 1 05:08 AST APR. 1 11:26 AST APR. 1 14:46 AST APR. 2 04:37 AST APR. 2 11:59 AST

APR. 2 13:57 AST APR. 2 17:11 AST APR. 2 17:43 AST APR. 2 17:57 AST APR. 2 17:43 HST
61.449 N. $150.039 \mathrm{~W} . \quad 45$ $51.285 \mathrm{~N} .177 .046 \mathrm{~W} .36$ $63.634 \mathrm{~N} .145 .435 \mathrm{~W} .33$ $\begin{array}{lllll}\text { GS } & - & - & 3.0 \mathrm{M}_{\mathrm{L}}(\mathrm{PM}) & \text { FELT } \\ \mathrm{EE} & 4.6 & - & 4.0 \mathrm{M}_{\mathrm{L}}(\mathrm{PM}) & - \\ \mathrm{GS} & - & - & 3.7 \mathrm{M}_{\mathrm{L}}(\mathrm{PM}) & \text { FELT }\end{array}$
APR. 3 01:02 AST APR. 3 02:20 HST APR. 3 12:11 AST 
Table 1. Summary of United States earthquakes for 1986-Continued

\begin{tabular}{|c|c|c|c|c|c|c|c|c|c|c|c|c|}
\hline \multirow[t]{2}{*}{ Date } & \multirow{2}{*}{$\begin{array}{l}\text { Origin time } \\
\text { (UTC) } \\
\text { hr min sec }\end{array}$} & \multirow{2}{*}{$\begin{array}{c}\text { Latitude } \\
\text { ()) }\end{array}$} & \multirow{2}{*}{$\begin{array}{c}\text { Longitude } \\
\text { (9) }\end{array}$} & \multirow{2}{*}{$\begin{array}{l}\text { Depth } \\
(\mathrm{km})\end{array}$} & \multirow{2}{*}{$\begin{array}{l}\text { Hypo- } \\
\text { center } \\
\text { Source }\end{array}$} & \multicolumn{3}{|c|}{ Magnitude } & \multirow{2}{*}{$\begin{array}{l}\text { Max. } \\
\text { inten- } \\
\text { sity }\end{array}$} & \multicolumn{3}{|c|}{ Local time } \\
\hline & & & & & & $\mathrm{m}_{\mathrm{b}}$ & $\mathrm{M}_{\mathrm{S}}$ & Local & & Date & $\overline{h r}$ & zone \\
\hline
\end{tabular}

ALASKA-Continued

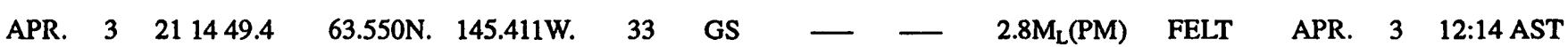

$\begin{array}{lllllllllllll}\text { APR. } & 3 & 211655.0 & 63.444 \mathrm{~N} & 145.564 \mathrm{~W} & 33 & \mathrm{GS} \quad- & - & -2 \mathrm{M}_{\mathrm{L}}(\mathrm{PM}) & \text { FELT } & \text { APR. } & 3 & 12: 16 \text { AST }\end{array}$

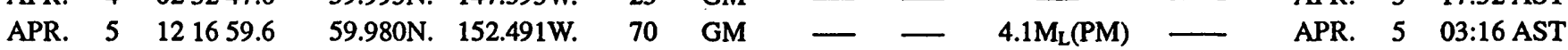

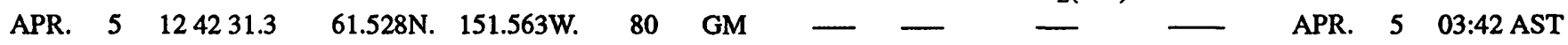

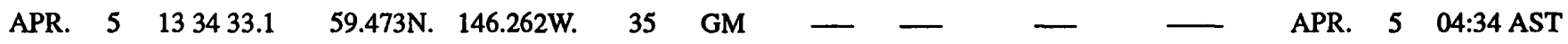
$\begin{array}{lllllllllllllll}\text { APR. } & 5 & 174150.7 & 54.055 \mathrm{~N} & 161.961 \mathrm{~W} & 33 & \mathrm{GS} & 5.0 & - & 5.0 \mathrm{~m}_{\mathrm{X}}(\mathrm{LD}) & - & \text { APR. } & 5 & 08: 41 \text { AST }\end{array}$

APR. $7 \quad 115554.1$ APR. $7 \quad 160656.0$ APR. $7 \quad 170746.8$ APR. $\quad 8 \quad 063648.0$ APR. $\quad 8 \quad 181226.7$

APR. $\quad 9 \quad 104101.5$ APR. $\quad 9 \quad 143200.3$ APR. $\quad 9 \quad 202010.8$ APR. $10 \quad 015037.9$ APR. $10 \quad 050954.6$

APR. $\quad 11 \quad 053209.3$ APR. $11 \quad 172220.8$ APR. $12 \quad 052732.1$ APR. $12 \quad 220232.9$ APR. $13 \quad 102539.9$

APR. $13 \quad 160507.0$ APR. $13 \quad 205908.6$ APR. $14 \quad 001627.1$ APR. $14 \quad 003402.4$ APR. $14 \quad 181709.4$

APR. $15 \quad 181245.2$ APR. $16 \quad 142250.8$ APR. $16 \quad 152452.7$ APR. $16 \quad 221322.8$ APR. $18 \quad 000638.2$

APR. $18 \quad 055122.0$ APR. $18 \quad 092239.5$ APR. $19 \quad 075704.8$ APR. $19 \quad 194225.8$ APR. $20 \quad 125400.9$
62.378N. $148.786 \mathrm{~W} . \quad 54 \quad$ GM 59.697N. 153.080W. 101 GM $54.058 \mathrm{~N}$. 167.343W. 33 GS $54.048 \mathrm{~N}$. $168.156 \mathrm{~W} .33$ GS 59.199N. 152.561W. 71 GM

63.193N. $150.535 \mathrm{~W} .142$ GS 50.982N. $173.376 \mathrm{E}$. 33 GS $60.207 \mathrm{~N} .152 .879 \mathrm{~W} .99 \quad \mathrm{GM}$ $51.070 \mathrm{~N}, 178.893 \mathrm{~W} .33$ GS $50.700 \mathrm{~N} .179 .128 \mathrm{~W} . \quad 33$ GS

$60.639 \mathrm{~N} .151 .672 \mathrm{~W} .68 \mathrm{GM}$ $54.164 \mathrm{~N} .167 .883 \mathrm{~W}, \quad 33$ $61.836 \mathrm{~N} .151 .980 \mathrm{~W} .113$ $67.263 \mathrm{~N} .161 .691 \mathrm{~W} . \quad 33$ $54.124 \mathrm{~N}$. 167.776W. 33 GS
$3.0 \mathrm{M}_{\mathrm{L}}(\mathrm{PM})$

-

$4.4 \mathrm{M}_{\mathrm{L}}(\mathrm{PM})$

4.6 $\mathrm{M}_{\mathrm{L}}(\mathrm{PM})$

$4.1 \mathrm{M}_{\mathrm{L}}(\mathrm{PM})$

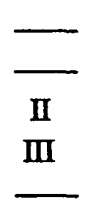

APR. 7 02:55 AST APR. 7 07:06 AST APR. 7 08:07 AST APR. 7 21:36 AST APR. 8 09:12 AST

APR. 9 01:41 AST APR. 9 04:32 HST APR. 9 11:20 AST APR. 9 15:50 HST APR. 9 19:09 HST

APR. $10 \quad 20: 32$ AST APR. 11 08:22 AST APR. $11 \quad 20: 27$ AST APR. 12 13:02 AST APR. 13 01:25 AST

APR. 13 07:05 AST APR. 13 11:59 AST APR. 13 15:16 AST APR. 13 15:34 AST APR. 14 09:17 AST

APR. $15 \quad 09: 12$ AST APR. 16 05:22 AST APR. 16 06:24 AST APR. 16 13:13 AST APR. 17 15:06 AST

APR. 17 20:51 AST APR. 18 00:22 AST APR. 18 22:57 AST APR. 19 10:42 AST APR. 20 03:54 AST 
Table 1. Summary of United States earthquakes for 1986-Continued

\begin{tabular}{|c|c|c|c|c|c|c|c|c|c|c|c|c|}
\hline \multirow[t]{2}{*}{ Date } & \multirow{2}{*}{$\begin{array}{l}\text { Origin time } \\
\text { (UTC) } \\
\text { hr min sec }\end{array}$} & \multirow{2}{*}{$\begin{array}{l}\text { Latitude } \\
\left({ }^{\circ}\right)\end{array}$} & \multirow{2}{*}{$\begin{array}{c}\text { Longitude } \\
\text { (9) }\end{array}$} & \multirow{2}{*}{$\begin{array}{l}\text { Depth } \\
(\mathrm{km})\end{array}$} & \multirow{2}{*}{$\begin{array}{l}\text { Hypo- } \\
\text { center } \\
\text { Source }\end{array}$} & \multicolumn{3}{|c|}{ Magnitude } & \multirow{2}{*}{$\begin{array}{l}\text { Max. } \\
\text { inten- } \\
\text { sity }\end{array}$} & \multicolumn{3}{|c|}{ Local time } \\
\hline & & & & & & $\mathbf{m}_{\mathrm{b}}$ & $\overline{M_{s}}$ & Local & & Date & hr & zone \\
\hline
\end{tabular}

\section{ALASKA-Continued}

\begin{tabular}{|c|c|c|c|c|c|c|c|c|c|c|c|c|}
\hline APR & 20 & 131416.7 & $64.902 \mathrm{~N}$. & 148.678W. & 33 & GS & - & - & $4.1 \mathrm{M}_{\mathrm{L}}(\mathrm{PM})$ & - & APR. 20 & 04:14 AST \\
\hline APR & 20 & 141411.3 & $53.363 \mathrm{~N}$. & $157.137 \mathrm{~W}$ & 16 & LD & - & - & $3.3 \mathrm{~m}_{\mathrm{x}}(\mathrm{LD})$ & & APR. 20 & 05:14 AST \\
\hline APR & 21 & 172025.5 & $62.545 \mathrm{~N}$. & $150.884 \mathrm{~W}$ & 79 & GM & - & - & - & & APR. 21 & 08:20 AST \\
\hline APR & 22 & 102651.4 & $53.659 \mathrm{~N}$. & $170.802 \mathrm{~W}$ & 33 & GS & 4.5 & - & - & & APR. & $00: 26 \mathrm{HST}$ \\
\hline APR & 23 & 033700.9 & $58.855 \mathrm{~N}$. & 153.079W. & 64 & GM & - & - & - & - & APR. 22 & $18: 37$ AST \\
\hline
\end{tabular}

APR. $25 \quad 034519.5 \quad 62.124 \mathrm{~N} .150 .304 \mathrm{~W} .56 \quad 56$

APR. $25 \quad 2357423$

APR. $26 \quad 035126.7$

APR. $26 \quad 121334.0$

APR. $26 \quad 235945.7$

APR. $27 \quad 060831.8$ APR. $27 \quad 105541.2$ APR. $27 \quad 133527.9$ APR. $27 \quad 195133.3$ APR. $27 \quad 220031.2$

APR. $28 \quad 002222.4$ APR. $28 \quad 073256.1$ APR. $29 \quad 103304.4$ APR. $29 \quad 153007.6$ APR. $30 \quad 015126.2$

MAY $\quad 1 \quad 090057.4$ MAY $\quad 2 \quad 005157.7$ MAY $2 \quad 130055.6$ MAY $3 \quad 060522.5$ MAY $3 \quad 101113.6$

\begin{tabular}{|c|c|c|}
\hline & 3 & \\
\hline $1 \mathrm{~A}$ & & \\
\hline & 2 & \\
\hline & & \\
\hline & & \\
\hline
\end{tabular}

53.733N. $170.932 \mathrm{~W} .237$ GS

$63.080 \mathrm{~N} .149 .866 \mathrm{~W} .161 \quad \mathrm{GM}$ $61.498 \mathrm{~N}$. 149.893W. $59 \mathrm{GM}$ $60.307 \mathrm{~N}$. $143.184 \mathrm{~W} .25 \mathrm{GM}$ $64.738 \mathrm{~N}$. $153.171 \mathrm{~W} .33$ GS $58.496 \mathrm{~N}$. $154.817 \mathrm{~W} . \quad 20 \quad \mathrm{GM}$

$65.823 \mathrm{~N} . \quad 155.169 \mathrm{~W}$. $61.238 \mathrm{~N} .149 .410 \mathrm{~W}$ $64.820 \mathrm{~N}$. $148.848 \mathrm{~W}$. $59.998 \mathrm{~N} . \quad 152.775 \mathrm{~W}$. $61.426 \mathrm{~N} .149 .859 \mathrm{~W}$.

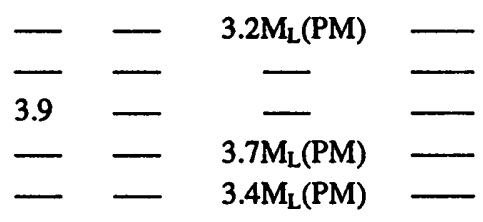

APR. 24 APR. 25 APR. 25 APR. 26 APR. 26

18:45 AST 14:57 AST 18:51 AST 03:13 AST 14:59 AST

APR. 26 21:08 AST APR. $27 \quad 01: 55$ AST APR. $27 \quad 04: 35$ AST APR. 27 10:51 AST APR. 27 13:00 AST

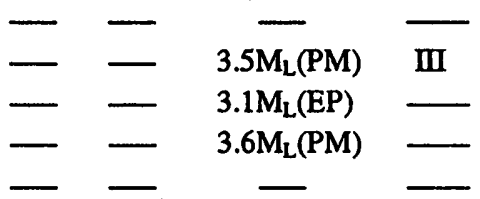

APR. $27 \quad$ 15:22 AST APR. $27 \quad 22: 32$ AST APR. 29 01:33 AST APR. 29 06:30 AST APR. 29 16:51 AST
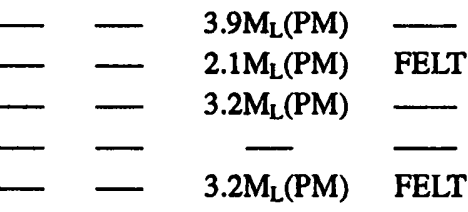

MAY 1 00:00 AST MAY 1 15:51 AST MAY 2 04:00 AST MAY 2 21:05 AST MAY 3 01:11 AST

$\begin{array}{lll}\text { MAY } & 5 & 115238.6\end{array}$ $\begin{array}{lll}\text { MAY } & 6 \quad 154458.3\end{array}$ MAY $\quad 7 \quad 164102.7$

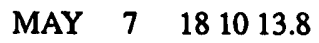
MAY $\quad 7 \quad 181259.5$

MAY $\quad 7 \quad 204311.0$ MAY $\quad 7 \quad 204333.3$ MAY $\quad 7 \quad 212313.6$ $66.326 \mathrm{~N}$. 141.662W. 33 GS $51.589 \mathrm{~N}$. 175.441E. 33 GS $57.772 \mathrm{~N}$. $156.542 \mathrm{~W} .142$ GS $57.805 \mathrm{~N}$. $156.609 \mathrm{~W} .142$ GS
$61.208 \mathrm{~N} .151 .756 \mathrm{~W} . \quad 88 \quad \mathrm{GM}$ $59.970 \mathrm{~N} . \quad 152.633 \mathrm{~W} . \quad 89 \quad$ GM $54.866 \mathrm{~N}$. 156.980W. 19 LD $55.149 \mathrm{~N} .157 .574 \mathrm{~W} . \quad 33 \quad$ GS 54.835N. 157.136W. $21 \quad$ LD
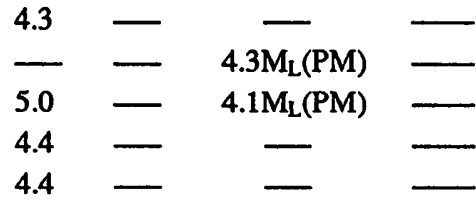

MAY

$\begin{array}{lll}\text { MAY } & 3 & 06: 35 \\ \text { AST }\end{array}$

MAY 3 06:56 HST

MAY 3 16:51 AST

MAY $4 \quad$ 00:07 AST
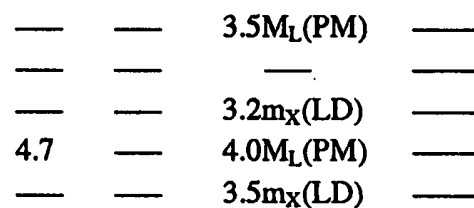

MAY

MAY

MAY

MAY

MAY

02:52 AST
06:44 AST
07:41 AST
09:10 AST
09:12 AST

$51.190 \mathrm{~N}$. 174.726W. $20 \quad$ EE

$51.234 \mathrm{~N}$. 174.741W. 25 EE

$5.0 \quad-$

6.1 $\mathrm{M}_{\mathrm{L}}(\mathrm{PM}) \quad \overline{\text { FELT }}$

MAY $7 \quad 10: 43$ HST

$\begin{array}{lll}\text { MAY } & 7 \quad 10: 43 \text { HST }\end{array}$

MAY $7 \quad$ 11:23 HST 
Table 1. Summary of United States earthquakes for 1986-Continued

\begin{tabular}{|c|c|c|c|c|c|c|c|c|c|c|c|c|c|}
\hline \multirow[t]{2}{*}{ Date } & & \multirow{2}{*}{$\begin{array}{l}\text { Origin time } \\
\text { (UTC) } \\
\mathrm{hr} \operatorname{min~sec}\end{array}$} & \multirow{2}{*}{$\begin{array}{l}\text { Latitude } \\
\text { ( ) }\end{array}$} & \multirow{2}{*}{$\begin{array}{l}\text { Longitude } \\
(9)\end{array}$} & \multirow{2}{*}{$\begin{array}{l}\text { Depth } \\
(\mathrm{km})\end{array}$} & \multirow{2}{*}{$\begin{array}{l}\text { Hypo- } \\
\text { center } \\
\text { Source }\end{array}$} & \multicolumn{3}{|c|}{ Magnitude } & \multirow{2}{*}{$\begin{array}{l}\text { Max. } \\
\text { inten- } \\
\text { sity }\end{array}$} & \multicolumn{3}{|c|}{ Local time } \\
\hline & & & & & & & $\mathrm{m}_{\mathrm{b}}$ & $\mathbf{M}_{\mathbf{s}}$ & Local & & Date & & zone \\
\hline \multicolumn{14}{|c|}{ ALASKA-Continued } \\
\hline MAY & 7 & 213042.4 & $51.385 \mathrm{~N}$. & 174.498W. & 33 & GS & 4.2 & - & - & - & MAY & 7 & 11:30 HST \\
\hline MAY & 7 & 213906.2 & $52.066 \mathrm{~N}$. & $174.911 \mathrm{~W}$ & 33 & GS & 4.4 & - & - & - & MAY & 7 & 11:39 HST \\
\hline MAY & 7 & 214229.3 & $51.148 \mathrm{~N}$. & 174.796W. & 20 & $\mathrm{EE}$ & 4.5 & - & - & - & MAY & 7 & 11:42 HST \\
\hline MAY & 7 & 222214.3 & $51.962 \mathrm{~N}$. & 174.721W. & 33 & GS & 4.1 & - & - & - & MAY & 7 & $12: 22 \mathrm{HST}$ \\
\hline MAY & 7 & 224712.3 & $51.325 \mathrm{~N}$. & 174.751W. & 31 & EE & 6.4 & 7.7 & - & VII & MAY & 7 & $12: 47$ HST \\
\hline MAY & 7 & 225505.0 & $51.500 \mathrm{~N}$. & $174.800 \mathrm{~W}$. & 33 & GS & 5.6 & - & - & - & MAY & 7 & 12:55 HST \\
\hline MAY & 7 & 225747.6 & $51.575 \mathrm{~N}$. & $174.219 \mathrm{~W}$ & 15 & EE & 5.7 & - & - & - & MAY & 7 & $12: 57 \mathrm{HST}$ \\
\hline MAY & 7 & 230041.0 & $51.500 \mathrm{~N}$. & $174.800 \mathrm{~W}$ & 33 & GS & 5.2 & - & - & - & MAY & 7 & 13:00 HST \\
\hline MAY & 7 & 230409.0 & $51.500 \mathrm{~N}$. & $174.800 \mathrm{~W}$. & 33 & GS & 4.9 & - & - & - & MAY & 7 & 13:04 HST \\
\hline MAY & 7 & 230452.0 & $51.500 \mathrm{~N}$. & $174.800 \mathrm{~W}$ & 33 & GS & 5.3 & - & - & - & MAY & 7 & 13:04 HST \\
\hline MAY & 7 & 230638.0 & $51.500 \mathrm{~N}$. & $174.800 \mathrm{~W}$ & 33 & GS & 5.0 & - & - & - & MAY & 7 & 13:06 HST \\
\hline MAY & 7 & 230745.0 & 51.317N. & $174.598 \mathrm{~W}$. & 20 & EE & 5.5 & - & - & & MAY & 7 & 13:07 HST \\
\hline MAY & 7 & 231235.0 & $51.500 \mathrm{~N}$. & $174.800 \mathrm{~W}$ & 33 & GS & 5.0 & - & - & - & MAY & 7 & 13:12 HST \\
\hline MAY & 7 & 231248.7 & $51.158 \mathrm{~N}$. & $174.005 \mathrm{~W}$. & 20 & EE & 5.3 & - & - & - & MAY & 7 & 13:12 HST \\
\hline MAY & 7 & 231316.0 & $51.500 \mathrm{~N}$. & $174.800 \mathrm{~W}$ & 33 & GS & 5.2 & - & - & - & MAY & 7 & $13: 13$ HST \\
\hline MAY & 7 & 231505.1 & $51.234 \mathrm{~N}$. & $175.334 \mathrm{~W}$ & 20 & EE & 5.4 & - & - & - & MAY & 7 & $13: 15 \mathrm{HST}$ \\
\hline MAY & 7 & 231716.0 & $51.500 \mathrm{~N}$. & $174.800 \mathrm{~W}$ & 33 & GS & 5.3 & - & - & & MAY & 7 & 13:17 HST \\
\hline MAY & 7 & 231823.0 & $51.500 \mathrm{~N}$. & $174.800 \mathrm{~W}$ & 33 & GS & 4.9 & - & - & - & MAY & 7 & 13:18 HST \\
\hline MAY & 7 & 232017.0 & $51.500 \mathrm{~N}$. & $174.800 \mathrm{~W}$. & 33 & GS & 5.2 & - & - & & MAY & 7 & $13: 20 \mathrm{HST}$ \\
\hline MAY & 7 & 232406.0 & $51.500 \mathrm{~N}$. & $174.800 \mathrm{~W}$. & 33 & GS & 5.2 & - & - & 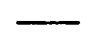 & MAY & 7 & $13: 24$ HST \\
\hline MAY & 7 & 232657.2 & $51.078 \mathrm{~N}$. & $174.028 \mathrm{~W}$. & 20 & $\mathrm{EE}$ & 5.4 & - & - & 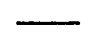 & MAY & 7 & 13:26 HST \\
\hline MAY & 7 & 232936.5 & $51.701 \mathrm{~N}$. & $176.439 \mathrm{~W}$. & 33 & GS & 4.9 & - & - & 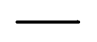 & MAY & 7 & $13: 29 \mathrm{HST}$ \\
\hline MAY & 7 & 233317.5 & $50.648 \mathrm{~N}$. & $174.879 \mathrm{~W}$. & 17 & $\mathrm{EE}$ & 4.7 & - & - & $\longrightarrow$ & MAY & 7 & 13:33 HST \\
\hline MAY & 7 & 233618.3 & $51.297 \mathrm{~N}$. & $174.132 \mathrm{~W}$. & 20 & $\mathrm{EE}$ & 5.7 & 一 & - & 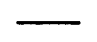 & MAY & 7 & $13: 36 \mathrm{HST}$ \\
\hline MAY & 7 & 234121.0 & $51.500 \mathrm{~N}$. & $174.800 \mathrm{~W}$ & 33 & GS & 5.1 & - & - & - & MAY & 7 & 13:41 HST \\
\hline MAY & 7 & 234807.4 & $51.228 \mathrm{~N}$. & $175.366 \mathrm{~W}$ & 20 & $\mathrm{EE}$ & 5.0 & - & - & - & MAY & 7 & 13:48 HST \\
\hline MAY & 7 & 235101.9 & $51.273 \mathrm{~N}$. & 174.836W. & 20 & $\mathrm{EE}$ & 5.8 & - & - & & MAY & 7 & $13: 51$ HST \\
\hline MAY & 7 & 235220.5 & $52: 300 \mathrm{~N}$. & $174.423 \mathrm{~W}$ & 15 & EE & 5.7 & - & - & & MAY & 7 & 13:52 HST \\
\hline MAY & 7 & 235935.2 & $51.465 \mathrm{~N}$. & 178.858E. & 33 & GS & 4.7 & - & - & & MAY & 7 & 13:59 HST \\
\hline MAY & 8 & 000040.0 & $51.500 \mathrm{~N}$. & $174.800 \mathrm{~W}$ & 33 & GS & 4.7 & - & 一 & & MAY & 7 & 14:00 HST \\
\hline MAY & 8 & 001058.8 & $51.313 \mathrm{~N}$. & $175.294 \mathrm{~W}$. & 25 & $\mathrm{EE}$ & 5.0 & - & - & & MAY & 7 & 14:10 HST \\
\hline MAY & 8 & 001827.7 & $51.910 \mathrm{~N}$. & 174.630W. & 33 & GS & 4.5 & - & - & - & MAY & 7 & $14: 18 \mathrm{HST}$ \\
\hline MAY & 8 & 002042.0 & $51.500 \mathrm{~N}$. & $174.800 \mathrm{~W}$ & 33 & GS & 4.4 & - & - & - & MAY & 7 & 14:20 HST \\
\hline MAY & 8 & 003059.3 & $51.562 \mathrm{~N}$. & 176.107W. & 33 & GS & 4.8 & - & 一 & 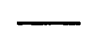 & MAY & 7 & $14: 30 \mathrm{HST}$ \\
\hline MAY & 8 & 003515.4 & $51.409 \mathrm{~N}$. & $175.490 \mathrm{~W}$. & 33 & GS & 4.2 & - & 一 & $\longrightarrow$ & MAY & 7 & $14: 35 \mathrm{HST}$ \\
\hline MAY & 8 & 004223.3 & $51.012 \mathrm{~N}$. & $176.233 \mathrm{~W}$ & 33 & GS & 4.5 & - & 一 & - & MAY & 7 & $14: 42 \mathrm{HST}$ \\
\hline MAY & 8 & 004547.1 & $51.226 \mathrm{~N}$. & $175.900 \mathrm{~W}$ & 33 & GS & 4.6 & - & - & - & MAY & 7 & $14: 45 \mathrm{HST}$ \\
\hline
\end{tabular}


Table 1. Summary of United States earthquakes for 1986 Continued

\begin{tabular}{|c|c|c|c|c|c|c|c|c|c|c|c|c|c|}
\hline \multirow[t]{2}{*}{ Date } & & \multirow{2}{*}{$\begin{array}{l}\text { Origin time } \\
\text { (UTC) } \\
\text { hr min sec }\end{array}$} & \multirow{2}{*}{$\begin{array}{l}\text { Latitude } \\
\left({ }^{\circ}\right)\end{array}$} & \multirow{2}{*}{$\begin{array}{l}\text { Longitude } \\
\text { (9) }\end{array}$} & \multirow{2}{*}{$\begin{array}{l}\text { Depth } \\
(\mathrm{km})\end{array}$} & \multirow{2}{*}{$\begin{array}{l}\text { Hypo- } \\
\text { center } \\
\text { Source }\end{array}$} & \multicolumn{3}{|c|}{ Magnitude } & \multirow{2}{*}{$\begin{array}{l}\text { Max. } \\
\text { inten- } \\
\text { sity }\end{array}$} & \multicolumn{3}{|c|}{ Local time } \\
\hline & & & & & & & $\mathrm{m}_{\mathrm{b}}$ & $\mathbf{M}_{\mathbf{s}}$ & Local & & Date & & zone \\
\hline \multicolumn{14}{|c|}{ ALASKA-Continued } \\
\hline MAY & 8 & 005518.9 & $51.268 \mathrm{~N}$ & 174.371W. & 20 & EE & 5.2 & - & - & & MAY & 7 & 14:55 HST \\
\hline MAY & 8 & 005911.9 & $52.204 \mathrm{~N}$ & $174.249 \mathrm{~W}$. & 15 & $\mathrm{EE}$ & 4.7 & - & - & & MAY & 7 & 14:59 HST \\
\hline MAY & 8 & 010239.0 & $51.792 \mathrm{~N}$ & $174.630 \mathrm{~W}$. & 33 & GS & 4.6 & - & - & & MAY & 7 & 15:02 HST \\
\hline MAY & 8 & 010550.4 & $51.199 \mathrm{~N}$. & $175.739 \mathrm{~W}$ & 33 & GS & 4.8 & - & - & & MAY & 7 & 15:05 HST \\
\hline MAY & 8 & 010616.5 & $51.400 \mathrm{~N}$ & $174.604 \mathrm{~W}$ & 25 & EE & 5.3 & - & - & & MAY & 7 & 15:06 HST \\
\hline MAY & 8 & 011102.1 & $50.960 \mathrm{~N}$ & $176.655 \mathrm{~W}$ & 20 & EE & 5.9 & - & $5.9 \mathrm{M}_{\mathrm{L}}(\mathrm{PM})$ & & MAY & 7 & 15:11 HST \\
\hline MAY & 8 & 011514.9 & $51.028 \mathrm{~N}$ & 176.778W. & 20 & EE & 5.6 & - & - & & MAY & 7 & 15:15 HST \\
\hline MAY & 8 & 011800.8 & $51.004 \mathrm{~N}$ & 176.573W. & 20 & EE & 5.4 & s. & 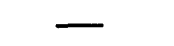 & & MAY & 7 & 15:18 HST \\
\hline MAY & 8 & 012056.9 & $51.003 \mathrm{~N}$ & $176.849 \mathrm{~W}$ & 20 & EE & 5.1 & 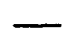 & - & & MAY & 7 & 15:20 HST \\
\hline MAY & 8 & 012246.9 & $51.037 \mathrm{~N}$ & $176.858 \mathrm{~W}$. & 20 & EE & 5.2 & & - & & MAY & 7 & 15:22 HST \\
\hline MAY & 8 & 013055.2 & $51.063 \mathrm{~N}$ & $176.882 \mathrm{~W}$ & 20 & EE & 5.1 & - & $5.2 \mathrm{M}_{\mathrm{L}}(\mathrm{PM})$ & & MAY & 7 & 15:30 HST \\
\hline MAY & 8 & 014011.8 & $51.381 \mathrm{~N}$ & 173.981W. & 33 & GS & 4.2 & - & - & & MAY & 7 & 15:40 HST \\
\hline MAY & 8 & 014519.3 & $51.002 \mathrm{~N}$ & $176.954 \mathrm{~W}$. & 33 & GS & 4.7 & & $5.0 \mathrm{M}_{\mathrm{L}}(\mathrm{PM})$ & & MAY & 7 & 15:45 HST \\
\hline MAY & 8 & 015413.1 & $51.090 \mathrm{~N}$ & 176.075W. & 20 & EE & 5.1 & $\longrightarrow$ & $4.5 \mathrm{M}_{\mathrm{L}}(\mathrm{PM})$ & & MAY & 7 & 15:54 HST \\
\hline MAY & 8 & 020400.2 & $51.002 \mathrm{~N}$ & $176.886 \mathrm{~W}$. & 20 & $\mathrm{EE}$ & 5.5 & & $5.5 \mathrm{M}_{\mathrm{L}}(\mathrm{PM})$ & & MAY & 7 & 16:04 HST \\
\hline MAY & 8 & 021634.4 & $51.293 \mathrm{~N}$. & 174.741W. & 20 & $\mathrm{EE}$ & 4.8 & - & - & & MAY & 7 & 16:16 HST \\
\hline MAY & 8 & 022741.1 & $51.040 \mathrm{~N}$ & $176.762 W$. & 33 & GS & 4.4 & & $4.4 \mathrm{M}_{\mathrm{L}}(\mathrm{PM})$ & & MAY & 7 & 16:27 HST \\
\hline MAY & 8 & 023254.6 & $51.145 \mathrm{~N}$ & 174.752W. & 20 & EE & 5.1 & & $\quad=$ & & MAY & 7 & 16:32 HST \\
\hline MAY & 8 & 024230.8 & $51.149 \mathrm{~N}$ & 175.950W. & 33 & GS & 3.9 & & - & & MAY & 7 & 16:42 HST \\
\hline MAY & 8 & 024435.0 & $51.257 \mathrm{~N}$ & $176.324 \mathrm{~W}$. & 33 & GS & 4.4 & - & - & & MAY & 7 & 16:44 HST \\
\hline MAY & 8 & 025940.4 & $51.217 \mathrm{~N}$ & $175.858 \mathrm{~W}$ & 20 & $\mathrm{EE}$ & 5.0 & & $4.7 \mathrm{M}_{\mathrm{L}}(\mathrm{PM})$ & & MAY & 7 & 16:59 HST \\
\hline MAY & 8 & 030825.7 & $50.967 \mathrm{~N}$ & $176.252 \mathrm{~W}$. & 33 & GS & 4.6 & & $4.6 \mathrm{M}_{\mathrm{L}}(\mathrm{PM})$ & & MAY & 7 & 17:08 HST \\
\hline MAY & 8 & 031427.4 & $51.220 \mathrm{~N}$ & $174.526 \mathrm{~W}$ & 20 & EE & 4.8 & - & - & & MAY & 7 & 17:14 HST \\
\hline MAY & 8 & 032014.2 & $51.032 \mathrm{~N}$ & $175.520 \mathrm{~W}$. & 20 & $\mathrm{EE}$ & 4.8 & - & - & & MAY & 7 & 17:20 HST \\
\hline MAY & 8 & 032340.5 & $51.005 \mathrm{~N}$ & $176.632 W$ & 20 & $\mathrm{EE}$ & 5.0 & - & $4.6 \mathrm{M}_{\mathrm{L}}(\mathrm{PM})$ & & MAY & 7 & $17: 23$ HST \\
\hline MAY & 8 & 034406.5 & $51.130 \mathrm{~N}$. & $175.233 \mathrm{~W}$ & 20 & $\mathrm{EE}$ & 5.1 & & 4.7 $\mathrm{M}_{\mathrm{L}}(\mathrm{PM})$ & & MAY & 7 & 17:44 HST \\
\hline MAY & 8 & 035251.2 & $50.975 \mathrm{~N}$ & $176.676 \mathrm{~W}$. & 20 & $\mathrm{EE}$ & 5.2 & - & - & & MAY & 7 & 17:52 HST \\
\hline MAY & 8 & 040349.7 & $50.971 \mathrm{~N}$ & 176.449W. & 20 & EE & 5.8 & 5.5 & $5.7 \mathrm{M}_{\mathrm{L}}(\mathrm{PM})$ & & MAY & 7 & 18:03 HST \\
\hline MAY & 8 & 041959.2 & $51.214 \mathrm{~N}$ & 175.840W. & 33 & GS & 4.6 & - & - & & MAY & 7 & 18:19 HST \\
\hline MAY & 8 & 042757.9 & $50.893 \mathrm{~N}$ & 176.160W. & 20 & $\mathrm{EE}$ & 5.1 & - & - & & MAY & 7 & 18:27 HST \\
\hline MAY & 8 & 043145.6 & $51.135 \mathrm{~N}$ & $176.424 \mathrm{~W}$. & 33 & GS & 4.3 & & & & MAY & 7 & 18:31 HST \\
\hline MAY & 8 & 043221.5 & $51.357 \mathrm{~N}$ & 174.642W. & 25 & $\mathrm{EE}$ & 5.0 & & . & & MAY & 7 & 18:32 HST \\
\hline MAY & 8 & 044517.6 & $51.217 \mathrm{~N}$ & 174.154W. & 20 & $\mathrm{EE}$ & 5.4 & - & - & & MAY & 7 & 18:45 HST \\
\hline MAY & 8 & 052023.5 & $51.333 \mathrm{~N}$ & $175.200 \mathrm{~W}$. & 33 & GS & 4.0 & - & $4.1 \mathrm{M}_{\mathrm{L}}(\mathrm{PM})$ & & MAY & 7 & 19:20 HST \\
\hline MAY & 8 & 053208.5 & $51.212 \mathrm{~N}$ & $174.878 \mathrm{~W}$. & 20 & $\mathrm{EE}$ & 4.9 & - & 4.7M $\mathrm{M}_{\mathrm{L}}(\mathrm{PM})$ & & MAY & 7 & 19:32 HST \\
\hline MAY & 8 & 053721.6 & $51.166 \mathrm{~N}$ & $175.329 \mathrm{~W}$. & 15 & EE & 6.0 & 6.2 & $6.2 \mathrm{M}_{\mathrm{L}}(\mathrm{PM})$ & & MAY & 7 & 19:37 HST \\
\hline MAY & 8 & 060418.7 & $50.985 \mathrm{~N}$ & $176.122 \mathrm{~W}$. & 20 & EE & 5.2 & - & $5.1 \mathrm{M}_{\mathrm{L}}(\mathrm{PM})$ & & MAY & 7 & 20:04 HST \\
\hline MAY & 8 & 062128.7 & $51.162 \mathrm{~N}$ & 175.275W. & 20 & $\mathrm{EE}$ & 4.8 & - & $4.2 \mathrm{M}_{\mathrm{L}}(\mathrm{PM})$ & - & MAY & 7 & 20:21 HST \\
\hline
\end{tabular}


Table 1. Summary of United States earthquakes for 1986-Continued

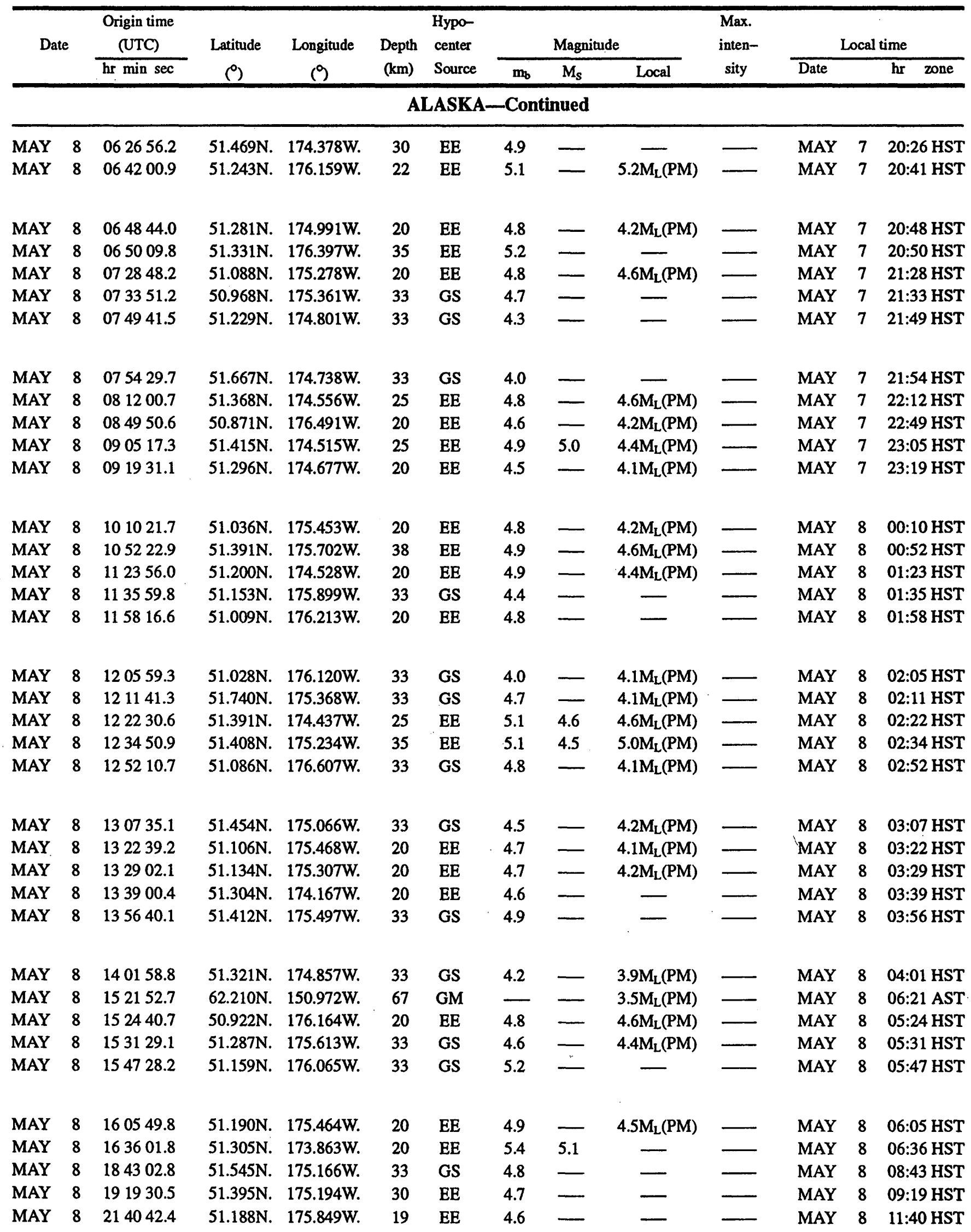


Table 1. Summary of United States earthquakes for 1986-Continued

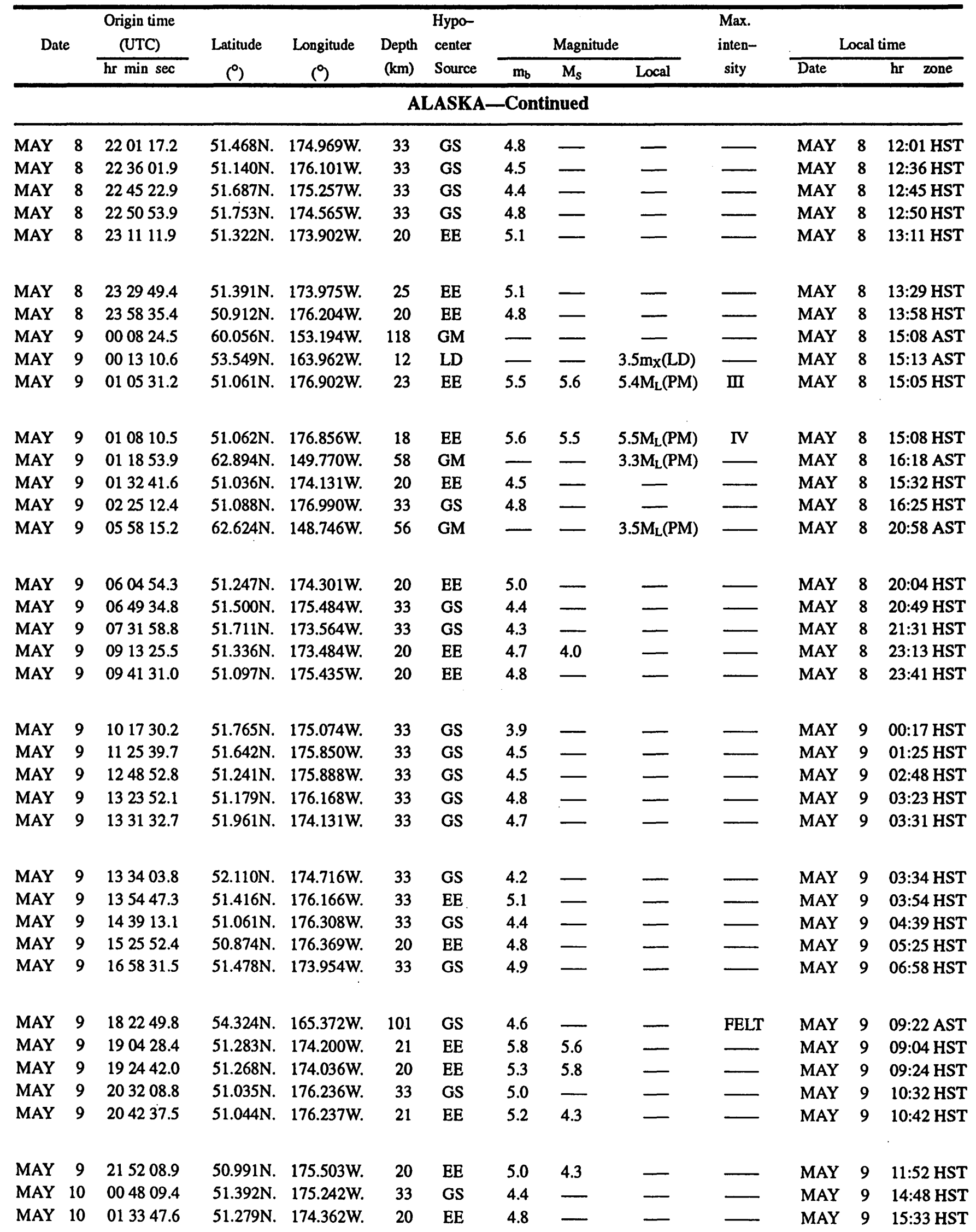


Table 1. Summary of United States earthquakes for 1986-Continued

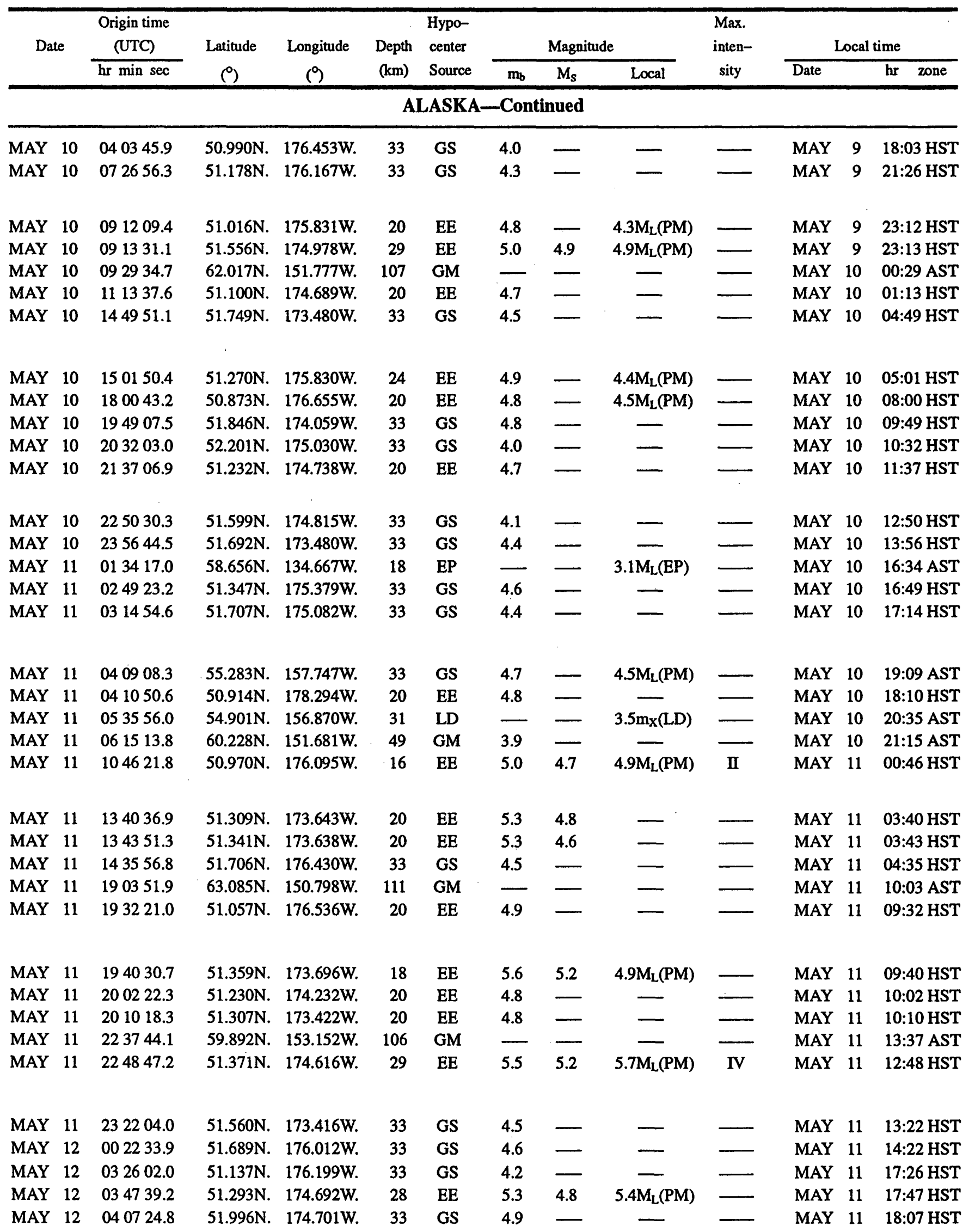


Table 1. Summary of United States earthquakes for 1986—Continued

\begin{tabular}{|c|c|c|c|c|c|c|c|c|c|c|c|c|c|}
\hline \multirow{2}{*}{\multicolumn{2}{|c|}{ Date }} & \multirow{3}{*}{$\begin{array}{l}\text { Origin time } \\
\text { (UTC) } \\
\text { hr min sec }\end{array}$} & \multirow{2}{*}{$\begin{array}{l}\text { Latitude } \\
\text { ( ) }\end{array}$} & \multirow{2}{*}{$\begin{array}{l}\text { Longitude } \\
\text { (9) }\end{array}$} & \multirow{2}{*}{$\begin{array}{l}\text { Depth } \\
(\mathbf{k m})\end{array}$} & \multirow{2}{*}{$\begin{array}{l}\text { Hypo- } \\
\text { center } \\
\text { Source }\end{array}$} & \multicolumn{3}{|c|}{ Magnitude } & \multirow{2}{*}{$\begin{array}{c}\text { Max. } \\
\text { inten- } \\
\text { sity }\end{array}$} & \multicolumn{3}{|c|}{ Local time } \\
\hline & & & & & & & $\mathrm{m}_{\mathrm{b}}$ & $\mathbf{M}_{\mathbf{S}}$ & Local & & Date & & zone \\
\hline \multicolumn{13}{|c|}{ ALASKA-Continued } & \\
\hline MAY & 12 & 051409.6 & $51.239 \mathrm{~N}$ & 176.266W. & 25 & $\mathbf{E E}$ & 4.4 & - & - & - & MAY & 11 & 19:14 HST \\
\hline MAY & 12 & 065950.3 & $57.601 \mathrm{~N}$ & $145.890 \mathrm{~W}$ & 15 & GM & - & - & - & - & MAY & 11 & 21:59 AST \\
\hline MAY & 12 & 081015.1 & $50.979 \mathrm{~N}$ & $176.085 \mathrm{~W}$. & 20 & EE & 4.8 & - & $4.4 \mathrm{M}_{\mathrm{L}}(\mathrm{PM})$ & & MAY & 11 & 22:10 HST \\
\hline MAY & 12 & 092954.6 & $51.456 \mathrm{~N}$ & $176.340 \mathrm{~W}$. & 39 & $\mathrm{EE}$ & 4.7 & 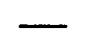 & $4.7 \mathrm{M}_{\mathrm{L}}(\mathrm{PM})$ & & MAY & 11 & 23:29 HST \\
\hline MAY & 12 & 104834.8 & $51.161 \mathrm{~N}$ & 175.983W. & 33 & GS & 4.2 & - & $4.5 \mathrm{M}_{\mathrm{L}}(\mathrm{PM})$ & & MAY & 12 & 00:48 HST \\
\hline MAY & 12 & 113745.5 & $51.154 \mathrm{~N}$ & 175.444W. & 20 & EE & 4.7 & - & $4.2 \mathrm{M}_{\mathrm{L}}(\mathrm{PM})$ & & MAY & 12 & 01:37 HST \\
\hline MAY & 12 & 141835.0 & $51.155 \mathrm{~N}$ & $176.084 \mathrm{~W}$. & 33 & GS & 3.8 & - & - & & MAY & 12 & 04:18 HST \\
\hline MAY & 12 & 170644.7 & $51.215 \mathrm{~N}$ & 174.794W. & 20 & $\mathrm{EE}$ & 4.7 & - & - & & MAY & 12 & 07:06 HST \\
\hline MAY & 12 & 201645.8 & $52.700 \mathrm{~N}$ & $172.432 \mathrm{E}$ & 33 & GS & 4.9 & 5.1 & - & III & MAY & 12 & 10:16 HST \\
\hline MAY & 12 & 202305.6 & $51.169 \mathrm{~N}$ & $174.550 \mathrm{~W}$ & 20 & EE & 5.0 & 5 & $5.0 \mathrm{M}_{\mathrm{L}}(\mathrm{PM})$ & & MAY & 12 & 10:23 HST \\
\hline MAY & 12 & 230839.4 & $57.685 \mathrm{~N}$ & 137.965W. & 5 & GM & - & - & $3.8 \mathrm{M}_{\mathrm{L}}(\mathrm{EP})$ & (- & MAY & 12 & 14:08 AST \\
\hline MAY & 13 & 015137.8 & $59.282 \mathrm{~N}$ & 153.776W. & 116 & GM & - & $\longrightarrow$ & - & & MAY & 12 & 16:51 AST \\
\hline MAY & 13 & 033337.0 & $51.741 \mathrm{~N}$ & 175.116W. & 33 & GS & 4.3 & - & - & & MAY & 12 & 17:33 HST \\
\hline MAY & 13 & 055624.0 & $51.246 \mathrm{~N}$ & $174.709 \mathrm{~W}$. & 20 & $\mathrm{EE}$ & 4.7 & - & - & & MAY & 12 & 19:56 HST \\
\hline MAY & 13 & 121742.3 & $53.543 \mathrm{~N}$ & 165.268W. & 40 & LD & $\longrightarrow$ & $\longrightarrow$ & $3.3 m_{X}(L D)$ & & MAY & 13 & 03:17 AST \\
\hline MAY . & 13 & 141456.1 & $51.390 \mathrm{~N}$ & $173.457 \mathrm{~W}$ & 20 & EE & 4.8 & 4.3 & 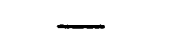 & & MAY & 13 & 04:14 HST \\
\hline MAY & 13 & 171520.8 & $50.957 \mathrm{~N}$ & 176.183W. & 20 & EE & 4.9 & 4.3 & & & MAY & 13 & 07:15 HST \\
\hline MAY & 14 & 015618.1 & $51.347 \mathrm{~N}$ & 173.401W. & 20 & $\mathrm{EE}$ & 4.8 & $\longrightarrow$ & $5.0 \mathrm{M}_{\mathrm{L}}(\mathrm{PM})$ & & MAY & 13 & 15:56 HST \\
\hline MAY & 14 & 015830.9 & $51.364 \mathrm{~N}$ & $173.437 \mathrm{~W}$ & 21 & $\mathrm{EE}$ & 5.5 & 4.7 & - & & MAY & 13 & 15:58 HST \\
\hline MAY & 14 & 032844.4 & $51.506 \mathrm{~N}$ & $175.771 \mathrm{~W}$. & 41 & $\mathrm{EE}$ & 4.8 & - & $4.2 \mathrm{M}_{\mathrm{L}}(\mathrm{PM})$ & & MAY & 13 & 17:28 HST \\
\hline MAY & 14 & 035424.9 & $51.350 \mathrm{~N}$ & 173.385W. & 20 & $\mathrm{EE}$ & 5.0 & - & $5.1 \mathrm{M}_{\mathrm{L}}(\mathrm{PM})$ & 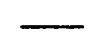 & MAY & 13 & 17:54 HST \\
\hline MAY & 14 & 040234.0 & $51.242 \mathrm{~N}$ & $178.405 \mathrm{~W}$ & 33 & $\mathrm{EE}$ & 5.4 & 4.6 & $4.9 \mathrm{M}_{\mathrm{L}}(\mathrm{PM})$ & & MAY & 13 & 18:02 HST \\
\hline MAY & 14 & 044910.9 & $51.321 \mathrm{~N}$ & 173.299W. & 20 & $\mathrm{EE}$ & 4.8 & - & $4.8 \mathrm{M}_{\mathrm{L}}(\mathrm{PM})$ & & MAY & 13 & 18:49 HST \\
\hline MAY & 14 & 075949.2 & $51.359 \mathrm{~N}$ & 173.442W. & 20 & EE & 4.8 & 4.1 & $5.0 \mathrm{M}_{\mathrm{L}}(\mathrm{PM})$ & & MAY & 13 & 21:59 HST \\
\hline MAY & 14 & 094900.7 & $56.127 \mathrm{~N}$ & $156.211 \mathrm{~W}$ & 99 & GS & 4.5 & - & - & & MAY & 14 & 00:49 AST \\
\hline MAY & 14 & 132832.8 & $51.149 \mathrm{~N}$. & $176.252 \mathrm{~W}$. & 33 & GS & 4.9 & - & - & & MAY & 14 & 03:28 HST \\
\hline MAY & 14 & 145825.8 & $51.334 \mathrm{~N}$ & 174.177W. & 20 & $\mathrm{EE}$ & 4.7 & 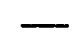 & $\longrightarrow$ & & MAY & 14 & 04:58 HST \\
\hline MAY & 14 & 201758.8 & $61.134 \mathrm{~N}$. & $152.027 \mathrm{~W}$. & 104 & GM & $\longrightarrow$ & - & $\bar{L}$ & & MAY & 14 & 11:17 AST \\
\hline MAY & 14 & 201917.5 & $51.400 \mathrm{~N}$ & 175.934W. & 35 & $\mathrm{EE}$ & 4.7 & & & & MAY & 14 & 10:19 HST \\
\hline MAY & 14 & 225853.3 & $51.340 \mathrm{~N}$ & 175.812W. & 33 & GS & 4.8 & - & - & & MAY & 14 & 12:58 HST \\
\hline MAY & 15 & 033009.8 & $51.172 \mathrm{~N}$ & $175.465 \mathrm{~W}$ & 20 & $\mathrm{EE}$ & 4.9 & - & $4.8 \mathrm{M}_{\mathrm{L}}(\mathrm{PM})$ & & MAY & 14 & 17:30 HST \\
\hline MAY & 15 & 063837.9 & $52.432 \mathrm{~N}$ & 174.719W. & 15 & EE & 5.7 & 6.4 & $5.5 \mathrm{M}_{\mathrm{L}}(\mathrm{PM})$ & VI & MAY & 14 & 20:38 HST \\
\hline MAY & 15 & 064232.1 & $51.248 \mathrm{~N}$ & $174.552 W$. & 20 & EE & 5.1 & & $4.9 \mathrm{M}_{\mathrm{L}}(\mathrm{PM})$ & & MAY & 14 & 20:42 HST \\
\hline MAY & 15 & 070125.6 & $52.373 \mathrm{~N}$ & $174.587 \mathrm{~W}$. & 15 & $\mathrm{EE}$ & 4.7 & - & - & & MAY & 14 & 21:01 HST \\
\hline MAY & 15 & 070615.8 & $52.045 \mathrm{~N}$ & 174.671W. & 33 & GS & 4.4 & & - & & MAY & 14 & 21:06 HST \\
\hline MAY & 15 & 070804.3 & $51.873 \mathrm{~N}$ & $174.383 W$. & 33 & GS & 4.3 & & - & & MAY & 14 & 21:08 HST \\
\hline MAY & 15 & 071020.6 & $52.335 \mathrm{~N}$ & $174.828 \mathrm{~W}$. & 33 & GS & 3.8 & & - & & MAY & 14 & 21:10 HST \\
\hline MAY & 15 & 071107.8 & $52.372 \mathrm{~N}$ & $174.702 W$. & 15 & $\mathrm{EE}$ & 4.8 & & - & & MAY & 14 & 21:11 HST \\
\hline MAY & 15 & 081215.0 & $52.049 \mathrm{~N}$ & 174.778W. & 33 & GS & 4.0 & & $\longrightarrow$ & & MAY & 14 & 22:12 HST \\
\hline MAY & 15 & 081318.3 & $52.152 \mathrm{~N}$ & $174.473 W$ & 33 & GS & 4.2 & - & - & $\longrightarrow$ & MAY & 14 & 22:13 HST \\
\hline
\end{tabular}


Table 1. Summary of United States earthquakes for 1986-Continued

\begin{tabular}{|c|c|c|c|c|c|c|c|c|c|c|c|c|c|}
\hline \multirow{2}{*}{\multicolumn{2}{|c|}{ Date }} & \multirow{2}{*}{$\begin{array}{l}\text { Origin time } \\
\text { (UTC) } \\
\text { hr min sec }\end{array}$} & \multirow{2}{*}{$\begin{array}{l}\text { Latitude } \\
\left({ }^{\circ}\right)\end{array}$} & \multirow{2}{*}{$\begin{array}{l}\text { Longitude } \\
\text { (9) }\end{array}$} & \multirow{2}{*}{$\begin{array}{l}\text { Depth } \\
\text { (km) }\end{array}$} & \multirow{2}{*}{$\begin{array}{l}\text { Hypo- } \\
\text { center } \\
\text { Source }\end{array}$} & \multicolumn{3}{|c|}{ Magnitude } & \multirow{2}{*}{$\begin{array}{l}\text { Max. } \\
\text { inten- } \\
\text { sity }\end{array}$} & \multicolumn{3}{|c|}{ Local time } \\
\hline & & & & & & & $\mathrm{m}_{\mathbf{b}}$ & $\mathrm{M}_{\mathrm{s}}$ & Local & & Date & & zone \\
\hline MAY & 15 & 081811.1 & $52.247 \mathrm{~N}$ & $174.517 \mathrm{~W}$. & 33 & GS & 4.1 & - & - & & MAY & 14 & $22: 18 \mathrm{HST}$ \\
\hline MAY & 15 & 081847.9 & $52.473 \mathrm{~N}$ & $174.560 \mathrm{~W}$. & 15 & EE & 5.1 & 5.3 & $5.2 \mathrm{M}_{\mathrm{L}}(\mathrm{PM})$ & & MAY & 14 & $22: 18$ HST \\
\hline MAY & 15 & 124713.7 & $52.074 \mathrm{~N}$ & $174.460 \mathrm{~W}$. & 33 & GS & 3.8 & - & - & & MAY & 15 & 02:47 HST \\
\hline MAY & 15 & 141943.3 & $63.283 \mathrm{~N}$ & $150.326 \mathrm{~W}$. & 112 & GM & 一 & - & - & & MAY & 15 & 05:19 AST \\
\hline MAY & 15 & 160947.7 & $51.259 \mathrm{~N}$. & 175.129W. & 33 & GS & 4.0 & - & - & - & MAY & 15 & 06:09 HST \\
\hline MAY & 15 & 232113.2 & $54.693 \mathrm{~N}$. & $163.089 \mathrm{~W}$. & 3 & LD & 4.5 & - & $4.4 \mathrm{~m}_{\mathrm{X}}(\mathrm{LD})$ & FELT & MAY & 15 & $14: 21$ AST \\
\hline MAY & .16 & 122056.0 & $51.277 \mathrm{~N}$. & $175.286 \mathrm{~W}$. & 33 & GS & 4.3 & - & - & & MAY & 16 & 02:20 HST \\
\hline MAY & 16 & 155953.1 & $51.563 \mathrm{~N}$. & 175.183W. & 36 & EE & 5.0 & 4.3 & 4.7M $\mathrm{M}_{\mathrm{L}}(\mathrm{PM})$ & & MAY & 16 & 05:59 HST \\
\hline MAY & 16 & 173132.8 & $51.485 \mathrm{~N}$. & $175.134 \mathrm{~W}$. & 35 & EE & 4.6 & & $4.3 \mathrm{M}_{\mathrm{L}}(\mathrm{PM})$ & & MAY & 16 & 07:31 HST \\
\hline MAY & 16 & 221227.5 & $51.530 \mathrm{~N}$. & 175.233W. & 38 & EE & 4.8 & & $4.2 \mathrm{M}_{\mathrm{L}}(\mathrm{PM})$ & & MAY & 16 & 12:12 HST \\
\hline MAY & 17 & 032429.5 & $58.889 \mathrm{~N}$. & $152.952 \mathrm{~W}$. & 75 & GM & 4.7 & - & $4.0 \mathrm{M}_{\mathrm{L}}(\mathrm{PM})$ & - & MAY & 16 & 18:24 AST \\
\hline MAY & 17 & 063114.2 & $62.038 \mathrm{~N}$ & 149.730W. & 51 & GM & - & - & $2.6 \mathrm{M}_{\mathrm{L}}(\mathrm{PM})$ & FELT & MAY & 16 & 21:31 AST \\
\hline MAY & 17 & 080010.1 & $50.848 \mathrm{~N}$ & 176.104W. & 20 & EE & 4.7 & & - & & MAY & 16 & 22:00 HST \\
\hline MAY & 17 & 082650.0 & $51.409 \mathrm{~N}$. & $175.743 W$. & 33 & GS & 3.8 & - & & & MAY & 16 & 22:26 HST \\
\hline MAY & 18 & 061500.9 & $51.398 \mathrm{~N}$. & $175.058 \mathrm{~W}$. & 33 & GS & 4.4 & - & $3.9 \mathrm{M}_{\mathrm{L}}(\mathrm{PM})$ & & MAY & 17 & 20:15 HST \\
\hline MAY & 18 & 080118.0 & $51.407 \mathrm{~N}$. & 176.770W. & 36 & EE & 4.5 & - & $4.5 \mathrm{M}_{\mathrm{L}}(\mathrm{PM})$ & & MAY & 17 & 22:01 HST \\
\hline MAY & 18 & 142238.4 & $51.228 \mathrm{~N}$. & 174.722W. & 20 & EE & 5.1 & 4.4 & $4.4 \mathrm{M}_{\mathrm{L}}(\mathrm{PM})$ & & MAY & 18 & 04:22 HST \\
\hline MAY & 18 & 152643.4 & $51.502 \mathrm{~N}$. & $175.148 \mathrm{~W}$. & 35 & $\mathrm{EE}$ & 4.9 & 4.5 & $4.2 \mathrm{M}_{\mathrm{L}}(\mathrm{PM})$ & & MAY & 18 & 05:26 HST \\
\hline MAY & 18 & 163449.3 & $59.577 \mathrm{~N}$. & $152.957 \mathrm{~W}$. & 88 & GM & - & 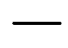 & - & & MAY & 18 & 07:34 AST \\
\hline MAY & 18 & 191400.9 & $51.367 \mathrm{~N}$. & $174.678 \mathrm{~W}$. & 25 & EE & 4.7 & & $4.7 \mathrm{M}_{\mathrm{L}}(\mathrm{PM})$ & & MAY & 18 & 09:14 HST \\
\hline MAY & 18 & 202426.7 & $51.350 \mathrm{~N}$. & $175.899 \mathrm{~W}$. & 33 & GS & 4.0 & & & & MAY & 18 & 10:24 HST \\
\hline MAY & 18 & 221907.7 & $52.252 \mathrm{~N}$ & $175.035 \mathrm{~W}$. & 33 & GS & 4.6 & - & - & & MAY & 18 & 12:19 HST \\
\hline MAY & 19 & 023734.7 & $52.359 \mathrm{~N}$ & 174.955W. & 15 & EE & 5.1 & 5.2 & $4.9 \mathrm{M}_{\mathrm{L}}(\mathrm{PM})$ & II & MAY & 18 & 16:37 HST \\
\hline MAY & 19 & 031905.9 & $52.397 \mathrm{~N}$ & $174.916 \mathrm{~W}$. & 15 & $\mathbf{E E}$ & 5.0 & - & $4.5 \mathrm{M}_{\mathrm{L}}(\mathrm{PM})$ & & MAY & 18 & 17:19 HST \\
\hline MAY & 19 & 054930.2 & $51.214 \mathrm{~N}$. & $175.527 \mathrm{~W}$ & 33 & GS & 4.8 & & - & & MAY & 18 & 19:49 HST \\
\hline MAY & 19 & 060359.7 & $60.216 \mathrm{~N}$ & $141.034 \mathrm{~W}$. & 5 & $\mathbf{G M}$ & - & & - & & MAY & 18 & 21:03 AST \\
\hline MAY & 19 & 065235.4 & $52.072 \mathrm{~N}$. & $174.434 \mathrm{~W}$. & 33 & GS & 4.0 & & - & & MAY & 18 & 20:52 HST \\
\hline MAY & 19 & 110434.4 & $61.964 \mathrm{~N}$ & $150.757 \mathrm{~W}$ & 67 & GM & - & - & - & & MAY & 19 & 02:04 AST \\
\hline MAY & 19 & 113548.8 & $52.122 \mathrm{~N}$ & $174.947 \mathrm{~W}$. & 33 & GS & 3.8 & - & $\longrightarrow$ & $\longrightarrow$ & MAY & 19 & 01:35 HST \\
\hline MAY & 19 & 202152.1 & $51.230 \mathrm{~N}$ & 179.348W. & 33 & GS & 4.7 & - & - & - & MAY & 19 & 10:21 HST \\
\hline
\end{tabular}


Table 1. Summary of United States earthquakes for 1986 - Continued

\begin{tabular}{|c|c|c|c|c|c|c|c|c|c|c|c|c|c|}
\hline \multirow[t]{2}{*}{ Date } & & \multirow{2}{*}{$\begin{array}{l}\text { Origin time } \\
\text { (UTC) } \\
\text { hr min sec }\end{array}$} & \multirow{2}{*}{$\begin{array}{c}\text { Latitude } \\
()^{\circ}\end{array}$} & \multirow{2}{*}{$\begin{array}{c}\text { Longitude } \\
(9 \\
\end{array}$} & \multirow{2}{*}{$\begin{array}{l}\text { Depth } \\
(\mathrm{km})\end{array}$} & \multirow{2}{*}{$\begin{array}{l}\text { Hypo- } \\
\text { center } \\
\text { Source }\end{array}$} & \multicolumn{3}{|c|}{ Magnitude } & \multirow{2}{*}{$\begin{array}{c}\text { Max. } \\
\text { inten- } \\
\text { sity } \\
\end{array}$} & \multicolumn{3}{|c|}{ Local time } \\
\hline & & & & & & & $\mathbf{m}_{\mathrm{b}}$ & $\mathrm{M}_{\mathrm{s}}$ & Local & & Date & & zone \\
\hline \multicolumn{14}{|c|}{ ALASKA-Continued } \\
\hline MAY & 19 & 205250.1 & $51.232 \mathrm{~N}$ & $174.849 \mathrm{~W}$ & 20 & EE & 4.9 & - & - & - & MAY & 19 & 10:52 HST \\
\hline MAY & 19 & 232646.2 & $51.801 \mathrm{~N}$ & 179.712W. & 33 & GS & 5.2 & - & - & - & MAY & 19 & 13:26 HST \\
\hline MAY & 20 & 020157.0 & $51.378 \mathrm{~N}$ & $175.080 \mathrm{~W}$ & 33 & GS & 4.0 & - & - & $\longrightarrow$ & MAY & 19 & 16:01 HST \\
\hline MAY & 20 & 101227.8 & $51.596 \mathrm{~N}$ & 175.070W. & 33 & GS & 4.3 & - & - & $\longrightarrow$ & MAY & 20 & 00:12 HST \\
\hline MAY & 20 & 105320.3 & $51.196 \mathrm{~N}$ & $175.198 \mathrm{~W}$ & 20 & $\mathrm{EE}$ & 4.9 & - & - & - & MAY & 20 & 00:53 HST \\
\hline MAY & 20 & 110831.1 & $51.003 \mathrm{~N}$ & $176.351 \mathrm{~W}$ & 33 & GS & 4.8 & - & - & $\longrightarrow$ & MAY & 20 & 01:08 HST \\
\hline MAY & 20 & 114959.9 & $51.346 \mathrm{~N}$ & $175.024 \mathrm{~W}$ & 33 & GS & 4.0 & - & - & - & MAY & 20 & 01:49 HST \\
\hline MAY & 20 & 115017.2 & $51.146 \mathrm{~N}$ & $175.082 \mathrm{~W}$ & 20 & EE & 4.6 & - & - & & MAY & 20 & 01:50 HST \\
\hline MAY & 20 & 134834.4 & $50.889 \mathrm{~N}$ & $176.448 \mathrm{~W}$. & 20 & $\mathrm{EE}$ & 4.8 & - & - & 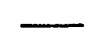 & MAY & 20 & 03:48 HST \\
\hline MAY & 20 & 141529.2 & $63.010 \mathrm{~N}$ & $150.480 \mathrm{~W}$ & 122 & GS & - & - & - & 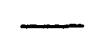 & MAY & 20 & 05:15 AST \\
\hline MAY & 20 & 144017.7 & $51.025 \mathrm{~N}$ & 176.391W. & 33 & GS & 4.7 & - & - & - & MAY & 20 & 04:40 HST \\
\hline MAY & 20 & 181006.8 & $51.360 \mathrm{~N}$ & $175.912 \mathrm{~W}$ & 33 & GS & 3.8 & - & - & - & MAY & 20 & 08:10 HST \\
\hline MAY & 20 & 204136.7 & $51.703 \mathrm{~N}$ & 179.564W. & 33 & GS & 4.2 & - & - & - & MAY & 20 & 10:41 HST \\
\hline MAY & 21 & 021409.2 & $65.483 \mathrm{~N}$ & $141.302 W$ & 33 & GS & 3.7 & - & $3.5 \mathrm{M}_{\mathrm{L}}(\mathrm{PM})$ & & MAY & 20 & $17: 14$ AST \\
\hline MAY & 21 & 041114.3 & $59.171 N$ & $153.635 \mathrm{~W}$ & 107 & GM & - & - & - & . & MAY & 20 & 19:11 AST \\
\hline MAY & 21 & 070515.7 & $51.351 \mathrm{~N}$ & $176.214 \mathrm{~W}$ & 33 & EE & 4.6 & - & $4.4 \mathrm{M}_{\mathrm{L}}(\mathrm{PM})$ & III & MAY & 20 & 21:05 HST \\
\hline MAY & 21 & 091413.0 & $52.669 \mathrm{~N}$ & $161.203 \mathrm{~W}$ & 4 & LD & - & - & 3.4 $\mathrm{m}_{\mathrm{X}}(\mathrm{LD})$ & & MAY & 21 & 00:14 AST \\
\hline MAY & 21 & 123853.4 & $59.780 \mathrm{~N}$ & $153.635 \mathrm{~W}$ & 135 & GM & - & - & - & - & MAY & 21 & 03:38 AST \\
\hline MAY & 21 & 173007.0 & $51.423 \mathrm{~N}$ & $175.988 \mathrm{~W}$ & 35 & EE & 4.8 & 4.7 & - & - & MAY & 21 & 07:30 HST \\
\hline $\begin{array}{c}\text { MAY } \\
-\end{array}$ & 21 & 221220.9 & $51.647 \mathrm{~N}$ & $175.325 \mathrm{~W}$ & 46 & EE & 4.8 & - & $4.6 \mathrm{M}_{\mathrm{L}}(\mathrm{PM})$ & II & MAY & 21 & 12:12 HST \\
\hline MAY & 22 & 072435.9 & $61.889 \mathrm{~N}$ & $147.357 \mathrm{~W}$ & 30 & GM & 3.5 & - & $3.8 \mathrm{M}_{\mathrm{L}}(\mathrm{PM})$ & 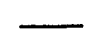 & MAY & 21 & 22:24 AST \\
\hline MAY & 22 & 114813.5 & $51.580 \mathrm{~N}$ & 175.683W. & 33 & GS & 4.5 & - & - & $\longrightarrow$ & MAY & 22 & 01:48 HST \\
\hline MAY & 22 & 144929.0 & $53.526 \mathrm{~N}$ & $164.698 \mathrm{~W}$ & 40 & $L D$ & 4.5 & - & $4.0 \mathrm{M}_{\mathrm{L}}(\mathrm{PM})$ & $\longrightarrow$ & MAY & 22 & 05:49 AST \\
\hline MAY & 22 & 145557.5 & $62.204 \mathrm{~N}$ & $148.887 \mathrm{~W}$ & 46 & GM & - & - & - & - & MAY & 22 & 05:55 AST \\
\hline MAY & 22 & 161800.2 & $50.394 \mathrm{~N}$ & 174.985W. & 33 & GS & 4.8 & - & - & & MAY & 22 & 06:18 HST \\
\hline MAY & 22 & 162452.3 & $51.201 \mathrm{~N}$ & $175.582 \mathrm{~W}$ & 33 & GS & 4.5 & - & - & $\longrightarrow$ & MAY & 22 & 06:24 HST \\
\hline MAY & 22 & 223255.2 & $54.799 \mathrm{~N}$ & 163.644W. & 118 & LD & - & - & $3.2 \mathrm{~m}_{\mathrm{X}}(\mathrm{LD})$ & & MAY & 22 & 13:32 AST \\
\hline MAY & 23 & 071906.4 & $51.139 \mathrm{~N}$ & 176.126W. & 33 & GS & 4.9 & - & - & & MAY & 22 & 21:19 HST \\
\hline MAY & 23 & 084526.0 & $62.988 \mathrm{~N}$ & $150.456 \mathrm{~W}$ & 115 & GS & 3.6 & - & - & $\ldots$ & MAY & 22 & 23:45 AST \\
\hline MAY & 23 & 231842.2 & $58.906 \mathrm{~N}$ & $153.377 \mathrm{~W}$ & 80 & GS & 5.0 & - & - & III & MAY & 23 & $14: 18$ AST \\
\hline MAY & 23 & 234000.3 & $51.257 \mathrm{~N}$ & 178.993E. & 33 & GS & 4.6 & - & $4.1 \mathrm{M}_{\mathrm{L}}(\mathrm{PM})$ & - & MAY & 23 & $13: 40$ HST \\
\hline MAY & 24 & 080753.3 & $63.096 \mathrm{~N}$ & $150.911 \mathrm{~W}$ & 129 & GS & 4.2 & - & - & - & MAY & 23 & 23:07 AST \\
\hline MAY & 24 & 132742.4 & $58.701 \mathrm{~N}$ & $155.325 \mathrm{~W}$ & 4 & GM & - & - & - & - & MAY & 24 & 04:27 AST \\
\hline MAY & 24 & 134547.7 & $61.179 \mathrm{~N}$ & $152.060 \mathrm{~W}$ & 93 & GM & - & - & - & - & MAY & 24 & 04:45 AST \\
\hline MAY & 24 & 164458.3 & $50.909 \mathrm{~N}$ & $176.202 \mathrm{~W}$ & 20 & $\mathrm{EE}$ & 4.6 & - & - & - & MAY & 24 & 06:44 HST \\
\hline MAY & 24 & 184049.4 & $52.183 \mathrm{~N}$ & 174.795W. & 15 & $\mathrm{EE}$ & 4.6 & - & $3.5 \mathrm{M}_{\mathrm{L}}(\mathrm{PM})$ & - & MAY & 24 & 08:40 HST \\
\hline MAY & 25 & 003304.6 & $51.341 \mathrm{~N}$ & $175.188 \mathrm{~W}$ & 25 & EE & 5.0 & 4.3 & - & $\longrightarrow$ & MAY & 24 & 14:33 HST \\
\hline MAY & 25 & 071658.9 & $50.884 \mathrm{~N}$ & $176.015 \mathrm{~W}$ & 20 & $\mathrm{EE}$ & 4.8 & - & $3.8 \mathrm{M}_{\mathrm{L}}(\mathrm{PM})$ & 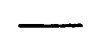 & MAY & 24 & 21:16 HST \\
\hline MAY & 25 & 125627.9 & $54.661 \mathrm{~N}$ & 161.706W. & 22 & LD & - & - & $3.2 \mathrm{~m}_{\mathrm{X}}(\mathrm{LD})$ & - & MAY & 25 & 03:56 AST \\
\hline MAY & 25 & 192310.4 & $51.148 \mathrm{~N}$ & $176.142 W$ & 20 & EE & 4.7 & - & $4.0 \mathrm{M}_{\mathrm{L}}(\mathrm{PM})$ & - & MAY & 25 & 09:23 HST \\
\hline
\end{tabular}


Table 1. Summary of United States earthquakes for 1986-Continued

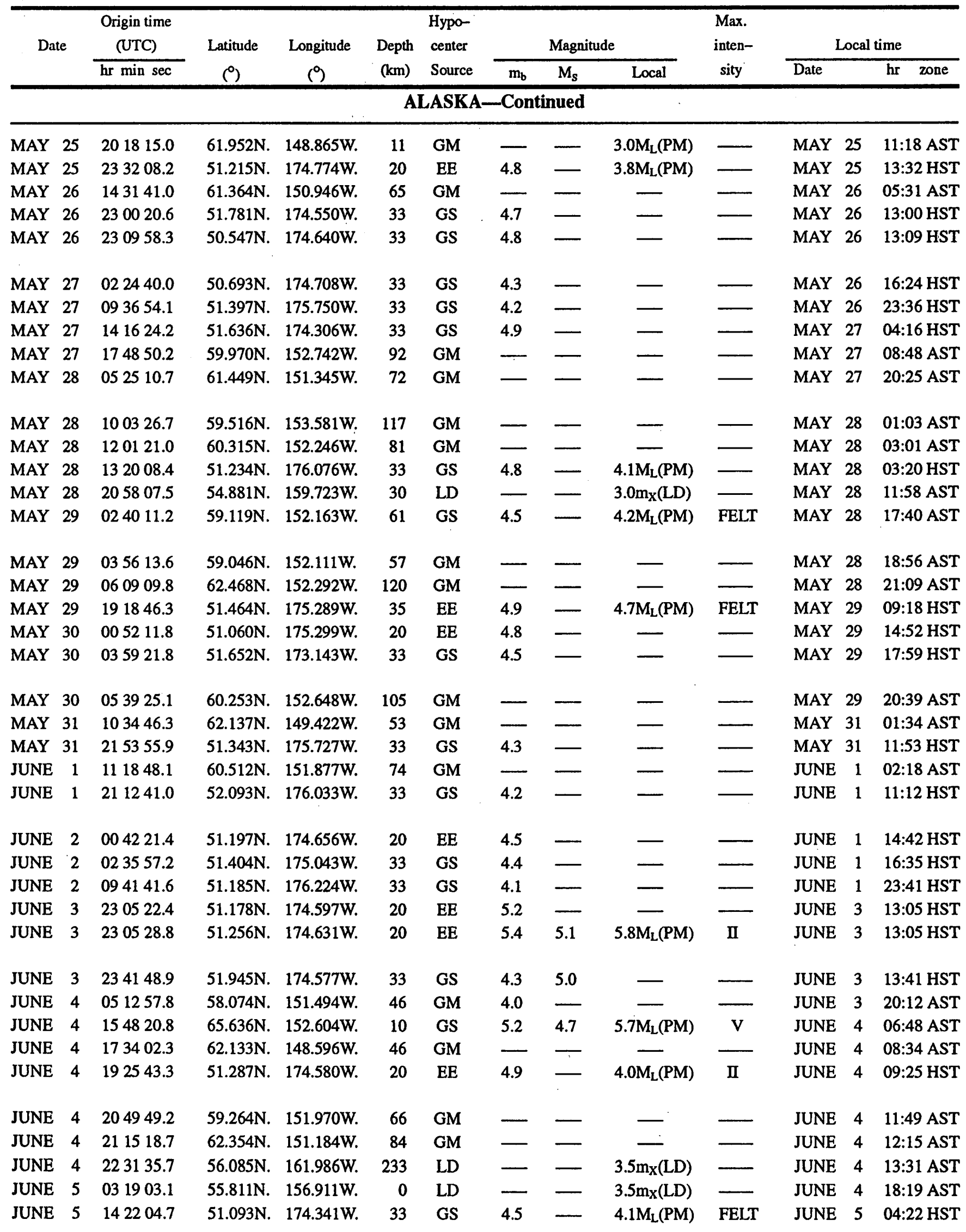


Table 1. Summary of United States earthquakes for 1986_Continued

\begin{tabular}{|c|c|c|c|c|c|c|c|c|c|c|c|c|c|}
\hline \multirow[t]{2}{*}{ Date } & & \multirow{2}{*}{$\begin{array}{l}\text { Origin time } \\
\text { (UTC) } \\
\text { hr min sec }\end{array}$} & \multirow{2}{*}{$\begin{array}{l}\text { Latitude } \\
\text { ()) }\end{array}$} & \multirow{2}{*}{$\begin{array}{l}\text { Longitude } \\
(9)\end{array}$} & \multirow{2}{*}{$\begin{array}{l}\text { Depth } \\
(\mathbf{k m})\end{array}$} & \multirow{2}{*}{$\begin{array}{l}\text { Hypo- } \\
\text { center } \\
\text { Source }\end{array}$} & \multicolumn{3}{|c|}{ Magnitude } & \multirow{2}{*}{$\begin{array}{l}\text { Max. } \\
\text { inten- } \\
\text { sity }\end{array}$} & \multicolumn{3}{|c|}{ Local time } \\
\hline & & & & & & & $\mathrm{m}_{\mathrm{b}}$ & $\mathrm{M}_{\boldsymbol{s}}$ & Local & & Date & & hr zone \\
\hline \multicolumn{14}{|c|}{ ALASKA-Continued } \\
\hline JUNE & 5 & 152453.7 & $51.138 \mathrm{~N}$ & 174.177W. & 20 & EE & 4.7 & - & $4.2 \mathrm{M}_{\mathrm{L}}(\mathrm{PM})$ & FELT & JUNE & 5 & 05:24 HST \\
\hline JUNE & 5 & 153919.1 & $61.775 \mathrm{~N}$ & $151.970 \mathrm{~W}$. & 108 & GM & - & - & - & - & JUNE & 5 & 06:39 AST \\
\hline JUNE & 5 & 173240.6 & $51.094 \mathrm{~N}$ & 175.350W. & 20 & EE & 5.1 & - & $4.5 \mathrm{M}_{\mathrm{L}}(\mathrm{PM})$ & FELT & JUNE & 5 & 07:32 HST \\
\hline JUNE & 5 & 191616.1 & $59.980 \mathrm{~N}$ & $152.302 \mathrm{~W}$ & 60 & GM & - & - & - & - & JUNE & 5 & 10:16 AST \\
\hline JUNE & 5 & 202703.2 & $51.296 \mathrm{~N}$. & $174.210 \mathrm{~W}$ & 22 & $\mathrm{EE}$ & 5.4 & 4.8 & $4.5 \mathrm{M}_{\mathrm{L}}(\mathrm{PM})$ & FELT & JUNE & 5 & 10:27 HST \\
\hline JUNE & 5 & 223503.7 & $56.090 \mathrm{~N}$ & $160.351 \mathrm{~W}$ & 163 & LD & - & - & $3.1 \mathrm{~m}_{\mathrm{X}}(\mathrm{LD})$ & - & JUNE & 5 & 13:35 AST \\
\hline JUNE & 5 & 231309.7 & $58.772 \mathrm{~N}$ & $152.521 \mathrm{~W}$ & 60 & GM & - & - & - & & JUNE & 5 & $14: 13$ AST \\
\hline JUNE & 5 & 233308.6 & $53.541 \mathrm{~N}$ & 164.096W. & 9 & LD & - & - & $3.2 \mathrm{~m}_{\mathrm{X}}(\mathrm{LD})$ & & JUNE & 5 & 14:33 AST \\
\hline JUNE & 6 & 034646.4 & $59.736 \mathrm{~N}$. & $153.398 \mathrm{~W}$. & 146 & GS & 4.3 & - & - & - & JUNE & 5 & 18:46 AST \\
\hline JUNE & 6 & 071101.9 & $63.252 \mathrm{~N}$ & 149.250W. & 87 & GS & - & - & $3.6 \mathrm{M}_{\mathrm{L}}(\mathrm{PM})$ & - & JUNE & 5 & 22:11 AST \\
\hline JUNE & 6 & 162737.6 & $61.852 \mathrm{~N}$ & $150.815 \mathrm{~W}$ & 64 & GM & - & - & $3.0 \mathrm{M}_{\mathrm{L}}(\mathrm{PM})$ & 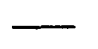 & JUNE & 6 & 07:27 AST \\
\hline JUNE & 6 & 204713.1 & $51.750 \mathrm{~N}$ & $174.724 \mathrm{~W}$. & 33 & GS & 4.4 & - & - & & JUNE & 6 & 10:47 HST \\
\hline JUNE & 7 & 155900.8 & $51.334 \mathrm{~N}$ & $174.843 \mathrm{~W}$. & 25 & EE & 4.8 & 4.6 & $4.6 \mathrm{M}_{\mathrm{L}}(\mathrm{PM})$ & & JUNE & 7 & 05:59 HST \\
\hline JUNE & 7 & 185420.7 & $58.012 \mathrm{~N}$ & $151.565 \mathrm{~W}$ & 91 & GM & - & - & - & $\longrightarrow$ & JUNE & 7 & 09:54 AST \\
\hline JUNE & 7. & 195056.2 & $52.518 \mathrm{~N}$ & $162.864 \mathrm{~W}$ & 6 & LD & $\div$ & - & $3.2 \mathrm{~m}_{\mathrm{X}}(\mathrm{LD})$ & . & JUNE & 7 & 10:50 AST \\
\hline JUNE & 7 & 210641.7 & $51.221 \mathrm{~N}$ & 179.992E. & 33 & GS & 5.0 & 4.1 & $4.6 \mathrm{M}_{\mathrm{L}}(\mathrm{PM})$ & - & JUNE & 7 & 11:06 HST \\
\hline JUNE & 8 & 051759.1 & $51.299 \mathrm{~N}$. & $174.768 \mathrm{~W}$. & 20 & $\mathrm{EE}$ & 4.8 & - & - & & JUNE & 7 & 19:17 HST \\
\hline JUNE & 8 & 113551.3 & $60.964 \mathrm{~N}$ & $152.488 \mathrm{~W}$ & 122 & GM & - & - & - & & JUNE & 8 & 02:35 AST \\
\hline JUNE & 8 & 124513.7 & $62.191 \mathrm{~N}$ & $150.199 \mathrm{~W}$ & 15 & GM & 一 & - & $3.2 \mathrm{M}_{\mathrm{L}}(\mathrm{PM})$ & & JUNE & 8 & 03:45 AST \\
\hline JUNE & 8 & 164307.0 & $50.858 \mathrm{~N}$. & $177.466 \mathrm{~W}$ & 20 & $\mathrm{EE}$ & 4.7 & - & - & - & JUNE & 8 & 06:43 HST \\
\hline JUNE & 8 & 170247.1 & $59.454 \mathrm{~N}$ & $153.662 \mathrm{~W}$ & 114 & GM & - & - & - & - & JUNE & 8 & 08:02 AST \\
\hline JUNE & 9 & 021738.2 & $54.142 \mathrm{~N}$. & $168.132 W$ & 33 & GS & 5.0 & 4.7 & $5.6 \mathrm{M}_{\mathrm{L}}(\mathrm{PM})$ & & JUNE & 8 & 17:17 AST \\
\hline JUNE & 9 & 135657.6 & $60.035 \mathrm{~N}$ & $153.528 \mathrm{~W}$ & 147 & GM & 一 & - & - & - & JUNE & 9 & 04:56 AST \\
\hline JUNE & 10 & 031724.5 & $55.889 \mathrm{~N}$ & 161.765W. & 192 & LD & - & - & $3.5 \mathrm{~m}_{\mathrm{X}}(\mathrm{LD})$ & & JUNE & 9 & 18:17 AST \\
\hline JUNE & 10 & 053309.0 & $51.277 \mathrm{~N}$ & $176.001 \mathrm{~W}$ & 33 & GS & 4.3 & - & - & & JUNE & 9 & 19:33 HST \\
\hline JUNE & 10 & 095846.0 & $61.005 \mathrm{~N}$ & $147.230 \mathrm{~W}$ & 16 & GM & - & 一 & - & . & JUNE & 10 & 00:58 AST \\
\hline JUNE & 10 & 120842.9 & $60.636 \mathrm{~N}$ & $150.406 \mathrm{~W}$ & 42 & GM & - & - & $3.4 \mathrm{M}_{\mathrm{L}}(\mathrm{PM})$ & $\longrightarrow$ & JUNE & 10 & 03:08 AST \\
\hline JUNE & 10 & 151546.0 & $51.572 \mathrm{~N}$ & $176.016 \mathrm{~W}$ & 44 & $\mathrm{EE}$ & 4.5 & - & $4.4 \mathrm{M}_{\mathrm{L}}(\mathrm{PM})$ & 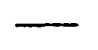 & JUNE & 10 & 05:15 HST \\
\hline JUNE & 10 & 175723.6 & $51.357 \mathrm{~N}$. & 175.776W. & 33 & GS & 4.6 & 一 & $4.5 \mathrm{M}_{\mathrm{L}}(\mathrm{PM})$ & - & JUNE & 10 & 07:57 HST \\
\hline JUNE & 11 & 075303.6 & $60.544 \mathrm{~N}$ & $145.090 \mathrm{~W}$ & 12 & GM & - & 一 & - & - & JUNE & 10 & 22:53 AST \\
\hline JUNE & 11 & 101909.9 & $62.219 \mathrm{~N}$ & $149.962 \mathrm{~W}$ & 65 & GM & - & 一 & - & 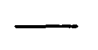 & JUNE & 11 & 01:19 AST \\
\hline JUNE & 12 & 000937.8 & $52.534 \mathrm{~N}$ & 176.220W. & 33 & GS & 4.4 & - & - & & JUNE & 11 & 14:09 HST \\
\hline JUNE 1 & 12 & 013248.6 & $54.859 \mathrm{~N}$. & $160.284 \mathrm{~W}$ & 33 & LD & - & - & $3.2 \mathrm{~m}_{\mathrm{X}}(\mathrm{LD})$ & - & JUNE & 11 & $16: 32$ AST \\
\hline JUNE 1 & 12 & 175755.0 & $62.379 \mathrm{~N}$ & 151.781W. & 108 & GM & - & - & - & - & JUNE & 12 & 08:57 AST \\
\hline JUNE 1 & 13 & 082847.4 & $53.325 \mathrm{~N}$ & $163.534 \mathrm{~W}$ & 19 & LD & 4.7 & - & $3.1 \mathrm{~m}_{\mathrm{X}}(\mathrm{LD})$ & - & JUNE & 12 & 23:28 AST \\
\hline JUNE 1 & 13 & 092550.0 & $53.289 \mathrm{~N}$ & 163.375W. & 13 & LD & 4.8 & - & $4.4 m_{X}(L D)$ & - & JUNE & 13 & 00:25 AST \\
\hline JUNE 1 & 13 & 132536.4 & $61.069 \mathrm{~N}$ & $151.057 \mathrm{~W}$. & 69 & GM & - & 一 & - & - & JUNE & 13 & 04:25 AST \\
\hline JUNE 1 & 13 & 140354.7 & $54.149 \mathrm{~N}$. & $164.355 \mathrm{~W}$ & 33 & GS & 4.7 & - & - & - & JUNE & 13 & 05:03 AST \\
\hline JUNE 1 & 13 & 200805.7 & $52.069 \mathrm{~N}$ & $178.038 \mathrm{E}$ & 106 & GS & 4.6 & - & - & - & JUNE & 13 & 10:08 HST \\
\hline JUNE 1 & 14 & 032651.4 & $56.033 \mathrm{~N}$. & 161.567W. & 202 & LD & 4.4 & - & $4.1 \mathrm{~m}_{\mathrm{X}}(\mathrm{LD})$ & - & JUNE & 13 & $18: 26$ AST \\
\hline
\end{tabular}


Table 1. Summary of United States earthquakes for 1986-Continued

\begin{tabular}{|c|c|c|c|c|c|c|c|c|c|c|c|c|c|}
\hline \multirow{2}{*}{\multicolumn{2}{|c|}{ Date }} & \multirow{2}{*}{$\begin{array}{l}\text { Origin time } \\
\text { (UTC) } \\
\text { hr min sec }\end{array}$} & \multirow{2}{*}{$\begin{array}{l}\text { Latitude } \\
\text { (?) }\end{array}$} & \multirow{2}{*}{$\begin{array}{l}\text { Longitude } \\
\text { ( } 9\end{array}$} & \multirow{2}{*}{$\begin{array}{l}\text { Depth } \\
(\mathrm{km})\end{array}$} & \multirow{2}{*}{$\begin{array}{l}\text { Hypo- } \\
\text { center } \\
\text { Source }\end{array}$} & \multicolumn{3}{|c|}{ Magnitude } & \multirow{2}{*}{$\begin{array}{l}\text { Max. } \\
\text { inten- } \\
\text { sity }\end{array}$} & \multicolumn{3}{|c|}{ Local time } \\
\hline & & & & & & & $\mathrm{m}_{\mathrm{b}}$ & $\mathbf{M}_{\mathbf{S}}$ & Local & & Date & & zone \\
\hline \multicolumn{14}{|c|}{ ALASKA-Continued } \\
\hline JUNE & 14 & 145456.7 & $60.626 \mathrm{~N}$ & $150.527 \mathrm{~W}$ & 54 & GM & - & 一 & - & - & JUNE & 14 & 05:54 AST \\
\hline JUNE & 14 & 221238.7 & $66.430 \mathrm{~N}$. & $149.756 \mathrm{~W}$ & 10 & GS & - & - & $3.2 \mathrm{M}_{\mathrm{L}}(\mathrm{PM})$ & $\longrightarrow$ & JUNE & 14 & 13:12 AST \\
\hline JUNE & 15 & 022252.9 & $51.396 \mathrm{~N}$. & $174.760 \mathrm{~W}$. & 33 & GS & 4.3 & - & $4.3 \mathrm{M}_{\mathrm{L}}(\mathrm{PM})$ & III & JUNE & 14 & $16: 22 \mathrm{HST}$ \\
\hline JUNE & 15 & 235416.8 & $51.587 \mathrm{~N}$. & $175.359 \mathrm{~W}$ & 33 & GS & 4.4 & 一 & - & - & JUNE & 15 & 13:54 HST \\
\hline JUNE & 16 & 010004.3 & $60.185 \mathrm{~N}$ & $152.532 \mathrm{~W}$. & 104 & GM & - & 一 & - & - & JUNE & 15 & $16: 00$ AST \\
\hline JUNE & 16 & 215402.0 & $61.838 \mathrm{~N}$. & 149.433W. & 43 & GM & - & - & $3.8 \mathrm{M}_{\mathrm{L}}(\mathrm{PM})$ & III & JUNE & 16 & 12:54 AST \\
\hline JUNE & 17 & 141039.2 & $59.791 \mathrm{~N}$. & $153.392 \mathrm{~W}$. & 118 & GM & - & - & - & - & JUNE & 17 & 05:10 AST \\
\hline JUNE & 17 & 160307.5 & $51.480 \mathrm{~N}$. & $175.186 \mathrm{~W}$. & 33 & GS & 4.5 & - & $4.5 \mathrm{M}_{\mathrm{L}}(\mathrm{PM})$ & 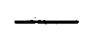 & JUNE & 17 & 06:03 HST \\
\hline JUNE & 17 & 174543.8 & 61.601N. & $151.008 \mathrm{~W}$ & 72 & GM & - & - & $3.0 \mathrm{M}_{\mathrm{L}}(\mathrm{PM})$ & $\longrightarrow$ & JUNE & 17 & 08:45 AST \\
\hline JUNE & 17 & 175947.9 & $61.282 \mathrm{~N}$ & $146.868 \mathrm{~W}$ & 32 & GM & - & - & - & - & JUNE & 17 & 08:59 AST \\
\hline JUNE & 18 & 033007.5 & $51.163 \mathrm{~N}$. & 173.793W. & 20 & $\mathrm{EE}$ & 4.6 & 4.9 & $\longrightarrow$ & - & JUNE & 17 & $17: 30 \mathrm{HST}$ \\
\hline JUNE & 18 & 043016.4 & $59.884 \mathrm{~N}$. & 153.180W. & 110 & GM & - & - & - & - & JUNE & 17 & 19:30 AST \\
\hline JUNE & 18 & 080516.4 & $51.465 \mathrm{~N}$. & $176.833 \mathrm{~W}$ & 41 & $\mathrm{EE}$ & 5.8 & 6.3 & $6.0 \mathrm{M}_{\mathrm{L}}(\mathrm{PM})$ & IV & JUNE & 17 & 22:05 HST \\
\hline JUNE & 18 & 103235.6 & $51.169 \mathrm{~N}$. & $176.015 \mathrm{~W}$ & 33 & GS & 4.5 & - & - & - & JUNE & 18 & 00:32 HST \\
\hline JUNE & 18 & 144620.8 & $50.945 \mathrm{~N}$. & 176.106W. & 20 & $\mathrm{EE}$ & 4.8 & - & - & - & JUNE & 18 & 04:46 HST \\
\hline JUNE & 18 & 182840.1 & $51.390 \mathrm{~N}$. & $175.950 \mathrm{~W}$ & 33 & GS & 4.2 & - & - & - & JUNE & 18 & 08:28 HST \\
\hline JUNE & 18 & 214749.8 & $51.613 \mathrm{~N}$. & $176.945 \mathrm{~W}$. & 33 & GS & 4.5 & - & - & - & JUNE & 18 & $11: 47$ HST \\
\hline JUNE & 18 & 231758.2 & $52.317 \mathrm{~N}$. & 179.615E. & 171 & GS & 5.1 & - & - & - & JUNE & 18 & 13:17 HST \\
\hline JUNE & 18 & 234304.4 & $63.067 \mathrm{~N}$. & $150.911 \mathrm{~W}$ & 131 & GS & - & - & - & FELT & JUNE & 18 & 14:43 AST \\
\hline JUNE & 19 & 023933.4 & $61.401 \mathrm{~N}$. & $150.664 \mathrm{~W}$ & 72 & GM & - & - & - & - & JUNE & 18 & 17:39 AST \\
\hline JUNE & 19 & 054056.8 & $53.253 \mathrm{~N}$. & 163.178W. & 21 & LD & 4.4 & 一 & $3.2 \mathrm{~m}_{\mathrm{X}}(\mathrm{LD})$ & - & JUNE & 18 & 20:41 AST \\
\hline JUNE & 19 & 090909.2 & $56.331 \mathrm{~N}$. & $152.914 \mathrm{~W}$ & 17 & GS & 6.0 & 6.3 & $5.4 \mathrm{M}_{\mathrm{L}}(\mathrm{PM})$ & IV & JUNE & 19 & 00:09 AST \\
\hline JUNE & 19 & 110837.3 & $56.303 \mathrm{~N}$. & $153.107 \mathrm{~W}$ & 33 & GS & 4.7 & - & $4.3 \mathrm{M}_{\mathrm{L}}(\mathrm{PM})$ & - & JUNE & 19 & 02:08 AST \\
\hline JUNE & 19 & 192558.4 & $50.251 \mathrm{~N}$. & $179.681 \mathrm{E}$ & 33 & GS & 4.4 & - & - & - & JUNE & 19 & 09:25 HST \\
\hline JUNE & 19 & 222838.8 & $54.644 \mathrm{~N}$. & $161.096 \mathrm{~W}$ & 15 & LD & 4.3 & - & $3.4 m_{x}(L D)$ & FELT & JUNE & 19 & 13:28 AST \\
\hline JUNE & 20 & 043629.8 & $56.482 \mathrm{~N}$. & $152.711 \mathrm{~W}$ & 33 & GS & 4.7 & 4.3 & $4.7 \mathrm{M}_{\mathrm{L}}(\mathrm{PM})$ & - & JUNE & 19 & 19:36 AST \\
\hline JUNE & 20 & 073932.8 & $60.676 \mathrm{~N}$. & $152.107 \mathrm{~W}$ & 82 & GM & - & - & $4.0 \mathrm{M}_{\mathrm{L}}(\mathrm{PM})$ & FELT & JUNE & 19 & 22:39 AST \\
\hline JUNE & 20 & 221349.6 & $62.248 \mathrm{~N}$. & $150.234 \mathrm{~W}$ & 10 & GM & 一 & - & $3.8 \mathrm{M}_{\mathrm{L}}(\mathrm{PM})$ & FELT & JUNE & 20 & 13:13 AST \\
\hline JUNE & 21 & 013228.2 & $50.828 \mathrm{~N}$. & $178.689 \mathrm{~W}$ & 20 & EE & 4.9 & 4.5 & $4.8 \mathrm{M}_{\mathrm{L}}(\mathrm{PM})$ & - & JUNE & 20 & 15:32 HST \\
\hline JUNE & 21 & 120730.2 & $59.947 \mathrm{~N}$. & $152.851 \mathrm{~W}$ & 101 & GS & 4.9 & - & - & FELT & JUNE & 21 & 03:07 AST \\
\hline JUNE & 21 & 130347.2 & $51.365 \mathrm{~N}$. & 175.881W. & 33 & GS & 4.5 & - & - & - & JUNE & 21 & 03:03 HST \\
\hline JUNE & 21 & 145457.5 & $55.698 \mathrm{~N}$. & 159.693W. & 80 & LD & - & - & $3.5 \mathrm{~m}_{\mathrm{X}}(\mathrm{LD})$ & $\longrightarrow$ & JUNE & 21 & 05:54 AST \\
\hline JUNE & 21 & 153853.8 & $51.237 \mathrm{~N}$. & 174.735W. & 20 & $\mathrm{EE}$ & 4.7 & 4.6 & $4.0 \mathrm{M}_{\mathrm{L}}(\mathrm{PM})$ & - & JUNE & 21 & 05:38 HST \\
\hline JUNE & 21 & 175823.1 & $59.313 \mathrm{~N}$. & $153.844 \mathrm{~W}$ & 121 & GM & - & - & - & - & JUNE & 21 & 08:58 AST \\
\hline JUNE & 21 & 192423.9 & $62.459 \mathrm{~N}$. & $151.744 \mathrm{~W}$ & 102 & GM & - & - & - & - & JUNE & 21 & $10: 24$ AST \\
\hline JUNE & 21 & 215825.9 & $66.113 \mathrm{~N}$. & $150.300 \mathrm{~W}$ & 10 & GS & 一 & 一 & $3.1 \mathrm{M}_{\mathrm{L}}(\mathrm{PM})$ & - & JUNE & 21 & 12:58 AST \\
\hline JUNE & 22 & 052852.4 & $51.108 \mathrm{~N}$. & 175.170W. & 16 & EE & 4.9 & 4.9 & $4.3 \mathrm{M}_{\mathrm{L}}(\mathrm{PM})$ & FELT & JUNE & 21 & 19:28 HST \\
\hline JUNE & 22 & 053353.1 & $51.082 \mathrm{~N}$. & $175.152 \mathrm{~W}$ & 33 & GS & 4.4 & - & - & - & JUNE & 21 & 19:33 HST \\
\hline JUNE & 22 & 053707.0 & $51.288 \mathrm{~N}$. & $175.259 \mathrm{~W}$ & 25 & $\mathrm{EE}$ & 4.8 & - & - & - & JUNE & 21 & 19:37 HST \\
\hline JUNE & 22 & 060337.2 & $52.077 \mathrm{~N}$. & $175.135 \mathrm{~W}$ & 33 & GS & 4.6 & - & - & - & JUNE & 21 & 20:03 HST \\
\hline
\end{tabular}


Table 1. Summary of United States earthquakes for 1986-Continued

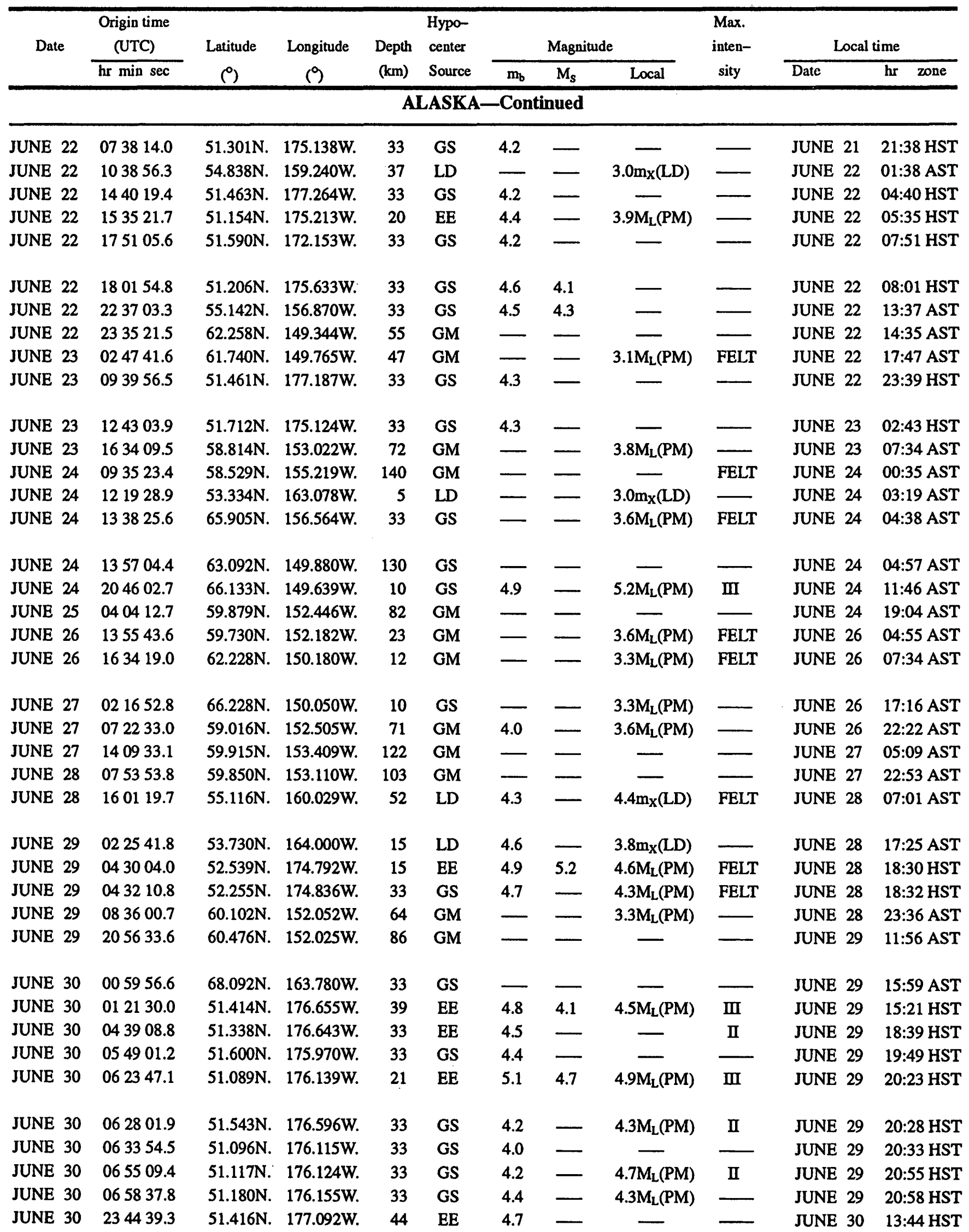


Table 1. Summary of United States earthquakes for 1986-Continued

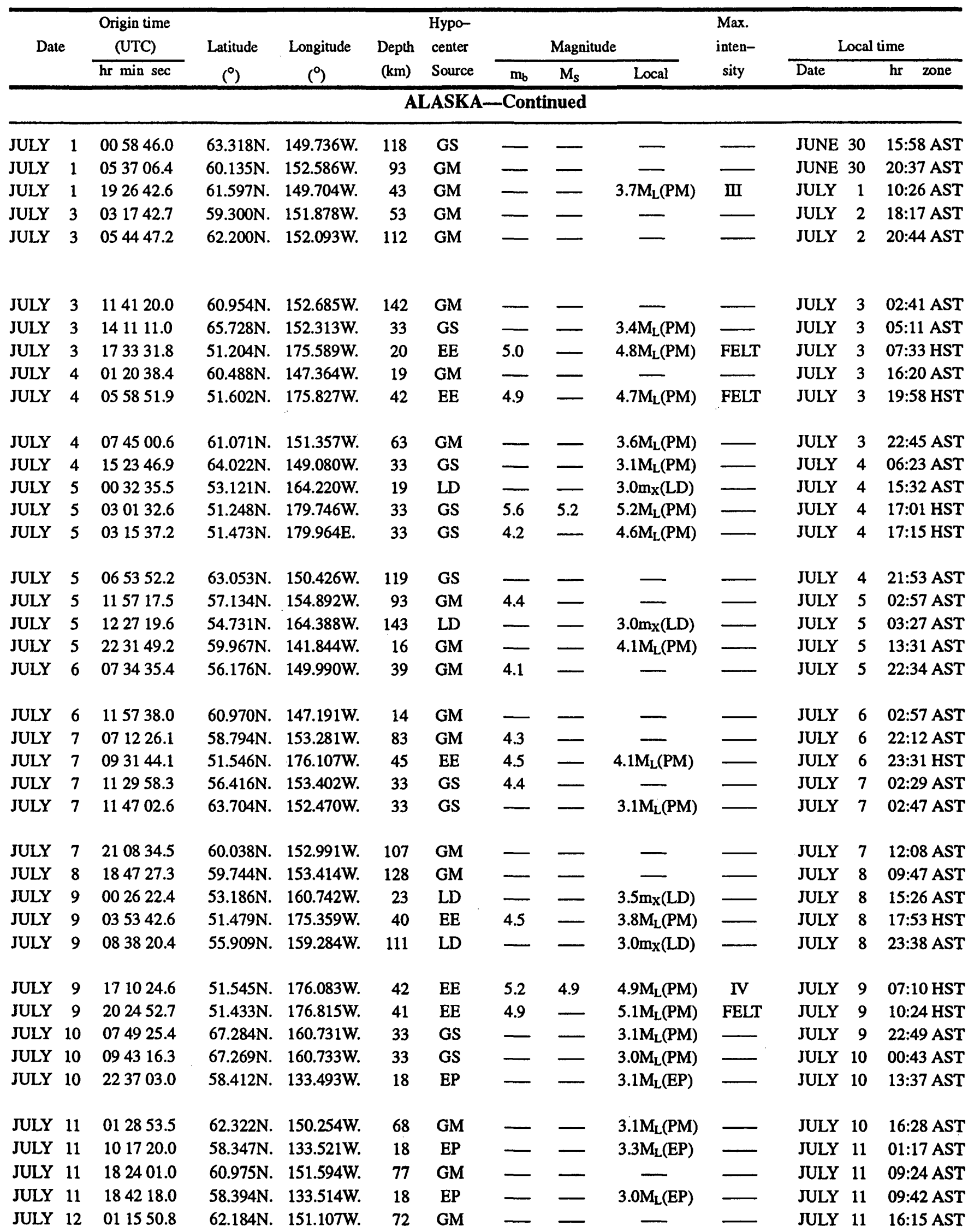


Table 1. Summary of United States earthquakes for 1986-Continued

\begin{tabular}{|c|c|c|c|c|c|c|c|c|c|c|c|c|c|}
\hline \multirow{2}{*}{\multicolumn{2}{|c|}{ Date }} & \multirow{3}{*}{$\begin{array}{l}\text { Origin time } \\
\text { (UTC) } \\
\text { hr min sec }\end{array}$} & \multirow{2}{*}{$\begin{array}{l}\text { Latitude } \\
\left({ }^{\circ}\right)\end{array}$} & \multirow{2}{*}{$\begin{array}{c}\text { Longitude } \\
\text { ( })\end{array}$} & \multirow{2}{*}{$\begin{array}{l}\text { Depth } \\
(\mathbf{k m})\end{array}$} & \multirow{2}{*}{$\begin{array}{l}\text { Hypo- } \\
\text { center } \\
\text { Source }\end{array}$} & \multicolumn{3}{|c|}{ Magnitude } & \multirow{3}{*}{$\begin{array}{l}\text { Max. } \\
\text { inten- } \\
\text { sity }\end{array}$} & \multicolumn{3}{|c|}{ Local time } \\
\hline & & & & & & & $\mathrm{m}_{\mathrm{b}}$ & $\mathbf{M}_{\mathbf{S}}$ & Local & & Date & & zone \\
\hline \multicolumn{12}{|c|}{ ALASKA-Continued } & & \\
\hline JULY & 13 & 011314.9 & $65.721 \mathrm{~N}$ & 152.274W. & 33 & GS & - & - & $3.0 \mathrm{M}_{\mathrm{L}}(\mathrm{PM})$ & - & JULY & 12 & 16:13 AST \\
\hline JULY & 13 & 035440.5 & $62.254 \mathrm{~N}$ & $150.228 \mathrm{~W}$. & 11 & GM & - & - & $3.9 \mathrm{M}_{\mathrm{L}}(\mathrm{PM})$ & FELT & JULY & 12 & 18:54 AST \\
\hline JULY & 13 & 035802.5 & $62.216 \mathrm{~N}$ & $150.286 \mathrm{~W}$. & 10 & GS & - & 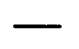 & $3.0 \mathrm{M}_{\mathrm{L}}(\mathrm{PM})$ & FELT & JULY & 12 & 18:58 AST \\
\hline JULY & 14 & 042909.1 & $60.633 \mathrm{~N}$ & $151.879 \mathrm{~W}$. & 69 & GM & - & & - & - & JULY & 13 & 19:29 AST \\
\hline JULY & 14 & 063715.8 & $59.872 \mathrm{~N}$ & $152.661 \mathrm{~W}$ & 77 & GM & - & & & & JULY & 13 & 21:37 AST \\
\hline JULY & 16 & 003816.6 & $60.062 \mathrm{~N}$ & 153.127W. & 106 & GM & - & - & - & - & JULY & 15 & 15:38 AST \\
\hline JULY & 17 & 010041.2 & $60.052 \mathrm{~N}$ & $153.448 \mathrm{~W}$. & 135 & GM & & & & & JULY & 16 & 16:00 AST \\
\hline JULY & 17 & 051211.0 & $58.173 \mathrm{~N}$ & $151.281 \mathrm{~W}$. & 45 & GM & & & & & JULY & 16 & 20:12 AST \\
\hline JULY & 17 & 164010.8 & $59.058 \mathrm{~N}$ & $152.689 \mathrm{~W}$. & 81 & GM & - & & & & JULY & 17 & 07:40 AST \\
\hline JULY & 17 & 183720.0 & $51.180 \mathrm{~N}$. & 174.494W. & 20 & $\mathrm{EE}$ & 4.8 & & & FELT & JULY & 17 & 08:37 HST \\
\hline JULY & 18 & 024448.6 & $62.081 \mathrm{~N}$ & 149.840W. & 52 & GM & - & - & - & - & JULY & 17 & 17:44 AST \\
\hline JULY & 18 & 182933.0 & $58.339 \mathrm{~N}$ & $133.563 \mathrm{~W}$. & 18 & EP & - & 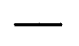 & $3.2 \mathrm{M}_{\mathrm{L}}(\mathrm{EP})$ & & JULY & 18 & 09:29 AST \\
\hline JULY & 18 & 220705.8 & $56.160 \mathrm{~N}$ & $152.762 \mathrm{~W}$. & 33 & GS & 4.2 & 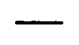 & $3.3 \mathrm{M}_{\mathrm{L}}(\mathrm{PM})$ & & JULY & 18 & 13:07 AST \\
\hline JULY & 19 & 014919.3 & $53.603 \mathrm{~N}$ & 167.273W. & 33 & GS & 4.6 & & $4.6 \mathrm{M}_{\mathrm{L}}(\mathrm{PM})$ & & JULY & 18 & 16:49 AST \\
\hline JULY & 19 & 022056.4 & $53.602 \mathrm{~N}$ & 167.291W. & 33 & GS & 4.4 & - & $4.4 \mathrm{M}_{\mathrm{L}}(\mathrm{PM})$ & & JULY & 18 & $17: 20 \mathrm{AST}$ \\
\hline JULY & 19 & 043155.9 & $53.352 \mathrm{~N}$ & $165.882 \mathrm{~W}$ & 33 & GS & 5.5 & 5.1 & $5.9 \mathrm{M}_{\mathrm{L}}(\mathrm{PM})$ & IV & JULY & 18 & 19:31 AST \\
\hline JULY & 19 & 050408.2 & $53.339 \mathrm{~N}$. & $165.859 \mathrm{~W}$ & 33 & GS & 5.1 & 4.5 & $5.6 \mathrm{M}_{\mathrm{L}}(\mathrm{PM})$ & IV & JULY & 18 & 20:04 AST \\
\hline JULY & 19 & 051833.3 & $60.136 \mathrm{~N}$ & $152.552 \mathrm{~W}$ & 87 & GM & - & $\longrightarrow$ & - & & JULY & 18 & 20:18 YST \\
\hline JULY & 19 & 065317.8 & $53.600 \mathrm{~N}$ & 167.171W. & 33 & GS & 5.5 & 5.7 & $5.8 \mathrm{M}_{\mathrm{L}}(\mathrm{PM})$ & IV & JULY & 18 & 21:53 AST \\
\hline JULY & 19 & 071610.6 & $53.403 \mathrm{~N}$ & $167.087 \mathrm{~W}$. & 33 & GS & 4.5 & - & $4.6 \mathrm{M}_{\mathrm{L}}(\mathrm{PM})$ & & JULY & 18 & 22:16 AST \\
\hline JULY & 19 & 075728.0 & $53.507 \mathrm{~N}$ & $167.242 \mathrm{~W}$ & 33 & GS & 4.8 & - & $4.4 \mathrm{M}_{\mathrm{L}}(\mathrm{PM})$ & & JULY & 18 & 22:57 AST \\
\hline JULY & 19 & 082024.4 & $53.527 \mathrm{~N}$. & 167.170W. & 33 & GS & 4.2 & - & 4.7M $\mathrm{M}(\mathrm{PM})$ & & JULY & 18 & 23:20 AST \\
\hline JULY & 19 & 111219.5 & $53.325 \mathrm{~N}$ & $164.915 \mathrm{~W}$. & 20 & LD & - & - & $4.2 \mathrm{~m}_{\mathrm{X}}(\mathrm{LD})$ & - & JULY & 19 & 02:12 AST \\
\hline JULY & 19 & 113107.5 & $53.617 \mathrm{~N}$ & $167.408 \mathrm{~W}$. & 33 & GS & 5.0 & 4.6 & $5.1 \mathrm{M}_{\mathrm{L}}(\mathrm{PM})$ & FELT & JULY & 19 & 02:31 AST \\
\hline JULY & 19 & 134255.5 & $51.499 \mathrm{~N}$ & 175.158W. & 33 & GS & 4.0 & $\longrightarrow$ & - & - & JULY & 19 & 03:42 HST \\
\hline JULY & 19 & 143559.2 & $62.114 \mathrm{~N}$ & $148.884 W$. & 40 & GM & - & - & 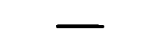 & - & JULY & 19 & 05:35 AST \\
\hline JULY & 19 & 173217.7 & $53.496 \mathrm{~N}$ & 167.248W. & 33 & GS & 4.4 & - & & - & JULY & 19 & 08:32 AST \\
\hline JULY & 19 & 205209.6 & $53.662 \mathrm{~N}$ & 167.184W. & 33 & GS & 4.9 & - & - & IIII & JULY & 19 & 11:52 AST \\
\hline JULY & 19 & 210804.6 & $52.713 \mathrm{~N}$ & 166.721W. & 24 & LD & 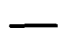 & & $3.9 \mathrm{~m}_{\mathrm{X}}(\mathrm{LD})$ & & JULY & 19 & 12:08 AST \\
\hline JULY & 19 & 220916.8 & $61.672 \mathrm{~N}$ & 152.019W. & 114 & GM & - & - & & & JULY & 19 & 13:09 AST \\
\hline JULY & 19 & 223236.0 & $53.521 \mathrm{~N}$ & 167.301W. & 33 & GS & 5.6 & 5.6 & - & V & JULY & 19 & 13:32 AST \\
\hline JULY & 20 & 015908.2 & $53.530 \mathrm{~N}$. & $167.344 \mathrm{~W}$. & 33 & GS & 4.9 & 4.5 & $5.2 \mathrm{M}_{\mathrm{L}}(\mathrm{PM})$ & FELT & JULY & 19 & 16:59 AST \\
\hline JULY & 20 & 050920.6 & $53.434 \mathrm{~N}$. & 167.155W. & 33 & GS & 4.4 & - & 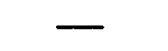 & & JULY & 19 & 20:09 AST \\
\hline JULY & 20 & 053509.8 & $53.661 \mathrm{~N}$ & 169.367W. & 33 & GS & 4.5 & & & & JULY & 19 & 20:35 AST \\
\hline JULY & 20 & 084824.2 & $53.494 \mathrm{~N}$. & 166.951W. & 33 & GS & 4.3 & - & - & & JULY & 19 & 23:48 AST \\
\hline JULY & 20 & 154023.6 & $58.752 \mathrm{~N}$ & $152.721 \mathrm{~W}$ & 80 & GM & - & - & - & - & JULY & 20 & 06:40 AST \\
\hline JULY & 20 & 161946.8 & $52.130 \mathrm{~N}$ & $174.708 \mathrm{~W}$. & 15 & $\mathrm{EE}$ & 4.6 & - & - & & JULY & 20 & 06:19 HST \\
\hline JULY & 20 & 162752.6 & $58.862 \mathrm{~N}$ & $154.614 \mathrm{~W}$. & 141 & GM & - & - & - & - & JULY & 20 & 07:27 AST \\
\hline JULY & 20 & 193747.4 & $61.996 \mathrm{~N}$ & $151.033 W$. & 71 & GM & - & - & - & & JULY & 20 & 10:37 AST \\
\hline JULY & 20 & 194429.7 & $57.042 \mathrm{~N}$ & $154.533 \mathrm{~W}$. & 92 & GM & 4.6 & - & - & - & JULY & 20 & 10:44 AST \\
\hline
\end{tabular}


Table 1. Summary of United States earthquakes for 1986-Continued

\begin{tabular}{|c|c|c|c|c|c|c|c|c|c|c|c|c|}
\hline \multirow[t]{2}{*}{ Date } & \multirow{2}{*}{$\begin{array}{l}\text { Origin time } \\
\text { (UTC) } \\
\text { hr min sec }\end{array}$} & \multirow{2}{*}{$\begin{array}{l}\text { Latitude } \\
()^{\circ}\end{array}$} & \multirow{2}{*}{$\begin{array}{l}\text { Longitude } \\
\text { (9) }\end{array}$} & \multirow{2}{*}{$\begin{array}{l}\text { Depth } \\
(\mathrm{km})\end{array}$} & \multirow{2}{*}{$\begin{array}{l}\text { Hypo- } \\
\text { center } \\
\text { Source }\end{array}$} & \multicolumn{3}{|c|}{ Magnitude } & \multirow{2}{*}{$\begin{array}{c}\text { Max. } \\
\text { inten- } \\
\text { sity } \\
\end{array}$} & \multicolumn{3}{|c|}{ Local time } \\
\hline & & & & & & $\mathbf{m}_{\mathbf{b}}$ & $\mathrm{M}_{s}$ & Local & & Date & & zone \\
\hline \multicolumn{13}{|c|}{ ALASKA-Continued } \\
\hline IULY 20 & 212723.7 & $55.802 \mathrm{~N}$. & $157.203 \mathrm{~W}$ & 33 & GS & 4.7 & - & $4.7 \mathrm{M}_{\mathrm{L}}(\mathrm{PM})$ & - & JULY & 20 & 12:27 AST \\
\hline JULY 21 & 002747.8 & $61.336 \mathrm{~N}$. & $151.518 \mathrm{~W}$. & 70 & GM & - & 一 & - & - & JULY & 20 & 15:27 AST \\
\hline JULY 21 & 083232.0 & $62.888 \mathrm{~N}$ & $149.622 \mathrm{~W}$ & 82 & GM & - & - & - & - & JULY & 20 & 23:32 AST \\
\hline JULY 21 & 152445.9 & $56.592 \mathrm{~N}$ & 153.361W. & 33 & GS & 5.0 & - & $5.1 \mathrm{M}_{\mathrm{L}}(\mathrm{PM})$ & - & JULY & 21 & 06:24 AST \\
\hline JULY 22 & 001107.6 & $59.874 \mathrm{~N}$. & $153.386 \mathrm{~W}$. & 126 & GM & - & 一 & - & - & JULY & 21 & 15:11 AST \\
\hline JULY 22 & 020009.4 & $51.466 \mathrm{~N}$. & $175.784 \mathrm{~W}$ & 35 & EE & 4.8 & - & - & - & JULY & 21 & $16: 00$ HST \\
\hline JULY 22 & 040922.4 & $53.203 \mathrm{~N}$ & $167.650 \mathrm{~W}$ & 33 & GS & 4.5 & - & - & - & JULY & 21 & 19:09 AST \\
\hline JULY 23 & 000840.7 & $51.022 \mathrm{~N}$. & $176.075 \mathrm{~W}$ & 20 & EE & 5.2 & - & - & - & JULY & 22 & 14:08 HST \\
\hline JULY 23 & 034740.1 & $50.994 \mathrm{~N}$. & 176.148W. & 20 & $\mathrm{EE}$ & 4.9 & 4.3 & - & 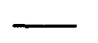 & JULY & 22 & $17: 47 \mathrm{HST}$ \\
\hline JULY 23 & 125932.7 & $60.344 \mathrm{~N}$ & $152.283 \mathrm{~W}$. & 75 & GM & - & - & - & - & JULY & 23 & 03:59 AST \\
\hline JULY 24 & 004200.8 & $51.012 \mathrm{~N}$ & $176.639 \mathrm{~W}$ & 20 & EE & 4.9 & 4.5 & $5.3 \mathrm{M}_{\mathrm{L}}(\mathrm{PM})$ & FELT & JULY & 23 & $14: 42 \mathrm{HST}$ \\
\hline JULY 24 & 030532.1 & $56.487 \mathrm{~N}$. & $153.067 \mathrm{~W}$ & 33 & GS & 4.3 & - & $3.6 \mathrm{M}_{\mathrm{L}}(\mathrm{PM})$ & - & JULY & 23 & 18:05 AST \\
\hline JULY 24 & 051354.6 & $59.896 \mathrm{~N}$. & $153.277 \mathrm{~W}$ & 115 & GM & - & - & - & - & JULY & 23 & 20:13 AST \\
\hline JULY 24 & 111609.0 & $53.330 \mathrm{~N}$. & $167.730 \mathrm{~W}$. & 33 & GS & 4.3 & - & - & - & JULY & 24 & 02:16 AST \\
\hline JULY 24 & 140330.0 & $51.492 \mathrm{~N}$. & $175.173 \mathrm{~W}$. & 40 & $\mathrm{EE}$ & 4.9 & 4.5 & $4.3 \mathrm{M}_{\mathrm{L}}(\mathrm{PM})$ & FELT & JULY & 24 & 04:03 HST \\
\hline JULY 24 & 163742.2 & $62.450 \mathrm{~N}$ & 148.196W. & 43 & GM & - & - & - & $\longrightarrow$ & JULY & 24 & 07:37 AST \\
\hline JULY 24 & 213015.3 & $58.425 \mathrm{~N}$ & $152.128 \mathrm{~W}$ & 55 & GM & 一 & 一 & - & - & JULY & 24 & $12: 30$ AST \\
\hline JULY 25 & 090132.6 & $51.079 \mathrm{~N}$. & $176.137 \mathrm{~W}$ & 21 & $\mathrm{EE}$ & 5.3 & 5.6 & $5.3 \mathrm{M}_{\mathrm{L}}(\mathrm{PM})$ & IV & JULY & 24 & 23:01 HST \\
\hline JULY 25 & 090416.3 & $51.056 \mathrm{~N}$. & $175.996 \mathrm{~W}$. & 20 & $\mathrm{EE}$ & 5.4 & 5.6 & - & FELT & JULY & 24 & 23:04 HST \\
\hline JULY 25 & 100633.0 & $51.754 \mathrm{~N}$ & $175.648 \mathrm{~W}$ & 33 & GS & 4.6 & - & - & - & JULY & 25 & 00:06 HST \\
\hline JULY 25 & 172118.5 & $63.121 \mathrm{~N}$ & $151.123 \mathrm{~W}$ & 33 & GS & - & - & $3.3 \mathrm{M}_{\mathrm{L}}(\mathrm{PM})$ & - & JULY & 25 & 08:21 AST \\
\hline JULY 25 & 232721.5 & $50.940 \mathrm{~N}$. & $175.968 \mathrm{~W}$. & 20 & $\mathrm{EE}$ & 4.7 & 一 & - & - & JULY & 25 & 13:27 HST \\
\hline JULY 26 & 025250.9 & $52.805 \mathrm{~N}$. & $166.539 \mathrm{~W}$. & 6 & LD & - & 一 & $4.0 \mathrm{~m}_{\mathrm{X}}(\mathrm{LD})$ & $\ldots$ & JULY & 25 & 17:52 AST \\
\hline JULY 26 & 032254.1 & $61.843 \mathrm{~N}$. & $150.671 \mathrm{~W}$ & 53 & GM & - & - & - & $\longrightarrow$ & JULY & 25 & 18:22 AST \\
\hline JULY 26 & 061537.1 & $60.004 \mathrm{~N}$ & $152.823 \mathrm{~W}$. & 92 & GM & - & - & $3.7 \mathrm{M}_{\mathrm{L}}(\mathrm{PM})$ & - & JULY & 25 & 21:15 AST \\
\hline JULY 26 & 091525.4 & $60.424 \mathrm{~N}$ & 151.724W. & 61 & GM & - & - & - & - & JULY & 26 & $00: 15$ AST \\
\hline JULY 26 & 183810.7 & $60.446 \mathrm{~N}$ & $148.846 \mathrm{~W}$ & 33 & GS & - & - & $3.5 \mathrm{M}_{\mathrm{L}}(\mathrm{PM})$ & - & JULY & 26 & 09:38 AST \\
\hline JULY 26 & 185244.8 & $53.280 \mathrm{~N}$. & 167.191W. & 33 & GS & 4.7 & 4.4 & $4.8 \mathrm{M}_{\mathrm{L}}(\mathrm{PM})$ & - & JULY & 26 & 09:52 AST \\
\hline JULY 26 & 221304.8 & $59.159 \mathrm{~N}$. & $136.084 \mathrm{~W}$ & 30 & GM & - & - & - & - & JULY & 26 & $13: 13$ AST \\
\hline JULY 27 & 000155.3 & $59.826 \mathrm{~N}$. & $152.946 \mathrm{~W}$ & 113 & GM & - & - & - & - & JULY & 26 & $15: 01$ AST \\
\hline JULY 27 & 031814.9 & $51.054 \mathrm{~N}$. & $176.132 \mathrm{~W}$ & 33 & GS & 4.4 & - & - & - & JULY & 26 & 17:18 HST \\
\hline JULY 27 & 045530.7 & $52.779 \mathrm{~N}$ & 169.725W. & 33 & GS & 4.4 & - & - & & JULY & 26 & $19: 55$ AST \\
\hline JULY 27 & 073107.2 & $60.581 \mathrm{~N}$ & $153.034 \mathrm{~W}$ & 139 & GM & - & - & - & $\longrightarrow$ & JULY & 26 & $22: 31$ AST \\
\hline JULY 27 & 100442.7 & $61.987 \mathrm{~N}$ & 149.680W. & 44 & GM & - & - & - & - & JULY & 27 & 01:04 AST \\
\hline JULY 27 & 175031.2 & $60.411 \mathrm{~N}$ & $152.973 \mathrm{~W}$ & 118 & GM & - & - & - & - & JULY & 27 & 08:50 AST \\
\hline JULY 28 & 002316.9 & $62.790 \mathrm{~N}$ & 151.286W. & 46 & GM & - & - & - & - & JULY & 27 & 15:23 AST \\
\hline JULY 28 & 040650.5 & $51.404 \mathrm{~N}$ & 174.016W. & 21 & $\mathrm{EE}$ & 5.4 & 4.8 & $4.5 \mathrm{M}_{\mathrm{L}}(\mathrm{PM})$ & III & JULY & 27 & 18:06 HST \\
\hline JULY 28 & 045419.3 & $51.533 \mathrm{~N}$ & $175.842 \mathrm{~W}$ & 40 & $\mathrm{EE}$ & 4.8 & 4.6 & - & - & JULY & 27 & 18:54 HST \\
\hline JULY 28 & 050159.6 & $52.862 \mathrm{~N}$ & $166.590 \mathrm{~W}$. & 33 & GS & 5.0 & 4.6 . & $4.7 \mathrm{M}_{\mathrm{L}}(\mathrm{PM})$ & FELT & JULY & 27 & 20:01 AST \\
\hline JULY 28 & 070054.0 & $58.342 \mathrm{~N}$ & 133.546W. & 18 & EP & - & - & $3.1 \mathrm{M}_{\mathrm{L}}(\mathrm{EP})$ & - & JULY & 27 & 22:00 AST \\
\hline
\end{tabular}


Table 1. Summary of United States earthquakes for 1986 - Continued

\begin{tabular}{|c|c|c|c|c|c|c|c|c|c|c|c|c|c|}
\hline \multirow{2}{*}{\multicolumn{2}{|c|}{ Date }} & \multirow{2}{*}{$\begin{array}{l}\text { Origin time } \\
\text { (UTC) } \\
\text { hr min sec }\end{array}$} & \multirow{2}{*}{$\begin{array}{l}\text { Latitude } \\
\left({ }^{\circ}\right)\end{array}$} & \multirow{2}{*}{$\begin{array}{l}\text { Longitude } \\
\text { (9) }\end{array}$} & \multirow{2}{*}{$\begin{array}{l}\text { Depth } \\
(\mathrm{km})\end{array}$} & \multirow{2}{*}{$\begin{array}{l}\text { Hypo- } \\
\text { center } \\
\text { Source }\end{array}$} & \multicolumn{3}{|c|}{ Magnitude } & \multirow{2}{*}{$\begin{array}{l}\text { Max. } \\
\text { inten- } \\
\text { sity }\end{array}$} & \multicolumn{3}{|c|}{ Local time } \\
\hline & & & & & & & $\mathrm{m}_{\mathrm{b}}$ & $\mathrm{M}_{\mathbf{s}}$ & Local & & Date & & zone \\
\hline \multicolumn{14}{|c|}{ ALASKA-Continued } \\
\hline JULY & 28 & 082820.8 & $62.135 \mathrm{~N}$. & 149.778W. & 50 & GM & 一 & - & $3.0 \mathrm{M}_{\mathrm{L}}(\mathrm{PM})$ & - & JULY & 27 & 23:28 AST \\
\hline JULY & 28 & 135855.3 & $59.848 \mathrm{~N}$. & $153.400 \mathrm{~W}$. & 124 & GM & - & - & - & - & JULY & 28 & 04:58 AST \\
\hline JULY & 28 & 143114.1 & $60.577 \mathrm{~N}$. & $150.386 \mathrm{~W}$. & 47 & GM & 4.4 & - & $4.6 \mathrm{M}_{\mathrm{L}}(\mathrm{PM})$ & IV & JULY & 28 & 05:31 AST \\
\hline JULY & 28 & 215716.6 & $51.573 \mathrm{~N}$. & $175.221 \mathrm{~W}$ & 42 & EE & 5.4 & 4.9 & $4.9 \mathrm{M}_{\mathrm{L}}(\mathrm{PM})$ & III & JULY & 28 & 11:57 HST \\
\hline JULY & 28 & 232920.7 & $51.512 \mathrm{~N}$. & 175.073W. & 35 & $\mathrm{EE}$ & 4.7 & 4.3 & - & - & JULY & 28 & 13:29 HST \\
\hline JULY & 29 & 031655.1 & $51.905 \mathrm{~N}$. & $175.097 \mathrm{~W}$ & 33 & GS & 4.4 & - & - & - & JULY & 28 & 17:16 HST \\
\hline JULY & 30 & 002950.8 & $62.549 \mathrm{~N}$. & $151.412 \mathrm{~W}$ & 95 & GM & - & - & - & 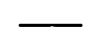 & JULY & 29 & 15:29 AST \\
\hline JULY & 30 & 132620.9 & $63.072 \mathrm{~N}$. & $151.021 \mathrm{~W}$. & 112 & GS & - & - & - & $\ldots$ & JULY & 30 & 04:26 AST \\
\hline JULY & 30 & 140353.6 & $62.439 \mathrm{~N}$. & $148.182 \mathrm{~W}$ & 42 & GM & - & - & - & $\ldots$ & JULY & 30 & 05:03 AST \\
\hline JULY & 30 & 183554.3 & $60.314 \mathrm{~N}$. & $153.466 \mathrm{~W}$. & 174 & GM & - & - & - & - & JULY & 30 & 09:35 AST \\
\hline JULY & 31 & 011726.4 & $62.346 \mathrm{~N}$. & $151.030 \mathrm{~W}$ & 76 & GM & - & - & $3.0 \mathrm{M}_{\mathrm{L}}(\mathrm{PM})$ & - & JULY & 30 & 16:17 AST \\
\hline JULY & 31 & 091632.4 & $60.717 \mathrm{~N}$. & 151.044W. & 50 & GM & - & - & - & - & JULY & 31 & 00:16 AST \\
\hline JULY & 31 & 093314.3 & $61.767 \mathrm{~N}$. & $149.567 \mathrm{~W}$. & 41 & GM & - & - & $3.2 \mathrm{M}_{\mathrm{L}}(\mathrm{PM})$ & FELT & JULY & 31 & 00:33 AST \\
\hline JULY & 31 & 140434.5 & $60.797 \mathrm{~N}$. & 149.614W. & 45 & GM & - & - & $3.7 \mathrm{M}_{\mathrm{L}}(\mathrm{PM})$ & FELT & JULY & 31 & 05:04 AST \\
\hline JULY & 31 & 172101.2 & $60.048 \mathrm{~N}$. & $140.718 W$ & 7 & GM & - & - & $3.2 \mathrm{M}_{\mathrm{L}}(\mathrm{EP})$ & - & JULY & 31 & 08:21 AST \\
\hline JULY & 31 & 191331.1 & $53.443 \mathrm{~N}$. & 167.171W. & 33 & GS & 4.7 & - & - & - & JULY & 31 & $10: 13$ AST \\
\hline AUG. & 1 & 132701.7 & $62.634 \mathrm{~N}$. & $148.558 \mathrm{~W}$ & 24 & GM & - & - & - & - & AUG. & 1 & 04:27 AST \\
\hline AUG. & 1 & 164306.4 & $53.495 \mathrm{~N}$. & $167.236 \mathrm{~W}$. & 33 & GS & 4.6 & - & $4.5 \mathrm{M}_{\mathrm{L}}(\mathrm{PM})$ & FELT & AUG. & 1 & 07:43 AST \\
\hline AUG. & 1 & 194634.1 & $50.872 \mathrm{~N}$. & 176.139W. & 20 & $\mathrm{EE}$ & 4.8 & - & $4.3 \mathrm{M}_{\mathrm{L}}(\mathrm{PM})$ & - & AUG. & 1 & 09:46 HST \\
\hline AUG. & 1 & 202459.3 & $50.954 \mathrm{~N}$. & 176.185W. & 20 & $\mathrm{EE}$ & 4.8 & 4.9 & $4.9 \mathrm{M}_{\mathrm{L}}(\mathrm{PM})$ & FELT & AUG. & 1 & 10:24 HST \\
\hline AUG. & 1 & 210540.1 & $51.262 \mathrm{~N}$. & 174.224W. & 22 & EE & 5.5 & 5.0 & $4.6 \mathrm{M}_{\mathrm{L}}(\mathrm{PM})$ & IV & AUG. & 1 & 11:05 HST \\
\hline AUG. & 1 & 214123.0 & $51.199 \mathrm{~N}$. & $174.177 \mathrm{~W}$ & 20 & EE & 4.8 & - & - & 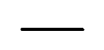 & AUG. & 1 & 11:41 HST \\
\hline AUG. & 1 & 214840.9 & $51.284 \mathrm{~N}$. & 174.171W. & 20 & $\mathrm{EE}$ & 4.9 & - & - & - & AUG. & 1 & 11:48 HST \\
\hline AUG. & 1 & 214918.9 & $51.442 \mathrm{~N}$. & $176.267 \mathrm{~W}$ & 33 & GS & 4.3 & - & - & $\longrightarrow$ & AUG. & 1 & 11:49 HST \\
\hline AUG. & 1 & 220926.5 & $51.550 \mathrm{~N}$. & 174.150W. & 33 & GS & 4.5 & - & - & $\longrightarrow$ & AUG. & 1 & 12:09 HST \\
\hline AUG. & 2 & 063706.4 & $51.314 \mathrm{~N}$. & $175.992 \mathrm{~W}$ & 33 & GS & 5.0 & - & 一 & - & AUG. & 1 & 20:37 HST \\
\hline AUG. & 2 & 131849.3 & $59.740 \mathrm{~N}$. & $153.464 \mathrm{~W}$ & 114 & GM & - & - & - & - & AUG. & 2 & 04:18 AST \\
\hline AUG. & 2 & 140101.9 & $52.802 \mathrm{~N}$. & $168.198 \mathrm{~W}$ & 33 & GS & 4.3 & - & - & - & AUG. & 2 & 05:01 AST \\
\hline AUG. & 2 & 195816.0 & $51.408 \mathrm{~N}$. & 174.757W. & 33 & GS & 4.7 & - & - & - & AUG. & 2 & 09:58 HST \\
\hline AUG. & 3 & 023928.7 & $51.244 \mathrm{~N}$. & 174.125W. & 20 & $\mathrm{EE}$ & 5.0 & - & $4.0 \mathrm{M}_{\mathrm{L}}(\mathrm{PM})$ & FELT & AUG. & 2 & 16:39 HST \\
\hline AUG. & 3 & 063017.2 & $56.602 \mathrm{~N}$. & $142.717 \mathrm{~W}$ & 31 & GM & - & - & $3.5 \mathrm{M}_{\mathrm{L}}(\mathrm{EP})$ & {[} & AUG. & 2 & 21:30 AST \\
\hline AUG. & 3 & 132910.4 & $51.026 \mathrm{~N}$. & 176.749W. & 22 & EE & 5.4 & 5.6 & $5.6 \mathrm{M}_{\mathrm{L}}(\mathrm{PM})$ & IV & AUG. & 3 & 03:29 HST \\
\hline AUG. & 3 & 134454.2 & $50.808 \mathrm{~N}$. & $176.671 \mathrm{~W}$ & 20 & $\mathrm{EE}$ & 4.7 & - & $5.1 \mathrm{M}_{\mathrm{L}}(\mathrm{PM})$ & FELT & AUG. & 3 & 03:44 HST \\
\hline AUG. & 3 & 200820.5 & $50.918 \mathrm{~N}$. & $176.638 \mathrm{~W}$ & 20 & EE & 4.8 & 4.9 & $4.2 \mathrm{M}_{\mathrm{L}}(\mathrm{PM})$ & FELT & AUG. & 3 & 10:08 HST \\
\hline AUG. & 3 & 204836.6 & $62.543 \mathrm{~N}$. & 149.775W. & 70 & GM & - & - & - & - & AUG. & 3 & 11:48 AST \\
\hline AUG. & 3 & 213621.5 & $51.147 \mathrm{~N}$. & $176.688 \mathrm{~W}$ & 33 & GS & 4.7 & - & - & - & AUG. & 3 & $11: 36$ HST \\
\hline AUG. & 4 & 044836.5 & $60.484 \mathrm{~N}$. & $147.247 \mathrm{~W}$. & 19 & GM & - & - & $3.6 \mathrm{M}_{\mathrm{L}}(\mathrm{PM})$ & - & AUG. & 3 & 19:48 AST \\
\hline AUG. & 4 & 193235.1 & $60.486 \mathrm{~N}$. & $151.967 \mathrm{~W}$ & 84 & GM & - & - & - & - & AUG. & 4 & $10: 32$ AST \\
\hline AUG. & 5 & 001733.0 & $51.221 \mathrm{~N}$. & 176.206W. & 33 & GS & - & - & - & - & AUG. & 4 & 14:17 HST \\
\hline AUG. & 5 & 045632.6 & $53.110 \mathrm{~N}$. & $163.969 \mathrm{~W}$ & 19 & LD & 4.5 & - & $4.3 \mathrm{M}_{\mathrm{L}}(\mathrm{PM})$ & - & AUG. & 4 & 19:56 AST \\
\hline
\end{tabular}

Table 1127 
Table 1. Summary of United States earthquakes for 1986-Continued

\begin{tabular}{|c|c|c|c|c|c|c|c|c|c|c|c|c|}
\hline \multirow[t]{2}{*}{ Date } & \multirow{2}{*}{$\begin{array}{l}\text { Origin time } \\
\text { (UTC) } \\
\text { hr min sec }\end{array}$} & \multirow{2}{*}{$\begin{array}{l}\text { Latitude } \\
\text { (?) }\end{array}$} & \multirow{2}{*}{$\begin{array}{l}\text { Longitude } \\
\text { (9) }\end{array}$} & \multirow{2}{*}{$\begin{array}{l}\text { Depth } \\
(\mathrm{km})\end{array}$} & \multirow{2}{*}{$\begin{array}{l}\text { Hypo- } \\
\text { center } \\
\text { Source } \\
\end{array}$} & \multicolumn{3}{|c|}{ Magnitude } & \multirow{2}{*}{$\begin{array}{l}\text { Max. } \\
\text { inten- } \\
\text { sity }\end{array}$} & \multicolumn{3}{|c|}{ Local time } \\
\hline & & & & & & $\mathbf{m}_{\mathrm{b}}$ & $\mathrm{M}_{\mathrm{s}}$ & Local & & Date & & zone \\
\hline \multicolumn{13}{|c|}{ ALASKA-Continued } \\
\hline AUG. 5 & 080135.6 & $59.781 \mathrm{~N}$ & $153.319 \mathrm{~W}$ & 112 & GM & - & - & - & - & AUG. & 4 & 23:01 AST \\
\hline AUG. 5 & 084813.2 & $52.929 \mathrm{~N}$. & $166.713 \mathrm{~W}$. & 33 & GS & 4.6 & - & - & - & AUG. & 4 & $23: 48$ AST \\
\hline AUG. 5 & 131243.8 & $60.323 \mathrm{~N}$ & $152.450 \mathrm{~W}$ & 99 & GM & - & - & - & - & AUG. & 5 & $04: 12$ AST \\
\hline AUG. 5 & 232744.3 & $61.783 \mathrm{~N}$ & $151.968 \mathrm{~W}$ & 116 & GM & - & - & - & - & AUG. & 5 & 14:27 AST \\
\hline AUG. & 013749.0 & $60.676 \mathrm{~N}$ & 147.091W. & 15 & GM & - & - & - & 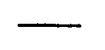 & AUG. & 5 & $16: 37$ AST \\
\hline AUG. & 082533.8 & $60.112 \mathrm{~N}$ & $153.467 \mathrm{~W}$ & 150 & GM & - & - & - & - & AUG. & 5 & 23:25 AST \\
\hline AUG. 6 & 124117.2 & $61.976 \mathrm{~N}$ & 148.188W. & 38 & GM & - & - & - & - & AUG. & 6 & 03:41 AST \\
\hline AUG. 6 & 133409.3 & $51.204 \mathrm{~N}$ & 179.387E. & 33 & GS & 4.6 & - & - & - & AUG. & 6 & 03:34 HST \\
\hline AUG. 6 & 180719.4 & $61.469 \mathrm{~N}$ & $152.072 \mathrm{~W}$. & 105 & GM & - & - & - & - & AUG. & 6 & 09:07 AST \\
\hline AUG. 6 & 231143.8 & $61.755 \mathrm{~N}$ & $151.978 \mathrm{~W}$ & 110 & GM & - & - & - & $\overline{-}$ & AUG. & 6 & $14: 11$ AST \\
\hline AUG. & 090744.6 & $61.207 \mathrm{~N}$ & $151.718 \mathrm{~W}$ & 85 & GM & - & - & - & & AUG. & 7 & 00:07 AST \\
\hline AUG. 7 & 231213.0 & $51.909 \mathrm{~N}$. & $178.174 \mathrm{~W}$. & 93 & EE & 4.5 & - & - & - & AUG. & 7 & 13:12 HST \\
\hline AUG. 8 & 011041.6 & $60.179 \mathrm{~N}$ & $151.473 \mathrm{~W}$ & 71 & GM & - & - & - & $\longrightarrow$ & AUG. & 7 & 16:10 AST \\
\hline AUG. 8 & 020644.0 & $58.369 \mathrm{~N}$. & $133.514 \mathrm{~W}$. & 18 & EP & - & 一 & $3.1 \mathrm{M}_{\mathrm{L}}(\mathrm{EP})$ & - & AUG. & 7 & 17:06 AST \\
\hline AUG. 8 & 043121.3 & $53.594 \mathrm{~N}$ & $167.320 \mathrm{~W}$ & 33 & GS & 4.5 & 4.3 & $5.0 \mathrm{M}_{\mathrm{L}}(\mathrm{PM})$ & FELT & AUG. & 7 & 19:31 AST \\
\hline AUG. & 054035.3 & $59.956 \mathrm{~N}$ & $153.369 \mathrm{~W}$. & 114 & GM & - & - & - & $\ldots$ & AUG. & 7 & 20:40 AST \\
\hline AUG. 8 & 134122.7 & $56.450 \mathrm{~N}$. & $153.308 \mathrm{~W}$. & 33 & GS & 4.3 & - & $3.5 \mathrm{M}_{\mathrm{L}}(\mathrm{PM})$ & & AUG. & 8 & 04:41 AST \\
\hline AUG. 8 & 152910.0 & $53.450 \mathrm{~N}$ & $164.592 \mathrm{~W}$. & 22 & LD & - & - & $3.1 \mathrm{~m}_{\mathrm{X}}(\mathrm{LD})$ & & AUG. & 8 & 06:29 AST \\
\hline AUG. 8 & 214349.5 & $50.320 \mathrm{~N}$ & 173.542W. & 33 & GS & 4.7 & - & - & & AUG. & 8 & $11: 43 \mathrm{HST}$ \\
\hline AUG. 9 & 042837.1 & $60.716 \mathrm{~N}$ & $151.672 \mathrm{~W}$ & 70 & GM & - & - & $3.1 \mathrm{M}_{\mathrm{L}}(\mathrm{PM})$ & & AUG. & 8 & 19:28 AST \\
\hline AUG. 9 & 142621.8 & $53.213 \mathrm{~N}$ & $161.549 \mathrm{~W}$ & 11 & LD & 4.7 & - & $3.6 m_{x}(L D)$ & - & AUG. & 9 & 05:26 AST \\
\hline AUG. 9 & 165738.7 & $51.331 \mathrm{~N}$ & 174.738W. & 33 & GS & 4.3 & - & $4.0 \mathrm{M}_{\mathrm{L}}(\mathrm{PM})$ & - & AUG. & 9 & 06:57 HST \\
\hline AUG. 9 & 174714.6 & $63.400 \mathrm{~N}$ & 147.315W. & 92 & GS & - & - & $3.4 \mathrm{M}_{\mathrm{L}}(\mathrm{PM})$ & - & AUG. & 9 & 08:47 AST \\
\hline AUG. 10 & 190756.3 & $60.092 \mathrm{~N}$ & $152.732 \mathrm{~W}$ & 96 & GM & - & - & $3.3 \mathrm{M}_{\mathrm{L}}(\mathrm{PM})$ & & AUG. & 10 & 10:07 AST \\
\hline AUG. 11 & 133454.0 & $60.399 \mathrm{~N}$ & 153.354W. & 148 & GM & - & - & - & - & AUG. & 11 & 04:34 AST \\
\hline AUG. 11 & 193447.3 & $62.048 \mathrm{~N}$ & $151.308 \mathrm{~W}$ & 81 & GM & - & - & - & - & AUG. & 11 & $10: 34$ AST \\
\hline AUG. 11 & 232237.6 & $60.575 \mathrm{~N}$ & $150.617 \mathrm{~W}$ & 43 & GM & - & - & - & $\longrightarrow$ & AUG. & 11 & $14: 22$ AST \\
\hline AUG. 12 & 040843.3 & $61.811 \mathrm{~N}$ & $151.608 \mathrm{~W}$. & 83 & GM & - & - & - & & AUG. & 11 & 19:08 AST \\
\hline AUG. 12 & 114629.5 & $63.124 \mathrm{~N}$ & $150.616 \mathrm{~W}$. & 33 & GS & - & - & $3.4 \mathrm{M}_{\mathrm{L}}(\mathrm{PM})$ & & AUG. & 12 & 02:46 AST \\
\hline AUG. 12 & 194717.5 & $61.882 \mathrm{~N}$ & $151.011 \mathrm{~W}$ & 65 & GM & - & - & - & & AUG. & 12 & $10: 47$ AST \\
\hline AUG. 13 & 134431.8 & $59.575 \mathrm{~N}$ & 152.971W. & 89 & GM & - & - & - & - & AUG. & 13 & 04:44 AST \\
\hline AUG. 13 & 210957.3 & $62.466 \mathrm{~N}$ & $149.662 W$ & 64 & GM & - & - & - & 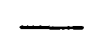 & AUG. & 13 & 12:09 AST \\
\hline AUG. 14 & 015428.2 & $59.559 \mathrm{~N}$ & $153.258 \mathrm{~W}$ & 97 & GM & - & - & - & $\longrightarrow$ & AUG. & 13 & 16:54 AST \\
\hline AUG. 14 & 021344.3 & $55.460 \mathrm{~N}$ & $163.425 \mathrm{~W}$ & 248 & LD & 4.6 & - & $3.7 m_{x}(L D)$ & 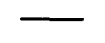 & AUG. & 13 & 17:13 AST \\
\hline AUG. 14 & 032536.0 & $58.358 \mathrm{~N}$ & $133.501 \mathrm{~W}$ & 18 & EP & - & - & $3.4 \mathrm{M}_{\mathrm{L}}(\mathrm{EP})$ & 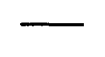 & AUG. & 13 & 18:25 AST \\
\hline AUG. 14 & 041328.1 & $59.502 \mathrm{~N}$ & 149.771W. & 43 & GM & - & - & $3.2 \mathrm{M}_{\mathrm{L}}(\mathrm{PM})$ & $\longrightarrow$ & AUG. & 13 & 19:13 AST \\
\hline AUG. 14 & 142854.5 & $51.449 \mathrm{~N}$ & 175.106W. & 35 & $\mathrm{EE}$ & 4.8 & - & - & - & AUG. & 14 & 04:28 HST \\
\hline AUG. 14 & 201529.3 & $60.103 \mathrm{~N}$ & $151.528 \mathrm{~W}$ & 61 & GM & - & - & - & - & AUG. & 14 & $11: 15$ AST \\
\hline AUG. 15 & 140241.0 & $58.356 \mathrm{~N}$ & $133.512 \mathrm{~W}$. & 18 & $\mathrm{EP}$ & - & - & $3.2 \mathrm{M}_{\mathrm{L}}(\mathrm{EP})$ & $\longrightarrow$ & AUG. & 15 & 05:02 AST \\
\hline AUG. 17 & 210258.9 & $60.685 \mathrm{~N}$ & $153.083 \mathrm{~W}$ & 163 & GM & - & - & - & - & AUG. & 17 & 12:02 AST \\
\hline
\end{tabular}


Table 1. Summary of United States earthquakes for 1986-Continued

\begin{tabular}{|c|c|c|c|c|c|c|c|c|c|c|c|}
\hline \multirow[t]{2}{*}{ Date } & \multirow{2}{*}{$\begin{array}{l}\text { Origin time } \\
\text { (UTC) } \\
\text { hr min sec }\end{array}$} & \multirow{2}{*}{$\begin{array}{l}\text { Latitude } \\
\left({ }^{\circ}\right)\end{array}$} & \multirow{2}{*}{$\begin{array}{c}\text { Longitude } \\
(9)\end{array}$} & \multirow{2}{*}{$\begin{array}{l}\text { Depth } \\
(\mathrm{km})\end{array}$} & \multirow{2}{*}{$\begin{array}{l}\text { Hypo- } \\
\text { center } \\
\text { Source }\end{array}$} & \multicolumn{3}{|c|}{ Magnitude } & \multirow{2}{*}{$\begin{array}{l}\text { Max. } \\
\text { inten- } \\
\text { sity }\end{array}$} & \multicolumn{2}{|c|}{ Local time } \\
\hline & & & & & & $\mathrm{m}_{\mathrm{b}}$ & $\mathrm{M}_{\mathrm{s}}$ & Local & & Date & hr zone \\
\hline \multicolumn{12}{|c|}{ ALASKA-Continued } \\
\hline AUG. 19 & 023407.3 & $51.838 \mathrm{~N}$ & $176.095 \mathrm{~W}$ & 33 & GS & 4.2 & - & - & $\longrightarrow$ & AUG. 18 & 16:34 HST \\
\hline AUG. 19 & 031934.9 & $62.131 \mathrm{~N}$ & $150.821 \mathrm{~W}$ & 65 & GM & - & - & - & - & AUG. 18 & 18:19 AST \\
\hline AUG. 19 & 175555.8 & $60.089 \mathrm{~N}$ & 153.234W. & 128 & GM & - & - & - & $\longrightarrow$ & AUG. 19 & 08:55 AST \\
\hline AUG. 20 & 044834.5 & $53.526 \mathrm{~N}$ & $162.940 \mathrm{~W}$. & 2 & LD & 4.6 & - & $4.1 \mathrm{~m}_{\mathrm{X}}(\mathrm{LD})$ & $\longrightarrow$ & AUG. 19 & 19:48 AST \\
\hline AUG. 20 & 083921.7 & $61.544 \mathrm{~N}$ & $146.420 \mathrm{~W}$ & 30 & GM & - & - & - & 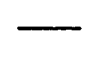 & AUG. 19 & 23:39 AST \\
\hline AUG. 20 & 171839.8 & $62.120 \mathrm{~N}$ & 151.191W. & 88 & GM & - & - & - & 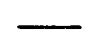 & AUG. 20 & 08:18 AST \\
\hline AUG. 20 & 185511.1 & $59.126 \mathrm{~N}$ & $153.782 \mathrm{~W}$. & 97 & GM & - & - & - & & AUG. 20 & 09:55 AST \\
\hline AUG. 20 & 224729.4 & $60.253 \mathrm{~N}$ & 141.122W. & 12 & GM & - & - & $4.1 \mathrm{M}_{\mathrm{L}}(\mathrm{EP})$ & & AUG. 20 & $13: 47$ AST \\
\hline AUG. 20 & 234419.7 & $65.896 \mathrm{~N}$ & $155.430 \mathrm{~W}$ & 33 & GS & - & - & $3.9 \mathrm{M}_{\mathrm{L}}(\mathrm{PM})$ & & AUG. 20 & $14: 44$ AST \\
\hline AUG. 21 & 041831.4 & $66.116 \mathrm{~N}$ & 155.746W. & 33 & GS & 一 & - & $4.0 \mathrm{M}_{\mathrm{L}}(\mathrm{PM})$ & & AUG. 20 & 19:18 AST \\
\hline AUG. 21 & 080945.3 & $62.298 \mathrm{~N}$ & $151.148 \mathrm{~W}$ & 81 & GM & - & - & - & - & AUG. 20 & 23:09 AST \\
\hline AUG. 21 & 100047.1 & $60.134 \mathrm{~N}$ & $140.994 \mathrm{~W}$. & 13 & GM & - & - & $2.8 \mathrm{M}_{\mathrm{L}}(\mathrm{EP})$ & & AUG. 21 & 01:00 AST \\
\hline AUG. 21 & 122431.7 & $51.689 \mathrm{~N}$ & 175.745W. & 33 & GS & 4.3 & - & - & & AUG. 21 & 02:24 HST \\
\hline AUG. 21 & 191935.3 & $60.408 \mathrm{~N}$ & 151.150W. & 51 & GM & - & - & - & & AUG. 21 & 10:19 AST \\
\hline AUG. 22 & 090122.9 & $60.398 \mathrm{~N}$ & $153.017 \mathrm{~W}$ & 120 & GM & - & - & - & & AUG. 22 & 00:01 AST \\
\hline AUG. 22 & 143152.6 & $52.081 \mathrm{~N}$ & $170.687 \mathrm{~W}$ & 33 & GS & 4.2 & - & - & & AUG. 22 & 04:31 HST \\
\hline AUG. 23 & 114043.8 & $60.061 \mathrm{~N}$ & $152.924 \mathrm{~W}$ & 99 & GM & - & - & - & & AUG. 23 & 02:40 AST \\
\hline AUG. 23 & 115512.0 & $53.547 \mathrm{~N}$ & $165.855 \mathrm{~W}$. & 33 & GS & 4.8 & - & $4.2 \mathrm{M}_{\mathrm{L}}(\mathrm{PM})$ & & AUG. 23 & 02:55 AST \\
\hline AUG. 24 & 104943.0 & $61.765 \mathrm{~N}$ & $150.035 \mathrm{~W}$ & 45 & GM & - & - & - & & AUG. 2 & 01:49 AST \\
\hline AUG. 24 & 143655.3 & $61.611 \mathrm{~N}$ & $-146.416 \mathrm{~W}$ & 30 & GM & - & - & - & & AUG. 2 & 05:36 AST \\
\hline AUG. 24 & 213556.1 & $59.810 \mathrm{~N}$ & $153.408 \mathrm{~W}$ & 117 & GM & - & - & - & - & AUG. 2 & $12: 35$ AST \\
\hline AUG. 24 & 213710.2 & $59.819 \mathrm{~N}$ & $153.415 \mathrm{~W}$ & 119 & GM & - & - & - & - & AUG. 2 & 12:37 AST \\
\hline AUG. 25 & 062130.1 & $62.711 \mathrm{~N}$ & $148.768 \mathrm{~W}$ & 75 & GM & - & - & $3.4 \mathrm{M}_{\mathrm{L}}(\mathrm{PM})$ & 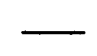 & AUG. 2 & $21: 21$ AST \\
\hline AUG. 25 & 232754.3 & $61.352 \mathrm{~N}$ & $150.333 \mathrm{~W}$. & 47 & GM & 4.5 & - & $4.4 \mathrm{M}_{\mathrm{L}}(\mathrm{PM})$ & III & AUG. 25 & $14: 27$ AST \\
\hline AUG. 26 & 215802.6 & $62.187 \mathrm{~N}$ & $148.503 \mathrm{~W}$ & 35 & GM & - & - & - & & AUG. 2 & $12: 58$ AST \\
\hline AUG. 27 & 035440.4 & $60.071 \mathrm{~N}$ & 153.073W. & 116 & GM & - & - & - & . & AUG. 2 & 18:54 AST \\
\hline AUG. 27 & 141210.4 & $59.025 \mathrm{~N}$ & $142.758 \mathrm{~W}$ & 30 & GM & 4.5 & - & $4.3 \mathrm{M}_{\mathrm{L}}(\mathrm{PM})$ & & AUG. 2 & 05:12 AST \\
\hline AUG. 29 & 051356.3 & $51.949 \mathrm{~N}$ & 171.413W. & 33 & GS & 4.3 & - & - & & AUG. 28 & 19:13 HST \\
\hline AUG. 29 & 080901.6 & $51.700 \mathrm{~N}$ & 171.797W. & 33 & GS & 4.4 & - & - & & AUG. 28 & 22:09 HST \\
\hline AUG. 29 & 140059.3 & $63.064 \mathrm{~N}$ & $150.793 \mathrm{~W}$ & 119 & GS & 4.4 & - & - & & AUG. 2 & 05:00 AST \\
\hline AUG. 29 & 144228.2 & $59.175 \mathrm{~N}$ & $153.620 \mathrm{~W}$ & 107 & GM & - & - & - & - & AUG. 2 & 05:42 AST \\
\hline SEPT. 1 & 183003.0 & $58.382 \mathrm{~N}$ & $133.517 \mathrm{~W}$ & 18 & $\mathrm{EP}$ & - & - & $3.2 \mathrm{M}_{\mathrm{L}}(\mathrm{EP})$ & & SEPT. & 09:30 AST \\
\hline SEPT. 1 & 184653.2 & $51.670 \mathrm{~N}$ & $175.167 \mathrm{~W}$ & 33 & GS & 4.4 & - & - & & SEPT. & 08:46 HST \\
\hline SEPT. 2 & 065856.0 & $58.355 \mathrm{~N}$ & 133.518W. & 18 & EP & - & - & $3.2 \mathrm{M}_{\mathrm{L}}(\mathrm{EP})$ & & SEPT. & 21:58 AST \\
\hline SEPT. 3 & 063930.0 & $.58 .352 \mathrm{~N}$ & $133.522 \mathrm{~W}$ & 18 & EP & - & - & $3.2 \mathrm{M}_{\mathrm{L}}(\mathrm{EP})$ & - & SEPT. & 21:39 AST \\
\hline SEPT. 3 & 104138.8 & $52.529 \mathrm{~N}$ & $168.322 \mathrm{~W}$ & 33 & GS & 4.7 & - & - & $\longrightarrow$ & SEPT. & 01:41 AST \\
\hline SEPT. 3 & 115106.4 & $51.106 \mathrm{~N}$ & 178.224W. & 30 & $\mathrm{EE}$ & 5.0 & - & $5.0 \mathrm{M}_{\mathrm{L}}(\mathrm{PM})$ & III & SEPT. & 01:51 HST \\
\hline SEPT. 3 & 132801.0 & $58.363 \mathrm{~N}$ & $133.503 \mathrm{~W}$ & 18 & EP & - & - & $3.1 \mathrm{M}_{\mathrm{L}}(\mathrm{EP})$ & 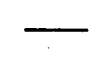 & SEPT. & $04: 28$ AST \\
\hline SEPT. 3 & 132901.3 & $54.700 \mathrm{~N}$ & $164.024 \mathrm{~W}$ & 122 & LD & - & - & $3.0 \mathrm{~m}_{\mathrm{x}}(\mathrm{LD})$ & $\longrightarrow$ & SEPT. & 04:29 AST \\
\hline SEPT. 3 & 162841.4 & $53.154 \mathrm{~N}$. & $163.543 \mathrm{~W}$ & 25 & LD & - & - & $3.3 m_{X}(L D)$ & - & SEPT. & 07:28 AST \\
\hline
\end{tabular}


Table 1. Summary of United States earthquakes for 1986-Continued

\begin{tabular}{|c|c|c|c|c|c|c|c|c|c|c|c|c|c|}
\hline \multicolumn{2}{|c|}{ Date } & \multirow{2}{*}{$\begin{array}{l}\text { Origin time } \\
\text { (UTC) } \\
\mathrm{hr} \operatorname{min~sec}\end{array}$} & $\begin{array}{c}\text { Latitude } \\
()^{\circ} \\
\end{array}$ & $\begin{array}{l}\text { Longitude } \\
\text { (9) }\end{array}$ & $\begin{array}{l}\text { Depth } \\
(\mathrm{km})\end{array}$ & $\begin{array}{l}\text { Hypo- } \\
\text { center } \\
\text { Source }\end{array}$ & \multicolumn{3}{|c|}{ Magnitude } & \multirow{2}{*}{$\begin{array}{l}\text { Max. } \\
\text { inten- } \\
\text { sity }\end{array}$} & \multicolumn{3}{|c|}{ Local time } \\
\hline \multicolumn{9}{|c|}{ ALASKA-Continued } & & & \multicolumn{2}{|l|}{ Date } & hr zone \\
\hline SEPT. & 3 & 172151.5 & $53.468 \mathrm{~N}$. & 167.138W. & 33 & GS & 4.5 & - & $4.6 \mathrm{M}_{\mathrm{L}}(\mathrm{PM})$ & & SEPT. & 3 & 08:21 AST \\
\hline SEPT. & 4 & 110007.6 & $51.783 \mathrm{~N}$. & 173.280W. & 33 & GS & $\longrightarrow$ & - & 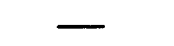 & & SEPT. & 4 & 01:00 HST \\
\hline SEPT. & 4 & 200816.7 & $59.726 \mathrm{~N}$. & 154.022W. & 154 & GM & - & & & & SEPT. & 4 & 11:08 AST \\
\hline SEPT. & 4 & 215401.0 & $61.476 \mathrm{~N}$. & 151.430W. & 79 & GM & - & - & 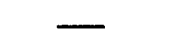 & & SEPT. & 4 & 12:54 AST \\
\hline SEPT. & 5 & 054801.7 & $62.994 \mathrm{~N}$. & $148.484 W$ & 102 & GS & 一 & - & & - & SEPT. & 4 & $20: 48$ AST \\
\hline SEPT. & 5 & 154226.0 & $60.000 \mathrm{~N}$ & 152.286W. & 72 & GM & - & - & $3.6 \mathrm{M}_{\mathrm{L}}(\mathrm{PM})$ & & SEPT. & 5 & 06:42 AST \\
\hline SEPT. & 6 & 113811.9 & $60.700 \mathrm{~N}$ & 152.120W. & 79 & GM & & & & & SEPT. & 6 & 02:38 AST \\
\hline SEPT. & 7 & 002232.5 & $60.244 \mathrm{~N}$ & $143.445 \mathrm{~W}$. & 18 & GM & 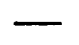 & & $3.8 \mathrm{M}_{\mathrm{L}}(\mathrm{PM})$ & & SEPT. & 6 & 15:22 AST \\
\hline SEPT. & 7 & 092957.0 & $60.027 \mathrm{~N}$ & $152.696 \mathrm{~W}$. & 89 & GM & $\overline{ }$ & & $3.1 \mathrm{M}_{\mathrm{L}}(\mathrm{PM})$ & & SEPT. & 7 & 00:29 AST \\
\hline SEPT. & 9 & 083431.5 & $53.884 \mathrm{~N}$. & $163.232 \mathrm{~W}$ & 4 & LD & - & & $3.6 \mathrm{~m}_{\mathrm{x}}(\mathrm{LD})$ & & SEPT. & 8 & 23:34 AST \\
\hline SEPT. & 9 & 093659.6 & $55.013 \mathrm{~N}$ & $159.677 \mathrm{~W}$. & 0 & LD & 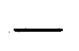 & & $3.1 \mathrm{~m}_{\mathrm{x}}(\mathrm{LD})$ & & SEPT. & 9 & 00:36 AST \\
\hline SEPT. & 9 & 151634.2 & $62.635 \mathrm{~N}$ & $151.145 \mathrm{~W}$. & 110 & GS & & & & & SEPT. & 9 & $06: 16$ AST \\
\hline SEPT. & & 215858.5 & $60.706 \mathrm{~N}$ & $151.198 \mathrm{~W}$. & 58 & GM & - & & & & SEPT. & 10 & 12:58 AST \\
\hline SEPT. & 10 & 231651.3 & $51.006 \mathrm{~N}$ & $171.882 W$. & 33 & GS & 4.6 & & & & SEPT. & 10 & 13:16 HST \\
\hline SEPT. & 11 & 022737.4 & $60.074 \mathrm{~N}$ & 153.144W. & 115 & GM & - & $\longrightarrow$ & & & SEPT. & 10 & 17:27 AST \\
\hline SEPT. & 11 & 222055.9 & $50.908 \mathrm{~N}$ & 178.995E. & 33 & GS & 4.4 & & & & SEPT. & 11 & 12:20 HST \\
\hline SEPT. & 12 & 200708.0 & $51.773 \mathrm{~N}$. & 178.490W. & 15 & $\mathrm{EE}$ & 4.3 & & & & SEPT. & 12 & 10:07 HST \\
\hline SEPT. & 12 & 201324.3 & $61.814 \mathrm{~N}$ & 149.935W. & 43 & GM & - & - & $3.4 \mathrm{M}_{\mathrm{L}}(\mathrm{PM})$ & & SEPT. & 12 & 11:13 AST \\
\hline SEPT. & 12 & 235715.6 & $56.201 \mathrm{~N}$ & 153.405W. & 31 . & GS & 6.1 & 6.3 & - & IV & SEPT. & 12 & 14:57 AST \\
\hline SEPT. & 13 & 004136.5 & $56.167 \mathrm{~N}$ & $153.804 \mathrm{~W}$ & 33 & GS & 4.6 & 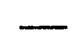 & $3.4 \mathrm{M}_{\mathrm{L}}(\mathrm{PM})$ & & SEPT. & 12 & 15:41 AST \\
\hline SEPT. & 13 & 030541.5 & $56.252 \mathrm{~N}$ & 153.594W. & 33 & GS & - & & $3.8 \mathrm{M}_{\mathrm{L}}(\mathrm{PM})$ & & SEPT. & 12 & 18:05 AST \\
\hline SEPT. & 13 & 031119.1 & $56.138 \mathrm{~N}$. & $153.639 \mathrm{~W}$. & 33 & GS & 4.8 & & $4.8 \mathrm{M}_{\mathrm{L}}(\mathrm{PM})$ & & SEPT. & 12 & 18:11 AST \\
\hline SEPT. & 13 . & 033810.2 & $56.523 \mathrm{~N}$ & 152.793W. & 33 & GS & $\longrightarrow$ & - & $3.4 \mathrm{M}_{\mathrm{L}}(\mathrm{PM})$ & & SEPT. & 12 & 18:38 AST \\
\hline SEPT. & 13 & 041959.7 & $56.102 \mathrm{~N}$. & $153.310 \mathrm{~W}$. & 33 & GS & 4.0 & - & $3.9 \mathrm{M}_{\mathrm{L}}(\mathrm{PM})$ & & SEPT. & 12 & 19:19 AST \\
\hline SEPT. & 13 & 050825.3 & $59.976 \mathrm{~N}$ & $152.824 \mathrm{~W}$ & 88 & GM & 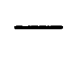 & - & 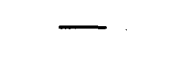 & & SEPT. & 12 & 20:08 AST \\
\hline SEPT. & 13 & 123040.7 & $61.245 \mathrm{~N}$ & $146.939 \mathrm{~W}$. & 42 & GM & & & $3.8 \mathrm{M}_{\mathrm{L}}(\mathrm{PM})$ & FELT & SEPT. & 13 & 03:30 AST \\
\hline SEPT. & 13 & 151615.0 & $54.984 \mathrm{~N}$ & $150.967 \mathrm{~W}$. & 33 & GS & & & $3.8 \mathrm{M}_{\mathrm{L}}(\mathrm{PM})$ & & SEPT. & 13 & 06:16 AST \\
\hline SEPT. & 13 & 162953.6 & $54.791 \mathrm{~N}$ & $161.838 \mathrm{~W}$. & 63 & LD & - & - & $3.8 \mathrm{M}_{\mathrm{L}}(\mathrm{PM})$ & & SEPT. & 13 & 07:29 AST \\
\hline SEPT. & 14 & 011129.5 & $56.319 \mathrm{~N}$ & 153.501W. & 33 & GS & 4.5 & - & - & & SEPT. & 13 & 16:11 AST \\
\hline SEPT. & 14 & 025838.1 & $61.015 \mathrm{~N}$ & $151.272 \mathrm{~W}$. & 70 & GM & - & & - & & SEPT. & 13 & 17:58 AST \\
\hline SEPT. & 14 & 113339.5 & $63.186 \mathrm{~N}$. & 147.191W. & 33 & GS & & & $3.1 \mathrm{M}_{\mathrm{L}}(\mathrm{PM})$ & - & SEPT. & 14 & 02:33 AST \\
\hline SEPT. & 14 & 113853.6 & $61.703 \mathrm{~N}$ & $149.682 W$. & 47 & GM & & & $3.4 \mathrm{M}_{\mathrm{L}}(\mathrm{PM})$ & FELT & SEPT. & 14 & 02:38 AST \\
\hline SEPT. & 14 & 174127.5 & $58.825 \mathrm{~N}$ & $137.022 \mathrm{~W}$. & 6 & GM & - & - & $3.7 \mathrm{M}_{\mathrm{L}}(\mathrm{EP})$ & $\longrightarrow$ & SEPT. & 14 & 08:41 AST \\
\hline SEPT. & 14 & 233316.0 & $61.228 \mathrm{~N}$ & $146.846 \mathrm{~W}$ & 16 & GM & - & - & - & $\longrightarrow$ & SEPT. & 14 & 14:33 AST \\
\hline SEPT. & 15 & 062938.6 & $51.368 \mathrm{~N}$ & 177.011W. & 35 & $\mathrm{EE}$ & 4.9 & 4.0 & $4.8 \mathrm{M}_{\mathrm{L}}(\mathrm{PM})$ & FELT & SEPT. & 14 & 20:29 HST \\
\hline SEPT. & 15 & 144822.1 & $61.528 \mathrm{~N}$ & 143.800W. & 52 & GM & 4.5 & & $4.7 \mathrm{M}_{\mathrm{L}}(\mathrm{PM})$ & IV & SEPT. & 15 & 05:48 AST \\
\hline SEPT. & 16 & 125319.7 & $59.892 \mathrm{~N}$. & 140.652W. & 4 & GM & $\longrightarrow$ & $\longrightarrow$ & $3.4 \mathrm{M}_{\mathrm{L}}(\mathrm{EP})$ & - & SEPT. & 16 & 03:53 AST \\
\hline SEPT. & 16 & 205721.9 & $56.222 \mathrm{~N}$ & $153.600 \mathrm{~W}$. & 33 & GS & 5.3 & 5.5 & $5.1 \mathrm{M}_{\mathrm{L}}(\mathrm{PM})$ & III & SEPT. & 16 & 11:57 AST \\
\hline SEPT. & 17 & 033707.8 & $65.900 \mathrm{~N}$ & 152.962W. & 33 & GS & $\longrightarrow$ & - & $3.9 \mathrm{M}_{\mathrm{L}}(\mathrm{PM})$ & $\longrightarrow$ & SEPT. & 16 & 18:37 AST \\
\hline SEPT. & 17 & 073057.6 & $62.216 \mathrm{~N}$ & $150.649 \mathrm{~W}$. & 72 & GM & - & - & - & - & SEPT. & 16 & 22:30 AST \\
\hline
\end{tabular}


Table 1. Summary of United States earthquakes for 1986-Continued

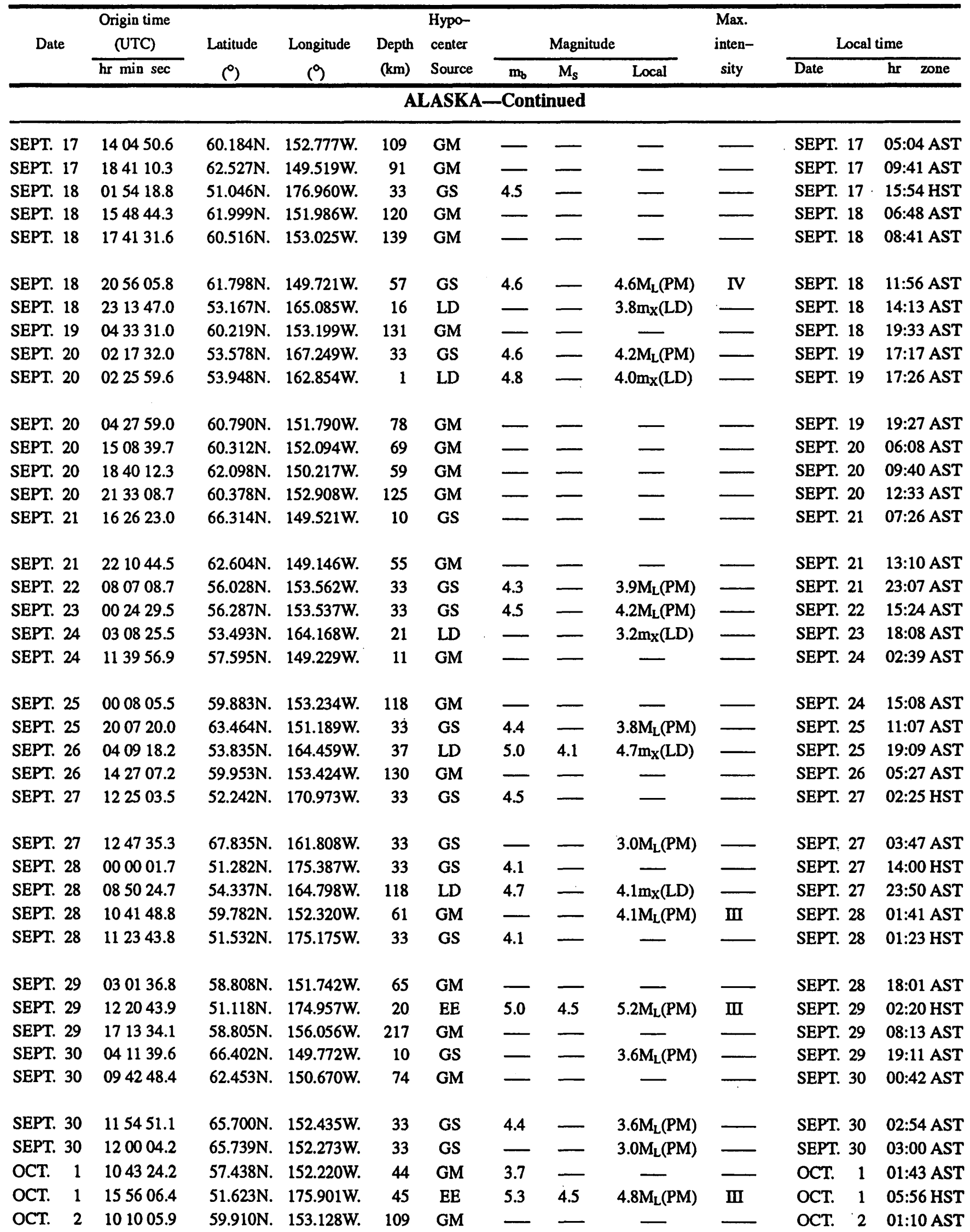


Table 1. Summary of United States earthquakes for 1986-Continued

\begin{tabular}{|c|c|c|c|c|c|c|c|c|c|c|c|c|c|}
\hline \multirow[t]{2}{*}{ Date } & & \multirow{2}{*}{$\begin{array}{l}\text { Origin time } \\
\text { (UTC) } \\
\text { hr min sec }\end{array}$} & \multirow{2}{*}{$\begin{array}{l}\text { Latitude } \\
\text { ()) }\end{array}$} & \multirow{2}{*}{$\begin{array}{l}\text { Longitude } \\
\text { (9) }\end{array}$} & \multirow{2}{*}{$\begin{array}{l}\text { Depth } \\
(\mathrm{km})\end{array}$} & \multirow{2}{*}{$\begin{array}{l}\text { Hypo- } \\
\text { center } \\
\text { Source }\end{array}$} & \multicolumn{3}{|c|}{ Magnitude } & \multirow{2}{*}{$\begin{array}{l}\text { Max. } \\
\text { inten- } \\
\text { sity }\end{array}$} & \multicolumn{3}{|c|}{ Local time } \\
\hline & & & & & & & $\mathbf{m}_{\mathrm{b}}$ & $\mathbf{M}_{s}$ & Local & & Date & & zone \\
\hline \multicolumn{14}{|c|}{ ALASKA-Continued } \\
\hline OCT. & 3 & 145204.1 & $54.479 \mathrm{~N}$. & $161.182 \mathrm{~W}$ & 4 & LD & - & - & $3.0 \mathrm{~m}_{\mathrm{X}}(\mathrm{LD})$ & - & OCT. & 3 & 05:52 AST \\
\hline OCT. & 3 & 152105.3 & $51.851 \mathrm{~N}$. & $178.587 \mathrm{~W}$. & 15 & $\mathrm{EE}$ & 5.0 & - & - & & OCT. & 3 & 05:21 HST \\
\hline OCT. & 5 & 032557.7 & $51.078 \mathrm{~N}$. & $176.179 \mathrm{~W}$. & 20 & EE & 4.9 & - & $4.5 \mathrm{M}_{\mathrm{L}}(\mathrm{PM})$ & II & OCT. & 4 & $17: 25 \mathrm{HST}$ \\
\hline OCT. & 5 & 050203.7 & $59.851 \mathrm{~N}$. & $152.964 \mathrm{~W}$ & 96 & GM & - & - & - & - & OCT. & 4 & 20:02 AST \\
\hline OCT. & 5 & 154158.9 & $60.194 \mathrm{~N}$. & $153.284 \mathrm{~W}$ & 134 & GM & - & - & - & - & OCT. & 5 & 06:41 AST \\
\hline OCT. & 5 & 212836.6 & $60.204 \mathrm{~N}$. & $152.729 \mathrm{~W}$. & 105 & GM & - & - & - & - & OCT. & 5 & $12: 28$ AST \\
\hline OCT. & 6 & 042148.9 & $51.513 \mathrm{~N}$. & $176.089 \mathrm{~W}$ & 48 & EE & 5.1 & 4.2 & $4.9 \mathrm{M}_{\mathrm{L}}(\mathrm{PM})$ & III & OCT. & 5 & 18:21 HST \\
\hline OCT. & 6 & 121326.1 & $51.397 \mathrm{~N}$. & $175.771 \mathrm{~W}$. & 33 & GS & 4.5 & - & - & - & OCT. & 6 & 02:13 HST \\
\hline OCT. & 7 & 142624.1 & $60.052 \mathrm{~N}$. & $153.401 \mathrm{~W}$ & 134 & GM & - & - & - & - & OCT. & 7 & 05:26 AST \\
\hline OCT. & 7 & 144015.5 & $51.573 \mathrm{~N}$. & $175.839 \mathrm{~W}$. & 45 & EE & 4.7 & - & - & 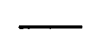 & OCT. & 7 & 04:40 HST \\
\hline OCT. & 7 & 222729.3 & $59.814 \mathrm{~N}$. & $153.588 \mathrm{~W}$ & 135 & GM & - & - & - & - & OCT. & 7 & $13: 27$ AST \\
\hline OCT. & 7 & 230512.4 & $61.377 \mathrm{~N}$. & $147.206 \mathrm{~W}$ & 35 & GM & - & - & - & - & OCT. & 7 & $14: 05$ AST \\
\hline OCT. & 8 & 004426.8 & $50.276 \mathrm{~N}$. & $176.383 \mathrm{~W}$ & 33 & GS & 4.4 & - & - & - & OCT. & 7 & 14:44 HST \\
\hline OCT. & 8 & 145807.9 & $57.208 \mathrm{~N}$. & $149.677 \mathrm{~W}$ & 44 & GM & - & - & - & $\longrightarrow$ & OCT. & 8 & 05:58 AST \\
\hline OCT. & 8 & 190050.0 & $61.561 \mathrm{~N}$. & $146.465 \mathrm{~W}$ & 37 & GM & - & - & - & - & OCT. & 8 & 10:00 AST \\
\hline OCT. & 8 & 192010.2 & $54.679 \mathrm{~N}$. & 164.020W. & 132 & LD & - & - & $3.3 \mathrm{~m}_{\mathrm{x}}(\mathrm{LD})$ & - & OCT. & 8 & 10:20 AST \\
\hline OCT. & 8 & 192938.0 & $59.545 \mathrm{~N}$. & $136.636 \mathrm{~W}$. & 18 & EP & - & - & $3.0 \mathrm{M}_{\mathrm{L}}(\mathrm{EP})$ & - & OCT. & 8 & $10: 29$ AST \\
\hline OCT. & 8 & 204301.0 & $58.323 \mathrm{~N}$. & $153.408 \mathrm{~W}$. & 67 & GM & - & 一 & - & 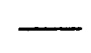 & OCT. & 8 & 11:43 AST \\
\hline OCT. & 9 & 005950.9 & $50.806 \mathrm{~N}$. & $172.814 \mathrm{~W}$. & 17 & EE & 4.7 & - & - & $\longrightarrow$ & OCT. & 8 & 14:59 HST \\
\hline OCT. & 9 & 012106.1 & $62.129 \mathrm{~N}$ & 149.544W. & 58 & GM & 4.5 & - & $4.3 \mathrm{M}_{\mathrm{L}}(\mathrm{PM})$ & III & OCT. & 8 & $16: 21$ AST \\
\hline OCT. & 9 & 105041.3 & $53.076 \mathrm{~N}$. & 166.126W. & 25 & LD & - & - & $3.9 m_{X}(L D)$ & - & OCT. & 9 & 01:50 AST \\
\hline OCT. & 9 & 1337.08 .7 & $61.689 \mathrm{~N}$. & $147.685 \mathrm{~W}$ & 26 & GM & - & - & - & & OCT. & 9 & 04:37 AST \\
\hline OCT. & 10 & 010605.0 & $58.369 \mathrm{~N}$. & $133.515 \mathrm{~W}$ & 18 & EP & 一 & - & $3.1 \mathrm{M}_{\mathrm{L}}(\mathrm{EP})$ & & OCT. & 9 & $16: 06$ AST \\
\hline OCT. & 10 & 090216.4 & $59.764 \mathrm{~N}$. & 138.394W. & 1 & GM & 3.8 & - & $3.6 \mathrm{M}_{\mathrm{L}}(\mathrm{EP})$ & & OCT. & 10 & 00:02 AST \\
\hline OCT. & 10 & 114854.1 & $60.332 \mathrm{~N}$. & 152.211W. & 73 & GM & - & - & - & & OCT. & 10 & 02:48 AST \\
\hline OCT. & 12 & 001525.3 & $60.455 \mathrm{~N}$ & $150.890 \mathrm{~W}$ & 61 & GM & - & - & - & - & OCT. & .11 & 15:15 AST \\
\hline OCT. & 12 & 071546.5 & $60.250 \mathrm{~N}$ & $152.714 \mathrm{~W}$ & 97 & GM & - & - & - & - & OCT. & 11 & $22: 15$ AST \\
\hline OCT. & 12 & 101756.9 & $57.251 \mathrm{~N}$. & 155.244W. & 63 & GM & - & - & - & - & OCT. & 12 & 01:17 AST \\
\hline OCT. & 12 & 144852.0 & $58.372 \mathrm{~N}$. & $133.532 \mathrm{~W}$ & 18 & EP & - & - & $3.1 \mathrm{M}_{\mathrm{L}}(\mathrm{EP})$ & & OCT. & 12 & 05:48 AST \\
\hline OCT. & 12 & 162732.8 & $61.558 \mathrm{~N}$ & $146.570 \mathrm{~W}$ & 25 & GM & - & - & $\longrightarrow$ & & OCT. & 12 & 07:27 AST \\
\hline OCT. & 12 & 185054.9 & $60.110 \mathrm{~N}$ & $147.074 \mathrm{~W}$ & 28 & GM & 一 & - & - & & OCT. & 12 & 09:50 AST \\
\hline OCT. & 12 & 190541.9 & $60.102 \mathrm{~N}$ & $147.070 \mathrm{~W}$. & 30 & GM & - & - & - & & OCT. & 12 & $10: 05$ AST \\
\hline OCT. & 13 & 022603.0 & $62.753 \mathrm{~N}$. & 143.388W. & 26 & GM & - & - & $3.6 \mathrm{M}_{\mathrm{L}}(\mathrm{PM})$ & & OCT. & 12 & $17: 26$ AST \\
\hline OCT. & 13 & 120530.0 & $62.148 \mathrm{~N}$. & $149.493 W$ & 48 & GM & - & - & - & & OCT. & 13 & 03:05 AST \\
\hline OCT. & 14 & 030710.0 & $58.345 \mathrm{~N}$ & $133.541 \mathrm{~W}$ & 18 & EP & - & - & $3.1 \mathrm{M}_{\mathrm{L}}(\mathrm{EP})$ & 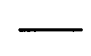 & OCT. & 13 & 18:07 AST \\
\hline OCT. & 14 & 045310.8 & $52.116 \mathrm{~N}$. & $168.762 \mathrm{~W}$ & 33 & GS & 4.9 & - & $4.7 \mathrm{M}_{\mathrm{L}}(\mathrm{PM})$ & & OCT. & 13 & 19:53 AST \\
\hline OCT. & 14 & 083621.8 & $63.302 \mathrm{~N}$. & 149.375W. & 11 & GS & - & - & $3.0 \mathrm{M}_{\mathrm{L}}(\mathrm{PM})$ & & OCT. & 13 & 23:36 AST \\
\hline OCT. & 14 & 104826.0 & $63.288 \mathrm{~N}$. & $149.439 \mathrm{~W}$ & 22 & GS & - & - & $3.8 \mathrm{M}_{\mathrm{L}}(\mathrm{PM})$ & & OCT. & 14 & 01:48 AST \\
\hline OCT. & 14 & 105701.7 & $63.300 \mathrm{~N}$. & 149.436W. & 29 & GS & - & - & $3.7 \mathrm{M}_{\mathrm{L}}(\mathrm{PM})$ & 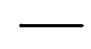 & OCT. & 14 & 01:57 AST \\
\hline OCT. & 15 & 044908.7 & $62.510 \mathrm{~N}$ & $147.057 \mathrm{~W}$ & 10 & GM & - & - & $3.1 \mathrm{M}_{\mathrm{L}}(\mathrm{PM})$ & - & OCT. & 14 & 19:49 AST \\
\hline
\end{tabular}


Table 1. Summary of United States earthquakes for 1986-Continued

\begin{tabular}{|c|c|c|c|c|c|c|c|c|c|c|c|c|c|}
\hline \multirow[t]{2}{*}{ Date } & & \multirow{2}{*}{$\begin{array}{l}\text { Origin time } \\
\text { (UTC) } \\
\text { hr min sec }\end{array}$} & \multirow{2}{*}{$\begin{array}{l}\text { Latitude } \\
\left({ }^{\circ}\right) \\
\end{array}$} & \multirow{2}{*}{$\begin{array}{c}\text { Longitude } \\
\text { (9) }\end{array}$} & \multirow{2}{*}{$\begin{array}{l}\text { Depth } \\
(\mathrm{km})\end{array}$} & \multirow{2}{*}{$\begin{array}{l}\text { Hypo- } \\
\text { center } \\
\text { Source }\end{array}$} & \multicolumn{3}{|c|}{ Magnitude } & \multirow{2}{*}{$\begin{array}{c}\text { Max. } \\
\text { inten- } \\
\text { sity }\end{array}$} & \multicolumn{3}{|c|}{ Local time } \\
\hline & & & & & & & $\mathrm{m}_{\mathrm{b}}$ & $\mathrm{M}_{\mathrm{S}}$ & Local & & Date & & zone \\
\hline \multicolumn{14}{|c|}{ ALASKA-Continued } \\
\hline OCT. & 15 & 165029.4 & $53.220 \mathrm{~N}$ & $166.069 \mathrm{~W}$. & 25 & LD & - & - & $3.4 m_{x}(L D)$ & & OCT. & 15 & 07:50 AST \\
\hline OCT. & 15 & 182306.0 & $59.990 \mathrm{~N}$ & $152.841 \mathrm{~W}$ & 100 & GM & - & - & - & - & OCT. & 15 & 09:23 AST \\
\hline OCT. & 15 & 233551.2 & $59.705 \mathrm{~N}$ & 153.072W. & 121 & GS & 4.6 & - & - & III & OCT. & 15 & $14: 35$ AST \\
\hline OCT. & 16 & 022816.0 & $58.394 \mathrm{~N}$ & 133.500W. & 18 & EP & - & - & $3.2 \mathrm{M}_{\mathrm{L}}(\mathrm{EP})$ & - & OCT. & 15 & $17: 28$ AST \\
\hline OCT. & 17 & 012005.1 & $60.146 \mathrm{~N}$ & $152.600 \mathrm{~W}$. & 74 & GM & - & - & - & - & OCT. & 16 & 16:20 AST \\
\hline OCT. & 17 & 233344.0 & $58.377 \mathrm{~N}$ & 133.503W. & 18 & EP & - & - & $3.2 \mathrm{M}_{\mathrm{L}}(\mathrm{EP})$ & 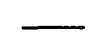 & OCT. & 17 & 14:33 AST \\
\hline OCT. & 18 & 010255.3 & $51.528 \mathrm{~N}$ & 175.213W. & 39 & $\mathrm{EE}$ & 5.4 & 4.9 & $5.3 \mathrm{M}_{\mathrm{L}}(\mathrm{PM})$ & & OCT. & 17 & 15:02 HST \\
\hline OCT. & 18 & 045109.1 & $51.005 \mathrm{~N}$ & 175.252W. & 33 & GS & 4.4 & - & - & - & OCT. & 17 & 18:51 HST \\
\hline OCT. & 18 & 192210.1 & $63.153 \mathrm{~N}$ & $150.443 \mathrm{~W}$ & 119 & GS & - & - & - & FELT & OCT. & 18 & 10:22 AST \\
\hline OCT. & 19 & 062811.4 & $52.630 \mathrm{~N}$ & $170.667 \mathrm{~W}$ & 33 & GS & 4.7 & - & - & 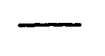 & OCT. & 18 & 20:28 HST \\
\hline OCT. & 19 & 183057.2 & 63.887N. & 178.727W. & 10 & GS & 5.4 & 4.9 & - & - & OCT. & 19 & 08:30 HST \\
\hline OCT. & 19 & 215334.0 & $59.509 \mathrm{~N}$ & $152.682 \mathrm{~W}$ & 81 & GM & - & - & 4.0M $(\mathrm{MM})$ & II & OCT. & 19 & $12: 53$ AST \\
\hline OCT. & 20 & 072553.8 & $52.165 \mathrm{~N}$ & $175.100 \mathrm{~W}$. & 15 & $\mathrm{EE}$ & 4.6 & - & - & $\longrightarrow$ & OCT. & 19 & $21: 25 \mathrm{HST}$ \\
\hline OCT. & 20 & 092452.0 & $59.046 \mathrm{~N}$ & $136.751 \mathrm{~W}$ & 18 & EP & - & - & $3.0 \mathrm{M}_{\mathrm{L}}(\mathrm{EP})$ & - & OCT. & 20 & 00:24 AST \\
\hline OCT. & 20 & 105156.4 & $62.117 \mathrm{~N}$ & 149.539W. & 51 & GM & - & - & - & - & OCT. & 20 & 01:51 AST \\
\hline OCT. & 20 & 125742.2 & $51.164 \mathrm{~N}$. & 174.732W. & 33 & GS & 4.8 & - & - & 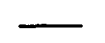 & OCT. & 20 & 02:57 HST \\
\hline OCT. & 20 & 125743.1 & $59.615 \mathrm{~N}$ & 151.012W. & 56 & GM & - & - & - & & OCT. & 20 & 03:57 AST \\
\hline OCT. & 20 & 194526.2 & $53.039 \mathrm{~N}$. & $166.782 \mathrm{~W}$ & 33 & GS & 4.8 & - & - & & OCT. & 20 & $10: 45$ AST \\
\hline OCT. & 21 & 105746.2 & $60.055 \mathrm{~N}$ & $152.684 \mathrm{~W}$ & 83 & GM & 一 & - & - & $\longrightarrow$ & OCT. & 21 & 01:57 AST \\
\hline OCT. & 22 & 015910.5 & $60.333 \mathrm{~N}$ & $150.488 \mathrm{~W}$ & 44 & GM & - & - & - & 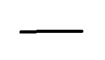 & OCT. & 21 & 16:59 AST \\
\hline OCT. & 22 & 152825.7 & $61.592 \mathrm{~N}$ & $151.500 \mathrm{~W}$ & 79 & $\mathrm{GM}$ & 一 & - & - & - & OCT. & 22 & 06:28 AST \\
\hline OCT. & 22 & 183134.1 & $61.339 \mathrm{~N}$ & $146.849 \mathrm{~W}$ & 38 & GM & - & - & $4.0 \mathrm{M}_{\mathrm{L}}(\mathrm{PM})$ & III & OCT. & 22 & 09:31 AST \\
\hline OCT. & 22 & 201427.2 & $59.529 \mathrm{~N}$ & $152.492 \mathrm{~W}$ & 75 & GM & - & - & - & - & OCT. & 22 & $11: 14$ AST \\
\hline OCT. & 23 & 004311.4 & $59.436 \mathrm{~N}$ & $152.324 \mathrm{~W}$ & 74 & GM & - & - & - & 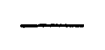 & OCT. & 22 & $15: 43$ AST \\
\hline OCT. & 23 & 004825.8 & $59.335 \mathrm{~N}$ & $138.802 \mathrm{~W}$ & 12 & GM & 一 & - & $3.6 \mathrm{M}_{\mathrm{L}}(\mathrm{EP})$ & - & OCT. & 22 & 15:48 AST \\
\hline OCT. & 23 & 014231.5 & $52.514 \mathrm{~N}$ & 168.161W. & 33 & GS & 5.0 & - & - & $\ldots$ & OCT. & 22 & $16: 42$ AST \\
\hline OCT. & 23 & 124036.5 & $56.027 \mathrm{~N}$ & $153.949 \mathrm{~W}$ & 33 & GS & 4.3 & - & - & & OCT. & 23 & 03:40 AST \\
\hline OCT. & 23 & 153327.3 & $60.183 \mathrm{~N}$ & 152.913W. & 116 & GM & - & - & - & & OCT. & 23 & 06:33 AST \\
\hline OCT. & 23 & 172442.3 & $62.656 \mathrm{~N}$ & $150.870 \mathrm{~W}$ & 100 & GM & - & - & - & - & OCT. & 23 & 08:24 AST \\
\hline OCT. & 24 & 075031.0 & $59.080 \mathrm{~N}$ & $154.406 \mathrm{~W}$ & 129 & GM & - & - & - & $\longrightarrow$ & OCT. & 23 & 22:50 AST \\
\hline OCT. & 24 & 110050.3 & $51.384 \mathrm{~N}$ & 176.750W. & 37 & $\mathrm{EE}$ & 5.2 & 4.3 & $4.7 \mathrm{M}_{\mathrm{L}}(\mathrm{PM})$ & III & OCT. & 24 & 01:00 HST \\
\hline OCT. & 24 & 115148.4 & $56.059 \mathrm{~N}$ & 153.198W. & 33 & GS & 4.3 & - & - & - & OCT. & 24 & 02:51 AST \\
\hline OCT. & 24 & 143453.8 & $60.946 \mathrm{~N}$ & $151.486 \mathrm{~W}$ & 69 & GM & 一 & - & $3.2 \mathrm{M}_{\mathrm{L}}(\mathrm{PM})$ & FELT & OCT. & 24 & 05:34 AST \\
\hline OCT. & 25 & 025145.3 & $51.092 \mathrm{~N}$ & $175.924 \mathrm{~W}$ & 20 & $\mathrm{EE}$ & 4.7 & - & - & - & OCT. & 24 & 16:51 HST \\
\hline OCT. & 25 & 104048.5 & $64.194 \mathrm{~N}$ & 154.191W. & 33 & GS & - & - & 3.7 $\mathrm{M}_{\mathrm{L}}(\mathrm{PM})$ & - & OCT. & 25 & 01:40 AST \\
\hline OCT. & 25 & 142326.2 & $60.228 \mathrm{~N}$ & $152.918 \mathrm{~W}$ & 109 & GM & - & 一 & - & - & OCT. & 25 & 05:23 AST \\
\hline OCT. & 25 & 200714.2 & $60.715 \mathrm{~N}$ & 152.112W. & 89 & GM & - & - & $3.0 \mathrm{M}_{\mathrm{L}}(\mathrm{PM})$ & 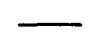 & OCT. & 25 & 11:07 AST \\
\hline OCT. & 26 & 033353.0 & $53.484 \mathrm{~N}$ & $163.215 \mathrm{~W}$ & 18 & LD & 4.7 & - & $3.8 \mathrm{~m}_{\mathrm{X}}(\mathrm{LD})$ & 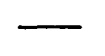 & OCT. & 25 & 18:33 AST \\
\hline OCT. & 26 & 033404.9 & $53.266 \mathrm{~N}$ & $163.442 \mathrm{~W}$ & 20 & LD & 5.1 & 5.0 & $5.1 \mathrm{M}_{\mathrm{L}}(\mathrm{PM})$ & - & OCT. & 25 & 18:34 AST \\
\hline OCT. & 26 & 044327.4 & $53.758 \mathrm{~N}$ & $170.049 \mathrm{~W}$ & 214 & GS & 5.4 & - & - & - & OCT. & 25 & 18:43 HST \\
\hline
\end{tabular}


Table 1. Summary of United States earthquakes for 1986 C Continued

\begin{tabular}{|c|c|c|c|c|c|c|c|c|c|c|c|c|c|}
\hline \multirow[t]{2}{*}{ Date } & & \multirow{2}{*}{$\begin{array}{l}\text { Origin time } \\
\text { (UTC) } \\
\text { hr min sec }\end{array}$} & \multirow{2}{*}{$\begin{array}{l}\text { Latitude } \\
\text { (?) }\end{array}$} & \multirow{2}{*}{$\begin{array}{l}\text { Longitude } \\
\text { (9) }\end{array}$} & \multirow{2}{*}{$\begin{array}{l}\text { Depth } \\
(\mathrm{km})\end{array}$} & \multirow{2}{*}{$\begin{array}{l}\text { Hypo- } \\
\text { center } \\
\text { Source }\end{array}$} & \multicolumn{3}{|c|}{ Magnitude } & \multirow{2}{*}{$\begin{array}{l}\text { Max. } \\
\text { inten- } \\
\text { sity }\end{array}$} & \multicolumn{3}{|c|}{ Local time } \\
\hline & & & & & & & $\mathrm{m}_{\mathrm{b}}$ & $\mathrm{M}_{\mathrm{s}}$ & Local & & Date & & zone \\
\hline \multicolumn{14}{|c|}{ ALASKA-Continued } \\
\hline OCT. & 26 & 055750.3 & $61.568 \mathrm{~N}$ & $146.357 \mathrm{~W}$ & 34 & GM & - & - & $3.5 \mathrm{M}_{\mathrm{L}}(\mathrm{PM})$ & - & OCT. & 25 & 20:57 AST \\
\hline OCT. & 26 & 082735.6 & $60.033 \mathrm{~N}$ & $152.676 \mathrm{~W}$ & 96 & GM & - & - & - & - & OCT. & 25 & 23:27 AST \\
\hline OCT. & 26 & 160940.2 & $59.803 \mathrm{~N}$ & $153.843 W$ & 145 & GM & - & - & - & - & OCT. & 26 & 07:09 AST \\
\hline OCT. & 26 & 221941.6 & $57.716 \mathrm{~N}$ & 156.290W. & 165 & GS & - & - & - & - & OCT. & 26 & 13:19 AST \\
\hline OCT. & 27 & 152715.4 & $52.332 \mathrm{~N}$ & $173.922 \mathrm{~W}$ & 15 & EE & 4.8 & - & - & $\square$ & OCT. & 27 & 05:27 HST \\
\hline OCT. & 27 & 184823.6 & $59.526 \mathrm{~N}$ & 153.411W. & 118 & GM & - & - & - & - & OCT. & 27 & 09:48 AST \\
\hline OCT. & 27 & 193936.5 & $60.928 \mathrm{~N}$ & $149.464 \mathrm{~W}$ & 39 & GM & - & - & $3.6 \mathrm{M}_{\mathrm{L}}(\mathrm{PM})$ & IV & OCT. & 27 & 10:39 AST \\
\hline OCT. & 27 & 233156.6 & $60.559 \mathrm{~N}$ & 151.396W. & 49 & GM & - & - & - & 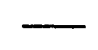 & OCT. & 27 & $14: 31$ AST \\
\hline OCT. & 28 & 031148.1 & $56.488 \mathrm{~N}$ & 154.113W. & 33 & GS & 4.3 & - & $3.3 \mathrm{M}_{\mathrm{L}}(\mathrm{PM})$ & - & OCT. & 27 & 18:11 AST \\
\hline OCT. & 28 & 061333.3 & $52.787 \mathrm{~N}$ & $165.874 \mathrm{~W}$ & 6 & LD & - & - & $3.6 \mathrm{~m}_{\mathrm{X}}(\mathrm{LD})$ & - & OCT. & 27 & $21: 13$ AST \\
\hline OCT. & 28 & 081517.0 & $56.721 \mathrm{~N}$ & 153.698W. & 33 & GS & 3.6 & - & $3.0 \mathrm{M}_{\mathrm{L}}(\mathrm{PM})$ & $\longrightarrow$ & OCT. & 27 & 23:15 AST \\
\hline OCT. & 28 & 082915.7 & $60.888 \mathrm{~N}$ & $147.146 \mathrm{~W}$ & 23 & GM & - & - & - & - & OCT. & 27 & 23:29 AST \\
\hline OCT. & 28 & 191942.0 & $63.552 \mathrm{~N}$ & $150.877 \mathrm{~W}$ & 33 & GS & - & - & $3.4 \mathrm{M}_{\mathrm{L}}(\mathrm{PM})$ & - & OCT. & 28 & 10:19 AST \\
\hline OCT. & 29 & 163521.6 & $52.491 \mathrm{~N}$ & 173.993W. & 33 & GS & 4.5 & - & - & - & OCT. & 29 & 06:35 HST \\
\hline OCT. & 29 & 191426.0 & $63.148 \mathrm{~N}$ & $150.630 \mathrm{~W}$ & 33 & GS & - & - & $3.0 \mathrm{M}_{\mathrm{L}}(\mathrm{PM})$ & 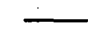 & OCT. & 29 & 10:14 AST \\
\hline OCT. & 30 & 082312.3 & $59.884 \mathrm{~N}$. & $152.423 \mathrm{~W}$ & 72 & GM & - & - & - & - & OCT. & 29 & 23:23 AST \\
\hline OCT. & 30 & 110502.3 & $54.436 \mathrm{~N}$ & $162.960 \mathrm{~W}$ & 24 & LD & - & - & $3.2 m_{x}(L D)$ & & OCT. & 30 & 02:05 AST \\
\hline OCT. & 30 & 224641.1 & $60.226 \mathrm{~N}$ & $153.039 \mathrm{~W}$ & 121 & GM & - & - & - & - & OCT. & 30 & 13:46 AST \\
\hline OCT. & 31 & 040723.3 & $51.439 \mathrm{~N}$ & $175.845 \mathrm{~W}$ & 35 & EE & 4.8 & 3.8 & $4.2 \mathrm{M}_{\mathrm{L}}(\mathrm{PM})$ & - & OCT. & 30 & 18:07 HST \\
\hline NOV. & 1 & 081815.1 & $61.504 \mathrm{~N}$ & $147.450 \mathrm{~W}$ & 19 & GM & - & - & $3.6 \mathrm{M}_{\mathrm{L}}(\mathrm{PM})$ & 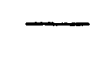 & OCT. & 31 & 23:18 AST \\
\hline NOV. & 1 & 142529.3 & $61.384 \mathrm{~N}$ & $151.854 \mathrm{~W}$ & 89 & GM & - & - & - & $\longrightarrow$ & NOV. & 1 & 05:25 AST \\
\hline NOV. & 1 & 162344.5 & $59.760 \mathrm{~N}$ & 153.349W. & 122 & GM & - & - & - & - & NOV. & 1 & 07:23 AST \\
\hline NOV. & 1 & 221701.8 & $62.009 \mathrm{~N}$ & $150.647 \mathrm{~W}$ & 63 & GM & - & - & - & - & NOV. & 1 & $13: 17$ AST \\
\hline NOV. & 1 & 222234.7 & $51.245 \mathrm{~N}$ & 179.755W. & 33 & GS & 5.0 & - & $4.9 \mathrm{M}_{\mathrm{L}}(\mathrm{PM})$ & - & NOV. & 1 & $12: 22$ HST \\
\hline NOV. & 2 & 103830.9 & $53.905 \mathrm{~N}$ & $162.990 \mathrm{~W}$ & 3 & LD & - & - & $3.7 \mathrm{~m}_{\mathrm{x}}(\mathrm{LD})$ & & NOV. & 2 & 01:38 AST \\
\hline NOV. & 3 & 063556.6 & $53.726 \mathrm{~N}$ & $162.096 \mathrm{~W}$ & 14 & LD & - & - & $3.0 \mathrm{~m}_{\mathrm{X}}(\mathrm{LD})$ & $\longrightarrow$ & NOV. & 2 & 21:35 AST \\
\hline NOV. & 4 & 061418.7 & $61.341 \mathrm{~N}$ & $151.900 \mathrm{~W}$ & 98 & GM & 4.7 & - & - & III & NOV. & 3 & $21: 14$ AST \\
\hline NOV. & 4 & 065555.0 & $61.317 \mathrm{~N}$ & $150.642 \mathrm{~W}$ & 47 & GM & 4.3 & - & $3.9 \mathrm{M}_{\mathrm{L}}(\mathrm{PM})$ & & NOV. & 3 & 21:55 AST \\
\hline Nov. & 4 & 070606.6 & $61.329 \mathrm{~N}$ & $150.660 \mathrm{~W}$ & 51 & GM & - & - & - & $\longrightarrow$ & NOV. & 3 & 22:06 AST \\
\hline NOV. & 4 & 181839.2 & $54.726 \mathrm{~N}$ & $160.534 \mathrm{~W}$ & 28 & LD & - & - & $3.3 m_{X}(L D)$ & $\longrightarrow$ & NOV. & 4 & 09:18 AST \\
\hline NOV. & 5 & 002638.7 & $60.059 \mathrm{~N}$ & $152.419 \mathrm{~W}$ & 89 & GM & - & - & $3.1 \mathrm{M}_{\mathrm{L}}(\mathrm{PM})$ & 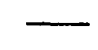 & NOV. & 4 & 15:26 AST \\
\hline NOV. & 5 & 031836.4 & $62.877 \mathrm{~N}$ & $148.802 \mathrm{~W}$ & 66 & GM & - & - & $3.5 \mathrm{M}_{\mathrm{L}}(\mathrm{PM})$ & & NOV. & 4 & 18:18 AST \\
\hline NOV. & 5 & 064207.4 & $59.296 \mathrm{~N}$ & 153.542W. & 100 & GM & - & - & - & & NOV. & 4 & 21:42 AST \\
\hline NOV. & 5 & 095809.3 & $60.223 \mathrm{~N}$ & 153.193W. & 135 & GM & - & - & - & - & NOV. & 5 & 00:58 AST \\
\hline NOV. & 5 & 174851.8 & $63.343 \mathrm{~N}$ & $151.507 \mathrm{~W}$ & 33 & GS & - & - & $4.0 \mathrm{M}_{\mathrm{L}}(\mathrm{PM})$ & 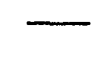 & NOV. & 5 & 08:48 AST \\
\hline NOV. & 5 & 225209.5 & $62.097 \mathrm{~N}$ & 151.096W. & 69 & GM & - & - & - & - & NOV. & 5 & $13: 52$ AST \\
\hline NOV. & 6 & 052108.1 & $61.656 \mathrm{~N}$ & $151.857 \mathrm{~W}$ & 102 & GM & - & - & - & - & NOV. & 5 & 20:21 AST \\
\hline NOV. & 6 & 064308.0 & $50.565 \mathrm{~N}$ & 175.256W. & 33 & GS & 4.7 & - & - & - & NOV. & 5 & 20:43 HST \\
\hline NOV. & 6 & 100820.0 & $59.406 \mathrm{~N}$ & $152.664 \mathrm{~W}$ & 71 & GM & - & - & $3.2 \mathrm{M}_{\mathrm{L}}(\mathrm{PM})$ & - & NOV. & 6 & 01:08 AST \\
\hline NOV. & 6 & 101302.5 & $59.907 \mathrm{~N}$ & 153.479W. & 130 & GM & - & - & - & - & NOV. & 6 & 01:13 AST \\
\hline
\end{tabular}


Table 1. Summary of United States earthquakes for 1986-Continued

\begin{tabular}{|c|c|c|c|c|c|c|c|c|c|c|c|c|c|}
\hline \multirow{2}{*}{\multicolumn{2}{|c|}{ Date }} & \multirow{3}{*}{$\begin{array}{l}\text { Origin time } \\
\text { (UTC) } \\
\text { hr min sec }\end{array}$} & \multirow{2}{*}{$\begin{array}{c}\text { Latitude } \\
\left({ }^{\circ}\right) \\
\end{array}$} & \multirow{2}{*}{$\begin{array}{c}\text { Longitude } \\
(9) \\
\end{array}$} & \multirow{2}{*}{$\begin{array}{l}\text { Depth } \\
(\mathrm{km})\end{array}$} & \multirow{2}{*}{$\begin{array}{l}\text { Hypo- } \\
\text { center } \\
\text { Source }\end{array}$} & \multicolumn{3}{|c|}{ Magnitude } & \multirow{2}{*}{$\begin{array}{l}\text { Max. } \\
\text { inten- } \\
\text { sity }\end{array}$} & \multicolumn{3}{|c|}{ Local time } \\
\hline & & & & & & & $\mathbf{m}_{\mathbf{b}}$ & $\mathbf{M}_{\mathbf{S}}$ & Local & & Date & & zone \\
\hline \multicolumn{13}{|c|}{ ALASKA-Continued } & \\
\hline NOV. & 6 & 182702.9 & $51.242 \mathrm{~N}$. & 176.631W. & 39 & $\mathrm{EE}$ & 5.1 & 5.5 & $5.2 \mathrm{M}_{\mathrm{L}}(\mathrm{PM})$ & IV & NOV. & 6 & 08:27 HST \\
\hline NOV. & 6 & 183624.9 & $62.842 \mathrm{~N}$ & 149.681W. & 100 & GS & - & - & - & & NOV. & 6 & 09:36 AST \\
\hline NOV. & 6 & 194540.5 & $51.072 \mathrm{~N}$ & $176.516 \mathrm{~W}$ & 20 & $\mathrm{EE}$ & 4.8 & - & - & IV & NOV. & 6 & 09:45 HST \\
\hline NOV. & 6 & 220820.8 & $61.997 \mathrm{~N}$ & $151.334 \mathrm{~W}$. & 89 & GM & - & - & - & & NOV. & 6 & 13:08 AST \\
\hline NOV. & 7 & 011612.4 & $62.661 \mathrm{~N}$ & 149.764W. & 119 & GS & $\longrightarrow$ & - & - & & NOV. & 6 & 16:16 AST \\
\hline NOV. & 8 & 020944.4 & $60.438 \mathrm{~N}$ & $151.878 \mathrm{~W}$. & 74 & GM & - & - & - & & NOV. & 7 & 17:09 AST \\
\hline NOV. & 8 & 040833.9 & $60.052 \mathrm{~N}$ & $140.794 \mathrm{~W}$ & 4 & GM & $\ldots$ & - & & & NOV. & 7 & 19:08 AST \\
\hline NOV. & 8 & 060311.2 & $60.064 \mathrm{~N}$ & $140.778 \mathrm{~W}$ & 2 & GM & - & - & . & & NOV. & 7 & 21:03 AST \\
\hline NOV. & 8 & 065458.6 & $60.760 \mathrm{~N}$ & $152.595 \mathrm{~W}$. & 138 & GM & - & - & & & NOV. & 7 & 21:54 AST \\
\hline NOV. & 8 & 074843.8 & $62.000 \mathrm{~N}$ & $152.028 \mathrm{~W}$. & 113 & GM & - & & & & NOV. & 7 & $22: 48$ AST \\
\hline NOV. & 8 & 094001.0 & $52.233 \mathrm{~N}$ & 169.617W. & 33 & GS & 4.9 & 4.4 & - & & NOV. & 7 & 23:40 HST \\
\hline NOV. & 9 & 021700.5 & $60.145 \mathrm{~N}$ & 141.026W. & 12 & $\mathrm{GM}$ & 4.1 & - & $4.2 \mathrm{M}_{\mathrm{L}}(\mathrm{PM})$ & & NOV. & 8 & 17:17 AST \\
\hline NOV. & 9 & 101545.3 & $61.679 \mathrm{~N}$ & $151.761 \mathrm{~W}$ & 89 & GM & - & - & - & & NOV. & 9 & 01:15 AST \\
\hline NOV. & 9 & 121624.4 & $52.263 \mathrm{~N}$ & 168.363W. & 33 & GS & 5.3 & 4.7 & & & NOV. & 9 & 03:16 AST \\
\hline NOV. & 9 & 124202.4 & $52.500 \mathrm{~N}$ & 168.467W. & 33 & GS & 4.8 & & & & NOV. & 9 & 03:42 AST \\
\hline NOV. & 9 & 210940.8 & $67.848 \mathrm{~N}$ & $165.552 W$. & 33 & GS & 4.2 & - & $4.1 \mathrm{M}_{\mathrm{L}}(\mathrm{PM})$ & & NOV. & 9 & 12:09 AST \\
\hline NOV. & 10 & 041355.3 & $50.831 \mathrm{~N}$ & 176.421W. & 20 & $\mathrm{EE}$ & 4.7 & & - & & NOV. & 9 & 18:13 HST \\
\hline NOV. & 10 & 214540.7 & $52.536 \mathrm{~N}$ & 169.503W. & 33 & GS & 4.8 & & - & & NOV. & 10 & 12:45 AST \\
\hline NOV. & 11 & 013826.9 & $59.953 \mathrm{~N}$. & 151.336W. & 44 & GM & - & & - & & NOV. & 10 & 16:38 AST \\
\hline NOV. & 11 & 042209.5 & $54.580 \mathrm{~N}$ & $165.286 \mathrm{~W}$ & 206 & LD & - & & $3.6 m_{x}(L D)$ & & NOV. & 10 & 19:22 AST \\
\hline NOV. & 11 & 081858.7 & 63.387N. & $150.241 \mathrm{~W}$. & 33 & GS & - & - & $3.5 \mathrm{M}_{\mathrm{L}}(\mathrm{PM})$ & & NOV. & 10 & 23:18 AST \\
\hline NOV. & 11 & 140516.8 & $51.246 \mathrm{~N}$ & 174.710W. & 20 & EE & 4.9 & & $4.6 \mathrm{M}_{\mathrm{L}}(\mathrm{PM})$ & & NOV. & 11 & 04:05 HST \\
\hline NOV. & 11 & 184651.2 & $62.240 \mathrm{~N}$ & 149.681W. & 56 & GM & - & & $3.0 \mathrm{M}_{\mathrm{L}}(\mathrm{PM})$ & & NOV. & 11 & 09:46 AST \\
\hline NOV. & 11 & 211903.6 & $61.975 \mathrm{~N}$ & $149.857 \mathrm{~W}$ & 23 & GS & 一 & & $3.1 \mathrm{M}_{\mathrm{L}}(\mathrm{PM})$ & & NOV. & 11 & 12:19 AST \\
\hline NOV. & 12 & 020137.6 & $51.114 \mathrm{~N}$ & 174.340W. & 20 & $\mathrm{EE}$ & 4.6 & & - & & NOV. & 11 & 16:01 HST \\
\hline NOV. & 12 & 043946.1 & $65.917 \mathrm{~N}$ & 156.440W. & 10 & GS & - & & $3.4 \mathrm{M}_{\mathrm{L}}(\mathrm{PM})$ & & NOV. & 11 & 19:39 AST \\
\hline NOV. & 12 & 124403.2 & $51.192 \mathrm{~N}$. & 179.412E. & 33 & GS & 4.8 & & $4.6 \mathrm{M}_{\mathrm{L}}(\mathrm{PM})$ & & NOV. & 12 & 02:44 HST \\
\hline NOV. & 13 & 043101.1 & 63.091N. & $150.799 \mathrm{~W}$. & 147 & GS & - & & - & & NOV. & 12 & 19:31 AST \\
\hline NOV. & 13 & 105944.3 & $61.987 \mathrm{~N}$ & $150.745 \mathrm{~W}$. & 62 & GM & $\longrightarrow$ & & $3.0 \mathrm{M}_{\mathrm{L}}(\mathrm{PM})$ & & NOV. & 13 & 01:59 AST \\
\hline NOV. & 13 & 141004.1 & $59.509 \mathrm{~N}$ & $152.399 \mathrm{~W}$ & 71 & GM & & & - & & NOV. & 13 & 05:10 AST \\
\hline NOV. & 13 & 145828.9 & $57.629 \mathrm{~N}$. & $156.549 \mathrm{~W}$. & 171 & GS & - & & & & NOV. & 13 & 05:58 AST \\
\hline NOV. & 13 & 152718.0 & $59.867 \mathrm{~N}$ & $152.359 \mathrm{~W}$ & 72 & GM & - & & - & & NOV. & 13 & 06:27 AST \\
\hline NOV. & 14 & 214245.9 & $51.442 \mathrm{~N}$ & $173.845 \mathrm{~W}$ & 25 & $\mathrm{EE}$ & 5.5 & & $4.9 \mathrm{M}_{\mathrm{L}}(\mathrm{PM})$ & & NOV. & 14 & 11:42 HST \\
\hline NOV. & 14 & 214903.8 & $60.405 \mathrm{~N}$ & 149.305W. & 38 & GM & - & & - & & NOV. & 14 & $12: 49$ AST \\
\hline NOV. & 15 & 070214.0 & $61.468 \mathrm{~N}$ & $146.441 \mathrm{~W}$. & 23 & GM & $\longrightarrow$ & & $3.8 \mathrm{M}_{\mathrm{L}}(\mathrm{PM})$ & & NOV. & 14 & 22:02 AST \\
\hline NOV. & 16 & 024800.7 & $61.898 \mathrm{~N}$. & $150.862 \mathrm{~W}$. & 70 & GM & - & & $3.0 \mathrm{M}_{\mathrm{L}}(\mathrm{PM})$ & & NOV. & 15 & 17:48 AST \\
\hline NOV. & 17 & 010534.9 & $60.581 \mathrm{~N}$ & $150.448 \mathrm{~W}$. & 47 & GM & $\longrightarrow$ & & - & & NOV. & 16 & 16:05 AST \\
\hline NOV. & 17 & 025206.5 & $61.598 \mathrm{~N}$. & $151.820 \mathrm{~W}$. & 93 & GM & $\longrightarrow$ & & - & & NOV. & 16 & 17:52 AST \\
\hline NOV. & 17 & 055051.1 & $58.927 \mathrm{~N}$ & $152.986 \mathrm{~W}$ & 75 & GM & - & - & - & & NOV. & 16 & 20:50 AST \\
\hline NOV. & 18 & 062751.7 & 63.191N. & $150.440 \mathrm{~W}$ & 141 & GS & $\longrightarrow$ & - & - & & NOV. & 17 & 21:27 AST \\
\hline
\end{tabular}


Table 1. Summary of United States earthquakes for 1986-Continued

\begin{tabular}{|c|c|c|c|c|c|c|c|c|c|c|c|c|c|}
\hline \multirow{2}{*}{\multicolumn{2}{|c|}{ Date }} & \multirow{3}{*}{$\begin{array}{l}\text { Origin time } \\
\text { (UTC) } \\
\text { hr min sec }\end{array}$} & \multirow{2}{*}{$\begin{array}{l}\text { Latitude } \\
\left({ }^{\circ}\right)\end{array}$} & \multirow{2}{*}{$\begin{array}{c}\text { Longitude } \\
\left({ }^{\circ}\right)\end{array}$} & \multirow{2}{*}{$\begin{array}{c}\text { Depth } \\
(\mathbf{k m})\end{array}$} & \multirow{2}{*}{$\begin{array}{l}\text { Hypo- } \\
\text { center } \\
\text { Source }\end{array}$} & \multicolumn{3}{|c|}{ Magnitude } & \multirow{2}{*}{$\begin{array}{l}\text { Max. } \\
\text { inten- } \\
\text { sity }\end{array}$} & \multicolumn{3}{|c|}{ Local time } \\
\hline & & & & & & & $\mathrm{m}_{\mathrm{b}}$ & $\mathbf{M}_{\mathbf{s}}$ & Local & & Date & & zone \\
\hline \multicolumn{13}{|c|}{ ALASKA-Continued } & \\
\hline NOV. & 19 & 024222.2 & $52.370 \mathrm{~N}$ & $170.727 \mathrm{~W}$ & 33 & GS & - & - & $4.3 \mathrm{M}_{\mathrm{L}}(\mathrm{PM})$ & & NOV. & 18 & $16: 42 \mathrm{HST}$ \\
\hline NOV. & 19 & 060043.7 & $60.659 \mathrm{~N}$ & 151.910W. & 92 & GM & - & - & - & - & NOV. & 18 & 21:00 AST \\
\hline NOV. & 19 & 153240.2 & $55.716 \mathrm{~N}$. & 159.508W. & 95 & LD & & & $3.3 m_{X}(L D)$ & & NOV. & 19 & 06:32 AST \\
\hline NOV. & 19 & 153842.5 & $57.561 \mathrm{~N}$. & $150.529 \mathrm{~W}$ & 33 & GS & & - & $3.6 \mathrm{M}_{\mathrm{L}}(\mathrm{PM})$ & - & NOV. & 19 & 06:38 AST \\
\hline NOV. & 19 & 190011.4 & $51.036 \mathrm{~N}$. & $176.001 \mathrm{~W}$ & 33 & GS & - & - & $4.5 \mathrm{M}_{\mathrm{L}}(\mathrm{PM})$ & IIII & NOV. & 19 & 09:00 HST \\
\hline NOV. & 19 & 204230.1 & $62.008 \mathrm{~N}$ & $150.910 \mathrm{~W}$. & 65 & GM & - & - & - & - & NOV. & 19 & 11:42 AST \\
\hline NOV. & 20 & 015216.5 & $52.971 \mathrm{~N}$ & 165.134W. & 21 & LD & - & - & $3.4 m_{x}(L D)$ & & NOV. & 19 & 16:52 AST \\
\hline NOV. & 20 & 073836.2 & $62.108 \mathrm{~N}$ & $150.848 \mathrm{~W}$ & 65 & GM & & & - & & NOV. & 19 & 22:38 AST \\
\hline NOV. & 20 & 124743.9 & $58.658 \mathrm{~N}$ & $136.282 \mathrm{~W}$ & 15 & GM & & & $3.8 \mathrm{M}_{\mathrm{L}}(\mathrm{EP})$ & & NOV. & 20 & 03:47 AST \\
\hline NOV. & 22 & 174150.1 & $62.988 \mathrm{~N}$ & 151.359W. & 33 & GS & - & . & $3.3 \mathrm{M}_{\mathrm{L}}(\mathrm{PM})$ & & NOV. & 22 & 08:41 AST \\
\hline NOV. & 22 & 221123.8 & $60.227 \mathrm{~N}$ & 153.230W. & 133 & GM & - & - & - & & NOV. & 22 & 13:11 AST \\
\hline NOV. & 22 & 221714.4 & $60.131 N$ & 153.106W. & 115 & GM & - & & - & & NOV. & 22 & 13:17 AST \\
\hline NOV. & 23 & 193403.4 & $61.394 \mathrm{~N}$ & $150.374 \mathrm{~W}$ & 47 & GM & - & & - & & NOV. & 23 & 10:34 AST \\
\hline NOV. & 24 & 112927.3 & $51.734 \mathrm{~N}$. & 178.349E. & 78 & GS & 5.0 & & $4.8 \mathrm{M}_{\mathrm{L}}(\mathrm{PM})$ & & NOV. & 24 & 01:29 HST \\
\hline NOV. & 25 & 024740.4 & $59.138 \mathrm{~N}$. & 153.965W. & 119 & GM & & & & & NOV. & 24 & 17:47 AST \\
\hline NOV. & 25 & 113758.8 & $60.110 \mathrm{~N}$ & 152.851W. & 91 & GM & - & - & - & & NOV. & 25 & 02:37 AST \\
\hline NOV. & 25 & 164419.2 & $61.408 \mathrm{~N}$ & $150.371 \mathrm{~W}$ & 16 & GM & & & $3.5 \mathrm{M}_{\mathrm{L}}(\mathrm{PM})$ & & NOV. & 25 & 07:44 AST \\
\hline NOV. & 25 & 165459.2 & $59.926 \mathrm{~N}$. & 152.854W. & 88 & GM & & & - & - & NOV. & 25 & 07:54 AST \\
\hline NOV. & 26 & 210443.4 & $61.774 \mathrm{~N}$ & $150.887 \mathrm{~W}$ & 62 & GM & $\underline{-}$ & & $3.6 \mathrm{M}_{\mathrm{L}}(\mathrm{PM})$ & III & NOV. & 26 & 12:04 AST \\
\hline NOV. & 27 & 103217.1 & $61.200 \mathrm{~N}$ & $149.867 \mathrm{~W}$ & 42 & GM & $\longrightarrow$ & & & & NOV. & 27 & 01:32 AST \\
\hline NOV. & 29 & 170556.9 & $59.047 \mathrm{~N}$. & 152.290W. & 78 & GM & - & - & - & & NOV. & 29 & 08:05 AST \\
\hline DEC. & 1 & 040037.2 & $63.582 \mathrm{~N}$ & 151.018W. & 33 & GS & - & & $3.4 \mathrm{M}_{\mathrm{L}}(\mathrm{PM})$ & & NOV. & 30 & 19:00 AST \\
\hline DEC. & 1 & 230710.5 & $51.264 \mathrm{~N}$. & 174.338W. & 20 & $\mathrm{EE}$ & 4.9 & & - & & DEC. & 1 & 13:07 HST \\
\hline DEC. & 2 & 133951.4 & $61.426 \mathrm{~N}$ & 151.270W. & 66 & GM & - & & - & 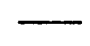 & DEC. & 2 & 04:39 AST \\
\hline DEC. & 3 & 050536.7 & $51.352 \mathrm{~N}$ & 176.465W. & 36 & EE & 4.6 & & - & III & DEC. & 2 & 19:05 HST \\
\hline DEC. & 3 & 175102.0 & $54.636 \mathrm{~N}$ & 162.371W. & 96 & LD & - & & $3.0 m_{X}(L D)$ & & DEC. & 3 & 08:51 AST \\
\hline DEC. & 4 & 081924.1 & 61.594N. & 151.077W. & 68 & GM & & & - & & DEC. & 3 & 23:19 AST \\
\hline DEC. & 4 & 173306.0 & $63.111 \mathrm{~N}$. & 148.778W. & 44 & GM & - & & - & & DEC. & 4 & 08:33 AST \\
\hline DEC. & 4 & 182247.5 & $54.696 \mathrm{~N}$. & 159.593W. & 30 & LD & & & $3.3 \mathrm{~m}_{\mathrm{X}}(\mathrm{LD})$ & & DEC. & 4 & 09:22 AST \\
\hline DEC. & 5 & 015958.2 & $59.664 \mathrm{~N}$. & 154.276W. & 178 & GM & - & & & & DEC. & 4 & 16:59 AST \\
\hline DEC. & 5 & 024704.7 & $63.013 \mathrm{~N}$ & 149.945W. & 33 & GS & & & $3.4 \mathrm{M}_{\mathrm{L}}(\mathrm{PM})$ & & DEC. & 4 & 17:47 AST \\
\hline DEC. & 6 & 173629.3 & $59.975 \mathrm{~N}$ & $151.548 \mathrm{~W}$ & 59 & GM & - & & - & & DEC. & 6 & 08:36 AST \\
\hline DEC. & 6 & 195619.0 & $52.687 \mathrm{~N}$ & 172.949E. & 33 & GS & 4.7 & & - & & DEC. & 6 & 09:56 HST \\
\hline DEC. & 7 & 013700.8 & $61.496 \mathrm{~N}$ & $151.692 \mathrm{~W}$ & 86 & GM & - & - & - & & DEC. & 6 & 16:37 AST \\
\hline DEC. & 7 & 014347.3 & $60.323 \mathrm{~N}$ & 153.738W. & 177 & GM & & & - & & DEC. & 6 & $16: 43$ AST \\
\hline DEC. & 7 & 051833.2 & $62.032 \mathrm{~N}$ & $152.044 \mathrm{~W}$ & 124 & GM & & & - & & DEC. & 6 & 20:18 AST \\
\hline DEC. & 8 & 061058.0 & $53.325 \mathrm{~N}$ & $166.835 \mathrm{~W}$. & 78 & GS & & & $4.6 \mathrm{M}_{\mathrm{L}}(\mathrm{PM})$ & & DEC. & 7 & 21:10 AST \\
\hline DEC. & 9 & 125609.0 & $57.890 \mathrm{~N}$. & $137.526 \mathrm{~W}$ & 18 & EP & & & $3.5 \mathrm{M}_{\mathrm{L}}(\mathrm{EP})$ & & DEC. & 9 & 03:56 AST \\
\hline DEC. & 9 & 193043.8 & $54.005 \mathrm{~N}$. & $165.059 \mathrm{~W}$ & 6 & LD & & & $3.4 m_{x}(L D)$ & & DEC. & 9 & $10: 30$ AST \\
\hline DEC. & 12 & 181043.0 & $61.901 \mathrm{~N}$ & 149.915W. & 49 & GM & - & - & $3.2 \mathrm{M}_{\mathrm{L}}(\mathrm{PM})$ & - & DEC. & 12 & 09:10 AST \\
\hline
\end{tabular}


Table 1. Summary of United States earthquakes for 1986 - Continued

\begin{tabular}{|c|c|c|c|c|c|c|c|c|c|c|c|c|}
\hline \multirow[t]{2}{*}{ Date } & \multirow{2}{*}{$\begin{array}{l}\text { Origin time } \\
\text { (UTC) } \\
\text { hr mill sec }\end{array}$} & \multirow{2}{*}{$\begin{array}{l}\text { Latitude } \\
\left({ }^{\circ}\right)\end{array}$} & \multirow{2}{*}{$\begin{array}{l}\text { Longitude } \\
\left({ }^{\circ}\right)\end{array}$} & \multirow{2}{*}{$\begin{array}{l}\text { Depth } \\
(\mathbf{k m})\end{array}$} & \multirow{2}{*}{$\begin{array}{l}\text { Hypo- } \\
\text { center } \\
\text { Source }\end{array}$} & \multicolumn{3}{|c|}{ Magnitude } & \multirow{2}{*}{$\begin{array}{l}\text { Max. } \\
\text { inten- } \\
\text { sity }\end{array}$} & \multicolumn{3}{|c|}{ Local time } \\
\hline & & & & & & $\mathrm{m}_{\mathrm{b}}$ & $\overline{\mathrm{M}_{s}}$ & Local & & Date & $\overline{h r}$ & zone \\
\hline
\end{tabular}

ALASKA-Continued

DEC. $12 \quad 184417.0$

DEC. $12 \quad 235147.4$

DEC. 14004145.3

DEC. $14 \quad 121734.9$

DEC. $15 \quad 044941.5$

DEC. $15 \quad 204213.2$

DEC. $16 \quad 053539.4$

DEC. $16 \quad 075824.2$

DEC. $16 \quad 102724.4$

DEC. $16 \quad 172629.8$

DEC. $17 \quad 035932.3$

DEC. $17 \quad 113203.8$

DEC. $17 \quad 215343.7$

DEC. $18 \quad 000845.0$

DEC. $18 \quad 015409.9$

DEC. $18 \quad 034630.6$

DEC. $18 \quad 094324.6$

DEC. 18201715.2

DEC. 19034746.9

DEC. $19 \quad 135013.3$

DEC. $20 \quad 000142.0$

DEC. 20

DEC. 20

DEC. 21

DEC. $22 \quad 062821.3$

DEC. $22 \quad 144215.5$

DEC. $23 \quad 034028.2$

DEC. 23

DEC. 23

DEC. 23

131922.2

171420.9

231429.9

DEC. $24 \quad 084846.1$

DEC. 26005540.5

DEC. 27033541.4

DEC. 27063626.2

DEC. $27 \quad 080408.1$

DEC. $27 \quad 130700.8$

DEC. $27 \quad 173841.4$

DEC. 28

DEC. 28

DEC. 29

045400.2

202151.7

041416.6
$62.681 \mathrm{~N} .150 .626 \mathrm{~W}$. $61.893 \mathrm{~N} .150 .123 \mathrm{~W} .56 \mathrm{GM}$ $60.233 \mathrm{~N} .153 .156 \mathrm{~W} .133 \mathrm{GM}$ $65.699 \mathrm{~N} .168 .831 \mathrm{~W} . \quad 33$ GS 59.831N. $152.922 \mathrm{~W} . \quad 86 \mathrm{GM}$

61.494N. 146.730W. 33 GS 63.653N. 147.458W. 107 GS 61.903N. $150.945 \mathrm{~W} . \quad 68 \mathrm{GM}$ $51.492 \mathrm{~N}$. 175.319W. 35 EE 60.958 N. $146.995 \mathrm{~W} .14 \mathrm{GM}$

59.688N. 153.231W. $104 \quad$ GM 66.299N. 150.133W. 10 GS 53.685N. 165.261W. 83 LD 61.445N. 151.439W. 72 GM 51.088N. 174.492W. 20 EE

51.708N. 179.033E. $62.356 \mathrm{~N} . \quad 151.208 \mathrm{~W}$

$58.890 \mathrm{~N} . \quad 152.275 \mathrm{~W}$

59.207N. 153.572W.

$51.391 \mathrm{~N} .176 .903 \mathrm{~W}$

$59.975 \mathrm{~N} . \quad 152.358 \mathrm{~W}$ $53.288 \mathrm{~N}$. $164.341 \mathrm{~W}$ $60.582 \mathrm{~N} . \quad 151.759 \mathrm{~W}$ $60.088 \mathrm{~N} . \quad 140.980 \mathrm{~W}$ $56.608 \mathrm{~N}$. $157.043 \mathrm{~W}$

59.683N. $153.075 W . \quad 102$ 60.433 N. $150.501 \mathrm{~W} . \quad 44$ 60.149 N. 153.159W. 124 $51.751 \mathrm{~N} .171 .602 \mathrm{~W}$. $58.474 \mathrm{~N}$. $148.174 \mathrm{~W}$. 33

$51.859 \mathrm{~N} .178 .229 \mathrm{~W} . \quad 15$ $62.520 \mathrm{~N} .151 .290 \mathrm{~W} .141$ $61.828 \mathrm{~N}$. 148.954W. 15 $61.406 \mathrm{~N} .151 .324 \mathrm{~W} . \quad 71$ 60.205 N. $151.052 \mathrm{~W} .76$

GS 84 GM

GM

GM

EE

GM

LD

GM

GM

LD

GM

GM

GM

GS

GM

EE

GM

GM

GM

GM

64.745N. 150.975W. 33 GS

$61.427 \mathrm{~N} .149 .966 \mathrm{~W} .52 \quad \mathrm{GM}$
$53.853 \mathrm{~N} .161 .748 \mathrm{~W} . \quad 2 \quad \mathrm{DD}$

$54.008 \mathrm{~N}, 162.816 \mathrm{~W}$

$53.779 \mathrm{~N}$. $163.879 \mathrm{~W}$.

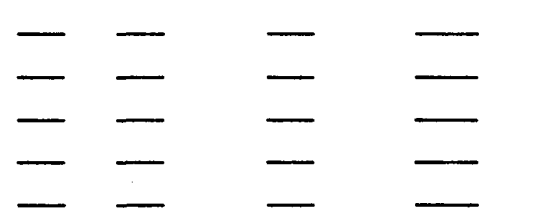

DEC. 12 09:44 AST

DEC. 12 14:51 AST

DEC. 13 15:41.AST

DEC. 14 03:17 AST

DEC. 14 19:49 AST

DEC. $15 \quad 11: 42$ AST

DEC. $15 \quad 20: 35$ AST

DEC. 15 22:58 AST

DEC. $16 \quad 00: 27$ HST

DEC. 16 08:26 AST

DEC. $16 \quad 18: 59$ AST

DEC. 17 02:32 AST

DEC. 17 12:53 AST

DEC. 17 15:08 AST

DEC. 17 15:54 HST

DEC. 17 17:46 HST

DEC. 18 00:43 AST

DEC. 18 11:17 AST

DEC. 18 18:47 AST

DEC. 19 03:50 HST

DEC. 19 15:01 AST

DEC. 19 22:23 AST

DEC. 20 07:10 AST

DEC. 21 08:45 AST

DEC. 21 21:28 AST

DEC. 22 05:42 AST

DEC. 22 18:40 AST

DEC. 23 04:19 AST

DEC. 23 07:14 HST

DEC. 23 14:14 AST

DEC. $2322: 48$ HST

DEC. 25 15:55 AST

DEC. 26 18:35 AST

DEC. 26 21:36 AST

DEC. 26 23:04 AST

DEC. $27 \quad 04: 07$ AST DEC. $27 \quad 08: 38$ AST DEC. $27 \quad$ 19:54 AST DEC. $28 \quad$ 11:21 AST DEC. $28 \quad$ 19:14 AST 
Table 1. Summary of United States earthquakes for 1986-Continued

\begin{tabular}{|c|c|c|c|c|c|c|c|c|c|c|c|c|}
\hline \multirow[t]{2}{*}{ Date } & \multirow{2}{*}{$\begin{array}{l}\text { Origin time } \\
\text { (UTC) }\end{array}$} & \multirow{2}{*}{$\begin{array}{l}\text { Latitude } \\
\text { (०) }\end{array}$} & \multirow{2}{*}{$\begin{array}{c}\text { Longitude } \\
\text { ( })\end{array}$} & \multirow{2}{*}{$\begin{array}{c}\text { Depth } \\
(\mathrm{km})\end{array}$} & \multirow{2}{*}{$\begin{array}{l}\text { Hypo- } \\
\text { center } \\
\text { Source }\end{array}$} & \multicolumn{3}{|c|}{ Magnitude } & \multirow{2}{*}{$\begin{array}{l}\text { Max. } \\
\text { inten- } \\
\text { sity }\end{array}$} & \multicolumn{3}{|c|}{ Local time } \\
\hline & & & & & & $\overline{m_{b}}$ & $\mathrm{M}_{\mathbf{S}}$ & Local & & Date & hr & zone \\
\hline
\end{tabular}

ALASKA-Continued

$\begin{array}{lllllll}\text { DEC. } 29 & 141744.0 & 63.206 \mathrm{~N} . & 150.497 \mathrm{~W} & 125 & \text { GS }\end{array}$

$66.466 \mathrm{~N} 149.908 \mathrm{~W} 125$ GS - - -

$\mathrm{GS}--\longrightarrow$

DEC. $29223139.5 \quad 60.176 \mathrm{~N}$. 153.254W. 142 GM - - - - $\quad$ - $\quad$ - DEC. 29 13:31 AST

DEC. $29230543.0 \quad 61.585 \mathrm{~N} .150 .894 \mathrm{~W} . \quad 73 \quad \mathrm{GM} \quad-\quad-\quad-\quad-\quad-\quad$ DEC. 29 14:05 AST

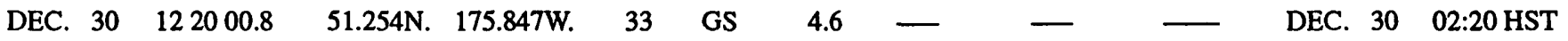

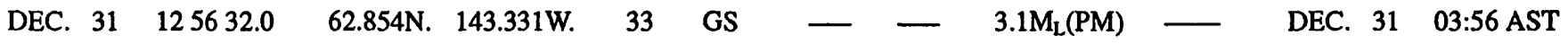

\section{ARIZONA}

\begin{tabular}{|c|c|c|c|c|c|c|c|c|c|c|c|}
\hline JAN. & 193500.1 & $32.550 \mathrm{~N}$. & $114.100 \mathrm{~W}$. & 0 & PS & - & — & $3.1 \mathrm{M}_{\mathrm{L}}(\mathrm{PS})$ & - & JAN. 19 & 11:35 PST \\
\hline MAR. 24 & 172957.4 & $32.476 \mathrm{~N}$. & $114.077 \mathrm{~W}$. & 0 & GS & - & - & $3.4 \mathrm{M}_{\mathrm{L}}(\mathrm{PS})$ & & MAR. 24 & 09:29 PST \\
\hline APR. & 180002.9 & $32.390 \mathrm{~N}$. & $113.800 \mathrm{~W}$. & 6 & PS & - & - & $3.1 \mathrm{M}_{\mathrm{L}}(\mathrm{PS})$ & & APR. & $11: 00 \mathrm{MST}$ \\
\hline JULY 17 & 211349.6 & $34.702 \mathrm{~N}$. & $111.149 \mathrm{~W}$. & 5 & GS & $\longrightarrow$ & - & $2.6 \mathrm{M}_{\mathrm{L}}(\mathrm{GS})$ & & JULY 17 & $14: 13$ MST \\
\hline AUG. 15 & 191506.2 & $34.620 \mathrm{~N}$. & $113.150 \mathrm{~W}$ & 0 & GP & 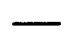 & - & $3.0 \mathrm{M}_{\mathrm{L}}(\mathrm{GP})$ & - & AUG. 15 & 12:15 MST \\
\hline AUG. 21 & 174239.7 & $34.620 \mathrm{~N}$. & $113.150 \mathrm{~W}$. & 0 & GP & - & - & $3.1 \mathrm{M}_{\mathrm{L}}(\mathrm{GP})$ & & AUG. 21 & 10:42 MST \\
\hline AUG. 27 & 182829.5 & $34.620 \mathrm{~N}$. & 113.150W. & 0 & GP & - & - & $3.1 \mathrm{M}_{\mathrm{L}}(\mathrm{GP})$ & - & AUG. 27 & $11: 28 \mathrm{MST}$ \\
\hline
\end{tabular}

ARKANSAS

\begin{tabular}{|c|c|c|c|c|c|c|c|c|c|c|c|}
\hline JAN. & 141322.5 & $35.87 \mathrm{~N}$. & $89.99 \mathrm{~W}$. & 1 & SL & - & - & $2.6 \mathrm{M}_{\mathrm{n}}(\mathrm{SL})$ & & JAN. & 08:13 CST \\
\hline $\mathrm{EB}$. & 133618.2 & $35.259 \mathrm{~N}$ & $92.273 \mathrm{~W}$ & 6 & TC & - & - & $2.5 \mathrm{M}_{\mathrm{v}}(\mathrm{TC})$ & & FEB. & 07:36 CST \\
\hline MAY & 081601.5 & $35.178 \mathrm{~N}$ & $92.217 \mathrm{~W}$ & 5 & TC & - & - & $3.0 \mathrm{M}_{\mathrm{D}}(\mathrm{TU})$ & 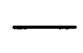 & MAY 2 & $02: 16 \mathrm{CST}$ \\
\hline SEPT. 2 & 085635.5 & $35.88 \mathrm{~N}$. & $89.98 \mathrm{~W}$. & 10 & SL & - & - & $2.8 \mathrm{M}_{\mathrm{t}}(\mathrm{SL})$ & & SEPT. 2 & 02:56 CST \\
\hline
\end{tabular}

\section{CALIFORNIA}

\begin{tabular}{|c|c|c|c|c|c|c|c|c|c|c|c|c|c|}
\hline JAN. & 2 & 214126.3 & $36.303 \mathrm{~N}$. & $120.335 \mathrm{~W}$ & 6 & GP & - & - & $3.0 \mathrm{M}_{\mathrm{L}}(\mathrm{GP})$ & - & JAN. & 2 & 13:41 PST \\
\hline JAN. & 3 & 123303.6 & $40.472 \mathrm{~N}$. & $124.578 \mathrm{~W}$. & 8 & BK & - & - & $3.1 \mathrm{M}_{\mathrm{L}}(\mathrm{BK})$ & & JAN. & 3 & 04:33 PST \\
\hline JAN. & 5 & 051849.1 & $37.262 \mathrm{~N}$. & $121.665 \mathrm{~W}$ & 6 & BK & - & & $2.7 \mathrm{M}_{\mathrm{L}}(\mathrm{BK})$ & FELT & JAN. & 4 & 21:18 PST \\
\hline JAN. & 6 & 195242.7 & $37.010 \mathrm{~N}$. & 121.483W. & 9 & BK & - & - & $3.7 \mathrm{M}_{\mathrm{L}}(\mathrm{BK})$ & III & JAN. & 6 & 11:52 PST \\
\hline JAN. & 8 & 041603.8 & $37.619 \mathrm{~N}$ & 118.854W. & 5 & GS & - & - & $3.0 \mathrm{M}_{\mathrm{L}}(\mathrm{PS})$ & & JAN. & 7 & 20:16 PST \\
\hline JAN. & 10 & 121358.6 & $37.468 \mathrm{~N}$. & $118.916 \mathrm{~W}$. & 6 & PS & - & & $3.1 \mathrm{M}_{\mathrm{L}}(\mathrm{PS})$ & & JAN. & 10 & 04:13 PST \\
\hline JAN. & 11 & 095205.3 & $38.788 \mathrm{~N}$ & $122.767 \mathrm{~W}$ & 3 & BK & - & - & $3.2 \mathrm{M}_{\mathrm{L}}(\mathrm{BK})$ & & JAN. & 11 & 01:52 PST \\
\hline JAN. & 12 & 094148.5 & $35.326 \mathrm{~N}$ & $118.524 W$ & 3 & PS & - & - & $3.2 \mathrm{M}_{\mathrm{L}}(\mathrm{GP})$ & & JAN. & 12 & 01:41 PST \\
\hline JAN. & 14 & 030754.9 & $36.563 \mathrm{~N}$. & $121.203 \mathrm{~W}$. & 7 & BK & - & & $3.4 \mathrm{M}_{\mathrm{L}}(\mathrm{BK})$ & FELT & JAN. & 13 & 19:07 PST \\
\hline JAN. & 14 & 030936.3 & $36.572 \mathrm{~N}$. & $121.205 \mathrm{~W}$ & 7 & BK & 5.0 & & $4.8 \mathrm{M}_{\mathrm{L}}(\mathrm{BK})$ & IV & JAN. & 13 & 19:09 PST \\
\hline JAN. & 14 & 053547.9 & $36.568 \mathrm{~N}$. & $121.202 \mathrm{~W}$ & 6 & BK & - & 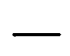 & $3.0 \mathrm{M}_{\mathrm{L}}(\mathrm{BK})$ & 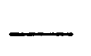 & JAN. & 13 & 21:35 PST \\
\hline JAN. & 14 & 131214.0 & $33.914 \mathrm{~N}$. & $116.697 \mathrm{~W}$. & 13 & PS & - & - & $3.4 \mathrm{M}_{\mathrm{L}}(\mathrm{GP})$ & FELT & JAN. & 14 & 05:12 PST \\
\hline JAN. & 16 & 093847.4 & $38.428 \mathrm{~N}$. & $122.645 \mathrm{~W}$ & 5 & BK & - & 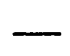 & $2.5 \mathrm{M}_{\mathrm{L}}(\mathrm{BK})$ & IV & JAN. & 16 & 01:38 PST \\
\hline JAN. & 16 & 190522.0 & $40.447 \mathrm{~N}$. & $124.588 \mathrm{~W}$. & 18 & BK & - & - & $3.0 \mathrm{M}_{\mathrm{L}}(\mathrm{BK})$ & & JAN. & 16 & 11:05 PST \\
\hline JAN. & 17 & 175200.8 & $36.203 \mathrm{~N}$. & $120.162 \mathrm{~W}$ & 6 & GP & - & - & $3.3 \mathrm{M}_{\mathrm{L}}(\mathrm{BK})$ & 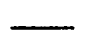 & JAN. & 17 & 09:52 PST \\
\hline
\end{tabular}


Table 1. Summary of United States earthquakes for 1986 Continued

\begin{tabular}{|c|c|c|c|c|c|c|c|c|c|c|c|c|c|}
\hline \multirow{2}{*}{\multicolumn{2}{|c|}{ Date }} & $\begin{array}{l}\text { Origin time } \\
\text { (UTC) }\end{array}$ & \multirow{2}{*}{$\begin{array}{l}\text { Latitude } \\
\text { (०) }\end{array}$} & \multirow{2}{*}{$\begin{array}{c}\text { Longitude } \\
(9)\end{array}$} & \multirow{2}{*}{$\begin{array}{l}\text { Depth } \\
(\mathbf{k m})\end{array}$} & \multirow{2}{*}{$\begin{array}{l}\text { Hypo- } \\
\text { center } \\
\text { Source }\end{array}$} & \multicolumn{3}{|c|}{ Magnitude } & \multirow{3}{*}{$\begin{array}{c}\text { Max. } \\
\text { inten- } \\
\text { sity }\end{array}$} & \multicolumn{3}{|c|}{ Local time } \\
\hline & & hr min sec & & & & & $\mathrm{m}_{\mathrm{b}}$ & $\mathrm{M}_{\mathrm{s}}$ & Local & & Date & & zone \\
\hline \multicolumn{13}{|c|}{ CALIFORNIA-Continued } & \\
\hline JAN. & 17 & 215838.0 & $37.522 \mathrm{~N}$ & $118.634 \mathrm{~W}$ & 5 & GS & 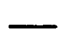 & - & $3.1 \mathrm{M}_{\mathrm{L}}(\mathrm{PS})$ & & JAN. & 17 & 13:58 PST \\
\hline JAN. & 18 & 065729.2 & $37.415 \mathrm{~N}$ & $118.647 \mathrm{~W}$ & 6 & PS & - & - & $3.4 \mathrm{M}_{\mathrm{L}}(\mathrm{PS})$ & & JAN. & 17 & 22:57 PST \\
\hline JAN. & 18 & 203842.4 & $36.264 \mathrm{~N}$. & $120.241 W$ & 6 & GP & - & 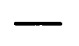 & $3.0 \mathrm{M}_{\mathrm{L}}(\mathrm{PS})$ & & JAN. & 18 & 12:38 PST \\
\hline JAN. & 21 & 005419.9 & $32.050 \mathrm{~N}$ & $116.368 \mathrm{~W}$ & 6 & GP & & $\longrightarrow$ & $3.0 \mathrm{M}_{\mathrm{L}}(\mathrm{PS})$ & & JAN. & 20 & 16:54 PST \\
\hline JAN. & 21 & 115731.3 & $40.437 \mathrm{~N}$ & $125.265 \mathrm{~W}$ & 10 & BK & & - & $3.5 \mathrm{M}_{\mathrm{L}}(\mathrm{BK})$ & & JAN. & 21 & 03:57 PST \\
\hline JAN. & 21 & 200730.8 & $38.543 \mathrm{~N}$ & 122.995W. & 1 & BK & - & - & $2.3 \mathrm{M}_{\mathrm{L}}(\mathrm{BK})$ & IV & JAN. & 21 & 12:07 PST \\
\hline JAN. & 22 & 162654.0 & $33.686 \mathrm{~N}$ & 119.134W. & 6 & PS & - & - & $3.6 \mathrm{M}_{\mathrm{L}}(\mathrm{PS})$ & & JAN. & 22 & 08:26 PST \\
\hline JAN. & 26 & 192051.2 & $36.810 \mathrm{~N}$ & $121.275 \mathrm{~W}$. & 7 & BK & 5.3 & 5.3 & $5.5 \mathrm{M}_{\mathrm{L}}(\mathrm{BK})$ & VII & JAN. & 26 & $11: 20$ PST \\
\hline JAN. & 26 & 214434.2 & 36.817N. & $121.265 \mathrm{~W}$. & 9 & BK & 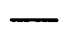 & $\longrightarrow$ & $3.2 \mathrm{M}_{\mathrm{L}}(\mathrm{BK})$ & & JAN. & 26 & 13:44 PST \\
\hline JAN. & 26 & 234654.9 & $36.828 \mathrm{~N}$ & $121.290 \mathrm{~W}$. & 6 & BK & & - & 4.0 $\mathrm{M}_{\mathrm{L}}(\mathrm{BK})$ & - & JAN. & 26 & 15:46 PST \\
\hline JAN. & 27 & 020201.1 & $37.638 \mathrm{~N}$ & $118.950 \mathrm{~W}$. & 6 & PS & & & $3.2 \mathrm{M}_{\mathrm{L}}(\mathrm{PS})$ & & JAN. & 26 & 18:02 PST \\
\hline JAN. & 27 & 100737.1 & $36.810 \mathrm{~N}$ & $121.263 \mathrm{~W}$ & 7 & BK & & - & $3.4 \mathrm{M}_{\mathrm{L}}(\mathrm{BK})$ & - & JAN. & 27 & 02:07 PST \\
\hline JAN. & 27 & 142606.4 & $36.835 \mathrm{~N}$ & $121.278 \mathrm{~W}$ & 5 & BK & & & $3.3 \mathrm{M}_{\mathrm{L}}(\mathrm{BK})$ & & JAN. & 27 & 06:26 PST \\
\hline JAN. & 27 & 195134.1 & $36.813 \mathrm{~N}$ & $121.255 \mathrm{~W}$ & 8 & BK & & & $3.4 \mathrm{M}_{\mathrm{L}}(\mathrm{BK})$ & & JAN. & 27 & 11:51 PST \\
\hline JAN. & 28 & 025350.5 & $34.480 \mathrm{~N}$ & $120.609 \mathrm{~W}$ & 11 & PS & & & $3.1 \mathrm{M}_{\mathrm{L}}(\mathrm{PS})$ & & JAN. & 27 & 18:53 PST \\
\hline JAN. & 30 & 174707.5 & $37.645 \mathrm{~N}$ & $121.837 \mathrm{~W}$. & 4 & BK & & & $2.9 \mathrm{M}_{\mathrm{L}}(\mathrm{BK})$ & FELT & JAN. & 30 & 09:47 PST \\
\hline JAN. & 31 & 163749.8 & $37.645 \mathrm{~N}$. & $121.837 \mathrm{~W}$ & 5 & BK & & & $3.1 \mathrm{M}_{\mathrm{L}}(\mathrm{BK})$ & & JAN. & 31 & 08:37 PST \\
\hline FEB. & 1 & 0232466 & $37.427 \mathrm{~N}$ & $118.472 W$. & 6 & PS & & & $3.4 \mathrm{M}_{\mathrm{L}}(\mathrm{PS})$ & & JAN. & 31 & 18:32 PST \\
\hline FEB. & 1 & 085125.8 & $37.460 \mathrm{~N}$ & $118.840 \mathrm{~W}$ & 11 & BK & & $\longrightarrow$ & $3.0 \mathrm{M}_{\mathrm{L}}(\mathrm{BK})$ & $\longrightarrow$ & FEB. & 1 & 00:51 PST \\
\hline FEB. & 2 & 193122.8 & $40.802 \mathrm{~N}$ & $124.063 \mathrm{~W}$. & 25 & BK & & & $3.2 \mathrm{M}_{\mathrm{L}}(\mathrm{BK})$ & FELT & FEB. & 2 & 11:31 PST \\
\hline FEB. & 5 & 120010.4 & $40.608 \mathrm{~N}$ & $124.727 \mathrm{~W}$ & 16 & BK & $\longrightarrow$ & - & $3.5 \mathrm{M}_{\mathrm{L}}(\mathrm{BK})$ & 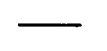 & FEB. & 5 & 04:00 PST \\
\hline FEB. & 10 & 164214.0 & $36.171 N$ & $120.174 \mathrm{~W}$ & 6 & GP & - & - & $3.2 \mathrm{M}_{\mathrm{L}}(\mathrm{GP})$ & & FEB. & 10 & 08:42 PST \\
\hline FEB. & 11 & 011557.2 & $41.634 \mathrm{~N}$ & $125.353 \mathrm{~W}$ & 10 & GS & 5.0 & 5.0 & $4.9 \mathrm{M}_{\mathrm{L}}(\mathrm{BK})$ & & FEB. & 10 & $17: 15$ PST \\
\hline FEB. & 11 & 043839.5 & $37.461 \mathrm{~N}$ & $118.904 \mathrm{~W}$ & 6 & PS & 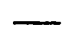 & - & $3.1 \mathrm{M}_{\mathrm{L}}(\mathrm{PS})$ & & FEB. & 10 & 20:38 PST \\
\hline FEB. & 14 & 222131.8 & $36.767 \mathrm{~N}$ & $121.265 \mathrm{~W}$ & 9 & BK & - & $\longrightarrow$ & $3.1 \mathrm{M}_{\mathrm{L}}(\mathrm{BK})$ & 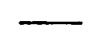 & FEB. & 14 & $14: 21$ PST \\
\hline FEB. & 15 & 222701.0 & $39.630 \mathrm{~N}$ & $122.072 \mathrm{~W}$ & 22 & BK & 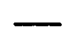 & 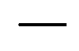 & $3.1 \mathrm{M}_{\mathrm{L}}(\mathrm{BK})$ & III & FEB. & 15 & 14:27 PST \\
\hline FEB. & 17 & 021233.5 & $34.116 \mathrm{~N}$. & $116.030 \mathrm{~W}$ & 11 & PS & 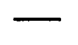 & & $3.8 \mathrm{M}_{\mathrm{L}}(\mathrm{GP})$ & FELT & FEB. & 16 & 18:12 PST \\
\hline FEB. & 17 & 105838.6 & $32.966 \mathrm{~N}$ & 115.553W. & 8 & PS & $\longrightarrow$ & & $3.4 \mathrm{M}_{\mathrm{L}}(\mathrm{GP})$ & IV & FEB. & 17 & 02:58 PST \\
\hline FEB. & 17 & 122805.7 & 40.329N. & 127.303W. & 10 & GS & 4.3 & & $4.3 \mathrm{M}_{\mathrm{L}}(\mathrm{BK})$ & & FEB. & 17 & 04:28 PST \\
\hline FEB. & 17 & 134642.4 & $33.554 \mathrm{~N}$ & 116.806W. & 0 & GP & & $\longrightarrow$ & $3.2 \mathrm{M}_{\mathrm{L}}(\mathrm{GP})$ & - & FEB. & 17 & 05:46 PST \\
\hline FEB. & 18 & 012529.0 & $32.962 \mathrm{~N}$ & 115.543W. & 5 & PS & & & $3.0 \mathrm{M}_{\mathrm{L}}(\mathrm{PS})$ & FELT & FEB. & 17 & $17: 25$ PST \\
\hline FEB. & 18 & 012629.0 & $32.957 \mathrm{~N}$ & 115.554W. & 5 & PS & & & $3.1 \mathrm{M}_{\mathrm{L}}(\mathrm{PS})$ & FELT & FEB. & 17 & 17:26 PST \\
\hline FEB. & 19 & 002540.1 & $40.730 \mathrm{~N}$ & $124.855 \mathrm{~W}$ & 8 & BK & & - & $4.0 \mathrm{M}_{\mathrm{L}}(\mathrm{BK})$ & - & FEB. & 18 & $16: 25$ PST \\
\hline FEB. & 19 & 004724.5 & $32.485 \mathrm{~N}$ & $117.567 \mathrm{~W}$ & 6 & GP & & 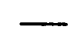 & $3.8 \mathrm{M}_{\mathrm{L}}(\mathrm{GP})$ & III & FEB. & 18 & 16:47 PST \\
\hline FEB. & 19 & 030109.1 & $36.827 \mathrm{~N}$ & $121.277 \mathrm{~W}$ & 9 & BK & & - & $3.1 \mathrm{M}_{\mathrm{L}}(\mathrm{BK})$ & FELT & FEB. & 18 & 19:01 PST \\
\hline FEB. & 19 & 234907.7 & $36.848 \mathrm{~N}$ & $121.297 \mathrm{~W}$. & 9 & BK & - & - & $3.1 \mathrm{M}_{\mathrm{L}}(\mathrm{BK})$ & FELT & FEB. & 19 & 15:49 PST \\
\hline FEB. & 28 & 103135.8 & $40.597 \mathrm{~N}$. & 124.728W. & 14 & BK & & - & $3.5 \mathrm{M}_{\mathrm{L}}(\mathrm{BK})$ & $\longrightarrow$ & FEB. & 28 & 02:31 PST \\
\hline MAR. & 2 & 230301.1 & $35.910 \mathrm{~N}$ & $118.352 \mathrm{~W}$ & 5 & GP & & - & $3.1 \mathrm{M}_{\mathrm{L}}(\mathrm{PS})$ & & MAR. & 2 & 15:03 PST \\
\hline MAR. & 3 & 131820.3 & $33.746 \mathrm{~N}$ & $117.525 \mathrm{~W}$ & 6 & PS & & - & $3.3 \mathrm{M}_{\mathrm{L}}(\mathrm{GP})$ & IV & MAR. & 3 & 05:18 PST \\
\hline MAR. & 3 & 144520.0 & $36.835 \mathrm{~N}$ & $121.268 \mathrm{~W}$ & 6 & BK & - & - & $2.7 \mathrm{M}_{\mathrm{L}}(\mathrm{BK})$ & FELT & MAR. & 3 & 06:45 PST \\
\hline
\end{tabular}


Table 1. Summary of United States earthquakes for 1986-Continued

\begin{tabular}{|c|c|c|c|c|c|c|c|c|c|c|c|c|c|}
\hline \multirow[t]{2}{*}{ Date } & & \multirow{2}{*}{$\begin{array}{l}\text { Origin time } \\
\text { (UTC) } \\
\text { hr min sec }\end{array}$} & \multirow{2}{*}{$\begin{array}{l}\text { Latitude } \\
\text { ( ) }\end{array}$} & \multirow{2}{*}{$\begin{array}{l}\text { Longitude } \\
\left({ }^{\circ}\right)\end{array}$} & \multirow{2}{*}{$\begin{array}{l}\text { Depth } \\
(\mathbf{k m})\end{array}$} & \multirow{2}{*}{$\begin{array}{l}\text { Hypo- } \\
\text { center } \\
\text { Source }\end{array}$} & \multicolumn{3}{|c|}{ Magnitude } & \multirow{2}{*}{$\begin{array}{l}\text { Max. } \\
\text { inten- } \\
\text { sity }\end{array}$} & \multicolumn{3}{|c|}{ Local time } \\
\hline & & & & & & & $m_{b}$ & $\mathbf{M}_{\mathbf{s}}$ & Local & & Date & & zone \\
\hline \multicolumn{14}{|c|}{ CALITORNIA-Continued } \\
\hline MAR. & 3 & 175618.5 & $40.613 \mathrm{~N}$ & $127.210 \mathrm{~W}$ & 5 & BK & & $\longrightarrow$ & $3.8 \mathrm{M}_{\mathrm{L}}(\mathrm{BK})$ & & MAR. & 3 & 09:56 PST \\
\hline MAR. & 3 & 175624.1 & $40.390 \mathrm{~N}$ & $127.348 \mathrm{~W}$. & 5 & BK & 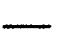 & - & $3.8 \mathrm{M}_{\mathrm{L}}(\mathrm{BK})$ & & MAR. & 3 & 09:56 PST \\
\hline MAR. & 7 & 222633.7 & $40.303 \mathrm{~N}$ & $124.501 \mathrm{~W}$. & 2 & BK & - & - & $3.8 \mathrm{M}_{\mathrm{L}}(\mathrm{BK})$ & $\longrightarrow$ & MAR. & 7 & $14: 26$ PST \\
\hline MAR. & 9 & 012813.0 & $37.672 \mathrm{~N}$ & $122.498 \mathrm{~W}$ & 7 & BK & - & - & $3.0 \mathrm{M}_{\mathrm{L}}(\mathrm{BK})$ & FELT & MAR. & 8 & 17:28 PST \\
\hline MAR. & 9 & 224142.6 & $34.113 \mathrm{~N}$. & $117.769 \mathrm{~W}$. & 5 & PS & - & - & $3.5 \mathrm{M}_{\mathrm{L}}(\mathrm{GP})$ & IV & MAR. & 9 & 14:41 PST \\
\hline MAR. & & 153316.0 & $34.403 \mathrm{~N}$ & $119.813 W$ & 24 & GP & 4.4 & - & $4.0 \mathrm{M}_{\mathrm{L}}(\mathrm{GP})$ & V & MAR. 1 & & 07:33 PST \\
\hline MAR. & & 000509.9 & $40.307 \mathrm{~N}$ & $124.388 \mathrm{~W}$. & 22 & BK & - & & $3.6 \mathrm{M}_{\mathrm{L}}(\mathrm{BK})$ & & MAR. 1 & 0 & 16:05 PST \\
\hline MAR. & & 071459.5 & $41.711 \mathrm{~N}$ & $127.258 \mathrm{~W}$. & 10 & GS & 4.0 & & - & 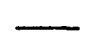 & MAR. 1 & 0 & 23:14 PST \\
\hline MAR. & & 153438.3 & $40.412 \mathrm{~N}$ & $125.433 W$. & 12 & BK & 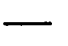 & & $3.9 \mathrm{M}_{\mathrm{L}}(\mathrm{BK})$ & & MAR. & 1 & 07:34 PST \\
\hline MAR. & & 033033.1 & $37.383 \mathrm{~N}$. & 118.699W. & 5 & PS & & & $3.1 \mathrm{M}_{\mathrm{L}}(\mathrm{PS})$ & & MAR. 1 & 2 & 19:30 PST \\
\hline MAR. & & 083700.7 & $36.288 \mathrm{~N}$. & 120.321W. & 6 & PS & & & $3.2 \mathrm{M}_{\mathrm{L}}(\mathrm{GP})$ & - & MAR. 1 & 3 & 00:37 PST \\
\hline MAR. & & 173516.8 & $37.613 N$ & $118.927 \mathrm{~W}$. & 5 & BK & & & $3.0 \mathrm{M}_{\mathrm{L}}(\mathrm{BK})$ & - & MAR. 1 & 4 & 09:35 PST \\
\hline MAR. & & 014545.3 & $34.150 \mathrm{~N}$ & 117.313W. & 5 & PS & 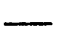 & & $3.0 \mathrm{M}_{\mathrm{L}}(\mathrm{GP})$ & FELT & MAR. & 5 & 17:45 PST \\
\hline MAR. & & 004324.6 & $33.619 \mathrm{~N}$ & $116.974 \mathrm{~W}$. & 14 & PS & & & $3.1 \mathrm{M}_{\mathrm{L}}(\mathrm{PS})$ & - & MAR. 1 & 6 & 16:43 PST \\
\hline MAR. & & 092739.2 & $37.468 \mathrm{~N}$ & $118.611 \mathrm{~W}$ & 5 & GS & - & - & $3.4 \mathrm{M}_{\mathrm{L}}(\mathrm{PS})$ & III & MAR. 1 & 9 & 01:27 PST \\
\hline MAR. & & 064940.3 & 33.794N. & $118.310 \mathrm{~W}$. & 10 & PS & - & $\longrightarrow$ & $3.3 \mathrm{M}_{\mathrm{L}}(\mathrm{GP})$ & FELT & MAR. & & 22:49 PST \\
\hline MAR. & & 224138.0 & $40.948 \mathrm{~N}$. & $123.685 \mathrm{~W}$. & 6 & BK & - & & $3.8 \mathrm{M}_{\mathrm{L}}(\mathrm{BK})$ & - & MAR. 2 & & 14:41 PST \\
\hline MAR. & & 224259.8 & $40.972 \mathrm{~N}$ & $123.688 \mathrm{~W}$ & 5 & BK & & & $3.4 \mathrm{M}_{\mathrm{L}}(\mathrm{BK})$ & & MAR. & 20 & 14:42 PST \\
\hline MAR. & & 224312.7 & $40.972 \mathrm{~N}$ & $123.688 \mathrm{~W}$. & 16 & BK & & & $3.3 \mathrm{M}_{\mathrm{L}}(\mathrm{BK})$ & & MAR. & 20 & 14:43 PST \\
\hline MAR. & & 012347.5 & 36.940N. & $120.998 \mathrm{~W}$. & 4 & BK & & & $3.0 \mathrm{M}_{\mathrm{L}}(\mathrm{BK})$ & & MAR. & 20 & 17:23 PST \\
\hline MAR. & & 063726.9 & $41.067 \mathrm{~N}$ & $125.220 \mathrm{~W}$ & 5 & BK & & & $3.7 \mathrm{M}_{\mathrm{L}}(\mathrm{BK})$ & & MAR. & 20 & 22:37 PST \\
\hline MAR. & & 045801.4 & $38.845 \mathrm{~N}$ & $122.887 \mathrm{~W}$ & 2 & BK & 3.7 & & $3.7 \mathrm{M}_{\mathrm{L}}(\mathrm{BK})$ & V & MAR. ? & & 20:58 PST \\
\hline MAR. & & 051440.0 & $33.785 \mathrm{~N}$ & $118.305 W$ & 8 & PS & - & & $2.8 \mathrm{M}_{\mathrm{L}}(\mathrm{PS})$ & IV & MAR. & & 21:14 PST \\
\hline MAR. & & 225534.0 & $36.557 \mathrm{~N}$. & $121.183 \mathrm{~W}$. & 4 & BK & $\longrightarrow$ & $\longrightarrow$ & $3.0 \mathrm{M}_{\mathrm{L}}(\mathrm{BK})$ & - & MAR. & 24 & 14:55 PST \\
\hline MAR. & & 162404.2 & $37.872 \mathrm{~N}$ & $122.201 \mathrm{~W}$ & 9 & BK & - & $\longrightarrow$ & $4.1 \mathrm{M}_{\mathrm{L}}(\mathrm{BK})$ & V & MAR. & & 08:24 PST \\
\hline MAR. & & 115540.1 & $37.488 \mathrm{~N}$. & $121.693 \mathrm{~W}$. & 8 & BK & 5.5 & 5.5 & $5.7 \mathrm{M}_{\mathrm{L}}(\mathrm{BK})$ & VI & MAR. ? & & 03:55 PST \\
\hline MAR. & & 115839.0 & $37.483 \mathrm{~N}$ & $121.683 \mathrm{~W}$. & 8 & BK & . & . & $3.2 \mathrm{M}_{\mathrm{L}}(\mathrm{BK})$ & & MAR. & & 03:58 PST \\
\hline MAR. & & 121748.4 & $37.507 \mathrm{~N}$ & $121.688 \mathrm{~W}$. & 8 & BK & & & $3.0 \mathrm{M}_{\mathrm{L}}(\mathrm{BK})$ & & MAR. & & 04:17 PST \\
\hline MAR. & & 123924.0 & $37.465 \mathrm{~N}$. & $121.700 \mathrm{~W}$. & 8 & BK & & & $3.1 \mathrm{M}_{\mathrm{L}}(\mathrm{BK})$ & & MAR. & & 04:39 PST \\
\hline MAR. & & 130538.2 & $37.513 \mathrm{~N}$ & $121.688 \mathrm{~W}$ & 7 & BK & - & - & $3.9 \mathrm{M}_{\mathrm{L}}(\mathrm{BK})$ & & MAR. & & 05:05 PST \\
\hline MAR. & & 142014.6 & $37.470 \mathrm{~N}$ & $121.697 \mathrm{~W}$ & 8 & BK & & & $3.2 \mathrm{M}_{\mathrm{L}}(\mathrm{BK})$ & & MAR. & & 06:20 PST \\
\hline APR. & 2 & 025037.3 & $37.465 \mathrm{~N}$ & $121.697 \mathrm{~W}$ & 7 & BK & - & & $3.3 \mathrm{M}_{\mathrm{L}}(\mathrm{BK})$ & & APR. & 1 & 18:50 PST \\
\hline APR. & 3 & 064037.5 & $41.212 \mathrm{~N}$ & $124.550 \mathrm{~W}$. & 12 & BK & 4.1 & & $3.6 \mathrm{M}_{\mathrm{L}}(\mathrm{BK})$ & & APR. & 2 & 22:40 PST \\
\hline APR. & 3 & 163335.9 & $37.515 \mathrm{~N}$. & $121.690 \mathrm{~W}$. & 7 & BK & - & - & $3.0 \mathrm{M}_{\mathrm{L}}(\mathrm{BK})$ & & APR. & 3 & 08:33 PST \\
\hline APR. & 4 & 101641.3 & $37.292 \mathrm{~N}$ & $121.685 \mathrm{~W}$. & 6 & BK & & & $3.2 \mathrm{M}_{\mathrm{L}}(\mathrm{BK})$ & & APR. & 4 & 02:16 PST \\
\hline APR. & 5 & 003050.6 & $33.975 \mathrm{~N}$ & $117.248 \mathrm{~W}$. & 13 & PS & - & - & $2.6 \mathrm{M}_{\mathrm{L}}(\mathrm{PS})$ & FELT & APR. & 4 & 16:30 PST \\
\hline APR. & 5 & 065040.4 & $33.730 \mathrm{~N}$ & $118.010 \mathrm{~W}$ & 14 & PS & - & - & $3.9 \mathrm{M}_{\mathrm{L}}(\mathrm{GP})$ & V & APR. & 4 & 22:50 PST \\
\hline APR. & 5 & 172149.5 & $33.336 \mathrm{~N}$. & 115.709W. & 3 & PS & - & - & $3.7 \mathrm{M}_{\mathrm{L}}(\mathrm{GP})$ & IV & APR. & 5 & 09:21 PST \\
\hline APR. & 5 & 184337.0 & $37.518 \mathrm{~N}$. & $121.692 \mathrm{~W}$. & 7 & BK & & - & $3.4 \mathrm{M}_{\mathrm{L}}(\mathrm{BK})$ & - & APR. & 5 & 10:43 PST \\
\hline APR. & 9 & 083453.7 & $37.480 \mathrm{~N}$. & $121.627 \mathrm{~W}$ & 9 & BK & - & - & $3.0 \mathrm{M}_{\mathrm{L}}(\mathrm{BK})$ & $\longrightarrow$ & APR. & 9 & 00:34 PST \\
\hline
\end{tabular}


Table 1. Summary of United States earthquakes for 1986_Continued

\begin{tabular}{|c|c|c|c|c|c|c|c|c|c|c|c|c|c|}
\hline \multirow[t]{2}{*}{ Date } & & \multirow{2}{*}{$\begin{array}{l}\text { Origin time } \\
\text { (UTC) } \\
\text { hr min sec }\end{array}$} & \multirow{2}{*}{$\begin{array}{l}\text { Latitude } \\
\text { ( })\end{array}$} & \multirow{2}{*}{$\begin{array}{c}\text { Longitude } \\
\left({ }^{\circ}\right) \\
\end{array}$} & \multirow{2}{*}{$\begin{array}{l}\text { Depth } \\
(\mathrm{km})\end{array}$} & \multirow{2}{*}{$\begin{array}{l}\text { Hypo- } \\
\text { center } \\
\text { Source }\end{array}$} & \multicolumn{3}{|c|}{ Magnitude } & \multirow{2}{*}{$\begin{array}{l}\text { Max. } \\
\text { inten- } \\
\text { sity }\end{array}$} & \multicolumn{3}{|c|}{ Local time } \\
\hline & & & & & & & $\mathrm{m}_{\mathrm{b}}$ & $\mathbf{M}_{s}$ & Local & & Date & & zone \\
\hline \multicolumn{14}{|c|}{ CALIFORNIA-Continued } \\
\hline APR. & 10 & 221259.8 & $37.518 \mathrm{~N}$ & $121.690 \mathrm{~W}$. & 8 & BK & - & - & $3.0 \mathrm{M}_{\mathrm{L}}(\mathrm{BK})$ & - & APR. & 10 & $14: 12$ PST \\
\hline APR. & 15 & 001316.9 & $40.450 \mathrm{~N}$ & $125.320 \mathrm{~W}$ & 5 & BK & 4.3 & - & $4.4 \mathrm{M}_{\mathrm{L}}(\mathrm{BK})$ & & APR. & 14 & 16:13 PST \\
\hline APR. & 15 & 092556.7 & $36.677 \mathrm{~N}$ & 121.347W. & 4 & BK & - & - & $3.6 \mathrm{M}_{\mathrm{L}}(\mathrm{BK})$ & II & APR. & 15 & 01:25 PST \\
\hline APR. & 15 & 140444.0 & $40.593 \mathrm{~N}$ & $125.223 \mathrm{~W}$ & 5 & BK & - & - & $3.5 \mathrm{M}_{\mathrm{L}}(\mathrm{BK})$ & - & APR. & 15 & 06:04 PST \\
\hline APR. & 18 & 093544.5 & $41.140 \mathrm{~N}$ & $123.367 \mathrm{~W}$. & 39 & BK & - & - & $3.0 \mathrm{M}_{\mathrm{L}}(\mathrm{BK})$ & - & APR. & 18 & 01:35 PST \\
\hline APR. & 18 & 110021.7 & $38.230 \mathrm{~N}$ & 122.178W. & 3 & BK & - & - & $2.3 \mathrm{M}_{\mathrm{L}}(\mathrm{BK})$ & FELT & APR. & 18 & 03:00 PST \\
\hline APR. & 20 & 124549.2 & $34.224 \mathrm{~N}$ & $117.469 \mathrm{~W}$ & 12 & PS & - & - & $2.7 \mathrm{M}_{\mathrm{L}}(\mathrm{PS})$ & FELT & APR. & 20 & 04:45 PST \\
\hline APR. & 21 & 063559.4 & $35.834 \mathrm{~N}$ & $117.766 \mathrm{~W}$. & 4 & PS & - & - & $3.3 \mathrm{M}_{\mathrm{L}}(\mathrm{GP})$ & 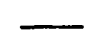 & APR. & 20 & 22:35 PST \\
\hline APR. & 21 & 085404.6 & $35.835 \mathrm{~N}$ & $117.766 \mathrm{~W}$. & 4 & PS & - & - & $3.0 \mathrm{M}_{\mathrm{L}}(\mathrm{PS})$ & - & APR. & 21 & 00:54 PST \\
\hline APR. & 21 & 091217.1 & $34.378 \mathrm{~N}$. & 119.768W. & 11 & PS & - & - & $3.0 \mathrm{M}_{\mathrm{L}}(\mathrm{PS})$ & FELT & APR. & 21 & 01:12 PST \\
\hline APR. & 22 & 174750.2 & $36.755 \mathrm{~N}$ & $121.495 \mathrm{~W}$ & 3 & BK & - & - & $3.0 \mathrm{M}_{\mathrm{L}}(\mathrm{BK})$ & 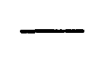 & APR. & 22 & 09:47 PST \\
\hline APR. & 22 & 234226.5 & $37.470 \mathrm{~N}$ & $118.807 \mathrm{~W}$ & 6 & PS & - & - & $3.0 \mathrm{M}_{\mathrm{L}}(\mathrm{PS})$ & - & APR. & 22 & $15: 42$ PST \\
\hline APR. & 23 & 011044.9 & $36.178 \mathrm{~N}$ & $120.236 \mathrm{~W}$ & 6 & PS & - & - & $3.2 \mathrm{M}_{\mathrm{L}}(\mathrm{PS})$ & - & APR. & 22 & $17: 10$ PST \\
\hline APR. & 23 & 163506.0 & $37.428 \mathrm{~N}$ & $121.800 \mathrm{~W}$ & 2 & BK & - & - & $2.2 \mathrm{M}_{\mathrm{L}}(\mathrm{BK})$ & FELT & APR. & 23 & 08:35 PST \\
\hline APR. & 25 & 190927.0 & $33.478 \mathrm{~N}$. & $115.649 \mathrm{~W}$ & 1 & GP & - & - & $3.0 \mathrm{M}_{\mathrm{L}}(\mathrm{GP})$ & & APR. & 25 & 11:09 PST \\
\hline APR. & 26 & 004355.0 & $36.145 \mathrm{~N}$ & $117.876 \mathrm{~W}$ & 7 & PS & - & - & $3.4 \mathrm{M}_{\mathrm{L}}(\mathrm{PS})$ & - & APR. & 25 & $16: 43$ PST \\
\hline APR. & 28 & 173347.8 & $37.478 \mathrm{~N}$ & 121.693W. & 7 & BK & - & - & $3.5 \mathrm{M}_{\mathrm{L}}(\mathrm{BK})$ & FELT & APR. & 28 & 09:33 PST \\
\hline APR. & 28 & 221840.6 & $36.815 \mathrm{~N}$ & 121.258W. & 8 & BK & - & - & $3.6 \mathrm{M}_{\mathrm{L}}(\mathrm{BK})$ & IV & APR. & 28 & 14:18 PST \\
\hline APR. & 30 & 223730.4 & $40.760 \mathrm{~N}$ & $124.560 \mathrm{~W}$. & 19 & BK & 3.5 & - & $3.9 \mathrm{M}_{\mathrm{L}}(\mathrm{BK})$ & III & APR. & 30 & 14:37 PST \\
\hline MAY & 1 & 010849.7 & $35.911 \mathrm{~N}$ & 117.264W. & 0 & GP & - & - & $3.0 \mathrm{M}_{\mathrm{L}}(\mathrm{GP})$ & - & APR & 30 & 17:08 PST \\
\hline MAY & 2 & 081907.9 & $33.677 \mathrm{~N}$ & $116.826 \mathrm{~W}$. & 12 & PS & - & - & $2.6 \mathrm{M}_{\mathrm{L}}(\mathrm{PS})$ & FELT & MAY & 2 & 00:19 PST \\
\hline MAY & 5 & 155144.4 & $35.047 \mathrm{~N}$ & $118.948 \mathrm{~W}$ & 6 & PS & - & - & $3.2 \mathrm{M}_{\mathrm{L}}(\mathrm{GP})$ & - & MAY & 5 & 07:51 PST \\
\hline MAY & 6 & 223835.4 & $40.375 \mathrm{~N}$ & $124.620 \mathrm{~W}$ & 22 & BK & - & - & $3.0 \mathrm{M}_{\mathrm{L}}(\mathrm{BK})$ & - & MAY & 6 & 14:38 PST \\
\hline MAY & 7 & 060841.9 & $40.240 \mathrm{~N}$ & 127.273W. & 5 & BK & 4.0 & - & $4.3 \mathrm{M}_{\mathrm{L}}(\mathrm{BK})$ & - & MAY & 6 & 22:08 PST \\
\hline MAY & 7 & 123409.0 & $34.196 \mathrm{~N}$ & $117.061 \mathrm{~W}$ & 12 & PS & - & - & $3.0 \mathrm{M}_{\mathrm{L}}(\mathrm{PS})$ & - & MAY & 7 & 04:34 PST \\
\hline MAY & 9 & 022942.9 & $36.885 \mathrm{~N}$ & 121.290W. & 7 & BK & - & - & $3.0 \mathrm{M}_{\mathrm{L}}(\mathrm{BK})$ & - & MAY & 8 & 18:29 PST \\
\hline MAY & 9 & 231646.1 & $32.410 \mathrm{~N}$ & $118.221 \mathrm{~W}$ & 6 & GP & - & - & $3.2 \mathrm{M}_{\mathrm{L}}(\mathrm{PS})$ & - & MAY & 9 & 15:16 PST \\
\hline MAY & 10 & 223013.1 & $37.348 \mathrm{~N}$ & $122.288 \mathrm{~W}$ & 13 & BK & - & - & $2.7 \mathrm{M}_{\mathrm{L}}(\mathrm{BK})$ & FELT & MAY & 10 & $14: 30$ PST \\
\hline MAY & 12 & 091709.3 & $37.462 \mathrm{~N}$ & $121.693 \mathrm{~W}$ & 6 & BK & - & - & $3.1 \mathrm{M}_{\mathrm{L}}(\mathrm{BK})$ & FELT & MAY & 12 & 01:17 PST \\
\hline MAY & 12 & 230019.7 & $36.848 \mathrm{~N}$ & $121.297 \mathrm{~W}$ & 6 & BK & - & - & $3.5 \mathrm{M}_{\mathrm{L}}(\mathrm{GP})$ & FELT & MAY & 12 & 15:00 PST \\
\hline MAY & 13 & 112931.2 & $35.251 \mathrm{~N}$ & $117.332 \mathrm{~W}$ & 6 & PS & - & - & $3.3 \mathrm{M}_{\mathrm{L}}(\mathrm{PS})$ & - & MAY & 13 & 03:29 PST \\
\hline MAY & 13 & 115540.3 & $33.793 \mathrm{~N}$ & $118.312 \mathrm{~W}$ & 10 & PS & - & - & $2.7 \mathrm{M}_{\mathrm{L}}(\mathrm{PS})$ & FELT & MAY & 13 & 03:55 PST \\
\hline MAY & 13 & 163545.0 & $33.791 \mathrm{~N}$ & $118.302 W$ & 8 & PS & - & - & $2.7 \mathrm{M}_{\mathrm{L}}(\mathrm{PS})$ & FELT & MAY & 13 & 08:35 PST \\
\hline MAY & 14 & 003009.6 & $37.363 \mathrm{~N}$ & $122.262 \mathrm{~W}$ & 14 & BK & - & - & $3.2 \mathrm{M}_{\mathrm{L}}(\mathrm{BK})$ & FELT & MAY & 13 & $16: 30$ PST \\
\hline MAY & 15 & 083202.1 & $37.477 \mathrm{~N}$ & 121.695W. & 7 & BK & - & - & $3.3 \mathrm{M}_{\mathrm{L}}(\mathrm{BK})$ & FELT & MAY & 15 & 00:32 PST \\
\hline MAY & 16 & 172130.1 & $40.370 \mathrm{~N}$ & $125.268 \mathrm{~W}$ & 21 & BK & - & - & $3.9 \mathrm{M}_{\mathrm{L}}(\mathrm{BK})$ & - & MAY & 16 & 09:21 PST \\
\hline MAY & 19 & 041253.3 & $33.892 \mathrm{~N}$ & $118.387 \mathrm{~W}$ & 10 & PS & - & - & $3.1 \mathrm{M}_{\mathrm{L}}(\mathrm{GP})$ & FELT & MAY & 18 & 20:12 PST \\
\hline MAY & 20 & 071140.2 & $33.940 \mathrm{~N}$ & $118.668 \mathrm{~W}$ & 6 & PS & - & - & $2.8 \mathrm{M}_{\mathrm{L}}(\mathrm{PS})$ & FELT & MAY & 19 & 23:11 PST \\
\hline MAY & 23 & 114155.1 & $35.806 \mathrm{~N}$. & 118.019W. & 10 & PS & 3.6 & - & $3.9 \mathrm{M}_{\mathrm{L}}(\mathrm{GP})$ & IV & MAY & 23 & 03:41 PST \\
\hline MAY & 24 & 085629.5 & $35.260 \mathrm{~N}$ & $118.585 \mathrm{~W}$ & 6 & PS & - & - & $3.2 \mathrm{M}_{\mathrm{L}}(\mathrm{GP})$ & - & MAY & 24 & 00:56 PST \\
\hline
\end{tabular}


Table 1. Summary of United States earthquakes for 1986-Continued

\begin{tabular}{|c|c|c|c|c|c|c|c|c|c|c|c|c|c|}
\hline \multirow{2}{*}{\multicolumn{2}{|c|}{ Date }} & \multirow{2}{*}{$\begin{array}{l}\text { Origin time } \\
\text { (UTC) } \\
\text { hr min sec }\end{array}$} & \multirow{2}{*}{$\begin{array}{c}\text { Latitude } \\
\left({ }^{\circ}\right) \\
\end{array}$} & \multirow{2}{*}{$\begin{array}{c}\text { Longitude } \\
\left({ }^{\circ}\right)\end{array}$} & \multirow{2}{*}{$\begin{array}{l}\text { Depth } \\
(\mathrm{km})\end{array}$} & \multirow{2}{*}{$\begin{array}{l}\text { Hypo- } \\
\text { center } \\
\text { Source }\end{array}$} & \multicolumn{3}{|c|}{ Magnitude } & \multirow{2}{*}{$\begin{array}{l}\text { Max. } \\
\text { inten- } \\
\text { sity }\end{array}$} & \multicolumn{3}{|c|}{ Local time } \\
\hline & & & & & & & $\mathrm{m}_{\mathrm{b}}$ & $\mathrm{M}_{\mathrm{s}}$ & Local & & Date & & hr zone \\
\hline \multicolumn{14}{|c|}{ CALIFORNIA-Continued } \\
\hline MAY & 25 & 034602.8 & $36.767 \mathrm{~N}$ & $121.247 \mathrm{~W}$. & 11 & BK & - & - & $3.0 \mathrm{M}_{\mathrm{L}}(\mathrm{BK})$ & - & MAY & 24 & 19:46 PST \\
\hline MAY & 25 & 095103.1 & $38.810 \mathrm{~N}$ & $122.798 \mathrm{~W}$. & 4 & BK & - & - & $3.2 \mathrm{M}_{\mathrm{L}}(\mathrm{BK})$ & FELT & MAY & 25 & 01:51 PST \\
\hline MAY & 31 & 014240.1 & $34.105 \mathrm{~N}$ & $116.611 W$ & 10 & PS & - & - & $3.5 \mathrm{M}_{\mathrm{L}}(\mathrm{PS})$ & - & MAY & 30 & 17:42 PST \\
\hline MAY & 31 & 084707.2 & $36.627 \mathrm{~N}$ & 121.273W. & 6 & BK & - & - & $3.7 \mathrm{M}_{\mathrm{L}}(\mathrm{BK})$ & - & MAY & 31 & 00:47 PST \\
\hline MAY & 31 & 084756.1 & $36.618 \mathrm{~N}$ & $121.255 \mathrm{~W}$ & 4 & BK & 4.6 & 3.7 & $4.7 \mathrm{M}_{\mathrm{L}}(\mathrm{BK})$ & IV & MAY & 31 & 00:47 PST \\
\hline JUNE & 1 & 061043.5 & $37.446 \mathrm{~N}$ & $118.840 \mathrm{~W}$ & 5 & PS & - & - & $3.1 \mathrm{M}_{\mathrm{L}}(\mathrm{PS})$ & - & MAY & 31 & 22:10 PST \\
\hline JUNE & 1 & 064935.0 & $36.607 \mathrm{~N}$. & $121.252 \mathrm{~W}$ & 7 & BK & - & - & $3.5 \mathrm{M}_{\mathrm{L}}(\mathrm{BK})$ & & MAY & 31 & 22:49 PST \\
\hline JUNE & 3 & 141449.3 & $33.788 \mathrm{~N}$ & $116.344 \mathrm{~W}$ & 11 & PS & - & - & $3.7 \mathrm{M}_{\mathrm{L}}(\mathrm{PS})$ & IV & JUNE & 3 & 06:14 PST \\
\hline JUNE & 5 & 001556.0 & $36.665 \mathrm{~N}$ & $121.340 \mathrm{~W}$. & 4 & BK & - & - & $3.1 \mathrm{M}_{\mathrm{L}}(\mathrm{BK})$ & - & JUNE & 4 & 16:15 PST \\
\hline JUNE & 9 & 044805.8 & $37.960 \mathrm{~N}$. & $121.668 \mathrm{~W}$. & 1 & BK & - & - & $2.1 \mathrm{M}_{\mathrm{L}}(\mathrm{BK})$ & FELT & JUNE & 8 & $20: 48$ PST \\
\hline JUNE & 10 & 013258.4 & $36.628 \mathrm{~N}$ & $121.300 \mathrm{~W}$. & 8 & BK & - & - & $3.3 \mathrm{M}_{\mathrm{L}}(\mathrm{BK})$ & - & JUNE & 9 & 17:32 PST \\
\hline JUNE & 10 & 103000.2 & $36.435 \mathrm{~N}$ & $120.454 \mathrm{~W}$ & 6 & PS & - & - & $3.0 \mathrm{M}_{\mathrm{L}}(\mathrm{PS})$ & - & JUNE & 10 & 02:29 PST \\
\hline JUNE & 11 & 130301.8 & $36.945 \mathrm{~N}$ & $121.618 \mathrm{~W}$ & 6 & BK & - & - & $2.7 \mathrm{M}_{\mathrm{L}}(\mathrm{BK})$ & FELT & JUNE & 11 & 05:03 PST \\
\hline JUNE & 11 & 150859.5 & $36.620 \mathrm{~N}$ & $121.277 \mathrm{~W}$ & 7 & BK & - & - & $3.5 \mathrm{M}_{\mathrm{L}}(\mathrm{BK})$ & FELT & JUNE & 11 & 07:08 PST \\
\hline JUNE & 13 & 132515.4 & $36.060 \mathrm{~N}$ & $119.938 \mathrm{~W}$ & 6 & GP & - & - & $3.6 \mathrm{M}_{\mathrm{L}}(\mathrm{GP})$ & II & JUNE & 13 & 05:25 PST \\
\hline JUNE & 14 & 095329.2 & $41.703 \mathrm{~N}$ & $126.543 \mathrm{~W}$ & 10 & GS & 3.7 & 4.1 & - & - & JUNE & 14 & 01:53 PST \\
\hline JUNE & 18 & 001059.3 & $37.475 \mathrm{~N}$ & $118.577 \mathrm{~W}$ & 5 & GS & - & - & $2.8 \mathrm{M}_{\mathrm{L}}(\mathrm{BK})$ & - & JUNE & 17 & 16:10 PST \\
\hline JUNE & 18 & 141326.4 & $33.935 \mathrm{~N}$ & $116.742 \mathrm{~W}$ & 17 & PS & - & - & $3.4 \mathrm{M}_{\mathrm{L}}(\mathrm{PS})$ & FELT & JUNE & 18 & 06:13 PST \\
\hline JUNE & 20 & 022813.9 & $40.415 \mathrm{~N}$ & $124.467 \mathrm{~W}$ & 20 & BK & - & - & $3.2 \mathrm{M}_{\mathrm{L}}(\mathrm{BK})$ & 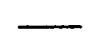 & JUNE & 19 & 18:28 PST \\
\hline JUNE & 22 & 203451.9 & $40.692 \mathrm{~N}$ & $124.673 \mathrm{~W}$ & 16 & BK & 4.1 & - & $4.0 \mathrm{M}_{\mathrm{L}}(\mathrm{BK})$ & 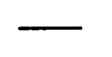 & JUNE & 22 & 12:34 PST \\
\hline JUNE & 22 & 235706.1 & $40.597 \mathrm{~N}$ & 123.450W. & 12 & BK & - & - & $3.5 \mathrm{M}_{\mathrm{L}}(\mathrm{BK})$ & - & JUNE & 22 & 15:57 PST \\
\hline JUNE & 26 & 053947.8 & $33.869 \mathrm{~N}$ & $118.450 \mathrm{~W}$ & 7 & PS & - & - & $3.4 \mathrm{M}_{\mathrm{L}}(\mathrm{GP})$ & $\mathbf{I V}$ & JUNE & 25 & 21:39 PST \\
\hline JUNE & 27 & 040643.4 & $37.745 \mathrm{~N}$ & $121.982 \mathrm{~W}$ & 4 & BK & - & - & $2.4 \mathrm{M}_{\mathrm{L}}(\mathrm{BK})$ & FELT & JUNE & 26 & 20:06 PST \\
\hline JUNE & 30 & 022120.4 & $37.437 \mathrm{~N}$ & $118.617 \mathrm{~W}$ & 6 & PS & - & - & $3.0 \mathrm{M}_{\mathrm{L}}(\mathrm{PS})$ & - & JUNE & 29 & 18:21 PST \\
\hline JUNE & 30 & 110005.9 & $37.842 \mathrm{~N}$ & $121.763 \mathrm{~W}$ & 11 & BK & - & - & $3.3 \mathrm{M}_{\mathrm{L}}(\mathrm{BK})$ & FELT & JUNE & 30 & 03:00 PST \\
\hline JULY & 1 & 084214.0 & $40.385 \mathrm{~N}$ & $127.440 \mathrm{~W}$ & 5 & BK & - & - & $3.9 \mathrm{M}_{\mathrm{L}}(\mathrm{BK})$ & - & JULY & 1 & 00:42 PST \\
\hline JULY & 2 & 081021.2 & $36.624 \mathrm{~N}$ & $116.322 \mathrm{~W}$. & 6 & PS & - & - & $3.0 \mathrm{M}_{\mathrm{L}}(\mathrm{PS})$ & - & JULY & 2 & $00: 10$ PST \\
\hline JULY & 2 & 081103.2 & $33.989 \mathrm{~N}$ & $117.229 \mathrm{~W}$ & 6 & GP & - & - & $3.1 \mathrm{M}_{\mathrm{L}}(\mathrm{GP})$ & - & JULY & 2 & 00:11 PST \\
\hline JULY & 4 & 195535.7 & $41.052 \mathrm{~N}$ & $124.603 \mathrm{~W}$ & 9 & BK & - & - & $3.7 \mathrm{M}_{\mathrm{L}}(\mathrm{BK})$ & - & JULY & 4 & 11:55 PST \\
\hline JULY & 5 & 032423.0 & $35.080 \mathrm{~N}$ & $119.091 \mathrm{~W}$ & 18 & GP & - & - & $3.1 \mathrm{M}_{\mathrm{L}}(\mathrm{GP})$ & - & JULY & 4 & 19:24 PST \\
\hline JULY & 5 & 141159.9 & $35.698 \mathrm{~N}$ & $117.645 \mathrm{~W}$ & 7 & GP & - & - & $3.1 \mathrm{M}_{\mathrm{L}}(\mathrm{PS})$ & - & JULY & 5 & 06:11 PST \\
\hline JULY & 7 & 034916.8 & $38.788 \mathrm{~N}$ & $122.780 \mathrm{~W}$ & 2 & BK & - & - & $3.2 \mathrm{M}_{\mathrm{L}}(\mathrm{BK})$ & - & JULY & 6 & 19:49 PST \\
\hline JULY & 7 & 091322.7 & $34.149 \mathrm{~N}$ & $117.744 \mathrm{~W}$. & 5 & GP & - & - & $3.0 \mathrm{M}_{\mathrm{L}}(\mathrm{PS})$ & FELT & JULY & 7 & 01:13 PST \\
\hline JULY & 8 & 004023.4 & $36.083 \mathrm{~N}$ & $121.827 \mathrm{~W}$ & 19 & BK & 4.4 & - & $4.4 \mathrm{M}_{\mathrm{L}}(\mathrm{BK})$ & IV & JULY & 7 & 16:40 PST \\
\hline JULY & 8 & 092044.5 & $33.998 \mathrm{~N}$ & $116.606 \mathrm{~W}$ & 12 & GP & 5.8 & 6.0 & $5.6 \mathrm{M}_{\mathrm{L}}(\mathrm{GP})$ & VII & JULY & 8 & 01:20 PST \\
\hline JULY & 8 & 092412.8 & $34.031 \mathrm{~N}$ & $116.657 \mathrm{w}$. & 6 & GP & - & - & $4.4 \mathrm{M}_{\mathrm{L}}(\mathrm{PS})$ & 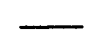 & JULY & 8 & 01:24 PST \\
\hline JULY & 8 & 092625.8 & $33.967 \mathrm{~N}$ & $116.617 \mathrm{~W}$ & 6 & PS & - & - & $3.2 \mathrm{M}_{\mathrm{L}}(\mathrm{PS})$ & - & JULY & 8 & 01:26 PST \\
\hline JULY & 8 & 092634.7 & $33.967 \mathrm{~N}$ & $116.617 \mathrm{~W}$ & 6 & PS & - & - & $3.1 \mathrm{M}_{\mathrm{L}}(\mathrm{PS})$ & - & JULY & 8 & 01:26 PST \\
\hline JULY & 8 & 092710.8 & $33.967 \mathrm{~N}$ & $116.617 \mathrm{~W}$ & 6 & PS & - & - & $3.5 \mathrm{M}_{\mathrm{L}}(\mathrm{PS})$ & - & JULY & 8 & 01:27 PST \\
\hline JULY & 8 & 092813.7 & $33.967 \mathrm{~N}$. & $116.617 \mathrm{~W}$ & 18 & PS & - & - & $4.0 \mathrm{M}_{\mathrm{L}}(\mathrm{PS})$ & - & JULY & 8 & 01:28 PST \\
\hline
\end{tabular}


Table 1. Summary of United States earthquakes for 1986-Continued

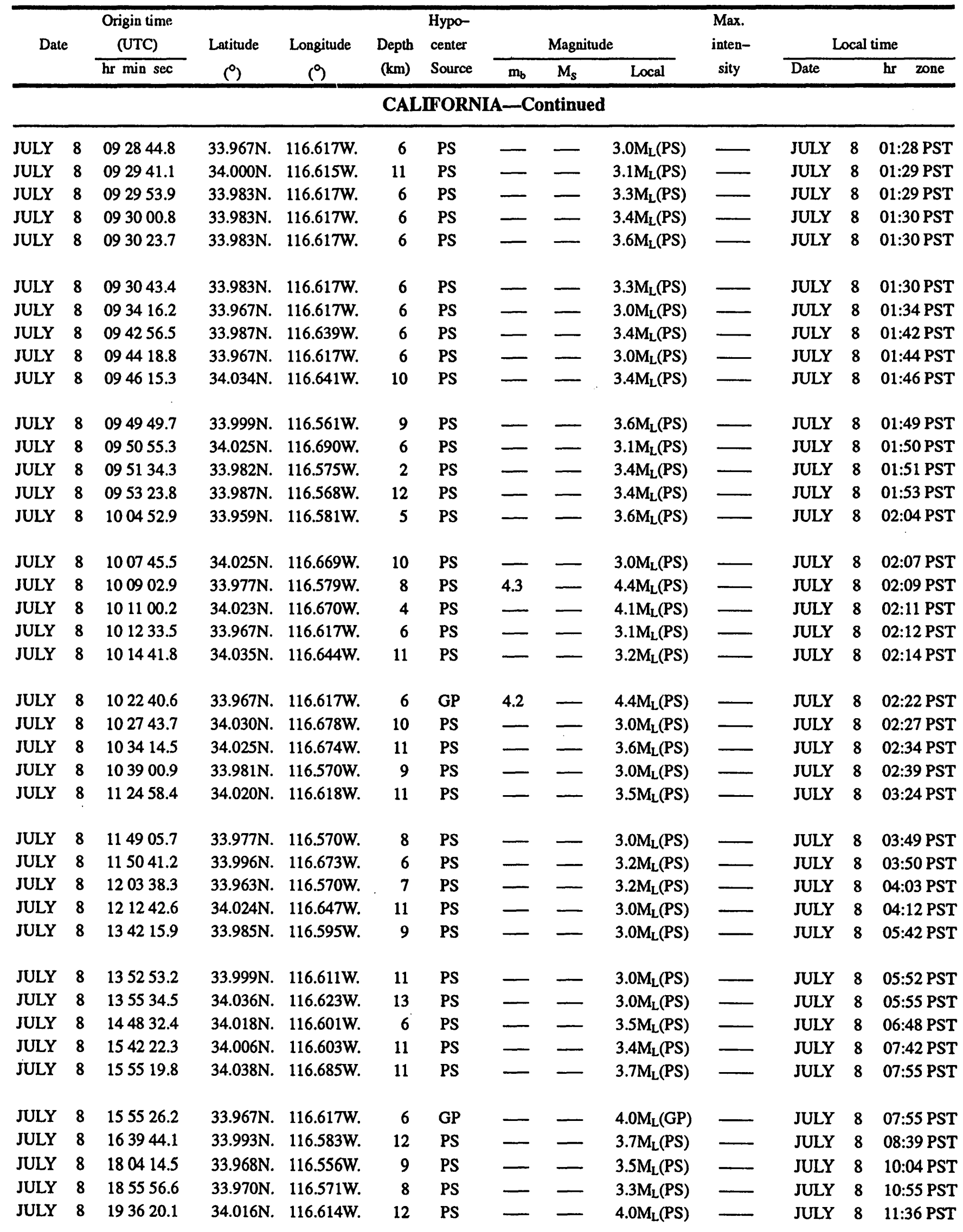


Table 1. Summary of United States earthquakes for 1986-Continued

\begin{tabular}{|c|c|c|c|c|c|c|c|c|c|c|c|c|c|}
\hline \multirow{2}{*}{\multicolumn{2}{|c|}{ Date }} & \multirow{2}{*}{$\begin{array}{l}\text { Origin time } \\
\text { (UTC) } \\
\text { hr min sec }\end{array}$} & \multirow{2}{*}{$\begin{array}{l}\text { Latitude } \\
\text { ( ) }\end{array}$} & \multirow{2}{*}{$\begin{array}{l}\text { Longitude } \\
\left({ }^{\circ}\right)\end{array}$} & \multirow{2}{*}{$\begin{array}{l}\text { Depth } \\
(\mathrm{km})\end{array}$} & \multirow{2}{*}{$\begin{array}{l}\text { Hypo- } \\
\text { center } \\
\text { Source }\end{array}$} & \multicolumn{3}{|c|}{ Magnitude } & \multirow{2}{*}{$\begin{array}{c}\text { Max. } \\
\text { inten- } \\
\text { sity }\end{array}$} & \multicolumn{3}{|c|}{ Local time } \\
\hline & & & & & & & $m_{b}$ & $\mathrm{M}_{\mathrm{s}}$ & Local & & Date & & zone \\
\hline \multicolumn{14}{|c|}{ CALIFORNIA-Continued } \\
\hline JULY & 8 & 215303.1 & $34.013 \mathrm{~N}$. & $116.588 \mathrm{~W}$ & 11 & PS & - & - & $3.1 \mathrm{M}_{\mathrm{L}}(\mathrm{PS})$ & - & JULY & 8 & $13: 53$ PST \\
\hline JULY & 9 & 001232.1 & $33.987 \mathrm{~N}$. & $116.569 \mathrm{~W}$ & 6 & GP & 4.2 & - & $4.4 \mathrm{M}_{\mathrm{L}}(\mathrm{GP})$ & FELT & JULY & 8 & 16:12 PST \\
\hline JULY & 9 & 003332.6 & $33.982 \mathrm{~N}$. & $116.557 \mathrm{~W}$ & 11 & PS & - & - & $3.1 \mathrm{M}_{\mathrm{L}}(\mathrm{PS})$ & - & JULY & 8 & 16:33 PST \\
\hline JULY & 9 & 093636.6 & $34.001 \mathrm{~N}$. & $116.573 \mathrm{~W}$ & 11 & PS & 一 & - & $3.2 \mathrm{M}_{\mathrm{L}}(\mathrm{PS})$ & - & JULY & 9 & 01:36 PST \\
\hline JULY & 9 & 094121.0 & $33.972 \mathrm{~N}$. & $116.574 \mathrm{~W}$ & 8 & PS & - & - & $3.7 \mathrm{M}_{\mathrm{L}}(\mathrm{PS})$ & - & JULY & 9 & 01:41 PST \\
\hline JULY & 9 & 113221.1 & $33.975 \mathrm{~N}$. & $116.565 \mathrm{~W}$ & 9 & PS & - & - & $3.6 \mathrm{M}_{\mathrm{L}}(\mathrm{PS})$ & - & JULY & 9 & 03:32 PST \\
\hline JULY & 9 & 125359.7 & $37.565 \mathrm{~N}$. & $118.435 \mathrm{~W}$ & 4 & RN & - & - & $3.4 \mathrm{M}_{\mathrm{D}}(\mathrm{RN})$ & FELT & JULY & 9 & 04:53 PST \\
\hline JULY & 9 & 141326.6 & $34.010 \mathrm{~N}$. & $116.615 \mathrm{~W}$ & 12 & PS & - & - & $3.0 \mathrm{M}_{\mathrm{L}}(\mathrm{PS})$ & 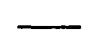 & JULY & 9 & 06:13 PST \\
\hline JULY & 10 & 042442.8 & $40.678 \mathrm{~N}$. & $124.753 \mathrm{~W}$. & 6 & BK & - & - & $3.2 \mathrm{M}_{\mathrm{L}}(\mathrm{BK})$ & - & JULY & 9 & 20:24 PST \\
\hline JULY & 10 & 094210.7 & $33.984 \mathrm{~N}$. & $116.636 \mathrm{~W}$ & 2 & PS & - & - & $3.0 \mathrm{M}_{\mathrm{L}}(\mathrm{PS})$ & - & JULY & 10 & 01:42 PST \\
\hline JULY & 10 & 120250.9 & $33.962 \mathrm{~N}$. & $116.593 \mathrm{~W}$ & 12 & PS & - & - & $3.4 \mathrm{M}_{\mathrm{L}}(\mathrm{PS})$ & V & JULY & 10 & 04:02 PST \\
\hline JULY & 10 & 205243.4 & $40.488 \mathrm{~N}$. & $124.705 \mathrm{~W}$ & 15 & BK & - & - & $3.7 \mathrm{M}_{\mathrm{L}}(\mathrm{BK})$ & - & JULY & 10 & 12:52 PST \\
\hline JULY & 10 & 234722.1 & $33.970 \mathrm{~N}$. & $116.550 \mathrm{~W}$ & 9 & PS & - & - & $3.1 \mathrm{M}_{\mathrm{L}}(\mathrm{PS})$ & - & JULY & 10 & 15:47 PST \\
\hline JULY & 11 & 001852.2 & $34.029 \mathrm{~N}$. & $116.642 \mathrm{~W}$ & 12 & PS & - & - & $3.0 \mathrm{M}_{\mathrm{L}}(\mathrm{PS})$ & - & JULY & 10 & 16:18 PST \\
\hline JULY & 11 & 074814.0 & $33.997 \mathrm{~N}$. & $116.572 \mathrm{~W}$ & 12 & PS & - & - & $3.1 \mathrm{M}_{\mathrm{L}}(\mathrm{PS})$ & III & JULY & 10 & 23:48 PST \\
\hline JULY & 11 & 085128.7 & $33.967 \mathrm{~N}$. & $116.575 \mathrm{~W}$ & 7 & PS & - & - & $3.3 \mathrm{M}_{\mathrm{L}}(\mathrm{PS})$ & III & JULY & 11 & 00:51 PST \\
\hline JULY & 11 & 151330.6 & $34.020 \mathrm{~N}$. & $116.653 \mathrm{~W}$ & 12 & PS & - & - & $3.2 \mathrm{M}_{\mathrm{L}}(\mathrm{PS})$ & III & JULY & 11 & 07:13 PST \\
\hline JULY & 11 & 155951.9 & $34.012 \mathrm{~N}$. & $116.617 \mathrm{~W}$ & 11 & PS & - & - & $3.1 \mathrm{M}_{\mathrm{L}}(\mathrm{PS})$ & & JULY & 11 & 07:59 PST \\
\hline JULY & 11 & 181231.5 & $34.025 \mathrm{~N}$. & $116.670 \mathrm{~W}$ & 10 & PS & - & - & $3.0 \mathrm{M}_{\mathrm{L}}(\mathrm{PS})$ & - & JULY & 11 & 10:12 PST \\
\hline JULY & 11 & 212852.5 & $34.298 \mathrm{~N}$. & $118.292 \mathrm{~W}$ & 8 & PS & - & - & $3.0 \mathrm{M}_{\mathrm{L}}(\mathrm{PS})$ & IV & JULY & 11 & 13:28 PST \\
\hline JULY & 12 & 035054.7 & $37.755 \mathrm{~N}$. & $119.010 \mathrm{~W}$ & 6 & PS & - & - & $3.4 \mathrm{M}_{\mathrm{L}}(\mathrm{PS})$ & 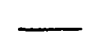 & JULY & 11 & 19:50 PST \\
\hline JULY & 12 & 054527.5 & $33.986 \mathrm{~N}$. & $116.652 \mathrm{~W}$ & 7 & PS & - & - & $4.0 \mathrm{M}_{\mathrm{L}}(\mathrm{PS})$ & III & JULY & 11 & 21:45 PST \\
\hline JULY & 12 & 172830.6 & $34.032 \mathrm{~N}$. & $116.673 \mathrm{~W}$ & 11 & PS & - & - & $3.4 \mathrm{M}_{\mathrm{L}}(\mathrm{PS})$ & - & JULY & 12 & 09:28 PST \\
\hline JULY & 13 & 014138.2 & $33.951 \mathrm{~N}$. & $116.613 \mathrm{~W}$ & 12 & PS & - & - & $3.7 \mathrm{M}_{\mathrm{L}}(\mathrm{PS})$ & FELT & JULY & 12 & 17:41 PST \\
\hline JULY & 13 & 112535.0 & $32.646 \mathrm{~N}$. & $117.138 \mathrm{~W}$ & 10 & GP & - & - & $3.0 \mathrm{M}_{\mathrm{L}}(\mathrm{GP})$ & - & JULY & 13 & 03:25 PST \\
\hline JULY & 13 & 134708.2 & $32.978 \mathrm{~N}$. & $117.858 \mathrm{~W}$ & 9 & $\mathrm{HJ}$ & 5.6 & 5.8 & $5.3 \mathrm{M}_{\mathrm{L}}(\mathrm{GP})$ & VI & JULY & 13 & 05:47 PST \\
\hline JULY & 13 & 135327.9 & $32.963 \mathrm{~N}$. & $117.828 \mathrm{~W}$ & 6 & PS & - & - & $3.9 \mathrm{M}_{\mathrm{L}}(\mathrm{PS})$ & & JULY & 13 & 05:53 PST \\
\hline JULY & 13 & 135850.4 & $32.990 \mathrm{~N}$. & $117.785 \mathrm{~W}$ & 7 & HJ & - & - & $3.2 \mathrm{M}_{\mathrm{L}}(\mathrm{PS})$ & & JULY & 13 & 05:58 PST \\
\hline JULY & 13 & 140132.8 & $33.003 \mathrm{~N}$. & $117.833 \mathrm{~W}$ & 7 & HJ & 4.8 & - & $4.6 \mathrm{M}_{\mathrm{L}}(\mathrm{GP})$ & & JULY & 13 & 06:01 PST \\
\hline JULY & 13 & 140252.1 & $33.062 \mathrm{~N}$. & $117.841 \mathrm{~W}$ & 6 & PS & - & - & $3.4 \mathrm{M}_{\mathrm{L}}(\mathrm{PS})$ & $\rightarrow$ & JULY & 13 & 06:02 PST \\
\hline JULY & 13 & 141100.5 & $32.977 \mathrm{~N}$. & $117.790 \mathrm{~W}$ & 5 & HJ & - & - & $3.7 \mathrm{M}_{\mathrm{L}}(\mathrm{PS})$ & - & JULY & 13 & 06:11 PST \\
\hline JULY & 13 & 141204.7 & $32.881 \mathrm{~N}$. & $117.059 \mathrm{~W}$ & 6 & PS & - & - & $3.1 \mathrm{M}_{\mathrm{L}}(\mathrm{PS})$ & 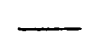 & JULY & 13 & 06:12 PST \\
\hline JULY & 13 & 142601.3 & $32.977 \mathrm{~N}$. & 117.760w. & 5 & HJ & - & - & $3.5 \mathrm{M}_{\mathrm{L}}(\mathrm{PS})$ & & JULY & 13 & 06:26 PST \\
\hline JULY & 13 & 152707.4 & $32.977 \mathrm{~N}$. & $117.725 \mathrm{~W}$. & 2 & HJ & - & - & $3.4 \mathrm{M}_{\mathrm{L}}(\mathrm{PS})$ & 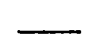 & JULY & 13 & 07:27 PST \\
\hline JULY & 13 & 155005.3 & $32.886 \mathrm{~N}$. & 117.746W. & 6 & PS & - & - & $3.6 \mathrm{M}_{\mathrm{L}}(\mathrm{PS})$ & & JULY & 13 & 07:50 PST \\
\hline JULY & 13 & 155209.2 & $32.970 \mathrm{~N}$. & $117.773 \mathrm{~W}$ & 4 & HJ & - & - & $3.3 \mathrm{M}_{\mathrm{L}}(\mathrm{PS})$ & - & JULY & 13 & 07:52 PST \\
\hline JULY & 13 & 161017.8 & $32.957 \mathrm{~N}$. & 117.785W. & 9 & HJ & - & - & $3.2 \mathrm{M}_{\mathrm{L}}(\mathrm{PS})$ & - & JULY & 13 & 08:10 PST \\
\hline JULY & 13 & 163701.6 & $32.965 \mathrm{~N}$. & $117.782 \mathrm{~W}$ & 6 & PS & - & - & $3.0 \mathrm{M}_{\mathrm{L}}(\mathrm{PS})$ & - & JULY & 13 & 08:37 PST \\
\hline JULY & 13 & 173935.7 & $33.972 \mathrm{~N}$. & $116.569 \mathrm{~W}$. & 8 & PS & - & - & $3.1 \mathrm{M}_{\mathrm{L}}(\mathrm{PS})$ & - & JULY & 13 & 09:39 PST \\
\hline JULY & 13 & 232513.3 & $32.973 \mathrm{~N}$. & 117.733W. & 6 & $\mathrm{HJ}$ & - & - & $3.2 \mathrm{M}_{\mathrm{L}}(\mathrm{PS})$ & - & JULY & 13 & 15:25 PST \\
\hline
\end{tabular}


Table 1. Summary of United States earthquakes for 1986_Continued

\begin{tabular}{|c|c|c|c|c|c|c|c|c|c|c|c|c|c|}
\hline \multirow{2}{*}{\multicolumn{2}{|c|}{ Date }} & \multirow{3}{*}{$\begin{array}{l}\text { Origin time } \\
\text { (UTC) } \\
\text { hr min sec }\end{array}$} & \multirow{3}{*}{$\begin{array}{c}\text { Latitude } \\
\left({ }^{\circ}\right)\end{array}$} & \multirow{3}{*}{$\begin{array}{c}\text { Longitude } \\
(9\end{array}$} & \multirow{2}{*}{$\begin{array}{l}\text { Depth } \\
(\mathrm{km})\end{array}$} & \multirow{2}{*}{$\begin{array}{l}\text { Hypo- } \\
\text { center } \\
\text { Source }\end{array}$} & \multicolumn{3}{|c|}{ Magnitude } & \multirow{3}{*}{$\begin{array}{l}\text { Max. } \\
\text { inten- } \\
\text { sity }\end{array}$} & \multicolumn{3}{|c|}{ Local time } \\
\hline & & & & & & & $\mathrm{m}_{\mathrm{b}}$ & $\mathrm{M}_{s}$ & Local & & Date & & zone \\
\hline \multicolumn{10}{|c|}{ CALIFORNIA-Continued } & & & & \\
\hline JULY & 13 & 235342.6 & $32.990 \mathrm{~N}$ & 117.750W. & 2 & HJ & $\longrightarrow$ & $\longrightarrow$ & $3.6 \mathrm{M}_{\mathrm{L}}(\mathrm{PS})$ & & JULY & 13 & 15:53 PST \\
\hline JULY & 14 & 003246.0 & $32.995 \mathrm{~N}$ & $117.787 \mathrm{~W}$. & 5 & HJ & 4.0 & - & $4.0 \mathrm{M}_{\mathrm{L}}(\mathrm{GP})$ & & JULY & 13 & $16: 32$ PST \\
\hline JULY & 14 & 011110.3 & $32.965 \mathrm{~N}$ & $117.820 \mathrm{~W}$. & 8 & HJ & - & $\longrightarrow$ & $3.7 \mathrm{M}_{\mathrm{L}}(\mathrm{PS})$ & - & JULY & 13 & 17:11 PST \\
\hline JULY & 14 & 014330.6 & $34.001 \mathrm{~N}$ & $116.589 \mathrm{~W}$. & 11 & PS & $\longrightarrow$ & - & $3.1 \mathrm{M}_{\mathrm{L}}(\mathrm{PS})$ & & JULY & 13 & 17:43 PST \\
\hline JULY & 14 & 053644.6 & $32.963 \mathrm{~N}$ & $117.837 \mathrm{~W}$. & 9 & $\mathrm{HJ}$ & 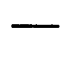 & & $3.3 \mathrm{M}_{\mathrm{L}}(\mathrm{PS})$ & & JULY & 13 & 21:36 PST \\
\hline JULY & 14 & 071734.7 & $32.993 \mathrm{~N}$ & 117.788W. & 0 & HJ & - & - & $3.7 \mathrm{M}_{\mathrm{L}}(\mathrm{PS})$ & - & JULY & 13 & 23:17 PST \\
\hline JULY & 14 & 090753.8 & $32.993 \mathrm{~N}$ & 117.795w. & 5 & $\mathrm{HJ}$ & - & - & $3.7 \mathrm{M}_{\mathrm{L}}(\mathrm{PS})$ & & JULY & 14 & 01:07 PST \\
\hline JULY & 14 & 144408.4 & $32.995 \mathrm{~N}$ & $117.817 \mathrm{~W}$. & 9 & $\mathrm{HJ}$ & & & $3.7 \mathrm{M}_{\mathrm{L}}(\mathrm{PS})$ & & JULY & 14 & 06:44 PST \\
\hline JULY & 14 & 162009.6 & $32.988 \mathrm{~N}$ & $117.827 \mathrm{~W}$. & 7 & $\mathrm{HJ}$ & & & $3.3 \mathrm{M}_{\mathrm{L}}(\mathrm{PS})$ & & JULY & 14 & 08:20 PST \\
\hline JULY & 14 & 171442.8 & $33.000 \mathrm{~N}$ & $117.752 \mathrm{~W}$. & 5 & $\mathrm{HJ}$ & & & $3.7 \mathrm{M}_{\mathrm{L}}(\mathrm{PS})$ & & JULY & 14 & 09:14 PST \\
\hline JUIJY & 14 & 212309.4 & $32.977 \mathrm{~N}$ & 117.738W. & 0 & HJ & 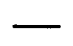 & - & $3.0 \mathrm{M}_{\mathrm{L}}(\mathrm{PS})$ & - & JULY & 14 & $13: 23$ PST \\
\hline JULY & 14 & 225849.8 & $32.953 \mathrm{~N}$ & 117.795W. & 8 & $\mathrm{HJ}$ & & & $3.0 \mathrm{M}_{\mathrm{L}}(\mathrm{PS})$ & & JULY & 14 & 14:58 PST \\
\hline JULY & 15 & 031740.4 & $34.005 \mathrm{~N}$ & $116.899 \mathrm{~W}$. & 11 & GP & & & $3.1 \mathrm{M}_{\mathrm{L}}(\mathrm{PS})$ & & JULY & 14 & 19:17 PST \\
\hline JULY & 15 & 034836.3 & $37.501 \mathrm{~N}$. & $118.633 W$. & 11 & BK & & & $3.3 \mathrm{M}_{\mathrm{L}}(\mathrm{BK})$ & & JULY & 14 & 19:48 PST \\
\hline JULY & 15 & 150245.2 & $32.982 \mathrm{~N}$ & $117.727 \mathrm{~W}$. & 5 & $\mathrm{HJ}$ & & & $3.1 \mathrm{M}_{\mathrm{L}}(\mathrm{PS})$ & & JULY & 15 & 07:02 PST \\
\hline JULY & 15 & 183214.5 & $33.980 \mathrm{~N}$. & $116.638 \mathrm{~W}$. & 7 & PS & & - & $3.1 \mathrm{M}_{\mathrm{L}}(\mathrm{PS})$ & & JULY & 15 & 10:32 PST \\
\hline JULY & 15 & 211038.0 & $32.962 \mathrm{~N}$. & $117.823 \mathrm{~W}$. & 6 & PS & & & $3.1 \mathrm{M}_{\mathrm{L}}(\mathrm{PS})$ & & JULY & 15 & 13:10 PST \\
\hline JULY & 16 & 003232.8 & $33.003 \mathrm{~N}$. & $117.835 \mathrm{~W}$. & 6 & HJ & & & $3.0 \mathrm{M}_{\mathrm{L}}(\mathrm{PS})$ & & JULY & 15 & 16:32 PST \\
\hline JULY & 16 & 052050.7 & $33.013 \mathrm{~N}$. & $117.760 \mathrm{~W}$. & 4 & $\mathrm{HJ}$ & & & $3.4 \mathrm{M}_{\mathrm{L}}(\mathrm{PS})$ & & JULY & 15 & 21:20 PST \\
\hline JULY & 16 & 124701.1 & $32.972 \mathrm{~N}$. & $117.805 \mathrm{~W}$. & 3 & $\mathrm{HJ}$ & - & - & $3.8 \mathrm{M}_{\mathrm{L}}(\mathrm{PS})$ & V & JULY & 16 & 04:47 PST \\
\hline JULY & 16 & 162749.9 & $37.747 \mathrm{~N}$. & $121.972 W$. & 4 & BK & & - & $2.4 \mathrm{M}_{\mathrm{L}}(\mathrm{BK})$ & FELT & JULY & 16 & 08:27 PST \\
\hline JULY & 16 & 205903.8 & $37.297 \mathrm{~N}$. & $121.662 \mathrm{~W}$. & 6 & BK & & & $3.0 \mathrm{M}_{\mathrm{L}}(\mathrm{BK})$ & FELT & JULY & 16 & 12:59 PST \\
\hline JULY & 17 & 032245.3 & $33.998 \mathrm{~N}$. & $116.624 \mathrm{~W}$. & 10 & PS & - & & $3.2 \mathrm{M}_{\mathrm{L}}(\mathrm{PS})$ & - & JULY & 16 & 19:22 PST \\
\hline JULY & 17 & 203515.0 & $33.989 \mathrm{~N}$. & $116.649 \mathrm{~W}$. & 6 & GP & 4.4 & & 4.0ML $(G P)$ & VI & JULY & 17 & 12:35 PST \\
\hline JULY & 17 & 215445.2 & $33.991 \mathrm{~N}$. & $116.641 \mathrm{~W}$ & 7 & GP & 4.1 & - & $4.4 \mathrm{M}_{\mathrm{L}}(\mathrm{PS})$ & IV & JULY & 17 & 13:54 PST \\
\hline JULY & 17 & 235111.4 & $33.994 \mathrm{~N}$. & $116.647 \mathrm{~W}$ & 7 & PS & & - & $3.2 \mathrm{M}_{\mathrm{L}}(\mathrm{PS})$ & 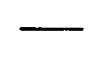 & JULY & 17 & 15:51 PST \\
\hline JULY & 18 & 033840.4 & $33.030 \mathrm{~N}$. & 117.713W. & 0 & $\mathrm{HJ}$ & & - & $3.3 \mathrm{M}_{\mathrm{L}}(\mathrm{PS})$ & & JULY & 17 & 19:37 PST \\
\hline JULY & 18 & 071805.5 & $37.557 \mathrm{~N}$. & $118.887 \mathrm{~W}$. & 3 & BK & & & $3.2 \mathrm{M}_{\mathrm{L}}(\mathrm{BK})$ & {[} & JULY & 17 & 23:18 PST \\
\hline JULY & 18 & 155834.1 & $37.576 \mathrm{~N}$. & $118.441 \mathrm{~W}$. & 6 & PS & & & $3.1 \mathrm{M}_{\mathrm{L}}(\mathrm{PS})$ & FELT & JULY & 18 & 07:58 PST \\
\hline JULY & 18 & 160008.5 & $37.570 \mathrm{~N}$. & $118.443 W$. & 4 & BK & & - & $3.9 \mathrm{M}_{\mathrm{L}}(\mathrm{BK})$ & FELT & JULY & 18 & 08:00 PST \\
\hline JULY & 18 & 170036.8 & $36.093 \mathrm{~N}$. & 117.849W. & 2 & GP & & - & 3.7 $\mathrm{M}_{\mathrm{L}}(\mathrm{GP})$ & IV & JULY & 18 & 09:00 PST \\
\hline JULY & 18 & 170227.8 & $36.112 \mathrm{~N}$. & $117.872 W$. & 6 & PS & & & $3.1 \mathrm{M}_{\mathrm{L}}(\mathrm{PS})$ & & JULY & 18 & 09:02 PST \\
\hline JULY & 18 & 170250.6 & $36.098 \mathrm{~N}$. & $117.849 \mathrm{~W}$. & 3 & GP & & & $3.4 \mathrm{M}_{\mathrm{L}}(\mathrm{GP})$ & & JULY & 18 & 09:02 PST \\
\hline JULY & 18 & 172547.5 & $37.675 \mathrm{~N}$. & $118.431 \mathrm{~W}$ & 6 & PS & & & $3.2 \mathrm{M}_{\mathrm{L}}(\mathrm{PS})$ & & JULY & 18 & 09:25 PST \\
\hline JULY & 18 & 185543.0 & $36.090 \mathrm{~N}$. & $117.855 \mathrm{~W}$. & 2 & GP & - & - & $3.1 \mathrm{M}_{\mathrm{L}}(\mathrm{GP})$ & - & JULY & 18 & 10:55 PST \\
\hline JULY & 18 & 195801.8 & $33.967 \mathrm{~N}$. & $116.569 \mathrm{~W}$. & 7 & PS & 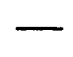 & - & $3.2 \mathrm{M}_{\mathrm{L}}(\mathrm{PS})$ & IV & JULY & 18 & 11:58 PST \\
\hline JULY & 19 & 022443.9 & $36.314 \mathrm{~N}$. & $120.364 \mathrm{~W}$ & 14 & GP & 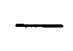 & 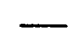 & $3.1 \mathrm{M}_{\mathrm{L}}(\mathrm{GP})$ & & JULY & 18 & 18:24 PST \\
\hline JULY & 19 & 030121.2 & $33.020 \mathrm{~N}$. & $117.737 \mathrm{~W}$. & 4 & $\mathrm{HJ}$ & & & $3.1 \mathrm{M}_{\mathrm{L}}(\mathrm{PS})$ & $\longrightarrow$ & JULY & 18 & 19:01 PST \\
\hline JULY & 19 & 082752.8 & $35.915 \mathrm{~N}$. & $117.716 \mathrm{~W}$ & 6 & GP & - & $\longrightarrow$ & $3.0 \mathrm{M}_{\mathrm{L}}(\mathrm{GP})$ & 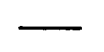 & JULY & 19 & 00:27 PST \\
\hline JULY & 19 & 102338.6 & $32.970 \mathrm{~N}$. & $117.805 \mathrm{~W}$. & 1 & $\mathrm{HJ}$ & 一 & - & $3.2 \mathrm{M}_{\mathrm{L}}(\mathrm{PS})$ & - & JULY & 19 & 02:23 PST \\
\hline
\end{tabular}


Table 1. Summary of United States earthquakes for 1986-Continued

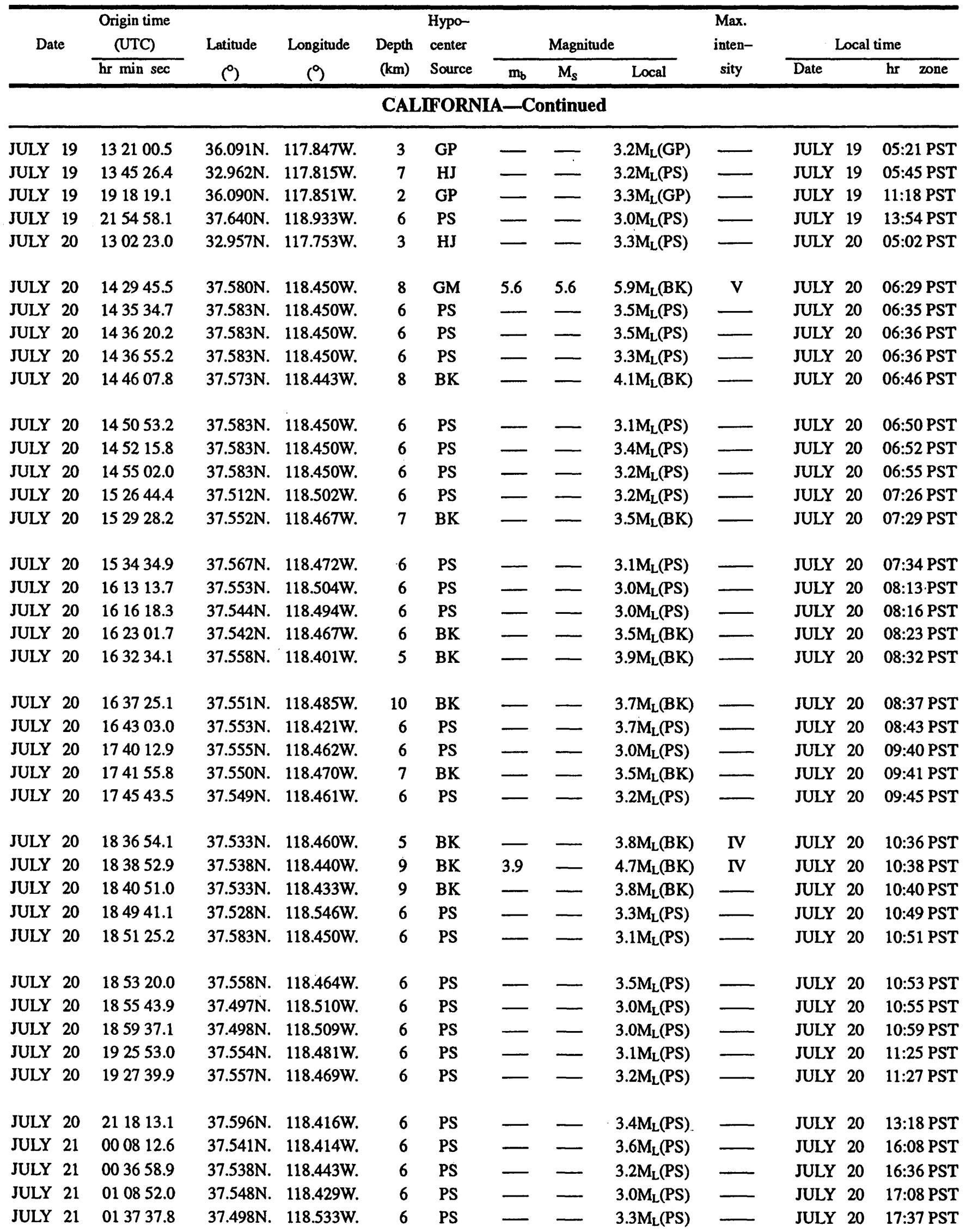


Table 1. Summary of United States earthquakes for 1986-Continued

\begin{tabular}{|c|c|c|c|c|c|c|c|c|c|c|c|c|c|}
\hline \multicolumn{2}{|c|}{ Date } & \multirow{2}{*}{$\begin{array}{l}\text { Origin time } \\
\text { (UTC) } \\
\text { hr min sec }\end{array}$} & $\begin{array}{l}\text { Latitude } \\
\text { ( ) }\end{array}$ & $\begin{array}{c}\text { Longitude } \\
(9)\end{array}$ & $\begin{array}{l}\text { Depth } \\
(\mathbf{k m})\end{array}$ & \multirow{2}{*}{$\begin{array}{l}\text { Hypo- } \\
\text { center } \\
\text { Source }\end{array}$} & \multicolumn{3}{|c|}{ Magnitude } & \multirow{2}{*}{$\begin{array}{l}\text { Max. } \\
\text { inten- } \\
\text { sity }\end{array}$} & \multicolumn{3}{|c|}{ Local time } \\
\hline \multicolumn{8}{|c|}{ CALIFORNIA-Continued } & & & & Date & \multicolumn{2}{|c|}{ hr zone } \\
\hline JULY & 21 & 015426.1 & $37.605 \mathrm{~N}$. & 118.456W. & 6 & PS & - & - & $3.1 \mathrm{M}_{\mathrm{L}}(\mathrm{PS})$ & & JULY & 20 & 17:54 PST \\
\hline JULY & 21 & 022754.3 & $37.533 \mathrm{~N}$. & $118.432 \mathrm{~W}$. & 8 & BK & - & - & $3.7 \mathrm{M}_{\mathrm{L}}(\mathrm{BK})$ & & JULY & 20 & 18:27 PST \\
\hline JULY & 21 & 030142.2 & $37.530 \mathrm{~N}$. & 118.516W. & 6 & PS & - & - & $3.0 \mathrm{M}_{\mathrm{L}}(\mathrm{PS})$ & 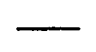 & JULY & 20 & 19:01 PST \\
\hline JULY & 21 & 031211.3 & $37.557 \mathrm{~N}$. & 118.473W. & 8 & BK & - & - & $4.4 \mathrm{M}_{\mathrm{L}}(\mathrm{BK})$ & & JULY & 20 & 19:12 PST \\
\hline JULY & 21 & 053921.8 & $37.516 \mathrm{~N}$. & 118.450W. & 6 & PS & - & - & $3.2 \mathrm{M}_{\mathrm{L}}(\mathrm{PS})$ & & JULY & 20 & 21:39 PST \\
\hline JULY & 21 & 060115.9 & $37.652 \mathrm{~N}$. & $118.412 \mathrm{~W}$ & 6 & PS & - & - & $3.0 \mathrm{M}_{\mathrm{L}}(\mathrm{PS})$ & & JULY & 20 & 22:01 PST \\
\hline JULY & 21 & 065042.0 & $37.609 \mathrm{~N}$. & $118.504 \mathrm{~W}$. & 6 & PS & 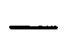 & - & $3.3 \mathrm{M}_{\mathrm{L}}(\mathrm{PS})$ & 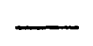 & JULY & 20 & 22:50 PST \\
\hline JULY & 21 & 072447.4 & $37.565 \mathrm{~N}$. & $118.447 \mathrm{~W}$ & 6 & PS & & & $3.0 \mathrm{M}_{\mathrm{L}}(\mathrm{PS})$ & & JULY & 20 & 23:24 PST \\
\hline JULY & 21 & 081031.1 & $37.530 \mathrm{~N}$. & $118.460 \mathrm{~W}$ & 5 & BK & & & $4.1 \mathrm{M}_{\mathrm{L}}(\mathrm{BK})$ & & JULY & 21 & 00:10 PST \\
\hline JULY & 21 & 082044.2 & $37.568 \mathrm{~N}$. & $118.450 \mathrm{~W}$ & 7 & BK & & & $3.6 \mathrm{M}_{\mathrm{L}}(\mathrm{BK})$ & & JULY & 21 & 00:20 PST \\
\hline JULY & 21 & 090242.0 & $37.571 \mathrm{~N}$. & $118.480 \mathrm{~W}$. & 6 & PS & - & - & $3.0 \mathrm{M}_{\mathrm{L}}(\mathrm{PS})$ & & JULY & 21 & 01:02 PST \\
\hline JULY & 21 & 111521.8 & $37.570 \mathrm{~N}$. & $118.463 \mathrm{~W}$ & 10 & BK & & - & $4.3 \mathrm{M}_{\mathrm{L}}(\mathrm{BK})$ & & JULY & 21 & 03:15 PST \\
\hline JULY & 21 & 132849.3 & $37.616 \mathrm{~N}$. & $118.357 w$ & 10 & GS & - & - & $3.4 \mathrm{M}_{\mathrm{L}}(\mathrm{BK})$ & & JULY & 21 & 05:28 PST \\
\hline JULY & 21 & 144226.5 & $37.537 \mathrm{~N}$. & $118.450 \mathrm{~W}$ & 9 & BK & 6.0 & 6.2 & $6.4 \mathrm{M}_{\mathrm{L}}(\mathrm{BK})$ & VI & JULY & 21 & 06:42 PST \\
\hline JULY & 21 & 144521.0 & $37.583 \mathrm{~N}$. & $118.417 \mathrm{~W}$ & 6 & PS & $\longrightarrow$ & & $4.6 \mathrm{M}_{\mathrm{L}}(\mathrm{PS})$ & & JULY & 21 & 06:45 PST \\
\hline JULY & 21 & 144652.7 & $37.583 \mathrm{~N}$ & $118.417 \mathrm{~W}$ & 6 & PS & - & & $3.8 \mathrm{M}_{\mathrm{L}}(\mathrm{PS})$ & & JULY & 21 & 06:46 PST \\
\hline JULY & 21 & 144757.7 & $37.583 \mathrm{~N}$. & $118.417 \mathrm{~W}$ & 6 & PS & - & & $3.9 \mathrm{M}_{\mathrm{L}}(\mathrm{PS})$ & & JULY & 21 & 06:47 PST \\
\hline JULY & 21 & 145110.1 & $37.570 \mathrm{~N}$. & $118.525 \mathrm{~W}$ & 1 & BK & 5.1 & & $5.7 \mathrm{M}_{\mathrm{L}}(\mathrm{BK})$ & V & JULY & 21 & 06:51 PST \\
\hline JULY & 21 & 145358.1 & $37.583 \mathrm{~N}$ & $118.583 W$ & 6 & PS & - & & $4.9 \mathrm{M}_{\mathrm{L}}(\mathrm{PS})$ & & JULY & 21 & 06:53 PST \\
\hline JULY & 21 & 145439.2 & $37.583 \mathrm{~N}$. & 118.417W: & 6 & PS & - & & $4.5 \mathrm{M}_{\mathrm{L}}(\mathrm{PS})$ & & JULY & 21 & 06:54 PST \\
\hline JULY & 21 & 145621.6 & $37.583 \mathrm{~N}$ & $118.417 \mathrm{~W}$ & 6 & PS & - & & $3.4 \mathrm{M}_{\mathrm{L}}(\mathrm{PS})$ & & JULY & 21 & 06:56 PST \\
\hline JULY & 21 & 145750.9 & $37.527 \mathrm{~N}$. & $118.357 \mathrm{~W}$ & 7 & BK & 4.7 & & $4.8 \mathrm{M}_{\mathrm{L}}(\mathrm{BK})$ & & JULY & 21 & 06:57 PST \\
\hline JULY & 21 & 145858.2 & $37.583 \mathrm{~N}$. & $118.417 \mathrm{~W}$ & 6 & PS & & & 4.0M $\mathrm{M}_{\mathrm{L}}(\mathrm{PS})$ & & JULY & 21 & 06:58 PST \\
\hline JULY & 21 & 150042.7 & $37.575 \mathrm{~N}$ & 118.420W. & 4 & BK & & & $3.9 \mathrm{M}_{\mathrm{L}}(\mathrm{PS})$ & & JULY & 21 & 07:00 PST \\
\hline JULY & 21 & 150302.4 & $37.632 \mathrm{~N}$ & 118.500W. & 8 & BK & & & $3.5 \mathrm{M}_{\mathrm{L}}(\mathrm{BK})$ & & JULY & 21 & 07:03 PST \\
\hline JULY & 21 & 150541.1 & $37.533 \mathrm{~N}$. & $118.482 W$ & 4 & BK & & & $4.1 \mathrm{M}_{\mathrm{L}}(\mathrm{BK})$ & & JULY & 21 & 07:05 PST \\
\hline JULY & 21 & 151015.0 & $37.547 \mathrm{~N}$ & $118.428 \mathrm{~W}$ & 6 & PS & & & $3.8 \mathrm{M}_{\mathrm{L}}(\mathrm{BK})$ & & JULY & 21 & 07:10 PST \\
\hline JULY & 21 & 151130.9 & $37.538 \mathrm{~N}$. & 118.473W. & 9 & BK & & & $4.4 \mathrm{M}_{\mathrm{L}}(\mathrm{BK})$ & & JULY & 21 & 07:11 PST \\
\hline JULY & 21 & 151433.5 & $37.583 \mathrm{~N}$ & $118.417 \mathrm{~W}$ & 6 & PS & & & $3.1 \mathrm{M}_{\mathrm{L}}(\mathrm{PS})$ & & JULY & 21 & 07:14 PST \\
\hline JULY & 21 & 151529.5 & $37.600 \mathrm{~N}$ & $118.455 \mathrm{~W}$ & 3 & BK & & & $3.5 \mathrm{M}_{\mathrm{L}}(\mathrm{BK})$ & & JULY & 21 & 07:15 PST \\
\hline JULY & 21 & 151934.9 & $37.488 \mathrm{~N}$ & 118.370W. & 16 & BK & & & $4.7 \mathrm{M}_{\mathrm{L}}(\mathrm{BK})$ & & JULY & 21 & 07:19 PST \\
\hline JULY & 21 & 152239.7 & $37.583 \mathrm{~N}$ & $118.417 \mathrm{~W}$ & 6 & PS & & & $3.4 \mathrm{M}_{\mathrm{L}}(\mathrm{PS})$ & & JULY & 21 & 07:22 PST \\
\hline JULY & 21 & 152649.2 & $37.533 \mathrm{~N}$. & 118.425W. & 18 & BK & & & $4.6 \mathrm{M}_{\mathrm{L}}(\mathrm{BK})$ & & JULY & 21 & 07:26 PST \\
\hline JULY & 21 & 152910.4 & $37.563 \mathrm{~N}$ & $118.467 \mathrm{~W}$ & 1 & BK & & & $3.7 \mathrm{M}_{\mathrm{L}}(\mathrm{BK})$ & & JULY & 21 & 07:29 PST \\
\hline JULY & 21 & 153104.8 & $37.597 \mathrm{~N}$ & $118.453 W$ & 4 & BK & & - & $3.6 \mathrm{M}_{\mathrm{L}}(\mathrm{BK})$ & & JULY & 21 & 07:31 PST \\
\hline JÜLY & 21 & 153649.9 & $37.553 \mathrm{~N}$ & 118.433W. & 5 & BK & & & $4.0 \mathrm{M}_{\mathrm{L}}(\mathrm{BK})$ & & JULY & 21 & 07:36 PST \\
\hline JULY & 21 & 154122.0 & $37.542 \mathrm{~N}$ & $118.405 W$ & 5 & BK & & & $4.2 \mathrm{M}_{\mathrm{L}}(\mathrm{BK})$ & & JULY & 21 & 07:41 PST \\
\hline JULY & 21 & 154623.0 & $37.551 \mathrm{~N}$ & $118.420 \mathrm{~W}$. & 1 & BK & & & $4.5 \mathrm{M}_{\mathrm{L}}(\mathrm{BK})$ & & JULY & 21 & 07:46 PST \\
\hline JULY & 21 & 155022.6 & $37.647 \mathrm{~N}$ & $118.401 \mathrm{~W}$ & 20 & BK & - & - & $3.8 \mathrm{M}_{\mathrm{L}}(\mathrm{BK})$ & & JULY & 21 & 07:50 PST \\
\hline JULY & 21 & 160401.5 & $37.688 \mathrm{~N}$ & $118.317 \mathrm{~W}$ & 6 & PS & - & - & $3.3 \mathrm{M}_{\mathrm{L}}(\mathrm{PS})$ & - & JULY & 21 & 08:04 PST \\
\hline
\end{tabular}


Table 1. Summary of United States earthquakes for 1986-Continued

\begin{tabular}{|c|c|c|c|c|c|c|c|c|c|c|c|c|c|}
\hline \multirow[t]{2}{*}{ Date } & & \multirow{2}{*}{$\begin{array}{l}\text { Origin time } \\
\text { (UTC) } \\
\text { hr min sec }\end{array}$} & \multirow{2}{*}{$\begin{array}{l}\text { Latitude } \\
\left({ }^{\circ}\right)\end{array}$} & \multirow{2}{*}{$\begin{array}{l}\text { Longitude } \\
\left({ }^{\circ}\right)\end{array}$} & \multirow{2}{*}{$\begin{array}{l}\text { Depth } \\
(\mathbf{k m})\end{array}$} & \multirow{2}{*}{$\begin{array}{l}\text { Hypo- } \\
\text { center } \\
\text { Source }\end{array}$} & \multicolumn{3}{|c|}{ Magnitude } & \multirow{2}{*}{$\begin{array}{l}\text { Max. } \\
\text { inten- } \\
\text { sity }\end{array}$} & \multicolumn{3}{|c|}{ Local time } \\
\hline & & & & & & & $\mathbf{m}_{\mathbf{b}}$ & $\mathrm{M}_{\boldsymbol{s}}$ & Local & & Date & & zone \\
\hline \multicolumn{14}{|c|}{ CALIFORNIA-Continued } \\
\hline JULY & 21 & 160849.8 & $37.639 \mathrm{~N}$ & 118.316W. & 6 & PS & - & - & $3.1 \mathrm{M}_{\mathrm{L}}(\mathrm{PS})$ & - & JULY & 21 & 08:08 PST \\
\hline JULY & 21 & 161904.7 & $37.583 \mathrm{~N}$. & 118.391W. & 6 & PS & - & - & $3.4 \mathrm{M}_{\mathrm{L}}(\mathrm{PS})$ & - & JULY & 21 & 08:19 PST \\
\hline JULY & 21 & 162644.7 & $37.487 \mathrm{~N}$ & $118.393 \mathrm{~W}$ & 8 & BK & - & - & $4.6 \mathrm{M}_{\mathrm{L}}(\mathrm{BK})$ & - & JULY & 21 & 08:26 PST \\
\hline JULY & 21 & 163643.8 & $37.533 \mathrm{~N}$ & $118.465 \mathrm{~W}$. & 10 & GS & - & - & $3.6 \mathrm{M}_{\mathrm{L}}(\mathrm{PS})$ & - & JULY & 21 & 08:36 PST \\
\hline JULY & 21 & 170533.4 & $37.532 \mathrm{~N}$. & $118.462 \mathrm{~W}$ & 9 & BK & 3.6 & - & $4.5 \mathrm{M}_{\mathrm{L}}(\mathrm{BK})$ & - & JULY & 21 & 09:05 PST \\
\hline JULY & 21 & 170832.9 & $37.547 \mathrm{~N}$ & $118.420 \mathrm{~W}$ & 6 & BK & - & - & $3.5 \mathrm{M}_{\mathrm{L}}(\mathrm{BK})$ & 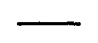 & JULY & 21 & 09:08 PST \\
\hline JULY & 21 & 172000.4 & $37.447 \mathrm{~N}$ & $118.367 \mathrm{~W}$ & 11 & BK & - & - & $4.5 \mathrm{M}_{\mathrm{L}}(\mathrm{BK})$ & - & JULY & 21 & 09:20 PST \\
\hline JULY & 21 & 173030.1 & $37.547 \mathrm{~N}$ & $118.405 \mathrm{~W}$ & 6 & BK & - & - & $3.9 \mathrm{M}_{\mathrm{L}}(\mathrm{BK})$ & - & JULY & 21 & 09:30 PST \\
\hline JULY & 21 & 174321.3 & $37.585 \mathrm{~N}$ & $118.442 \mathrm{~W}$ & 6 & PS & - & - & $3.0 \mathrm{M}_{\mathrm{L}}(\mathrm{PS})$ & - & JULY & 21 & 09:43 PST \\
\hline JULY & 21 & 174855.8 & $37.598 \mathrm{~N}$. & $118.448 \mathrm{~W}$ & 3 & BK & - & 一 & $4.3 \mathrm{M}_{\mathrm{L}}(\mathrm{BK})$ & & JULY & 21 & 09:48 PST \\
\hline JULY & 21 & 175303.0 & $37.572 \mathrm{~N}$. & $118.482 \mathrm{~W}$ & 11 & BK & - & - & $3.5 \mathrm{M}_{\mathrm{L}}(\mathrm{BK})$ & - & JULY & 21 & 09:53 PST \\
\hline JULY & 21 & 180227.4 & $37.564 \mathrm{~N}$. & 118.498W. & 6 & PS & - & - & $3.0 \mathrm{M}_{\mathrm{L}}(\mathrm{PS})$ & - & JULY & 21 & 10:02 PST \\
\hline JULY & 21 & 181324.1 & $37.580 \mathrm{~N}$. & 118.439W. & 6 & PS & - & - & $3.1 \mathrm{M}_{\mathrm{L}}(\mathrm{PS})$ & - & JULY & 21 & 10:13 PST \\
\hline JULY & 21 & 181357.6 & $37.623 \mathrm{~N}$ & $118.472 \mathrm{~W}$ & 3 & BK & - & - & $4.0 \mathrm{M}_{\mathrm{L}}(\mathrm{BK})$ & - & JULY & 21 & 10:13 PST \\
\hline JULY & 21 & 181401.3 & $37.424 \mathrm{~N}$ & $118.639 \mathrm{~W}$ & 6 & PS & - & - & $3.4 \mathrm{M}_{\mathrm{L}}(\mathrm{PS})$ & & JULY & 21 & 10:14 PST \\
\hline JULY & 21 & 181833.2 & $37.525 \mathrm{~N}$ & $118.427 \mathrm{~W}$ & 7 & BK & - & - & $3.5 \mathrm{M}_{\mathrm{L}}(\mathrm{BK})$ & - & JULY & 21 & 10:18 PST \\
\hline JULY & 21 & 182008.5 & $37.583 \mathrm{~N}$ & $118.417 \mathrm{~W}$ & 6 & PS & - & - & $3.1 \mathrm{M}_{\mathrm{L}}(\mathrm{PS})$ & - & JULY & 21 & 10:20 PST \\
\hline JULY & 21 & 182930.8 & $33.018 \mathrm{~N}$ & $117.802 \mathrm{~W}$ & 8 & HJ & - & - & $3.8 \mathrm{M}_{\mathrm{L}}(\mathrm{PS})$ & FELT & JULY & 21 & 10:29 PST \\
\hline JULY & 21 & 183705.5 & $37.533 \mathrm{~N}$ & $118.524 \mathrm{~W}$ & 6 & PS & - & 一 & $3.1 \mathrm{M}_{\mathrm{L}}(\mathrm{PS})$ & & JULY & 21 & 10:37 PST \\
\hline JULY & 21 & 195122.6 & $37.513 \mathrm{~N}$ & 118.395W. & 6 & PS & - & - & $3.0 \mathrm{M}_{\mathrm{L}}(\mathrm{PS})$ & & JULY & 21 & 11:51 PST \\
\hline JULY & 21 & 202019.7 & $37.497 \mathrm{~N}$. & 118.341W. & 6 & PS & - & - & $3.0 \mathrm{M}_{\mathrm{L}}(\mathrm{PS})$ & — & JULY & 21 & 12:20 PST \\
\hline JULY & 21 & 202624.6 & $37.521 \mathrm{~N}$ & $118.432 \mathrm{~W}$ & 6 & PS & - & - & $3.1 \mathrm{M}_{\mathrm{L}}(\mathrm{PS})$ & & JULY & 21 & 12:26 PST \\
\hline JULY & 21 & 203605.2 & $37.523 \mathrm{~N}$. & $118.455 \mathrm{~W}$ & 9 & BK & - & - & $4.0 \mathrm{M}_{\mathrm{L}}(\mathrm{BK})$ & - & JULY & 21 & 12:36 PST \\
\hline JULY & 21 & 203708.7 & $37.538 \mathrm{~N}$. & $118.457 \mathrm{w}$ & 6 & PS & - & - & $3.1 \mathrm{M}_{\mathrm{L}}(\mathrm{PS})$ & - & JULY & 21 & 12:37 PST \\
\hline JULY & 21 & 204024.8 & $37.442 \mathrm{~N}$. & $118.372 \mathrm{~W}$ & 4 & BK & - & - & $3.6 \mathrm{M}_{\mathrm{L}}(\mathrm{BK})$ & - & JULY & 21 & $12: 40 \mathrm{PST}$ \\
\hline JULY & 21 & 204044.2 & $37.473 \mathrm{~N}$ & 118.444W. & 6 & PS & - & - & $3.5 \mathrm{M}_{\mathrm{L}}(\mathrm{PS})$ & 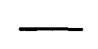 & JULY & 21 & $12: 40$ PST \\
\hline JULY & 21 & 205749.2 & $37.505 \mathrm{~N}$. & $118.425 \mathrm{~W}$ & 6 & PS & - & - & $3.0 \mathrm{M}_{\mathrm{L}}(\mathrm{PS})$ & - & JULY & 21 & 12:57 PST \\
\hline JULY & 21 & 205800.6 & $37.597 \mathrm{~N}$. & $118.431 \mathrm{~W}$ & 6 & PS & - & - & $3.1 \mathrm{M}_{\mathrm{L}}(\mathrm{PS})$ & - & JULY & 21 & 12:58 PST \\
\hline JULY & 21 & 210343.5 & $37.500 \mathrm{~N}$. & $118.642 \mathrm{~W}$ & 6 & PS & - & - & $3.2 \mathrm{M}_{\mathrm{L}}(\mathrm{PS})$ & - & JULY & 21 & 13:03 PST \\
\hline JULY & 21 & 210718.1 & $37.541 \mathrm{~N}$ & $118.429 \mathrm{~W}$ & 6 & PS & - & - & $3.2 \mathrm{M}_{\mathrm{L}}(\mathrm{PS})$ & & JULY & 21 & $13: 07$ PST \\
\hline JULY & 21 & 210841.8 & $37.585 \mathrm{~N}$. & 118.453W. & 5 & BK & 一 & - & $3.6 \mathrm{M}_{\mathrm{L}}(\mathrm{BK})$ & $\longrightarrow$ & JULY & 21 & 13:08 PST \\
\hline JULY & 21 & 211012.0 & $37.498 \mathrm{~N}$. & $118.438 \mathrm{~W}$ & 6 & PS & - & - & $3.2 \mathrm{M}_{\mathrm{L}}(\mathrm{PS})$ & - & JULY & 21 & 13:10 PST \\
\hline JULY & 21 & 213937.0 & $37.520 \mathrm{~N}$ & $118.366 \mathrm{~W}$ & 6 & PS & - & - & $3.3 \mathrm{M}_{\mathrm{L}}(\mathrm{PS})$ & - & JULY & 21 & 13:39 PST \\
\hline JULY & 21 & 215144.9 & $37.603 \mathrm{~N}$ & $118.438 \mathrm{~W}$ & 3 & BK & - & 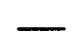 & $3.7 \mathrm{M}_{\mathrm{L}}(\mathrm{BK})$ & - & JULY & 21 & 13:51 PST \\
\hline JULY & 21 & 220701.2 & $37.480 \mathrm{~N}$. & $118.373 W$ & 6 & BK & - & - & $3.1 \mathrm{M}_{\mathrm{L}}(\mathrm{BK})$ & - & JULY & 21 & 14:07 PST \\
\hline JULY & 21 & 220717.0 & $37.483 \mathrm{~N}$ & $118.367 \mathrm{~W}$ & 6 & BK & 5.5 & 5.0 & $5.6 \mathrm{M}_{\mathrm{L}}(\mathrm{BK})$ & FELT & JULY & 21 & 14:07 PST \\
\hline JULY & 21 & 220922.1 & $37.613 \mathrm{~N}$. & $118.569 \mathrm{~W}$ & 6 & PS & - & 一 & $4.7 \mathrm{M}_{\mathrm{L}}(\mathrm{PS})$ & - & JULY & 21 & 14:09 PST \\
\hline JULY & 21 & 222831.1 & $37.625 \mathrm{~N}$ & $118.501 \mathrm{~W}$ & 6 & PS & - & - & $3.2 \mathrm{M}_{\mathrm{L}}(\mathrm{PS})$ & - & JULY & 21 & $14: 28$ PST \\
\hline JULY & 21 & 223917.2 & $37.603 \mathrm{~N}$ & $118.447 \mathrm{~W}$ & 6 & PS & - & - & $3.2 \mathrm{M}_{\mathrm{L}}(\mathrm{PS})$ & - & JULY & 21 & 14:39 PST \\
\hline JULY & 21 & 224401.9 & $37.503 \mathrm{~N}$ & $118.369 \mathrm{~W}$ & 6 & PS & - & - & $3.0 \mathrm{M}_{\mathrm{L}}(\mathrm{PS})$ & - & JULY & 21 & 14:44 PST \\
\hline
\end{tabular}


Table 1. Summary of United States earthquakes for 1986-Continued

\begin{tabular}{|c|c|c|c|c|c|c|c|c|c|c|c|c|c|}
\hline \multirow{2}{*}{\multicolumn{2}{|c|}{ Date }} & \multirow{2}{*}{$\begin{array}{l}\text { Origin time } \\
\text { (UTC) } \\
\text { hr } \min \mathrm{sec}\end{array}$} & \multirow{2}{*}{$\begin{array}{l}\text { Latitude } \\
\text { () }\end{array}$} & \multirow{2}{*}{$\begin{array}{c}\text { Longitude } \\
(9)\end{array}$} & \multirow{2}{*}{$\begin{array}{l}\text { Depth } \\
(\mathrm{km})\end{array}$} & \multirow{2}{*}{$\begin{array}{l}\text { Hypo- } \\
\text { center } \\
\text { Source }\end{array}$} & \multicolumn{3}{|c|}{ Magnitude } & \multirow{2}{*}{$\begin{array}{l}\text { Max. } \\
\text { inten- } \\
\text { sity }\end{array}$} & \multicolumn{3}{|c|}{ Local time } \\
\hline & & & & & & & $\mathbf{m}_{\mathrm{b}}$ & $\mathrm{M}_{\mathrm{s}}$ & Local & & Date & & zone \\
\hline \multicolumn{14}{|c|}{ CALIFORNIA-Continued } \\
\hline JULY & 21 & 225201.4 & $37.599 \mathrm{~N}$. & $118.454 \mathrm{~W}$ & 6 & PS & - & - & $3.0 \mathrm{M}_{\mathrm{L}}(\mathrm{PS})$ & & JULY & 21 & 14:52 PST \\
\hline JULY & 21 & 234144.1 & $37.707 \mathrm{~N}$ & $118.424 \mathrm{~W}$ & 6 & PS & - & - & $3.0 \mathrm{M}_{\mathrm{L}}(\mathrm{PS})$ & & JULY & 21 & 15:41 PST \\
\hline JULY & 21 & 234221.5 & $37.775 \mathrm{~N}$ & 118.345W. & 6 & PS & - & - & $3.2 \mathrm{M}_{\mathrm{L}}(\mathrm{PS})$ & & JULY & 21 & 15:42 PST \\
\hline JULY & 21 & 234306.7 & $37.601 \mathrm{~N}$. & $118.490 \mathrm{~W}$ & 7 & BK & - & - & $4.2 \mathrm{M}_{\mathrm{L}}(\mathrm{BK})$ & & JULY & 21 & 15:43 PST \\
\hline JULY & 22 & 000953.8 & $37.610 \mathrm{~N}$ & $118.430 \mathrm{~W}$ & 4 & BK & 4.0 & - & $4.5 \mathrm{M}_{\mathrm{L}}(\mathrm{BK})$ & & JULY & 21 & 16:09 PST \\
\hline JULY & 22 & 002519.0 & $36.077 \mathrm{~N}$ & $117.862 \mathrm{~W}$ & 3 & PS & - & - & $3.3 \mathrm{M}_{\mathrm{L}}(\mathrm{PS})$ & & JULY & 21 & 16:25 PST \\
\hline JULY & 22 & 010054.9 & $37.527 \mathrm{~N}$ & $118.418 \mathrm{~W}$ & 6 & PS & - & - & $3.0 \mathrm{M}_{\mathrm{L}}(\mathrm{PS})$ & & JULY & 21 & 17:00 PST \\
\hline JULY & 22 & 014907.3 & $37.585 \mathrm{~N}$ & $118.451 \mathrm{~W}$ & 6 & PS & - & - & $3.0 \mathrm{M}_{\mathrm{L}}(\mathrm{PS})$ & & JULY & 21 & $17: 49$ PST \\
\hline JULY & 22 & 020314.6 & $37.465 \mathrm{~N}$ & 118.369W. & 6 & PS & - & - & $3.1 \mathrm{M}_{\mathrm{L}}(\mathrm{PS})$ & & JULY & 21 & 18:03 PST \\
\hline JULY & 22 & 021705.9 & $37.602 \mathrm{~N}$ & $118.490 \mathrm{~W}$ & 7 & BK & - & - & $3.6 \mathrm{M}_{\mathrm{L}}(\mathrm{BK})$ & & JULY & 21 & 18:17 PST \\
\hline JULY & 22 & 022131.5 & $37.640 \mathrm{~N}$ & $118.417 \mathrm{~W}$ & 10 & GS & - & - & $3.6 \mathrm{M}_{\mathrm{L}}(\mathrm{BK})$ & & JULY & 21 & 18:21 PST \\
\hline JULY & 22 & 024843.8 & $37.559 \mathrm{~N}$ & $118.432 \mathrm{~W}$ & 6 & PS & - & - & $3.2 \mathrm{M}_{\mathrm{L}}(\mathrm{PS})$ & & JULY & 21 & 18:48 PST \\
\hline JULY & 22 & 030210.7 & $37.580 \mathrm{~N}$ & 118.483W. & 7 & BK & - & - & $3.8 \mathrm{M}_{\mathrm{L}}(\mathrm{BK})$ & & JULY & 21 & 19:02 PST \\
\hline JULY & 22 & 030841.9 & $37.538 \mathrm{~N}$ & 118.380W. & 6 & PS & - & - & $3.3 \mathrm{M}_{\mathrm{L}}(\mathrm{PS})$ & & JULY & 21 & 19:08 PST \\
\hline JULY & 22 & 031745.4 & $37.454 \mathrm{~N}$. & $118.674 \mathrm{~W}$ & 6 & PS & - & - & $3.1 \mathrm{M}_{\mathrm{L}}(\mathrm{PS})$ & & JULY & 21 & 19:17 PST \\
\hline JULY & 22 & 031848.1 & $37.528 \mathrm{~N}$ & $118.445 \mathrm{~W}$ & 10 & BK & - & - & $3.9 \mathrm{M}_{\mathrm{L}}(\mathrm{BK})$ & & JULY & 21 & 19:18 PST \\
\hline JULY & 22 & 032604.5 & $37.670 \mathrm{~N}$ & 118.293W. & 6 & PS & - & - & $3.1 \mathrm{M}_{\mathrm{L}}(\mathrm{PS})$ & & JULY & 21 & 19:26 PST \\
\hline JULY & 22 & 032912.8 & $37.468 \mathrm{~N}$ & $118.384 \mathrm{~W}$ & 6 & PS & - & - & $3.1 \mathrm{M}_{\mathrm{L}}(\mathrm{PS})$ & & JULY & 21 & $19: 29$ PST \\
\hline JULY & 22 & 034245.2 & $37.540 \mathrm{~N}$ & $118.421 \mathrm{~W}$ & 6 & PS & - & - & $3.2 \mathrm{M}_{\mathrm{L}}(\mathrm{PS})$ & & JULY & 21 & 19:42 PST \\
\hline JULY & 22 & 034710.2 & $37.616 \mathrm{~N}$ & $118.459 \mathrm{~W}$ & 6 & PS & - & - & $3.2 \mathrm{M}_{\mathrm{L}}(\mathrm{PS})$ & & JULY & 21 & 19:47 PST \\
\hline JULY & 22 & 035105.4 & $37.499 \mathrm{~N}$ & 118.395W. & 6 & PS & - & - & $3.1 \mathrm{M}_{\mathrm{L}}(\mathrm{PS})$ & & JULY & 21 & 19:51 PST \\
\hline JULY & 22 & 035920.0 & $37.527 \mathrm{~N}$ & $118.415 \mathrm{~W}$ & 6 & PS & - & - & $3.0 \mathrm{M}_{\mathrm{L}}(\mathrm{PS})$ & & JULY & 21 & 19:59 PST \\
\hline JULY & 22 & 040927.4 & $37.562 \mathrm{~N}$ & $118.393 \mathrm{~W}$ & 6 & PS & - & - & $3.0 \mathrm{M}_{\mathrm{L}}(\mathrm{PS})$ & & JULY & 21 & 20:09 PST \\
\hline JULY & 22 & 041135.9 & $37.614 \mathrm{~N}$ & $118.528 \mathrm{~W}$ & 6 & PS & - & - & $3.1 \mathrm{M}_{\mathrm{L}}(\mathrm{PS})$ & & JULY & 21 & 20:11 PST \\
\hline JULY & 22 & 042249.6 & $37.657 \mathrm{~N}$ & $118.382 \mathrm{~W}$ & 6 & PS & - & - & $3.0 \mathrm{M}_{\mathrm{L}}(\mathrm{PS})$ & & JULY & 21 & 20:22 PST \\
\hline JULY & 22 & 050521.6 & $37.647 \mathrm{~N}$ & $118.428 \mathrm{~W}$ & 6 & PS & - & - & $3.3 \mathrm{M}_{\mathrm{L}}(\mathrm{PS})$ & & JULY & 21 & 21:05 PST \\
\hline JULY & 22 & 051732.1 & $37.433 \mathrm{~N}$ & $118.510 \mathrm{~W}$ & 6 & PS & - & - & $3.1 \mathrm{M}_{\mathrm{L}}(\mathrm{PS})$ & & JULY & 21 & 21:17 PST \\
\hline JULY & 22 & 052408.0 & $37.550 \mathrm{~N}$ & $118.427 \mathrm{~W}$ & 6 & BK & - & - & $3.6 \mathrm{M}_{\mathrm{L}}(\mathrm{BK})$ & - & JULY & 21 & 21:24 PST \\
\hline JULY & 22 & 054043.9 & $37.525 \mathrm{~N}$ & $118.580 \mathrm{~W}$ & 6 & BK & - & - & $4.1 \mathrm{M}_{\mathrm{L}}(\mathrm{BK})$ & & JULY & 21 & 21:40 PST \\
\hline JULY & 22 & 055135.9 & $37.523 \mathrm{~N}$ & $118.396 \mathrm{~W}$ & 6 & PS & - & - & $3.2 \mathrm{M}_{\mathrm{L}}(\mathrm{PS})$ & & JULY & 21 & 21:51 PST \\
\hline JULY & 22 & 061823.3 & $37.646 \mathrm{~N}$ & 118.513W. & 6 & PS & - & - & $3.0 \mathrm{M}_{\mathrm{L}}(\mathrm{PS})$ & & JULY & 21 & 22:18 PST \\
\hline JULY & 22 & 062152.8 & $37.442 \mathrm{~N}$ & $118.388 \mathrm{~W}$ & 12 & BK & - & - & $4.2 \mathrm{M}_{\mathrm{L}}(\mathrm{BK})$ & & JULY & 21 & 22:21 PST \\
\hline JULY & 22 & 063339.2 & $37.528 \mathrm{~N}$ & $118.440 \mathrm{~W}$ & 9 & BK & - & - & $3.5 \mathrm{M}_{\mathrm{L}}(\mathrm{BK})$ & & JULY & 21 & 22:33 PST \\
\hline JULY & 22 & 064329.5 & $37.404 \mathrm{~N}$ & $118.629 \mathrm{~W}$ & 6 & PS & - & - & $3.1 \mathrm{M}_{\mathrm{L}}(\mathrm{PS})$ & & JULY & 21 & 22:43 PST \\
\hline JULY & 22 & 065811.2 & $37.601 \mathrm{~N}$ & $118.482 \mathrm{~W}$ & 3 & BK & - & - & $3.6 \mathrm{M}_{\mathrm{L}}(\mathrm{BK})$ & & JULY & 21 & 22:58 PST \\
\hline JULY & 22 & 075529.9 & $37.618 \mathrm{~N}$ & $118.492 \mathrm{~W}$ & 6 & PS & - & - & $3.0 \mathrm{M}_{\mathrm{L}}(\mathrm{PS})$ & $\longrightarrow$ & JULY & 21 & 23:55 PST \\
\hline JULY & 22 & 082916.3 & $37.527 \mathrm{~N}$ & $118.450 \mathrm{~W}$ & 3 & BK & - & - & $4.0 \mathrm{M}_{\mathrm{L}}(\mathrm{BK})$ & & JULY & 22 & 00:29 PST \\
\hline JULY & 22 & 083810.7 & $37.576 \mathrm{~N}$ & $118.423 \mathrm{~W}$ & 6 & PS & - & - & $3.1 \mathrm{M}_{\mathrm{L}}(\mathrm{PS})$ & 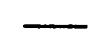 & JULY & 22 & 00:38 PST \\
\hline JULY & 22 & 084804.2 & $37.549 \mathrm{~N}$ & $118.423 \mathrm{~W}$ & 6 & PS & - & - & $3.1 \mathrm{M}_{\mathrm{L}}(\mathrm{PS})$ & 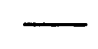 & JULY & 22 & 00:48 PST \\
\hline JULY & 22 & 093416.1 & $37.440 \mathrm{~N}$ & $118.377 \mathrm{~W}$ & 9 & BK & - & - & $3.5 \mathrm{M}_{\mathrm{L}}(\mathrm{BK})$ & - & JULY & 22 & 01:34 PST \\
\hline
\end{tabular}


Table 1. Summary of United States earthquakes for 1986-Continued

\begin{tabular}{|c|c|c|c|c|c|c|c|c|c|c|c|c|c|}
\hline \multirow{2}{*}{\multicolumn{2}{|c|}{ Date }} & $\begin{array}{l}\text { Origin time } \\
\text { (UTC) }\end{array}$ & \multirow{3}{*}{$\begin{array}{l}\text { Latitude } \\
\text { ( ) }\end{array}$} & \multirow{3}{*}{$\begin{array}{c}\text { Longitude } \\
\text { (9) }\end{array}$} & \multirow{2}{*}{$\begin{array}{l}\text { Depth } \\
(\mathrm{km})\end{array}$} & \multirow{2}{*}{$\begin{array}{l}\text { Hypo- } \\
\text { center } \\
\text { Source }\end{array}$} & \multicolumn{3}{|c|}{ Magnitude } & \multirow{3}{*}{$\begin{array}{l}\text { Max. } \\
\text { inten- } \\
\text { sity }\end{array}$} & \multicolumn{3}{|c|}{ Local time } \\
\hline & & hr min sec & & & & & $\mathbf{m}_{\mathbf{b}}$ & $\mathrm{M}_{\mathrm{s}}$ & Local & & Date & & zone \\
\hline \multicolumn{11}{|c|}{ CALIFORNIA-Continued } & & & \\
\hline JULY & 22 & 100941.1 & $37.574 \mathrm{~N}$. & $118.453 \mathrm{~W}$. & 6 & PS & & & $3.2 \mathrm{M}_{\mathrm{L}}(\mathrm{PS})$ & & JULY & 22 & 02:09 PST \\
\hline JULY & 22 & 101327.4 & $37.505 \mathrm{~N}$. & $118.487 \mathrm{~W}$. & 6 & PS & 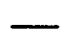 & - & $3.0 \mathrm{M}_{\mathrm{L}}(\mathrm{PS})$ & & JULY & 22 & 02:13 PST \\
\hline JULY & 22 & 102613.4 & $37.473 \mathrm{~N}$. & 118.378W. & 6 & PS & - & - & $3.3 \mathrm{M}_{\mathrm{L}}(\mathrm{PS})$ & & JULY & 22 & 02:26 PST \\
\hline JULY & 22 & 110100.3 & $37.521 \mathrm{~N}$. & 118.401W. & 6 & PS & - & - & $3.0 \mathrm{M}_{\mathrm{L}}(\mathrm{PS})$ & & JULY & 22 & 03:01 PST \\
\hline JULY & 22 & 111241.1 & $37.556 \mathrm{~N}$. & $118.451 \mathrm{~W}$ & 6 & PS & - & - & $3.0 \mathrm{M}_{\mathrm{L}}(\mathrm{PS})$ & & JULY & 22 & 03:12 PST \\
\hline JULY & 22 & 120251.2 & $37.640 \mathrm{~N}$. & $118.476 \mathrm{~W}$. & 6 & PS & - & & $3.0 \mathrm{M}_{\mathrm{L}}(\mathrm{PS})$ & & JULY & 22 & 04:02 PST \\
\hline JULY & 22 & 121548.2 & $37.652 \mathrm{~N}$. & $118.486 \mathrm{~W}$. & 6 & PS & - & & $3.1 \mathrm{M}_{\mathrm{L}}(\mathrm{PS})$ & & JULY & 22 & 04:15 PST \\
\hline JULY & 22 & 122450.2 & $37.522 \mathrm{~N}$. & $118.482 \mathrm{~W}$ & 9 & BK & 3.7 & & $4.4 \mathrm{M}_{\mathrm{L}}(\mathrm{BK})$ & & JULY & 22 & 04:24 PST \\
\hline JULY & 22 & 122615.5 & $37.583 \mathrm{~N}$. & $118.417 \mathrm{~W}$ & 6 & PS & - & & $4.0 \mathrm{M}_{\mathrm{L}}(\mathrm{PS})$ & & JULY & 22 & $04: 26$ PST \\
\hline JULY & 22 & 123135.6 & $37.545 \mathrm{~N}$. & $118.504 \mathrm{~W}$ & 6 & PS & - & - & $3.1 \mathrm{M}_{\mathrm{L}}(\mathrm{PS})$ & & JULY & 22 & 04:31 PST \\
\hline JULY & 22 & 123329.2 & $37.536 \mathrm{~N}$. & $118.428 \mathrm{~W}$ & 6 & PS & - & & $3.3 \mathrm{M}_{\mathrm{L}}(\mathrm{PS})$ & & JULY & 22 & 04:33 PST \\
\hline JULY & 22 & 123937.8 & $37.496 \mathrm{~N}$. & 118.410W. & 6 & PS & - & 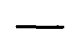 & $3.0 \mathrm{M}_{\mathrm{L}}(\mathrm{PS})$ & & JULY & 22 & 04:39 PST \\
\hline JULY & 22 & 132835.2 & $37.538 \mathrm{~N}$. & $118.565 \mathrm{~W}$. & 6 & PS & - & & $3.1 \mathrm{M}_{\mathrm{L}}(\mathrm{PS})$ & - & JULY & 22 & 05:28 PST \\
\hline JULY & 22 & 133359.8 & $37.517 \mathrm{~N}$. & $118.477 \mathrm{~W}$ & 9 & BK & 4.2 & & $4.7 \mathrm{M}_{\mathrm{L}}(\mathrm{BK})$ & IV & JULY & 22 & 05:33 PST \\
\hline JULY & 22 & 134404.8 & $37.549 \mathrm{~N}$. & 118.493W. & 6 & PS & - & - & $3.1 \mathrm{M}_{\mathrm{L}}(\mathrm{PS})$ & & JULY & 22 & 05:44 PST \\
\hline JULY & 22 & 134900.3 & $37.498 \mathrm{~N}$. & $118.520 \mathrm{~W}$. & 19 & BK & 4.5 & & $5.0 \mathrm{M}_{\mathrm{L}}(\mathrm{BK})$ & & JULY & 22 & 05:49 PST \\
\hline JULY & 22 & 135734.9 & $37.533 \mathrm{~N}$. & $118.483 \mathrm{~W}$. & 9 & BK & & & $3.6 \mathrm{M}_{\mathrm{L}}(\mathrm{BK})$ & & JULY & 22 & 05:57 PST \\
\hline JULY & 22 & 144046.1 & $37.641 \mathrm{~N}$. & $118.478 \mathrm{~W}$. & 6 & PS & - & & $3.1 \mathrm{M}_{\mathrm{L}}(\mathrm{PS})$ & & JULY & 22 & 06:40 PST \\
\hline JULY & 22 & 162839.0 & $37.511 \mathrm{~N}$. & $118.414 \mathrm{~W}$. & 6 & PS & & & 3.1 $\mathrm{M}_{\mathrm{L}}(\mathrm{PS})$ & & JULY & 22 & 08:28 PST \\
\hline JULY & 22 & 163810.9 & $37.623 \mathrm{~N}$. & $118.532 \mathrm{~W}$. & 6 & PS & $\longrightarrow$ & - & $3.1 \mathrm{M}_{\mathrm{L}}(\mathrm{PS})$ & & JULY & 22 & 08:38 PST \\
\hline JULY & 22 & 171201.7 & $37.536 \mathrm{~N}$. & $118.507 \mathrm{~W}$ & 6 & PS & . & 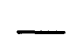 & $3.2 \mathrm{M}_{\mathrm{L}}(\mathrm{PS})$ & & JULY & 22 & 09:12 PST \\
\hline JULY & 22 & 171721.5 & $37.510 \mathrm{~N}$. & 118.440W. & 10 & BK & & & 3.7 $\mathrm{M}_{\mathrm{L}}(\mathrm{BK})$ & & JULY & 22 & 09:17 PST \\
\hline JULY & 22 & 173918.5 & $37.567 \mathrm{~N}$. & $118.485 \mathrm{~W}$. & 9 & BK & & & $3.6 \mathrm{M}_{\mathrm{L}}(\mathrm{BK})$ & & JULY & 22 & 09:39 PST \\
\hline JULY & 22 & 181653.1 & $37.578 \mathrm{~N}$. & $118.483 \mathrm{~W}$. & 8 & BK & & & $3.5 \mathrm{M}_{\mathrm{L}}(\mathrm{BK})$ & & JULY & 22 & 10:16 PST \\
\hline JULY & 22 & 181936.3 & $37.483 \mathrm{~N}$. & $118.377 \mathrm{~W}$ & 7 & BK & & & $4.2 \mathrm{M}_{\mathrm{L}}(\mathrm{BK})$ & & JULY & 22 & 10:19 PST \\
\hline JULY & 22 & 182943.8 & $37.493 \mathrm{~N}$. & 118.382W. & 7 & BK & 3.7 & . & $4.7 \mathrm{M}_{\mathrm{L}}(\mathrm{BK})$ & & JULY & 22 & 10:29 PST \\
\hline JULY & 22 & 190542.1 & $37.574 \mathrm{~N}$. & 118.554W: & 6 & PS & - & & $3.1 \mathrm{M}_{\mathrm{L}}(\mathrm{PS})$ & & JULY & 22 & 11:05 PST \\
\hline JULY & 22 & 201134.2 & $37.636 \mathrm{~N}$. & 118.477W. & 6 & PS & - & & $3.4 \mathrm{M}_{\mathrm{L}}(\mathrm{PS})$ & & JULY & 22 & 12:11 PST \\
\hline JULY & 22 & 201659.6 & $37.607 \mathrm{~N}$. & $118.463 \mathrm{~W}$. & 9 & BK & 3.7 & & $4.2 \mathrm{M}_{\mathrm{L}}(\mathrm{BK})$ & & JULY & 22 & 12:16 PST \\
\hline JULY & 22 & 202226.3 & $37.607 \mathrm{~N}$. & 118.473W. & 8 & BK & $\longrightarrow$ & & $4.3 \mathrm{M}_{\mathrm{L}}(\mathrm{BK})$ & & JULY & 22 & $12: 22$ PST \\
\hline JULY & 22 & 204914.9 & $37.483 \mathrm{~N}$. & 118.378W. & 7 & BK & & & $3.5 \mathrm{M}_{\mathrm{L}}(\mathrm{BK})$ & & JULY & 22 & $12: 49$ PST \\
\hline JULY & 22 & 220641.7 & $37.493 \mathrm{~N}$. & $118.375 \mathrm{~W}$ & 7 & BK & & & $4.2 \mathrm{M}_{\mathrm{L}}(\mathrm{BK})$ & & JULY & 22 & 14:06 PST \\
\hline JULY & 22 & 221552.3 & $37.500 \mathrm{~N}$. & 118.365W. & 6 & PS & - & 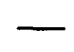 & $3.0 \mathrm{M}_{\mathrm{L}}(\mathrm{PS})$ & & JULY & 22 & $14: 15$ PST \\
\hline JULY & 22 & 223808.5 & $37.450 \mathrm{~N}$. & $118.514 \mathrm{~W}$. & 6 & PS & & - & $3.2 \mathrm{M}_{\mathrm{L}}(\mathrm{PS})$ & & JULY & 22 & 14:38 PST \\
\hline JULY & 22 & 233816.8 & $37.503 \mathrm{~N}$. & $118.362 \mathrm{~W}$ & 6 & PS & & & $3.4 \mathrm{M}_{\mathrm{L}}(\mathrm{PS})$ & & JULY & 22 & $15: 38$ PST \\
\hline JULY & 23 & 011926.8 & $37.493 \mathrm{~N}$. & $118.387 \mathrm{~W}$. & 6 & PS & - & - & $3.0 \mathrm{M}_{\mathrm{L}}(\mathrm{PS})$ & & JULY & 22 & $17: 19$ PST \\
\hline JULY & 23 & 014522.3 & $37.560 \mathrm{~N}$. & $118.458 \mathrm{~W}$. & 6 & PS & & 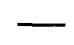 & $3.0 \mathrm{M}_{\mathrm{L}}(\mathrm{PS})$ & & JULY & 22 & 17:45 PST \\
\hline JULY & 23 & 021214.0 & $37.502 \mathrm{~N}$. & $118.482 \mathrm{~W}$. & 17 & BK & - & - & $3.6 \mathrm{M}_{\mathrm{L}}(\mathrm{BK})$ & & JULY & 22 & $18: 12$ PST \\
\hline JULY & 23 & 024121.5 & $37.521 \mathrm{~N}$ & 118.455W. & 6 & PS & - & - & $3.3 \mathrm{M}_{\mathrm{L}}(\mathrm{PS})$ & & JULY & 22 & 18:41 PST \\
\hline JULY & 23 & 025758.2 & $32.998 \mathrm{~N}$. & $117.788 \mathrm{~W}$. & 4 & HJ & - & - & $3.5 \mathrm{M}_{\mathrm{L}}(\mathrm{PS})$ & FELT & JULY & 22 & 18:57 PST \\
\hline
\end{tabular}


Table 1. Summary of United States earthquakes for 1986-Continued

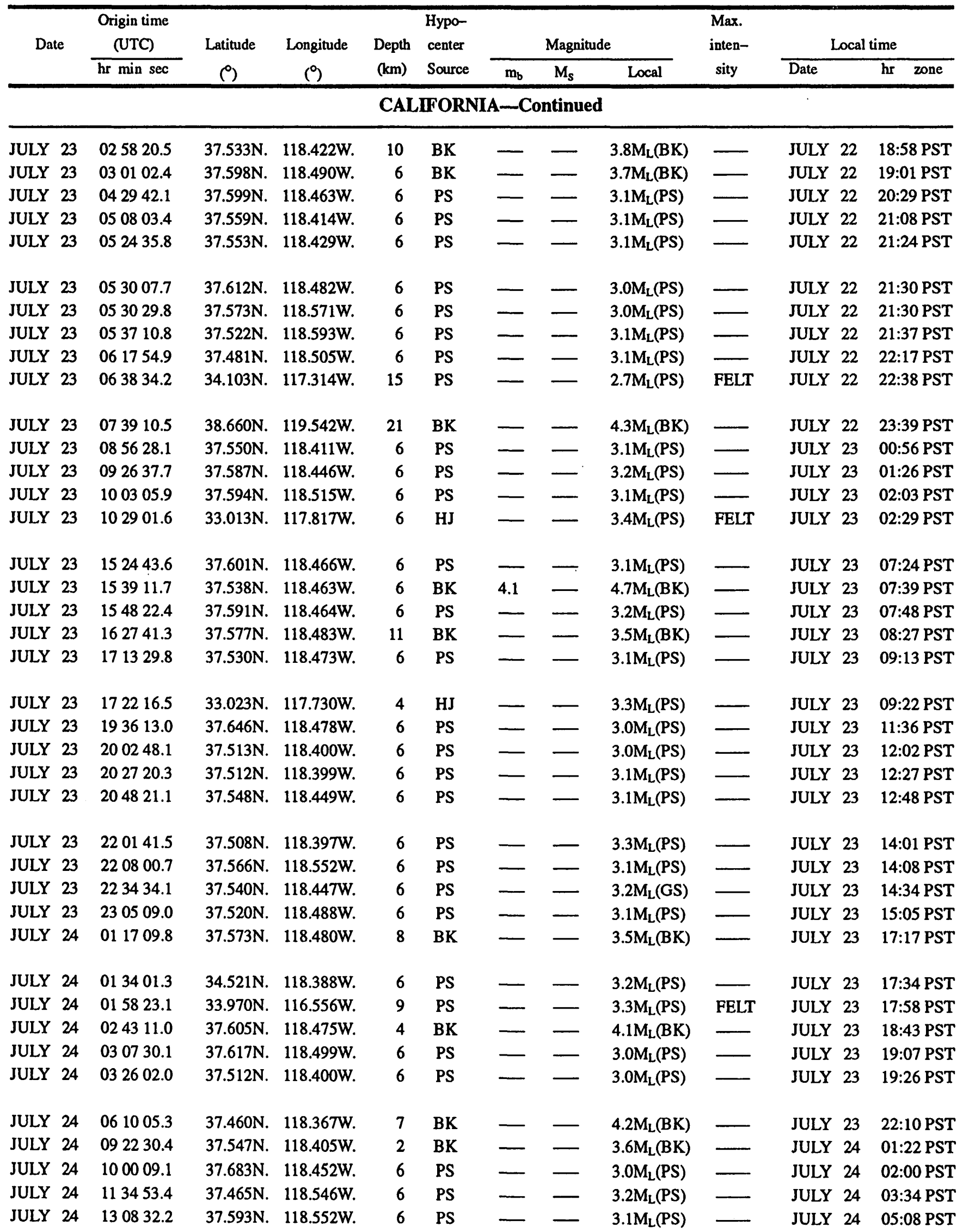


Table 1. Summary of United States earthquakes for 1986 — Continued

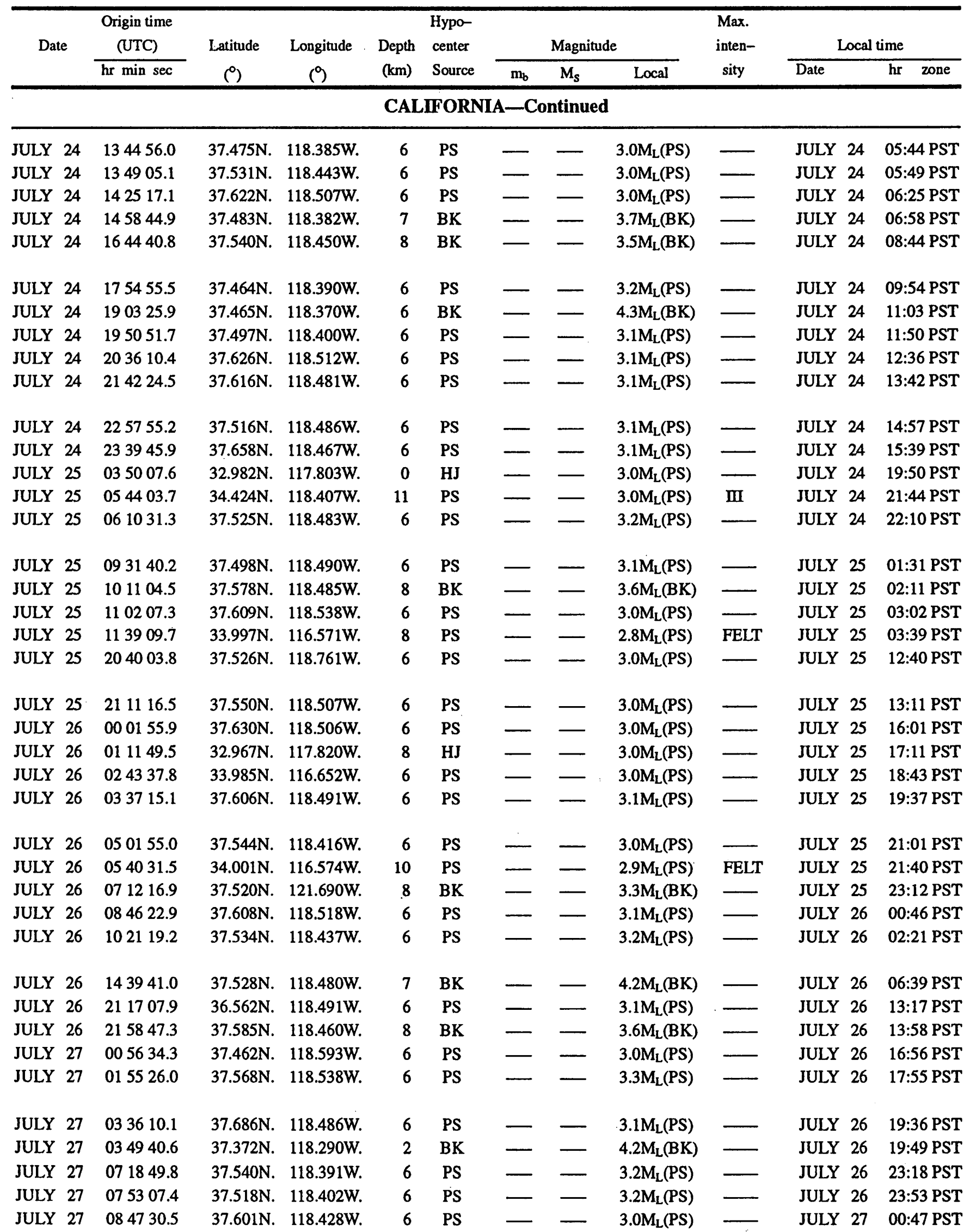


Table 1. Summary of United States earthquakes for 1986-Continued

\begin{tabular}{|c|c|c|c|c|c|c|c|c|c|c|c|c|}
\hline \multirow[t]{2}{*}{ Date } & \multirow{2}{*}{$\begin{array}{l}\text { Origin time } \\
\text { (UTC) } \\
\text { hr min sec }\end{array}$} & \multirow{2}{*}{$\begin{array}{l}\text { Latitude } \\
\text { ()) }\end{array}$} & \multirow{2}{*}{$\begin{array}{l}\text { Longitude } \\
\text { (9) }\end{array}$} & \multirow{2}{*}{$\begin{array}{l}\text { Depth } \\
(\mathrm{km})\end{array}$} & \multirow{2}{*}{$\begin{array}{l}\text { Hypo- } \\
\text { center } \\
\text { Source }\end{array}$} & \multicolumn{3}{|c|}{ Magnitude } & \multirow{2}{*}{$\begin{array}{l}\text { Max. } \\
\text { inten- } \\
\text { sity }\end{array}$} & \multicolumn{3}{|c|}{ Local time } \\
\hline & & & & & & $\mathrm{m}_{\mathrm{b}}$ & $\mathbf{M}_{\mathbf{s}}$ & Local & & Date & & zone \\
\hline \multicolumn{13}{|c|}{ CALIFORNIA-Continued } \\
\hline JULY 27 & 084851.4 & $37.671 \mathrm{~N}$ & $118.515 \mathrm{~W}$ & 6 & PS & - & - & $3.3 \mathrm{M}_{\mathrm{L}}(\mathrm{PS})$ & - & JULY & 27 & 00:48 PST \\
\hline JULY 27 & 100802.2 & $37.505 \mathrm{~N}$ & $118.398 \mathrm{~W}$. & 7 & BK & - & - & $3.9 \mathrm{M}_{\mathrm{L}}(\mathrm{BK})$ & $\longrightarrow$ & JULY & 27 & 02:08 PST \\
\hline JULY 28 & 022602.0 & $37.513 \mathrm{~N}$ & $118.461 \mathrm{~W}$ & 6 & PS & - & - & $3.0 \mathrm{M}_{\mathrm{L}}(\mathrm{PS})$ & & JULY & 27 & $18: 26$ PST \\
\hline JULY 28 & 025445.7 & $32.953 \mathrm{~N}$ & $117.812 \mathrm{~W}$ & 5 & HJ & - & - & $3.6 \mathrm{M}_{\mathrm{L}}(\mathrm{PS})$ & FELT & JULY & 27 & 18:54 PST \\
\hline JULY 28 & 080331.3 & $37.622 \mathrm{~N}$ & $118.605 \mathrm{~W}$. & 6 & PS & - & - & $3.0 \mathrm{M}_{\mathrm{L}}(\mathrm{PS})$ & & JULY & 28 & 00:03 PST \\
\hline JULY 28 & 103309.2 & $32.972 \mathrm{~N}$ & $117.812 \mathrm{~W}$ & 4 & HJ & - & - & $3.2 \mathrm{M}_{\mathrm{x}}(\mathrm{PS})$ & - & JULY & 28 & 02:33 PST \\
\hline JULY 28 & 104842.6 & $37.597 \mathrm{~N}$ & $118.419 \mathrm{~W}$ & 6 & PS & - & - & $3.2 \mathrm{M}_{\mathrm{L}}(\mathrm{PS})$ & & JULY & 28 & 02:48 PST \\
\hline JULY 28 & 130012.9 & $36.169 \mathrm{~N}$ & $120.204 \mathrm{~W}$ & 6 & PS & - & - & $3.0 \mathrm{M}_{\mathrm{L}}(\mathrm{PS})$ & & JULY & 28 & 05:00 PST \\
\hline JULY 28 & 205042.8 & $37.501 \mathrm{~N}$ & 118.396W. & 6 & PS & - & - & $3.1 \mathrm{M}_{\mathrm{L}}(\mathrm{PS})$ & 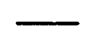 & JULY & 28 & 12:50 PST \\
\hline JULY 28 & 211458.0 & $37.452 \mathrm{~N}$ & $118.384 W$ & 6 & PS & - & - & $3.2 \mathrm{M}_{\mathrm{L}}(\mathrm{PS})$ & 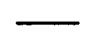 & JULY & 28 & 13:14 PST \\
\hline JULY 29 & 064350.3 & $33.965 \mathrm{~N}$ & $116.590 \mathrm{~W}$. & 7 & PS & - & - & $3.2 \mathrm{M}_{\mathrm{L}}(\mathrm{PS})$ & FELT & JULY & 28 & 22:43 PST \\
\hline JULY 29 & 071158.8 & $37.543 \mathrm{~N}$ & $118.445 \mathrm{~W}$ & 8 & BK & 一 & - & $4.2 \mathrm{M}_{\mathrm{L}}(\mathrm{BK})$ & FELT & JULY & 28 & 23:11 PST \\
\hline JULY 29 & 081741.4 & $32.945 \mathrm{~N}$ & $117.828 \mathrm{~W}$ & 6 & $\mathrm{HJ}$ & 3.9 & - & $4.3 \mathrm{M}_{\mathrm{L}}(\mathrm{PS})$ & V & JULY & 29 & 00:17 PST \\
\hline JULY 29 & 095757.2 & $37.595 \mathrm{~N}$ & 118.477W. & 7 & BK & 3.7 & - & $4.6 \mathrm{M}_{\mathrm{L}}(\mathrm{BK})$ & IV & JULY & 29 & 01:57 PST \\
\hline JULY 29 & 112222.4 & $32.957 \mathrm{~N}$ & $117.815 \mathrm{~W}$ & 5 & HJ & - & - & $3.5 \mathrm{M}_{\mathrm{L}}(\mathrm{PS})$ & FELT & JULY & 29 & 03:22 PST \\
\hline JULY 29 & 120319.5 & $33.966 \mathrm{~N}$ & $116.568 \mathrm{~W}$ & 7 & PS & - & - & $3.0 \mathrm{M}_{\mathrm{L}}(\mathrm{PS})$ & FELT & JULY & 29 & 04:03 PST \\
\hline JULY 29 & 133205.9 & $32.970 \mathrm{~N}$ & $117.810 \mathrm{~W}$ & 10 & PS & - & - & $3.2 \mathrm{M}_{\mathrm{L}}(\mathrm{PS})$ & - & JULY & 29 & 05:32 PST \\
\hline JULY 29 & 151425.4 & $37.452 \mathrm{~N}$ & 118.375W. & 8 & BK & - & - & $4.2 \mathrm{M}_{\mathrm{L}}(\mathrm{BK})$ & - & JULY & 29 & 07:14 PST \\
\hline JULY 30 & 002629.7 & $32.963 \mathrm{~N}$ & $117.807 \mathrm{~W}$ & 2 & HJ & - & - & $3.4 \mathrm{M}_{\mathrm{L}}(\mathrm{PS})$ & - & JULY & 29 & 16:26 PST \\
\hline JULY 30 & 004650.7 & $37.528 \mathrm{~N}$ & 118.515W. & 11 & BK & - & - & $3.6 \mathrm{M}_{\mathrm{L}}(\mathrm{BK})$ & - & JULY & 29 & 16:46 PST \\
\hline JULY 30 & 011401.1 & $34.000 \mathrm{~N}$ & $118.378 \mathrm{~W}$ & 4 & PS & - & - & $2.8 \mathrm{M}_{\mathrm{L}}(\mathrm{PS})$ & FELT & JULY & 29 & 17:14 PST \\
\hline JULY 30 & 060332.2 & $37.605 \mathrm{~N}$ & $118.480 \mathrm{~W}$ & 8 & BK & - & - & $4.0 \mathrm{M}_{\mathrm{L}}(\mathrm{BK})$ & - & JULY & 29 & 22:03 PST \\
\hline JULY 30 & 064152.9 & $37.582 \mathrm{~N}$ & $118.468 \mathrm{~W}$ & 7 & BK & 4.1 & - & $4.8 \mathrm{M}_{\mathrm{L}}(\mathrm{BK})$ & IV & JULY & 29 & 22:41 PST \\
\hline JULY 30 & 172907.5 & $37.582 \mathrm{~N}$ & $118.487 \mathrm{~W}$ & 6 & PS & - & - & $3.0 \mathrm{M}_{\mathrm{L}}(\mathrm{PS})$ & - & JULY & 30 & 09:29 PST \\
\hline JULY 30 & 225113.0 & $33.012 \mathrm{~N}$ & $117.782 \mathrm{~W}$ & 7 & HJ & - & - & $3.9 \mathrm{M}_{\mathrm{L}}(\mathrm{PS})$ & FELT & JULY & 30 & 14:51 PST \\
\hline JULY 31 & 003224.7 & $37.590 \mathrm{~N}$ & $118.490 \mathrm{~W}$ & 6 & PS & - & - & $3.2 \mathrm{M}_{\mathrm{L}}(\mathrm{PS})$ & - & JULY & 30 & 16:32 PST \\
\hline JULY 31 & 035308.9 & $37.594 \mathrm{~N}$ & $118.488 \mathrm{~W}$ & 6 & PS & - & - & $3.0 \mathrm{M}_{\mathrm{L}}(\mathrm{PS})$ & - & JULY & 30 & 19:53 PST \\
\hline JULY 31 & 045014.3 & $34.098 \mathrm{~N}$ & $116.631 \mathrm{~W}$ & 10 & PS & - & - & $3.1 \mathrm{M}_{\mathrm{L}}(\mathrm{PS})$ & - & JULY & 30 & 20:50 PST \\
\hline JULY 31 & $07 \cdot 2240.2$ & $37.463 \mathrm{~N}$ & $118.367 \mathrm{~W}$ & 7 & BK & 5.5 & 5.2 & $5.8 \mathrm{M}_{\mathrm{L}}(\mathrm{BK})$ & VI & JULY & 30 & 23:22 PST \\
\hline JULY 31 & 072804.7 & $37.445 \mathrm{~N}$ & $118.377 \mathrm{~W}$ & 11 & BK & - & - & $4.5 \mathrm{M}_{\mathrm{L}}(\mathrm{BK})$ & 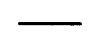 & JULY & 30 & 23:28 PST \\
\hline JULY 31 & 073418.8 & 37.494N. & $118.400 \mathrm{~W}$ & 6 & PS & - & - & $3.4 \mathrm{M}_{\mathrm{L}}(\mathrm{PS})$ & $\longrightarrow$ & JULY & 30 & 23:34 PST \\
\hline JULY 31 & 073602.4 & $37.470 \mathrm{~N}$ & 118.375W. & 8 & BK & - & - & $4.0 \mathrm{M}_{\mathrm{L}}(\mathrm{BK})$ & - & JULY & 30 & 23:36 PST \\
\hline JULY 31 & 074148.7 & $37.455 \mathrm{~N}$ & 118.370W. & 6 & PS & - & - & $3.0 \mathrm{M}_{\mathrm{L}}(\mathrm{PS})$ & 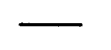 & JULY & 30 & 23:41 PST \\
\hline JULY 31 & 075046.2 & $37.488 \mathrm{~N}$ & 118.372W. & 6 & BK & - & - & $3.8 \mathrm{M}_{\mathrm{L}}(\mathrm{BK})$ & $\longrightarrow$ & JULY & 30 & 23:50 PST \\
\hline JULY 31 & 075142.9 & $33.966 \mathrm{~N}$ & $116.574 \mathrm{~W}$ & 7 & PS & - & - & $3.3 \mathrm{M}_{\mathrm{L}}(\mathrm{PS})$ & FELT & JULY & 30 & 23:51 PST \\
\hline JULY 31 & 075352.8 & $37.460 \mathrm{~N}$. & 118.399W. & 6 & PS & - & - & $3.2 \mathrm{M}_{\mathrm{L}}(\mathrm{PS})$ & & JULY & 30 & 23:53 PST \\
\hline JULY 31 & 075818.6 & $37.504 \mathrm{~N}$ & $118.404 \mathrm{~W}$ & 6 & PS & - & - & $3.0 \mathrm{M}_{\mathrm{L}}(\mathrm{PS})$ & & JULY & 30 & 23:58 PST \\
\hline JULY 31 & 081042.8 & $37.433 \mathrm{~N}$ & $118.355 \mathrm{~W}$ & 9 & BK & - & - & $3.9 \mathrm{M}_{\mathrm{L}}(\mathrm{BK})$ & - & JULY & 31 & 00:10 PST \\
\hline JULY 31 & 081539.3 & $37.478 \mathrm{~N}$ & 118.373W. & 7 & BK & - & - & $4.0 \mathrm{M}_{\mathrm{L}}(\mathrm{BK})$ & $\longrightarrow$ & JULY & 31 & 00:15 PST \\
\hline JULY 31 & 112550.6 & $37.595 \mathrm{~N}$. & 118.456W. & 6 & PS & - & - & $3.2 \mathrm{M}_{\mathrm{L}}(\mathrm{PS})$ & & JULY & 31 & 03:25 PST \\
\hline
\end{tabular}


Table 1. Summary of United States earthquakes for 1986-Continued

\begin{tabular}{|c|c|c|c|c|c|c|c|c|c|c|c|c|}
\hline \multirow[t]{2}{*}{ Date } & \multirow{2}{*}{\multicolumn{2}{|c|}{$\begin{array}{l}\text { Origin time } \\
\text { (UTC) } \\
\text { hr min sec }\end{array}$}} & \multirow{2}{*}{$\begin{array}{l}\text { Longitude } \\
\left.\text { ( }{ }^{\circ}\right)\end{array}$} & \multirow{2}{*}{$\begin{array}{c}\text { Depth } \\
(\mathrm{km})\end{array}$} & \multirow{2}{*}{$\begin{array}{l}\text { Hypo- } \\
\text { center } \\
\text { Source }\end{array}$} & \multicolumn{3}{|c|}{ Magnitude } & \multirow{2}{*}{$\begin{array}{l}\text { Max. } \\
\text { inten- } \\
\text { sity }\end{array}$} & \multicolumn{3}{|c|}{ Local time } \\
\hline & & & & & & $\mathbf{m}_{\mathrm{b}}$ & $\mathbf{M}_{\mathbf{s}}$ & Local & & Date & $\mathrm{hr}$ & zone \\
\hline
\end{tabular}

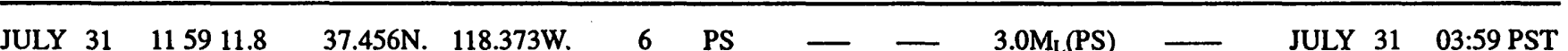

JULY $31 \quad 151925.3$

JULY $31 \quad 181339.7$

JULY $31 \quad 191645.9$

JULY $31 \quad 220353.4$

$40.325 \mathrm{~N} .124 .742 \mathrm{~W}$.

$32.962 \mathrm{~N} .117 .810 \mathrm{~W}$.

$33.995 \mathrm{~N} .118 .368 \mathrm{~W}$.

$37.560 \mathrm{~N}$. $118.478 \mathrm{~W}$.

AUG. $1 \quad 050441.7$

AUG. 1060955.8

AUG. 1063443.3

AUG. 1142716.4

AUG. $1 \quad 142819.6$

$37.539 \mathrm{~N} .118 .405 \mathrm{~W}$.

$37.645 \mathrm{~N}$. $118.536 \mathrm{~W}$.

$37.555 \mathrm{~N} .118 .458 \mathrm{~W}$.

$37.501 \mathrm{~N}$. $118.398 \mathrm{~W}$.

$37.468 \mathrm{~N}$. $118.448 \mathrm{~W}$.

10

PS
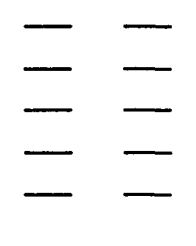

$3.0 \mathrm{M}_{\mathrm{L}}(\mathrm{PS})$

3.4M $\mathrm{M}_{\mathrm{L}}(\mathrm{BK})$

$3.0 \mathrm{M}_{\mathrm{L}}(\mathrm{PS})$

$2.8 \mathrm{M}_{\mathrm{L}}$ (PS)

$3.1 \mathrm{M}_{\mathrm{L}}$ (PS)

$\begin{array}{ll}- & - \\ 4.2 & - \\ 4.9 & -\end{array}$

$3.0 \mathrm{M}_{\mathrm{L}}$ (GS)

$3.0 \mathrm{M}_{\mathrm{L}}$ (PS)

$3.9 \mathrm{M}_{\mathrm{L}}(\mathrm{BK})$

4.8 $\mathrm{M}_{\mathrm{L}}(\mathrm{BK})$

$5.1 \mathrm{M}_{\mathrm{L}}(\mathrm{BK})$

$-$

FELT

-

AUG. 1151044.2

AUG. 2050502.1

AUG. $2 \quad 113657.8$

$37.505 \mathrm{~N} .118 .392 \mathrm{~W}$.

$34.025 \mathrm{~N} .116 .677 \mathrm{~W}$.

$34.036 \mathrm{~N}$. $116.696 \mathrm{~W}$.

$37.622 \mathrm{~N} .118 .503 \mathrm{~W}$.

AUG. $2 \quad 123126.2$

$37.526 \mathrm{~N}$. $118.455 \mathrm{~W}$.

AUG. $2 \quad 145137.0$

$37.615 \mathrm{~N}$. 118.433W.

$37.551 \mathrm{~N}$. 118.423W.

$37.622 \mathrm{~N}$. $118.466 \mathrm{~W}$.

AUG. 3

013732.

073931.4

AUG. $3 \quad 090014.2$

$36.585 \mathrm{~N} .121 .260 \mathrm{~W}$.

AUG. $3 \quad 103305.3$

$37.615 \mathrm{~N}$. 118.455W.

AUG. $3 \quad 103721.1$

$37.437 \mathrm{~N}$. $119.530 \mathrm{~W}$.

$37.506 \mathrm{~N} .118 .398 \mathrm{~W}$.

$40.430 \mathrm{~N}$. $125.365 \mathrm{~W}$.

$37.432 \mathrm{~N} .121 .773 \mathrm{~W}$.

$37.548 \mathrm{~N}$. $118.447 \mathrm{~W}$.

AUG. 4034141.

123108.1

AUG. $5 \quad 010041.8$

$37.421 \mathrm{~N} .118 .335 \mathrm{~W}$.

AUG. $5 \quad 023208.1$

$37.428 \mathrm{~N}$. $118.348 \mathrm{~W}$.

$37.487 \mathrm{~N}$. $118.389 \mathrm{~W}$.

$32.912 \mathrm{~N} .117 .840 \mathrm{~W}$.

$37.533 \mathrm{~N}$. $118.436 \mathrm{~W}$.

AUG. $5 \quad 135035.3$

AUG. $\quad 6 \quad 090939.7$

$37.604 \mathrm{~N} .118 .435 \mathrm{~W}$.

AUG. $7 \quad 055140.6$

AUG. 7200432.0

$37.596 \mathrm{~N} . \quad 118.441 \mathrm{~W}$.

$37.542 \mathrm{~N} .118 .437 \mathrm{~W}$.

$37.584 \mathrm{~N}$. $118.427 \mathrm{~W}$.

$40.862 \mathrm{~N}$. $123.725 \mathrm{~W}$.

$32.545 \mathrm{~N} .117 .338 \mathrm{~W}$.

$32.500 \mathrm{~N}$. $117.398 \mathrm{~W}$.

$37.560 \mathrm{~N}$. $118.403 \mathrm{~W}$.

$32.536 \mathrm{~N}$. $117.346 \mathrm{~W}$.

$32.553 \mathrm{~N}$. $117.332 \mathrm{~W}$.
$3.8 \mathrm{M}_{\mathrm{L}}(\mathrm{BK})$

$3.1 \mathrm{M}_{\mathrm{L}}$ (PS)

$3.4 \mathrm{M}_{\mathrm{L}}$ (PS)

3.1 $\mathrm{M}_{\mathrm{L}}(\mathrm{PS})$

$3.0 \mathrm{M}_{\mathrm{L}}$ (PS)

$3.6 \mathrm{M}_{\mathrm{L}}(\mathrm{BK})$

$3.2 \mathrm{M}_{\mathrm{L}}(\mathrm{PS})$

$3.2 \mathrm{M}_{\mathrm{L}}$ (PS)

3.4 $\mathrm{M}_{\mathrm{L}}(\mathrm{BK})$

$4.3 \mathrm{M}_{\mathrm{L}}(\mathrm{BK})$

3.5 $\mathrm{M}_{\mathrm{L}}(\mathrm{PS})$

$3.1 \mathrm{M}_{\mathrm{L}}$ (PS)

4.3M $\mathrm{M}_{\mathrm{L}}(\mathrm{BK})$

3.4M $\mathrm{M}_{\mathrm{L}}(\mathrm{BK})$

$3.2 \mathrm{M}_{\mathrm{L}}(\mathrm{PS})$

$3.1 \mathrm{M}_{\mathrm{L}}(\mathrm{PS})$

3.7 $\mathrm{M}_{\mathrm{L}}$ (BK)

$3.0 \mathrm{M}_{\mathrm{L}}$ (PS)

$3.3 \mathrm{M}_{\mathrm{L}}$ (PS)

$3.0 \mathrm{M}_{\mathrm{L}}(\mathrm{PS})$

3.1 $\mathrm{M}_{\mathrm{L}}$ (PS)

$3.1 \mathrm{M}_{\mathrm{L}}$ (PS)

$3.0 \mathrm{M}_{\mathrm{L}}$ (PS)

$3.0 \mathrm{M}_{\mathrm{L}}$ (PS)

$3.8 \mathrm{M}_{\mathrm{L}}(\mathrm{BK})$

3.0M $(\mathrm{PS})$

$3.4 \mathrm{M}_{\mathrm{L}}(\mathrm{PS})$

$3.0 \mathrm{M}_{\mathrm{L}}(\mathrm{PS})$

$3.1 \mathrm{M}_{\mathrm{L}}(\mathrm{PS})$

$3.1 \mathrm{M}_{\mathrm{L}}(\mathrm{PS})$
JULY $31 \quad$ 07:19 PST

JULY $31 \quad$ 10:13 PST

JULY $31 \quad 11: 16$ PST

JULY $31 \quad$ 14:03 PST

JULY $31 \quad 21: 04$ PST JULY $31 \quad 22: 09$ PST JULY $31 \quad 22: 34$ PST AUG. 1 06:27 PST AUG. 1 06:28 PST

AUG. 1 07:10 PST AUG. 1 21:05 PST AUG. 2 03:36 PST AUG. 2 04:31 PST AUG. 2 06:08 PST

AUG. 2 06:51 PST AUG. 2 17:37 PST AUG. 2 23:39 PST AUG. 3 01:00 PST AUG. 3 02:33 PST

AUG. $3 \quad 02: 37$ PST AUG. 3 04:05 PST AUG. 3 08:15 PST AUG. $3 \quad$ 19:41 PST AUG. 4 04:31 PST

AUG. 4 17:00 PST AUG. 4 18:32 PST AUG. 4 18:35 PST AUG. 5 00:35 PST AUG. 5 05:50 PST

AUG. 6 01:09 PST AUG. $6 \quad 21: 51$ PST AUG. 7 12:04 PST AUG. 8 06:30 PST AUG. 8 09:31 PST

AUG. 8 12:51 PST AUG. 8 16:52 PST AUG. 9 05:20 PST AUG. $10 \quad 16: 19$ PST AUG. $10 \quad 16: 26$ PST 
Table 1. Summary of United States earthquakes for 1986 -Continued

\begin{tabular}{|c|c|c|c|c|c|c|c|c|c|c|c|c|}
\hline \multirow{3}{*}{ Date } & \multirow{3}{*}{$\begin{array}{l}\text { Origin time } \\
\text { (UTC) } \\
\text { hr min sec }\end{array}$} & \multirow{3}{*}{$\begin{array}{l}\text { Latitude } \\
\text { ()) }\end{array}$} & \multirow{3}{*}{$\begin{array}{c}\text { Longitude } \\
\left(^{\circ}\right)\end{array}$} & \multirow{3}{*}{$\begin{array}{l}\text { Depth } \\
\text { (km) }\end{array}$} & \multirow{3}{*}{$\begin{array}{l}\text { Hypo- } \\
\text { center } \\
\text { Source }\end{array}$} & \multirow{2}{*}{\multicolumn{3}{|c|}{ Magnitude }} & \multirow{3}{*}{$\begin{array}{l}\text { Max. } \\
\text { inten- } \\
\text { sity }\end{array}$} & \multirow{2}{*}{\multicolumn{3}{|c|}{ Local time }} \\
\hline & & & & & & & & & & & & \\
\hline & & & & & & $\mathrm{m}_{\mathrm{b}}$ & $\mathrm{M}_{\mathrm{s}}$ & Local & & Date & $\mathrm{hr}$ & zone \\
\hline
\end{tabular}

AUG. $11 \quad 042648.2$

AUG. $12 \quad 092948.3$

AUG. $12 \quad 153728.1$

AUG. $13 \quad 023736.6$

AUG. $13 \quad 173223.2$

AUG. $14 \quad 005040.8$

AUG. $14 \quad 043122.8$

AUG. $14 \quad 075338.9$

AUG. $14 \quad 080146.2$

AUG. $14 \quad 083602.8$

AUG. $15 \quad 130946.9$

AUG. $15 \quad 184516.1$

AUG. 16

094856.2

AUG. 16

193143.7

AUG. 16

211917.8

AUG. $17 \quad 133536.6$

AUG. 18

AUG. 19

AUG. 19

AUG. 20

AUG. 20

AUG. 20

AUG. 20

AUG. 20

AUG. 20

AUG. 20

AUG. 21

AUG. 21

AUG. 23

AUG. 23

104939.0

203846.9

235340.0

034946.3

070216.8

095021.0

095705.0

144655.7

144929.2

151655.4

010429.3

112832.1

030130.3

144649.6

AUG. $23 \quad 1652013$

AUG. 24

124809.2

AUG. 24

164609.5

AUG. $24 \quad 172943.2$

AUG. $24 \quad 205353.4$

AUG. $25 \quad 081328.4$

AUG. $25 \quad 082058.7$

AUG. $25 \quad 114224.5$
$37.502 \mathrm{~N} .118 .392 \mathrm{~W}$.

$37.492 \mathrm{~N}$. $118.382 \mathrm{~W}$.

$37.502 \mathrm{~N} .118 .487 \mathrm{~W}$.

$37.203 \mathrm{~N}$. 118.378W.

$37.590 \mathrm{~N}$. $118.445 \mathrm{~W}$.

$40.377 \mathrm{~N}$. $124.328 \mathrm{~W}$.

$37.511 \mathrm{~N}$. $118.389 \mathrm{~W}$

$37.491 \mathrm{~N} . \quad 118.413 \mathrm{~W}$

$33.003 \mathrm{~N} . \quad 117.799 \mathrm{~W}$

$37.501 \mathrm{~N}$. $118.432 \mathrm{~W}$.

$33.012 \mathrm{~N} .117 .782 \mathrm{~W}$.

$32.948 \mathrm{~N}$. $117.797 \mathrm{~W}$

$37.503 \mathrm{~N}$. $118.342 \mathrm{~W}$.

$37.552 \mathrm{~N} . \quad 118.408 \mathrm{~W}$.

$33.572 \mathrm{~N} .118 .954 \mathrm{~W}$.

$37.552 \mathrm{~N} .118 .455 \mathrm{~W}$

$37.540 \mathrm{~N}$. $118.453 \mathrm{~W}$

$37.460 \mathrm{~N}$. $118.364 \mathrm{~W}$

$37.489 \mathrm{~N} . \quad 118.397 \mathrm{~W}$.

$37.243 \mathrm{~N} .118 .192 \mathrm{~W}$.

$37.122 \mathrm{~N} . \quad 121.563 \mathrm{~W}$.

$37.120 \mathrm{~N}$. $121.562 \mathrm{~W}$

$37.118 \mathrm{~N}$. $121.565 \mathrm{~W}$

$32.950 \mathrm{~N}$. 117.834W

$32.971 N .117 .788 \mathrm{~W}$.

$32.936 \mathrm{~N} . \quad 117.842 \mathrm{~W}$

$37.527 \mathrm{~N} .121 .685 \mathrm{~W}$

$32.922 \mathrm{~N} .117 .749 \mathrm{~W}$

37.493N. $118.383 \mathrm{~W}$

$37.457 \mathrm{~N} .118 .410 \mathrm{~W}$

32.944N. $117.847 \mathrm{~W} . \quad 10$ PS

$32.979 \mathrm{~N} \quad 115.879 \mathrm{~W}-13$ PS

$32.926 \mathrm{~N}$ 117.751W. 10 PS

$37.580 \mathrm{~N}, 118.413 \mathrm{~W}$

$37.616 \mathrm{~N}$

$37.624 \mathrm{~N}$. 118.430W.

$37.618 \mathrm{~N}$. $118.430 \mathrm{~W}$.

$32.971 \mathrm{~N} .117 .784 \mathrm{~W}$.

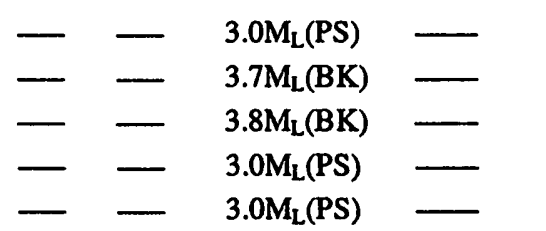

AUG. $10 \quad 20: 26$ PST

AUG. 12 01:29 PST

AUG. 12 07:37 PST

AUG. $12 \quad 18: 37$ PST

AUG. $13 \quad 09: 32$ PST

AUG. 13 16:50 PST

AUG. 13 20:31 PST

AUG. 13 23:53 PST

AUG. 14 00:01 PST

AUG. $1400: 36$ PST

AUG. 15 05:09 PST

AUG. 15 10:45 PST

AUG. 16 01:48 PST

AUG. 16 11:31 PST

AUG. 16 13:19 PST

AUG. $17 \quad 05: 35$ PST

AUG. 18 02:49 PST

AUG. $19 \quad 12: 38$ PST

AUG. 19 15:53 PST

AUG. 19 19:49 PST

AUG. 19 23:02 PST

AUG. 20 01:50 PST

AUG. 20 01:57 PST

AUG. 20 06:46 PST

AUG. 20 06:49 PST

AUG. $20 \quad 07: 16$ PST

AUG. 20 17:04 PST

AUG. $21 \quad 03: 28$ PST

AUG. 22 19:01 PST

AUG. 23 06:46 PST

AUG. 23 08:52 PST

AUG. $24 \quad 04: 48$ PST

AUG. 24 08:46 PST

AUG. $24 \quad 09: 29$ PST

AUG. $24 \quad 12: 53$ PST

AUG. $25 \quad 00: 13$ PST

AUG. $25 \quad 00: 20$ PST

AUG. 25 03:42 PST 
Table 1. Summary of United States earthquakes for 1986 - Continued

\begin{tabular}{|c|c|c|c|c|c|c|c|c|c|c|c|c|}
\hline \multirow[t]{2}{*}{ Date } & \multirow{2}{*}{\multicolumn{2}{|c|}{ 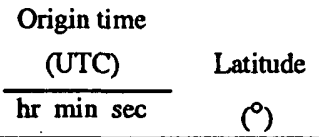 }} & \multirow{2}{*}{$\begin{array}{l}\text { Longitude } \\
\text { (9) }\end{array}$} & \multirow{2}{*}{$\begin{array}{l}\text { Depth } \\
(\mathrm{km})\end{array}$} & \multirow{2}{*}{$\begin{array}{l}\text { Hypo- } \\
\text { center } \\
\text { Source }\end{array}$} & \multicolumn{3}{|c|}{ Magnitude } & \multirow{2}{*}{$\begin{array}{l}\text { Max. } \\
\text { inten- } \\
\text { sity }\end{array}$} & \multicolumn{3}{|c|}{ Local time } \\
\hline & & & & & & $\mathbf{m}_{\mathbf{b}}$. & $\mathrm{M}_{\mathrm{S}}$ & Local & & Date & hr & zone \\
\hline
\end{tabular}

AUG. $25 \quad 114751.2 \quad 37.747 \mathrm{~N}, 121.968 \mathrm{~W} \quad 450$

AUG. $25 \quad 114751.2 \quad 37.747 \mathrm{~N} .121 .968 \mathrm{~W}$. AUG. $26 \quad 122444.140 .278 \mathrm{~N}$. $127.247 \mathrm{~W}$.

AUG. $26 \quad 190721.1$ AUG. $26 \quad 191149.9$

AUG. $26 \quad 214826.7$

AUG. $27 \quad 061035.3$

AUG. $27 \quad 092040.8$

AUG. $28 \quad 101416.3$

AUG. $28 \quad 163214.6$

AUG. 29064442.5

AUG. 29074654.5

AUG. 29230309.9

AUG. 30

AUG. 31

205823.2

043619.6

AUG. 31

AUG. 31

175707.0

232933.9

SEPT. $1 \quad 115552.4$

SEPT. $1 \quad 194303.9$

SEPT. $2 \quad 004949.1$

SEPT. $2 \quad 135956.1$

SEPT. $2 \quad 212403.9$

SEPT. $3 \quad 043114.8$

SEPT. $3 \quad 123516.2$

SEPT. $4 \quad 004236.6$

SEPT. $4 \quad 013145.2$

SEPT. $4 \quad 102916.6$

SEPT. $4 \quad 215446.2$

SEPT. $5 \quad 181341.4$ SEPT. $6 \quad 013059.7$

SEPT. $8 \quad 033741.3$

SEPT. $8 \quad 110729.3$

SEPT. $8 \quad 162244.8$

SEPT. $9 \quad 073008.3$

SEPT. 9132940.4

SEPT. 9162250.6

SEPT. 9222939.4

SEPT. $10 \quad 155152.4$

SEPT. $11 \quad 132626.8 \quad 37.577 \mathrm{~N} .118 .476 \mathrm{~W}$. SEPT. $12233811.2 \quad 36.243 \mathrm{~N} . \quad 120.485 \mathrm{~W}$.

$37.525 \mathrm{~N} .118 .442 \mathrm{~W}$.

$32.935 \mathrm{~N} .117 .825 \mathrm{~W}$.

$32.941 \mathrm{~N} .117 .847 \mathrm{~W}$.

$37.572 \mathrm{~N} .118 .490 \mathrm{~W}$.

$37.610 \mathrm{~N}$. $118.467 \mathrm{~W}$.

$32.491 \mathrm{~N} .117 .420 \mathrm{~W}$.

$33.919 \mathrm{~N} .116 .273 \mathrm{~W}$.

$35.888 \mathrm{~N}$. $120.475 \mathrm{~W}$.

$33.956 \mathrm{~N} .116 .599 \mathrm{~W}$.

$40.607 \mathrm{~N} .124 .782 \mathrm{~W}$.

$34.212 \mathrm{~N} . \quad 119.525 \mathrm{~W}$.

$37.401 \mathrm{~N}$. $118.426 \mathrm{~W}$.

$37.538 \mathrm{~N} .118 .871 \mathrm{~W}$.

$41.832 \mathrm{~N} . \quad 126.501 \mathrm{~W}$.

$37.442 \mathrm{~N}$. $118.358 \mathrm{~W}$.

$35.801 \mathrm{~N} .120 .375 \mathrm{~W}$.

$37.611 \mathrm{~N}$. $118.463 \mathrm{~W}$.

$41.128 \mathrm{~N} .123 .755 \mathrm{~W}$.

$37.511 \mathrm{~N}$. $118.671 \mathrm{~W}$.

$37.295 \mathrm{~N}$. $121.667 \mathrm{~W}$.

$37.539 \mathrm{~N} .118 .404 \mathrm{~W}$.

$37.539 \mathrm{~N} .118 .442 \mathrm{~W}$.

$37.510 \mathrm{~N}$. $118.427 \mathrm{~W}$.

$37.522 \mathrm{~N} .118 .420 \mathrm{~W}$.

$37.574 \mathrm{~N}$. $118.876 \mathrm{~W}$.

$38.828 \mathrm{~N} . \quad 122.802 \mathrm{~W}$.

$37.613 \mathrm{~N} .118 .465 \mathrm{~W}$.

$40.407 \mathrm{~N} . \quad 124.380 \mathrm{~W}$.

$32.943 \mathrm{~N} . \quad 117.765 \mathrm{~W}$.

$36.838 \mathrm{~N}$. $121.395 \mathrm{~W}$.

$37.622 \mathrm{~N} .118 .436 \mathrm{~W}$. $37.457 \mathrm{~N}$. $118.458 \mathrm{~W}$.

$33.966 \mathrm{~N} .116 .567 \mathrm{~W}$.

$37.465 \mathrm{~N}$. $118.581 \mathrm{~W}$.

$33.958 \mathrm{~N}$. $116.669 \mathrm{~W}$.

$\begin{array}{ll}4 & \text { BK } \\ 5 & \text { BK }\end{array}$

$-$

$-$

2.6 $\mathrm{M}_{\mathrm{L}}(\mathrm{BK}) \quad$ FELT

AUG. 26

04:24 PST

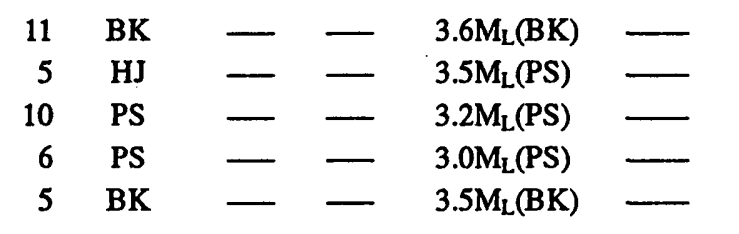

AUG. 26 11:07 PST

AUG. $26 \quad 11: 11$ PST

AUG. 26 13:48 PST

AUG. 26 22:10 PST

AUG. 27 01:20 PST

$\begin{array}{rllllr}6 & \mathrm{PS} & - & - & 3.6 \mathrm{M}_{\mathrm{L}}(\mathrm{PS}) & \mathrm{V} \\ 8 & \mathrm{PS} & - & - & 3.2 \mathrm{M}_{\mathrm{L}}(\mathrm{PS}) & \mathrm{FEL} \\ 5 & \mathrm{BK} & - & - & 3.2 \mathrm{M}_{\mathrm{L}}(\mathrm{BK}) & \mathrm{III} \\ 8 & \mathrm{PS} & - & - & 3.7 \mathrm{M}_{\mathrm{L}}(\mathrm{PS}) & \mathrm{V} \\ 11 & \mathrm{BK} & - & - & 3.3 \mathrm{M}_{\mathrm{L}}(\mathrm{BK}) & \end{array}$

V

ELT

III

V

(P.OM)

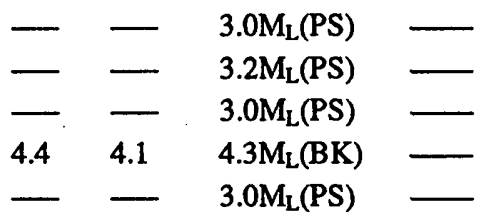

3.0ML(PS)

$3.0 \mathrm{M}_{\mathrm{L}}$ (PS)

$3.5 \mathrm{M}_{\mathrm{L}}(\mathrm{BK})$

$3.4 \mathrm{M}_{\mathrm{L}}$ (BK)

3.4M $\mathrm{M}(\mathrm{RN})$

4.0 $\mathrm{M}_{\mathrm{L}}(\mathrm{BK})$

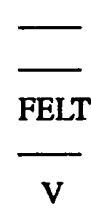

AUG. 28

AUG. 28

AUG. 28

AUG. 28

AUG. 29

02:14 PST

08:32 PST

22:44 PST

23:46 PST

AUG. $30 \quad 12: 58$ PST

AUG. $30 \quad 20: 36$ PST

AUG. 31

09:57 PST

AUG. 31

15:29 PST

SEPT. 1 03:55 PST

SEPT. 1 11:43 PST

SEPT. 1 16:49 PST

SEPT. 2 05:59 PST

SEPT. 2 13:24 PST

SEPT. 2 20:31 PST

SEPT. $3 \quad 04: 35$ PST SEPT. 3 16:42 PST SEPT. 3 17:31 PST SEPT. 4 02:29 PST SEPT. 4 13:54 PST

SEPT. $5 \quad 10: 13$ PST SEPT. 5 17:30 PST SEPT. 7 19:37 PST SEPT. 8 03:07 PST SEPT. 8 08:22 PST

SEPT. 8 23:30 PST SEPT. 9 05:29 PST SEPT. 9 08:22 PST SEPT. 9 14:29 PST SEPT. 10 07:51 PST

SEPT. $11 \quad$ 05:26 PST SEPT. $12 \quad$ 15:38 PST 
Table 1. Summary of United States earthquakes for 1986 — Continued

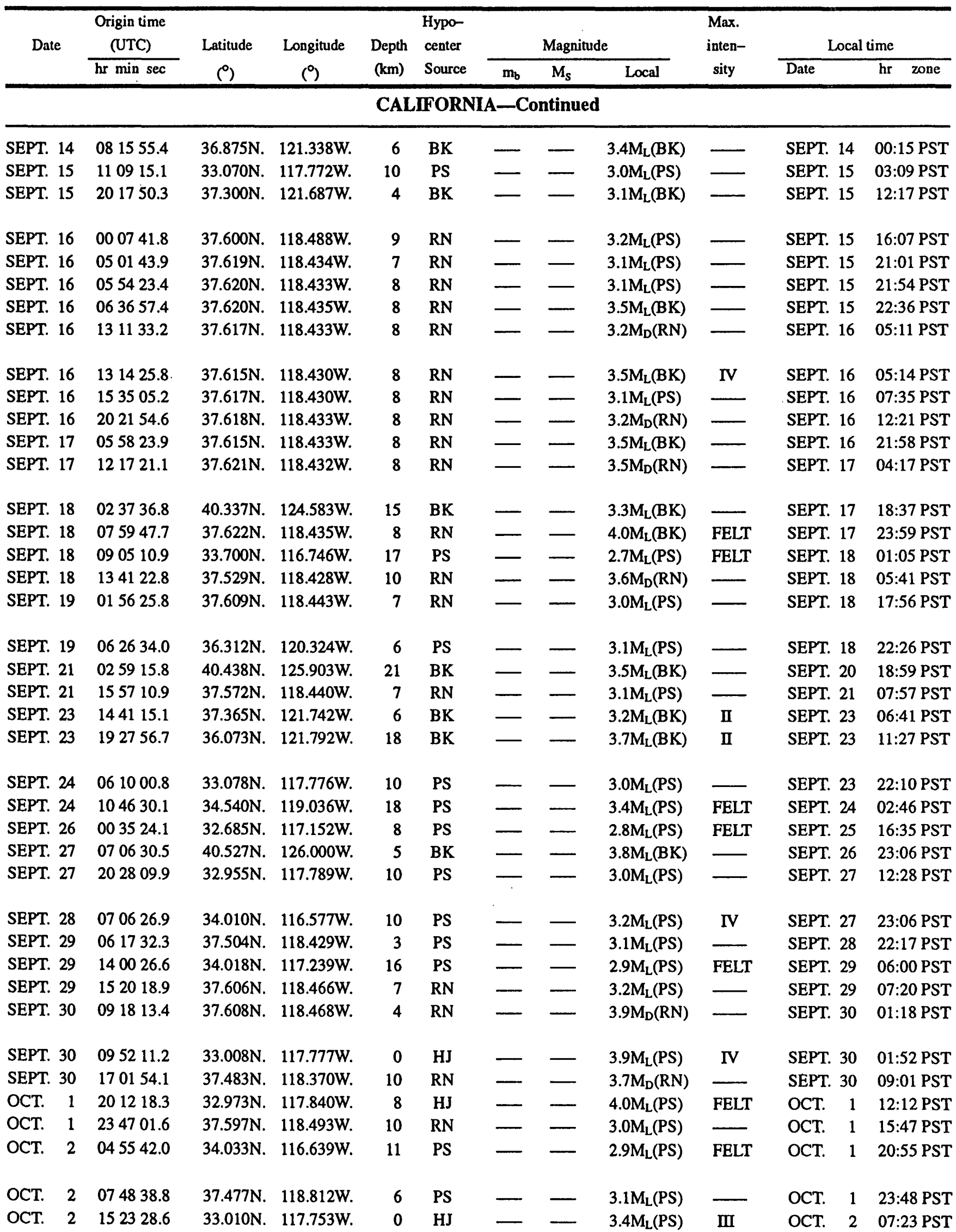


Table 1. Summary of United States earthquakes for 1986 - Continued

\begin{tabular}{|c|c|c|c|c|c|c|c|c|c|c|c|c|c|}
\hline \multirow[t]{2}{*}{ Dat } & & \multirow{2}{*}{$\begin{array}{l}\text { Origin time } \\
\text { (UTC) } \\
\text { hr min sec }\end{array}$} & \multirow{2}{*}{$\begin{array}{l}\text { Latitude } \\
\left({ }^{\circ}\right)\end{array}$} & \multirow{2}{*}{$\begin{array}{l}\text { Longitude } \\
\left({ }^{\circ}\right)\end{array}$} & \multirow{2}{*}{$\begin{array}{l}\text { Depth } \\
(\mathbf{k m})\end{array}$} & \multirow{2}{*}{$\begin{array}{l}\text { Hypo- } \\
\text { center } \\
\text { Source }\end{array}$} & \multicolumn{3}{|c|}{ Magnitude } & \multirow{2}{*}{$\begin{array}{l}\text { Max. } \\
\text { inten- } \\
\text { sity }\end{array}$} & \multicolumn{3}{|c|}{ Local time } \\
\hline & & & & & & & $\mathbf{m}_{\mathbf{b}}$ & $\mathbf{M}_{\mathbf{S}}$ & Local & & Date & & zone \\
\hline \multicolumn{14}{|c|}{ CALIFORNIA-Continued } \\
\hline OCT. & 2 & 225643.9 & $37.527 \mathrm{~N}$. & $118.439 \mathrm{~W}$. & 12 & RN & - & & $3.2 \mathrm{M}_{\mathrm{L}}(\mathrm{PS})$ & - & OCT. & 2 & 14:56 PST \\
\hline OCT. & 3 & 133351.9 & $40.293 \mathrm{~N}$ & $125.122 \mathrm{~W}$. & 4 & BK & & - & $3.0 \mathrm{M}_{\mathrm{L}}(\mathrm{BK})$ & & OCT. & 3 & 05:33 PST \\
\hline OCT. & 3 & 214931.2 & $37.622 \mathrm{~N}$. & $118.475 W$ & 10 & RN & 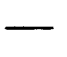 & & $3.4 \mathrm{M}_{\mathrm{D}}(\mathrm{RN})$ & & OCT. & 3 & 13:49 PST \\
\hline OCT. & 3 & 235327.7 & $37.521 \mathrm{~N}$. & $118.431 W$ & 12 & $\mathbf{R N}$ & $\longrightarrow$ & $\longrightarrow$ & $3.4 \mathrm{M}_{\mathrm{D}}(\mathrm{RN})$ & & OCT. & 3 & 15:53 PST \\
\hline OCT. & 6 & 034648.7 & $37.555 \mathrm{~N}$. & $118.465 \mathrm{~W}$. & 11 & RN & & & $3.4 \mathrm{M}_{\mathrm{D}}(\mathrm{RN})$ & & OCT. & 5 & 19:46 PST \\
\hline OCT. & 6 & 134247.1 & $37.605 \mathrm{~N}$ & $118.911 \mathrm{~W}$ & 4 & RN & & 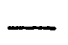 & $3.2 \mathrm{M}(\mathrm{RN})$ & & OCT. & 6 & 05:42 PST \\
\hline OCT. & 6 & 181440.7 & $37.590 \mathrm{~N}$. & $118.460 \mathrm{~W}$. & 9 & RN & & 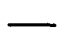 & $3.2 \mathrm{M}_{\mathrm{D}}(\mathrm{RN})$ & & OCT. & 6 & 10:14 PST \\
\hline OCT. & 6 & 233237.9 & $37.596 \mathrm{~N}$. & $118.467 \mathrm{~W}$. & 9 & RN & - & 一 & $3.2 \mathrm{M}_{\mathrm{L}}(\mathrm{PS})$ & & OCT. & 6 & 15:32 PST \\
\hline OCT. & 9 & 020623.0 & $37.297 \mathrm{~N}$. & $121.688 \mathrm{~W}$. & 5 & BK & & & $3.0 \mathrm{M}_{\mathrm{L}}(\mathrm{BK})$ & & OCT. & 8 & 18:06 PST \\
\hline OCT. & 9 & 053725.0 & $37.348 \mathrm{~N}$. & $118.370 \mathrm{~W}$. & 15 & RN & & & $4.4 \mathrm{M}_{\mathrm{L}}(\mathrm{BK})$ & III & OCT. & 8 & 21:37 PST \\
\hline OCT. & 10 & 152302.6 & $33.948 \mathrm{~N}$. & $116.786 \mathrm{~W}$. & 2 & PS & 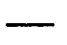 & - & $3.5 \mathrm{M}_{\mathrm{L}}(\mathrm{PS})$ & IV & OCT. & 10 & 07:23 PST \\
\hline OCT. & 11 & 051736.4 & $37.827 \mathrm{~N}$ & $121.960 \mathrm{~W}$. & 6 & BK & & & $4.2 \mathrm{M}_{\mathrm{L}}(\mathrm{BK})$ & V & OCT. & 10 & 21:17 PST \\
\hline OCT. & 11 & 063938.2 & $37.827 \mathrm{~N}$. & $121.952 \mathrm{~W}$. & 5 & BK & - & - & $3.2 \mathrm{M}_{\mathrm{L}}(\mathrm{BK})$ & FELT & OCT. & 10 & 22:39 PST \\
\hline OCT. & 12 & 064301.9 & $38.718 \mathrm{~N}$ & $123.500 \mathrm{~W}$. & 11 & BK & 4.2 & - & $4.0 \mathrm{M}_{\mathrm{L}}(\mathrm{BK})$ & V & OCT. & 11 & 22:43 PST \\
\hline OCT. & 12 & 163702.8 & $37.523 \mathrm{~N}$. & 118.423W. & 10 & RN & - & & $3.1 \mathrm{M}_{\mathrm{L}}(\mathrm{PS})$ & 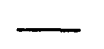 & OCT. & 12 & 08:37 PST \\
\hline OCT. & 15 & 022847.8 & $33.953 \mathrm{~N}$. & $116.572 \mathrm{~W}$. & 9 & PS & 4.3 & & $4.7 \mathrm{M}_{\mathrm{L}}(\mathrm{GP})$ & V & OCT. & 14 & $18: 28$ PST \\
\hline OCT. & 15 & 054618.8 & $34.165 \mathrm{~N}$ & $118.274 \mathrm{~W}$ & 3 & PS & & - & $2.5 \mathrm{M}_{\mathrm{L}}(\mathrm{PS})$ & FELT & OCT. & 14 & 21:46 PST \\
\hline OCT. & 15 & 081917.9 & $34.986 \mathrm{~N}$ & $119.207 \mathrm{~W}$ & 1 & GP & & & $3.2 \mathrm{M}_{\mathrm{L}}(\mathrm{PS})$ & $\longrightarrow$ & OCT. & 15 & 00:19 PST \\
\hline OCT. & 16 & 061640.7 & $40.327 \mathrm{~N}$ & 123.927W. & 6 & BK & & & $2.4 \mathrm{M}_{\mathrm{L}}(\mathrm{BK})$ & III & OCT. & 15 & 22:16 PST \\
\hline OCT. & 16 & 101617.0 & $33.977 \mathrm{~N}$ & $116.565 \mathrm{~W}$ & 8 & PS & & & $2.9 \mathrm{M}_{\mathrm{L}}(\mathrm{PS})$ & FELT & OCT. & 16 & 02:16 PST \\
\hline OCT. & 16 & 112037.6 & $37.755 \mathrm{~N}$. & $122.138 \mathrm{~W}$. & 7 & BK & - & - & $2.2 \mathrm{M}_{\mathrm{L}}(\mathrm{BK})$ & FELT & OCT. & 16 & 03:20 PST \\
\hline OCT. & 17 & 130605.4 & $41.700 \mathrm{~N}$ & $126.988 \mathrm{~W}$. & 10 & GS & 4.3 & 3.9 & - & - & OCT. & 17 & 05:06 PST \\
\hline OCT. & 17 & 185616.6 & $34.370 \mathrm{~N}$ & $116.385 \mathrm{~W}$. & 7 & PS & & 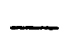 & $3.5 \mathrm{M}_{\mathrm{L}}(\mathrm{PS})$ & - & OCT. & 17 & 10:56 PST \\
\hline OCT. & 19 & 004240.3 & $38.418 \mathrm{~N}$ & $119.312 W$ & 5 & BK & & ـ & 3.7M $\mathrm{M}_{\mathrm{L}}(\mathrm{BK})$ & $\ldots$ & OCT. & 18 & $16: 42$ PST \\
\hline OCT. & 19 & 145026.1 & $34.015 \mathrm{~N}$ & $116.687 \mathrm{~W}$. & 8 & PS & & & $2.9 \mathrm{M}_{\mathrm{L}}(\mathrm{PS})$ & FELT & OCT. & 19 & 06:50 PST \\
\hline OCT. & 19 & 180956.6 & $36.098 \mathrm{~N}$. & $117.845 \mathrm{~W}$. & 4 & PS & & & $3.2 \mathrm{M}_{\mathrm{L}}(\mathrm{PS})$ & - & OCT. & 19 & 10:09 PST \\
\hline OCT. & 19 & 205131.2 & $37.470 \mathrm{~N}$ & $118.506 \mathrm{~W}$. & 6 & RN & & & $3.1 \mathrm{M}_{\mathrm{D}}(\mathrm{RN})$ & & OCT. & 19 & $12: 51$ PST \\
\hline OCT. & 20 & 223530.0 & $32.955 \mathrm{~N}$ & 117.786W. & 6 & PS & & & $3.2 \mathrm{M}_{\mathrm{L}}(\mathrm{PS})$ & & OCT. & 20 & 14:35 PST \\
\hline OCT. & 21 & 052626.9 & $37.537 \mathrm{~N}$. & $118.464 \mathrm{~W}$. & 7 & RN & & & $3.0 \mathrm{M}_{\mathrm{L}}(\mathrm{PS})$ & & OCT. & 20 & 21:26 PST \\
\hline OCT. & 21 & 083625.0 & $37.489 \mathrm{~N}$ & $118.363 \mathrm{~W}$. & 7 & RN & & & $3.1 \mathrm{M}_{\mathrm{L}}(\mathrm{PS})$ & & OCT. & 21 & 00:36 PST \\
\hline OCT. & 21 & 093004.6 & $37.591 \mathrm{~N}$. & $118.457 \mathrm{~W}$. & 9 & RN & & & $3.2 \mathrm{M}_{\mathrm{L}}(\mathrm{PS})$ & - & OCT. & 21 & 01:30 PST \\
\hline OCT. & 21 & 131458.6 . & $36.760 \mathrm{~N}$. & $121.367 \mathrm{~W}$. & 11 & BK & & & $3.3 \mathrm{M}_{\mathrm{L}}(\mathrm{BK})$ & FELT & OCT. & 21 & 05:14 PST \\
\hline OCT. & 21 & 205119.9 & $37.468 \mathrm{~N}$. & $118.503 W$ & 4 & RN & & & $3.2 \mathrm{M}_{\mathrm{L}}(\mathrm{PS})$ & 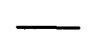 & OCT. & 21 & 12:51 PST \\
\hline OCT. & 22 & 080712.3 & $35.061 \mathrm{~N}$ & 119.087W. & 16 & PS & & & $3.0 \mathrm{M}_{\mathrm{L}}(\mathrm{PS})$ & & OCT. & 22 & 00:07 PST \\
\hline OCT. & 22 & 150321.8 & $37.476 \mathrm{~N}$. & 118.511W. & 4 & RN & & - & $3.0 \mathrm{M}_{\mathrm{L}}(\mathrm{PS})$ & & OCT. & 22 & 07:03 PST \\
\hline OCT. & 22 & 225835.3 & $37.471 \mathrm{~N}$. & $118.512 \mathrm{~W}$. & 5 & RN & & & $3.1 \mathrm{M}_{\mathrm{D}}(\mathrm{RN})$ & & OCT. & 22 & 14:58 PST \\
\hline OCT. & 23 & 162333.8 & $37.594 \mathrm{~N}$. & $118.488 \mathrm{~W}$. & 9 & RN & & & $3.2 \mathrm{M}_{\mathrm{D}}(\mathrm{RN})$ & & OCT. & 23 & 08:23 PST \\
\hline OCT. & 24 & 194257.2 & $32.964 \mathrm{~N}$. & $117.821 \mathrm{~W}$. & 10 & PS & & - & $3.1 \mathrm{M}_{\mathrm{L}}(\mathrm{PS})$ & m & OCT. & 24 & $11: 42$ PST \\
\hline OCT. & 25 & 003526.1 & $37.413 \mathrm{~N}$. & $116.868 \mathrm{~W}$. & 6 & PS & - & - & $3.1 \mathrm{M}_{\mathrm{L}}(\mathrm{PS})$ & - & OCT. & 24 & $16: 35$ PST \\
\hline OCT. & 25 & 164028.5 & $36.095 \mathrm{~N}$ & $117.848 \mathrm{~W}$. & 4 & PS & - & - & $3.1 \mathrm{M}_{\mathrm{L}}(\mathrm{PS})$ & - & OCT. & 25 & 08:40 PST \\
\hline
\end{tabular}


Table 1. Summary of United States earthquakes for 1986-Continued

\begin{tabular}{|c|c|c|c|c|c|c|c|c|c|c|c|c|c|}
\hline \multirow[t]{2}{*}{ Date } & & \multirow{2}{*}{$\begin{array}{l}\text { Origin time } \\
\text { (UTC) } \\
\text { hr min sec }\end{array}$} & \multirow{2}{*}{$\begin{array}{l}\text { Latitude } \\
\left({ }^{\circ}\right)\end{array}$} & \multirow{2}{*}{$\begin{array}{c}\text { Longitude } \\
(9)\end{array}$} & \multirow{2}{*}{$\begin{array}{l}\text { Depth } \\
(\mathrm{km})\end{array}$} & \multirow{2}{*}{$\begin{array}{l}\text { Hypo- } \\
\text { center } \\
\text { Source }\end{array}$} & \multicolumn{3}{|c|}{ Magnitude } & \multirow{2}{*}{$\begin{array}{l}\text { Max. } \\
\text { inten- } \\
\text { sity }\end{array}$} & \multicolumn{3}{|c|}{ Local time } \\
\hline & & & & & & & $\mathrm{m}_{\mathrm{b}}$ & $\mathrm{M}_{\mathrm{s}}$ & Local & & Date & & zone \\
\hline \multicolumn{14}{|c|}{ CALIFORNIA-Continued } \\
\hline OCT. & 26 & 011147.1 & $37.598 \mathrm{~N}$ & $118.923 W$ & 3 & RN & - & - & $3.1 \mathrm{M}_{\mathrm{D}}(\mathrm{RN})$ & - & OCT. & 25 & 17:11 PST \\
\hline OCT. & 26 & 051733.2 & $37.293 \mathrm{~N}$. & $116.151 \mathrm{~W}$ & 6 & PS & - & - & $3.1 \mathrm{M}_{\mathrm{L}}(\mathrm{PS})$ & $\longrightarrow$ & OCT. & 25 & 21:17 PST \\
\hline OCT. & 26 & 051756.2 & $35.575 \mathrm{~N}$ & $117.229 \mathrm{~W}$ & 6 & PS & - & - & $3.0 \mathrm{M}_{\mathrm{L}}(\mathrm{PS})$ & 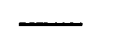 & OCT. & 25 & 21:17 PST \\
\hline OCT. & 26 & 102013.8 & $37.472 \mathrm{~N}$ & 118.384W. & 10 & $\mathbf{R N}$ & - & - & $3.2 \mathrm{M}_{\mathrm{L}}(\mathrm{PS})$ & & OCT. & 26 & 02:20 PST \\
\hline OCT. & 27 & 020645.5 & $37.175 \mathrm{~N}$ & $121.583 \mathrm{~W}$. & 8 & BK & - & - & $3.6 \mathrm{M}_{\mathrm{L}}(\mathrm{BK})$ & II & OCT. & 26 & 18:06 PST \\
\hline OCT. & 28 & 114723.8 & $37.531 \mathrm{~N}$ & $118.450 \mathrm{~W}$ & 6 & $\mathbf{R N}$ & - & - & $3.2 \mathrm{M}_{\mathrm{L}}(\mathrm{BK})$ & & OCT. & 28 & 03:47 PST \\
\hline OCT. & 28 & 212900.8 & $38.772 \mathrm{~N}$. & $122.790 \mathrm{~W}$ & 2 & BK & - & - & $3.2 \mathrm{M}_{\mathrm{L}}(\mathrm{BK})$ & & OCT. & 28 & 13:29 PST \\
\hline OCT. & 29 & 023815.3 & $32.615 \mathrm{~N}$ & $117.152 W$. & 15 & PS & 3.9 & - & $4.1 \mathrm{M}_{\mathrm{L}}(\mathrm{PS})$ & $\mathbf{v}$ & OCT. & 28 & 18:38 PST \\
\hline OCT. & 29 & 071019.2 & $32.967 \mathrm{~N}$ & $117.821 \mathrm{~W}$ & 6 & PS & - & - & $3.0 \mathrm{M}_{\mathrm{L}}(\mathrm{PS})$ & 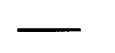 & OCT. & 28 & 23:10 PST \\
\hline OCT. & 29 & 081534.5 & $34.734 \mathrm{~N}$. & $120.144 \mathrm{~W}$ & 0 & PS & - & - & $3.1 \mathrm{M}_{\mathrm{L}}(\mathrm{PS})$ & 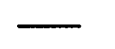 & OCT. & 29 & $00: 15$ PST \\
\hline OCT. & 30 & 040153.2 & $38.093 \mathrm{~N}$ & 119.268W. & 4 & BK & - & - & $3.6 \mathrm{M}_{\mathrm{L}}(\mathrm{BK})$ & & OCT. & 29 & 20:01 PST \\
\hline OCT. & 30 & 181208.3 & $36.827 \mathrm{~N}$ & 121.578W. & 4 & BK & - & - & $3.1 \mathrm{M}_{\mathrm{L}}(\mathrm{BK})$ & IV & OCT. & 30 & 10:12 PST \\
\hline OCT. & 31 & 035728.9 & $38.420 \mathrm{~N}$ & $119.323 \mathrm{~W}$ & 1 & BK & - & - & $4.6 \mathrm{M}_{\mathrm{L}}(\mathrm{BK})$ & IV & OCT. & 30 & 19:57 PST \\
\hline OCT. & 31 & 142705.2 & $35.578 \mathrm{~N}$ & $117.178 \mathrm{~W}$ & 6 & PS & - & - & $3.8 \mathrm{M}_{\mathrm{L}}(\mathrm{PS})$ & III & OCT. & 31 & 06:27 PST \\
\hline OCT. & 31 & 184614.2 & $36.947 \mathrm{~N}$ & $121.572 \mathrm{~W}$ & 7 & BK & - & - & $3.5 \mathrm{M}_{\mathrm{L}}(\mathrm{BK})$ & $\mathbf{v}$ & OCT. & 31 & 10:46 PST \\
\hline Nov. & 1 & 084012.7 & $38.767 \mathrm{~N}$ & $122.732 \mathrm{~W}$ & 3 & BK & - & - & $3.0 \mathrm{M}_{\mathrm{L}}(\mathrm{BK})$ & & NOV. & 1 & 00:40 PST \\
\hline NOV. & 1 & 145057.4 & $37.347 \mathrm{~N}$ & $121.730 \mathrm{~W}$ & 6 & BK & - & - & $3.4 \mathrm{M}_{\mathrm{L}}(\mathrm{BK})$ & IV & NOV. & 1 & 06:50 PST \\
\hline NOV. & 2 & 034614.4 & $37.630 \mathrm{~N}$ & $122.483 \mathrm{~W}$ & 10 & BK & - & - & $3.0 \mathrm{M}_{\mathrm{L}}(\mathrm{BK})$ & FELT & NOV. & 1 & 19:46 PST \\
\hline NOV. & 3 & 002707.8 & $37.554 \mathrm{~N}$ & 118.486W. & 6 & PS & - & - & $3.1 \mathrm{M}_{\mathrm{L}}(\mathrm{PS})$ & - & NOV. & 2 & 16:27 PST \\
\hline NOV. & 3 & 184040.4 & $33.689 \mathrm{~N}$ & $116.813 \mathrm{~W}$ & 18 & PS & - & - & $2.9 \mathrm{M}_{\mathrm{L}}(\mathrm{PS})$ & III & NOV. & 3 & 10:40 PST \\
\hline NOV. & 3 & 210401.4 & $33.874 \mathrm{~N}$ & $116.859 \mathrm{~W}$ & 11 & GP & - & - & $3.1 \mathrm{M}_{\mathrm{L}}(\mathrm{PS})$ & III & NOV. & 3 & 13:04 PST \\
\hline NOV. & 6 & 091958.3 & $34.735 \mathrm{~N}$ & $120.147 \mathrm{~W}$ & 0 & PS & 4.0 & - & $4.0 \mathrm{M}_{\mathrm{L}}(\mathrm{PS})$ & $\mathrm{V}$ & NOV. & 6 & 01:19 PST \\
\hline NOV. & 6 & 230250.5 & $34.366 \mathrm{~N}$ & $116.383 \mathrm{~W}$ & 2 & PS & - & - & $3.1 \mathrm{M}_{\mathrm{L}}(\mathrm{PS})$ & & NOV. & 6 & 15:02 PST \\
\hline NOV. & 7 & 010403.1 & $40.597 \mathrm{~N}$ & $124.555 \mathrm{~W}$ & 9 & BK & - & - & $3.3 \mathrm{M}_{\mathrm{L}}(\mathrm{BK})$ & - & NOV. & 6 & 17:04 PST \\
\hline NOV. & 7 & 020800.8 & $32.983 \mathrm{~N}$ & $117.825 \mathrm{~W}$ & 6 & PS & - & - & $3.1 \mathrm{M}_{\mathrm{L}}(\mathrm{PS})$ & 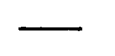 & NOV. & 6 & 18:08 PST \\
\hline NOV. & 10 & 013512.5 & $32.976 \mathrm{~N}$ & $117.791 \mathrm{~W}$ & 6 & PS & - & - & $3.2 \mathrm{M}_{\mathrm{L}}(\mathrm{PS})$ & - & NOV. & 9 & 17:35 PST \\
\hline NOV. & 10 & 152234.4 & $38.805 \mathrm{~N}$ & $122.822 \mathrm{~W}$ & 2 & BK & - & - & $3.3 \mathrm{M}_{\mathrm{L}}(\mathrm{BK})$ & - & NOV. & 10 & 07:22 PST \\
\hline NOV. & 12 & 220717.2 & $36.112 \mathrm{~N}$ & $120.162 W$ & 3 & BK & - & - & $3.1 \mathrm{M}_{\mathrm{L}}(\mathrm{BK})$ & $\longrightarrow$ & NOV. & 12 & 14:07 PST \\
\hline NOV. & 12 & 232647.9 & $34.528 \mathrm{~N}$ & $118.488 \mathrm{~W}$ & 2 & PS & - & - & $2.7 \mathrm{M}_{\mathrm{L}}(\mathrm{PS})$ & FELT & NOV. & 12 & 15:26 PST \\
\hline NOV. & 13 & 051228.0 & $33.959 \mathrm{~N}$ & 116.734W. & 10 & PS & - & - & $3.2 \mathrm{M}_{\mathrm{L}}(\mathrm{PS})$ & IV & NOV. & 12 & $21: 12$ PST \\
\hline NOV. & 13 & 150035.5 & $37.486 \mathrm{~N}$. & $118.374 \mathrm{~W}$ & 9 & $\mathrm{RN}$ & - & - & $3.2 \mathrm{M}_{\mathrm{D}}(\mathrm{RN})$ & - & NOV. & 13 & 07:00 PST \\
\hline NOV. & 13 & 165538.3 & $37.479 \mathrm{~N}$ & 118.510W. & 3 & RN & - & - & $3.9 \mathrm{M}_{\mathrm{L}}(\mathrm{BK})$ & FELT & NOV. & 13 & 08:55 PST \\
\hline NOV. & 13 & 184522.3 & $37.476 \mathrm{~N}$ & $118.511 \mathrm{~W}$ & 4 & RN & - & - & $3.5 \mathrm{M}_{\mathrm{L}}(\mathrm{BK})$ & - & NOV. & 13 & $10: 45$ PST \\
\hline NOV. & 14 & 042620.4 & $38.528 \mathrm{~N}$ & $122.977 \mathrm{~W}$ & 2 & BK & - & 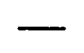 & $2.6 \mathrm{M}_{\mathrm{L}}(\mathrm{BK})$ & FELT & NOV. & 13 & 20:26 PST \\
\hline NOV. & 15 & 013958.1 & $37.581 \mathrm{~N}$. & $118.451 \mathrm{~W}$ & 2 & $\mathrm{RN}$ & - & & $3.6 \mathrm{M}_{\mathrm{D}}(\mathrm{RN})$ & $\bar{L}$ & NOV. & 14 & $17: 39$ PST \\
\hline NOV. & 16 & 205148.0 & $39.197 \mathrm{~N}$. & $120.414 \mathrm{~W}$. & 11 & $\mathrm{RN}$ & - & - & $3.0 \mathrm{M}_{\mathrm{D}}(\mathrm{RN})$ & & NOV. & 16 & 12:51 PST \\
\hline NOV. & 17 & 124022.7 & $37.577 \mathrm{~N}$ & 118.433W. & 7 & RN & - & - & $3.7 \mathrm{M}_{\mathrm{D}}(\mathrm{RN})$ & & NOV. & 17 & 04:40 PST \\
\hline NOV. & 17 & 144322.8 & $37.579 \mathrm{~N}$ & $118.439 \mathrm{~W}$ & 5 & RN & - & - & $3.2 \mathrm{M}_{\mathrm{D}}(\mathrm{RN})$ & & NOV. & 17 & 06:43 PST \\
\hline NOV. & 19 & 045600.5 & $32.964 \mathrm{~N}$ & $117.811 \mathrm{~W}$ & 6 & PS & - & 一 & $3.1 \mathrm{M}_{\mathrm{L}}(\mathrm{PS})$ & 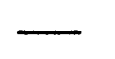 & NOV. & 18 & 20:56 PST \\
\hline NOV. & 19 & 161906.0 & $40.375 \mathrm{~N}$ & $125.060 \mathrm{~W}$ & 8 & BK & 4.2 & - & $3.9 \mathrm{M}_{\mathrm{L}}(\mathrm{BK})$ & IV & Nov. & 19 & $08: 19 \mathrm{Y}$ \\
\hline
\end{tabular}


Table 1. Summary of United States earthquakes for 1986 - Continued

\begin{tabular}{|c|c|c|c|c|c|c|c|c|c|c|c|c|}
\hline \multirow[t]{2}{*}{ Date } & \multirow{2}{*}{$\begin{array}{l}\text { Origin time } \\
\text { (UTC) } \\
\text { hr min sec }\end{array}$} & \multirow{2}{*}{$\begin{array}{c}\text { Latitude } \\
\text { (०) }\end{array}$} & \multirow{2}{*}{$\begin{array}{l}\text { Longitude } \\
\text { (9) }\end{array}$} & \multirow{2}{*}{$\begin{array}{l}\text { Depth } \\
(\mathbf{k m})\end{array}$} & \multirow{2}{*}{$\begin{array}{l}\text { Hypo- } \\
\text { center } \\
\text { Source }\end{array}$} & \multicolumn{3}{|c|}{ Magnitude } & \multirow{2}{*}{$\begin{array}{l}\text { Max. } \\
\text { inten- } \\
\text { sity }\end{array}$} & \multicolumn{3}{|c|}{ Local time } \\
\hline & & & & & & $m_{b}$ & $\mathbf{M}_{\mathbf{S}}$ & Local & & Date & $\mathrm{hr}$ & zone \\
\hline
\end{tabular}

CALIFORNIA-Continued

\begin{tabular}{|c|c|c|c|c|c|c|c|c|c|c|c|c|c|}
\hline NOV. & 19 & 174037.9 & $37.530 \mathrm{~N}$ & $118.454 \mathrm{~W}$ & 7 & RN & - & - & $3.1 \mathrm{M}_{\mathrm{L}}(\mathrm{PS})$ & - & NOV. & 19 & 09:40 PST \\
\hline NOV. & 21 & 021350.4 & $32.486 \mathrm{~N}$ & $119.527 \mathrm{~W}$ & 6 & PS & - & - & $3.3 \mathrm{M}_{\mathrm{L}}(\mathrm{PS})$ & & NOV. & 20 & 18:13 PST \\
\hline NOV. & 21 & 233301.7 & $40.372 \mathrm{~N}$ & 124.443W. & 15 & BK & 5.3 & 5.1 & $5.1 \mathrm{M}_{\mathrm{L}}(\mathrm{BK})$ & VII & NOV. & 21 & 15:33 PST \\
\hline NOV. & 21 & 233418.0 & $40.367 \mathrm{~N}$ & $124.450 \mathrm{~W}$ & 15 & BK & 5.1 & - & $5.1 \mathrm{M}_{\mathrm{L}}(\mathrm{BK})$ & FELT & NOV. & 21 & 15:34 PST \\
\hline NOV. & 22 & 000524.7 & $40.480 \mathrm{~N}$ & $124.477 \mathrm{~W}$ & 23 & BK & - & - & $3.5 \mathrm{M}_{\mathrm{L}}(\mathrm{BK})$ & - & NOV. & 21 & 16:05 PST \\
\hline NOV. & 22 & 005752.4 & $40.447 \mathrm{~N}$ & $124.482 \mathrm{~W}$ & 24 & BK & - & - & $3.2 \mathrm{M}_{\mathrm{L}}(\mathrm{BK})$ & - & NOV. & 21 & 16:57 PST \\
\hline NOV. & 22 & 012955.9 & 40.378N. & $124.575 W$ & 19 & BK & - & - & $3.2 \mathrm{M}_{\mathrm{L}}(\mathrm{BK})$ & - & NOV. & 21 & 17:29 PST \\
\hline NOV. & 22 & 031758.4 & $40.370 \mathrm{~N}$ & $124.493 \mathrm{~W}$ & 18 & BK & - & - & $3.8 \mathrm{M}_{\mathrm{L}}(\mathrm{BK})$ & - & NOV. & 21 & 19:17 PST \\
\hline NOV. & 22 & 171422.2 & $37.516 \mathrm{~N}$ & 118.395W. & 7 & RN & - & - & $3.4 \mathrm{M}_{\mathrm{D}}(\mathrm{RN})$ & 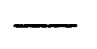 & NOV. & 22 & 09:14 PST \\
\hline NOV. & 22 & 183658.2 & 36.087N. & $120.050 \mathrm{~W}$ & 6 & BK & - & - & $3.2 \mathrm{M}_{\mathrm{L}}(\mathrm{BK})$ & & NOV. & 22 & 10:36 PST \\
\hline NOV. & 22 & 223127.4 & $36.098 \mathrm{~N}$ & $119.987 \mathrm{~W}$ & 6 & PS & - & - & $3.0 \mathrm{M}_{\mathrm{L}}(\mathrm{PS})$ & & NOV. & 22 & $14: 31$ PST \\
\hline NOV. & 23 & 012148.3 & $40.367 \mathrm{~N}$ & 124.515W. & 19 & BK & - & - & $3.5 \mathrm{M}_{\mathrm{L}}(\mathrm{BK})$ & & NOV. & 22 & $17: 21$ PST \\
\hline NOV. & 23 & 020855.9 & $34.096 \mathrm{~N}$ & $120.848 \mathrm{~W}$ & 6 & PS & - & - & $3.2 \mathrm{M}_{\mathrm{L}}(\mathrm{PS})$ & & NOV. & 22 & 18:08 PST \\
\hline NOV. & 23 & 054106.3 & $40.360 \mathrm{~N}$ & $124.528 \mathrm{~W}$ & 20 & BK & 4.0 & - & $4.0 \mathrm{M}_{\mathrm{L}}(\mathrm{BK})$ & III & NOV. & 22 & 21:41 PST \\
\hline NOV. & 23 & 091337.7 & $40.408 \mathrm{~N}$ & $124.310 \mathrm{~W}$ & 17 & BK & - & - & $3.2 \mathrm{M}_{\mathrm{L}}(\mathrm{BK})$ & & NOV. & 23 & 01:13 PST \\
\hline NOV. & 23 & 160045.0 & $40.378 \mathrm{~N}$ & 124.383W. & 18 & BK & - & - & $3.0 \mathrm{M}_{\mathrm{L}}(\mathrm{BK})$ & & NOV. & 23 & 08:00 PST \\
\hline NOV. & 24 & 043149.5 & $36.113 \mathrm{~N}$ & $120.015 \mathrm{~W}$ & 7 & BK & - & - & $3.1 \mathrm{M}_{\mathrm{L}}(\mathrm{BK})$ & 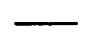 & Nov. & 23 & 20:31 PST \\
\hline NOV. & 24 & 150801.3 & $36.602 \mathrm{~N}$ & $121.240 \mathrm{~W}$ & 4 & BK & - & - & $3.1 \mathrm{M}_{\mathrm{L}}(\mathrm{BK})$ & & NOV. & 24 & 07:08 PST \\
\hline NOV. & 24 & 181525.2 & $34.369 \mathrm{~N}$ & 116.384W. & 3 & PS & - & - & $3.0 \mathrm{M}_{\mathrm{L}}(\mathrm{PS})$ & - & NOV. & 24 & 10:15 PST \\
\hline NOV. & 26 & 082215.4 & $40.357 \mathrm{~N}$ & $124.475 \mathrm{~W}$ & 18 & BK & - & - & $3.5 \mathrm{M}_{\mathrm{L}}(\mathrm{BK})$ & & NOV. & 26 & 00:22 PST \\
\hline Nov. & 28 & 034557.0 & $38.842 \mathrm{~N}$ & 122.790W. & 2 & BK & - & - & $3.0 \mathrm{M}_{\mathrm{L}}(\mathrm{BK})$ & & NOV. & 27 & 19:45 PST \\
\hline NOV. & 29 & 131026.1 & $37.584 \mathrm{~N}$ & 118.438W. & 5 & RN & - & - & $3.6 \mathrm{M}_{\mathrm{D}}(\mathrm{RN})$ & - & NOV. & 29 & 05:10 PST \\
\hline NOV. & 29 & 131051.6 & $37.582 \mathrm{~N}$. & $118.481 \mathrm{~W}$ & 6 & PS & - & - & $3.2 \mathrm{M}_{\mathrm{L}}(\mathrm{PS})$ & & NOV. & 29 & 05:10 PST \\
\hline DEC. & 2 & 230901.2 & $36.143 \mathrm{~N}$ & $120.043 \mathrm{~W}$ & 6 & PS & - & - & $3.1 \mathrm{M}_{\mathrm{L}}(\mathrm{PS})$ & - & DEC. & 2 & 15:09 PST \\
\hline DEC. & 4 & 105519.6 & $33.713 \mathrm{~N}$ & $116.836 \mathrm{~W}$ & 16 & PS & - & - & $2.6 \mathrm{M}_{\mathrm{L}}(\mathrm{PS})$ & FELT & DEC. & 4 & 02:55 PST \\
\hline DEC. & 4 & 195616.0 & $37.478 \mathrm{~N}$ & $118.365 \mathrm{~W}$ & 7 & RN & - & - & $3.0 \mathrm{M}_{\mathrm{D}}(\mathrm{RN})$ & & DEC. & 4 & 11:56 PST \\
\hline DEC. & 6 & 042732.2 & $37.514 \mathrm{~N}$ & $118.438 \mathrm{~W}$ & 10 & RN & - & - & $3.0 \mathrm{M}_{\mathrm{L}}(\mathrm{PS})$ & & DEC. & 5 & 20:27 PST \\
\hline DEC. & 6 & 052416.0 & $32.962 \mathrm{~N}$ & $117.802 \mathrm{~W}$ & 6 & PS & - & - & $3.1 \mathrm{M}_{\mathrm{L}}(\mathrm{PS})$ & & DEC. & 5 & 21:24 PST \\
\hline DEC. & 6 & 052436.5 & $32.930 \mathrm{~N}$ & 117.771W. & 6 & PS & - & - & $3.1 \mathrm{M}_{\mathrm{L}}(\mathrm{PS})$ & & DEC. & 5 & $21: 24$ PST \\
\hline DEC. & 7 & 123308.8 & $35.352 \mathrm{~N}$ & $120.980 \mathrm{~W}$ & 6 & BK & - & - & $3.3 \mathrm{M}_{\mathrm{L}}(\mathrm{BK})$ & IV & DEC. & 7 & 04:33 PST \\
\hline DEC. & 8 & 062903.8 & $38.113 \mathrm{~N}$ & $118.947 \mathrm{~W}$ & 10 & RN & - & - & $3.0 \mathrm{M}_{\mathrm{D}}(\mathrm{RN})$ & 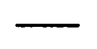 & DEC. & 5 & $22: 29$ PST \\
\hline DEC. & 8 & 074352.2 & $38.107 \mathrm{~N}$ & $118.941 \mathrm{~W}$ & 9 & RN & - & - & $3.3 \mathrm{M}_{\mathrm{D}}(\mathrm{RN})$ & & DEC. & 7 & 23:43 PST \\
\hline DEC. & 9 & 041612.9 & $38.111 \mathrm{~N}$ & $118.947 \mathrm{~W}$ & 10 & RN & - & - & $3.4 \mathrm{M}_{\mathrm{L}}(\mathrm{BK})$ & & DEC. & 8 & 20:16 PST \\
\hline DEC. & 10 & 150352.4 & $36.748 \mathrm{~N}$ & $116.320 \mathrm{~W}$ & 6 & PS & - & - & $3.0 \mathrm{M}_{\mathrm{L}}(\mathrm{PS})$ & - & DEC. & 10 & 07:03 PST \\
\hline DEC. & 11 & 141805.3 & $37.568 \mathrm{~N}$ & $121.665 \mathrm{~W}$ & 4 & BK & - & - & $4.1 \mathrm{M}_{\mathrm{L}}(\mathrm{BK})$ & IV & DEC. & 11 & 06:18 PST \\
\hline DEC. & 11 & 165247.7 & $38.110 \mathrm{~N}$ & $118.945 \mathrm{~W}$. & 6 & RN & - & - & $3.3 \mathrm{M}_{\mathrm{D}}(\mathrm{RN})$ & & DEC. & 11 & 08:52 PST \\
\hline DEC. & 11 & 165832.3 & $38.110 \mathrm{~N}$ & $118.945 \mathrm{~W}$ & 7 & RN & - & - & $3.0 \mathrm{M}_{\mathrm{D}}(\mathrm{RN})$ & & DEC. & 11 & 08:58 PST \\
\hline DEC. & 11 & 230723.9 & $37.570 \mathrm{~N}$ & $121.655 \mathrm{~W}$ & 6 & BK & - & - & $3.3 \mathrm{M}_{\mathrm{L}}(\mathrm{BK})$ & & DEC. & 11 & 15:07 PST \\
\hline DEC. & 12 & 021940.4 & $37.480 \mathrm{~N}$ & $118.512 \mathrm{~W}$ & 4 & $\mathrm{RN}$ & - & - & $3.8 \mathrm{M}_{\mathrm{L}}(\mathrm{BK})$ & 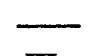 & DEC. & 11 & 18:19 PST \\
\hline DEC. & 13 & 014706.5 & $38.298 \mathrm{~N}$ & $122.153 \mathrm{~W}$ & 7 & BK & - & - & $2.9 \mathrm{M}_{\mathrm{L}}(\mathrm{BK})$ & III & DEC. & 12 & 17:47 PS \\
\hline
\end{tabular}


Table 1. Summary of United States earthquakes for 1986-Continued

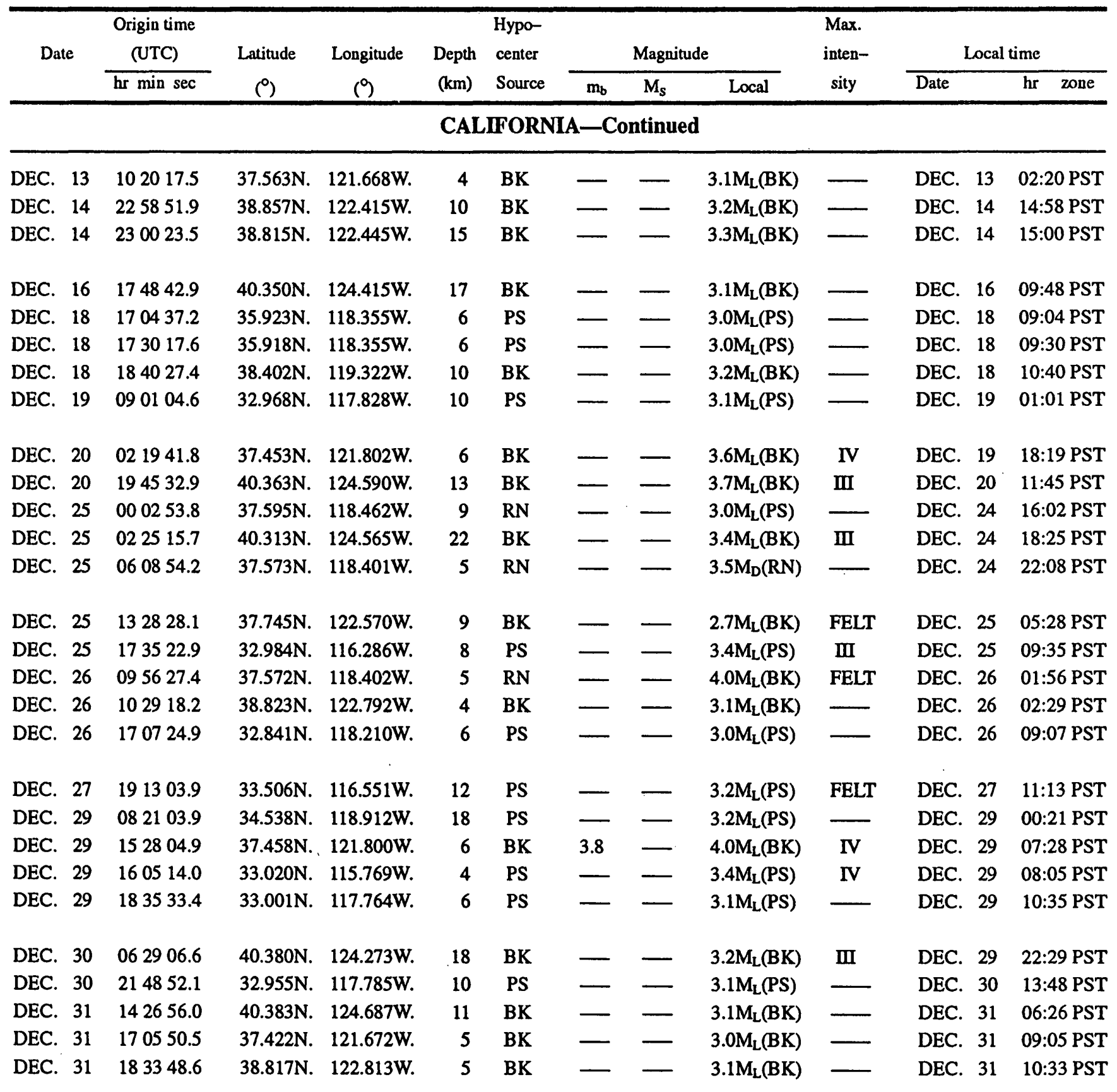

\section{COLORADO}

\begin{tabular}{|c|c|c|c|c|c|c|c|c|c|c|c|c|}
\hline APR. & 11 & 061714.7 & $38.982 \mathrm{~N}$. & $106.940 \mathrm{~W}$. & 5 & GS & - & - & $2.9 \mathrm{M}_{\mathrm{L}}(\mathrm{GS})$ & III & APR. 10 & 23:17 MST \\
\hline MAY & 9 & 215526.7 & $38.887 \mathrm{~N}$. & 106.884W. & 5 & GS & - & & $2.7 \mathrm{M}_{\mathrm{L}}(\mathrm{GS})$ & III & MAY & 14:55 MST \\
\hline AUG. & 13 & 024255.6 & $38.814 \mathrm{~N}$ & 106.996W. & 5 & GS & & & $2.6 \mathrm{M}_{\mathrm{L}}(\mathrm{GS})$ & FELT & AUG. 12 & 19:42 MST \\
\hline AUG. & 13 & 120831.4 & $38.858 \mathrm{~N}$ & 107.035W. & 5 & GS & & & $2.1 \mathrm{M}_{\mathrm{L}}(\mathrm{GS})$ & $\longrightarrow$ & AUG. 13 & 05:08 MST \\
\hline AUG. & 13 & 121343.9 & $38.879 \mathrm{~N}$. & $107.039 \mathrm{~W}$. & 5 & GS & & & $2.4 \mathrm{M}_{\mathrm{L}}(\mathrm{GS})$ & FELT & AUG. 13 & 05:13 MST \\
\hline & 14 & 173925.9 & $38.908 \mathrm{~N}$ & $107.082 \mathrm{~W}$. & 5 & GS & & & $2.6 \mathrm{M}_{\mathrm{L}}(\mathrm{GS})$ & FELT & AUG. 14 & 10:39 MS \\
\hline AUG & 17 & 221028.3 & $38.897 \mathrm{~N}$. & $107.076 \mathrm{~W}$ & 5 & GS & - & - & $2.4 \mathrm{M}_{\mathrm{L}}(\mathrm{GS})$ & FELT & AUG. 17 & $15: 10 \mathrm{MST}$ \\
\hline
\end{tabular}


Table 1. Summary of United States earthquakes for 1986-Continued

\begin{tabular}{|c|c|c|c|c|c|c|c|c|c|c|c|}
\hline \multirow[t]{2}{*}{ Date } & \multirow{2}{*}{$\begin{array}{l}\text { Origin time } \\
\text { (UTC) } \\
\text { hr min sec }\end{array}$} & \multirow{2}{*}{$\begin{array}{l}\text { Latitude } \\
\text { (0) }\end{array}$} & \multirow{2}{*}{$\begin{array}{l}\text { Longitude } \\
\text { ( })\end{array}$} & \multirow{2}{*}{$\begin{array}{l}\text { Depth } \\
(\mathrm{km})\end{array}$} & \multirow{2}{*}{$\begin{array}{l}\text { Hypo- } \\
\text { center } \\
\text { Source }\end{array}$} & \multicolumn{3}{|c|}{ Magnitude } & \multirow{2}{*}{$\begin{array}{l}\text { Max. } \\
\text { inten- } \\
\text { sity }\end{array}$} & \multicolumn{2}{|c|}{ Local time } \\
\hline & & & & & & $\mathbf{m}_{\mathrm{b}}$ & $\mathbf{M}_{s}$ & Local & & Date & zone \\
\hline \multicolumn{12}{|c|}{ COLORADO_Continued } \\
\hline AUG. 18 & 011515.0 & $38.914 \mathrm{~N}$ & $107.087 \mathrm{~W}$. & 5 & GS & 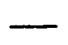 & & $3.0 \mathrm{M}_{\mathrm{L}}(\mathrm{GS})$ & III & AUG. 17 & 18:15 MST \\
\hline AUG. 20 & 044340.1 & $38.892 \mathrm{~N}$ & $107.077 \mathrm{~W}$ & 5 & GS & & . & $2.3 \mathrm{M}_{\mathrm{L}}(\mathrm{GS})$ & FELT & AUG. 19 & 21:43 MST \\
\hline AUG. 20 & 202132.9 & $38.892 \mathrm{~N}$. & 107.068W. & 5 & GS & . & - & $2.7 \mathrm{M}_{\mathrm{L}}(\mathrm{GS})$ & FELT & AUG. 20 & 13:21 MST \\
\hline AUG. 21 & 141131.6 & $38.903 \mathrm{~N}$. & $107.063 \mathrm{~W}$. & 5 & GS & 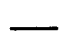 & - & $1.9 \mathrm{M}_{\mathrm{L}}(\mathrm{GS})$ & FELT & AUG. 21 & 07:11 MST \\
\hline AUG. 23 & 051303.0 & $38.905 \mathrm{~N}$ & $107.095 \mathrm{~W}$ & 5 & GS & & & $2.4 \mathrm{M}_{\mathrm{L}}(\mathrm{GS})$ & FELT & AUG. 22 & 22:13 MST \\
\hline AUG. 24 & 035917.5 & $38.967 \mathrm{~N}$. & 107.141W. & 5 & GS & - & & $2.1 \mathrm{M}_{\mathrm{L}}(\mathrm{GS})$ & FELT & AUG. 23 & 20:59 MST \\
\hline AUG. 26 & 020602.6 & $38.900 \mathrm{~N}$. & 107.041W. & 5 & GS & 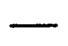 & & $3.1 \mathrm{M}_{\mathrm{L}}(\mathrm{GS})$ & IV & AUG. 25 & 19:06 MST \\
\hline AUG. 30 & 114228.6 & $38.881 \mathrm{~N}$ & $107.053 \mathrm{~W}$. & 5 & GS & 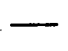 & & $2.5 \mathrm{M}_{\mathrm{L}}(\mathrm{GS})$ & & AUG. 30 & 04:42 MST \\
\hline SEPT. 3 & 062050.9 & $38.912 \mathrm{~N}$ & $107.090 \mathrm{~W}$. & 5 & GS & & & $3.5 \mathrm{M}_{\mathrm{L}}(\mathrm{GS})$ & $\mathbf{V}$ & SEPT. 2 & 23:20 MST \\
\hline SEPT. 18 & 045321.6 & $38.937 \mathrm{~N}$. & 107.116W. & 5 & GS & & & $3.2 \mathrm{M}_{\mathrm{L}}(\mathrm{GS})$ & FELT & SEPT. 17 & 21:53 MST \\
\hline SEPT. 18 & 092638.1 & $38.925 \mathrm{~N}$ & $107.086 \mathrm{~W}$. & 5 & GS & 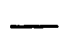 & 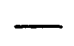 & $3.4 \mathrm{M}_{\mathrm{L}}(\mathrm{GS})$ & III & SEPT. 18 & 02:26 MST \\
\hline SEPT. 21 & 092046.6 & $39.597 \mathrm{~N}$ & $105.285 \mathrm{~W}$. & 5 & GS & - & & $2.5 \mathrm{M}_{\mathrm{L}}(\mathrm{GS})$ & FELT & SEPT. 21 & 02:20 MST \\
\hline SEPT. 22 & 062016.9 & $38.930 \mathrm{~N}$ & $107.097 \mathrm{~W}$. & 5 & GS & - & - & $2.5 \mathrm{M}_{\mathrm{L}}(\mathrm{GS})$ & - & SEPT. 21 & 23:20 MST \\
\hline OCT. 7 & 123503.2 & $38.947 \mathrm{~N}$ & 107.090W. & 5 & GS & - & $\longrightarrow$ & $1.8 \mathrm{M}_{\mathrm{L}}(\mathrm{GS})$ & FELT & OCT. & 05:35 MST \\
\hline
\end{tabular}

DELAWARE

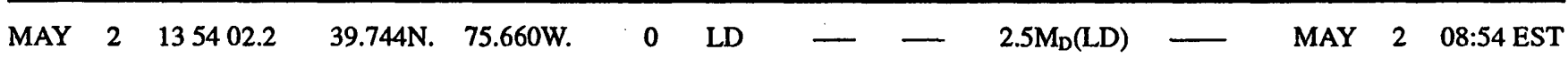

GEORGIA

\begin{tabular}{|c|c|c|c|c|c|c|c|c|c|c|c|}
\hline FEB. & 041257.9 & $33.296 \mathrm{~N}$. & 83.245W. & 1 & GT & - & - & $1.7 \mathrm{M}_{\mathrm{D}}(\mathrm{GT})$ & IV & FEB. 27 & 23:12 EST \\
\hline MAR. 13 & 022931.0 & $33.356 \mathrm{~N}$. & 83.394W. & 1 & GT & & & $2.2 \mathrm{M}_{\mathrm{D}}(\mathrm{GT})$ & IV & MAR. 12 & 21:29 ES \\
\hline JULY 11 & 142614.8 & $34.937 \mathrm{~N}$. & $84.987 \mathrm{~W}$. & 13 & TC & 3.7 & - & $3.8 \mathrm{M}_{\mathrm{n}}(\mathrm{GS})$ & VI & JULY 11 & 09:26 ES \\
\hline
\end{tabular}

\section{HAWAII}

\begin{tabular}{|c|c|c|c|c|c|c|c|c|c|c|c|c|c|}
\hline JAN. & 17 & 234831.6 & $19.359 \mathrm{~N}$. & $155.063 \mathrm{~W}$ & 10 & HV & - & - & $3.6 \mathrm{M}_{\mathrm{L}}(\mathrm{HV})$ & II & JAN. & 17 & $13: 48$ HST \\
\hline JAN. & 17 & 235215.1 & $19.363 \mathrm{~N}$. & $155.057 \mathrm{~W}$ & 0 & HV & - & - & $3.0 \mathrm{M}_{\mathrm{L}}(\mathrm{HV})$ & & JAN. & 17 & $13: 52$ HST \\
\hline JAN. & 23 & 223557.9 & $19.340 \mathrm{~N}$. & 155.199W. & 8 & HV & - & - & $3.7 \mathrm{M}_{\mathrm{L}}(\mathrm{HV})$ & III & JAN. & 23 & 12:35 HST \\
\hline JAN. & 27 & 063842.0 & $18.814 \mathrm{~N}$. & 155.239W. & 11 & HV & - & - & $3.3 \mathrm{M}_{\mathrm{L}}(\mathrm{HV})$ & & JAN. & 26 & 20:38 HST \\
\hline JAN. & 27 & 233628.1 & $19.315 \mathrm{~N}$. & $155.228 \mathrm{~W}$ & 6 & HV & - & - & $4.0 \mathrm{M}_{\mathrm{L}}(\mathrm{HV})$ & III & JAN. & 27 & 13:36 HST \\
\hline JAN. & 27 & 233757.0 & $19.311 \mathrm{~N}$. & $155.227 \mathrm{~W}$ & 7 & HV & - & - & $3.0 \mathrm{M}_{\mathrm{L}}(\mathrm{HV})$ & & JAN. & 27 & 13:37 HST \\
\hline JAN. & 30 & 151008.5 & $19.313 \mathrm{~N}$. & $155.223 \mathrm{~W}$ & 0 & HV & - & - & $3.1 \mathrm{M}_{\mathrm{L}}(\mathrm{HV})$ & & JAN. & 30 & 05:10 HST \\
\hline FEB. & 3 & 210122.2 & $19.353 \mathrm{~N}$. & $155.021 \mathrm{~W}$ & 7 & HV & - & - & $3.7 \mathrm{M}_{\mathrm{L}}(\mathrm{HV})$ & II & FEB. & 3 & 11:01 HST \\
\hline FEB. & 4 & 205633.0 & $19.553 \mathrm{~N}$. & 155.234W. & 25 & HV & - & - & $3.3 \mathrm{M}_{\mathrm{L}}(\mathrm{HV})$ & II & FEB. & 4 & 10:56 HST \\
\hline FEB. & 6 & 230353.1 & $19.397 \mathrm{~N}$. & 155.611W. & 28 & HV & - & - & $3.0 \mathrm{M}_{\mathrm{L}}(\mathrm{HV})$ & & FEB. & 6 & 13:03 HST \\
\hline $\mathbf{F}$ & 8 & 120706.9 & $19.338 \mathrm{~N}$. & $155.186 \mathrm{~W}$. & 6 & HV & - & - & $3.0 \mathrm{M}_{\mathrm{L}}(\mathrm{HV})$ & & FEB. & 8 & 02:07 HST \\
\hline FEB. & 14 & 142527.3 & $19.327 \mathrm{~N}$. & 155.194W. & 3 & HV & - & - & $3.5 \mathrm{M}_{\mathrm{L}}(\mathrm{HV})$ & 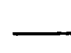 & FEB. & 14 & 04:25 HST \\
\hline
\end{tabular}


Table 1. Summary of United States earthquakes for 1986 — Continued

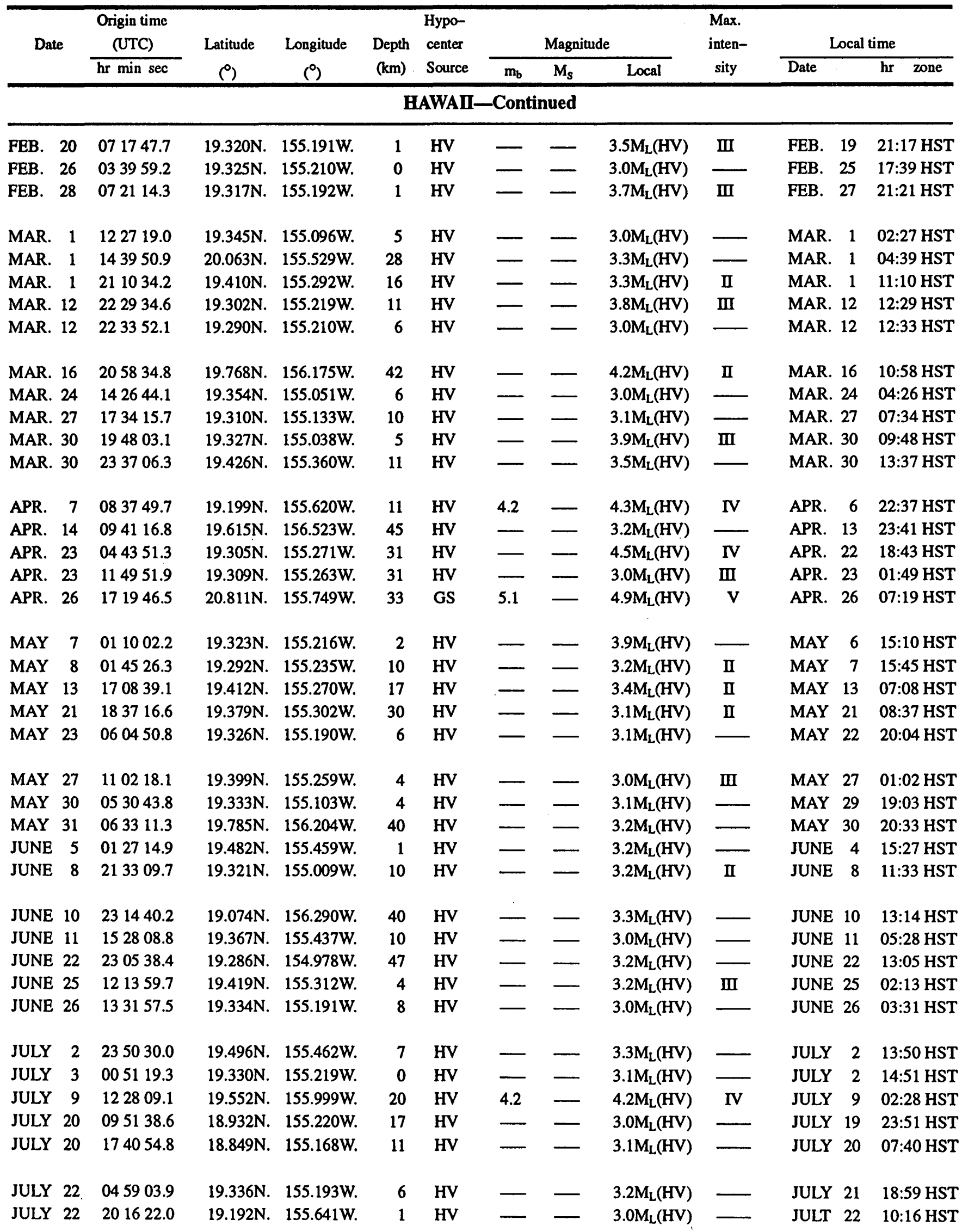


Table 1. Summary of United States earthquakes for 1986 - Continued

\begin{tabular}{|c|c|c|c|c|c|c|c|c|c|c|c|c|c|}
\hline \multirow[t]{2}{*}{ Date } & & \multirow{2}{*}{$\begin{array}{c}\text { Origin time } \\
\text { (UTC) } \\
\text { hr min sec }\end{array}$} & \multirow{2}{*}{$\begin{array}{l}\text { Latitude } \\
\text { (?) }\end{array}$} & \multirow{2}{*}{$\begin{array}{l}\text { Longitude } \\
\text { (9) }\end{array}$} & \multirow{2}{*}{$\begin{array}{r}\text { Depth } \\
(\mathrm{km})\end{array}$} & \multirow{2}{*}{$\begin{array}{l}\text { Hypo- } \\
\text { center } \\
\text { Source }\end{array}$} & \multicolumn{3}{|c|}{ Magnitude } & \multirow{2}{*}{$\begin{array}{l}\text { Max. } \\
\text { inten- } \\
\text { sity }\end{array}$} & \multicolumn{3}{|c|}{ Local time } \\
\hline & & & & & & & $\mathrm{m}_{\mathrm{b}}$ & $\mathrm{M}_{\mathbf{s}}$ & Local & & Date & & zone \\
\hline \multicolumn{14}{|c|}{ HAWAII-Continued } \\
\hline JULY & 28 & 124525.4 & $19.537 \mathrm{~N}$. & 155.970W. & 11 & HV & - & - & $3.1 \mathrm{M}_{\mathrm{L}}(\mathrm{HV})$ & II & JULY & 28 & 02:45 HST \\
\hline JULY & 30 & 080426.0 & $19.344 \mathrm{~N}$. & $155.102 \mathrm{~W}$ & 8 & HV & - & - & $3.3 \mathrm{M}_{\mathrm{L}}(\mathrm{HV})$ & & JULY & 29 & 22:04 HST \\
\hline AUG. & 11 & 031612.2 & $19.372 \mathrm{~N}$. & $155.079 \mathrm{~W}$ & 10 & HV & - & - & $3.7 \mathrm{M}_{\mathrm{L}}(\mathrm{HV})$ & II & AUG. & 10 & 17:16 HST \\
\hline AUG. & 11 & 161113.5 & $19.339 \mathrm{~N}$. & $155.041 \mathrm{~W}$ & 9 & HV & - & - & $3.8 \mathrm{M}_{\mathrm{L}}(\mathrm{HV})$ & 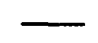 & AUG. & 11 & 06:11 HST \\
\hline AUG. & 21 & 231451.9 & $19.634 \mathrm{~N}$. & 155.155W. & 15 & HV & - & - & $3.1 \mathrm{M}_{\mathrm{L}}(\mathrm{HV})$ & & AUG. & 21 & $13: 14 \mathrm{HST}$ \\
\hline SEPT. & 4 & 025511.2 & $19.352 \mathrm{~N}$. & $155.503 \mathrm{~W}$ & 10 & HV & - & - & $3.6 \mathrm{M}_{\mathrm{L}}(\mathrm{HV})$ & & SEPT. & 3 & 16:55 HST \\
\hline SEPT. & 5 & 211206.1 & $19.306 \mathrm{~N}$. & $155.309 \mathrm{~W}$ & 32 & HV & - & - & $3.1 \mathrm{M}_{\mathrm{L}}(\mathrm{HV})$ & & SEPT. & 5 & $11: 12 \mathrm{HST}$ \\
\hline SEPT. & 8 & 141621.0 & $21.284 \mathrm{~N}$. & $156.782 \mathrm{~W}$ & 1 & HV & - & - & $3.3 \mathrm{M}_{\mathrm{L}}(\mathrm{HV})$ & II & SEPT. & 8 & 04:16 HST \\
\hline SEPT. & 9 & 061255.2 & $19.305 \mathrm{~N}$. & $155.197 \mathrm{~W}$ & 8 & HV & - & - & $3.2 \mathrm{M}_{\mathrm{L}}(\mathrm{HV})$ & - & SEPT. & 8 & 20:12 HST \\
\hline SEPT. & 13 & 220637.7 & $19.430 \mathrm{~N}$. & $155.324 \mathrm{~W}$. & 6 & HV & 一 & - & $3.1 \mathrm{M}_{\mathrm{L}}(\mathrm{HV})$ & - & SEPT. & 13 & 12:06 HST \\
\hline SEPT. & 15 & 043916.8 & $18.789 \mathrm{~N}$. & $155.235 \mathrm{~W}$ & 43 & HV & - & - & $3.0 \mathrm{M}_{\mathrm{L}}(\mathrm{HV})$ & 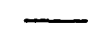 & SEPT. & 14 & 18:39 HST \\
\hline SEPT. & & 144442.5 & $19.333 \mathrm{~N}$. & $155.349 \mathrm{~W}$ & 31 & HV & - & - & $4.2 \mathrm{M}_{\mathrm{L}}(\mathrm{HV})$ & IV & SEPT. & 19 & 04:44 HST \\
\hline SEPT. & & 053137.1 & $17.696 \mathrm{~N}$. & $154.839 \mathrm{~W}$ & 40 & HV & - & - & $3.7 \mathrm{M}_{\mathrm{L}}(\mathrm{HV})$ & & SEPT. & 20 & 19:31 HST \\
\hline SEPT. & & 063501.7 & $18.844 \mathrm{~N}$. & $155.237 \mathrm{~W}$ & 12 & HV & - & - & $3.4 \mathrm{M}_{\mathrm{L}}(\mathrm{HV})$ & $\longrightarrow$ & SEPT. & 20 & 20:35 HST \\
\hline SEPT. & 21 & 065722.2 & $18.766 \mathrm{~N}$. & $155.228 \mathrm{~W}$ & 12 & HV & - & - & $3.6 \mathrm{M}_{\mathrm{L}}(\mathrm{HV})$ & 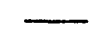 & SEPT. & 20 & 20:57 HST \\
\hline SEPT. & 21 & 070246.0 & $18.823 \mathrm{~N}$. & 155.263W. & 12 & HV & - & - & $3.3 \mathrm{M}_{\mathrm{L}}(\mathrm{HV})$ & 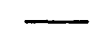 & SEPT. & 20 & 21:02 HST \\
\hline SEPT. & 21 & 071504.8 & $18.793 \mathrm{~N}$. & 155.273W. & 12 & HV & - & - & $3.6 \mathrm{M}_{\mathrm{L}}(\mathrm{HV})$ & 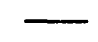 & SEPT. & 20 & 21:15 HST \\
\hline SEPT. & 21 & 072806.5 & $18.787 \mathrm{~N}$. & $155.265 \mathrm{~W}$ & 12 & HV & 一 & - & $3.2 \mathrm{M}_{\mathrm{L}}(\mathrm{HV})$ & - & SEPT. & 20 & 21:28 HST \\
\hline SEPT. & 21 & 081016.5 & $18.843 \mathrm{~N}$. & $155.268 \mathrm{~W}$ & 12 & HV & - & - & $3.3 \mathrm{M}_{\mathrm{L}}(\mathrm{HV})$ & 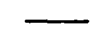 & SEPT. & 20 & 22:10 HST \\
\hline SEPT. & 21 & 093033.6 & $18.795 \mathrm{~N}$. & $155.268 \mathrm{~W}$ & 12 & HV & - & - & $3.7 \mathrm{M}_{\mathrm{L}}(\mathrm{HV})$ & IV & SEPT. & 20 & 23:30 HST \\
\hline SEPT. & 21 & 145845.9 & $18.829 \mathrm{~N}$. & 155.352W. & 25 & HV & - & - & $3.1 \mathrm{M}_{\mathrm{L}}(\mathrm{HV})$ & & SEPT. & 21 & 04:58 HST \\
\hline SEPT. & 21 & 174926.6 & $18.813 \mathrm{~N}$. & 155.324W. & 35 & HV & - & - & $3.4 \mathrm{M}_{\mathrm{L}}(\mathrm{HV})$ & & SEPT. & 21 & 07:49 HST \\
\hline SEPT. & 22 & 111047.6 & $19.301 \mathrm{~N}$. & $155.257 \mathrm{~W}$ & 6 & HV & - & - & $3.3 \mathrm{M}_{\mathrm{L}}(\mathrm{HV})$ & - & SEPT. & 22 & 01:10 HST \\
\hline SEPT. & 23 & 171602.0 & $19.979 \mathrm{~N}$. & $155.502 \mathrm{~W}$. & 37 & HV & - & - & $3.9 \mathrm{M}_{\mathrm{L}}(\mathrm{HV})$ & IV & SEPT. & 23 & 07:16 HST \\
\hline OCT. & 1 & 080216.0 & $19.702 \mathrm{~N}$. & $155.224 \mathrm{~W}$ & 36 & HV & - & - & $3.7 \mathrm{M}_{\mathrm{L}}(\mathrm{HV})$ & IV & SEPT. & 30 & 22:02 HST \\
\hline OCT. & 7 & 091745.3 & $19.368 \mathrm{~N}$. & 155.487W. & 11 & HV & - & - & $3.0 \mathrm{M}_{\mathrm{L}}(\mathrm{HV})$ & & OCT. & 6 & 23:17 HST \\
\hline OCT. & 15 & 161518.2 & $19.367 \mathrm{~N}$. & 155.481W. & 10 & HV & - & - & $3.0 \mathrm{M}_{\mathrm{L}}(\mathrm{HV})$ & 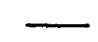 & OCT. & 15 & 06:15 HST \\
\hline OCT. & 20 & 195304.3 & $19.330 \mathrm{~N}$. & 155.139W. & 0 & HV & - & - & $3.0 \mathrm{M}_{\mathrm{L}}(\mathrm{HV})$ & - & OCT. & 20 & 09:53 HST \\
\hline OCT. & 27 & 024151.0 & $19.329 \mathrm{~N}$. & $155.020 \mathrm{~W}$ & 6 & HV & - & - & $3.0 \mathrm{M}_{\mathrm{L}}(\mathrm{HV})$ & & OCT. & 26 & 16:41 HST \\
\hline NOV. & 2 & 030818.2 & $19.380 \mathrm{~N}$. & $155.247 \mathrm{~W}$ & 31 & HV & - & - & $3.0 \mathrm{M}_{\mathrm{L}}(\mathrm{HV})$ & & NOV. & 1 & 17:08 HST \\
\hline NOV. & 6 & 092518.2 & $19.319 \mathrm{~N}$. & 155.213W. & 11 & HV & 一 & - & $3.7 \mathrm{M}_{\mathrm{L}}(\mathrm{HV})$ & & NOV. & 5 & 23:25 HST \\
\hline NOV. & 6 & 220244.1 & $19.177 \mathrm{~N}$. & 155.694W. & 0 & HV & - & - & $3.1 \mathrm{M}_{\mathrm{L}}(\mathrm{HV})$ & & NOV. & 6 & 12:02 HST \\
\hline NOV. & 15 & 205854.3 & $19.344 \mathrm{~N}$. & 155.218W. & 8 & HV & - & - & $4.0 \mathrm{M}_{\mathrm{L}}(\mathrm{HV})$ & III & NOV. & 15 & 10:58 HST \\
\hline NOV. & 18 & 024022.6 & $20.189 \mathrm{~N}:$ & $155.774 \mathrm{~W}$ & 37 & HV & - & - & $3.7 \mathrm{M}_{\mathrm{L}}(\mathrm{HV})$ & III & NOV. & 17 & 16:40 HST \\
\hline NOV. & 19 & 191341.7 & $19.307 \mathrm{~N}$. & $155.137 \mathrm{~W}$ & 9 & $\mathrm{HV}$ & - & - & $3.2 \mathrm{M}_{\mathrm{L}}(\mathrm{HV})$ & - & NOV. & 19 & 09:13 HST \\
\hline DEC. & 5 & 155334.9 & $19.325 \mathrm{~N}$. & 155.198W. & 6 & HV & - & - & $3.1 \mathrm{M}_{\mathrm{L}}(\mathrm{HV})$ & 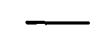 & DEC. & 5 & 05:53 HST \\
\hline DEC. & 6 & 221040.6 & $19.360 \mathrm{~N}$. & $155.038 \mathrm{~W}$ & 1 & HV & - & - & $3.8 \mathrm{M}_{\mathrm{L}}(\mathrm{HV})$ & IV & DEC. & 6 & 12:10 HST \\
\hline DEC. & 7 & 024530.1 & $20.864 \mathrm{~N}$. & $156.036 \mathrm{~W}$ & 38 & HV & - & - & $4.2 \mathrm{M}_{\mathrm{L}}(\mathrm{HV})$ & IV & DEC. & 6 & 16:45 HST \\
\hline DEC. & 8 & 170753.5 & $19.315 \mathrm{~N}$. & 155.225W. & 0 & HV & - & - & $3.1 \mathrm{M}_{\mathrm{L}}(\mathrm{HV})$ & - & DEC. & 8 & 07:07 HST \\
\hline DEC. & 11 & 004742.9 & $19.335 \mathrm{~N}$. & 155.036W. & 4 & HV & - & - & $3.2 \mathrm{M}_{\mathrm{L}}(\mathrm{HV})$ & III & DEC. & 10 & 14:47 HST \\
\hline
\end{tabular}


Table 1. Summary of United States earthquakes for 1986_-Continued

\begin{tabular}{|c|c|c|c|c|c|c|c|c|c|c|c|c|}
\hline \multirow{3}{*}{ Date } & \multirow{2}{*}{$\begin{array}{c}\text { Origin time } \\
\text { (UTC) }\end{array}$} & \multirow{3}{*}{$\begin{array}{l}\text { Latitude } \\
\text { (9) }\end{array}$} & \multirow{3}{*}{$\begin{array}{l}\text { Longitude } \\
\text { (9) }\end{array}$} & \multirow{3}{*}{$\begin{array}{c}\text { Depth } \\
(\mathrm{km})\end{array}$} & \multirow{3}{*}{$\begin{array}{l}\text { Hypo- } \\
\text { center } \\
\text { Source }\end{array}$} & \multirow{2}{*}{\multicolumn{3}{|c|}{ Magnitude }} & Max. & \multirow{2}{*}{\multicolumn{3}{|c|}{ Local time }} \\
\hline & & & & & & & & & inten- & & & \\
\hline & hr $\min \sec$ & & & & & $\mathbf{m}_{\mathrm{b}}$ & $\mathbf{M}_{S}$ & Local & sity & Date & hr & zone \\
\hline
\end{tabular}

IDAHO

\begin{tabular}{|c|c|c|c|c|c|c|c|c|c|c|c|c|c|}
\hline JAN. & 5 & 015433.0 & $44.305 \mathrm{~N}$ & $114.138 \mathrm{~W}$. & 13 & BU & - & - & $2.8 \mathrm{M}_{\mathrm{L}}(\mathrm{BU})$ & - & JAN. & 4 & 18:54 MST \\
\hline JAN. & 6 & 045204.6 & $44.092 \mathrm{~N}$ & $113.941 \mathrm{~W}$ & 24 & BU & - & - & $2.5 \mathrm{M}_{\mathrm{L}}(\mathrm{BU})$ & & JAN. & 5 & 21:52 MST \\
\hline JAN. & 10 & 031215.1 & $44.623 \mathrm{~N}$ & $116.001 \mathrm{~W}$ & 10 & BU & & - & $2.9 \mathrm{M}_{\mathrm{D}}(\mathrm{BU})$ & & JAN. & 9 & 20:12 MST \\
\hline JAN. & 11 & 192455.5 & $44.654 \mathrm{~N}$ & $113.902 \mathrm{~W}$ & 1 & BU & & & $2.7 \mathrm{M}_{\mathrm{L}}(\mathrm{BU})$ & & JAN. & 11 & 12:24 MST \\
\hline JAN. & 15 & 160504.9 & $44.813 \mathrm{~N}$ & $114.489 \mathrm{~W}$ & 10 & $\mathrm{BU}$ & & & $2.8 \mathrm{M}_{\mathrm{L}}(\mathrm{BU})$ & & JAN. & 15 & 09:16 MST \\
\hline JAN. & 16 & 020158.4 & $44.447 \mathrm{~N}$ & $114.232 \mathrm{~W}$ & 5 & GS & & - & $3.1 \mathrm{M}_{\mathrm{L}}(\mathrm{GS})$ & & JAN. & 15 & 19:01 MST \\
\hline JAN. & 16 & 112754.0 & $44.476 \mathrm{~N}$ & $114.108 \mathrm{~W}$ & 13 & BU & - & - & $2.8 \mathrm{M}_{\mathrm{L}}(\mathrm{BU})$ & & JAN. & 16 & 04:27 MST \\
\hline JAN. & 18 & 143950.3 & $44.412 \mathrm{~N}$ & $113.802 W$ & 28 & BU & & - & $2.7 \mathrm{M}_{\mathrm{L}}(\mathrm{BU})$ & & JAN. & 18 & 07:39 MST \\
\hline JAN. & 28 & 054501.5 & $44.153 \mathrm{~N}$ & 113.946W. & 5 & GS & 一 & 一 & $4.0 \mathrm{M}_{\mathrm{L}}(\mathrm{GS})$ & IV & JAN. & 27 & $22: 45$ MST \\
\hline JAN. & 28 & 071532.8 & $44.184 \mathrm{~N}$ & $113.955 \mathrm{~W}$ & 5 & GS & 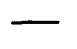 & 一 & $3.7 \mathrm{M}_{\mathrm{L}}(\mathrm{GS})$ & & JAN. & 28 & 00:15 MST \\
\hline JAN. & 28 & 072657.5 & $44.111 \mathrm{~N}$ & $113.901 \mathrm{~W}$ & 24 & BU & - & 一 & $3.3 \mathrm{M}_{\mathrm{L}}(\mathrm{GS})$ & & JAN. & 27 & 00:26 MST \\
\hline JAN. & 30 & 114755.3 & $44.780 \mathrm{~N}$. & $111.491 \mathrm{~W}$ & 20 & BU & - & - & $2.5 \mathrm{M}_{\mathrm{L}}(\mathrm{BU})$ & & JAN. & 30 & 04:47 MST \\
\hline FEB. & 1 & 121244.0 & $44.278 \mathrm{~N}$ & $114.036 \mathrm{~W}$. & 21 & BU & & - & $2.9 \mathrm{M}_{\mathrm{L}}(\mathrm{BU})$ & & FEB. & 1 & 05:12 MST \\
\hline FEB. & 5 & 152528.1 & $44.234 \mathrm{~N}$ & $114.006 \mathrm{~W}$. & 18 & BU & & - & $3.3 \mathrm{M}_{\mathrm{L}}(\mathrm{BU})$ & & FEB. & 5 & 08:25 MST \\
\hline FEB. & 9 & 070351.3 & $44.363 \mathrm{~N}$ & $113.930 \mathrm{~W}$ & 29 & BU & & - & $3.1 \mathrm{M}_{\mathrm{L}}(\mathrm{BU})$ & & FEB. & 8 & 00:03 MST \\
\hline FEB. & 10 & 001743.7 & $44.644 \mathrm{~N}$. & $114.712 \mathrm{~W}$ & 21 & BU & - & - & $2.6 \mathrm{M}_{\mathrm{L}}(\mathrm{BU})$ & & FEB. & 9 & 17:17 MST \\
\hline FEB. & 16 & 125444.0 & $44.627 \mathrm{~N}$ & $114.088 \mathrm{~W}$ & 13 & BU & & - & $2.8 \mathrm{M}_{\mathrm{L}}(\mathrm{BU})$ & & FEB. & 16 & 05:54 MST \\
\hline FEB. & 17 & 085338.6 & $42.596 \mathrm{~N}$ & $111.301 \mathrm{~W}$ & 5 & GS & & - & $3.0 \mathrm{M}_{\mathrm{L}}(\mathrm{GS})$ & III & FEB. & 17 & 01:53 MST \\
\hline FEB. & 22 & 180718.7 & $44.578 \mathrm{~N}$ & $114.232 \mathrm{~W}$ & 10 & BU & & - & $2.7 \mathrm{M}_{\mathrm{L}}(\mathrm{BU})$ & & FEB. & 22 & $11: 07$ MST \\
\hline FEB. & 24 & 031333.0 & 43.081N. & $111.224 \mathrm{~W}$ & 5 & GS & 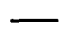 & - & $2.8 \mathrm{M}_{\mathrm{L}}(\mathrm{GS})$ & III & FEB. & 23 & 20:13 MST \\
\hline FEB. & 26 & 150549.3 & $44.627 \mathrm{~N}$ & 114.192W. & 5 & GS & - & 一 & $3.7 \mathrm{M}_{\mathrm{L}}(\mathrm{GS})$ & & FEB. & 26 & 08:05 MST \\
\hline FEB. & 28 & 220910.1 & $44.328 \mathrm{~N}$ & $114.080 \mathrm{~W}$ & 22 & BU & - & - & $2.6 \mathrm{M}_{\mathrm{L}}(\mathrm{BU})$ & & FEB. & 28 & 15:09 MST \\
\hline MAR. & 8 & 205835.2 & $44.397 \mathrm{~N}$ & $113.999 \mathrm{~W}$ & 4 & BU & - & - & $2.8 \mathrm{M}_{\mathrm{L}}(\mathrm{BU})$ & & MAR. & 8 & 13:58 MST \\
\hline MAR. & 11 & 225704.9 & $44.485 \mathrm{~N}$ & 114.146W. & 19 & BU & - & - & $3.2 \mathrm{M}_{\mathrm{L}}(\mathrm{BU})$ & & MAR. & 11 & $15: 57$ MST \\
\hline MAR. & 12 & 124235.1 & $44.771 \mathrm{~N}$ & $112.812 \mathrm{~W}$ & 5 & GS & & - & $2.6 \mathrm{M}_{\mathrm{L}}(\mathrm{GS})$ & & MAR. & 12 & 05:42 MST \\
\hline MAR. & 12 & 163256.0 & $47.470 \mathrm{~N}$ & $115.800 \mathrm{~W}$ & 1 & GS & - & - & $2.6 \mathrm{M}_{\mathrm{L}}(\mathrm{BU})$ & FELT & MAR. & 12 & 08:32 PST \\
\hline MAR. & 23 & 142611.8 & $44.407 \mathrm{~N}$ & $114.213 \mathrm{~W}$ & 5 & GS & - & - & $3.1 \mathrm{M}_{\mathrm{L}}(\mathrm{GS})$ & & MAR. & 23 & 07:26 MST \\
\hline MAR. & 31 & 181351.3 & $44.251 \mathrm{~N}$ & $114.668 \mathrm{~W}$ & 34 & BU & - & - & $2.7 \mathrm{M}_{\mathrm{L}}(\mathrm{BU})$ & & MAR. & 31 & 11:13 MST \\
\hline APR. & 7 & 140725.8 & $44.337 \mathrm{~N}$ & $114.177 \mathrm{~W}$ & 5 & GS & - & & $4.1 \mathrm{M}_{\mathrm{L}}(\mathrm{GS})$ & III & APR. & 7 & 07:07 MST \\
\hline APR. & 11 & 093129.1 & $44.240 \mathrm{~N}$ & $114.004 \mathrm{~W}$ & 22 & BU & 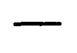 & - & $2.7 \mathrm{M}_{\mathrm{L}}(\mathrm{BU})$ & & APR. & 11 & 02:31 MST \\
\hline APR. & 11 & 101358.7 & $44.572 \mathrm{~N}$ & $114.294 \mathrm{~W}$ & 18 & BU & - & - & $2.8 \mathrm{M}_{\mathrm{L}}(\mathrm{BU})$ & & APR. & 11 & 03:13 MST \\
\hline APR. & 13 & 050250.1 & $44.283 \mathrm{~N}$. & $114.153 \mathrm{~W}$ & 5 & GS & - & - & $3.0 \mathrm{M}_{\mathrm{L}}(\mathrm{GS})$ & & APR. & 12 & 22:02 MST \\
\hline APR. & 14 & 100255.5 & $44.619 \mathrm{~N}$ & $113.992 \mathrm{~W}$ & 23 & BU & - & - & $3.0 \mathrm{M}_{\mathrm{L}}(\mathrm{BU})$ & & APR. & 14 & 03:02 MST \\
\hline APR. & 15 & 060550.4 & $44.310 \mathrm{~N}$ & $114.172 \mathrm{~W}$ & 17 & BU & $\longrightarrow$ & - & $3.7 \mathrm{M}_{\mathrm{L}}(\mathrm{BU})$ & - & APR. & 14 & 23:05 MST \\
\hline APR. & 16 & 062527.6 & $44.271 \mathrm{~N}$ & $114.099 \mathrm{~W}$ & 5 & GS & 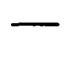 & - & $3.5 \mathrm{M}_{\mathrm{L}}(\mathrm{GS})$ & & APR. & 15 & 23:25 MST \\
\hline APR. & 16 & 175447.2 & $44.613 \mathrm{~N}$ & 114.423W. & 17 & BU & - & - & $2.5 \mathrm{M}_{\mathrm{L}}(\mathrm{BU})$ & & APR. & 16 & 10:54 MST \\
\hline APR. & 17 & 031038.9 & $44.323 \mathrm{~N}$ & $114.119 \mathrm{~W}$ & 5 & GS & - & - & $3.0 \mathrm{M}_{\mathrm{L}}(\mathrm{GS})$ & & APR. & 16 & 20:10 MST \\
\hline APR. & 20 & 023155.3 & $44.136 \mathrm{~N}$ & $114.925 \mathrm{~W}$ & 5 & GS & 一 & - & $2.8 \mathrm{M}_{\mathrm{L}}(\mathrm{GS})$ & & A.PR. & 19 & 19:31 MST \\
\hline APR. & 20 & 171225.4 & $44.105 \mathrm{~N}$ & 113.823W. & 21 & $\mathrm{BU}$ & - & - & $2.6 \mathrm{M}_{\mathrm{L}}(\mathrm{BU})$ & & APR. & 20 & 10:12 MST \\
\hline APR. & 26 & 013016.5 & $44.665 \mathrm{~N}$ & $112.869 \mathrm{~W}$. & 1 & BU & - & - & $2.6 \mathrm{M}_{\mathrm{L}}(\mathrm{GS})$ & & APR. & 25 & 18:30 MST \\
\hline
\end{tabular}


Table 1. Summary of United States earthquakes for 1986—Continued

\begin{tabular}{|c|c|c|c|c|c|c|c|c|c|c|c|c|c|}
\hline \multirow[t]{2}{*}{ Date } & & \multirow{2}{*}{$\begin{array}{l}\text { Origin time } \\
\text { (UTC) } \\
\text { hr min sec }\end{array}$} & \multirow{2}{*}{$\begin{array}{l}\text { Latitude } \\
\text { (?) }\end{array}$} & \multirow{2}{*}{$\begin{array}{l}\text { Longitude } \\
\text { (9) }\end{array}$} & \multirow{2}{*}{$\begin{array}{l}\text { Depth } \\
(\mathrm{km})\end{array}$} & \multirow{2}{*}{$\begin{array}{l}\text { Hypo- } \\
\text { center } \\
\text { Source }\end{array}$} & \multicolumn{3}{|c|}{ Magnitude } & \multirow{2}{*}{$\begin{array}{l}\text { Max. } \\
\text { inten- } \\
\text { sity }\end{array}$} & \multicolumn{3}{|c|}{ Local time } \\
\hline & & & & & & & $m_{b}$ & $\mathrm{M}_{\boldsymbol{s}}$ & Local & & Date & & zone \\
\hline \multicolumn{14}{|c|}{ DAHO_Continued } \\
\hline APR. & 28 & 120023.8 & $44.492 \mathrm{~N}$. & $114.843 \mathrm{~W}$ & 8 & BU & - & - & $2.8 \mathrm{M}_{\mathrm{L}}(\mathrm{BU})$ & & APR. & 28 & 05:00 MST \\
\hline APR. & 28 & 125108.3 & $44.573 \mathrm{~N}$. & $114.879 \mathrm{~W}$ & 5 & GS & - & - & $2.5 \mathrm{M}_{\mathrm{L}}(\mathrm{GS})$ & & APR. & 28 & 05:51 MST \\
\hline MAY & 15 & 150626.3 & $44.687 \mathrm{~N}$. & $114.444 \mathrm{~W}$ & 5 & BU & - & - & $2.6 \mathrm{M}_{\mathrm{L}}(\mathrm{BU})$ & & MAY & 15 & 08:06 MST \\
\hline MAY & 17 & 040748.7 & $44.187 \mathrm{~N}$ & $113.947 \mathrm{~W}$ & 12 & BU & - & - & $3.4 \mathrm{M}_{\mathrm{L}}(\mathrm{BU})$ & - & MAY & 16 & 21:07 MST \\
\hline MAY & 19 & 021710.0 & $44.583 \mathrm{~N}$. & $115.180 \mathrm{~W}$ & 32 & BU & - & - & $2.9 \mathrm{M}_{\mathrm{L}}(\mathrm{BU})$ & & MAY & 18 & 19:17 MST \\
\hline MAY & 21 & 030457.6 & $44.658 \mathrm{~N}$ & $113.984 \mathrm{~W}$. & 6 & BU & - & - & $2.6 \mathrm{M}_{\mathrm{L}}(\mathrm{BU})$ & - & MAY & 20 & 20:04 MST \\
\hline JUNE & 2 & 225638.8 & $44.259 \mathrm{~N}$ & $114.061 \mathrm{~W}$ & 18 & BU & - & - & $2.7 \mathrm{M}_{\mathrm{L}}(\mathrm{BU})$ & - & JUNE & 2 & 15:56 MST \\
\hline JUNE & 21 & 203053.5 & $42.793 \mathrm{~N}$ & $111.153 \mathrm{~W}$ & 5 & GS & - & - & $3.5 \mathrm{M}_{\mathrm{L}}(\mathrm{GS})$ & III & JUNE & 21 & 13:30 MST \\
\hline JUNE & 22 & 020247.1 & $44.950 \mathrm{~N}$. & $112.784 \mathrm{~W}$ & 5 & GS & - & - & $2.7 \mathrm{M}_{\mathrm{L}}(\mathrm{GS})$ & 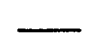 & JUNE & 21 & 19:02 MST \\
\hline JULY & 7 & 115317.2 & $43.248 \mathrm{~N}$ & $111.090 \mathrm{~W}$ & 5 & GS & - & - & $3.3 \mathrm{M}_{\mathrm{L}}(\mathrm{GS})$ & & JULY & 7 & 04:53 MST \\
\hline JULY & 20 & 022920.3 & $44.415 \mathrm{~N}$ & $116.002 \mathrm{~W}$ & 5 & GS & - & - & $3.2 \mathrm{M}_{\mathrm{L}}(\mathrm{GS})$ & & JULY & 19 & 19:29 MST \\
\hline JULY & 20 & 190532.6 & $44.455 \mathrm{~N}$ & $116.033 \mathrm{~W}$ & 5 & GS & - & - & $3.6 \mathrm{M}_{\mathrm{L}}(\mathrm{GS})$ & & JULY & 20 & 12:05 MST \\
\hline JULY & 29 & 190418.9 & $44.247 \mathrm{~N}$ & 114.136W. & 5 & GS & - & - & $3.5 \mathrm{M}_{\mathrm{L}}(\mathrm{GS})$ & & JULY & 29 & 12:04 MST \\
\hline JULY & 30 & 081907.8 & $42.437 \mathrm{~N}$ & $111.242 \mathrm{~W}$ & 7 & UU & - & - & $3.5 \mathrm{M}_{\mathrm{L}}(\mathrm{GS})$ & & JULY & 30 & 01:19 MST \\
\hline AUG. & 3 & 044816.4 & $44.469 \mathrm{~N}$ & 114.150W. & 14 & BU & - & - & $2.9 \mathrm{M}_{\mathrm{L}}(\mathrm{BU})$ & & AUG. & 2 & 21:48 MST \\
\hline AUG. & 6 & 093742.1 & $44.152 \mathrm{~N}$ & $114.612 \mathrm{~W}$ & 23 & BU & - & - & $2.9 \mathrm{M}_{\mathrm{L}}(\mathrm{BU})$ & & AUG. & 6 & 02:37 MST \\
\hline AUG. & 10 & 100558.1 & $44.512 \mathrm{~N}$ & 114.261W. & 20 & BU & - & - & $3.0 \mathrm{M}_{\mathrm{L}}(\mathrm{BU})$ & & AUG. & 10 & 03:05 MST \\
\hline AUG. & 17 & 085920.7 & $44.118 \mathrm{~N}$ & $113.967 \mathrm{~W}$ & 16 & BU & - & - & $2.6 \mathrm{M}_{\mathrm{L}}(\mathrm{BU})$ & & AUG. & 17 & 01:59 MST \\
\hline AUG. & 18 & 203021.7 & $44.460 \mathrm{~N}$ & 114.190W. & 18 & BU & - & - & $2.8 \mathrm{M}_{\mathrm{L}}(\mathrm{BU})$ & & AUG. & 18 & $13: 30 \mathrm{MST}$ \\
\hline AUG. & 28 & 151258.9 & $44.215 \mathrm{~N}$ & $114.260 \mathrm{~W}$ & 19 & BU & - & - & $2.8 \mathrm{M}_{\mathrm{L}}(\mathrm{BU})$ & & AUG. & 28 & 08:12 MST \\
\hline AUG. & 29 & 082624.3 & $42.095 \mathrm{~N}$ & $111.649 \mathrm{~W}$ & 4 & UU & - & - & $3.2 \mathrm{M}_{\mathrm{L}}(\mathrm{UU})$ & 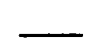 & AUG. & 29 & 01:26 MST \\
\hline AUG. & 29 & 093734.7 & $42.096 \mathrm{~N}$ & $111.650 \mathrm{~W}$ & 1 & UU & - & - & $2.4 \mathrm{M}_{\mathrm{L}}(\mathrm{UU})$ & III & AUG. & 29 & 02:37 MST \\
\hline SEPT. & 1 & 020324.6 & $44.460 \mathrm{~N}$ & 114.280W. & 21 & BU & - & - & $2.7 \mathrm{M}_{\mathrm{L}}(\mathrm{BU})$ & & AUG. & 31 & 19:03 MST \\
\hline SEPT. & 3 & 061111.3 & $44.010 \mathrm{~N}$ & 114.792W. & 5 & GS & - & - & $3.2 \mathrm{M}_{\mathrm{L}}(\mathrm{GS})$ & & SEPT. & 2 & 23:11 MST \\
\hline SEPT. & 3 & 185349.1 & $44.039 \mathrm{~N}$ & 114.764W. & 5 & GS & - & - & $3.9 \mathrm{M}_{\mathrm{L}}(\mathrm{GS})$ & III & SEPT. & 3 & 11:53 MST \\
\hline SEPT. & 4 & 001458.3 & $43.970 \mathrm{~N}$ & $114.658 \mathrm{~W}$ & 24 & BU & 一 & - & $2.9 \mathrm{M}_{\mathrm{L}}(\mathrm{BU})$ & & SEPT. & 3 & 17:14 MST \\
\hline SEPT. & 4 & 041555.8 & $43.993 \mathrm{~N}$ & $114.803 \mathrm{~W}$ & 5 & GS & - & - & $3.4 \mathrm{M}_{\mathrm{L}}(\mathrm{GS})$ & & SEPT. & 3 & $21: 15$ MST \\
\hline SEPT. & 4 & 043820.0 & $44.066 \mathrm{~N}$ & $114.745 \mathrm{~W}$ & 5 & GS & - & - & $4.0 \mathrm{M}_{\mathrm{L}}(\mathrm{GS})$ & & SEPT. & 3 & $21: 38 \mathrm{MST}$ \\
\hline SEPT. & 4 & 124347.6 & $44.036 \mathrm{~N}$ & $114.719 \mathrm{~W}$ & 5 & GS & - & - & $3.3 \mathrm{M}_{\mathrm{L}}(\mathrm{GS})$ & & SEPT. & 4 & 05:43 MST \\
\hline SEPT. & 5 & 160505.5 & $43.989 \mathrm{~N}$ & $114.656 \mathrm{~W}$ & 19 & $\mathrm{BU}$ & - & - & $3.7 \mathrm{M}_{\mathrm{L}}(\mathrm{BU})$ & & SEPT. & 5 & 09:05 MST \\
\hline SEPT. & 5 & 191505.2 & $43.984 \mathrm{~N}$ & $114.645 \mathrm{~W}$ & 21 & BU & - & - & $3.2 \mathrm{M}_{\mathrm{L}}(\mathrm{BU})$ & & SEPT. & 5 & $12: 15 \mathrm{MST}$ \\
\hline SEPT. & 5 & 192051.0 & $44.008 \mathrm{~N}$ & 114.752W. & 5 & GS & - & - & $4.0 \mathrm{M}_{\mathrm{L}}(\mathrm{GS})$ & & SEPT. & 5 & $12: 20 \mathrm{MST}$ \\
\hline SEPT. & 5 & 192351.3 & $44.017 \mathrm{~N}$ & 114.693W. & 19 & BU & - & - & $3.4 \mathrm{M}_{\mathrm{L}}(\mathrm{GS})$ & & SEPT. & 5 & $12: 23 \mathrm{MST}$ \\
\hline SEPT. & 5 & 194005.3 & $43.986 \mathrm{~N}$ & $114.673 \mathrm{~W}$ & 19 & BU & - & - & $3.3 \mathrm{M}_{\mathrm{L}}(\mathrm{BU})$ & & SEPT. & 5 & $12: 40 \mathrm{MST}$ \\
\hline SEPT. & 6 & 023134.9 & $43.974 \mathrm{~N}$ & $114.649 \mathrm{~W}$. & 21 & BU & - & - & $3.0 \mathrm{M}_{\mathrm{L}}(\mathrm{BU})$ & $\longrightarrow$ & SEPT. & 5 & 19:31 MST \\
\hline SEPT. & 6 & 175522.7 & $44.012 \mathrm{~N}$ & $114.659 \mathrm{~W}$ & 19 & BU & - & - & $3.5 \mathrm{M}_{\mathrm{L}}(\mathrm{GS})$ & - & SEPT. & 6 & 10:55 MST \\
\hline SEPT. & 6 & 214027.5 & $43.984 \mathrm{~N}$ & $114.656 \mathrm{~W}$ & 20 & BU & - & - & $3.2 \mathrm{M}_{\mathrm{L}}(\mathrm{BU})$ & $\longrightarrow$ & SEPT. & 6 & $14: 40 \mathrm{MST}$ \\
\hline SEPT. & 7 & 103553.7 & $43.960 \mathrm{~N}$ & $114.647 \mathrm{~W}$ & 22 & BU & - & - & $3.2 \mathrm{M}_{\mathrm{L}}(\mathrm{BU})$ & & SEPT. & 7 & 03:35 MST \\
\hline SEPT. & 7 & 110747.8 & $43.984 \mathrm{~N}$ & $114.657 \mathrm{~W}$ & 20 & BU & - & - & $3.1 \mathrm{M}_{\mathrm{L}}(\mathrm{BU})$ & 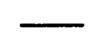 & SEPT. & 7 & 04:07 MST \\
\hline SEPT. & 11 & 020728.4 & $44.053 \mathrm{~N}$ & 114.705W. & 5 & GS & - & - & $3.5 \mathrm{M}_{\mathrm{L}}(\mathrm{GS})$ & & SEPT. & 10 & 19:07 MST \\
\hline
\end{tabular}


Table 1. Summary of United States earthquakes for 1986_Continued

\begin{tabular}{|c|c|c|c|c|c|c|c|c|c|c|c|c|}
\hline \multirow{3}{*}{ Date } & \multirow{3}{*}{$\begin{array}{l}\text { Origin time } \\
\text { (UTC) } \\
\text { hr min sec }\end{array}$} & \multirow{3}{*}{$\begin{array}{c}\text { Latitude } \\
\left({ }^{\circ}\right)\end{array}$} & \multirow{3}{*}{$\begin{array}{l}\text { Longitude } \\
\left({ }^{\circ}\right)\end{array}$} & \multirow{3}{*}{$\begin{array}{c}\text { Depth } \\
(\mathbf{k m})\end{array}$} & \multirow{3}{*}{$\begin{array}{l}\text { Hypo- } \\
\text { center } \\
\text { Source }\end{array}$} & \multirow{2}{*}{\multicolumn{3}{|c|}{ Magnitude }} & Max. & \multirow{2}{*}{\multicolumn{3}{|c|}{ Local time }} \\
\hline & & & & & & & & & inten- & & & \\
\hline & & & & & & $\mathbf{m}_{\mathbf{b}}$ & $\mathrm{M}_{\mathbf{S}}$ & Local & sity & Date & $\mathrm{hr}$ & zone \\
\hline
\end{tabular}

IDAHO-Continued

\begin{tabular}{|c|c|c|c|c|c|c|c|c|c|c|c|c|c|}
\hline SEPT. 1 & & $02 \quad 1234.2$ & $44.057 \mathrm{~N}$ & $114.757 \mathrm{~W}$ & 10 & BU & - & - & $3.3 \mathrm{M}_{\mathrm{L}}(\mathrm{BU})$ & - & SEPT. & 10 & 19:12 MST \\
\hline SEPT. 11 & & 034911.6 & 43.960N. & $114.660 \mathrm{~W}$. & 20 & BU & - & - & $3.4 \mathrm{M}_{\mathrm{L}}(\mathrm{GS})$ & - & SEPT. & 10 & 20:49 MST \\
\hline SEPT. 11 & 11 & 035512.7 & $44.067 \mathrm{~N}$ & $114.701 \mathrm{~W}$ & 5 & GS & 一 & - & $4.0 \mathrm{M}_{\mathrm{L}}(\mathrm{GS})$ & & SEPT. & 10 & 20:55 MST \\
\hline SEPT. 11 & 11 & 070925.6 & $43.990 \mathrm{~N}$ & $114.665 \mathrm{~W}$. & 19 & BU & - & - & $3.3 \mathrm{M}_{\mathrm{L}}(\mathrm{BU})$ & & SEPT. & 11 & 00:09 MST \\
\hline SEPT. 1 & 11 & 083532.1 & $43.968 \mathrm{~N}$ & $114.647 \mathrm{~W}$ & 21 & BU & - & - & $3.1 \mathrm{M}_{\mathrm{L}}(\mathrm{BU})$ & & SEPT. & 11 & 01:35 MST \\
\hline SEPT. 1 & & 160149.4 & $43.968 \mathrm{~N}$ & 114.751W. & 5 & GS & - & - & $3.1 \mathrm{M}_{\mathrm{L}}(\mathrm{GS})$ & 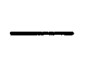 & SEPT. & 14 & 09:01 MST \\
\hline SEPT. 1 & 14 & 215204.5 & $43.979 \mathrm{~N}$ & $114.679 \mathrm{~W}$ & 19 & BU & - & - & $3.1 \mathrm{M}_{\mathrm{L}}(\mathrm{BU})$ & & SEPT. & 14 & 14:52 MST \\
\hline SEPT. 1 & 16 & 210021.0 & $43.978 \mathrm{~N}$ & $114.672 \mathrm{~W}$ & 20 & BU & - & - & $3.5 \mathrm{M}_{\mathrm{L}}(\mathrm{GS})$ & & SEPT. & 16 & $14: 00 \mathrm{MST}$ \\
\hline SEPT. 2 & 22 & 053851.9 & $44.040 \mathrm{~N}$ & $114.756 \mathrm{~W}$. & 5 & GS & - & - & $3.4 \mathrm{M}_{\mathrm{L}}(\mathrm{GS})$ & & SEPT. & 21 & 22:38 MST \\
\hline SEPT. 2 & & 153226.7 & $44.003 \mathrm{~N}$ & $114.755 \mathrm{~W}$ & 5 & GS & - & - & $3.7 \mathrm{M}_{\mathrm{L}}(\mathrm{GS})$ & FELT & SEPT. & 24 & 08:32 MST \\
\hline SEPT. 2 & & 155047.8 & $43.992 \mathrm{~N}$ & $114.677 \mathrm{~W}$ & 20 & BU & - & - & $3.2 \mathrm{M}_{\mathrm{L}}(\mathrm{BU})$ & & SEPT. & 24 & 08:50 MST \\
\hline SEPT. 2 & 26 & 212808.5 & $44.016 \mathrm{~N}$ & $114.750 \mathrm{~W}$ & 5 & GS & - & - & $4.3 \mathrm{M}_{\mathrm{L}}(\mathrm{GS})$ & IV & SEPT. & 26 & 14:28 MST \\
\hline SEPT. 2 & 26 & 220948.3 & 43.959N. & 114.780W. & 5 & GS & - & - & $3.6 \mathrm{M}_{\mathrm{L}}(\mathrm{BU})$ & & SEPT. & 26 & 15:09 MST \\
\hline SEPT. 2 & 26 & 224857.9 & $44.043 \mathrm{~N}$ & $114.756 \mathrm{~W}$. & 5 & GS & 4.6 & - & $4.5 \mathrm{M}_{\mathrm{L}}(\mathrm{GS})$ & IV & SEPT. & 26 & $15: 48 \mathrm{MST}$ \\
\hline SEPT. 2 & 26 & 225612.1 & $43.975 \mathrm{~N}$ & $114.668 \mathrm{~W}$ & 20 & BU & - & - & $3.2 \mathrm{M}_{\mathrm{L}}(\mathrm{BU})$ & & SEPT. & 26 & $15: 56 \mathrm{MST}$ \\
\hline SEPT. 2 & 27 & 130201.7 & $44.017 \mathrm{~N}$ & $114.780 \mathrm{~W}$ & 5 & GS & - & - & $3.3 \mathrm{M}_{\mathrm{L}}(\mathrm{GS})$ & & SEPT. & 27 & 06:02 MST \\
\hline SEPT. 2 & 27 & 185617.7 & $44.055 \mathrm{~N}$ & $114.781 \mathrm{~W}$ & 5 & GS & - & - & $3.3 \mathrm{M}_{\mathrm{L}}(\mathrm{GS})$ & & SEPT. & 27 & 11:56 MST \\
\hline SEPT. 2 & 27 & 185912.5 & $43.996 \mathrm{~N}$ & $114.680 \mathrm{~W}$ & 19 & BU & - & - & $3.8 \mathrm{M}_{\mathrm{L}}(\mathrm{GS})$ & & SEPT. & 27 & 11:59 MST \\
\hline SEPT. 3 & 30 & 113333.8 & $44.380 \mathrm{~N}$ & $114.222 \mathrm{~W}$ & 12 & BU & - & - & $3.0 \mathrm{M}_{\mathrm{L}}(\mathrm{BU})$ & - & SEPT. & 30 & 04:33 MST \\
\hline SEPT. 3 & 30 & 161153.5 & $43.996 \mathrm{~N}$ & $113.942 \mathrm{~W}$ & 5 & GS & - & - & $3.6 \mathrm{M}_{\mathrm{L}}(\mathrm{GS})$ & . & SEPT. & 30 & 09:11 MST \\
\hline OCT. & 1 & 072029.6 & 43.978N. & $114.780 \mathrm{~W}$ & 5 & GS & - & - & $3.0 \mathrm{M}_{\mathrm{L}}(\mathrm{GS})$ & & SEPT. & 30 & 00:20 MST \\
\hline OCT. & 2 & 090224.6 & $44.405 \mathrm{~N}$ & $114.054 \mathrm{~W}$. & 22 & BU & - & - & $3.4 \mathrm{M}_{\mathrm{L}}(\mathrm{BU})$ & & OCT. & 2 & 02:02 MST \\
\hline OCT. & 9 & 094241.5 & $43.961 \mathrm{~N}$ & $114.756 \mathrm{~W}$ & 5 & GS & - & - & $3.1 \mathrm{M}_{\mathrm{L}}(\mathrm{GS})$ & & OCT. & 9 & 02:42 MST \\
\hline OCT. 1 & 10 & 234504.9 & $43.976 \mathrm{~N}$ & $114.675 \mathrm{~W}$ & 20 & BU & - & - & $2.9 \mathrm{M}_{\mathrm{L}}(\mathrm{BU})$ & - & OCT. & 10 & $16: 45 \mathrm{MST}$ \\
\hline OCT. 1 & 14 & 121753.3 & $44.023 \mathrm{~N}$ & $114.674 \mathrm{~W}$ & 5 & GS & 一 & - & $3.9 \mathrm{M}_{\mathrm{L}}(\mathrm{GS})$ & IV & OCT. & 14 & 05:17 MST \\
\hline OCT. & 14 & 131009.8 & $44.058 \mathrm{~N}$ & $114.712 \mathrm{~W}$ & 5 & GS & - & - & $3.9 \mathrm{M}_{\mathrm{L}}(\mathrm{GS})$ & 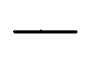 & OCT. & 14 & 06:10 MST \\
\hline OCT. & 14 & 134347.5 & $44.066 \mathrm{~N}$ & $114.684 \mathrm{~W}$ & 5 & GS & - & - & $3.6 \mathrm{M}_{\mathrm{L}}(\mathrm{GS})$ & & OCT. & 14 & 06:43 MST \\
\hline OCT. & 18 & 212129.0 & $42.014 \mathrm{~N}$ & $111.448 \mathrm{~W}$ & 7 & UU & - & - & $3.5 \mathrm{M}_{\mathrm{L}}(\mathrm{GS})$ & IV & OCT. & 18 & 14:21 MST \\
\hline OCT. & 25 & 202725.4 & $43.985 \mathrm{~N}$ & $114.673 \mathrm{~W}$ & 20 & BU & - & - & $2.9 \mathrm{M}_{\mathrm{L}}(\mathrm{BU})$ & & OCT. & 25 & 13:27 MST \\
\hline NOV. & 1 & 013912.6 & 44.399N. & $114.049 \mathrm{~W}$ & 27 & BU & - & - & $2.9 \mathrm{M}_{\mathrm{L}}(\mathrm{BU})$ & & OCT. & 31 & 18:39 MST \\
\hline NOV. & 3 & 170819.5 & $44.010 \mathrm{~N}$ & $114.579 \mathrm{~W}$. & 15 & BU & - & - & $3.4 \mathrm{M}_{\mathrm{L}}(\mathrm{BU})$ & & NOV. & 3 & 10:08 MST \\
\hline NOV. & 4 & 084601.7 & $44.187 \mathrm{~N}$ & $114.065 \mathrm{~W}$. & 5 & GS & - & - & $3.5 \mathrm{M}_{\mathrm{L}}(\mathrm{GS})$ & & Nov. & 4 & 01:46 MST \\
\hline NOV. & 7 & 124414.2 & $44.073 \mathrm{~N}$ & $114.482 \mathrm{~W}$. & 5 & GS & - & - & $3.0 \mathrm{M}_{\mathrm{L}}(\mathrm{GS})$ & & NOV. & 7 & 05:44 PST \\
\hline NOV. & 9 & 140226.4 & $43.979 \mathrm{~N}$ & $114.740 \mathrm{~W}$ & 5 & GS & - & - & $3.8 \mathrm{M}_{\mathrm{L}}(\mathrm{GS})$ & 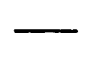 & NOV. & 9 & 07:02 MST \\
\hline NOV. & 9 & 141537.8 & $44.024 \mathrm{~N}$ & $114.723 W$ & 5 & GS & - & - & $3.3 \mathrm{M}_{\mathrm{L}}(\mathrm{GS})$ & & NOV. & 9 & 07:15 MST \\
\hline NOV. & 9 & 171642.0 & $43.979 \mathrm{~N}$ & $114.640 \mathrm{~W}$ & 17 & BU & - & - & $2.9 \mathrm{M}_{\mathrm{L}}(\mathrm{BU})$ & & NOV. & 9 & 10:16 MST \\
\hline NOV. 12 & 12 & 071247.5 & $44.004 \mathrm{~N}$ & $114.715 \mathrm{~W}$ & 5 & GS & - & - & $3.0 \mathrm{M}_{\mathrm{L}}(\mathrm{GS})$ & $\ldots$ & NOV. & 11 & $00: 12$ MST \\
\hline NOV. 1 & 15 & 090013.2 & $42.706 \mathrm{~N}$ & $111.667 \mathrm{~W}$ & 5 & GS & - & - & $3.3 \mathrm{M}_{\mathrm{L}}(\mathrm{GS})$ & IV & NOV. & 15 & 02:00 MST \\
\hline NOV. 18 & 18 & 160940.3 & $43.976 \mathrm{~N}$ & $114.754 \mathrm{~W}$. & 18 & BU & 一 & - & $3.6 \mathrm{M}_{\mathrm{D}}(\mathrm{BU})$ & & NOV. & 18 & 09:09 MST \\
\hline NOV. 2 & 21 & 080755.0 & $44.523 \mathrm{~N}$ & $114.025 \mathrm{~W}$ & 6 & BU & - & - & $2.6 \mathrm{M}_{\mathrm{L}}(\mathrm{BU})$ & - & NOV. & 21 & 01:07 MST \\
\hline
\end{tabular}


Table 1. Summary of United States earthquakes for 1986-Continued

\begin{tabular}{|c|c|c|c|c|c|c|c|c|c|c|c|}
\hline \multirow[t]{2}{*}{ Date } & \multirow{2}{*}{$\begin{array}{l}\text { Origin time } \\
\text { (UTC) } \\
\text { hr min sec }\end{array}$} & \multirow{2}{*}{$\begin{array}{l}\text { Latitude } \\
\text { ( ) }\end{array}$} & \multirow{2}{*}{$\begin{array}{l}\text { Longitude } \\
\text { ( })\end{array}$} & \multirow{2}{*}{$\begin{array}{l}\text { Depth } \\
(\mathrm{km})\end{array}$} & \multirow{2}{*}{$\begin{array}{l}\text { Hypo- } \\
\text { center } \\
\text { Source }\end{array}$} & \multicolumn{3}{|c|}{ Magnitude } & \multirow{2}{*}{$\begin{array}{l}\text { Max. } \\
\text { inten- } \\
\text { sity }\end{array}$} & \multicolumn{2}{|c|}{ Local time } \\
\hline & & & & & & $\mathbf{m}_{\mathrm{b}}$ & $\mathbf{M}_{\mathbf{s}}$ & Local & & Date & zone \\
\hline \multicolumn{12}{|c|}{ IDAHO_Continued } \\
\hline NOV. 23 & 060309.9 & $44.758 \mathrm{~N}$. & $114.387 \mathrm{~W}$. & 16 & BU & & 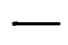 & $2.5 \mathrm{M}_{\mathrm{L}}(\mathrm{BU})$ & & NOV. 22 & 23:03 MST \\
\hline NOV. 26 & 001309.9 & $42.476 \mathrm{~N}$. & 111.204W. & 6 & UU & & & $3.5 \mathrm{M}_{\mathrm{L}}(\mathrm{UU})$ & & NOV. 25 & 17:13 MST \\
\hline DEC. 2 & 152456.9 & $43.982 \mathrm{~N}$. & $114.756 \mathrm{~W}$. & 16 & BU & & & $2.8 \mathrm{M}_{\mathrm{L}}(\mathrm{BU})$ & & DEC. & 08:24 MST \\
\hline DEC. & 235809.8 & $43.965 \mathrm{~N}$. & $114.627 \mathrm{~W}$. & 26 & BU & & & $3.0 \mathrm{M}_{\mathrm{L}}(\mathrm{BU})$ & & DEC. & 16:58 MST \\
\hline DEC. 11 & 123553.1 & $44.638 \mathrm{~N}$. & $114.093 \mathrm{~W}$. & 10 & BU & - & - & $2.6 \mathrm{M}_{\mathrm{L}}(\mathrm{BU})$ & & DEC. 11 & 05:35 MST \\
\hline DEC. 13 & 181206.3 & $44.213 \mathrm{~N}$. & 114.010W. & 5 & GS & 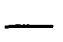 & L & $3.2 \mathrm{M}_{\mathrm{L}}(\mathrm{GS})$ & & DEC. 13 & 11:12 MST \\
\hline DEC. 15 & 205027.6 & $44.377 \mathrm{~N}$. & $114.152 W$. & 5 & GS & - & 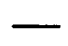 & $2.8 \mathrm{M}_{\mathrm{L}}(\mathrm{GS})$ & 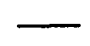 & DEC. 15 & 13:50 MST \\
\hline DEC. 20 & 071847.5 & $44.335 \mathrm{~N}$. & $114.454 \mathrm{~W}$. & 5 & GS & . & & $3.0 \mathrm{M}_{\mathrm{L}}(\mathrm{GS})$ & & DEC. 19 & 00:18 MST \\
\hline DEC. 24 & 072858.4 & $43.878 \mathrm{~N}$. & $114.848 \mathrm{~W}$. & 5 & GS & - & - & $3.0 \mathrm{M}_{\mathrm{L}}(\mathrm{GS})$ & _ & DEC. 23 & 00:28 MST \\
\hline DEC. 30 & 095128.3 & $42.720 \mathrm{~N}$ & $111.275 \mathrm{~W}$. & 5 & GS & - & - & $3.2 \mathrm{M}_{\mathrm{L}}(\mathrm{GS})$ & - & DEC. 30 & 02:51 MST \\
\hline
\end{tabular}

\section{ILLINOIS}

\begin{tabular}{|c|c|c|c|c|c|c|c|c|c|c|c|c|}
\hline FEB. & 194448.3 & $37.46 \mathrm{~N}$ & $89.19 \mathrm{~W}$. & 20 & SL & - & - & $2.5 \mathrm{M}_{\mathrm{D}}(\mathrm{SL})$ & $\longrightarrow$ & FEB. & 8 & 13:44 CST \\
\hline FEB. 15 & 110112.8 & $38.25 \mathrm{~N}$ & $89.77 \mathrm{~W}$. & 5 & SL & - & - & $2.7 \mathrm{M}_{\mathrm{n}}(\mathrm{SL})$ & & FEB. & 15 & 03:01 CST \\
\hline 26 & 150300.5 & $38.39 \mathrm{~N}$ & $89.10 \mathrm{~W}$. & 5 & SL & & & $2.7 \mathrm{M}_{\mathrm{n}}(\mathrm{SL})$ & & FEB. & 26 & 09:03 CST \\
\hline AY $\quad 20$ & 064459.0 & $38.00 \mathrm{~N}$ & $89.90 \mathrm{~W}$ & 5 & SL & & & $2.5 \mathrm{M}_{\mathrm{D}}(\mathrm{SL})$ & & MAY & 20 & 00:44 CST \\
\hline JG. 26 & 164124.8 & $38.32 \mathrm{~N}$ & $89.79 \mathrm{~W}$ & 5 & SL & & & $3.7 \mathrm{M}_{\mathrm{n}}(\mathrm{GS})$ & $\mathbf{V}$ & AUG. & 26 & $10: 41 \mathrm{CST}$ \\
\hline T. 29 & 050341.3 & $38.44 \mathrm{~N}$ & $89.04 \mathrm{~W}$. & 5 & SL & - & - & $2.7 \mathrm{M}_{\mathrm{n}}(\mathrm{GS})$ & III & OCT. & 28 & $23: 03 \mathrm{C}$ \\
\hline
\end{tabular}

\section{INDIANA}

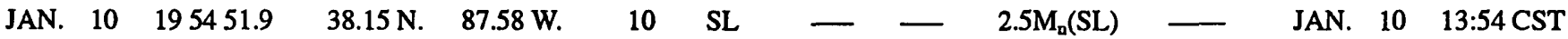

\section{KANSAS}

\begin{tabular}{|c|c|c|c|c|c|c|c|c|c|c|c|}
\hline JUNE 2 & 040405.2 & $39.344 \mathrm{~N}$. & 99.781W. & 5 & GS & $\longrightarrow$ & - & $3.0 \mathrm{M}_{\mathrm{D}}(\mathrm{GS})$ & IV & JUNE & 22:04 CST \\
\hline OCT. 20 & 043249.0 & $37.918 \mathrm{~N}$. & 101.372W. & 5 & GS & - & - & $3.0 \mathrm{M}_{\mathrm{p}}(\mathrm{GS})$ & IV & OCT. 19 & 22:32 CST \\
\hline
\end{tabular}

\section{KENTUCKY}

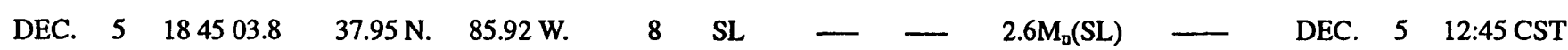

\section{MAINE}

\begin{tabular}{|c|c|c|c|c|c|c|c|c|c|c|c|c|}
\hline MAR. 19 & 020933.0 & $45.158 \mathrm{~N}$. & $69.059 \mathrm{~W}$. & 13 & WO & - & - & $2.5 \mathrm{M}_{\mathrm{n}}(\mathrm{WO})$ & - & MAR. & 18 & 21:09 EST \\
\hline APR. 29 & 093924.4 & $45.359 \mathrm{~N}$. & $70.166 \mathrm{~W}$. & 5 & Wo & & & $2.5 \mathrm{M}_{\mathrm{D}}(\mathrm{WO})$ & & APR. & 29 & 04:39 EST \\
\hline JUNE 24 & 024015.7 & $45.203 \mathrm{~N}$. & $69.177 \mathrm{~W}$. & 2 & WO & & & $2.5 \mathrm{M}_{\mathrm{n}}(\mathrm{WO})$ & FELT & JUNE & 23 & 21:40 EST \\
\hline JULY 12 & 203248.4 & $46.170 \mathrm{~N}$. & 68.198W. & 9 & Wo & & & $3.4 \mathrm{M}_{\mathrm{n}}(\mathrm{WO})$ & FELT & JULY & 12 & 15:32 EST \\
\hline UG. 15 & 200236.4 & $45.083 \mathrm{~N}$. & 69.437W. & 3 & WO & & & $2.5 \mathrm{M}_{\mathrm{n}}(\mathrm{WO})$ & & AUG. & 15 & 16:01 EST \\
\hline & 005611.6 & $45.175 \mathrm{~N}$. & $68.415 \mathrm{~W}$. & 1 & WO & & - & $2.6 \mathrm{M}_{\mathrm{v}}(\mathrm{WO})$ & - & AUG. & 21 & 19:56 EST \\
\hline UG. 31 & 040156.9 & $44.618 \mathrm{~N}$. & $68.970 \mathrm{~W}$. & 6 & WO & - & - & $2.6 \mathrm{M}_{\mathrm{n}}(\mathrm{WO})$ & - & AUG. & 30 & 23:01 EST \\
\hline
\end{tabular}


Table 1. Summary of United States earthquakes for 1986-Continued

\begin{tabular}{|c|c|c|c|c|c|c|c|c|c|c|c|}
\hline \multirow[t]{2}{*}{ Date } & \multirow{2}{*}{$\begin{array}{l}\text { Origin time } \\
\text { (UTC) } \\
\text { hr min sec }\end{array}$} & \multirow{2}{*}{$\begin{array}{l}\text { Latitude } \\
\text { ()) }\end{array}$} & \multirow{2}{*}{$\begin{array}{l}\text { Longitude } \\
\left({ }^{\circ}\right)\end{array}$} & \multirow{2}{*}{$\begin{array}{l}\text { Depth } \\
(\mathbf{k m})\end{array}$} & \multirow{2}{*}{$\begin{array}{l}\text { Hypo- } \\
\text { center } \\
\text { Source }\end{array}$} & \multicolumn{3}{|c|}{ Magnitude } & \multirow{2}{*}{$\begin{array}{l}\text { Max. } \\
\text { inten- } \\
\text { sity }\end{array}$} & \multicolumn{2}{|c|}{ Local time } \\
\hline & & & & & & $\mathrm{m}_{\mathrm{b}}$ & $\mathbf{M}_{\boldsymbol{S}}$ & Local & & Date & zone \\
\hline \multicolumn{12}{|c|}{ MARYLAND } \\
\hline MAY 23 & 174812.2 & $38.689 \mathrm{~N}$. & 77.038W. & 0 & VP & - & - & $2.5 \mathrm{M}_{\mathrm{D}}(\mathrm{VP})$ & - & MAY 23 & 12:48 EST \\
\hline \multicolumn{12}{|c|}{ MASSACHUSETTS } \\
\hline APR. 16 & 042142.7 & $42.847 \mathrm{~N}$ & $70.982 \mathrm{~W}$ & 5 & WO & - & - & $2.6 \mathrm{M}_{\mathrm{D}}(\mathrm{WO})$ & III & APR. 15 & 23:21 EST \\
\hline \multicolumn{12}{|c|}{ MISSOURI } \\
\hline JAN. 21 & 182034.4 & $36.57 \mathrm{~N}$. & $89.60 \mathrm{~W}$ & 7 & SL & $\longrightarrow$ & - & $2.8 \mathrm{M}_{\mathrm{D}}(\mathrm{SL})$ & - & JAN. & $12: 20 \mathrm{CST}$ \\
\hline FEB. $\quad 17$ & 191306.7 & $37.94 \mathrm{~N}$. & $90.40 \mathrm{~W}$ & 4 & SL & - & - & $2.8 \mathrm{M}_{\mathrm{r}}(\mathrm{SL})$ & & FEB. & $13: 13 \mathrm{CST}$ \\
\hline MAY 11 & 235914.3 & $36.17 \mathrm{~N}$. & $89.65 \mathrm{~W}$. & 5 & SL & & & $2.7 \mathrm{M}_{\mathrm{n}}(\mathrm{SL})$ & & MAY 11 & 17:59 CST \\
\hline MAY 20 & 050029.6 & $36.66 \mathrm{~N}$ & $89.56 \mathrm{~W}$ & 16 & SL & & 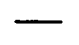 & $2.5 \mathrm{M}_{\mathrm{n}}(\mathrm{SL})$ & $=$ & MAY 19 & 23:00 CST \\
\hline MAY 24 & 124813.5 & $36.58 \mathrm{~N}$ & $89.88 \mathrm{~W}$. & 10 & SL & - & - & $3.4 \mathrm{M}_{\mathrm{D}}(\mathrm{SL})$ & IV & MAY 24 & 06:48 CST \\
\hline JULY & 062947.4 & $36.78 \mathrm{~N}$ & $89.92 \mathrm{~W}$ & 5 & SL & & & $2.8 \mathrm{M}_{\mathrm{D}}(\mathrm{SL})$ & - & JULY & 00:29 CST \\
\hline JULY 18 & 144253.6 & $36.01 \mathrm{~N}$ & $89.88 \mathrm{~W}$. & 9 & SL & - & - & $2.6 \mathrm{M}_{\mathrm{n}}(\mathrm{SL})$ & - & JULY 18 & 08:42 CST \\
\hline AUG. 21 & 192506.0 & $36.78 \mathrm{~N}$ & $89.35 \mathrm{~W}$. & 5 & SL & - & 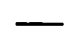 & $2.8 \mathrm{M}_{\mathrm{n}}(\mathrm{SL})$ & - & AUG. 21 & $13: 25 \mathrm{CST}$ \\
\hline OCT. 8 & 081346.4 & $36.52 \mathrm{~N}$ & $89.56 \mathrm{~W}$. & 7 & SL & - & & $2.5 \mathrm{M}_{\mathrm{n}}(\mathrm{SL})$ & & OCT. & 02:13 CST \\
\hline OCT. 24 & 055745.8 & $36.17 \mathrm{~N}$ & $89.66 \mathrm{~W}$. & 9 & SL & - & - & $2.9 \mathrm{M}_{\mathrm{D}}(\mathrm{SL})$ & IV & OCT. 23 & 23:57 CST \\
\hline NOV. & 192147.2 & $38.11 \mathrm{~N}$. & $90.42 \mathrm{~W}$ & 9 & SL & - & - & $2.7 \mathrm{M}_{\mathrm{D}}(\mathrm{SL})$ & III & NOV. 6 & $13: 21 \mathrm{CST}$ \\
\hline DEC. 12 & 235148.5 & $36.84 \mathrm{~N}$. & $89.22 \mathrm{~W}$ & 5 & SL & & & $2.5 \mathrm{M}_{\mathrm{n}}(\mathrm{SL})$ & $\longrightarrow$ & DEC. 12 & 17:51 CST \\
\hline DEC. 16 & 000213.3 & $36.73 \mathrm{~N}$ & $89.52 \mathrm{~W}$ & 5 & SL & $\longrightarrow$ & - & $2.5 \mathrm{M}_{\mathrm{n}}(\mathrm{SL})$ & 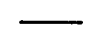 & DEC. 15 & $18: 02$ CST \\
\hline DEC. 30 & 071519.1 & $36.42 \mathrm{~N}$ & $89.58 \mathrm{~W}$ & 14 & SL & - & - & $3.5 \mathrm{M}_{\mathrm{D}}(\mathrm{GS})$ & IV & DEC. 30 & 01:15 CST \\
\hline
\end{tabular}

\section{MONTANA}

\begin{tabular}{|c|c|c|c|c|c|c|c|c|c|c|c|c|c|}
\hline JAN. & 9 & 063453.8 & $47.477 \mathrm{~N}$. & 115.771W. & 6 & BU & - & - & $2.5 \mathrm{M}_{\mathrm{L}}(\mathrm{BU})$ & - & JAN. & 8 & 23:34 MST \\
\hline JAN. & 24 & 114815.2 & $47.632 \mathrm{~N}$ & $114.320 \mathrm{~W}$. & 5 & BU & & & $2.6 \mathrm{M}_{\mathrm{D}}(\mathrm{BU})$ & & JAN. & 24 & 04:48 MST \\
\hline JAN. & 28 & 132400.3 & $47.486 \mathrm{~N}$ & 115.722W. & 7 & BU & & & $2.6 \mathrm{M}_{\mathrm{L}}(\mathrm{BU})$ & & JAN. & 28 & 06:24 MST \\
\hline FEB. & 6 & 022419.5 & $47.642 \mathrm{~N}$ & $115.647 \mathrm{~W}$ & 1 & BU & & & $2.6 \mathrm{M}_{\mathrm{L}}(\mathrm{BU})$ & & FEB. & 5 & $19: 24 \mathrm{MST}$ \\
\hline MAR. & 6 & 151154.5 & $44.697 \mathrm{~N}$. & $112.500 \mathrm{~W}$ & 9 & $\mathrm{BU}$ & & & $2.9 \mathrm{M}_{\mathrm{L}}(\mathrm{BU})$ & & MAR. & 6 & 08:11 MST \\
\hline APR. & 12 & 050737.8 & $46.068 \mathrm{~N}$ & $111.484 \mathrm{~W}$. & 7 & BU & 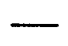 & - & $2.5 \mathrm{M}_{\mathrm{L}}(\mathrm{BU})$ & & APR. & 11 & 22:07 MST \\
\hline APR. & 21 & 113552.3 & $47.792 \mathrm{~N}$ & $114.036 \mathrm{~W}$. & 32 & $\mathrm{BU}$ & & & $3.0 \mathrm{M}_{\mathrm{L}}(\mathrm{BU})$ & & APR. & 21 & 04:35 MST \\
\hline MAY & 2 & 021745.6 & $44.638 \mathrm{~N}$. & $112.079 \mathrm{~W}$ & 13 & BU & & & $2.7 \mathrm{M}_{\mathrm{L}}(\mathrm{BU})$ & & MAY & 1 & 19:17 MST \\
\hline MAY & 11 & 100456.4 & $44.648 \mathrm{~N}$ & $111.994 \mathrm{~W}$. & 5 & GS & & & $3.1 \mathrm{M}_{\mathrm{L}}(\mathrm{GS})$ & & MAY & 11 & 03:04 MST \\
\hline MAY & 12 & 095828.2 & $45.348 \mathrm{~N}$ & $112.415 \mathrm{~W}$ & 11 & BU & & & $3.2 \mathrm{M}_{\mathrm{L}}(\mathrm{GS})$ & & MAY & 12 & 02:58 MST \\
\hline JUNE & 13 & 201626.8 & $44.837 \mathrm{~N}$. & $111.527 \mathrm{~W}$. & 5 & GS & $\longrightarrow$ & - & $3.1 \mathrm{M}_{\mathrm{L}}(\mathrm{GS})$ & & JUNE & 13 & 13:16 MST \\
\hline JUNE & 27 & 040930.5 & $44.648 \mathrm{~N}$ & $111.076 \mathrm{~W}$. & 5 & GS & - & & $2.8 \mathrm{M}_{\mathrm{L}}(\mathrm{GS})$ & & JUNE & 26 & 21:09 MST \\
\hline JUNE & 28 & 162657.1 & $44.660 \mathrm{~N}$. & $111.055 \mathrm{~W}$. & 27 & BU & & & $2.9 \mathrm{M}_{\mathrm{L}}(\mathrm{BU})$ & & JUNE & 28 & 09:26 MST \\
\hline JULY & 9 & 104822.3 & $47.434 \mathrm{~N}$ & $114.806 \mathrm{~W}$. & 1 & BU & & & $3.0 \mathrm{M}_{\mathrm{L}}(\mathrm{BU})$ & & JULY & 9 & 03:48 MST \\
\hline JULY & 16 & 221402.5 & $46.297 \mathrm{~N}$. & $112.044 \mathrm{~W}$. & 5 & BU & - & & $3.6 \mathrm{M}_{\mathrm{L}}(\mathrm{GS})$ & & JULY & 16 & 15:14 MST \\
\hline JULY & 16 & 223711.8 & $46.292 \mathrm{~N}$. & $112.038 \mathrm{~W}$. & 9 & BU & - & - & $3.6 \mathrm{M}_{\mathrm{L}}(\mathrm{GS})$ & & JULY & 16 & 15:37 MST \\
\hline JULY & 16 & 231200.2 & $46.300 \mathrm{~N}$ & $112.050 \mathrm{~W}$. & 6 & BU & - & - & $2.5 \mathrm{M}_{\mathrm{L}}(\mathrm{BU})$ & & JULY & 16 & $16: 12$ MST \\
\hline
\end{tabular}


Table 1. Summary of United States earthquakes for 1986-Continued

\begin{tabular}{|c|c|c|c|c|c|c|c|c|c|c|c|}
\hline \multirow[t]{2}{*}{ Date } & \multirow{2}{*}{$\begin{array}{l}\text { Origin time } \\
\text { (UTC) } \\
\text { hr min sec }\end{array}$} & \multirow{2}{*}{$\begin{array}{l}\text { Latitude } \\
\text { ( })\end{array}$} & \multirow{2}{*}{$\begin{array}{l}\text { Longitude } \\
\text { (9) }\end{array}$} & \multirow{2}{*}{$\begin{array}{l}\text { Depth } \\
(\mathrm{km})\end{array}$} & \multirow{2}{*}{$\begin{array}{l}\text { Hypo- } \\
\text { center } \\
\text { Source }\end{array}$} & \multicolumn{3}{|c|}{ Magnitude } & \multirow{2}{*}{$\begin{array}{l}\text { Max. } \\
\text { inten- } \\
\text { sity }\end{array}$} & \multicolumn{2}{|c|}{ Local time } \\
\hline & & & & & & $\mathbf{m}_{\mathbf{b}}$ & $\mathbf{M}_{\boldsymbol{s}}$ & Local & & Date & zone \\
\hline \multicolumn{12}{|c|}{ MONTANA-Continued } \\
\hline JULY 17 & 021624.1 & $46.300 \mathrm{~N}$ & $112.045 \mathrm{~W}$. & 1 & BU & & - & $2.5 \mathrm{M}_{\mathrm{L}}(\mathrm{BU})$ & & JULY 16 & 19:16 MST \\
\hline AUG. 3 & 180740.5 & $47.754 \mathrm{~N}$ & 113.204W. & 12 & BU & . & & $3.1 \mathrm{M}_{\mathrm{L}}(\mathrm{BU})$ & & AUG. 3 & 11:07 MST \\
\hline AUG. 11 & 051446.2 & $48.275 \mathrm{~N}$ & $114.582 W$. & 33 & BU & & & $3.4 \mathrm{M}_{\mathrm{L}}(\mathrm{BU})$ & & AUG. 10 & 22:14 MST \\
\hline AUG. 12 & 002352.5 & $44.786 \mathrm{~N}$ & $111.391 \mathrm{~W}$. & 21 & BU & - & $\longrightarrow$ & $2.8 \mathrm{M}_{\mathrm{L}}(\mathrm{BU})$ & {[} & AUG. 11 & 17:23 MST \\
\hline AUG. 22 & 050341.3 & $47.376 \mathrm{~N}$. & $114.095 \mathrm{~W}$. & 36 & BU & - & & $2.6 \mathrm{M}_{\mathrm{L}}(\mathrm{BU})$ & & AUG. 21 & 22:03 MST \\
\hline AUG. 24 & 180425.5 & $45.802 \mathrm{~N}$ & $111.594 \mathrm{~W}$. & 13 & BU & & & $3.9 \mathrm{M}_{\mathrm{L}}(\mathrm{GS})$ & IV & AUG. 24 & 11:04 MST \\
\hline SEPT. 4 & 234734.8 & $47.740 \mathrm{~N}$ & $113.756 \mathrm{~W}$. & 20 & $\mathbf{B U}$ & & & $2.7 \mathrm{M}_{\mathrm{D}}(\mathrm{BU})$ & & SEPT. 4 & 16:47 MST \\
\hline SEPT. 12 & 131701.4 & $45.140 \mathrm{~N}$ & $111.882 \mathrm{~W}$. & 8 & $\mathrm{BU}$ & & & $2.9 \mathrm{M}_{\mathrm{L}}(\mathrm{BU})$ & & SEPT. 12 & 06:17 MST \\
\hline OCT. 3 & 185835.2 & $44.358 \mathrm{~N}$ & $112.596 \mathrm{~W}$ & 16 & BU & & & $2.5 \mathrm{M}_{\mathrm{L}}(\mathrm{BU})$ & - & OCT. & 11:58 MST \\
\hline OCT. 18 & 142046.9 & $46.304 \mathrm{~N}$. & $112.060 \mathrm{~W}$. & 3 & BU & & & $3.1 \mathrm{M}_{\mathrm{L}}(\mathrm{BU})$ & IV & OCT. 18 & 07:20 MST \\
\hline OCT. 18 & 185538.7 & 46.293N. & $112.026 \mathrm{~W}$. & 0 & BU & & & $3.4 \mathrm{M}_{\mathrm{L}}(\mathrm{BU})$ & IV & OCT. 18 & 11:55 MST \\
\hline OCT. 19 & 100143.4 & $46.292 \mathrm{~N}$ & $112.025 \mathrm{~W}$. & 0 & BU & & & $3.3 \mathrm{M}_{\mathrm{L}}(\mathrm{BU})$ & IV & OCT. 19 & 03:01 MST \\
\hline OCT. 24 & 045447.7 & $46.305 \mathrm{~N}$ & $112.046 \mathrm{~W}$. & 3 & BU & & - & $3.2 \mathrm{M}_{\mathrm{L}}(\mathrm{BU})$ & FELT & OCT. 23 & 21:54 MST \\
\hline OCT. 27 & 141429.6 & $46.729 \mathrm{~N}$ & $111.981 \mathrm{~W}$. & 9 & $\mathrm{BU}$ & & & $2.8 \mathrm{M}_{\mathrm{L}}(\mathrm{BU})$ & - & OCT. 27 & 07:14 MST \\
\hline NOV. 9 & 120635.3 & $46.205 \mathrm{~N}$ & $112.112 \mathrm{~W}$ & 10 & BU & & & $3.2 \mathrm{M}_{\mathrm{L}}(\mathrm{BU})$ & FELT & NOV. 9 & 05:06 MST \\
\hline NOV. 17 & 152220.5 & $44.657 \mathrm{~N}$ & $111.095 \mathrm{~W}$. & 18 & BU & - & - & $2.5 \mathrm{M}_{\mathrm{L}}(\mathrm{BU})$ & & NOV. 17 & 08:22 MST \\
\hline NOV. 30 & 162915.1 & $47.882 \mathrm{~N}$ & $114.306 \mathrm{~W}$. & 17 & $\mathrm{BU}$ & - & - & $2.7 \mathrm{M}_{\mathrm{L}}(\mathrm{BU})$ & - & NOV. 30 & 09:29 MST \\
\hline DEC. 3 & 234657.8 & $46.442 \mathrm{~N}$ & $112.199 \mathrm{~W}$. & 0 & GS & & - & $3.1 \mathrm{M}_{\mathrm{L}}(\mathrm{GS})$ & & DEC. 3 & 16:46 MST \\
\hline DEC. 19 & 011346.4 & $46.722 \mathrm{~N}$ & $111.908 \mathrm{~W}$. & 5 & BU & - & - & $2.7 \mathrm{M}_{\mathrm{L}}(\mathrm{BU})$ & - & DEC. 18 & 18:13 MST \\
\hline
\end{tabular}

NEVADA

\begin{tabular}{|c|c|c|c|c|c|c|c|c|c|c|c|c|}
\hline JAN. & 040743.3 & $39.626 \mathrm{~N}$ & 119.380W. & - & RN & - & - & $3.1 \mathrm{M}_{\mathrm{D}}(\mathrm{RN})$ & FELT & JAN. & 11 & 20:07 PST \\
\hline FEB. & 100406.9 & $39.240 \mathrm{~N}$. & 119.417W. & 5 & GS & - & - & $2.8 \mathrm{M}_{\mathrm{L}}(\mathrm{BK})$ & - & FEB. & 6 & 02:04 PST \\
\hline MAR. 6 & 201652.4 & $37.173 \mathrm{~N}$ & $117.306 \mathrm{~W}$. & 5 & GS & - & - & $3.9 \mathrm{M}_{\mathrm{L}}(\mathrm{BK})$ & . & MAR. & 6 & 12:16 PST \\
\hline MAR. 22 & 161500.0 & $37.083 \mathrm{~N}$ & $116.066 \mathrm{~W}$ & 0 & EN & 5.1 & - & $5.1 \mathrm{M}_{\mathrm{L}}(\mathrm{BK})$ & & MAR. & 22 & 08:15 PST \\
\hline APR. 3 & 054107.4 & $40.142 \mathrm{~N}$ & $115.946 \mathrm{~W}$. & 5 & GS & - & - & $3.5 \mathrm{M}_{\mathrm{L}}(\mathrm{GS})$ & & APR. & 2 & 21:41 PST \\
\hline APR. & 122640.2 & $38.263 \mathrm{~N}$ & $118.618 \mathrm{~W}$ & 3 & BK & - & - & $3.6 \mathrm{M}_{\mathrm{L}}(\mathrm{BK})$ & 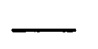 & APR. & 8 & 04:26 PST \\
\hline APR. 10 & 140830.1 & $37.218 \mathrm{~N}$ & $116.183 \mathrm{~W}$. & 0 & EN & 4.9 & - & $4.8 \mathrm{M}_{\mathrm{L}}(\mathrm{BK})$ & & APR. & 10 & 06:08 PST \\
\hline APR. 20 & 231229.9 & $37.010 \mathrm{~N}$ & $116.027 \mathrm{~W}$ & 5 & GS & 4.0 & & $4.0 \mathrm{M}_{\mathrm{L}}(\mathrm{BK})$ & & APR. & 20 & 15:12 PST \\
\hline APR. 21 & 000324.0 & $37.023 \mathrm{~N}$ & $115.940 \mathrm{~W}$. & 5 & GS & - & - & $2.8 \mathrm{M}_{\mathrm{L}}(\mathrm{GS})$ & & APR. & 20 & 16:03 PST \\
\hline APR. 22 & 143000.0 & $37.264 \mathrm{~N}$. & $116.440 \mathrm{~W}$ & 0 & EN & 5.3 & 4.2 & $5.4 \mathrm{M}_{\mathrm{L}}(\mathrm{BK})$ & & APR. & 22 & 06:30 PST \\
\hline MAY 21 & 135900.0 & $37.125 \mathrm{~N}$. & $116.060 \mathrm{~W}$ & 0 & EN & - & - & $4.1 \mathrm{M}_{\mathrm{L}}(\mathrm{BK})$ & & MAY & 21 & 05:59 PST \\
\hline JUNE 4 & 150738.4 & $37.326 \mathrm{~N}$. & $117.187 \mathrm{~W}$. & 5 & GS & - & - & $3.5 \mathrm{M}_{\mathrm{L}}(\mathrm{BK})$ & - & JUNE & 4 & 07:07 PST \\
\hline JUNE & 150400.0 & $37.098 \mathrm{~N}$. & $116.016 \mathrm{~W}$ & 0 & EN & 5.3 & 4.2 & $5.2 \mathrm{M}_{\mathrm{L}}(\mathrm{BK})$ & - & JUNE & 5 & 07:04 PST \\
\hline JUNE 5 & 152411.2 & $37.137 \mathrm{~N}$. & $115.998 \mathrm{~W}$. & 1 & GS & 4.2 & - & - & - & JUNE & 5 & 07:24 PST \\
\hline JUNE 15 & 140051.3 & $38.766 \mathrm{~N}$ & $119.402 W$. & 5 & RN & - & - & $3.2 \mathrm{M}_{\mathrm{D}}(\mathrm{RN})$ & IV & JUNE & 15 & 06:00 PST \\
\hline JUNE 25 & 202745.1 & $37.265 \mathrm{~N}$ & $116.499 \mathrm{~W}$. & 0 & EN & 5.5 & 4.2 & $5.4 \mathrm{M}_{\mathrm{L}}(\mathrm{BK})$ & - & JUNE & 25 & 12:27 PST \\
\hline JUNE 28 & 020629.6 & $39.518 \mathrm{~N}$ & 119.761W. & - & RN & - & - & $3.7 \mathrm{M}_{\mathrm{L}}(\mathrm{BK})$ & v & JUNE & 27 & 18:06 PST \\
\hline JULY 17 & 210000.0 & $37.279 \mathrm{~N}$. & $116.356 \mathrm{~W}$ & 0 & EN & 5.7 & - & $5.6 \mathrm{M}_{\mathrm{L}}(\mathrm{BK})$ & - & JULY & 17 & $13: 00$ PST \\
\hline
\end{tabular}


Table 1. Summary of United States earthquakes for 1986-Continued

\begin{tabular}{|c|c|c|c|c|c|c|c|c|c|c|c|}
\hline \multirow[t]{2}{*}{ Date } & \multirow{2}{*}{$\begin{array}{l}\text { Origin time } \\
\text { (UTC) } \\
\text { hr min sec }\end{array}$} & \multirow{2}{*}{$\begin{array}{c}\text { Latitude } \\
\text { (?) }\end{array}$} & \multirow{2}{*}{$\begin{array}{l}\text { Longitude } \\
\text { ( })\end{array}$} & \multirow{2}{*}{$\begin{array}{l}\text { Depth } \\
(\mathrm{km})\end{array}$} & \multirow{2}{*}{$\begin{array}{l}\text { Hypo- } \\
\text { center } \\
\text { Source }\end{array}$} & \multicolumn{3}{|c|}{ Magnitude } & \multirow{2}{*}{$\begin{array}{l}\text { Max. } \\
\text { inten- } \\
\text { sity }\end{array}$} & \multicolumn{2}{|c|}{ Local time } \\
\hline & & & & & & $\mathrm{m}_{b}$ & $\mathbf{M}_{\mathbf{S}}$ & Local & & Date & zone \\
\hline \multicolumn{12}{|c|}{ NEVADA-Continued } \\
\hline JULY 24 & 150500.0 & $37.143 \mathrm{~N}$. & $116.071 W$ & 0 & EN & 4.4 & - & $4.5 \mathrm{M}_{\mathrm{L}}(\mathrm{BK})$ & & JULY 24 & 07:05 PST \\
\hline AUG. 4 & 040030.6 & $37.979 \mathrm{~N}$. & $117.877 \mathrm{~W}$ & 6 & PS & - & - & $3.2 \mathrm{M}_{\mathrm{L}}(\mathrm{PS})$ & & AUG. 3 & 20:00 PST \\
\hline AUG. 8 & 140610.4 & $39.521 \mathrm{~N}$ & $119.753 \mathrm{~W}$. & & RN & - & - & $2.7 \mathrm{M}_{\mathrm{L}}(\mathrm{GS})$ & FELT & AUG. 8 & 06:06 PST \\
\hline AUG. 22 & 144417.0 & $36.611 \mathrm{~N}$ & $116.366 \mathrm{~W}$. & 5 & GS & - & 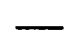 & $3.0 \mathrm{M}_{\mathrm{L}}(\mathrm{PS})$ & - & AUG. 22 & 06:44 PST \\
\hline AUG. 25 & 170636.9 & $39.638 \mathrm{~N}$. & 119.803W. & 5 & RN & - & . & $2.8 \mathrm{M}_{\mathrm{L}}(\mathrm{GS})$ & III & AUG. 25 & 09:06 PST \\
\hline AUG. 25 & 175742.1 & $39.638 \mathrm{~N}$. & $119.803 W$. & 5 & RN & 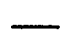 & 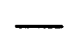 & $2.5 \mathrm{M}_{\mathrm{L}}(\mathrm{GS})$ & FELT & AUG. 25 & 09:57 PST \\
\hline SEPT. 4 & 160900.1 & $37.236 \mathrm{~N}$. & $116.352 \mathrm{~W}$. & 5 & GS & 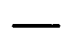 & - & $3.5 \mathrm{M}_{\mathrm{L}}(\mathrm{GS})$ & & SEPT. 4 & 08:09 PST \\
\hline SEPT. 7 & 125512.4 & $37.394 \mathrm{~N}$. & 117.070W. & 6 & PS & & & $3.1 \mathrm{M}_{\mathrm{L}}(\mathrm{PS})$ & & SEPT. 7 & 04:55 PST \\
\hline SEPT. 11 & 145700.1 & $37.069 \mathrm{~N}$ & $116.050 \mathrm{~W}$ & 0 & EN & & & $3.2 \mathrm{M}_{\mathrm{L}}(\mathrm{GS})$ & & SEPT. 11 & 06:57 PST \\
\hline SEPT. 11 & 234008.7 & $38.528 \mathrm{~N}$ & $117.055 \mathrm{~W}$ & 0 & RN & & & 3.7 $\mathrm{M}_{\mathrm{D}}(\mathrm{RN})$ & & SEPT. 11 & 15:40 PST \\
\hline SEPT. 24 & 142033.6 & $37.374 \mathrm{~N}$. & 117.199W. & 5 & GS & & & $4.3 \mathrm{M}_{\mathrm{L}}(\mathrm{BK})$ & & SEPT. 24 & 06:20 PST \\
\hline SEPT. 24 & 143555.9 & $37.351 \mathrm{~N}$ & $117.206 \mathrm{~W}$. & 5 & GS & . & . & $4.1 \mathrm{M}_{\mathrm{L}}(\mathrm{BK})$ & & SEPT. 24 & 06:35 PST \\
\hline SEPT. 30 & 223000.1 & $37.300 \mathrm{~N}$ & $116.307 \mathrm{~W}$ & 0 & EN & 5.5 & 4.5 & $5.3 \mathrm{M}_{\mathrm{L}}(\mathrm{BK})$ & & SEPT. 30 & 14:30 PST \\
\hline OCT. 1 & 153423.8 & $40.721 \mathrm{~N}$ & $116.370 \mathrm{~W}$. & 5 & GS & - & - & $3.7 \mathrm{M}_{\mathrm{L}}(\mathrm{GS})$ & & OCT. 1 & 07:34 PST \\
\hline OCT. 5 & 055509.8 & $40.431 N$ & $116.804 \mathrm{~W}$. & 0 & RN & - & & $3.2 \mathrm{M}_{\mathrm{D}}(\mathrm{RN})$ & & OCT. & 21:55 PST \\
\hline OCT. 16 & 192500.0 & $37.220 \mathrm{~N}$. & $116.462 \mathrm{~W}$. & 0 & EN & 5.6 & & $5.4 \mathrm{M}_{\mathrm{L}}(\mathrm{BK})$ & & OCT. 16 & 11:25 PST \\
\hline OCT. 23 & 133657.5 & $39.160 \mathrm{~N}$. & 118.046W. & 8 & $\mathbf{R N}$ & - & - & 3.0 $\mathrm{M}_{\mathrm{D}}(\mathrm{RN})$ & & OCT. & 05:36 PST \\
\hline OCT. 26 & 051735.3 & $37.224 \mathrm{~N}$ & $116.448 \mathrm{~W}$ & 5 & GS & - & $\longrightarrow$ & $3.1 \mathrm{M}_{\mathrm{L}}(\mathrm{PS})$ & & OCT. 25 & 21:17 PST \\
\hline NOV. 1 & 192338.3 & $38.712 \mathrm{~N}$. & $119.540 \mathrm{~W}$. & 17 & BK & - & - & $4.6 \mathrm{M}_{\mathrm{L}}(\mathrm{BK})$ & V & NOV. 1 & 11:23 PST \\
\hline NOV. 14 & 160000.0 & $37.100 \mathrm{~N}$. & 116.048W. & 0 & EN & 5.8 & 4.5 & $5.5 \mathrm{M}_{\mathrm{L}}(\mathrm{BK})$ & III & NOV. 14 & 08:00 PST \\
\hline NOV. 14 & 200238.7 & $37.081 \mathrm{~N}$ & $116.014 \mathrm{~W}$. & 0 & GS & 4.0 & - & - & 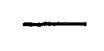 & NOV. 14 & $12: 02$ PST \\
\hline NOV. 26 & 033922.8 & $39.097 \mathrm{~N}$. & 115.621W. & 5 & GS & - & & $3.2 \mathrm{M}_{\mathrm{L}}(\mathrm{GS})$ & & NOV. 25 & 19:39 PST \\
\hline NOV. 26 & 052403.1 & $40.065 \mathrm{~N}$ & $117.956 \mathrm{~W}$. & 6 & RN & - & - & $3.3 \mathrm{M}_{\mathrm{D}}(\mathrm{RN})$ & & NOV. 25 & 21:24 PST \\
\hline DEC. 13 & 175005.0 & $37.263 \mathrm{~N}$ & $116.412 \mathrm{~W}$. & 0 & EN & 5.5 & 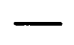 & $5.4 \mathrm{M}_{\mathrm{L}}(\mathrm{BK})$ & & DEC. 13 & 09:50 PST \\
\hline
\end{tabular}

\section{NEW HAMPSHIRE}

\begin{tabular}{|c|c|c|c|c|c|c|c|c|c|c|c|c|c|}
\hline JAN. & 23 & 143357.5 & $43.500 \mathrm{~N}$. & $71.568 \mathrm{~W}$. & 5 & WO & - & - & $2.6 \mathrm{M}_{\mathrm{L}}(\mathrm{WO})$ & III & JAN. & 23 & 09:33 EST \\
\hline MAR. & 14 & 134407.0 & $43.460 \mathrm{~N}$. & $71.591 \mathrm{~W}$ & 3 & Wo & - & - & $2.5 \mathrm{M}_{\mathrm{b}}(\mathrm{WO})$ & & MAR. & 14 & 08:44 EST \\
\hline OCT. & 25 & 171638.4 & $43.399 \mathrm{~N}$. & $71.590 \mathrm{~W}$. & 5 & GS & 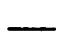 & & $3.9 \mathrm{M}_{\mathrm{D}}(\mathrm{GS})$ & V & OCT. & 25 & $12: 16$ EST \\
\hline OCT. & 25 & 182114.4 & $43.420 \mathrm{~N}$. & $71.588 \mathrm{~W}$. & 9 & WO & 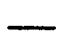 & - & $2.8 \mathrm{M}_{\mathrm{D}}(\mathrm{WO})$ & - & OCT. & 25 & $13: 21 \mathrm{EST}$ \\
\hline
\end{tabular}

NEW JERSEY

$\begin{array}{lllllllllll}\text { NOV. } 23 & 212938.8 & 40.956 \mathrm{~N} . & 74.820 \mathrm{~W} . & 7 & \mathrm{LD} & - & - & -8 \mathrm{M}_{\mathrm{D}}(\mathrm{LD}) & \text { IV } & \text { NOV. } 23 \text { 16:29 EST }\end{array}$

\section{NEW MEXICO}

\begin{tabular}{|c|c|c|c|c|c|c|c|c|c|c|c|}
\hline APR. 17 & 210430.3 & $32.587 \mathrm{~N}$. & $106.912 \mathrm{~W}$. & 5 & GS & - & - & $2.7 \mathrm{M}_{\mathrm{D}}(\mathrm{GS})$ & FELT & APR. 17 & 14:04 MST \\
\hline APR. 28 & 130016.0 & $34.009 \mathrm{~N}$. & $106.821 \mathrm{~W}$ & 5 & GS & & & $2.6 \mathrm{M}_{\mathrm{D}}(\mathrm{GS})$ & FELT & APR. & 06:00 MST \\
\hline AUG. 27 & 180656.3 & $35.160 \mathrm{~N}$. & 105.094W. & 5 & GS & - & - & $3.2 \mathrm{M}_{\mathrm{L}}(\mathrm{GS})$ & FELT & AUG. 27 & 11:06 MST \\
\hline
\end{tabular}


Table 1. Summary of United States earthquakes for 1986-Continued

\begin{tabular}{|c|c|c|c|c|c|c|c|c|c|c|c|}
\hline \multirow[t]{2}{*}{ Date } & \multirow{2}{*}{$\begin{array}{l}\text { Origin time } \\
\text { (UTC) } \\
\text { hr min sec }\end{array}$} & \multirow{2}{*}{$\begin{array}{l}\text { Latitude } \\
\text { ()) }\end{array}$} & \multirow{2}{*}{$\begin{array}{c}\text { Longitude } \\
\left({ }^{\circ}\right)\end{array}$} & \multirow{2}{*}{$\begin{array}{l}\text { Depth } \\
(\mathrm{km})\end{array}$} & \multirow{2}{*}{$\begin{array}{l}\text { Hypo- } \\
\text { center } \\
\text { Source }\end{array}$} & \multicolumn{3}{|c|}{ Magnitude } & \multirow{2}{*}{$\begin{array}{l}\text { Max. } \\
\text { inten- } \\
\text { sity }\end{array}$} & \multicolumn{2}{|c|}{ Local time } \\
\hline & & & & & & $m_{b}$ & $\mathrm{M}_{\mathbf{s}}$ & Local & & Date & zone \\
\hline \multicolumn{12}{|c|}{ NEW YORK } \\
\hline JAN. 5 & 033556.2 & $40.996 \mathrm{~N}$. & $73.833 W$. & 6 & LD & - & - & $2.5 \mathrm{M}_{\mathrm{n}}(\mathrm{LD})$ & IV & JAN. & 22:35 EST \\
\hline JAN. 31 & 231625.3 & $43.775 \mathrm{~N}$. & $73.427 \mathrm{~W}$. & 19 & WO & - & - & $2.6 \mathrm{M}_{\mathrm{D}}(\mathrm{WO})$ & & JAN. 31 & 18:16 EST \\
\hline APR. 18 & 125016.7 & $43.981 \mathrm{~N}$. & $74.240 \mathrm{~W}$. & 11 & WO & - & - & $2.5 \mathrm{M}_{\mathrm{n}}(\mathrm{WO})$ & $\ldots$ & APR. 18 & 07:50 EST \\
\hline APR. 22 & 072823.7 & $40.980 \mathrm{~N}$ & $73.834 W$. & 6 & LD & - & - & $2.7 \mathrm{M}_{\mathrm{D}}(\mathrm{LD})$ & IV & APR. 22 & 02:28 EST \\
\hline NOV. 17 & 125432.1 & $44.746 \mathrm{~N}$ & $73.914 W$. & 6 & LD & - & - & $2.8 \mathrm{M}_{\mathrm{D}}(\mathrm{LD})$ & - & NOV. 17 & 07:54 EST \\
\hline DEC. 20 & 131531.0 & $40.999 \mathrm{~N}$. & $73.831 \mathrm{~W}$. & 5 & LD & - & - & $1.9 \mathrm{M}_{\mathrm{D}}(\mathrm{LD})$ & FELT & DEC. 20 & 08:15 EST \\
\hline
\end{tabular}

\section{NORTH CAROLINA}

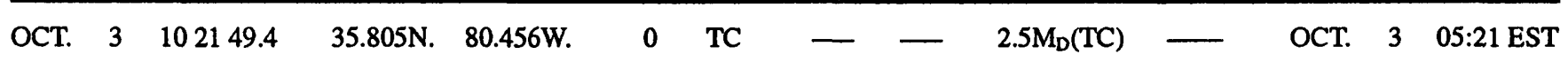

\section{OHIO}

\begin{tabular}{|c|c|c|c|c|c|c|c|c|c|c|c|c|}
\hline JAN. & 31 & 164642.3 & $41.650 \mathrm{~N}$ & $81.162 \mathrm{~W}$. & 2 & NI & 5.0 & - & $4.9 \mathrm{M}_{\mathrm{n}}(\mathrm{SL})$ & VI & JAN. 31 & 11:46 EST \\
\hline FEB. & 7 & 183622.3 & $41.645 \mathrm{~N}$ & $81.157 \mathrm{~W}$. & 6 & GS & $\longrightarrow$ & $\longrightarrow$ & $2.5 \mathrm{M}_{\mathrm{n}}(\mathrm{GS})$ & IV & FEB. & 13:36 EST \\
\hline JULY & 12 & 081937.9 & $40.537 \mathrm{~N}$ & 84.371W. & 10 & GS & 4.5 & - & $4.6 \mathrm{M}_{\mathrm{n}}(\mathrm{SL})$ & VI & JULY 12 & 03:19 EST \\
\hline
\end{tabular}

\section{OKLAHOMA}

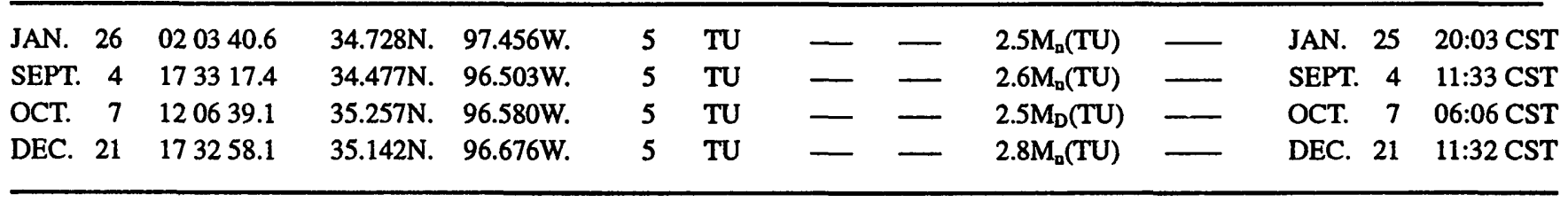

\section{OREGON}

\begin{tabular}{|c|c|c|c|c|c|c|c|c|c|c|c|c|c|}
\hline JAN. & 22 & 115024.5 & $44.642 \mathrm{~N}$ & $130.872 \mathrm{~W}$ & 10 & GS & 4.5 & - & - & & JAN. & 22 & 02:50 YST \\
\hline JAN. & 30 & 071533.5 & $43.601 \mathrm{~N}$. & $127.339 \mathrm{~W}$ & 10 & GS & 5.2 & - & & & JAN. & 29 & 23:15 PST \\
\hline MAR. & 19 & 085007.7 & $43.938 \mathrm{~N}$. & $128.422 \mathrm{~W}$ & 10 & GS & 4.8 & 4.7 & & & MAR. & 19 & 00:50 PST \\
\hline MAR. & 26 & 200333.9 & $43.390 \mathrm{~N}$ & $126.878 \mathrm{~W}$ & 10 & GS & 4.5 & - & & & MAR. & 26 & 12:03 PST \\
\hline MAR. & 31 & 181518.9 & $44.079 \mathrm{~N}$ & $128.319 \mathrm{~W}$ & 10 & GS & 3.9 & - & & & MAR. & 31 & 10:15 PST \\
\hline MAY & 4 & 151604.1 & $44.219 \mathrm{~N}$ & 129.218W. & 10 & GS & 4.6 & - & - & & MAY & 4 & 07:16 PST \\
\hline MAY & 4 & 154207.5 & $44.206 \mathrm{~N}$. & 129.299W. & 10 & GS & 4.5 & 4.2 & & & MAY & 4 & 07:42 PST \\
\hline MAY & 20 & 102257.6 & $42.093 \mathrm{~N}$ & 126.741W. & 10 & GS & 4.6 & 4.3 & & & MAY & 20 & 02:22 PST \\
\hline JUNE & 13 & 183704.2 & $43.628 \mathrm{~N}$ & 127.380W. & 10 & GS & 4.4 & - & & & JUNE & 13 & $10: 37$ PST \\
\hline JUNE & 29 & 093852.5 & $44.251 \mathrm{~N}$ & 129.128W. & 10 & GS & 4.0 & - & & & JUNE & 29 & 01:38 PST \\
\hline JULY & 18 & 204239.7 & $44.207 \mathrm{~N}$ & 128.240W. & 10 & GS & 4.0 & - & & & JULY & 18 & 12:42 PST \\
\hline JULY & 19 & 092548.5 & $42.564 \mathrm{~N}$ & 127.355W. & 10 & GS & 4.1 & & & & JULY & 19 & 01:25 PST \\
\hline JULY & 21 & 142523.9 & $43.682 \mathrm{~N}$ & 127.411W. & 10 & GS & 4.1 & - & - & & JULY & 21 & 06:25 PST \\
\hline SEPT. & 12 & 011912.3 & 43.991N. & 128.921W. & 10 & GS & 4.3 & 4.5 & & & SEPT. & 11 & 17:19 PST \\
\hline SEPT. & 23 & 214305.0 & $44.580 \mathrm{~N}$ & 129.685W. & 10 & GS & 4.1 & - & & & SEPT. & 23 & 13:43 PST \\
\hline OCT. & 5 & 215720.5 & $43.493 \mathrm{~N}$ & $127.252 W$ & 10 & GS & 5.1 & 5.1 & & & OCT. & 5 & 13:57 PST \\
\hline OCT. & 6 & 070320.7 & $44.229 \mathrm{~N}$ & $129.524 \mathrm{~W}$ & 10 & GS & 4.5 & - & - & & OCT. & 5 & 23:03 PST \\
\hline
\end{tabular}


Table 1. Summary of United States earthquakes for 1986 - Continued

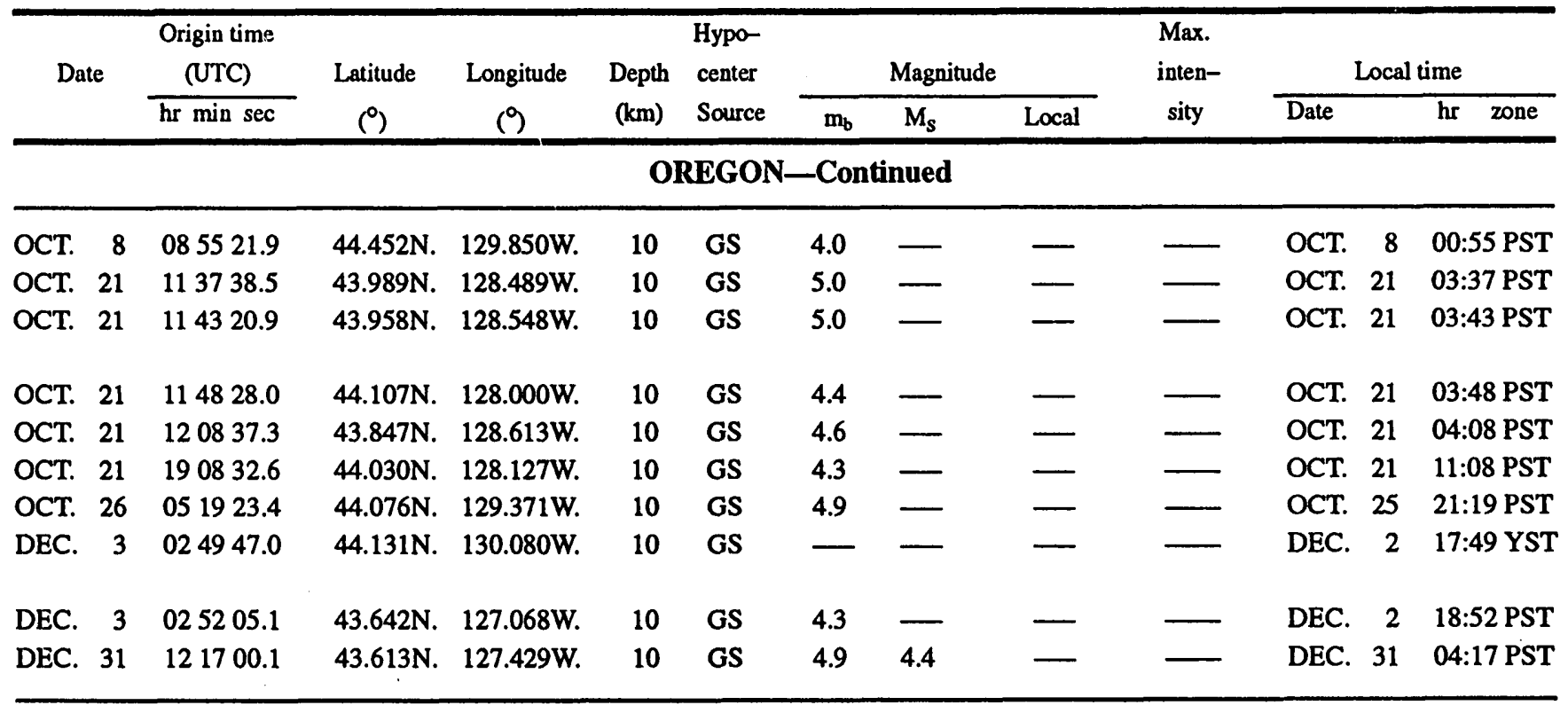

PENNSYLVANIA

\begin{tabular}{llllllllllllll}
\hline MAY & 2 & 135352.6 & $39.925 N$ & $76.293 W$. & 5 & GS & - & - & $2.5 \mathrm{M}_{\mathrm{D}}(\mathrm{DE})$ & IV & MAY & 2 & $08: 53 \mathrm{EST}$
\end{tabular}

\section{SOUTH CAROLINA}

\begin{tabular}{|c|c|c|c|c|c|c|c|c|c|c|c|}
\hline FEB. 13 & 113545.3 & $34.793 \mathrm{~N}$. & $82.907 \mathrm{~W}$. & 5 & TC & - & - & $3.5 \mathrm{M}_{\mathrm{n}}(\mathrm{GS})$ & V & FEB. 13 & 06:35 EST \\
\hline MAR. 9 & 234915.3 & $32.968 \mathrm{~N}$. & $80.169 \mathrm{~W}$ & 6 & GS & & - & $2.2 \mathrm{M}_{\mathrm{D}}(\mathrm{GS})$ & III & MAR. 9 & 18:49 EST \\
\hline JUNE 11 & 161200.5 & $34.777 \mathrm{~N}$. & $82.916 \mathrm{~W}$ & 4 & SC & & & $2.8 \mathrm{M}_{\mathrm{D}}(\mathrm{SC})$ & . & JUNE 11 & $11: 12$ EST \\
\hline JULY 14 & 223121.1 & $34.760 \mathrm{~N}$ & $82.936 \mathrm{~W}$. & 4 & SC & & & $2.6 \mathrm{M}_{\mathrm{D}}(\mathrm{SC})$ & - & JULY 14 & 17:31 EST \\
\hline SEPT. 17 & 093349.4 & $32.928 \mathrm{~N}$ & $80.152 W$. & 8 & GS & & & $2.6 \mathrm{M}_{\mathrm{D}}(\mathrm{GS})$ & IV & SEPT. 17 & 04:33 EST \\
\hline OCT. 18 & 083138.8 & $34.946 \mathrm{~N}$. & 81.172W. & 23 & TC & & & $2.9 \mathrm{M}_{\mathrm{D}}(\mathrm{TC})$ & FELT & OCT. 18 & 03:31 EST \\
\hline DEC. 11 & 140550.2 & $34.889 \mathrm{~N}$ & $82.887 \mathrm{~W}$ & 9 & TC & & & $2.9 \mathrm{M}_{\mathrm{D}}(\mathrm{TC})$ & FELT & DEC. 11 & 09:05 EST \\
\hline DEC. 11 & 140711.5 & $34.898 \mathrm{~N}$. & $82.880 \mathrm{~W}$. & 9 & TC & $\longrightarrow$ & - & $3.0 \mathrm{M}_{\mathrm{D}}(\mathrm{TC})$ & FELT & DEC. 11 & 09:07 EST \\
\hline
\end{tabular}

\section{SOUTH DAKOTA}

\begin{tabular}{llllllllllllll}
\hline MAY & 25 & 071322.1 & $43.937 \mathrm{~N}$. & $98.289 \mathrm{~W}$. & 5 & GS & - & - & $3.4 \mathrm{M}_{\mathrm{p}}(\mathrm{GS})$ & IV & MAY & 25 & $01: 13 \mathrm{CST}$
\end{tabular}

TENNESSEE

\begin{tabular}{|c|c|c|c|c|c|c|c|c|c|c|c|c|c|}
\hline JAN. & 7 & 012643.3 & $35.609 \mathrm{~N}$. & $84.762 W$. & 22 & TC & - & - & $3.2 \mathrm{M}_{\mathrm{D}}(\mathrm{TC})$ & V & JAN. & 6 & 20:26 EST \\
\hline JAN. & 27 & 064426.8 & $35.926 \mathrm{~N}$. & $83.636 \mathrm{~W}$. & 20 & TC & & & $2.7 \mathrm{M}_{\mathrm{D}}(\mathrm{TC})$ & & JAN. & 27 & 01:44 EST \\
\hline APR. & 19 & 074053.0 & $35.187 \mathrm{~N}$. & $85.510 \mathrm{~W}$. & 27 & TC & & & $3.0 \mathrm{M}_{\mathrm{D}}(\mathrm{TC})$ & & APR. & 19 & 02:40 EST \\
\hline MAY & 19 & 234647.0 & $35.516 \mathrm{~N}$. & 84.529W. & 11 & TC & & & $2.8 \mathrm{M}_{\mathrm{D}}(\mathrm{TC})$ & & MAY & 19 & 18:46 EST \\
\hline JUNE & 2 & 074612.4 & $35.441 \mathrm{~N}$ & $84.498 W$. & 19 & TC & & & $2.5 \mathrm{M}_{\mathrm{D}}(\mathrm{TC})$ & & JUNE & 2 & 02:46 EST \\
\hline JUNE & 24 & 192242.0 & $35.990 \mathrm{~N}$. & 83.931W. & 24 & TC & & & $2.9 \mathrm{M}_{\mathrm{D}}(\mathrm{TC})$ & & JUNE & 24 & 14:22 EST \\
\hline AUG. & 7 & 123646.0 & $35.506 \mathrm{~N}$. & $84.561 \mathrm{~W}$. & 20 & TC & & & $2.7 \mathrm{M}_{\mathrm{D}}(\mathrm{TC})$ & & AUG. & 7 & 07:36 EST \\
\hline AUG. & 19 & 205126.0 & $36.291 \mathrm{~N}$. & $85.020 \mathrm{~W}$. & 30 & TC & & & $2.9 \mathrm{M}_{\mathrm{D}}(\mathrm{TC})$ & FELT & AUG. & 19 & 15:51 EST \\
\hline SEPT. & 12 & 174156.0 & $35.65 \mathrm{~N}$ & $89.66 \mathrm{~W}$. & 9 & SL & & & $2.9 \mathrm{M}_{\mathrm{v}}(\mathrm{SL})$ & 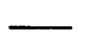 & SEPT. & 12 & 11:41 CST \\
\hline OCT. & 18 & 225329.2 & $36.24 \mathrm{~N}$. & $89.51 \mathrm{~W}$ & 5 & SL & - & 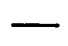 & $2.5 \mathrm{M}_{\mathrm{n}}(\mathrm{SL})$ & 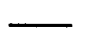 & OCT. & 18 & $16: 53 \mathrm{CST}$ \\
\hline
\end{tabular}


Table 1. Summary of United States earthquakes for 1986-Continued

\begin{tabular}{|c|c|c|c|c|c|c|c|c|c|c|c|}
\hline \multirow[t]{2}{*}{ Date } & \multirow{2}{*}{$\begin{array}{l}\text { Origin time } \\
\text { (UTC) } \\
\text { hr min sec }\end{array}$} & \multirow{2}{*}{$\begin{array}{c}\text { Latitude } \\
\text { ( ) }\end{array}$} & \multirow{2}{*}{$\begin{array}{l}\text { Longitude } \\
\text { ( })^{\circ}\end{array}$} & \multirow{2}{*}{$\begin{array}{c}\text { Depth } \\
(\mathbf{k m})\end{array}$} & \multirow{2}{*}{$\begin{array}{l}\text { Hypo- } \\
\text { center } \\
\text { Source }\end{array}$} & \multicolumn{3}{|c|}{ Magnitude } & \multirow{2}{*}{$\begin{array}{l}\text { Max. } \\
\text { inten- } \\
\text { sity }\end{array}$} & \multicolumn{2}{|c|}{ Local time } \\
\hline & & & & & & $\mathrm{m}_{\mathrm{b}}$ & $\mathbf{M}_{\boldsymbol{S}}$ & Local & & Date & zone \\
\hline \multicolumn{12}{|c|}{ TEXAS } \\
\hline JAN. 25 & 225024.9 & $32.064 \mathrm{~N}$ & $100.733 \mathrm{~W}$. & 5 & GS & - & - & $2.9 \mathrm{M}_{\mathrm{D}}(\mathrm{GS})$ & - & JAN. 25 & $16: 50 \mathrm{CST}$ \\
\hline JAN. 30 & 222637.0 & $32.066 \mathrm{~N}$. & $100.693 \mathrm{~W}$. & 5 & GS & - & $\longrightarrow$ & $3.3 \mathrm{M}_{\mathrm{n}}(\mathrm{GS})$ & III & JAN. $\quad 30$ & $16: 26 \mathrm{CST}$ \\
\hline MAR. 3 & 114517.4 & $35.308 \mathrm{~N}$ & $102.514 \mathrm{~W}$ & 5 & GS & - & - & $3.1 \mathrm{M}_{\mathrm{n}}(\mathrm{TU})$ & 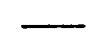 & MAR. 3 & 05:45 CST \\
\hline DEC. 11 & 012300.6 & $35.090 \mathrm{~N}$. & 101.605W. & 5 & TU & - & - & $2.5 \mathrm{M}_{\mathrm{n}}(\mathrm{TU})$ & - & DEC. 10 & 19:23 CST \\
\hline
\end{tabular}

UTAH

\begin{tabular}{|c|c|c|c|c|c|c|c|c|c|c|c|}
\hline JAN. & 123204.6 & $41.715 \mathrm{~N}$ & $111.665 \mathrm{~W}$. & 7 & UU & - & - & $3.3 \mathrm{M}_{\mathrm{L}}(\mathrm{UU})$ & IV & JAN. & 05:32 MST \\
\hline JAN. & 192901.1 & $41.351 \mathrm{~N}$ & $111.626 \mathrm{~W}$. & 9 & UU & & & $2.7 \mathrm{M}_{\mathrm{L}}(\mathrm{UU})$ & & JAN. & 12:29 MST \\
\hline JAN. & 045628.1 & $41.919 \mathrm{~N}$ & $112.535 \mathrm{~W}$. & 6 & UU & & & $2.5 \mathrm{M}_{\mathrm{L}}(\mathrm{UU})$ & & JAN. & 21:56 MST \\
\hline JAN. & 095052.8 & $42.345 \mathrm{~N}$ & $111.359 \mathrm{~W}$. & 1 & UU & & & $2.5 \mathrm{M}_{\mathrm{L}}(\mathrm{UU})$ & & JAN. 30 & 02:50 MST \\
\hline FEB. & 230913.1 & $39.703 \mathrm{~N}$ & $110.565 \mathrm{~W}$ & 0 & UU & & & $2.7 M_{D}(U U)$ & & FEB. 11 & 16:09 MST \\
\hline FEB. 14 & 005621.3 & $39.686 \mathrm{~N}$. & $110.525 \mathrm{~W}$. & 0 & UU & & & $3.2 \mathrm{M}_{\mathrm{D}}(\mathrm{UU})$ & & FEB. 13 & 17:56 MST \\
\hline FEB. & 232012.5 & $41.745 \mathrm{~N}$ & $112.852 \mathrm{~W}$ & 7 & UU & & & $3.6 \mathrm{M}_{\mathrm{L}}(\mathrm{UU})$ & & FEB. 21 & 16:20 MST \\
\hline MAR. 4 & 200234.9 & $40.768 \mathrm{~N}$ & $110.557 \mathrm{~W}$ & 0 & UU & & - & $2.7 \mathrm{M}_{\mathrm{L}}(\mathrm{UU})$ & & MAR. 4 & 13:02 MST \\
\hline MAR. 9 & 204806.3 & $40.682 \mathrm{~N}$ & 109.567W. & 3 & UU & & & $2.5 \mathrm{M}_{\mathrm{D}}(\mathrm{UU})$ & & MAR. 9 & 13:48 MST \\
\hline MAR. 12 & 061724.7 & $39.326 \mathrm{~N}$. & $111.094 \mathrm{~W}$ & 0 & UU & & & 2.6ML(UU) & & MAR. 11 & 23:17 MST \\
\hline MAR. 24 & 223341.2 & $39.221 \mathrm{~N}$ & $111.998 \mathrm{~W}$. & 0 & UU & - & 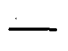 & $3.3 \mathrm{M}_{\mathrm{L}}(\mathrm{UU})$ & & MAR. 24 & 15:33 MST \\
\hline MAR. 24 & 224023.4 & $39.234 \mathrm{~N}$. & $112.062 \mathrm{~W}$ & 1 & UU & 4.7 & & $4.4 \mathrm{M}_{\mathrm{L}}(\mathrm{UU})$ & V & MAR. 24 & $15: 40 \mathrm{MST}$ \\
\hline MAR. 25 & 024906.5 & $39.228 \mathrm{~N}$ & $112.062 \mathrm{~W}$ & 1 & UU & & & $2.8 \mathrm{M}_{\mathrm{L}}(\mathrm{UU})$ & & MAR. 24 & 19:49 MST \\
\hline MAR. 25 & 025301.3 & $39.225 \mathrm{~N}$ & $112.013 W$ & 1 & UU & 4.5 & & $3.9 \mathrm{M}_{\mathrm{L}}(\mathrm{UU})$ & V & MAR. 24 & 19:53 MST \\
\hline MAY 14 & 150255.7 & $37.294 \mathrm{~N}$ & $110.319 \mathrm{~W}$ & 8 & UU & & & $3.2 \mathrm{M}_{\mathrm{L}}(\mathrm{GS})$ & & MAY 14 & 08:02 MST \\
\hline MAY 28 & 001754.3 & $39.773 \mathrm{~N}$. & $112.791 \mathrm{~W}$ & 3 & UU & & & $2.8 \mathrm{M}_{\mathrm{L}}(\mathrm{UU})$ & & MAY 27 & 17:17 MST \\
\hline JUNE 5 & 074121.0 & $41.266 \mathrm{~N}$ & $111.679 \mathrm{~W}$. & 12 & UU & & & $2.8 \mathrm{M}_{\mathrm{L}}(\mathrm{UU})$ & & JUNE 5 & 00:41 MST \\
\hline JUNE & 080541.7 & $41.266 \mathrm{~N}$ & $111.684 W$. & 10 & UU & & & $3.6 \mathrm{M}_{\mathrm{L}}(\mathrm{UU})$ & FELT & JUNE & 01:05 MST \\
\hline JUNE 5 & 193402.6 & $41.382 \mathrm{~N}$ & $109.677 \mathrm{~W}$ & 7 & UU & & & $2.7 \mathrm{M}_{\mathrm{D}}(\mathrm{UU})$ & & JUNE 5 & 12:34 MST \\
\hline JUNE 28 & 211624.0 & $40.325 \mathrm{~N}$ & $111.379 \mathrm{~W}$ & 7 & UU & & & $2.5 \mathrm{M}_{\mathrm{L}}(\mathrm{UU})$ & & JUNE 28 & $14: 16 \mathrm{MST}$ \\
\hline JULY 31 & 033328.2 & $38.225 \mathrm{~N}$ & $112.556 \mathrm{~W}$ & 1 & UU & & & $2.6 \mathrm{M}_{\mathrm{D}}(\mathrm{UU})$ & & JULY 30 & 20:33 MST \\
\hline AUG. 7 & 223122.9 & $39.697 \mathrm{~N}$. & $110.736 \mathrm{~W}$ & 0 & UU & & & $2.5 \mathrm{M}_{\mathrm{L}}(\mathrm{UU})$ & & AUG. 7 & 15:31 MST \\
\hline AUG. 22 & 132633.3 & $37.420 \mathrm{~N}$. & $110.574 \mathrm{~W}$ & 5 & GS & & & $4.0 \mathrm{M}_{\mathrm{L}}(\mathrm{UU})$ & V & AUG. 22 & 06:26 MST \\
\hline AUG. 25 & 052925.7 & $41.490 \mathrm{~N}$ & $111.945 \mathrm{~W}$ & 18 & UU & & & $2.5 \mathrm{M}_{\mathrm{D}}(\mathrm{UU})$ & & AUG. 24 & 22:29 MST \\
\hline AUG. 31 & 044701.3 & $38.966 \mathrm{~N}$ & $111.419 \mathrm{~W}$. & 2 & UU & & & $2.5 \mathrm{M}_{\mathrm{D}}(\mathrm{UU})$ & & AUG. 30 & 21:47 MST \\
\hline SEPT. 14 & 034025.6 & $41.295 \mathrm{~N}$ & $111.474 \mathrm{~W}$ & 9 & UU & & & $2.8 \mathrm{M}_{\mathrm{D}}(\mathrm{UU})$ & & SEPT. 13 & 20:40 MST \\
\hline SEPT. 19 & 104128.2 & $41.466 \mathrm{~N}$ & $111.702 W$ & 7 & UU & & & $3.4 \mathrm{M}_{\mathrm{L}}(\mathrm{UU})$ & III & SEPT. 19 & 03:41 MST \\
\hline SEPT. 24 & 023836.0 & $38.585 \mathrm{~N}$ & $112.562 \mathrm{~W}$ & 0 & UU & & & $2.5 \mathrm{M}_{\mathrm{D}}(\mathrm{UU})$ & & SEPT. 23 & 19:38 MST \\
\hline SEPT. 24 & 172808.3 & $40.703 \mathrm{~N}$ & 109.447W. & 7 & UU & & & $2.6 \mathrm{M}_{\mathrm{D}}(\mathrm{UU})$ & & SEPT. 24 & 10:28 MST \\
\hline SEPT. 25 & 124531.3 & $38.614 \mathrm{~N}$ & $112.553 \mathrm{~W}$. & 1 & UU & & & $2.8 \mathrm{M}_{\mathrm{L}}(\mathrm{GS})$ & & SEPT. 25 & 05:45 MST \\
\hline SEPT. 25 & 223115.0 & $38.602 \mathrm{~N}$. & $112.555 \mathrm{~W}$. & 0 & UU & & & $3.1 \mathrm{M}_{\mathrm{D}}(\mathrm{UU})$ & & SEPT. 25 & 15:31 MST \\
\hline SEPT. 26 & 074120.6 & $41.848 \mathrm{~N}$. & $112.320 \mathrm{~W}$. & 20 & UU & & & $2.5 \mathrm{M}_{\mathrm{D}}(\mathrm{UU})$ & & SEPT. 26 & 00:41 MST \\
\hline SEPT. 27 & 073414.8 & $39.561 \mathrm{~N}$. & $110.403 \mathrm{~W}$ & 0 & UU & & & $2.6 \mathrm{M}_{\mathrm{L}}(\mathrm{UU})$ & & SEPT. 27 & 00:34 MST \\
\hline OCT. 1 & 115146.7 & $40.818 \mathrm{~N}$ & $111.821 \mathrm{~W}$ & 5 & UU & & & $2.7 \mathrm{M}_{\mathrm{L}}(\mathrm{UU})$ & III & OCT. 1 & 04:51 MST \\
\hline OCT. & 154733.4 & $38.631 \mathrm{~N}$. & $112.558 \mathrm{~W}$ & $\mathbf{0}$ & UU & - & - & $3.3 \mathrm{M}_{\mathrm{L}}(\mathrm{UU})$ & III & OCT. & 08:47 MST \\
\hline
\end{tabular}


Table 1. Summary of United States earthquakes for 1986_Continued

\begin{tabular}{|c|c|c|c|c|c|c|c|c|c|c|c|c|c|}
\hline \multirow[t]{2}{*}{ Date } & & \multirow{2}{*}{$\begin{array}{l}\text { Origin time } \\
\text { (UTC) } \\
\text { hr min sec }\end{array}$} & \multirow{2}{*}{$\begin{array}{l}\text { Latitude } \\
\text { ( ) }\end{array}$} & \multirow{2}{*}{$\begin{array}{l}\text { Longitude } \\
\text { ( })\end{array}$} & \multirow{2}{*}{$\begin{array}{c}\text { Depth } \\
(\mathbf{k m})\end{array}$} & \multirow{2}{*}{$\begin{array}{l}\text { Hypo- } \\
\text { center } \\
\text { Source }\end{array}$} & \multicolumn{3}{|c|}{ Magnitude } & \multirow{2}{*}{$\begin{array}{l}\text { Max. } \\
\text { inten- } \\
\text { sity }\end{array}$} & \multicolumn{3}{|c|}{ Local time } \\
\hline & & & & & & & $\mathbf{m}_{\mathbf{b}}$ & $\mathbf{M}_{s}$ & Local & & Date & & zone \\
\hline \multicolumn{14}{|c|}{ UTAH-Continued } \\
\hline OCT. & 5 & 155948.5 & $38.622 \mathrm{~N}$. & $112.557 \mathrm{~W}$ & 1 & UU & & - & $2.9 \mathrm{M}_{\mathrm{L}}(\mathrm{UU})$ & - & OCT. & 5 & 08:59 MST \\
\hline OCT. & 25 & 100325.1 & $41.825 \mathrm{~N}$. & $112.317 \mathrm{~W}$ & 5 & UU & - & - & $2.6 \mathrm{M}_{\mathrm{L}}(\mathrm{UU})$ & & OCT. & 25 & 03:03 MST \\
\hline OCT. & 26 & 123857.7 & $41.825 \mathrm{~N}$ & $112.321 \mathrm{~W}$ & 4 & UU & - & - & $2.5 \mathrm{M}_{\mathrm{D}}(\mathrm{UU})$ & & OCT. & 26 & 05:38 MST \\
\hline OCT. & 26 & 143156.7 & $41.824 \mathrm{~N}$ & $112.316 \mathrm{~W}$ & 4 & UU & & & $3.0 \mathrm{M}_{\mathrm{L}}(\mathrm{UU})$ & & OCT. & 26 & 07.31 MST \\
\hline OCT. & 27 & 083448.3 & $41.825 \mathrm{~N}$. & $112.318 \mathrm{~W}$. & 2 & UU & - & - & $2.5 \mathrm{M}_{\mathrm{D}}(\mathrm{UU})$ & & OCT. & 27 & 01:34 MST \\
\hline OCT. & 29 & 221314.5 & $41.821 \mathrm{~N}$. & $112.318 \mathrm{~W}$. & 5 & UU & - & - & $3.6 \mathrm{M}_{\mathrm{L}}(\mathrm{UU})$ & IV & OCT. & 29 & 15:13 MST \\
\hline OCT. & 30 & 000542.8 & $39.735 \mathrm{~N}$. & $110.965 \mathrm{~W}$. & 6 & UU & - & - & $2.8 \mathrm{M}_{\mathrm{L}}(\mathrm{UU})$ & - & OCT. & 29 & 17:05 MST \\
\hline OCT. & 31 & 115828.2 & $41.823 \mathrm{~N}$ & 112.316W. & 4 & UU & $\ldots$ & & $3.5 \mathrm{M}_{\mathrm{L}}(\mathrm{UU})$ & IV & OCT. & 31 & 04:58 MST \\
\hline NOV. & 7 & 013153.7 & $37.430 \mathrm{~N}$. & $110.297 \mathrm{~W}$ & 1 & UU & - & & $3.0 \mathrm{M}_{\mathrm{D}}(\mathrm{UU})$ & & NOV. & 6 & 18:31 MST \\
\hline NOV. & 8 & 044829.4 & $41.828 \mathrm{~N}$ & 112.316W. & 4 & UU & - & - & $2.7 \mathrm{M}_{\mathrm{L}}(\mathrm{UU})$ & & NOV. & 7 & 21:48 MST \\
\hline NOV. & 13 & 232805.3 & $40.711 \mathrm{~N}$ & $112.085 \mathrm{~W}$. & 13 & UU & - & - & $2.6 \mathrm{M}_{\mathrm{L}}(\mathrm{UU})$ & & NOV. & 13 & 16:28 MST \\
\hline DEC. & 31 & 112156.5 & $41.822 \mathrm{~N}$ & $112.316 \mathrm{~W}$. & 5 & UU & - & - & $3.3 \mathrm{M}_{\mathrm{L}}(\mathrm{UU})$ & IV & DEC. & 31 & 04:21 MST \\
\hline
\end{tabular}

\section{VIRGINIA}

\begin{tabular}{|c|c|c|c|c|c|c|c|c|c|c|c|}
\hline MAR. 26 & 163623.9 & $37.245 \mathrm{~N}$. & 80.494W. & 12 & VP & - & - & $2.9 \mathrm{M}_{\mathrm{D}}(\mathrm{VP})$ & IV & MAR. 26 & 11:36 EST \\
\hline DEC. 3 & 094421.1 & $37.580 \mathrm{~N}$. & $77.458 \mathrm{~W}$. & 1 & VP & & & $1.5 \mathrm{M}_{\mathrm{D}}(\mathrm{VP})$ & IV & DEC. 3 & 04:44 EST \\
\hline DEC. 10 & 113006.1 & $37.584 \mathrm{~N}$. & $77.468 \mathrm{~W}$. & 1 & VP & & - & $2.2 \mathrm{M}_{\mathrm{D}}(\mathrm{VP})$ & V & DEC. 10 & 06:30 EST \\
\hline DEC. 24 & 175838.2 & $37.583 \mathrm{~N}$. & $77.458 \mathrm{~W}$. & 1 & VP & - & - & $1.5 \mathrm{M}_{\mathrm{D}}(\mathrm{VP})$ & IV & DEC. & $12: 58 \mathrm{EST}$ \\
\hline
\end{tabular}

\section{WASHINGTON}

\begin{tabular}{|c|c|c|c|c|c|c|c|c|c|c|c|c|}
\hline JAN. & 3 & 161104.6 & $46.932 \mathrm{~N}$. & 121.924W. & 11 & WA & - & - & 3.0M $\mathrm{D}(\mathrm{WA})$ & - & JAN. & 08:11 PST \\
\hline JAN. & 12 & 203013.6 & $48.074 \mathrm{~N}$. & $121.674 \mathrm{~W}$. & 2 & WA & & & $2.8 \mathrm{M}_{\mathrm{D}}(\mathrm{WA})$ & & JAN. 12 & 12:30 PST \\
\hline FEB. & 4 & 015907.2 & $46.044 \mathrm{~N}$ & $118.810 \mathrm{~W}$. & 8 & WA & & & $3.2 \mathrm{M}_{\mathrm{D}}(\mathrm{WA})$ & & FEB. 3 & 17:59 PST \\
\hline FEB. 1 & 10 & 171207.4 & $48.395 \mathrm{~N}$. & $121.955 \mathrm{~W}$. & 4 & WA & & & $3.1 \mathrm{M}_{\mathrm{D}}(\mathrm{WA})$ & IV & FEB. 10 & 09:12 PST \\
\hline FEB. & 10 & 180508.0 & $48.397 \mathrm{~N}$. & $121.941 \mathrm{~W}$. & 0 & WA & & & $3.7 \mathrm{M}_{\mathrm{L}}(\mathrm{GS})$ & V & FEB. 10 & 10:05 PST \\
\hline MAR. & 2 & 045836.0 & $48.747 \mathrm{~N}$. & $124.907 \mathrm{~W}$. & 40 & WA & & & $2.7 \mathrm{M}_{\mathrm{D}}(\mathrm{WA})$ & - & MAR. 1 & 20:58 PST \\
\hline MAR. 11 & 11 & 072321.0 & $47.335 \mathrm{~N}$. & $122.488 \mathrm{~W}$. & 7 & WA & & & $2.9 \mathrm{M}_{\mathrm{D}}(\mathrm{WA})$ & III & MAR. 10 & 23:23 PST \\
\hline MAR. 11 & 11 & 104810.4 & $45.941 \mathrm{~N}$. & $122.411 \mathrm{~W}$ & 15 & WA & & & $3.1 \mathrm{M}_{\mathrm{D}}(\mathrm{WA})$ & V & MAR. 11 & 02:48 PST \\
\hline MAR. 2 & 27 & 121012.8 & $48.265 \mathrm{~N}$ & $121.732 \mathrm{~W}$. & 2 & WA & & & $2.8 \mathrm{M}_{\mathrm{L}}(\mathrm{GS})$ & IV & MAR. 27 & 04:10 PST \\
\hline MAR. 28 & 28 & 034834.7 & $48.256 \mathrm{~N}$. & $121.736 \mathrm{~W}$. & 2 & WA & & & $3.1 \mathrm{M}_{\mathrm{L}}(\mathrm{GS})$ & IV & MAR. 27 & 19:48 PST \\
\hline MAR. 28 & 28 & 041246.7 & $48.260 \mathrm{~N}$. & 121.734W. & 2 & WA & & & $3.6 \mathrm{M}_{\mathrm{L}}(\mathrm{GS})$ & V & MAR. 27 & 20:12 PST \\
\hline MAR. 28 & 28 & 054055.3 & $48.254 \mathrm{~N}$. & $121.740 \mathrm{~W}$. & 3 & WA & & & $2.4 \mathrm{M}_{\mathrm{D}}(\mathrm{WA})$ & FELT & MAR. 27 & 21:40 PST \\
\hline MAR. 28 & 28 & 121114.9 & $48.258 \mathrm{~N}$ & $121.732 \mathrm{~W}$. & 2 & WA & & & $2.1 \mathrm{M}_{\mathrm{D}}(\mathrm{WA})$ & III & MAR. 28 & 04:11 PST \\
\hline MAR. 25 & 29 & 130924.0 & $48.258 \mathrm{~N}$. & $121.732 \mathrm{~W}$. & 2 & WA & & & $3.1 \mathrm{M}_{\mathrm{L}}(\mathrm{GS})$ & FELT & MAR. 29 & 05:09 PST \\
\hline MAR. 3 & 31 & 071127.2 & $48.260 \mathrm{~N}$. & $121.736 \mathrm{~W}$. & 2 & WA & & & $2.3 \mathrm{M}_{\mathrm{D}}(\mathrm{WA})$ & FELT & MAR. 30 & 23:11 PST \\
\hline APR. & 8 & 105735.6 & $47.770 \mathrm{~N}$ & $120.230 \mathrm{~W}$. & 14 & WA & & & $2.9 \mathrm{M}_{\mathrm{L}}(\mathrm{GS})$ & FELT & APR. & 02:57 PST \\
\hline APR. & 15 & 114327.5 & $46.211 \mathrm{~N}$. & $122.187 \mathrm{~W}$. & 0 & WA & & & $2.7 \mathrm{M}_{\mathrm{D}}(\mathrm{WA})$ & - & APR. 15 & 03:43 PST \\
\hline APR. & 20 & 164033.1 & $48.840 \mathrm{~N}$ & $122.526 \mathrm{~W}$ & 18 & WA & & & $2.8 \mathrm{M}_{\mathrm{L}}(\mathrm{GS})$ & III & APR. 20 & 08:40 PST \\
\hline APR. & 27 & 002914.5 & $46.208 \mathrm{~N}$. & $122.188 \mathrm{~W}$. & 0 & WA & $\longrightarrow$ & & $2.8 \mathrm{M}_{\mathrm{D}}(\mathrm{WA})$ & & APR. 26 & 16:29 PST \\
\hline MAY & 4 & 212456.6 & $46.201 \mathrm{~N}$. & $122.188 \mathrm{~W}$. & 1 & WA & & & 2.7 $\mathrm{M}_{\mathrm{D}}(\mathrm{WA})$ & 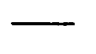 & MAY & 13:24 PST \\
\hline
\end{tabular}


Table 1. Summary of United States earthquakes for 1986 - Continued

\begin{tabular}{|c|c|c|c|c|c|c|c|c|c|c|c|c|c|}
\hline \multirow{2}{*}{\multicolumn{2}{|c|}{ Date }} & \multirow{2}{*}{$\begin{array}{l}\text { Origin time } \\
\text { (UTC) } \\
\mathrm{hr} \operatorname{min~sec}\end{array}$} & \multirow{2}{*}{$\begin{array}{l}\text { Latitude } \\
()^{\circ}\end{array}$} & \multirow{2}{*}{$\begin{array}{l}\text { Longitude } \\
\text { (9) }\end{array}$} & \multirow{2}{*}{$\begin{array}{l}\text { Depth } \\
(\mathrm{km})\end{array}$} & \multirow{2}{*}{$\begin{array}{l}\text { Hypo- } \\
\text { center } \\
\text { Source }\end{array}$} & \multicolumn{3}{|c|}{ Magnitude } & \multirow{2}{*}{$\begin{array}{l}\text { Max. } \\
\text { inten- } \\
\text { sity }\end{array}$} & \multicolumn{3}{|c|}{ Local time } \\
\hline & & & & & & & $\mathrm{m}_{\mathrm{b}}$ & $\mathrm{M}_{\mathrm{s}}$ & Local & & Date & & zone \\
\hline \multicolumn{14}{|c|}{ WASHINGTON_Continued } \\
\hline MAY & 7 & 040043.6 & $46.200 \mathrm{~N}$ & $122.187 \mathrm{~W}$ & 2 & WA & - & - & $2.7 \mathrm{M}_{\mathrm{D}}(\mathrm{WA})$ & - & MAY & 6 & 20:00 PST \\
\hline MAY & 7 & 154707.8 & $46.199 \mathrm{~N}$ & 122.190W. & 1 & WA & - & - & $2.7 \mathrm{M}_{\mathrm{D}}(\mathrm{WA})$ & & MAY & 7 & 07:47 PST \\
\hline MAY & 7 & 214320.9 & $46.199 \mathrm{~N}$ & 122.188W. & 1 & WA & - & - & $3.0 \mathrm{M}_{\mathrm{D}}(\mathrm{WA})$ & & MAY & 7 & 13:43 PST \\
\hline MAY & 8 & 140353.9 & $46.200 \mathrm{~N}$ & 122.191W. & 1 & WA & - & - & $3.2 \mathrm{M}_{\mathrm{D}}(\mathrm{WA})$ & & MAY & 8 & 06:03 PST \\
\hline MAY & 8 & 174817.7 & $46.200 \mathrm{~N}$ & $122.189 \mathrm{~W}$. & 2 & WA & - & - & $2.7 \mathrm{M}_{\mathrm{D}}(\mathrm{WA})$ & 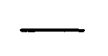 & MAY & 8 & 09:48 PST \\
\hline MAY & 8 & 202715.5 & $46.200 \mathrm{~N}$ & $122.190 \mathrm{~W}$ & 2 & WA & 一 & - & $2.9 \mathrm{M}_{\mathrm{D}}(\mathrm{WA})$ & 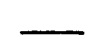 & MAY & 8 & 12:27 PST \\
\hline MAY & 9 & 083146.7 & $46.203 \mathrm{~N}$ & 122.185W. & 2 & WA & - & - & $2.8 \mathrm{M}_{\mathrm{D}}(\mathrm{WA})$ & & MAY & 9 & 00:31 PST \\
\hline MAY & 10 & 041133.1 & $46.196 \mathrm{~N}$ & 122.193W. & 0 & WA & - & - & $2.7 \mathrm{M}_{\mathrm{D}}(\mathrm{WA})$ & & MAY & 9 & 20:11 PST \\
\hline JUNE & 11 & 061242.3 & $47.776 \mathrm{~N}$ & $120.168 \mathrm{~W}$. & 9 & WA & 一 & - & $2.7 \mathrm{M}_{\mathrm{D}}(\mathrm{WA})$ & III & JUNE & 10 & 22:12 PST \\
\hline JUNE & 20 & 165550.9 & $46.470 \mathrm{~N}$ & $120.913 W$. & 3 & WA & 一 & - & $2.8 \mathrm{M}_{\mathrm{D}}(\mathrm{WA})$ & & JUNE & 20 & 08:55 PST \\
\hline JUNE & 29 & 234025.3 & $47.407 \mathrm{~N}$ & 123.155W. & 15 & WA & - & - & $2.7 \mathrm{M}_{\mathrm{D}}(\mathrm{WA})$ & & JUNE & 29 & 15:40 PST \\
\hline JUNE & 30 & 010327.2 & $47.842 \mathrm{~N}$ & $122.577 \mathrm{~W}$ & 20 & WA & - & - & $2.7 \mathrm{M}_{\mathrm{D}}(\mathrm{WA})$ & $\longrightarrow$ & JUNE & 29 & 17:03 PST \\
\hline JULY & 8 & 051632.4 & $48.264 \mathrm{~N}$ & $122.512 \mathrm{~W}$ & 63 & WA & - & - & $3.5 \mathrm{M}_{\mathrm{D}}(\mathrm{WA})$ & IV & JULY & 7 & 21:16 PST \\
\hline AUG. & 6 & 105946.9 & $45.837 \mathrm{~N}$ & 121.916W. & 10 & WA & - & - & $2.7 \mathrm{M}_{\mathrm{D}}(\mathrm{WA})$ & & AUG. & 6 & 02:59 PST \\
\hline AUG. & 28 & 032111.3 & $46.832 \mathrm{~N}$ & $121.938 W$. & 12 & WA & - & - & $2.8 \mathrm{M}_{\mathrm{D}}(\mathrm{WA})$ & - & AUG. & 27 & 19:21 PST \\
\hline AUG. & 28 & 043413.5 & $45.835 \mathrm{~N}$ & $121.923 \mathrm{~W}$ & 9 & WA & - & - & $2.7 \mathrm{M}_{\mathrm{D}}(\mathrm{WA})$ & FELT & AUG. & 27 & 20:34 PST \\
\hline AUG. & 31 & 111451.0 & $47.281 \mathrm{~N}$ & $123.447 \mathrm{~W}$ & 41 & WA & 一 & - & $2.8 \mathrm{M}_{\mathrm{D}}(\mathrm{WA})$ & - & AUG. & 31 & 03:14 PST \\
\hline AUG. & 31 & 183159.1 & $47.374 \mathrm{~N}$ & $122.810 \mathrm{~W}$. & 27 & WA & - & - & $2.9 \mathrm{M}_{\mathrm{D}}(\mathrm{WA})$ & - & AUG. & 31 & $10: 31$ PST \\
\hline SEPT. & 1 & 213244.0 & $46.718 \mathrm{~N}$ & 119.285W. & 14 & WA & - & - & $3.4 \mathrm{M}_{\mathrm{D}}(\mathrm{WA})$ & - & SEPT. & 1 & $13: 32$ PST \\
\hline SEPT. & 16 & 231949.5 & $48.221 \mathrm{~N}$ & 121.643W. & 2 & WA & - & - & $1.6 \mathrm{M}_{\mathrm{D}}(\mathrm{WA})$ & FELT & SEPT. & 16 & 15:19 PST \\
\hline SEPT. & 16 & 233857.8 & $48.065 \mathrm{~N}$ & 121.523W. & 6 & WA & - & - & $2.8 \mathrm{M}_{\mathrm{D}}(\mathrm{WA})$ & FELT & SEPT. & 16 & $15: 38$ PST \\
\hline SEPT. & 16 & 234937.1 & $48.067 \mathrm{~N}$ & $121.542 \mathrm{~W}$ & 8 & WA & - & - & $2.4 \mathrm{M}_{\mathrm{D}}(\mathrm{WA})$ & FELT & SEPT. & 16 & 15:49 PST \\
\hline SEPT. & 26 & 233454.7 & $48.552 \mathrm{~N}$ & $121.989 \mathrm{~W}$ & 0 & WA & - & - & $2.4 \mathrm{M}_{\mathrm{D}}(\mathrm{WA})$ & FELT & SEPT. & 26 & 15:34 PST \\
\hline SEPT. & 29 & 193706.9 & $48.551 \mathrm{~N}$ & $121.983 \mathrm{~W}$. & $\mathbf{0}$ & WA & - & - & $2.2 \mathrm{M}(\mathrm{WA})$ & FELT & SEPT. & 29 & 11:37 PST \\
\hline OCT. & 12 & 185851.0 & $46.357 \mathrm{~N}$ & 122.655W. & 67 & WA & - & - & $3.2 \mathrm{M}_{\mathrm{D}}(\mathrm{WA})$ & - & OCT. & 12 & 10:58 PST \\
\hline OCT. & 19 & 052947.0 & $46.201 \mathrm{~N}$ & $122.187 \mathrm{~W}$ & 1 & WA & - & - & $2.7 \mathrm{M}_{\mathrm{D}}(\mathrm{WA})$ & 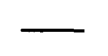 & OCT. & 18 & 21:29 PST \\
\hline OCT. & 19 & 163137.3 & $46.204 \mathrm{~N}$ & $122.185 \mathrm{~W}$ & 1 & WA & - & - & $2.9 \mathrm{M}_{\mathrm{D}}(\mathrm{WA})$ & & OCT. & 19 & 08:31 PST \\
\hline OCT. & 20 & 131749.3 & $46.200 \mathrm{~N}$ & $122.186 \mathrm{~W}$. & 1 & WA & - & $\longrightarrow$ & $2.7 \mathrm{M}_{\mathrm{D}}(\mathrm{WA})$ & 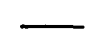 & OCT. & 20 & 05:17 PST \\
\hline OCT. & 20 & 163350.1 & $46.202 \mathrm{~N}$ & $122.184 \mathrm{~W}$ & 1 & WA & - & - & $2.9 \mathrm{M}_{\mathrm{D}}(\mathrm{WA})$ & - & OCT. & 20 & 08:33 PST \\
\hline OCT. & 20 & 212120.8 & $46.199 \mathrm{~N}$ & 122.180W. & 1 & WA & - & - & $3.0 \mathrm{M}_{\mathrm{D}}(\mathrm{WA})$ & - & OCT. & 20 & 13:21 PST \\
\hline OCT. & 21 & 004522.8 & $46.201 \mathrm{~N}$ & 122.186W. & 1 & WA & - & - & $3.1 \mathrm{M}_{\mathrm{D}}(\mathrm{WA})$ & & OCT. & 20 & 16:45 PST \\
\hline OCT. & 21 & 031129.0 & $46.201 \mathrm{~N}$ & $122.188 \mathrm{~W}$. & 1 & WA & - & - & $3.1 \mathrm{M}_{\mathrm{D}}(\mathrm{WA})$ & & OCT. & 20 & 19:11 PST \\
\hline OCT. & 21 & 034550.6 & $46.200 \mathrm{~N}$ & 122.188W. & 1 & WA & - & - & $2.8 \mathrm{M}_{\mathrm{D}}(\mathrm{WA})$ & & OCT. & 20 & 19:45 PST \\
\hline OCT. & 21 & 054202.0 & $46.200 \mathrm{~N}$ & $122.187 \mathrm{~W}$ & 1 & WA & - & - & $3.1 \mathrm{M}_{\mathrm{D}}(\mathrm{WA})$ & & OCT. & 20 & 21:42 PST \\
\hline OCT. & 21 & 082955.6 & $46.199 \mathrm{~N}$ & $122.188 \mathrm{~W}$ & 1 & WA & - & - & $3.1 \mathrm{M}_{\mathrm{D}}(\mathrm{WA})$ & & OCT. & 21 & 00:29 PST \\
\hline OCT. & 21 & 084313.8 & $46.200 \mathrm{~N}$ & $122.188 \mathrm{~W}$ & 1 & WA & - & - & $3.0 \mathrm{M}_{\mathrm{D}}(\mathrm{WA})$ & - & OCT. & 21 & 00:43 PST \\
\hline OCT. & 21 & 094938.1 & $46.200 \mathrm{~N}$ & $122.187 \mathrm{~W}$ & 1 & WA & - & - & $3.0 \mathrm{M}_{\mathrm{D}}(\mathrm{WA})$ & - & OCT. & 21 & 01:49 PST \\
\hline OCT. & 21 & 110935.2 & $46.200 \mathrm{~N}$ & $122.187 \mathrm{~W}$ & 1 & WA & - & - & $3.2 \mathrm{M}_{\mathrm{D}}(\mathrm{WA})$ & 工 & OCT. & 21 & 03:09 PST \\
\hline OCT. & 21 & 133452.8 & $46.200 \mathrm{~N}$ & $122.187 \mathrm{~W}$ & 1 & WA & - & - & $3.1 \mathrm{M}_{\mathrm{D}}(\mathrm{WA})$ & $\longrightarrow$ & OCT. & 21 & 05:34 PST \\
\hline OCT. & 21 & 143256.8 & $46.200 \mathrm{~N}$ & 122.186W. & 1 & WA & - & - & 3.2 $\mathrm{M}_{\mathrm{D}}(\mathrm{WA})$ & - & OCT. & 21 & 06:32 PST \\
\hline
\end{tabular}


Table 1. Summary of United States earthquakes for 1986_-Continued

\begin{tabular}{|c|c|c|c|c|c|c|c|c|c|c|c|c|c|}
\hline \multirow[t]{2}{*}{ Date } & & \multirow{2}{*}{$\begin{array}{l}\text { Origin time } \\
\text { (UTC) } \\
\text { hr min sec }\end{array}$} & \multirow{2}{*}{$\begin{array}{l}\text { Latitude } \\
\text { ()) }\end{array}$} & \multirow{2}{*}{$\begin{array}{l}\text { Longitude } \\
\text { (9) }\end{array}$} & \multirow{2}{*}{$\begin{array}{l}\text { Depth } \\
(\mathrm{km})\end{array}$} & \multirow{2}{*}{$\begin{array}{l}\text { Hypo- } \\
\text { center } \\
\text { Source }\end{array}$} & \multicolumn{3}{|c|}{ Magnitude } & \multirow{2}{*}{$\begin{array}{l}\text { Max. } \\
\text { inten- } \\
\text { sity }\end{array}$} & \multicolumn{3}{|c|}{ Local time } \\
\hline & & & & & & & $\mathbf{m}_{\mathbf{b}}$ & $\mathbf{M}_{\mathbf{S}}$ & Local & & Date & & zone \\
\hline \multicolumn{14}{|c|}{ WASHINGTON_Continued } \\
\hline OCT. & 21 & 151156.6 & $46.200 \mathrm{~N}$ & 122.186W. & 1 & WA & . & - & $2.8 \mathrm{M}_{\mathrm{D}}(\mathrm{WA})$ & & OCT. & 21 & 07:11 PST \\
\hline OCT. & 21 & 154203.5 & $46.201 \mathrm{~N}$ & $122.189 \mathrm{~W}$. & 1 & WA & & & $3.0 \mathrm{M}_{\mathrm{D}}(\mathrm{WA})$ & & OCT. & 21 & 07:42 PST \\
\hline OCT. & 21 & 171541.4 & $46.199 \mathrm{~N}$. & $122.187 \mathrm{~W}$. & 1 & WA & & & $3.0 \mathrm{M}(\mathrm{WA})$ & & OCT. & 21 & 09:15 PST \\
\hline OCT. & 21 & 173223.0 & $46.200 \mathrm{~N}$ & $122.188 \mathrm{~W}$ & 2 & WA & & & $2.8 \mathrm{M}_{\mathrm{D}}(\mathrm{WA})$ & & OCT. & 21 & 09:32 PST \\
\hline OCT. & 21 & 181348.9 & $46.200 \mathrm{~N}$. & $122.187 \mathrm{~W}$ & 2 & WA & {[} & & $2.8 \mathrm{M}_{\mathrm{D}}(\mathrm{WA})$ & & OCT. & 21 & 10:13 PST \\
\hline OCT. & 21 & 182113.7 & $46.200 \mathrm{~N}$ & $122.187 \mathrm{~W}$. & 1 & WA & & - & $3.1 \mathrm{M}_{\mathrm{D}}(\mathrm{WA})$ & & OCT. & 21 & 10:21 PST \\
\hline OCT. & 21 & 190302.6 & $46.200 \mathrm{~N}$. & $122.186 \mathrm{~W}$. & 1 & WA & & & 2.7 $\mathrm{M}_{\mathrm{D}}(\mathrm{WA})$ & & OCT. & 21 & 11:03 PST \\
\hline OCT. & 21 & 192112.7 & $46.201 \mathrm{~N}$ & $122.189 \mathrm{~W}$. & 2 & WA & & & 2.7 $\mathrm{M}_{\mathrm{D}}(\mathrm{WA})$ & & OCT. & 21 & 11:21 PST \\
\hline OCT. & 21 & 193524.0 & $46.198 \mathrm{~N}$. & $122.189 \mathrm{~W}$. & 1 & WA & & & $3.0 \mathrm{M}_{\mathrm{D}}(\mathrm{WA})$ & & OCT. & 21 & 11:35 PST \\
\hline OCT. & 21 & 195620.4 & $46.200 \mathrm{~N}$. & $122.187 \mathrm{~W}$ & 1 & WA & & & $3.1 \mathrm{M}_{\mathrm{D}}(\mathrm{WA})$ & & OCT. & 21 & 11:56 PST \\
\hline OCT. & 21 & 204237.0 & $46.202 \mathrm{~N}$ & $122.188 \mathrm{~W}$. & 1 & WA & & & $3.2 \mathrm{M}_{\mathrm{D}}(\mathrm{WA})$ & & OCT. & 21 & 12:42 PST \\
\hline OCT. & 21 & 214704.1 & $46.201 \mathrm{~N}$ & 122.188W. & 1 & WA & & & $3.1 \mathrm{M}_{\mathrm{D}}(\mathrm{WA})$ & & OCT. & 21 & 13:47 PST \\
\hline OCT. & 21 & 224041.2 & $46.203 \mathrm{~N}$ & 122.191W. & 1 & WA & & & 3.0 $\mathrm{M}_{\mathrm{D}}(\mathrm{WA})$ & & OCT. & 21 & 14:40 PST \\
\hline OCT. & 22 & 000135.4 & $46.203 \mathrm{~N}$ & $122.192 \mathrm{~W}$. & 2 & WA & & & 3.0 $\mathrm{M}_{\mathrm{D}}(\mathrm{WA})$ & & OCT. & 21 & 16:01 PST \\
\hline OCT. & 22 & 123504.5 & $46.204 \mathrm{~N}$ & $122.200 \mathrm{~W}$ & 2 & WA & 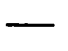 & & $2.8 \mathrm{M}_{\mathrm{D}}(\mathrm{WA})$ & & OCT. & 22 & 04:35 PST \\
\hline OCT. & 24 & 072058.2 & $46.200 \mathrm{~N}$ & 122.193W. & 0 & WA & & & $2.8 \mathrm{M}_{\mathrm{D}}(\mathrm{WA})$ & & OCT. & 23 & 23:20 PST \\
\hline NOV. & 7 & 103554.0 & $48.120 \mathrm{~N}$. & $123.317 \mathrm{~W}$. & 38 & EP & - & & $3.9 \mathrm{M}_{\mathrm{L}}(\mathrm{EP})$ & FELT & NOV. & 7 & 02:35 PST \\
\hline NOV. & 8 & 145413.1 & $46.859 \mathrm{~N}$. & $120.580 \mathrm{~W}$. & 8 & WA & - & - & $2.9 \mathrm{M}_{\mathrm{L}}(\mathrm{GS})$ & - & NOV. & 8 & 06:54 PST \\
\hline NOV. & 11 & 131256.0 & $47.816 \mathrm{~N}$. & $128.542 \mathrm{~W}$. & 10 & GS & 4.3 & - & - & $\square$ & NOV. & 11 & 05:12 PST \\
\hline
\end{tabular}

\section{WYOMING}

\begin{tabular}{|c|c|c|c|c|c|c|c|c|c|c|c|c|c|}
\hline JAN. & 2 & 155340.9 & $44.620 \mathrm{~N}$ & $110.997 \mathrm{~W}$ & 6 & UU & - & - & $3.0 \mathrm{M}_{\mathrm{L}}(\mathrm{GS})$ & II & JAN. & 2 & 08:53 MST \\
\hline JAN. & 2 & 200405.6 & $44.639 \mathrm{~N}$. & $110.982 \mathrm{~W}$ & 10 & UU & - & - & $2.9 \mathrm{M}_{\mathrm{L}}(\mathrm{BU})$ & & JAN. & 2 & 13:04 MST \\
\hline JAN. & 4 & 031441.7 & $44.640 \mathrm{~N}$ & $110.990 \mathrm{~W}$ & 12 & UU & - & - & $2.7 \mathrm{M}_{\mathrm{L}}(\mathrm{BU})$ & & JAN. & 3 & 20:14 MST \\
\hline JAN. & 8 & 073225.7 & $44.620 \mathrm{~N}$. & $111.003 \mathrm{~W}$. & 8 & UU & - & - & $2.8 \mathrm{M}_{\mathrm{L}}(\mathrm{GS})$ & II & JAN. & 8 & 00:32 MST \\
\hline JAN. & 8 & 110815.7 & $44.621 \mathrm{~N}$ & $110.998 \mathrm{~W}$ & 7 & UU & 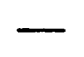 & - & $3.0 \mathrm{M}_{\mathrm{L}}(\mathrm{GS})$ & III & JAN. & 8 & 04:08 MST \\
\hline AN. & 8 & 133628.5 & $44.635 \mathrm{~N}$ & $110.999 \mathrm{~W}$ & 7 & UU & - & - & $2.9 \mathrm{M}_{\mathrm{L}}(\mathrm{GS})$ & III & JAN. & 8 & 06:36 MST \\
\hline JAN. & 9 & 191647.6 & $44.654 \mathrm{~N}$ & $111.008 \mathrm{~W}$ & 7 & UU & - & - & $2.6 \mathrm{M}_{\mathrm{L}}(\mathrm{BU})$ & & JAN. & 9 & $12: 16 \mathrm{MST}$ \\
\hline JAN. & 9 & 204640.5 & $44.647 \mathrm{~N}$ & $111.007 \mathrm{~W}$ & 7 & UU & - & - & $2.9 \mathrm{M}_{\mathrm{L}}(\mathrm{BU})$ & & JAN. & 9 & 13:46 MST \\
\hline JAN. & 14 & 015037.8 & $44.637 \mathrm{~N}$ & $111.002 \mathrm{~W}$ & 6 & UU & $\longrightarrow$ & $\longrightarrow$ & $2.9 \mathrm{M}_{\mathrm{L}}(\mathrm{GS})$ & II & JAN. & 13 & 18:50 MST \\
\hline JAN. & 14 & 164629.9 & $44.659 \mathrm{~N}$ & $111.018 \mathrm{~W}$ & 7 & UU & - & - & $3.2 \mathrm{M}_{\mathrm{L}}(\mathrm{GS})$ & II & JAN. & 14 & 09:46 MST \\
\hline AN. & 15 & 211018.0 & $44.633 \mathrm{~N}$ & $111.002 \mathrm{~W}$. & 7 & UU & - & - & GS) & III & JAN. & 15 & MST \\
\hline JAN. & 16 & 102947.7 & $44.621 \mathrm{~N}$ & $111.001 \mathrm{~W}$ & 6 & UU & - & - & $3.4 \mathrm{M}_{\mathrm{L}}(\mathrm{GS})$ & III & JAN. & 16 & 03:29 MST \\
\hline JAN. & 18 & 052721.4 & $44.628 \mathrm{~N}$ & $110.997 \mathrm{~W}$ & 7 & UU & - & - & $2.8 \mathrm{M}_{\mathrm{L}}(\mathrm{BU})$ & & JAN. & 17 & 22:27 MST \\
\hline JAN. & 18 & 225141.5 & $44.625 \mathrm{~N}$ & $110.993 \mathrm{~W}$ & 7 & UU & - & - & $2.8 \mathrm{M}_{\mathrm{L}}(\mathrm{BU})$ & & JAN. & 18 & 15:51 MST \\
\hline JAN. & 25 & 125104.5 & $44.660 \mathrm{~N}$ & $110.995 \mathrm{~W}$ & 11 & UU & - & - & $2.8 \mathrm{M}_{\mathrm{L}}(\mathrm{BU})$ & 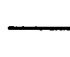 & JAN. & 25 & 05:51 MS \\
\hline JAN. & 25 & 200156.0 & $44.657 \mathrm{~N}$ & $110.998 \mathrm{~W}$ & 9 & UU & - & - & $2.7 \mathrm{M}_{\mathrm{L}}(\mathrm{BU})$ & 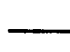 & JAN. & 25 & 13:01 MS \\
\hline FEB. & 1 & 060954.8 & $44.626 \mathrm{~N}$ & $110.996 \mathrm{~W}$. & 7 & UU & - & - & $3.1 \mathrm{M}_{\mathrm{L}}(\mathrm{BU})$ & III & JAN. & 31 & 23:09 MS \\
\hline FEB & 4 & 054145.3 & $44.665 \mathrm{~N}$ & $111.012 \mathrm{~W}$ & 5 & UU & - & - & $2.7 \mathrm{M}_{\mathrm{L}}(\mathrm{BU})$ & 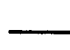 & FEB. & 3 & $22: 41 \mathrm{MS}$ \\
\hline
\end{tabular}


Table 1. Summary of United States earthquakes for 1986_Continued

\begin{tabular}{|c|c|c|c|c|c|c|c|c|c|c|c|}
\hline \multirow[t]{2}{*}{ Date } & \multirow{2}{*}{$\begin{array}{l}\text { Origin time } \\
\text { (UTC) } \\
\text { hr min sec }\end{array}$} & \multirow{2}{*}{$\begin{array}{l}\text { Latitude } \\
\text { ( ) }\end{array}$} & \multirow{2}{*}{$\begin{array}{l}\text { Longitude } \\
\text { (9) }\end{array}$} & \multirow{2}{*}{$\begin{array}{l}\text { Depth } \\
(\mathbf{k m})\end{array}$} & \multirow{2}{*}{$\begin{array}{l}\text { Hypo- } \\
\text { center } \\
\text { Source }\end{array}$} & \multicolumn{3}{|c|}{ Magnitude } & \multirow{2}{*}{$\begin{array}{l}\text { Max. } \\
\text { inten- } \\
\text { sity }\end{array}$} & \multicolumn{2}{|c|}{ Local time } \\
\hline & & & & & & $\mathbf{m}_{\mathbf{b}}$ & $\mathrm{M}_{\mathrm{s}}$ & Local & & Date & zone \\
\hline \multicolumn{12}{|c|}{ WYOMING-Continued } \\
\hline FEB. 5 & 142619.3 & $44.644 \mathrm{~N}$. & $111.016 \mathrm{~W}$. & 4 & UU & - & - & $3.4 \mathrm{M}_{\mathrm{L}}(\mathrm{GS})$ & FELT & FEB. & 07:26 MST \\
\hline FEB. & 143701.7 & $44.640 \mathrm{~N}$. & $111.014 \mathrm{~W}$ & 3 & UU & 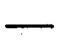 & - & $3.3 \mathrm{M}_{\mathrm{L}}(\mathrm{BU})$ & FELT & FEB. & 07:37 MST \\
\hline FEB. & 132202.9 & $44.622 \mathrm{~N}$. & $111.038 \mathrm{~W}$. & 1 & UU & - & - & $3.4 \mathrm{M}_{\mathrm{L}}(\mathrm{GS})$ & - & FEB. & 06:22 MST \\
\hline FEB. & 142911.2 & $44.653 N$. & 111.013W. & 7 & $\mathbf{U U}$ & - & - & $2.8 \mathrm{M}_{\mathrm{L}}(\mathrm{BU})$ & & FEB. & 07:29 MST \\
\hline FEB. 11 & 222609.1 & $44.645 \mathrm{~N}$. & $111.015 W$ & 9 & UU & & - & $3.2 \mathrm{M}_{\mathrm{L}}(\mathrm{BU})$ & - & FEB. & 15:26 MST \\
\hline FEB. 11 & 222655.6 & $44.647 \mathrm{~N}$. & $111.016 \mathrm{~W}$ & 7 & UU & & 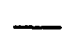 & $4.2 \mathrm{M}_{\mathrm{L}}(\mathrm{GS})$ & FELT & FEB. & 15:26 MST \\
\hline FEB. 11 & 222756.7 & $44.623 \mathrm{~N}$ & $111.012 \mathrm{~W}$. & 8 & $\mathbf{U U}$ & & & $4.0 \mathrm{M}_{\mathrm{L}}(\mathrm{BU})$ & & FEB. 11 & 15:27 MST \\
\hline FEB. & 223407.0 & $44.659 \mathrm{~N}$. & $111.002 W$. & 4 & UU & & & $2.9 \mathrm{M}_{\mathrm{L}}(\mathrm{BU})$ & & FEB. & 15:34 MST \\
\hline FEB. 16 & 021110.0 & $44.648 \mathrm{~N}$. & $111.012 \mathrm{~W}$. & 7 & UU & & & $2.9 \mathrm{M}_{\mathrm{L}}(\mathrm{BU})$ & & FEB. 15 & 19:11 MST \\
\hline MAR. 2 & 105525.4 & $44.652 \mathrm{~N}$ & $111.018 \mathrm{~W}$ & 7 & UU & & $\longrightarrow$ & $3.2 \mathrm{M}_{\mathrm{L}}(\mathrm{GS})$ & II & MAR. 2 & 03:55 MST \\
\hline MAR. & 112326.5 & $44.646 \mathrm{~N}$. & $111.019 \mathrm{~W}$. & 6 & UU & & & $2.9 \mathrm{M}_{\mathrm{L}}(\mathrm{BU})$ & & MAR. 2 & 04:23 MST \\
\hline MAR. & 125936.5 & $44.653 \mathrm{~N}$. & $111.022 \mathrm{~W}$ & 7 & UU & & 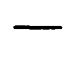 & $3.0 \mathrm{M}_{\mathrm{L}}(\mathrm{GS})$ & II & MAR. 2 & 05:59 MST \\
\hline MAR. 7 & 193209.3 & $44.647 \mathrm{~N}$ & $111.027 \mathrm{~W}$ & 7 & UU & & - & $3.4 \mathrm{M}_{\mathrm{L}}(\mathrm{BU})$ & & MAR. 7 & 12:32 MST \\
\hline MAR. 18 & 235337.0 & $44.638 \mathrm{~N}$ & $111.007 \mathrm{~W}$ & 7 & $\mathbf{U U}$ & & & $3.1 \mathrm{M}_{\mathrm{L}}(\mathrm{GS})$ & & MAR. 18 & 16:53 MST \\
\hline MAR. 21 & 020656.5 & $44.227 \mathrm{~N}$. & $110.691 W$ & $\mathbf{0}$ & UU & & & $3.1 \mathrm{M}_{\mathrm{D}}(\mathrm{BU})$ & & MAR. 20 & 19:06 MST \\
\hline MAR. 22 & 073340.3 & $44.729 \mathrm{~N}$. & $111.042 \mathrm{~W}$. & 9 & BU & & & $2.5 \mathrm{M}_{\mathrm{L}}(\mathrm{BU})$ & & MAR. 22 & 00:33 MST \\
\hline MAR. 26 & 041543.3 & $44.640 \mathrm{~N}$. & $111.004 W$ & 8 & UU & & & $2.8 \mathrm{M}_{\mathrm{L}}(\mathrm{BU})$ & & MAR. 25 & 21:15 MST \\
\hline APR. & 141635.8 & $44.627 \mathrm{~N}$. & $110.996 \mathrm{~W}$. & 4 & $\mathbf{U U}$ & & & $3.0 \mathrm{M}_{\mathrm{L}}(\mathrm{GS})$ & & APR. & 07:16 MST \\
\hline APR. & 110531.8 & $44.717 \mathrm{~N}$. & $110.967 \mathrm{~W}$ & 19 & BU & & & $2.8 \mathrm{M}_{\mathrm{L}}(\mathrm{BU})$ & & APR. & 04:05 MST \\
\hline APR. & 110623.6 & $44.632 \mathrm{~N}$. & $110.966 \mathrm{~W}$. & 3 & UU & & & $3.2 \mathrm{M}_{\mathrm{L}}(\mathrm{BU})$ & & APR. & 04:06 MST \\
\hline APR. & 185427.2 & $44.646 \mathrm{~N}$. & $111.004 \mathrm{~W}$. & 8 & UU & & & $2.8 \mathrm{M}_{\mathrm{L}}(\mathrm{BU})$ & & APR. & 11:54 MST \\
\hline APR. & 185525.6 & $44.647 \mathrm{~N}$. & $110.989 \mathrm{~W}$. & 11 & UU & & & $2.7 \mathrm{M}_{\mathrm{L}}(\mathrm{BU})$ & & APR. & 11:55 MST \\
\hline APR. 12 & 230547.8 & $44.644 \mathrm{~N}$. & $111.006 \mathrm{~W}$. & 8 & UU & & & $3.0 \mathrm{M}_{\mathrm{L}}(\mathrm{GS})$ & II & APR. 12 & 16:05 MST \\
\hline APR. 17 & 011526.8 & $44.647 \mathrm{~N}$ & $111.009 \mathrm{~W}$. & 8 & UU & & & $2.8 \mathrm{M}_{\mathrm{L}}(\mathrm{BU})$ & & APR. 16 & 18:15 MST \\
\hline APR. 18 & 141755.6 & $44.642 \mathrm{~N}$. & $111.004 W$. & 6 & UU & & & $3.2 \mathrm{M}_{\mathrm{L}}(\mathrm{GS})$ & II & APR. 18 & 07:17 MST \\
\hline APR. 26 & 025134.7 & $44.640 \mathrm{~N}$. & $110.996 \mathrm{~W}$. & 8 & UU & & & $3.0 \mathrm{M}_{\mathrm{L}}(\mathrm{BU})$ & & APR. 25 & 19:51 MST \\
\hline APR. 29 & 075544.3 & $41.047 \mathrm{~N}$. & $108.928 \mathrm{~W}$. & 5 & GS & & & $2.5 \mathrm{M}_{\mathrm{L}}(\mathrm{GS})$ & & APR. 29 & 00:55 MST \\
\hline JUNE 12 & 151434.0 & 42.397N. & 105.694W. & 20 & GS & & - & $3.0 \mathrm{M}_{\mathrm{L}}(\mathrm{GS})$ & & JUNE 12 & 08:14 MST \\
\hline JUNE 20 & 205548.0 & $44.787 \mathrm{~N}$. & $111.000 \mathrm{~W}$ & 5 & UU & & & $3.4 \mathrm{M}_{\mathrm{L}}(\mathrm{GS})$ & & JUNE 20 & 13:55 MST \\
\hline JULY 5 & 021805.6 & $44.666 \mathrm{~N}$. & $111.024 \mathrm{~W}$. & 9 & UU & & & $3.6 \mathrm{M}_{\mathrm{L}}(\mathrm{GS})$ & II & JULY 4 & 19:18 MST \\
\hline NOV. & 002345.0 & $41.922 \mathrm{~N}$ & 108.896W. & 5 & GS & & & $3.3 \mathrm{M}_{\mathrm{L}}(\mathrm{GS})$ & IV & NOV. & 17:23 MST \\
\hline NOV. & 022615.4 & $44.547 \mathrm{~N}$. & $110.937 \mathrm{~W}$ & 9 & UU & & & $2.5 \mathrm{M}_{\mathrm{L}}(\mathrm{BU})$ & & NOV. & 19:26 MST \\
\hline NOV. 9 & 191148.0 & $44.644 \mathrm{~N}$. & $110.238 \mathrm{~W}$ & 0 & UU & & & $3.0 \mathrm{M}_{\mathrm{L}}(\mathrm{BU})$ & & NOV. & 12:11 MST \\
\hline NOV. 15 & 005657.0 & $44.676 \mathrm{~N}$ & $111.030 \mathrm{~W}$. & 7 & UU & & & $3.4 \mathrm{M}_{\mathrm{L}}(\mathrm{GS})$ & IV & NOV. 14 & 17:56 MST \\
\hline NOV. 17 & 083413.3 & $43.156 \mathrm{~N}$. & $110.812 \mathrm{~W}$ & 5 & GS & & & $3.9 \mathrm{M}_{\mathrm{L}}(\mathrm{GS})$ & III & NOV. 17 & 01:34 MST \\
\hline NOV. 17 & 090627.3 & $43.157 \mathrm{~N}$. & $110.798 \mathrm{~W}$. & 5 & GS & - & & $3.7 \mathrm{M}_{\mathrm{L}}(\mathrm{GS})$ & 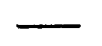 & NOV. 17 & 02:06 MST \\
\hline NOV. 21 & 161100.0 & $44.678 \mathrm{~N}$. & $111.024 \mathrm{~W}$ & 7 & UU & & & $2.7 \mathrm{M}_{\mathrm{L}}(\mathrm{BU})$ & — & NOV. 21 & 09:11 MST \\
\hline NOV. 24 & 021058.7 & $44.674 \mathrm{~N}$. & 111.021W. & 7 & UU & & & $2.6 \mathrm{M}_{\mathrm{L}}(\mathrm{BU})$ & II & NOV. 23 & 19:10 MST \\
\hline NOV. 24 & 063150.4 & $44.670 \mathrm{~N}$. & $111.029 \mathrm{~W}$. & 7 & UU & & & $3.2 \mathrm{M}_{\mathrm{L}}(\mathrm{GS})$ & IV & NOV. 23 & 23:31 MST \\
\hline NOV. 25 & 204525.4 & $44.671 \mathrm{~N}$. & $111.027 \mathrm{~W}$. & 7 & UU & - & - & $3.5 \mathrm{M}_{\mathrm{L}}(\mathrm{GS})$ & IV & NOV. 25 & 13:45 MST \\
\hline
\end{tabular}


Table 1. Summary of United States earthquakes for 1986_Continued

\begin{tabular}{|c|c|c|c|c|c|c|c|c|c|c|c|}
\hline \multirow[t]{2}{*}{ Date } & \multirow{2}{*}{$\begin{array}{l}\text { Origin time } \\
\text { (UTC) } \\
\text { hr min sec }\end{array}$} & \multirow{2}{*}{$\begin{array}{l}\text { Latitude } \\
\text { (0) }\end{array}$} & \multirow{2}{*}{$\begin{array}{l}\text { Longitude } \\
\text { (9) }\end{array}$} & \multirow{2}{*}{$\begin{array}{l}\text { Depth } \\
(\mathrm{km})\end{array}$} & \multirow{2}{*}{$\begin{array}{l}\text { Hypo- } \\
\text { center } \\
\text { Source }\end{array}$} & \multicolumn{3}{|c|}{ Magnitude } & \multirow{2}{*}{$\begin{array}{l}\text { Max. } \\
\text { inten- } \\
\text { sity }\end{array}$} & \multicolumn{2}{|c|}{ Local time } \\
\hline & & & & & & $\mathbf{m}_{\mathbf{b}}$ & $\mathbf{M}_{S}$ & Local & & Date & zone \\
\hline NOV. 30 & 140304.4 & $44.668 \mathrm{~N}$. & $111.028 \mathrm{~W}$. & 7 & UU & & & $2.7 \mathrm{M}_{\mathrm{L}}(\mathrm{BU})$ & & NOV. 30 & 07:03 MST \\
\hline NOV. 30 & 200727.3 & $44.666 \mathrm{~N}$. & $111.032 \mathrm{~W}$. & 8 & UU & - & - & $3.1 \mathrm{M}_{\mathrm{L}}(\mathrm{BU})$ & & NOV. 30 & 13:07 MST \\
\hline NOV 30 & 221652.8 & $44.669 \mathrm{~N}$. & $111.038 \mathrm{~W}$. & 7 & UU & . & $\bar{\tau}$ & $2.5 \mathrm{M}_{\mathrm{L}}(\mathrm{BU})$ & & NOV. 30 & 15:16 MST \\
\hline DEC. 1 & 160938.0 & $44.673 \mathrm{~N}$. & $111.022 \mathrm{~W}$ & 6 & UU & - & $\longrightarrow$ & $3.1 \mathrm{M}_{\mathrm{L}}(\mathrm{GS})$ & & DEC. 1 & 09:09 MST \\
\hline DEC. & 194226.1 & $44.652 \mathrm{~N}$. & $111.032 \mathrm{~W}$. & 2 & UU & 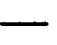 & & $2.7 \mathrm{M}_{\mathrm{L}}(\mathrm{BU})$ & & DEC. & $12: 42 \mathrm{MST}$ \\
\hline DEC. & 224520.2 & $44.670 \mathrm{~N}$. & $111.020 \mathrm{~W}$. & 7 & UU & 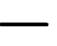 & $\longrightarrow$ & $2.8 \mathrm{M}_{\mathrm{L}}(\mathrm{BU})$ & 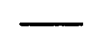 & DEC. & 15:45 MST \\
\hline DEC. & 144008.6 & $42.234 \mathrm{~N}$ & $109.646 \mathrm{~W}$ & 10 & UU & - & - & $2.9 \mathrm{M}_{\mathrm{L}}(\mathrm{UU})$ & $\ldots$ & DEC. & 07:40 MST \\
\hline
\end{tabular}




\section{NETWORK OPERATIONS}

\section{Eastern Aleutians Seismicity}

By J.J. Taber, M.A. Luckman, and S. Rosen

Lamont-Doherty Geological Observatory

of Columbia University

Palisades, NY 10964

The Shumagin seismic network in the eastern Aleutians, Alaska, was used to locate 611 earthquakes in 1986. The seismicity of the region for this time period is shown in map view and cross section in figures 23 and 24. Most of the seismicity is related to the subduction of the Pacific plate beneath the North American plate.

The largest earthquake in this time period within the network had a local magnitude of $4.5\left(\mathrm{~m}_{\mathrm{b}} 5.0\right)$ and was located over the main thrust zone at a depth of $4.5 \mathrm{~km}$. A magnitude $4.1\left(\mathrm{~m}_{\mathrm{b}} 4.4\right)$ earthquake occurred at a depth of $201 \mathrm{~km}$. This was the first deep event larger than magnitude 4.0 since 1981.

Otherwise, the overall pattern during this time period is similar to the long-term seismicity. Concentrations of events occur at the base of the main thrust zone and in the shallow crust directly above it. The continuation of the thrust zone towards the trench is poorly defined. West of the network (which ends at $163^{\circ}$ ) the seismicity is more diffuse in map view and extends closer to the trench. Nine of the 13 located events larger than magnitude 4.0 occurred in this western region. Below the base of the main thrust zone (about $45 \mathrm{~km}$ ), the dip of the Benioff zone steepens. A double Wadati-Benioff zone is evident near $100-\mathrm{km}$ depth. The base of the seismogenic part of the subducting Pacific plate lies near $250-\mathrm{km}$ depth.

Network Configuration

The Shumagin seismic network consists of shortperiod, high-gain seismic stations, a few low-gain stations, and strong-motion accelerographs. The data from the different sets of instruments are being applied to ground motion, seismic source, earthquake prediction, tectonic, and volcanological studies. The network includes 13 remote stations plus three stations in the Pavlof volcano subarray, four repeater stations, and the local station at Sand Point (SAN). Each remote station has a single, short-period vertical seismometer except San Diego Bay (SGB), Chernabura (CNB), Pavlof Volcano (PVV), and Black Hills (BLH), which are three-component stations. Station SQF uses a three-component force-balanced accelerometer (FBA) sensor. The analog signals from the high-gain remote stations have a dynamic range of approximately $42-60 \mathrm{~dB}$ and are transmitted via radio links to a central recording site at Sand Point.

Sensors local to the recording center at Sand Point consist of a three-component set of short-period seismometers $\left(f_{0}=1 \mathrm{~Hz}\right)$ and a triaxial, force-balanced accelerometer $(\mathrm{FBA})\left(f_{0}=50 \mathrm{~Hz}\right)$. The seismometers are recorded on the digital system with a 72-dB dynamic range at both medium and high gains, whereas the FBA is recorded independently on a digital strong-motion recorder (PDR-1).

Within the region of the Shumagin network there are 11 analog strong-motion accelerographs (SMA's) (Kinemetrics SMA-1, $1 g$ or $1 / 2 g$ ), nine of which are located with remote stations of the network. These nine SMA's are connected to the telemetry system, and a $400-\mathrm{Hz}$ trigger signal is sent to the central recording site indicating the exact time at which the SMA began recording a given earthquake. This start time is combined with an internal time code to determine absolute timing within an earthquake.

This range of instrumentation permits the digital recording and locating of events as small as $\mathrm{M}_{\mathrm{L}} 0.4$, with uniform coverage at $\mathrm{M}_{\mathrm{L}} 2.0$, and onscale recording to about $M_{S}$ 6.5. Larger events are recorded digitally by the PDR-1 and on photographic film by the 11 SMA's.

\section{Northern and Central California Earthquakes, 1986}

By Robert A. Urhammer

Seismographic Station

475 Earth Sciences Building

University of California

Berkeley, CA 94720

The year 1986 was very productive in terms of upgrading the University of California seismographic 


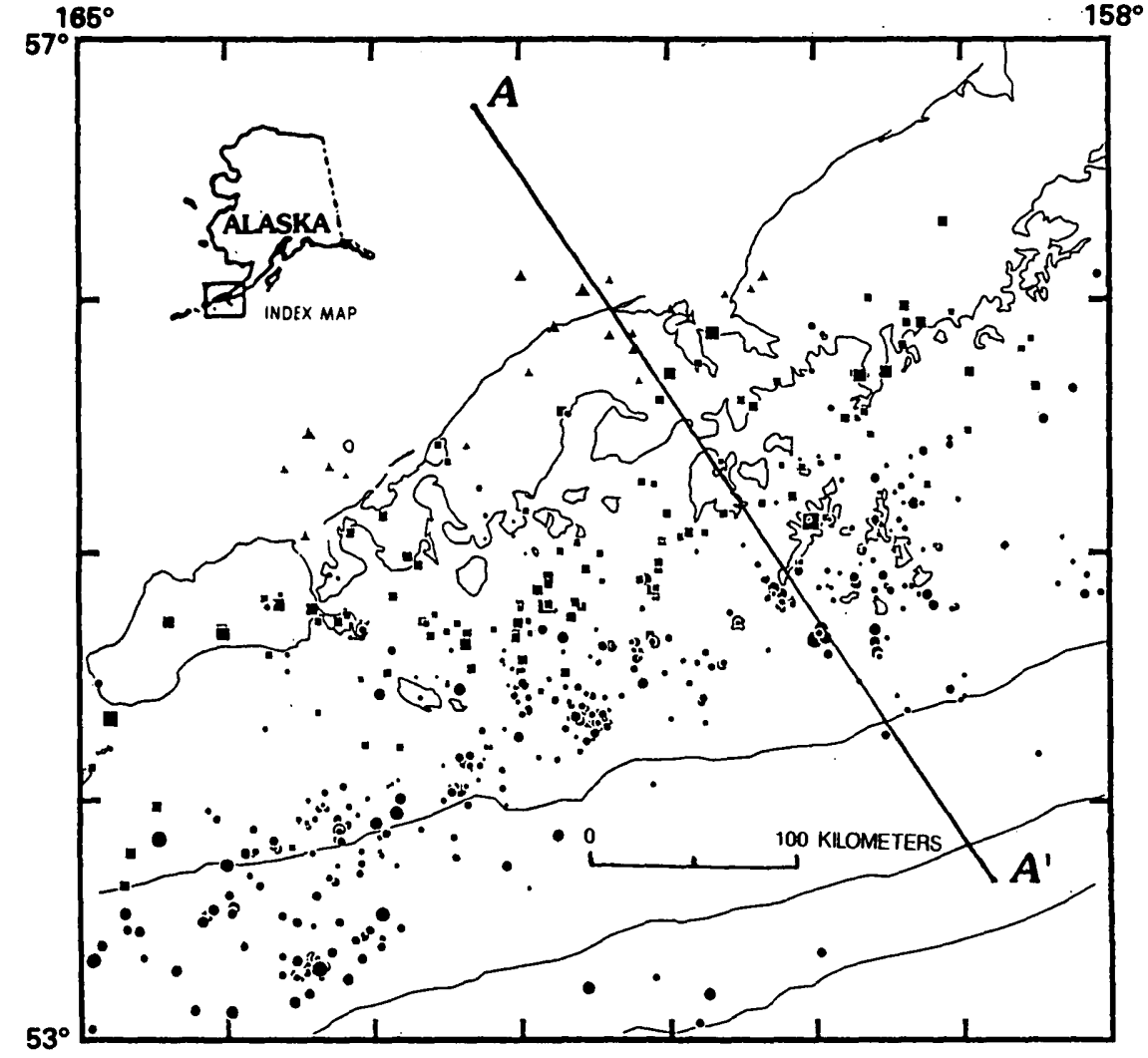

Figure 23. Seismicity located by the Shumagin, Alaska, seismic network from January 1 to December 30, 1986. Earthquakes are represented by filled symbols with white borders. Depth is shown by symbol type and magnitude by symbol size. Dots show earthquakes less than $50 \mathrm{~km}$. Squares show earthquakes between 50 and $150 \mathrm{~km}$; triangles show earthquakes below $150 \mathrm{~km}$. Northeast-southwast trending lines across the bottom of the map indicate increasing depth of the Aleutian trench toward the northwest.

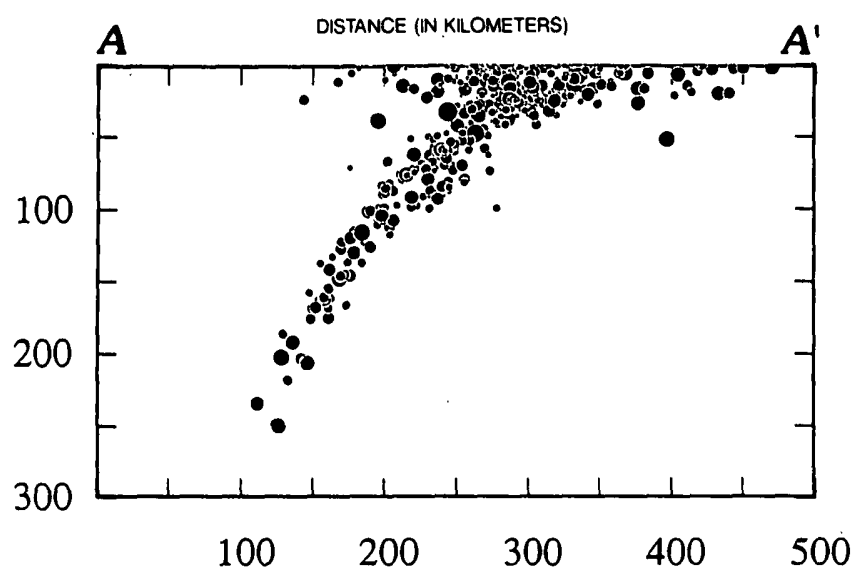

Figure 24. Cross section of Shumagin, Alaska, network seismicity along line $A-A^{\prime}$ in figure 23 . Symbol size shows earthquake size.

stations' instrumentation and facilities. New broadband seismometers (Streckeisen STS-1) were installed at the Oroville (ORV) and San Andreas Observatory (SAO) stations (fig. 25). The Berkeley network now has threecomponent broadband seismographs at five stations (BKS, $\mathrm{CMB}, \mathrm{MHC}, \mathrm{ORV}$, and SAO) and a broadband vertical seismograph at station WDC. The instruments in the
Byerly seismographic vault (BKS, at Berkeley) were temporarily removed from service (starting on July 10 ) to facilitate renovation and waterproofing of the vault. The instruments were reinstalled, calibrated, and returned to service by October 3. A microcomputer-based digital dataacquisition and telemetry system was installed at Mount Hamilton (station MHC) on September 10 as the first step in setting up the newly developed Berkeley Digital Seismograph Network (BDSN) (Bolt, Friday, and Uhrhammer, 1988). A new vault was constructed at Columbia College (150 $\mathrm{km}$ east of Berkeley) to house the seismometers and digital recording system originally installed at the Jamestown station (JAS), which was closed. The Columbia College seismographic station (CMB) began operation on November 6. $\mathrm{CMB}$ is sited on hard rock (crystallized limestone) and is an excellent location with a very low ambient noise level (about $0.3 \mathrm{~nm}$ root-mean-square in the $1-10 \mathrm{~Hz}$ band). The CMB short-period vertical component is recorded with a magnification of $300 \mathrm{k}$ at $1 \mathrm{~Hz}$.

During 1986, the occurrences of approximately 6,300 seismic events were catalogued on summary sheets, and 1,100 teleseisms and 623 local earthquakes were 


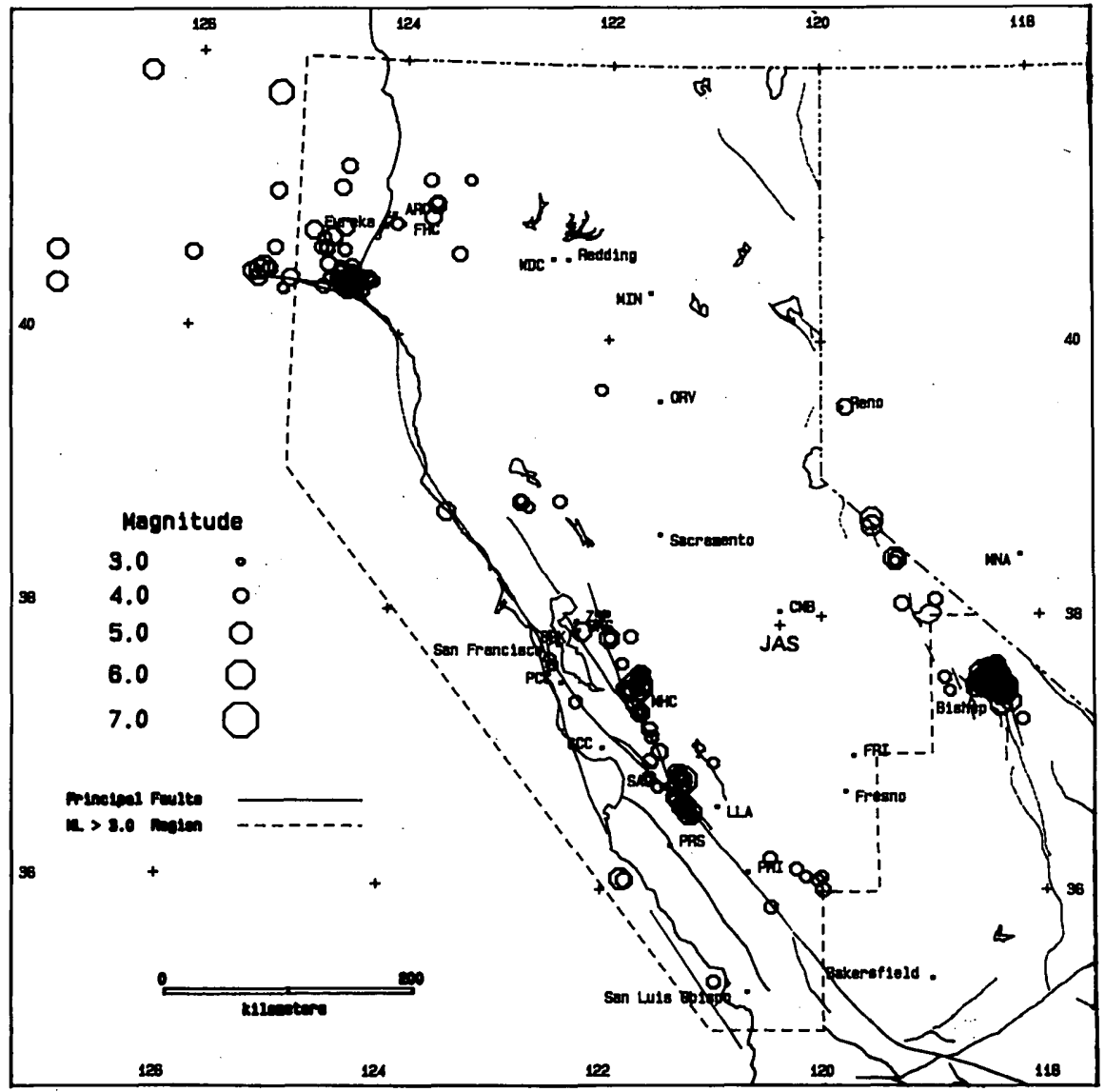

Figure 25. University of California seismographic station network and northern and central California seismicity during 1986. Plotted are 289 earthquakes $\left(3.0 \leq M_{L} \leq 6.4\right)$. Squares show stations. Station abbreviations are defined in text.

analyzed. The "Bulletin of the University of California Seismographic Stations", v. 56 (McKenzie and Uhrhammer, 1988), contains location and magnitude information for 289 earthquakes $\left(3.0 \leq \mathrm{M}_{\mathrm{L}} \leq 6.4\right)$ located in northern and central California and adjoining regions. The epicentral locations are plotted in figure 25 .

Four earthquake sequences during the year contained a total of eleven shocks of $\mathrm{M}_{\mathrm{L}} 5.0$ or larger; most notable were a $M_{L}-5.5$ shock near Hollister on January 26 , and a $\mathrm{M}_{\mathrm{L}}-5.7$ shock at Mount Lewis on March 31, both in the central coast ranges; a $\mathrm{M}_{\mathrm{L}}-6.4$ shock on the east side of the Sierra Nevada mountains in Chalfant Valley on July 21; and a $\mathrm{M}_{\mathrm{L}}-5.1$ shock off the coast of northern California along the Mendocino Escarpment on November 21.

The most significant earthquake $\left(\mathrm{M}_{\mathrm{L}}\right.$ 6.4) in northern and central California during the year occurred on July 21 in Chalfant Valley $20 \mathrm{~km}$ north-northwest of Bishop. Several buildings were damaged in Bishop (MMI VI), and the earthquake was felt throughout a large area of California and Nevada from San Francisco to Reno and south to Los Angeles and Las Vegas. The sequence began on July
18 with the occurrence of a $\mathrm{M}_{\mathrm{L}}-3.9$ earthquake and 16 foreshocks $\left(3.5 \leq M_{L} \leq 5.9\right)$ in the three days prior to the mainshock. One-hundred and ten aftershocks $\left(3.5 \leq \mathrm{M}_{\mathrm{L}} \leq\right.$ 5.8) occurred by the end of the year. The sequence contained seven earthquakes with $M_{L} \geq 5.0$.

\section{Seismicity and Volcanic Activity in Hawaii, 1986}

By Robert Y. Koyanagi and Jennifer S. Nakata

U.S. Geological Survey

Hawaiian Volcano Observatory

Hawaii National Park, HI 96718

The U.S. Geological Survey's Hawaiian Volcano Observatory (HVO) operated an island-wide network (fig. 26) of short-period seismograph stations consisting of 12 three-component and 34 single-component vertical FM systems, radio-telemetered to HVO, and monitored continuously on 13 revolving drum recorders, two develocorders, and a magnetic-tape recorder. The discriminated signals were also transmitted to an analog-to-digital converter and VAX 750 computer for event detection and digitization. 


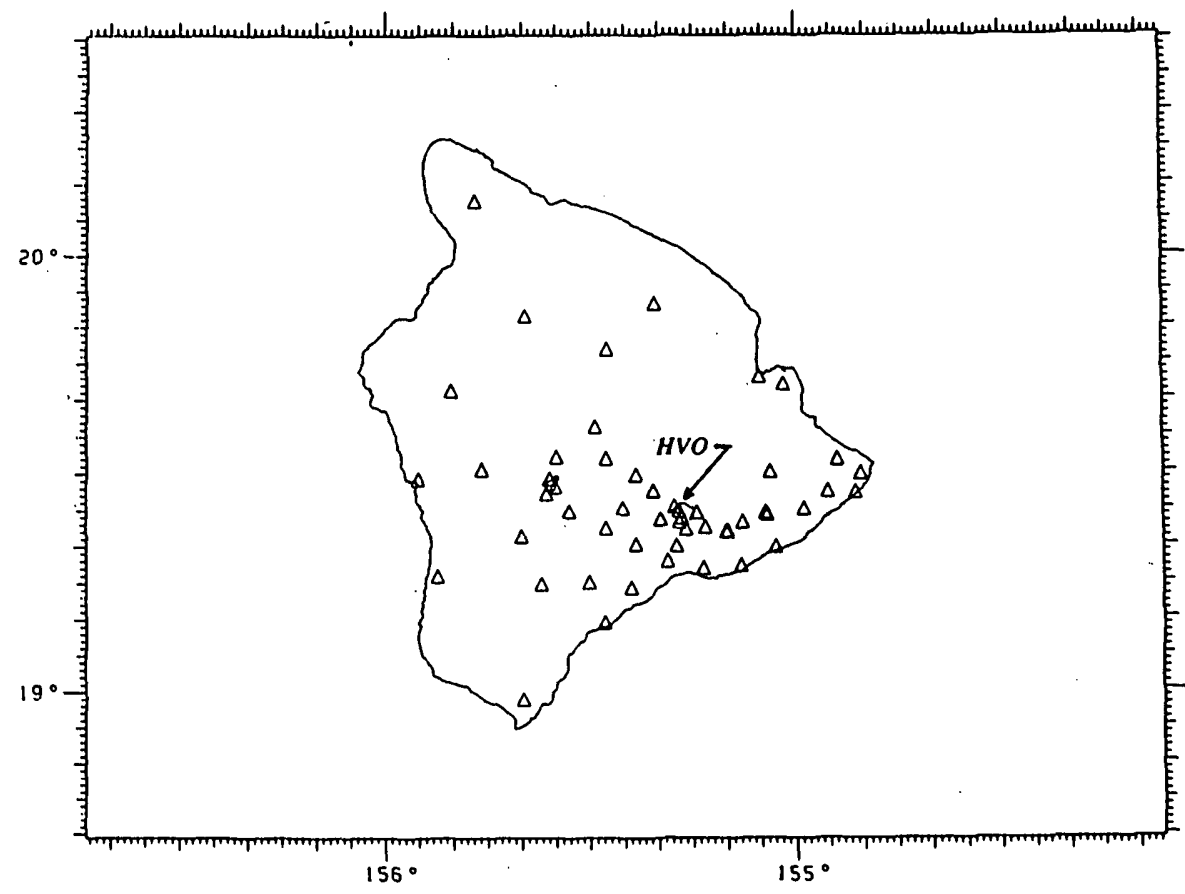

Figure 26. Seismograph stations (triangles) on the island of Hawaii during 1986.

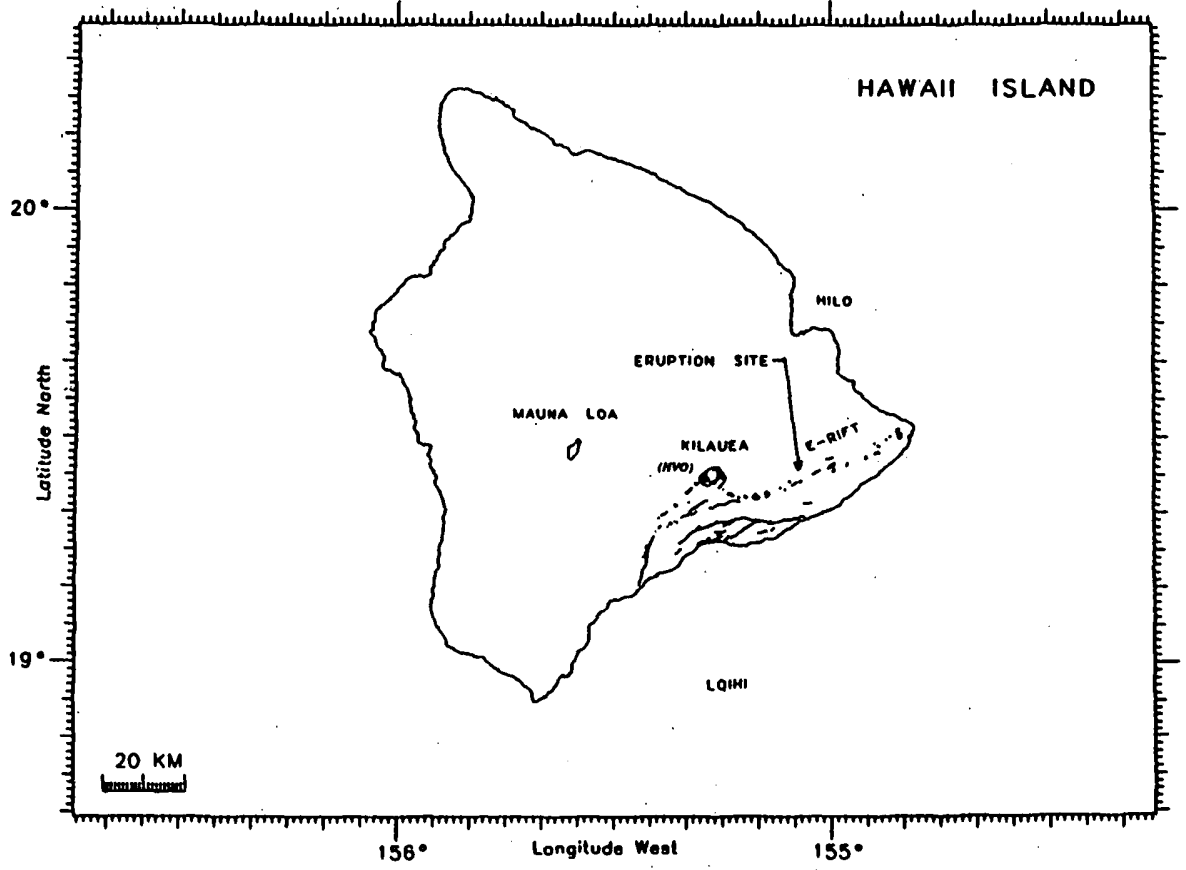

Figure 27. Map of the island of Hawaii showing locations of the active volcanoes Mauna Loa, Kilauea, and Loihi (submarine). The active vent associated with the continuing enuption of Kilauea is in the middle east rift zone. 


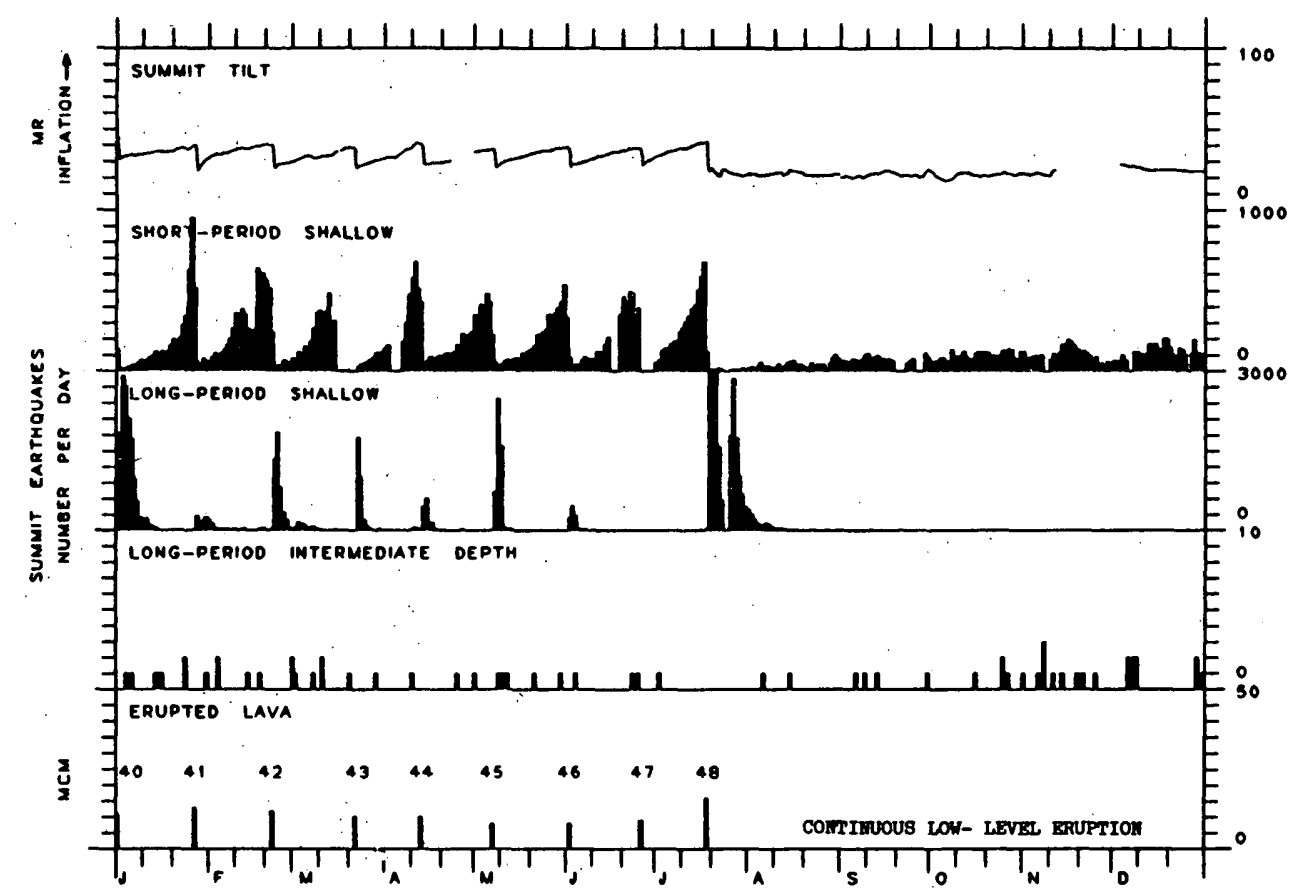

Figure 28. Ground tilt, number of earthquakes, and lava volume associated with the eruption of Kilauea in 1986. Summit tilt is in microradians (MR, west-east) derived from hourly readings of a continuously recording tiltmeter near the observatory. Daily number of short-period and long-period earthquakes at the summit includes instrumentally detected events of 0.1 magnitude or greater. Volume of lava shown in millions of cubic meters (MCM) produced during the major episodes of eruption numerically identified from 40 to 48 and extended from the continuing sequence since 1983 . Since the eruption site migrated $3 \mathrm{~km}$ downrift of Pu' u O'o in July 1986, the new vent (Kupaianaha) has produced approximately $0.5 \mathrm{MCM}$ of lava per day.

Optical-drum seismographs were also operated independently on Hawaii, Maui, and Oahu. These included one three-component, short-period and one three-component, long-period systems at HVO, two short-period systems, each with one high-gain vertical component and two Wood-Anderson horizontal components at Hilo on Hawaii and Haleakala on Maui, and one short-period vertical component at Kipapa on Oahu.

In 1986, Kilauea Volcano (fig. 27) began its fourth year of eruption with eight more episodes of lava erupting in fountains followed by a marked change in behavior during the second half of the year (fig. 28). The episodic pattern of eruption during the first half year was accompanied by changes in tremor amplitude near the eruptive vent in the east rift zone and the number of small earthquakes near the inflation center at the summit. The episodes of high lava output with fountains reaching several hundreds of meters in height were instrumentally characterized by high-amplitude tremor near the eruptive vent, and shallow, long-period microshocks and low-amplitude tremor at the summit.

The vigorous output of lava from the east-rift vent was accompanied by rapid deflation of the summit. The longer repose periods between episodes were marked by weak tremor near the eruptive vent, and a gradually increasing number of shallow microearthquakes and inflation at the summit.

Seismometers near the eruptive area also detected a variety of seismic signals associated with explosive degassing and rockfalls at the vent, microfracturing of cooling and contracting lava flows, and explosive combustion of organic gases at forested boundaries of active lava flows.

The changed mode of eruption from July 20 through the end of the year was marked by relatively steady production of lava, low-level tremor from the eruptive vent in the east rift zone, and small, irregular changes in the number of shallow microearthquakes and amount of ground tilting at the summit.

Intermittent bursts of long-period earthquakes and volcanic tremor occurred at intermediate depths of about 5 to $15 \mathrm{~km}$ beneath the summit of Kilauea and in the upper mantle at about 40 to $60 \mathrm{~km}$ beneath south Hawaii. Intermediate-depth sequences beneath Kilauea generally lasted several days, and individual bursts of deeper tremor beneath south Hawaii were limited to a few hours in duration. 


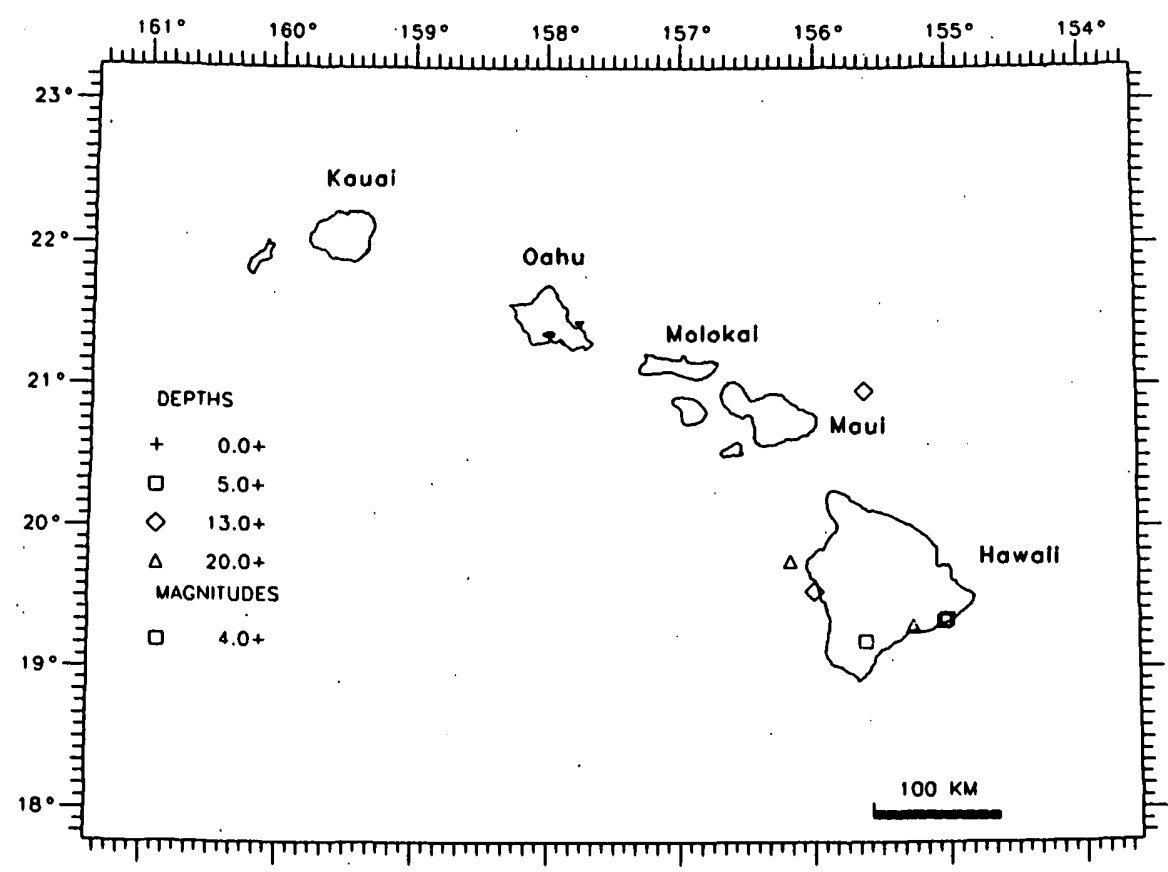

Figure 29. Earthquakes in Hawaii during 1986 (0-20 km depths; $M \geq 4.0$ ). Depth is shown by symbol type and magnitude by symbol size.

Regional seismicity of Hawaii consisted of several thousands of earthquakes of magnitude 1.5-4.9; eight of the largest ones were 4.0 or higher in magnitude (fig. 29). The stresses induced locally by volcanic and related tectonic processes in Hawaii were more definitively outlined by concentrations of smaller earthquakes beneath Kilauea, Mauna Loa, and Loihi (fig. 30). Most of the earthquakes were located at $5-10 \mathrm{~km}$ beneath the south flank of Kilauea and the southeast flank of Mauna Loa (fig. 31). These earthquakes were generally dispersed throughout the year and were attributed to strain release, induced tectonically by the gradual accumulation of stresses along the flanks of the active volcanoes, in contrast to swarms of shallower earthquakes that occur in eruptive zones immediately before renewed eruptions.

\section{Kansas and Nebraska Earthquakes, 1986}

\section{By Choon Byong Park and Don W. Steeples \\ University of Kansas, Kansas Geological Survey 1930 Constant Avenue, Campus West \\ Lawrence, KS 66044}

During 1986 the Kansas Geological Survey (KGS) operated a network consisting of 15 stations in the eastern half of Kansas and Nebraska. All stations from which signals were received at any time in 1986 are shown in figure 32. The data from the network were transmitted via telephone lines to the KGS where they were recorded in both drum analog and triggered digital form. In the digital system the stations in the network were grouped by threes so that storage of data took place when the short-term average amplitudes at three stations exceeded the long-term average amplitude by some preset threshold. The sampling rate established for all stations was 100 samples per second per station.

The KGS network located 18 events in 1986 using the HYPO71 algorithm of Lee and Lahr (1975) (table 2, fig. 33). These events are presented in table 2 and figure 33. The magnitudes in figure 33 range from a low of 1.0 $\left(\mathrm{M}_{\mathrm{D}}\right)$ in Pottawatomie County, Kans., to a high of $3.0\left(\mathrm{M}_{\mathrm{D}}\right)$ in Graham and Kearny Counties, Kans.

Of 18 events, 7 seismic events were astride the $\mathrm{Ne}$ maha ridge (fig. 33), a buried Precambrian uplift that extends roughly from Omaha, Neb., southward across Kansas to near Oklahoma City, Okla. These seven events can be genetically related to the faults associated with or bounding this ridge (Humboldt fault zone, fig. 33) (Hildebrand and others, 1988). This geologic structure has been the site 


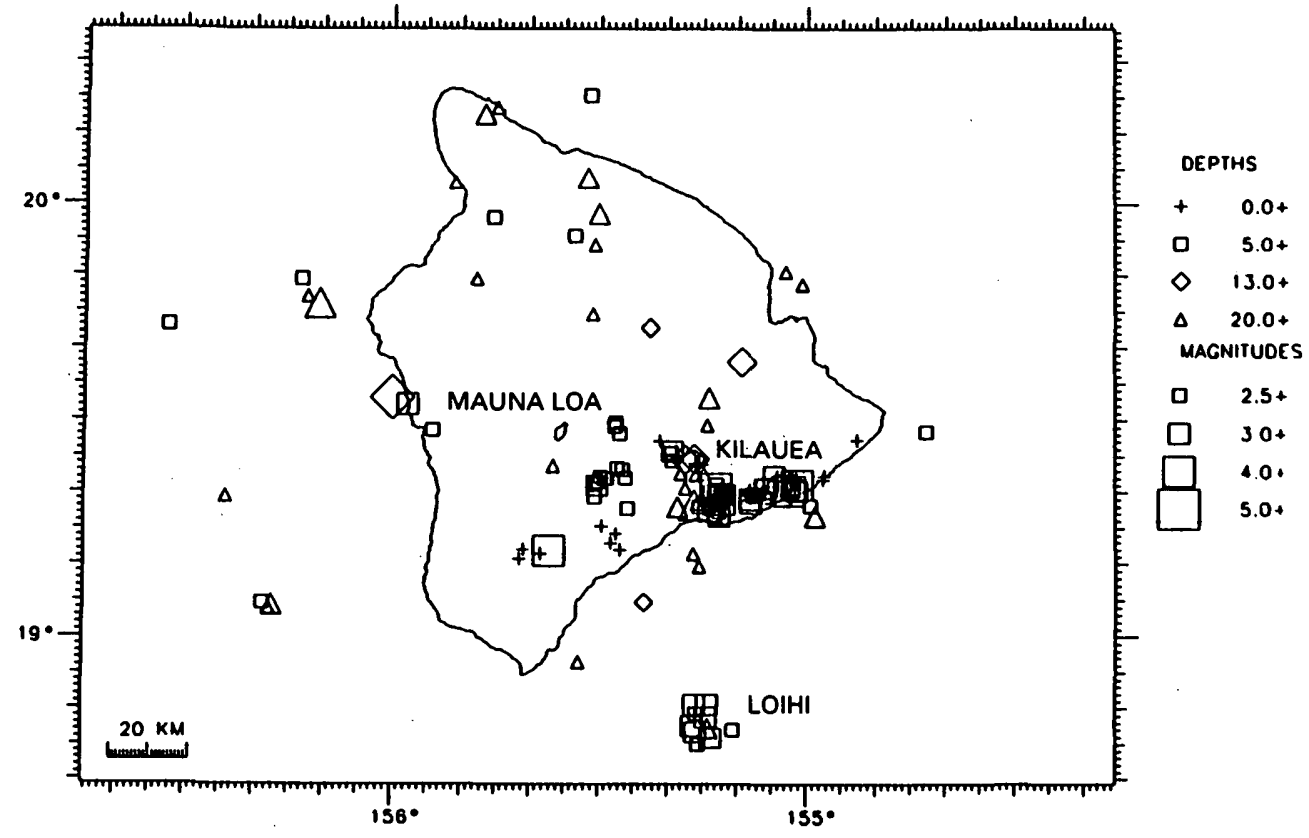

Figure 30. Locations of earthquakes of magnitude 2.5 or greater beneath the island of Hawaii region in 1986. Depth is shown by symbol type and magnitude by symbol size.

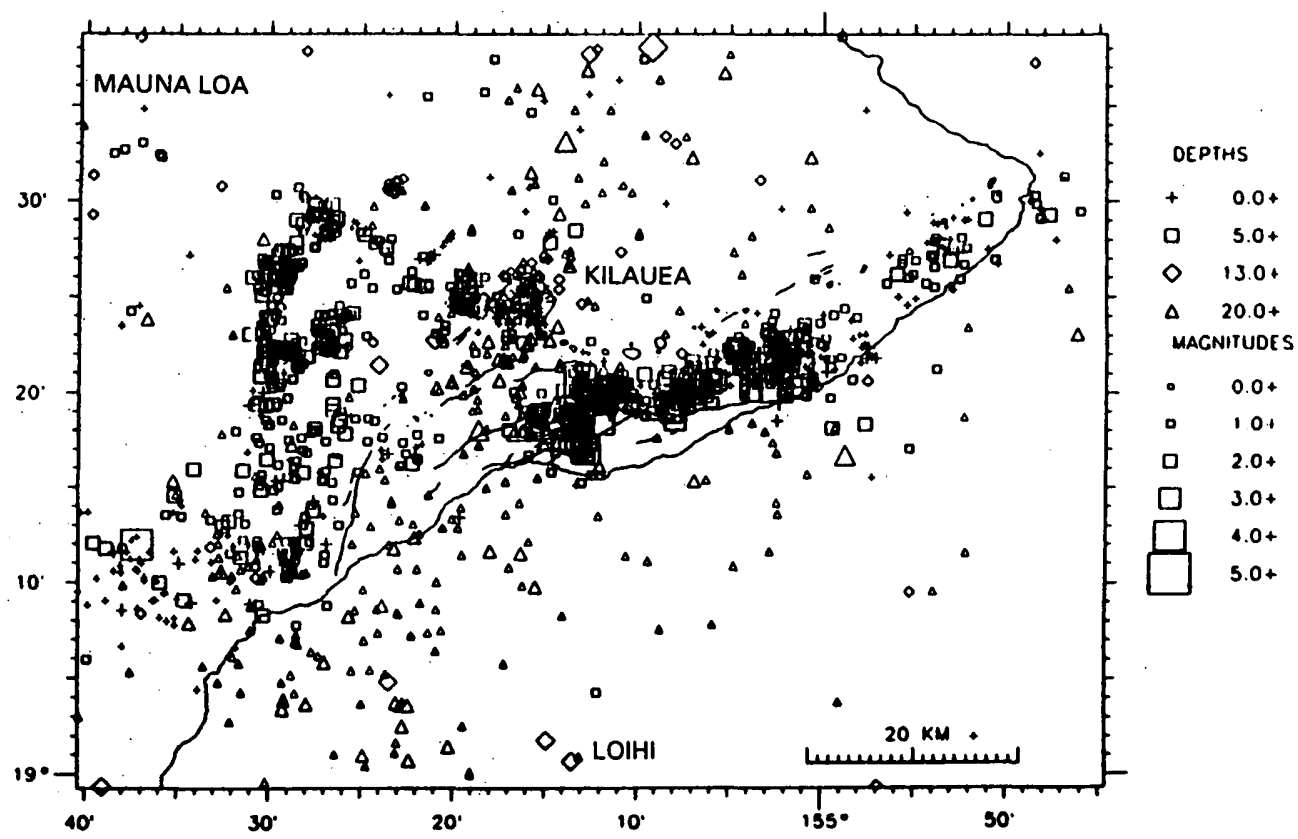

Figure 31: Locations of earthquakes of magnitude 1.5 or greater and standard errors of $2.0 \mathrm{~km}$ or less beneath Kilauea and Mauna Loa volcanoes in the southeastern region of the island of Hawaii for 1986. Depth is shown by symbol type and magnitude by symbol size. 


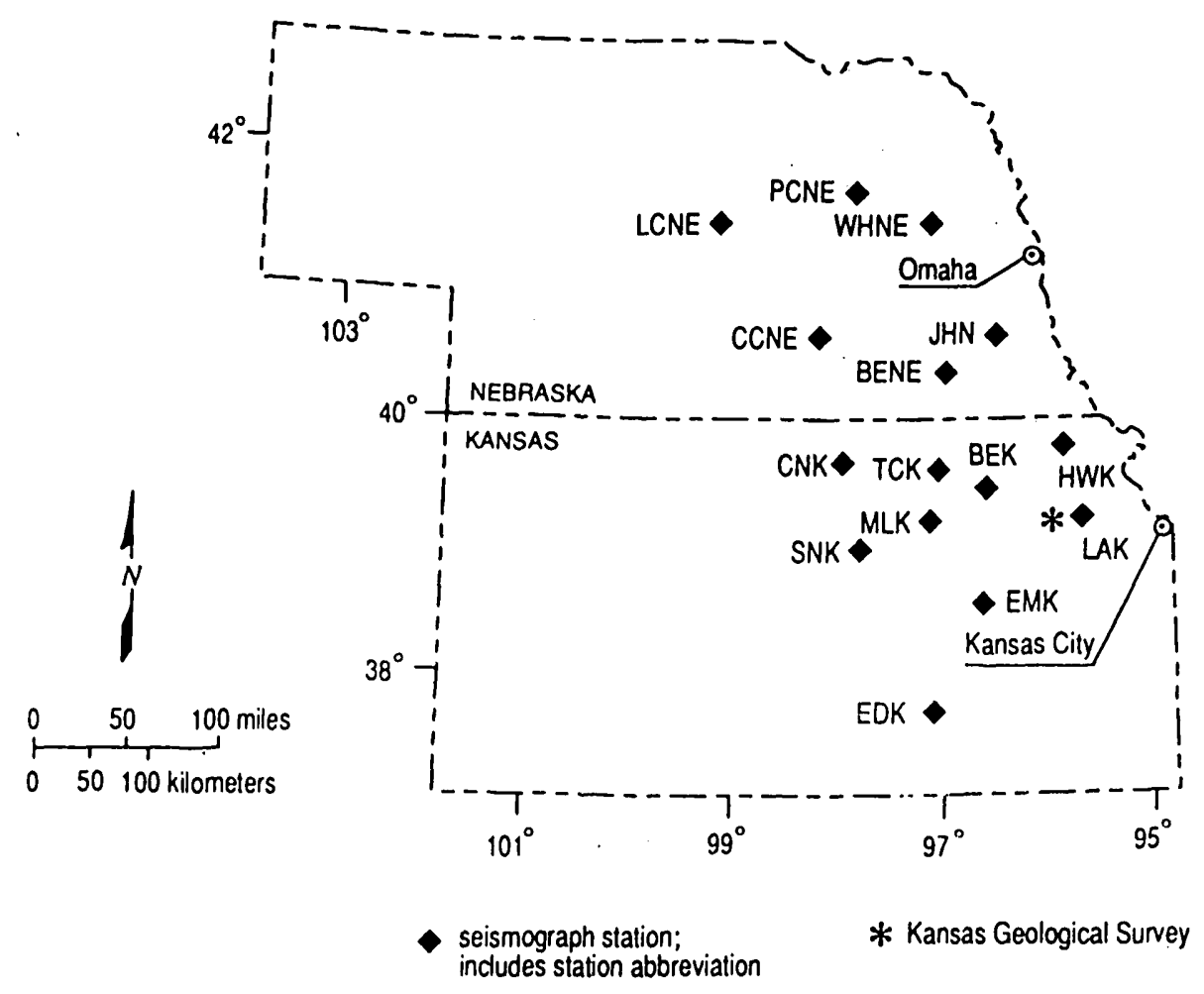

Figure 32. Active seismograph stations in the Kansas-Nebraska network during 1986.

of several earthquakes of Modified Mercalli Intensity VII-VIII (p. 5) over the past 123 years.

Another seven events occurred along the central Kansas uplift, which is a buried anticline similar in age to

Table 2. Kansas and Nebraska earthquakes for 1986.

[UTC, Coordinated Universal Time ; N, north; W, west, (MD)-Duration or coda-length magnitude, $(\mathrm{km})$, kilometers.]

\begin{tabular}{|c|c|c|c|c|c|c|}
\hline $\begin{array}{r}\text { Date } \\
\text { (1986) }\end{array}$ & $\begin{array}{l}\text { Origin Time } \\
\text { (UTC) }\end{array}$ & County & $\begin{array}{l}\text { Lalitude } \\
\text { (N) }\end{array}$ & $\begin{array}{l}\text { Longitude } \\
\text { (W) }\end{array}$ & $\begin{array}{l}\text { Magnitude } \\
\text { (MDUR) }\end{array}$ & $\begin{array}{l}\text { Depth } \\
(\mathrm{km})\end{array}$ \\
\hline FEB 2 & 110445.87 & Greeley, NE & $41-24.76$ & 98-32.62 & 1.9 & 9.90 \\
\hline MAR 4 & 075103.19 & Buchanan, MO & 39-33.37 & 94.55 .50 & 1.9 & 5.0 \\
\hline MAR 13 & 202136.29 & Miaml, KS & $38 \cdot 26.39$ & 95. .47 & 2.1 & 5.0 \\
\hline JUN 2 & 040406.46 & Graham, KS & 39.22 .85 & $99-42.05$ & 3.0 & 16.0 \\
\hline JUN 4 & 223721.99 & Butler, KS & 37.35 .32 & $96-35.14$ & 2.1 & 5.0 \\
\hline JUL 28 & 221507.04 & Jackson, KS & $39-15.89$ & $95-54.82$ & 1.4 & 5.0 \\
\hline SEP 1 & 022402.64 & Pottawatomie, KS & $39-17.84$ & $96 \cdot 10.89$ & 1.6 & 8.8 \\
\hline SEP 24 & 073458.52 & Otoe, NE & 40.37 .80 & 95.54 .09 & 2.0 & 5.0 \\
\hline SEP 27 & 093402.24 & Norton, KS & 39.55 .29 & 99.54 .64 & 2.2 & 0.1 \\
\hline От 8 & 111031.74 & Rooks, KS & $39-11.54$ & $99 \cdot 6.82$ & 1.9 & 12.4 \\
\hline От 9 & 154129.10 & Osborne, KS & $39-10.96$ & 99. 1.99 & 1.9 & 0.01 \\
\hline ○Т 11 & 081735.40 & Rooks, KS & 39- 8.91 & $99 \cdot 11.48$ & 1.9 & 5.0 \\
\hline Ост 20 & 043249.57 & Kearny, KS & 37.55 .86 & 101.21 .81 & 3.0 & 0.4 \\
\hline NOV 5 & 054148.44 & Morris, KS & $38-39.76$ & 96.32 .99 & 1.4 & 5.0 \\
\hline NOV 9 & 090507.24 & Poltawatomie, KS & $39 \cdot 26.44$ & $96 \cdot 5.53$ & 1.0 & 7.5 \\
\hline NOV 25 & 063926.53 & Rooks, KS & $39 \cdot 6.53$ & $99 \cdot 8.60$ & 2.3 & 12.8 \\
\hline DEC 9 & 111145.53 & Osborne, KS & 39.8 .79 & $99 \cdot 1.53$ & 2.1 & 5.0 \\
\hline DEC 31 & 215704.95 & Harper, KS & 37. .97 & $98 \cdot 1.00$ & 2.0 & 5.0 \\
\hline
\end{tabular}

the Nemaha ridge, and are related to the faults flanking it (Hildebrand and others, 1988). Recently, a marked increase in earthquake activity along this uplift has been observed; during the first 5-6 years of network operation (1977-83) it had only sparse activity. The period from October through about mid-December was especially active, when five events occurred with epicenters clustered in an area of roughly $20 \mathrm{~km}$ by $20 \mathrm{~km}$, northwest of Russell, Kans.

One event occurred in central Nebraska, near a pair of normal faults that are oriented northeast-southwest (fig. 33) (Hildebrand and others, 1988). A single event recorded on October 20,1986, with an epicenter near Lakin, Kans., was of sufficient size ( $\left.M_{D} 3.0\right)$ to be felt by people in a relatively small area.

The seismicity in 1986 was not particularly strong in terms of energy release; only nine earthquakes with local magnitude greater than 2.0 occurred. The two largest earthquakes had magnitudes of 3.0. 


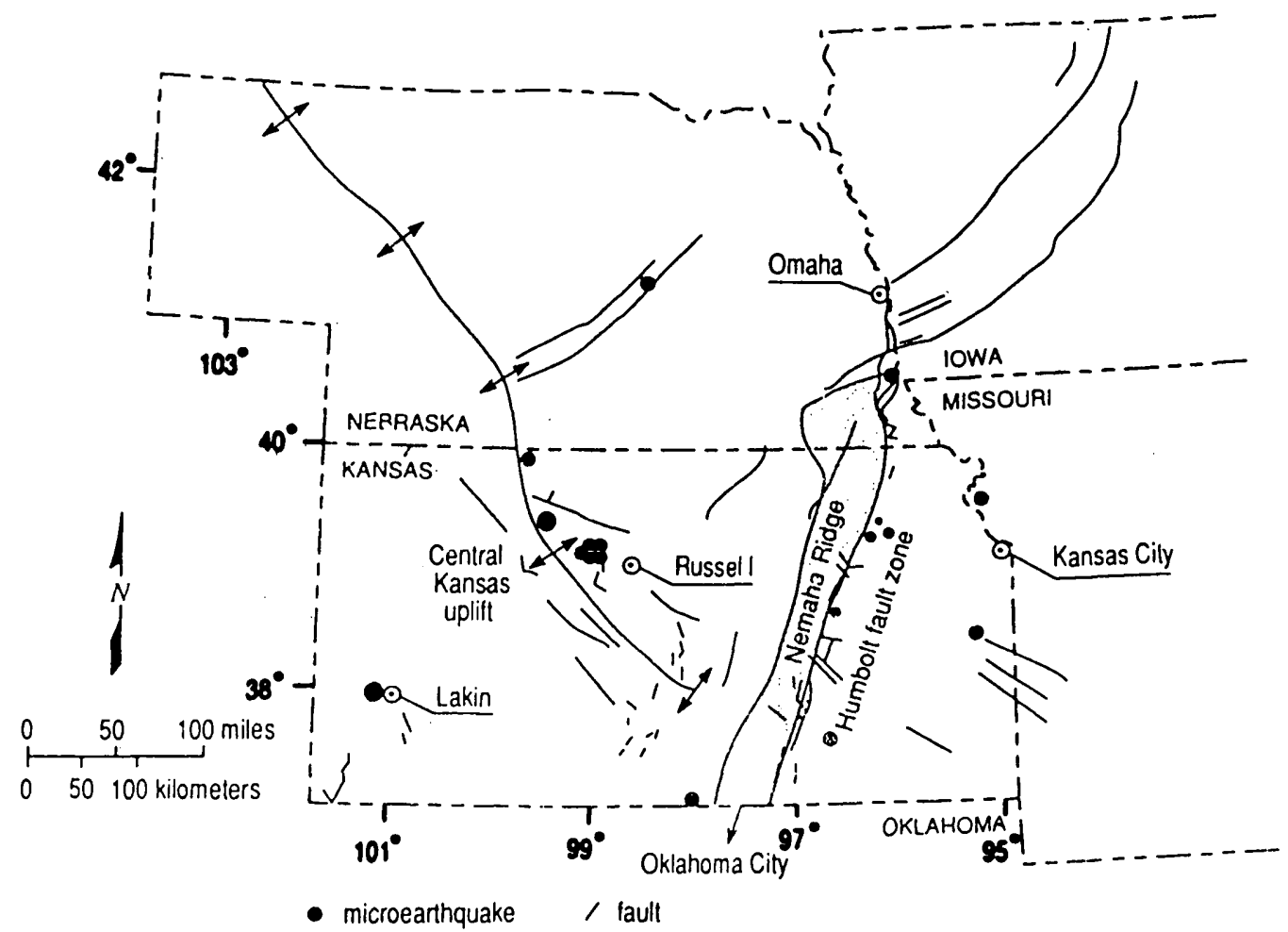

Figure 33. Size-coded microearthquakes recorded by the Kansas Geological Survey during 1986 and major regional tectonic features that are apparently related to earthquake activity. Magnitudes (table 2) are shown by the size of the blackened epicenter symbols. Open circles with dots represent cities and towns.

\section{Mississippi Valley Earthquakes, 1986}

By W. Stauder, R. Herrmann, S. Neiers, S. Horton, C. Carr, C. Finn, and L. Li

Department of Earth and Atmospheric Sciences

Saint Louis University, 3507 Laclede

St. Louis, MO 63103

In 1986, 262 earthquakes were located and 78 other nonlocatable earthquakes were detected by the 40 -station network (a regional telemetered microearthquake network) operated by Saint Louis University under contract with the U.S. Geological Survey and the U.S. Nuclear Regulatory Commission. The location and station readings for these seismic events are published in the quarterly "Central Mississippi Valley Earthquake Bulletin," nos. 47-50.

The locations of 245 of these events within a $4^{\circ}$ by $5^{\circ}$ region of southeast Missouri and Southern Illinois are shown on figure 34 .
The locations of 203 earthquakes located within the $1.5^{\circ}$ by $1.5^{\circ}$ area of the immediate New Madrid region are shown on figure 35 .

In addition to local earthquakes, 183 teleseisms were recorded by the network's microcomputer (PDP 11/34) during 1986 . The apparent velocity of the $P$-wave propagating across the network was used to determine the azimuth of approach and distance and hence the epicentral coordinates of these events. The arrival times for teleseismic $P$ and $P K P$ phases and a map comparing epicenter locations by this method with those determined by the National Earthquake Information Service (NEIS) are published in the "Central Mississippi Valley Earthquake Bulletin."

The earthquake of January 31, 1986, 16:46 UTC, $41.46 \mathrm{~N} ., 81.25 \mathrm{~W}$., was large enough $\left(m_{b} 5.0, N E I S\right)$ to warrant an aftershock study. Four smoked-paper (MEQ-800) recorders were deployed within 12 hours after the main shock and operated for 60 hours. 


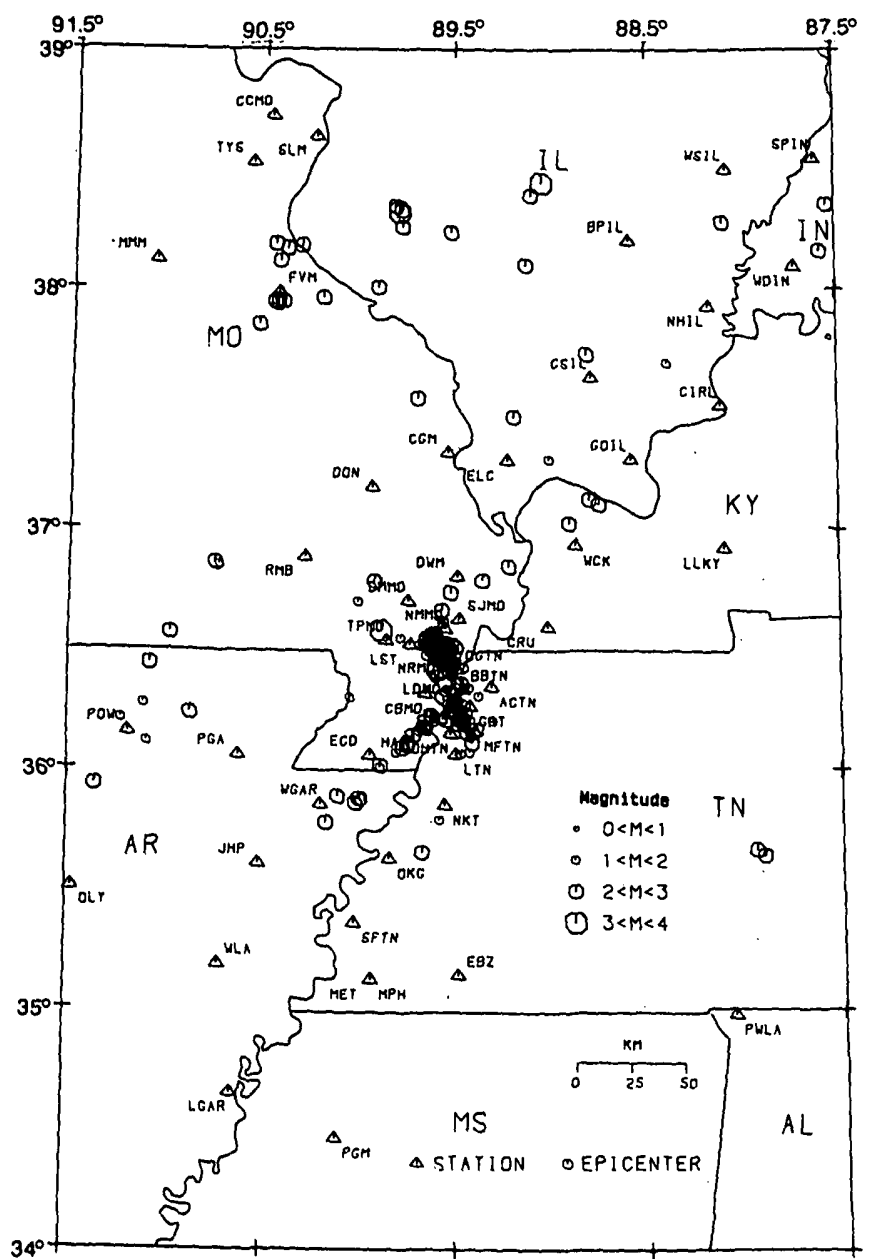

Figure 34. Central Mississippi Valley earthquakes during 1986 within $a 4^{\circ}$ by $5^{\circ}$ region centered at $36.55^{\circ} \mathrm{N}$. and $89.55^{\circ} \mathrm{W}$. The locations of seismograph stations are shown by triangles and are labeled by the station code. Magnitudes are shown by the size of the epicenter symbols.

\section{Montana and adjacent area earthquakes, 1986}

By Michael C. Stickney

Earthquake Studies Office

Montana Bureau of Mines and Geology

Montana College of Mineral Science and Technology

Butte, MT 59701

The Earthquake Studies Office (ESO) of the Montana Bureau of Mines and Geology (MBMG) operated a nine-station seismograph network in southwestern Montana during 1986 (fig. 36). Eight stations were shortperiod, vertical components at remote locations with data radio-telemetered to the ESO in Butte and recorded on helicorders. The Butte station (BUT) consists of two shortperiod horizontal components that are electronically fil-

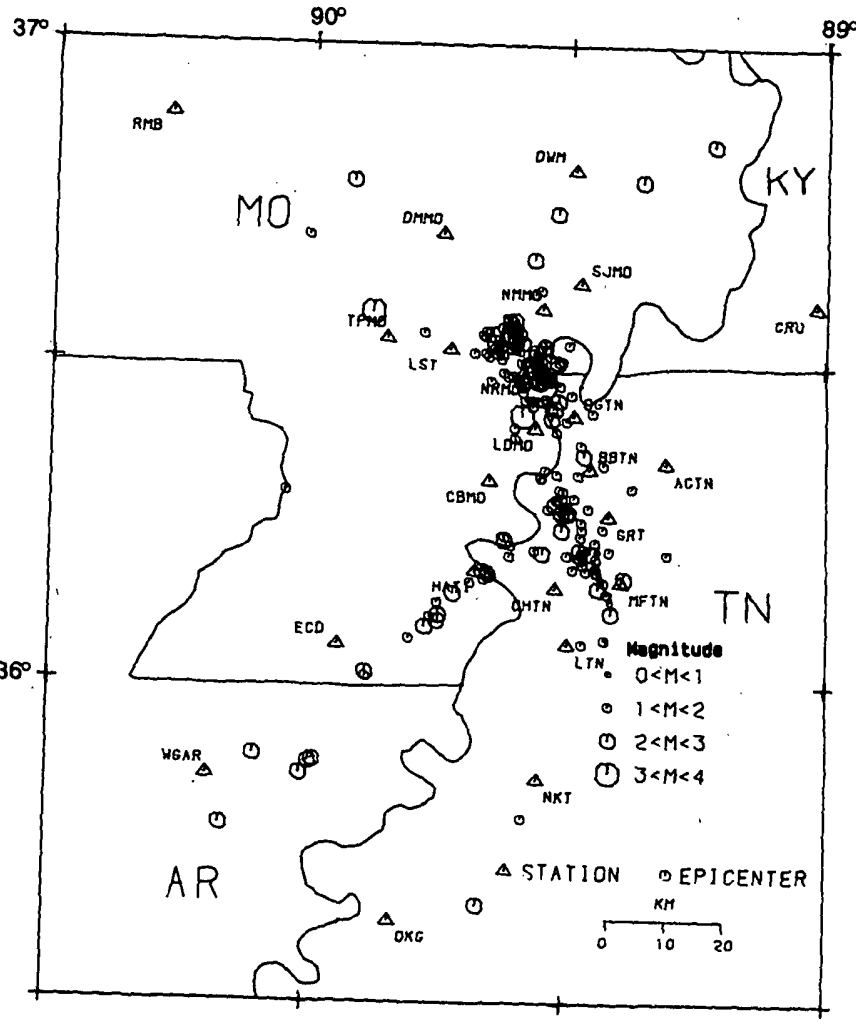

Figure 35. Central Mississippi Valley earthquakes during 1986 within $a 1.5^{\circ}$ by $1.5^{\circ}$ area of the immediate New Madrid region. The locations of seismograph stations are shown by triangles and are labeled by the station code. Magnitudes are shown by the size of the epicenter symbols.

tered to emulate the frequency response of a Wood-Anderson seismograph.

These Wood-Anderson equivalent components are recorded with pen and ink on paper at a magnification of 25,000 and are used to calculate Richter magnitudes for most seismic events in the region with magnitudes of 2.0 or greater. Coda-duration measurements from the shortperiod vertical seismographs were also used to estimate Richter magnitudes. Seismograms from nine stations in northwestern Montana and northern Idaho operated by other agencies were analyzed at the ESO. Phase picks from these nine stations and readings from 26 additional stations operated by other agencies in southeast Idaho and northwest Wyoming were combined with ESO data and used to locate regional seismicity.

During 1986, the ESO reported hypocenter locations for 792 earthquakes (Stickney, 1988) in Montana and adjacent parts of Idaho and Wyoming (fig. 37) within the Intermountain seismic belt and the Centennial tectonic belt (Stickney and Bartholomew, 1987). Of these, 88 events were located in the Yellowstone Park-Hebgen Lake region by the University of Utah. Earthquake magnitudes ranged 


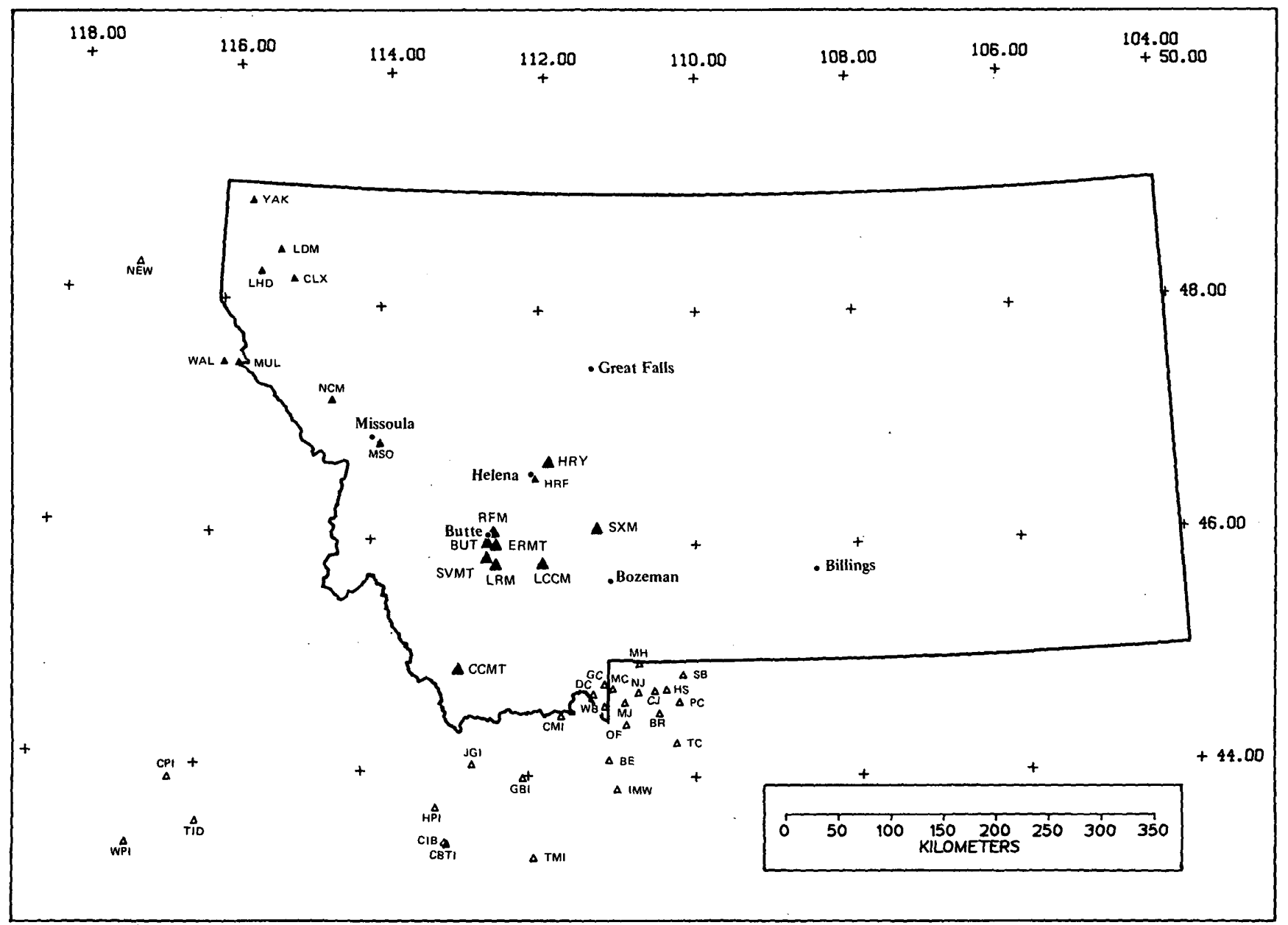

Figure 36. Permanent seismograph stations used to locate 1986 seismicity in Montana, parts of Idaho, and part of Wyoming. Large solid triangles show seismograph stations operated by the Earthquake Studies Office (ESO). Small solid triangles show stations operated by other institutions which contributed seismograms to the ESO for analysis. Small open triangles show stations operated by other institutions which contributed arrival-time data to the ESO.

from about 0.5 to 4.6 , with 146 events of magnitude $\geq 3.0$ and 15 events with magnitudes 24.0 (fig. 38). A total of 25 earthquakes were reported as felt. Regions with significant seismicity during 1986 are listed below.

Seismicity continued in western Yellowstone Park $10 \mathrm{~km}$ east of West Yellowstone, Mont, after an intense earthquake swarm beginning October 10, 1985. Between January 1 and April 30, 1986, 25 earthquakes with magnitudes $\geq 3.0$ (12 reported felt) occurred in the epicentral region of the swarm. The largest of these was a $\mathrm{M}_{\mathrm{L}}-4.3$ (BUT) event on February 11.

On March 12, 1986, a $\mathrm{MD}_{\mathrm{D}}-2.6$ rockburst in the Lucky Friday Mine near Wallace, Idaho, killed one miner and injured two others.

Late aftershock activity of the 1983 Borah Peak, Idaho, earthquake (MS 7.3) continued through 1986 with 30 events of magnitude 3.0 or greater. Three aftershocks had magnitudes of 4.0 or greater; the largest occurred April 7 with $\mathrm{M}_{\mathrm{L}} 4.5$ (BUT).

On August 24 , an $\mathrm{M}_{\mathrm{D}}-3.9$ earthquake occurred 10 km southwest of Three Forks, Mont. The entire aftershock sequence of this event consisted of one $M_{L}-1.6$ event the following day.

A swarm of earthquakes occurred near Boulder, Mont., beginning July 16. A total of 75 locatable events occurred, with two peak periods of activity. The first on July 16-17 included two events of $\mathrm{M}_{\mathrm{L}}$ 3.3. The second peak of activity occurred from October 18 to 24 and included four events of $M_{L}$ 3.1-3.4. Sixteen days later on November 9 , a $\mathrm{M}_{\mathrm{L}}-3.2$ event occurred $5 \mathrm{~km}$ south of the swarm epicenter at an active hot spring.

A swarm of earthquakes in the White Cloud Range of east-central Idaho began in early September and continued through late December. The White Cloud 


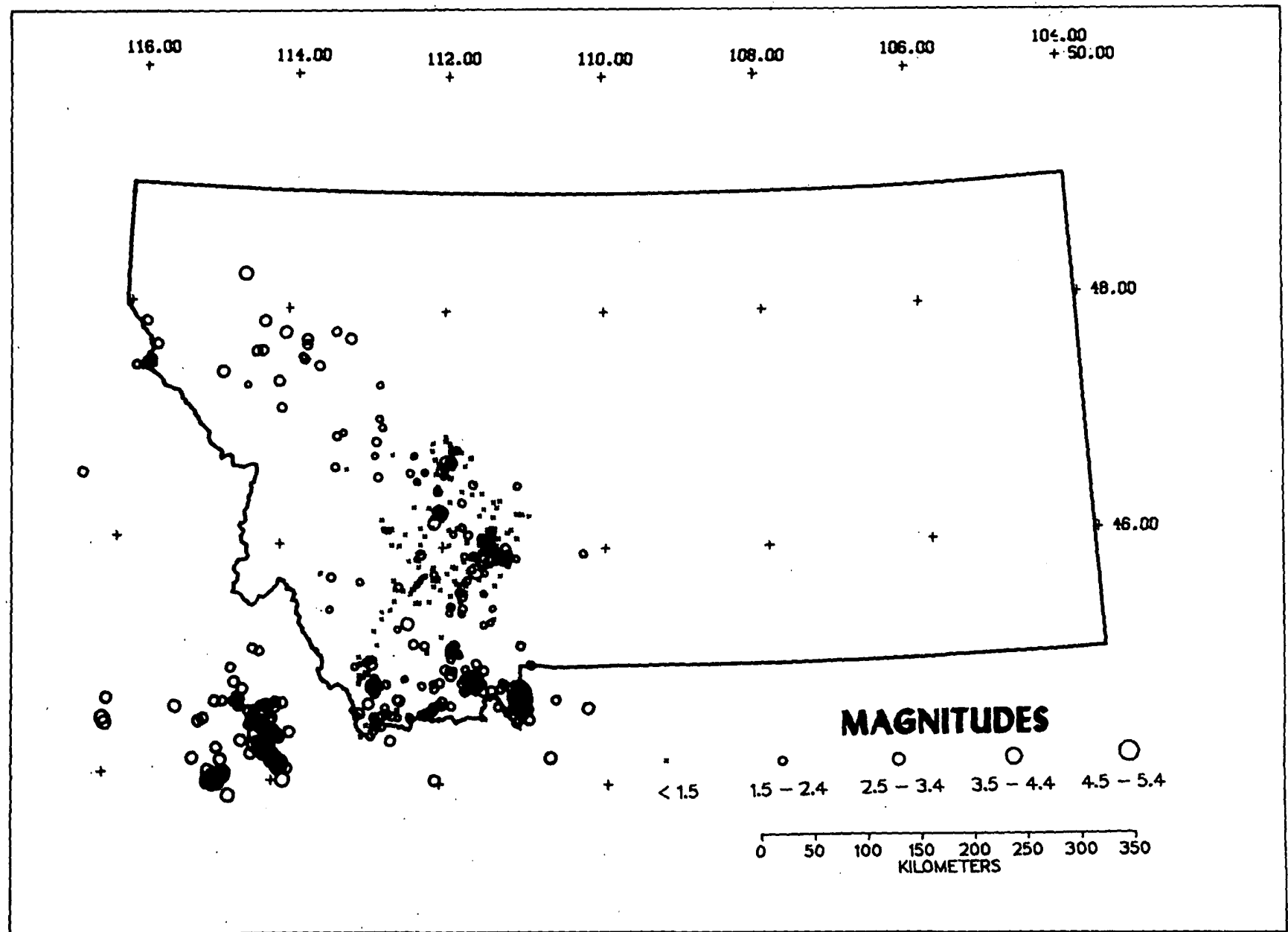

Figure 37. Seismicity of Montana and adjacent regions during 1986. Magnitudes are shown by the size of the epicenter symbols.

swarm included 51 events with magnitudes of $\geq 3.0$ and seven events of magnitude 24.0 . The largest event occurred September 26 with $M_{L} 4.6$ (BUT). Only five events were reported felt.

\section{Western Nevada and Eastern California Earthquakes, 1986}

By Arturo Aburto

Seismological Laboratory

University of Nevada

Reno, NV 89557-0018

This section contains notes on significant earthquake activity from January to December, 1986. It includes all earthquakes with magnitude $\geq 4.0$ and all earthquakes that were reported felt (fig. 39). It is interesting that some of the stronger earthquakes with magnitude $\geq 4.0$ were not reported felt, probably because of the sparse population density in many parts of Nevada. Forty earthquakes had mag- nitudes of $\geq M_{L} 4.0$, the two largest had magnitudes of $M_{L}$ 6.6 (mainshock) and $M_{L}$ 5.6. This sequence was located in the northern part of the White Mountain seismic gap.

\section{Now England Earthquakes, 1986}

By James P. McCaffrey, S.J.

Boston College, Weston Observatory Weston, MA 02193

During 1986 Weston Observatory operated a total of 30 stations in New England. Twenty-nine stations were remote, telemetering their signals to the home station, Weston.

The data were recorded on develocorders as well as in digital computer form at Weston Observatory. The digital system sampled the signals from each station and computed the long-term average (LTA) and the short-term average (STA) of the signal amplitudes. When the STA LTA ratio exceeded some threshold value at two different 


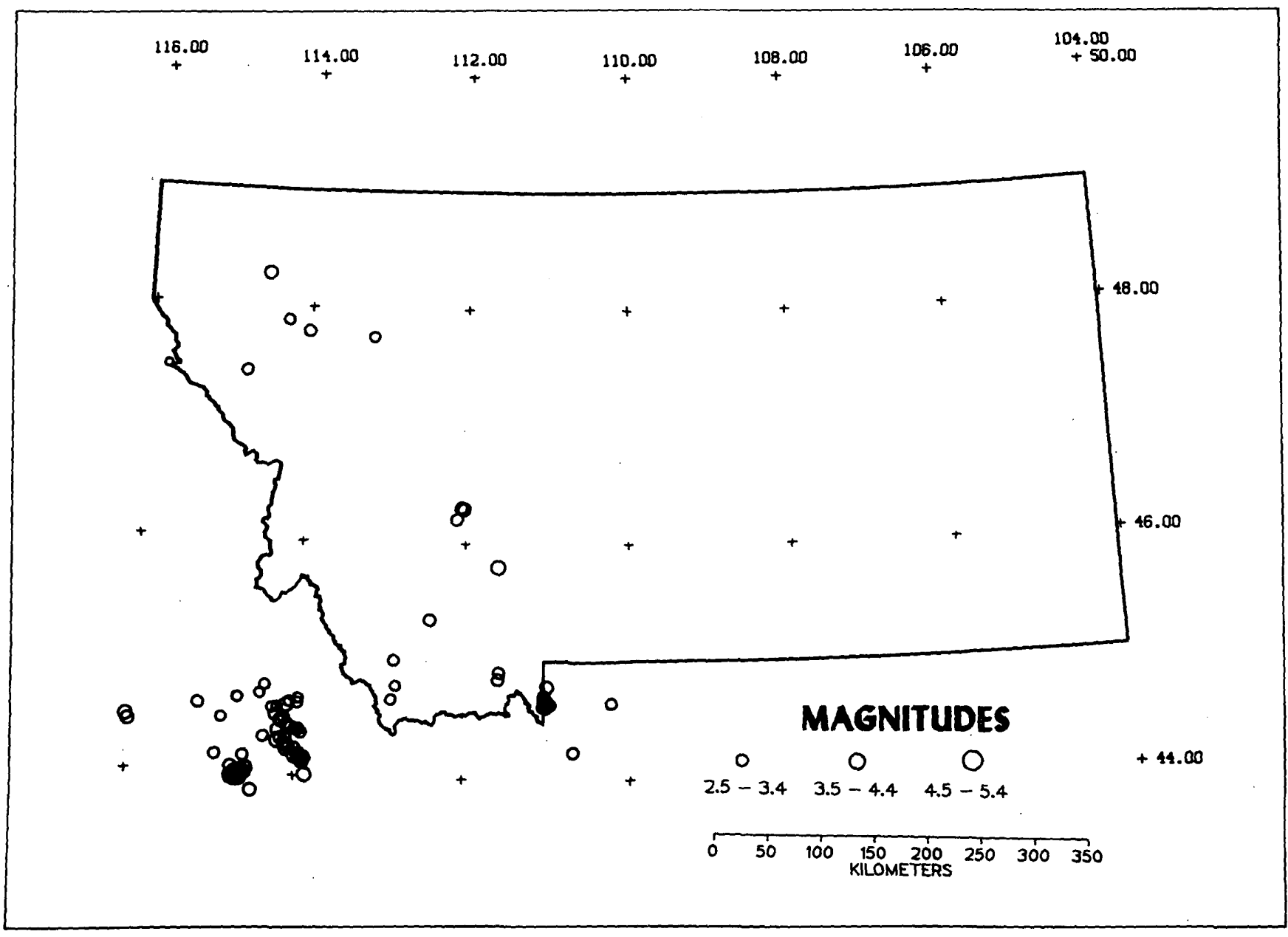

Figure 38. Earthquakes in Montana and adjacent regions that had magnitudes greater than or equal to 3.0 , or reported felt if the magnitude was less than 3.0. Magnitudes are shown by the size of the epicenter symbols.

stations, a seismic event was declared by the computer, and 2.5 minutes of the signal were recorded. In order to analyze the data accurately, step-function and calibration signals from some of the stations were taken.

During the year 67 earthquakes were detected and located in the northeastern United States. In addition, 110 earthquakes that have epicenters in Canada were recorded and located. Of the latter, 50 earthquakes had epicenters within $100 \mathrm{~km}$ of the Canadian-United States border. An additional 184 microearthquakes, foreshocks, and aftershocks were recorded. The magnitudes of these earthquakes ranged from 1.0 to 5.3, and the magnitude range of the foreshocks, aftershocks, and microearthquakes was from -1.9 to 3.4 (fig. 40 ).

Research at Weston Observatory included study of the $\mathrm{Rg}$ waves produced by quarry blasts in southeastern New England, an analysis of the signals recorded by the network from the 1984 Maine Seismic Refraction Experi- ment, and estimations of the return times of earthquakes of various magnitudes in the northeastern United States.

\section{Socorro, New Mexico, Area Earthquakes, 1986}

By Allan Sanford, Lawrence Jaksha, and Roderick Flores Geoscience Department and Geophysical Research Center New Mexico Institute of Mining and Technology Socorro, NM 87801

The Socorro, New Mexico, network is a collaborative project of New Mexico Institute of Mining and Technology and the U.S. Geological Survey. For 1986, the network usually consisted of 11 stations; all are shown in figure 41 except one outside the map area to the north (MIM) and another one just west of Socorro (WTX). Station BMT was substituted for MAG in 1987. Earthquakes recorded by the Socorro network were located using the algorithm HYPO71 Revised (Lee and Lahr, 1975) with a 


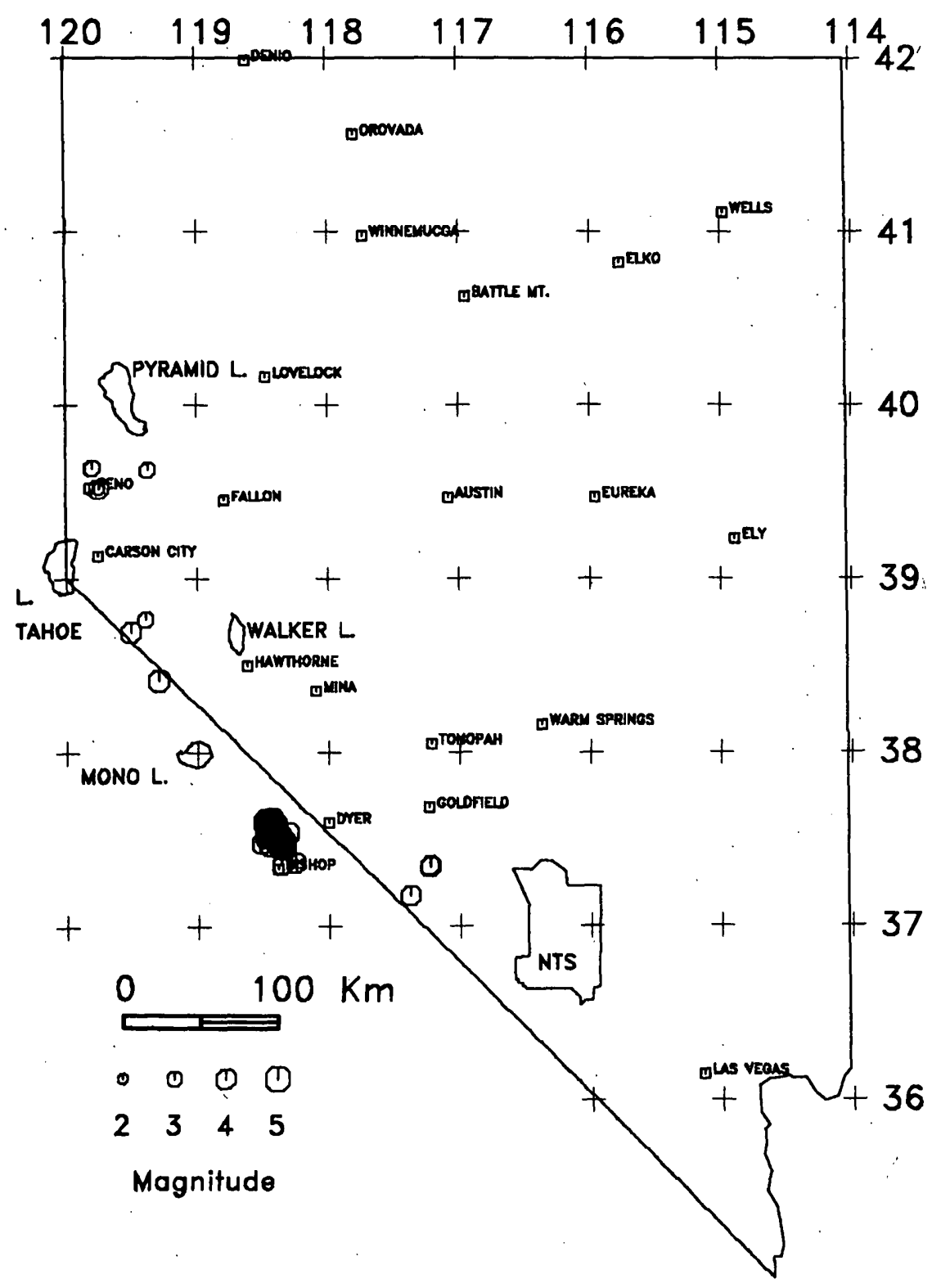

Figure 39. Earthquakes in western Nevada and eastern California during 1986 with magnitudes greater than or equal to 4.0 or reported felt if less than magnitude 4.0. Magnitudes are shown by the size of the epicenter symbols.

half-space velocity model of $5.85 \mathrm{~km} / \mathrm{s}$ and a Poisson's ratio of 0.25 (Ward and others, 1981). Station corrections ranging from -0.33 to 0.28 seconds were used to account for differences in near-surface geology and elevation within the network (Ward, 1980; and Ake, 1984).

Magnitudes of the earthquakes were calculated from durations of recorded signals using an empirical equation based on northern New Mexico earthquakes (Newton and others, 1976). The same equation was found to be applicable to earthquakes in the Socorro area in the magnitude range 1.0 to 4.0 (Ake and others, 1983).
Epicenters for 301 earthquakes are plotted in figure 41. In general, the location quality is best for epicenters towards the center of the instument array. Duration magnitudes for these 301 events range from -0.5 to 2.8. During 1986, the Socorro area had 17 earthquakes with magnitudes $\geq 1.5 ; 7$ of these exceeded or equaled magnitude 2.0.

The total number of earthquakes recorded by the Socorro network in 1986 was far greater than the 301 events shown in figure 41. A characteristic of seismic activity in the Socorro area is that the majority of earthquakes occur in swarms. On figure 41, these swarm sequences appear as 


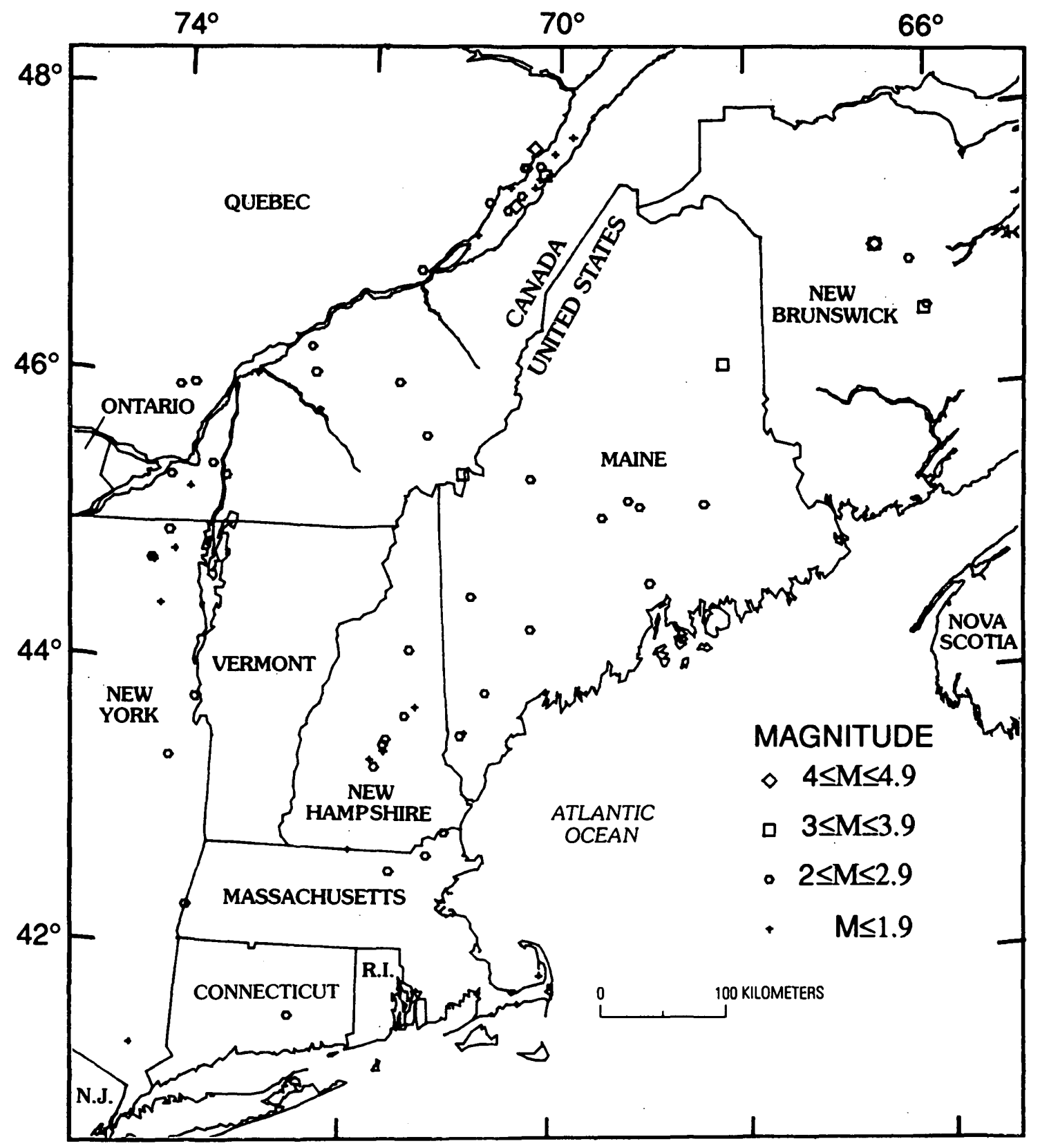

Figure 40. Northeastern United States earthquakes during 1986. Magnitudes are shown by the type of epicenter symbol.

tight clusters of epicenters; an example is the cluster centered at $34.02^{\circ} \mathrm{N}$., $106.83^{\circ} \mathrm{W}$. Activity in this area appears to have commenced on April 19 but was most vigorous from April 27 through May 1. In routine analysis of swarms, we usually make no effort to locate all events, only the strongest. Thus, the April-May swarm (fig. 41) is represented by about a dozen closely spaced epicenters. In a detailed study of this swarm, Petrillo (1987) was able to obtain HYPO71 quality B or better locations for 51 earthquakes.

The tight cluster of epicenters centered at $34.15^{\circ} \mathrm{N}$., $106.73^{\circ} \mathrm{W}$ shows for a swarm that started on September 24 and continued into the early part of December. This swarm produced the strongest earthquake within the Rio Grande rift in 1986, a magnitude-2.8 event at 15:55 UTC on Octo- 

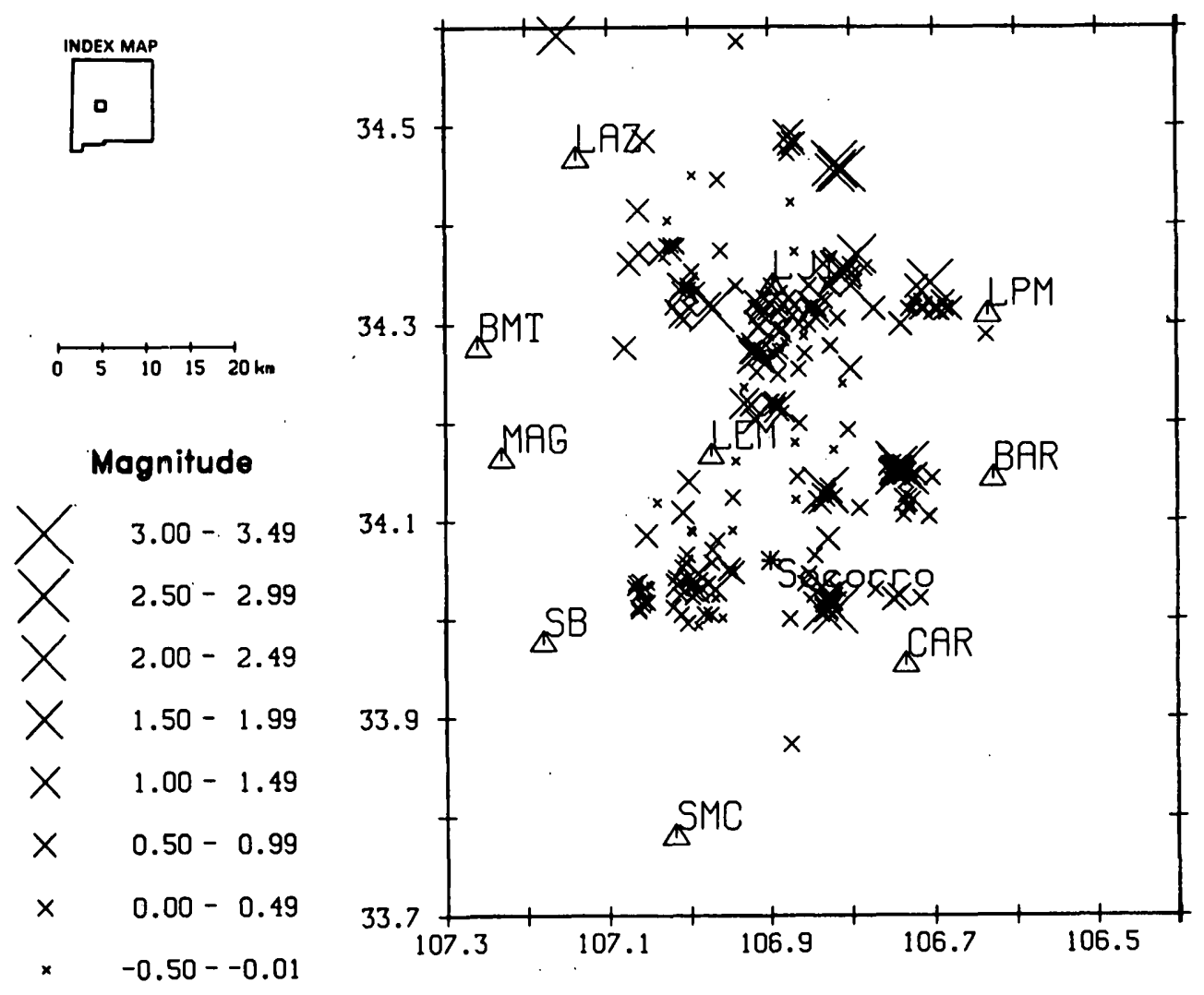

Figure 41. Seismicity of the Socorro, New Mexico, area for 1986. Stations are shown by triangles and abbreviations, and epicenters are shown by $x$ symbols. Magnitudes are shown by the size of the epicenter symbols.

ber 5 . All locatable shocks (41) of this swarm are included in the 301 epicenters plotted on figure 41 .

\section{Selsmicity of New Mexico, 1986}

By Allan Sanford and Lawrence Jaksha

Geoscience Department and Geophysical Research Center New Mexico Institute of Mining and Technology

Socorro, NM 87801 and

\section{Dan Cash}

Geophysics Group, ESS-3

Los Alamos National Laboratory

Los Alamos, NM 87845

The distribution of earthquakes in New Mexico during 1986 is shown in figure 42 . Epicenters for 40 shocks were plotted whose duration magnitudes were $\geq 1.5$. Data are primarily from networks of seismic stations operated by New Mexico Institute of Mining and Technology (NMIMT) in collaboration with the U. S. Geological Survey (USGS) and Los Alamos National Laboratory (LANL). The LANL network is centered near $36.00^{\circ} \mathrm{N}$, $106.30^{\circ} \mathrm{W}$., on the Rio Grande rift in north-central New Mexico; the NMIMT/USGS network centered near $34.10^{\circ} \mathrm{N}$., $106.90^{\circ} \mathrm{W}$., is also on the rift but near the middle of the state. An additional small network of stations in southeastern New Mexico, centered near $32.40^{\circ} \mathrm{N}$, $103.9^{\circ} \mathrm{W}$., is operated by NMIMT.

Although the recording stations are concentrated in the central regions of the State, the geographical distribution of earthquake activity (fig. 42) is believed to be nearly free of station-location bias. Earthquakes with magnitudes of 1.5 in the farthest regions of the state are well within the detection capabilities of all stations in the two principal networks. However, because the azimuthal distribution of 


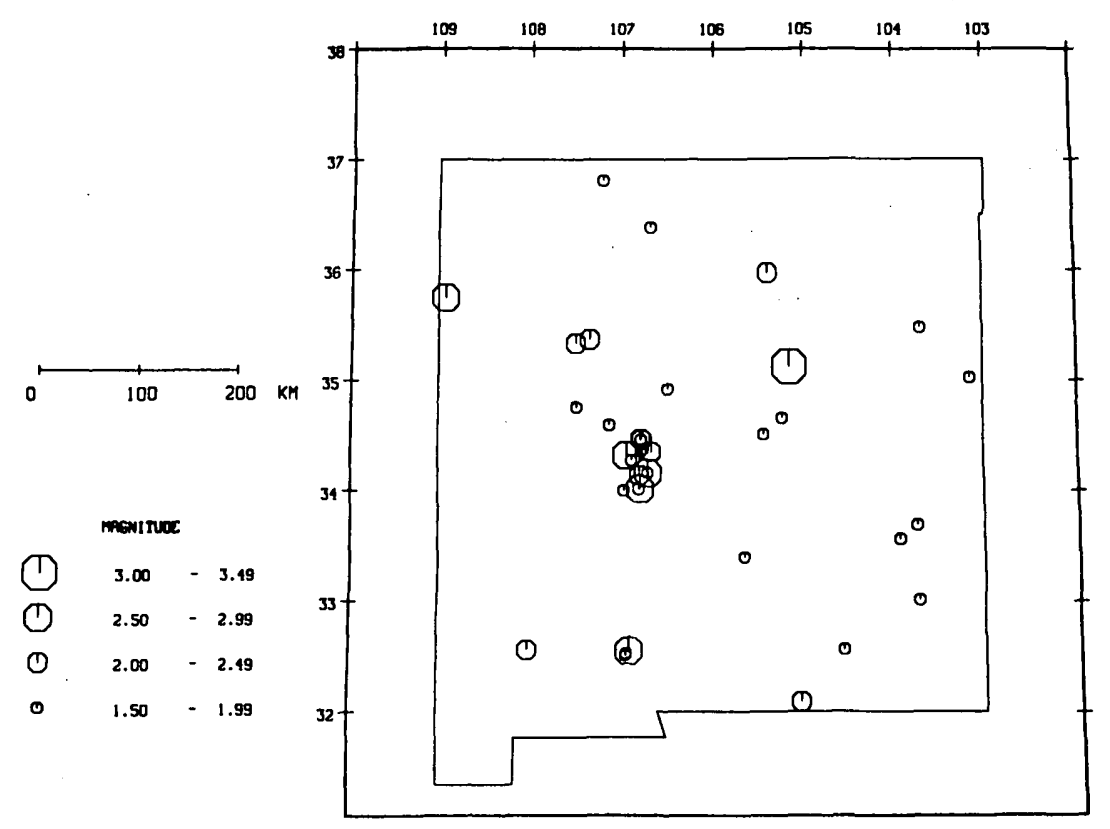

Figure 42. Earthquakes in New Mexico during 1986 with magnitudes greater than or equal to 1.5. Epicenters are shown by octagon symbols. Magnitudes are shown by the size of the epicenter symbols.

stations narrows as distance from the networks increases, the accuracy of earthquake locations diminishes progressively towards the State boundaries.

For the most part, the distribution of the 40 earthquakes in 1986 was similar to what has been observed since instrumental studies began in New Mexico (Sanford, 1965; Sanford and Cash, 1969; Toppozada and Sanford, 1972; Sanford and others, 1981). More than half the seismic events were within or near the Rio Grande rift, a major extensional structure extending north-south through the center of the state between longitudes $105.5^{\circ} \mathrm{W}$. and $107.3^{\circ} \mathrm{W}$.

However, as in the previous 24 years of recording, relatively strong earthquakes occurred in the presumably stable physiographic provinces bordering the rift. Two examples are a magnitude- 2.6 earthquake on the Colorado Plateau at $35.75^{\circ}$ N., $108.96^{\circ} \mathrm{W}$., on May 22 and a magnitude-3.1 earthquake on the western edge of the Great Plains at $35.12^{\circ} \mathrm{N} ., 105.18^{\circ} \mathrm{W}$, on August 21. The latter earthquake was the strongest in New Mexico during 1986. The strongest earthquake in the Rio Grande rift occurred $20 \mathrm{~km}$ northeast of Socorro on October 5. The shock, which had a magnitude of 2.8, was the strongest of a swarm that commenced September 24 and continued into early December. A separate discussion of the 1986 seismic activity in the Socorro area, which accounts for over 40 percent of the earthquake epicenters (fig. 42), appears elsewhere in this report (p. 192).

\section{Oklahoma Earthquakes, 1986}

By James E. Lawson, Jr.

Oklahoma Geological Survey

Oklahoma Geophysical Observatory

Leonard, OK 74043 and

Kenneth V. Luza

Oklahoma Geological Survey

University of Oklahoma

Norman, OK 73019

In 1986, 52 earthquakes recorded on seismograms from three or more stations were located (fig. 43, table 3, and fig. 44) (see Stover and Brewer, 1991, United States Earthquakes, 1985, p. 143 for a description of the seismic network). No earthquakes were reported felt. Garvin and McClain Counties continued to be among the most active in the state, as they have been since 1979. For the thind 


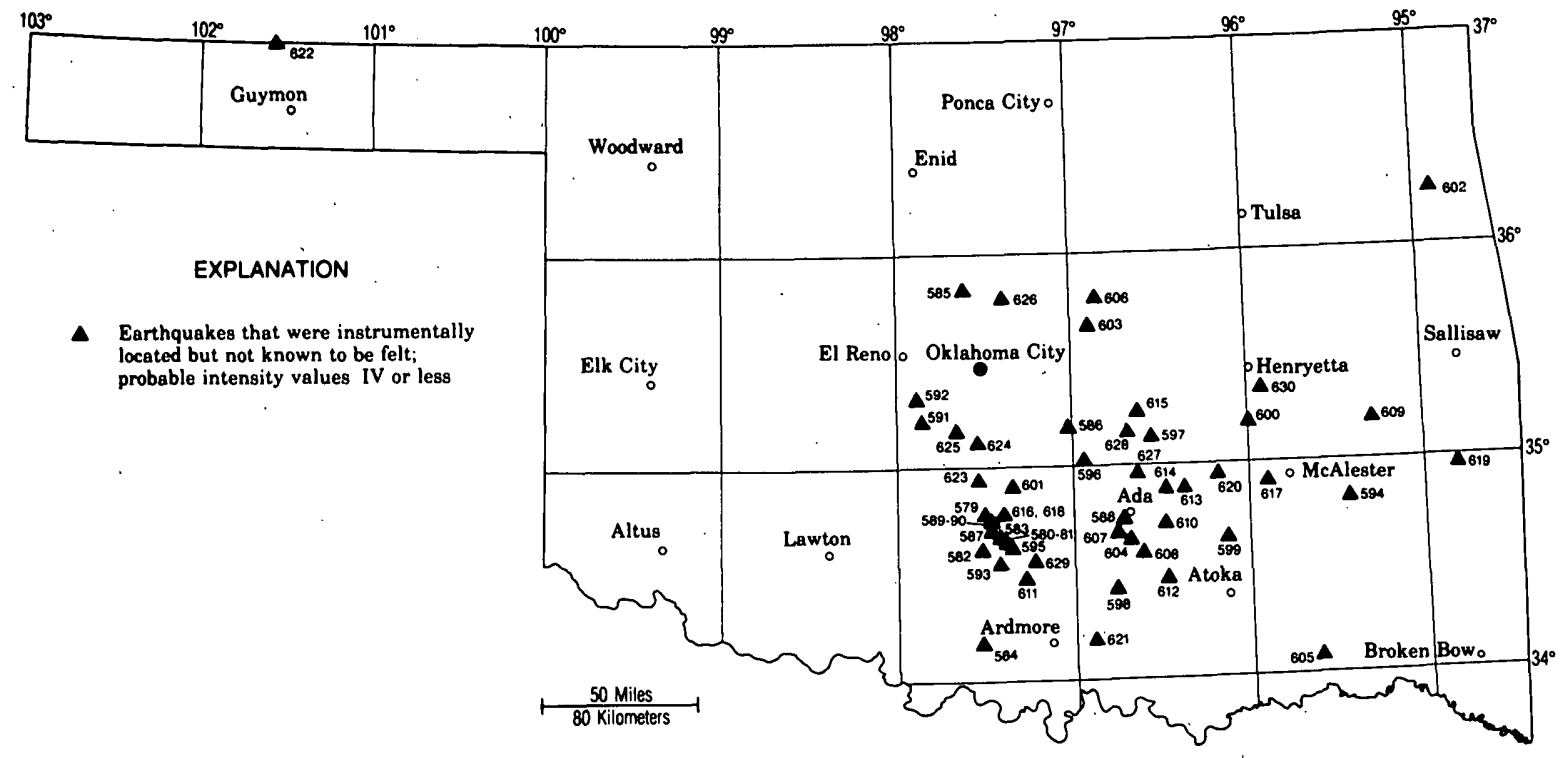

Figure 43. Distribution of Oklahoma earthquakes for 1986. Numbers correspond to event numbers in table 3.

Table 3. Oklahoma earthquakes for 1986.

\begin{tabular}{|c|c|c|c|c|c|c|c|c|c|c|}
\hline Event & Date and & Prigin Time & & Intensity & & gnitud & & Latitude & Longitude & Depth \\
\hline & & & County & & $3 \mathbf{H z}$ & bL8 & DUR & & & \\
\hline 579 & IAN 1 & 013407.41 & GARVIN & & 2.2 & 1.9 & 2.3 & 34.764 & 97.473 & $5.0 \mathrm{R}$ \\
\hline 580 & IAN 1 & 022046.17 & GARVIN & & 1.9 & 1.9 & 2.0 & 34.656 & 97.436 & $5.0 R$ \\
\hline 581 & IAN 1 & 064044.52 & GARVIN & & 1.6 & & 1.8 & 34.683 & 97.451 & $5.0 R$ \\
\hline 582 & IAN 1 & 080807.63 & CARVIN & & 1.7 & & 2.1 & 34.625 & 97.537 & $5.0 \mathrm{R}$ \\
\hline 583 & IAN 1 & 094638.73 & GARVIN & & 1.5 & & 2.1 & 34.703 & 97.459 & $5.0 R$ \\
\hline 584 & $\operatorname{IAN} 3$ & 061213.41 & CARTER & & & & 2.2 & 34.197 & 97.554 & $5.0 R$ \\
\hline 585 & IAN 3 & 192434.00 & LOCAN & & & & 2.2 & 35.851 & 97.646 & $5.0 R$ \\
\hline 586 & |AN 7 & 122510.81 & POTTAWATOMIE & & 1.8 & & 1.6 & 35.274 & 96.987 & $5.0 R$ \\
\hline 587 & IAN 9 & 034926.82 & CARVIN & & 1.8 & & 2.2 & 34.726 & 97.464. & $5.0 R$ \\
\hline 588 & IAN 25 & 223307.40 & PONTOTOC & & 2.0 & & 2.0 & 34.753 & 96.720 & $5.0 R$ \\
\hline 589 & IAN 26 & 020340.65 & CARVIN & & 2.5 & 2.5 & 2.4 & 34.728 & 97.456 & $5.0 R$ \\
\hline 590 & IAN 26 & 121212.62 & GARVIN & & & & 2.2 & 34.748 & 97.472 & $5.0 R$ \\
\hline 591 & IAN 27 & 023723.85 & GRADY & & 2.2 & 2.1 & 2.3 & 35.238 & 97.858 & $5.0 R$ \\
\hline 592 & IAN 27 & 050350.38 & GRADY & & 2.5 & 2.4 & 2.4 & 35.348 & 97.878 & $5.0 R$ \\
\hline 593 & FEB 6 & 105013.75 & GARVIN & & 1.6 & & 1.8 & 34.560 & 97.446 & $5.0 R$ \\
\hline 594 & FEB 14 & 060905.41 & LATIMER & & 2.5 & 1.8 & 2.4 & 34.821 & 95.442 & $5.0 R$ \\
\hline 595 & FEB 24 & 235222.43 & GARVIN & & 2.6 & 2.3 & 2.1 & 34.626 & 97.417 & S.OR \\
\hline 596 & FEB 25 & 052620.33 & POTTAWATOMIE & & 1.7 & & 1.6 & 35.023 & 96.923 & $5.0 R$ \\
\hline 597 & MAR 13 & 094626.55 & SEMINOLE & & 1.6 & & 1.6 & 35.117 & 96.546 & $5.0 R$ \\
\hline 598 & APR 5 & 145453.00 & IOHNSTON & & 1.6 & 1.6 & 1.9 & 34.446 & 96.749 & $5.0 R$ \\
\hline 599 & APR 16 & 195208.80 & COAL & & 2.3 & 2.0 & 2.1 & 34.631 & 96.149 & $5.0 R$ \\
\hline 600 & APR 29 & 235718.65 & HUGHES & & 1.9 & & 1.6 & 35.165 & 96.003 & $5.0 \mathrm{R}$ \\
\hline 601 & APR 30 & 033610.71 & MCCLAIN & & 2.0 & & 2.2 & 34.931 & 97.360 & $5.0 R$ \\
\hline 602 & MAY 25 & 102744.82 & DELAWARE & & 2.1 & 1.4 & 2.2 & 36.230 & 94.877 & $5.0 \mathrm{R}$ \\
\hline 603 & IUN 1 & 195238.19 & LINCOLN & & 2.1 & 1.6 & 2.0 & 35.656 & 96.897 & $5.0 \mathrm{R}$ \\
\hline 604 & IUN 2 & 070811.21 & PONTOTOC & & 1.3 & & 1.1 & 34.652 & 96.651 & $5.0 R$ \\
\hline 605 & JUN 10 & 074801.66 & CHOCTAW & & 2.0 & 1.5 & 1.9 & 34.056 & 95.592 & $5.0 \mathrm{R}$ \\
\hline 606 & JUN 15 & 220054.27 & LINCOLN & & 1.3 & & 1.6 & 35.767 & 96.859 & $5.0 R$ \\
\hline 607 & JUN 30 & 195551.16 & PONTOTOC & & 2.7 & 2.1 & 2.3 & 34.706 & 96.752 & $5.0 R$ \\
\hline 608 & JUL 26 & 041723.83 & PONTOTOC & & 2.6 & 2.3 & 2.3 & 34.591 & 96.620 & $5.0 \mathrm{R}$ \\
\hline 609 & $A \cup C 4$ & 233606.82 & HASKELL & & 1.2 & & 1.7 & 35.165 & 95.296 & $5.0 R$ \\
\hline 610 & SEP 2 & 131959.04 & PONTOTOC & & 2.1 & & 2.1 & 34.684 & 96.483 & $5.0 \mathrm{R}$ \\
\hline 611 & SEP 2 & 153709.90 & MURRAY & & 1.9 & & 1.7 & 34.489 & 97.270 & $5.0 R$ \\
\hline 612 & SEP 4 & 173317.41 & COAL & & 2.9 & 2.6 & 2.5 & 34.477 & 96.503 & $5.0 \mathrm{R}$ \\
\hline 613 & SEP 16 & 010516.94 & HUGHES & & 2.5 & & 2.3 & 34.884 & 96.370 & $5.0 R$ \\
\hline 614 & SEP 23 & 054927.96 & PONTOTOC & & 2.0 & & 1.8 & 34.903 & 96.468 & $5.0 \mathrm{R}$ \\
\hline 615 & OCT 7 & 120639.12 & SEMINOLE & & 2.2 & & 2.5 & 35.257 & 96.580 & $5.0 R$ \\
\hline 616 & OCT 13 & 174244.71 & CARVIN & & 2.6 & 2.3 & 2.0 & 34.750 & 97.421 & $5.0 R$ \\
\hline 617 & OCT 18 & 211216.49 & PITTSBURC & & & & 1.1 & 34.915 & 95.909 & $5.0 R$ \\
\hline 618 & OCT 30 & 012434.80 & GARVIN & & 2.0 & & 1.8 & 34.759 & 97.409 & $5.0 R$ \\
\hline 619 & NOV 1 & 013035.93 & LE FLORE & & 1.6 & & 1.5 & 34.962 & 94.747 & $5.0 R$ \\
\hline 620 & NOV 2 & 012403.59 & HUCHES & & 1.5 & & 1.4 & 34.940 & 96.179 & $5.0 R$ \\
\hline 621 & NOV 2 & 040011.97 & JOHNSTON & & 1.9 & & 1.7 & 34.192 & 96.855 & $5.0 R$ \\
\hline 622 & NOV 5 & 133446.18 & TEXAS & & 2.8 & & 2.4 & 36.993 & 101.561 & $5.0 R$ \\
\hline 623 & NOV 26 & 205338.63 & MCCLAIN & & 2.2 & 1.8 & 1.8 & 34.957 & 97.526 & $5.0 R$ \\
\hline 624 & NOV 26 & 221656.53 & MCCLAIN & & 2.0 & 1.9 & 2.0 & 35.125 & 97.541 & $5.0 \mathrm{R}$ \\
\hline 625 & NOV 27 & 061215.90 & CRADY & & 1.6 & 1.8 & 2.0 & 35.158 & 97.671 & $5.0 \mathrm{R}$ \\
\hline 626 & DEC 4 & 175011.83 & LOGAN & & 2.7 & 2.4 & 2.2 & 35.766 & 97.328 & $5.0 R$ \\
\hline 627 & DEC 14 & 115618.54 & SEMINOLE & & 1.7 & & 1.6 & 34.959 & 96.642 & $5.0 R$ \\
\hline 628 & DEC 21 & 173258.13 & SEMINOLE & & 2.8 & 2.8 & 2.6 & 35.142 & 96.676 & $5.0 R$ \\
\hline 629 & DEC 23 & 211047.62 & GARVIN & & & & 1.6 & 34.572 & 97.204 & $5.0 R$ \\
\hline 630 & DEC 25 & 084617.38 & MCINTOSH & & 1.9 & 1.4 & 1.7 & 35.399 & 95.839 & $5.0 \mathrm{R}$ \\
\hline
\end{tabular}

"UTC refers to Coordinated Universal Time, formerly Creenwich Mean Time. The first two digits refer to the hour on a 24-hour clock. The next two digits refer to the minute, and the remaining digits are the second. To covert the local Central Standard Time, subtract 6 hours. Modified Mercalli (MM) eanthauake-intensity scale

3The hypoce
origin time. 


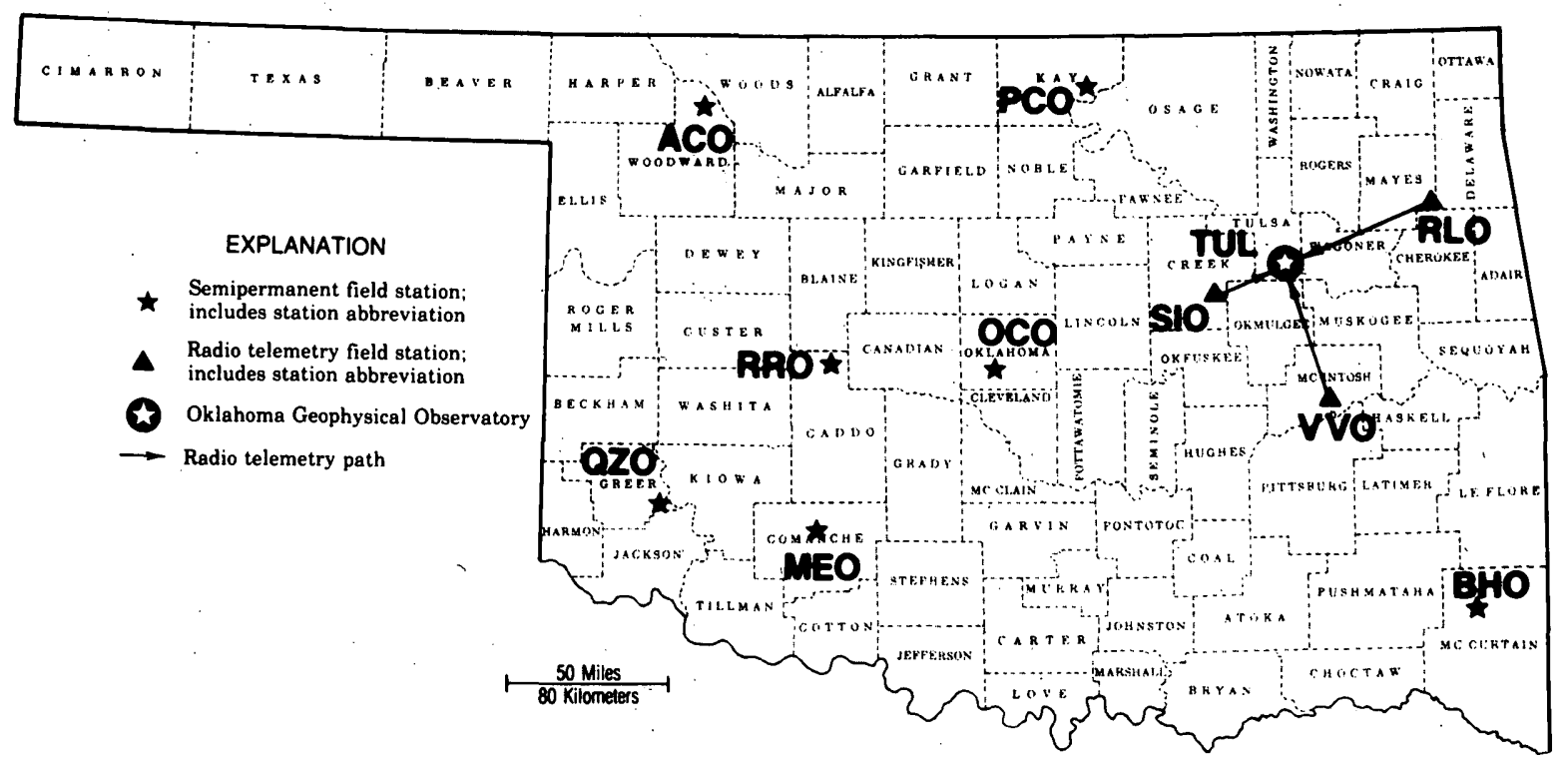

Figure 44. Active seismographs in Oklahoma for 1986.

year in a row, the Canadian County area contained no locatable earthquakes. The Arkoma basin, which includes all or parts of Pontotoc, Coal, Hughes, McIntosh, Pittsburg, Latimer, and Le Flore Counties, had many low-magnitude earthquakes. The first known earthquakes in Texas and Delaware Counties were recorded in 1986. Except for the Texas County earthquake, western Oklahoma was conspicuously quiet in 1986.

Earthquake detection and location accuracy have greatly improved since the installation of the statewide network of seismograph stations (fig. 44). The frequency of earthquakes and the possible correlation of earthquakes to specific tectonic elements in Oklahoma are being studied. It is hoped that this information will provide a more complete data base that can be used to develop numerical estimates of earthquake risk, giving the approximate frequency of the earthquakes of any given size for various regions of Oklahoma. Numerical-risk estimates could be used for better design of large-scale structures, such as dams, highrise buildings, and powerplants, as well as to provide the necessary information to evaluate insurance rates.

\section{Southeastern United States Earthquakes, 1986}

By M.S. Sibol, M.C. Chapman, and G.A. Bollinger Seismological Observatory

Virginia Polytechnic Institute and State University Blacksburg, VA 24061

Ninety-three earthquakes $(0.0 \leq \mathrm{M} \leq 3.8)$ were detected and located in the southeastern United States during 1986 (fig. 45). Of those, 12 were either felt and (or) had magnitudes greater than 3.0 (table 4). The largest shock in the

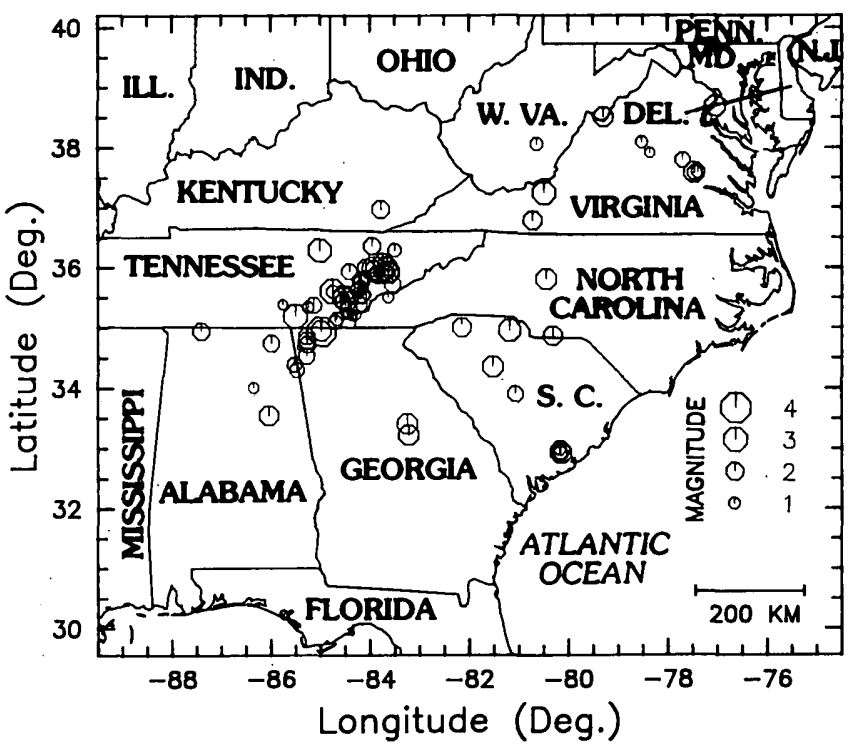

Figure 45. Southeastern United States earthquakes during 1986. Epicenters are octogons. Magnitudes are shown by the size of the epicenter symbols.

region during the year was a magnitude- 3.8 event that was felt (MMI VI) in Cohutta, Ga. The area having the largest number of earthquakes was southeastern Tennessee. Additionally, 43 reservoir-associated earthquakes $(0.0 \leq \mathrm{M} \leq 3.5)$ were detected and located within the region. Most of those earthquakes were in South Carolina and Georgia. The largest reservoir-associated event was near Lake Keowee, S. C. on January 13 (M 3.5; MMI V; table 4). Data listings of 1986 earthquakes (including earthquake locations, magnitudes, arrival times, and earthquake statistics) are presented in Seismicity of the Southeastern United States, Bulletins 18 and 19 by Sibol and others (1987a,b). A printed and (or) magnetic 


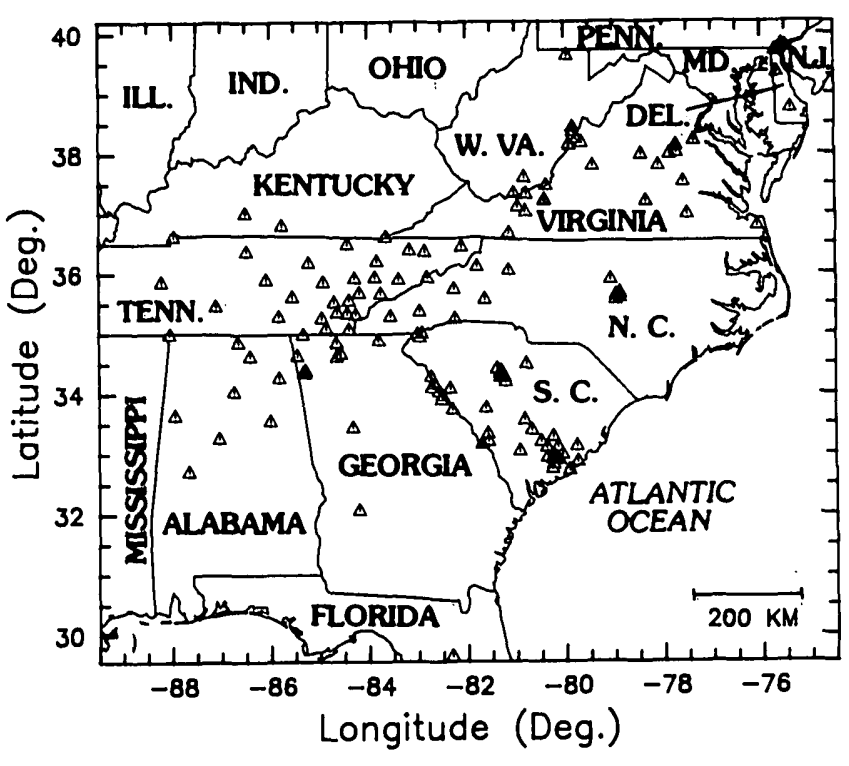

Figure 46. Southeastern United States Seismic Network (SEUSSN) stations (triangles) operating at the end of 1986.
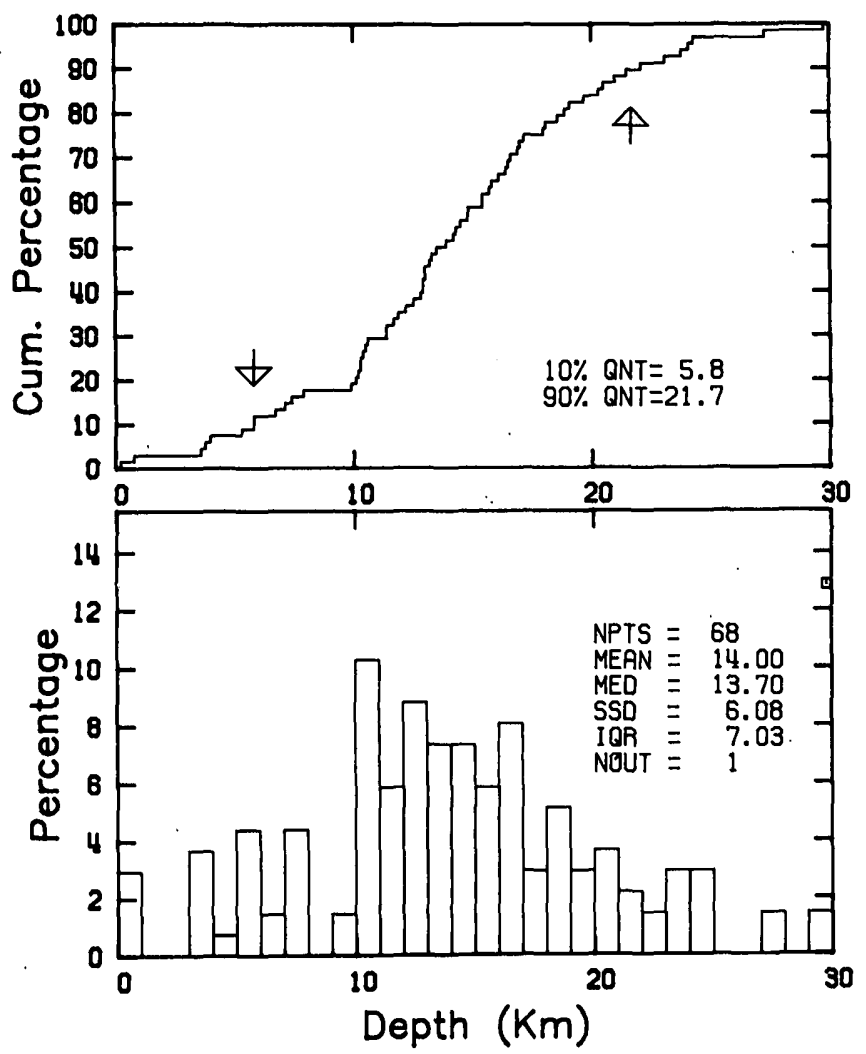

Figure 47. Distribution of focal depths of 1986 earthquakes in the southeastern United States for which standard errors of epicenter and depth were $5 \mathrm{~km}$ or less. The arrows mark the 10 percent and 90 percent quantile of the cumulative distribution.

tape listing of earthquakes from Seismicity of the Southeastem United States, Bulletins 1 through 19 (July 1977 through December 1986) is available from the authors of this Network Operations report.

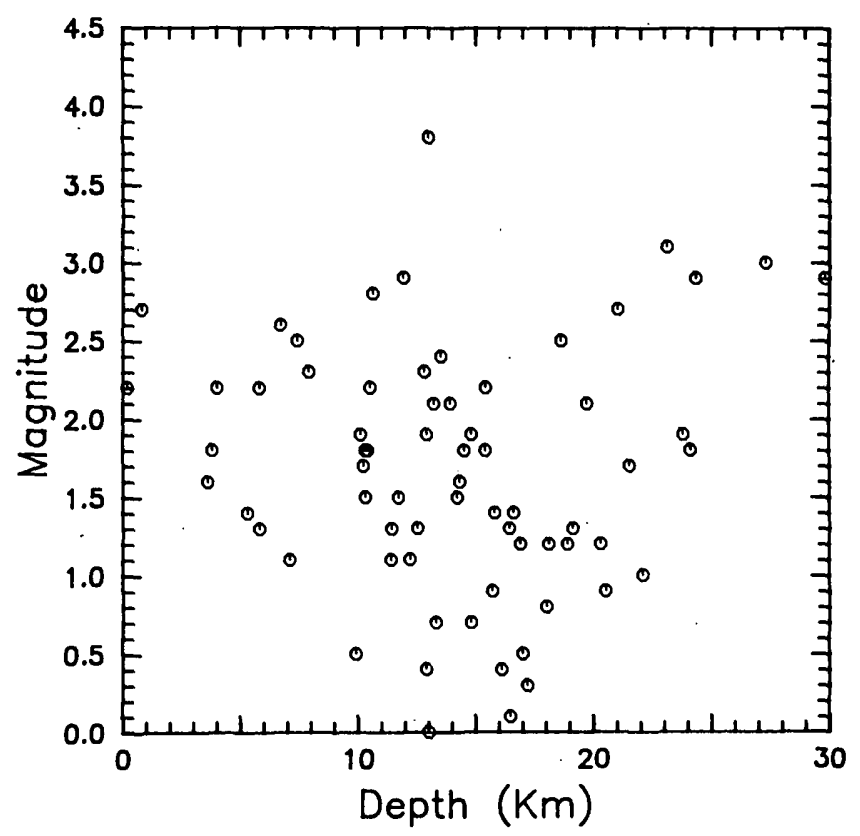

Figure 48. A plot of magnitude versus depth for 1986 earthquakes in the southeastern United States for which standard errors of epicenter and depth were $5 \mathrm{~km}$ or less.

A total of 140 seismograph stations operated in the region at the end of 1986 (fig. 46, table 5). Figure 47 shows the distribution of 1986 earthquake focal depths for events with ERH (standard error of epicenter, in kilometers) and ERZ (standard error of depth in kilometers) of 5 $\mathrm{km}$ or less. Figure 48 shows the distribution of magnitude versus depth for those same events.

\section{Washington Earthquakes, 1986}

By R.S. Ludwin, S.D. Malone, R.S. Crosson, and A.I. Qamar

University of Washington

Seattle, WA 98195

During 1986, the University of Washington operated more than 100 telemetered seismic stations in Washington and northern Oregon (fig. 49). The eastern part of the network was reconfigured in 1985 and 1986 to take advantage of low-cost telemetry provided by the Bonneville Power Administration microwave network. Station coordinates and a description of data-acquisition and data-processing procedures are given in Qamar and others (1987).

No damaging earthquakes occurred in Washington or Oregon during 1986. The largest earthquake within our network was a very shallow $\mathrm{M}_{\mathrm{D}}-3.9 \mathrm{M}_{\mathrm{D}}=$ coda-length magnitude) event near Darrington in the northern Cascade Range on February 10 at 18:05 UTC.

During 1986, 1,587 earthquakes were located within Washington and northern Oregon. Epicenters of 
Table 4. Southeastern United States earthquakes for 1986.

[UT, Universal Time; ( ${ }^{\circ} \mathrm{N}$.), degrees latitude north; ( ${ }^{\circ} \mathrm{W}$.), degrees longitude west; $(\mathrm{km})$, kilometers; $(\mathrm{mb}(\mathrm{Lg}))$ magnitude, see page 3; $\left(M_{D}\right)$, duration magnitude; $I_{0}$, epicentral; (MM), Modified Mercalli Intensity. Magnitudes are greater than 3.0 or reported felt]

\begin{tabular}{|c|c|c|c|c|c|c|c|c|}
\hline \multicolumn{2}{|c|}{$\begin{array}{c}\text { Date } \\
\text { (19866) }\end{array}$} & $\begin{array}{l}\text { Origin } \\
\text { (UT) }\end{array}$ & $\begin{array}{l}\text { Lat. } \\
\left({ }^{\circ} \text { N. }\right)\end{array}$ & $\begin{array}{l}\text { Long. } \\
\text { (००w.; }\end{array}$ & $\begin{array}{r}\text { Depth } \\
(\mathrm{Xm})\end{array}$ & $\begin{array}{l}\text { Magnitude } \\
\left(m_{\mathrm{d}}\left(\mathrm{I}_{\mathrm{g}}\right) / \mathrm{M}_{\mathrm{d}}\right)\end{array}$ & (Mo) & State \\
\hline 7 & Jan. & $01: 26$ & 35.61 & 84.76 & 23.1 & $3.1 \mathrm{~d}$ & $\mathbf{F}$ & $\mathrm{TN}$ \\
\hline 13 & Jan, * & $11: 35$ & 34.79 & 82.94 & 1.2 & $3.5 \mathrm{~b} / 3.2 \mathrm{~d}$ & $v$ & sc \\
\hline & Mar. & $23: 49$ & 32.97 & 80.17 & 5.8 & $2.2 \mathrm{~d}$ & III & sc \\
\hline $13 \%$ & $\operatorname{Max}$. & $02: 29$ & 33.23 & 83.23 & $5.0 \mathrm{~F}$ & $2.4 d$ & IV & GA \\
\hline 26 & Mar. & $16: 36$ & 37.25 & 80.49 & 11.9 & $2.9 \mathrm{~d}$ & IV & VA \\
\hline 19 & Apx. & $07: 40$ & 35.19 & 85.51 & 27.3 & $3.0 d$ & - & TN \\
\hline 113 & Ju1. & $14: 26$ & 34.94 & 84.99 & 13.0 & $3.8 b / 3.7 d$ & vI & GA \\
\hline 223 & Jul. & $22: 49$ & 32.93 & 80.17 & 5.8 & $1.3 d$ & $\mathrm{~F}$ & sc \\
\hline $17 \pi$ & Aug. & $20: 36$ & 32.91 & 80.18 & 20.2 & $2.7 d$ & $\mathbf{F}$ & sc \\
\hline $19 \pi$ & Aug. & $20: 51$ & 36.29 & 85. 02 & 29.8 & $2.9 d$ & $\mathbf{F}$ & TN \\
\hline 17 ? & sep. & $09: 33$ & 32.93 & 80.16 & 6.7 & $2.6 \mathrm{~d}$ & IV & sc \\
\hline 3 & Dec. & $09: 44$ & 37.58 & 77.46 & $1.6 \mathrm{~F}$ & $1.5 d$ & IV & VA \\
\hline 10 & Dec. & $11: 30$ & 37.59 & 77.47 & $1.2 \mathrm{~F}$ & $2.5 b / 2.2 d$ & $\mathrm{v}$ & $\mathrm{vA}_{\mathrm{A}}$ \\
\hline
\end{tabular}

Table 5. Southestern United States Seismic Network earthquake statistics, 1986.

[M, magnitude; ERH, standard error of epicenter; ERZ, standard error of depth; $\mathrm{km}$, kilometers; $(\mathrm{mb}(\mathrm{Lg}))$ magnitude, see page 3; MD, duration magnitude; MMI, Modified Mercalli Intensity]

\begin{tabular}{|c|c|c|c|c|}
\hline \multirow{3}{*}{ Number of earthquakes with... } & \multicolumn{2}{|c|}{1986} & \multicolumn{2}{|c|}{ JuL $1972-\operatorname{Dec} 1986$} \\
\hline & Tectonic & Reservoir & Tectonic & Reservois \\
\hline & 93 & 43 & 567 & 620 \\
\hline (implied rate per year)........ & 93 & 43 & 59.7 & 65.3 \\
\hline$M \geq 2.0 \ldots \ldots \ldots \ldots \ldots \ldots$ & 31 & 11 & 255 & 165 \\
\hline (Implied rate per year)....... & 31 & 11 & 26.8 & 17.4 \\
\hline$M \geq 3.0 \ldots \ldots \ldots \ldots \ldots \ldots \ldots$ & 3 & 1 & 45 & 4 \\
\hline (implied rate per year) $\ldots \ldots$ & 3 & 1 & 4.7 & 0.4 \\
\hline$M \geq 4.0 \ldots \ldots \ldots \ldots \ldots \ldots \ldots \ldots \ldots \ldots$ & 1 & 0 & 3 & 0 \\
\hline (implied rate per year) ....... & 1 & 0 & 0.3 & 0.0 \\
\hline 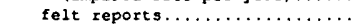 & 12 & 1 & 82 & 4 \\
\hline (implied rate per year\}...... & 12 & 1 & 8.6 & 0.04 \\
\hline known Dmin $s 10 \mathrm{~km} \ldots \ldots \ldots \ldots$ & 23 & 24 & 112 & 72 \\
\hline known ERH $55 \mathrm{~km}, \ldots \ldots \ldots \ldots$ & 90 & 42 & 446 & 220 \\
\hline known ERZ $\leq 5 \mathrm{~km}, \ldots \ldots \ldots \ldots$ & 69 & 19 & 391 & 91 \\
\hline Maximum magnitude $\ldots \ldots \ldots \ldots \ldots \ldots$ & 3.8 & 3.5 & 5.2 & 3.7 \\
\hline 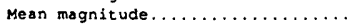 & 1.7 & 1.6 & 1.8 & 1.4 \\
\hline \multicolumn{5}{|c|}{ Number of seismographs operating in $1986 \ldots \ldots \ldots: 140$} \\
\hline Largest earthquake in 1986 : & \multicolumn{4}{|c|}{$\begin{array}{r}11 \text { July 1986; } 14: 26-\text { Conutta, GA } \\
m_{b}\left(L_{g}\right)=3.8, M_{d}=3.7, \text { MMI }=V I\end{array}$} \\
\hline $\begin{array}{l}\text { Lorgest earthquake for } \\
\text { Ju1 } 1977 \text { - Dec 1986: }\end{array}$ & \multicolumn{4}{|c|}{$\begin{aligned} & 20 \text { July } 1980 ; 18: 52 \text { - Sharpsburg, } K Y \\
& \mathrm{~m}_{\mathrm{b}}\left(1_{\mathrm{g}}\right)=5.2, \text { MMI }=\text { VII }\end{aligned}$} \\
\hline
\end{tabular}

earthquakes which were reported felt or had magnitudes $\left(M_{D}\right) \geq 2.7$ are shown in figure 50. During 1986, 21 felt earthquakes were located in the area of figure 50, and 55 additional events had magnitudes $\geq M_{D} 2.7$. Forty-two of these unfelt earthquakes of magnitude $\geq 2.7$ were located at Mount St. Helens during the two nonviolent eruptive episodes in April-May and October. Of all earthquakes located by the University of Washington network in 1986, 59 percent were near Mount St. Helens, reflecting both a greater station density and a higher level of activity there. In 1986, 19 percent of the earthquakes were located west of the Cascade Range outside of the Mount St. Helens area, 12 percent occurred within the Cascade Range and the remaining 11 percent occurred east of the Cascade Range.

A small earthquake swarm began on February 10 about $15 \mathrm{~km}$ south of Concrete, Wash., and included two felt earthquakes of $M_{D} 3.1$ and 3.9. The larger one was felt at Concrete, Mount Vernon, Sedro-Woolley, Cape Horn, Clear Lake, and Day Creek. Eight additional unfelt earthquakes $>1.0$ were located nearby during February, and all the earthquakes were located at depths $<5 \mathrm{~km}$.

Seven of the earthquakes felt in 1986 occurred in a swarm about $10 \mathrm{~km}$ west of Darrington, Wash., between March 27 and 31. The magnitudes ranged from 2.1 to 3.6, and depths were shallower than $4 \mathrm{~km}$. The $\mathrm{M}_{\mathrm{D}-3.6}$ earthquake occurred on March 28 and was felt in Darrington, Concrete, and Duvall. During March and April, 18 additional unfelt earthquakes larger than magnitude 1.0 were located in this swarm. In 1985, a single felt earthquake was located in the same area. Seismicity in the vicinity of Darrington is described in more detail by Zollweg and Johnson (1989).

Several more felt earthquakes were located in northwestern Washington. An $\mathrm{M}_{\mathrm{D}}-3.0$ earthquake was located at a depth of about $18 \mathrm{~km}$ near Bellingham on April 20 and was reported felt. On July 8 , a deep earthquake (depth $\sim 63$ $\mathrm{km}$ ) of $\mathrm{M}_{\mathrm{D}} 3.5$ occurred beneath the Saratoga passage between Whidbey and Camano Islands in the Puget basin. It was felt on both Camano and Whidbey Islands, and in Mount Vernon and Marysville. Three earthquakes at depths less than $10 \mathrm{~km}$ were felt on September 16, one near the site of the March activity and the other two about 25 $\mathrm{km}$ southeast of Darrington. These earthquakes had $\mathrm{M}_{\mathrm{D}}$ 1.6, 2.8, and 2.4, respectively. On September 26 and 29 two very shallow (depths $<1 \mathrm{~km}$ ) earthquakes of magnitudes 2.4 and 2.2 were reported felt at Sedro-Woolley.

In southwestern Washington, a $\mathrm{M}_{\mathrm{D}}-3.1$ earthquake was felt in Cougar and Vancouver, Wash., on March 11. It was located at a depth of about $15 \mathrm{~km}$. Earlier on the same day, a smaller earthquake of $M_{D} 2.9$ was reported felt in the Puget basin at Gig Harbor near Tacoma and located at a depth of less than $10 \mathrm{~km}$. Earthquakes of $M_{D} 3.3$ ( $14-\mathrm{km}$ depth) and 2.7 (depth $\sim 9 \mathrm{~km}$ ) were located near Lake Chelan on April 8 and June 11, respectively. On August 28 a shallow earthquake (depth $<10 \mathrm{~km}$ ) of $\mathrm{M}_{\mathrm{D}} 2.7$ was felt in southwestern Washington.

Mount St. Helens had an eruptive phase during April and May of 1986. Degassing events, both steam emissions and ash plumes, began on April 17 and continued almost daily into May. Seismic activity increased rapidly from a background level prior to May 3 to a high level of activity on May 7 and 8, then began to decrease, again reaching background level on May 19th. Eleven earthquakes of magnitude $\geq 2.7$ were located at very shallow depths (less than $2 \mathrm{~km}$ ) beneath the crater during April and May. The largest earthquake in this sequence was a $\mathrm{MD}_{\mathrm{D}} \mathbf{3 . 2}$ event of May 8th. 


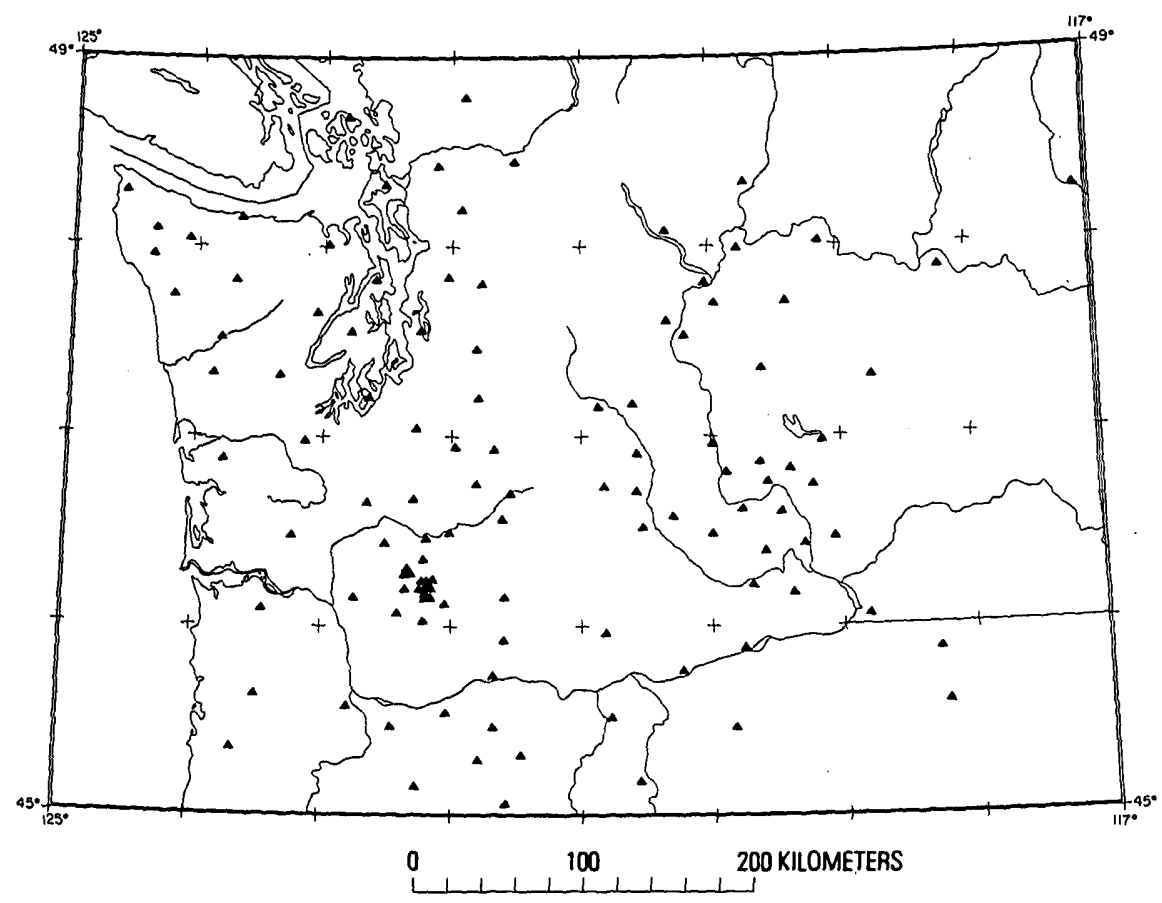

Figure 49. Seismograph stations (triangles) in Washington and Oregon operated by the University of Washington during 1986.

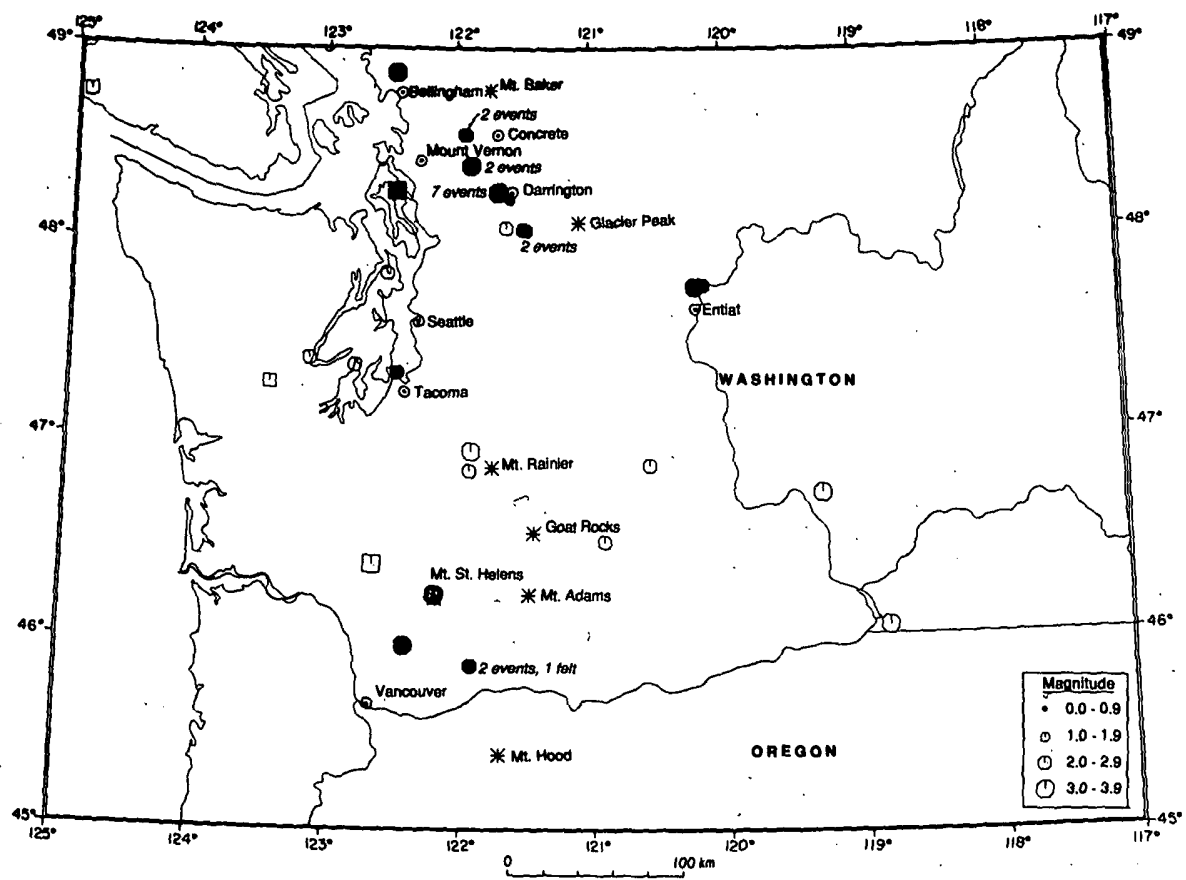

Figure 50. Felt earthquakes in Washington and northern Oregon during 1986, plus earthquakes with coda-length magnitudes greater than or equal to 2.7 which were not felt. Earthquakes reported felt in Washington and northern Oregon during 1986 are shown as solid symbols. Open symbols show all other earthquakes having magnitudes greater than or equal to 2.7. Octagon symbols represent events with depths shallower than $30 \mathrm{~km}$, and square symbols indicate event depths of $30 \mathrm{~km}$ or more. 
The second eruption of Mount St. Helens took place during October. Seismicity began to rise above background levels during the weekend of Oct. 4-5 but fell to a background level by Oct. 7. During the weekend of Oct. 11-12, the seismicity began to increase again, and by Oct. 19, seismicity was at a high level. This eruption was accompanied by the greatest release of seismic energy since the eruption of May 18, 1980. Thirty-one earthquakes $2 \mathrm{M}_{\mathrm{D}} 2.7$ were located at depths $<2 \mathrm{~km}$ directly beneath the crater. The three largest earthquakes of this sequence $\left(M_{D}\right.$ 3.2) occurred on Oct. 21. A discussion of the magnitude and distribution of earthquakes during eruptive episodes at Mount St. Helens since 1980 is given in Qamar and others (1987). During the afternoon (PST, Pacific Standard Time) of Oct. 21, seismicity decreased and tremor began, which continued for several hours. At first, tremor amplitude increased and then decreased until, shortly after midnight (PST), only large, low-frequency earthquakes were occurring. The U.S. Geological Survey confirmed the extrusion of a new lobe of lava at the top of the lava dome early in the morning (PST) of Oct. 22. Seismicity returned to background levels by Oct. 27 . 


\section{PRINCIPAL EARTHQUAKES OF THE WORLD}

Table 6. Principal earthquakes of the World during 1986

This table includes all earthquakes of magnitude 6.8 or larger, those of smaller magnitude that caused loss of lives and significant damages, and events of unusual interest. The primary source for this table is the National Earthquake Information Center publication Preliminary Determination of Epicenters, Monthly Listing.

[(UTC), Coordinated Universal Time; (KM), kilometers; $\left(m_{b}\right)$, body-wave magnitude; $\left(M_{L}\right)$, local magnitude; (Ms), surface-wave magnitude; (AT), Athens Observatory, Greece; (BK), University of California, Berkeley; (GS), U.S. Geological Survey, Golden, Colo.; (PS), California Institute of Technology, Pasadena; (TG), Titograd Observatory, Yugoslavia]

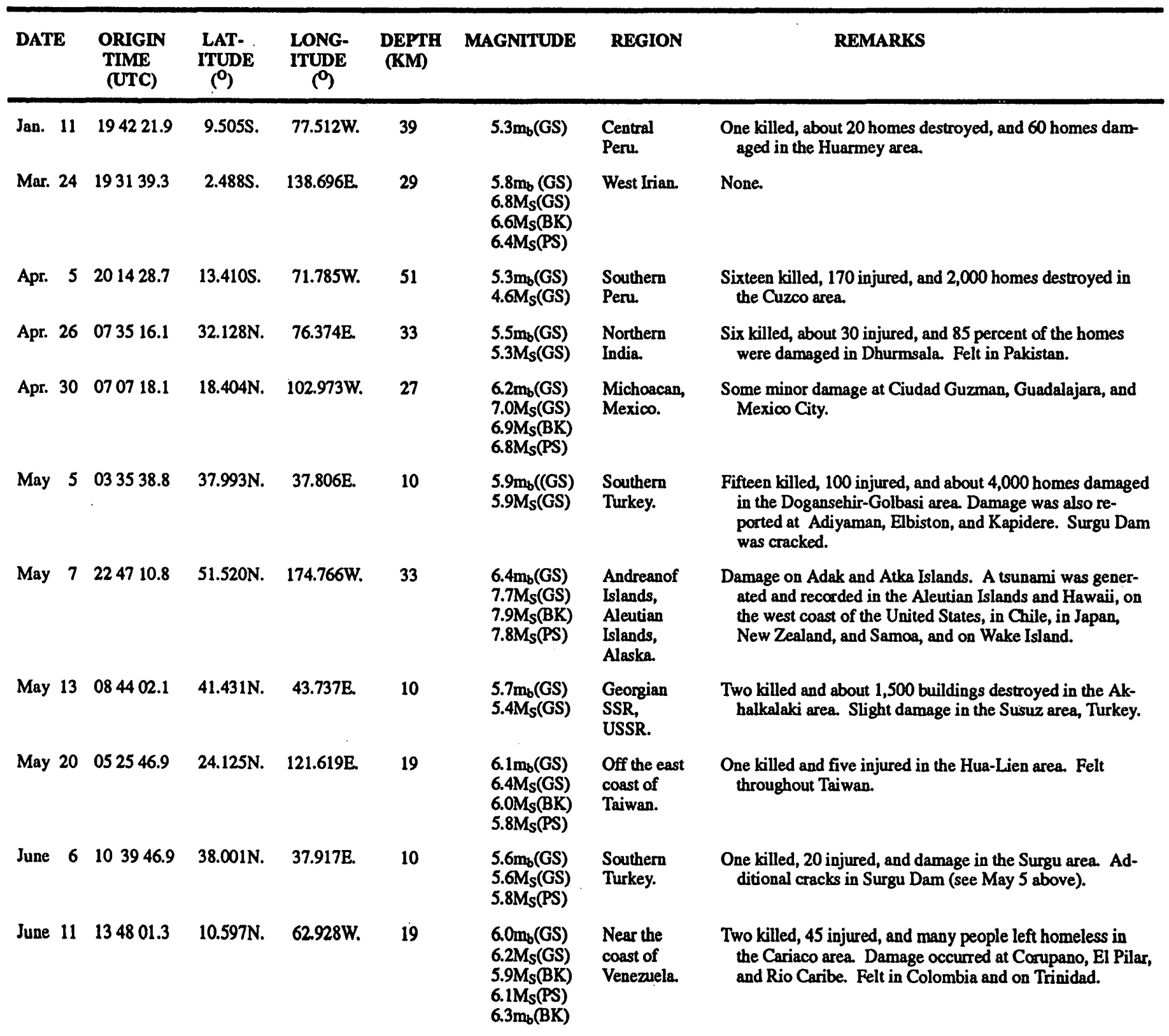


Table 6. Principal earthquakes of the World during 1986_-Continued

\begin{tabular}{|c|c|c|c|c|c|c|c|}
\hline DATE & $\begin{array}{l}\text { ORIGIN } \\
\text { TIME } \\
\text { (UTC) }\end{array}$ & $\begin{array}{l}\text { LAT- } \\
\text { ITUDE } \\
(\mathbf{0})\end{array}$ & $\begin{array}{l}\text { LONG- } \\
\text { ITUDE } \\
\left({ }^{\circ}\right)\end{array}$ & $\begin{array}{l}\text { DEPTH } \\
\text { (KM) }\end{array}$ & MAGNITUDE & REGION & REMARKS \\
\hline June 24 & 031130.9 & 4.448S. & 143.943E. & 102 & $\begin{array}{l}6.6 \mathrm{~m}_{\mathrm{b}}(\mathrm{GS}) \\
7.1 \mathrm{M}_{\mathrm{s}}(\mathrm{GS}) \\
6.9 \mathrm{~m}_{\mathrm{b}}(\mathrm{PS}) \\
7.4 \mathrm{M}_{\mathrm{S}}(\mathrm{BK})\end{array}$ & $\begin{array}{l}\text { Papua, } \\
\text { New Guinea. }\end{array}$ & $\begin{array}{l}\text { Damage and landslides occurred throughout the Papua, } \\
\text { New Guinea, highlands. Submarine cables from Madang } \\
\text { to Guam and Cairns, Australia, were damaged. Damage } \\
\text { was estimated at U.S. } \$ 500,000 \text {. }\end{array}$ \\
\hline July 12 & 075426.8 & $29.962 \mathrm{~N}$ & $51.582 \mathrm{E}$ & 10 & $\begin{array}{l}5.7 \mathrm{~m}_{b}(\mathrm{GS}) \\
5.6 \mathrm{MS}(\mathrm{GS})\end{array}$ & $\begin{array}{l}\text { Southern } \\
\text { Iran. }\end{array}$ & $\begin{array}{l}\text { One killed, } 4 \text { injured, and about } 300 \text { homes damaged in the } \\
\text { Mamasani area. }\end{array}$ \\
\hline July 18 & 172238.2 & $10.770 \mathrm{~N}$ & $69.428 W$. & 7 & $\begin{array}{l}5.9 \mathrm{~m}_{b}(\mathrm{GS}) \\
4.9 \mathrm{M}_{\mathrm{s}}(\mathrm{GS})\end{array}$ & $\begin{array}{l}\text { Northwestern } \\
\text { Venezuela. }\end{array}$ & $\begin{array}{l}\text { One person died from a heart attack, and } 30 \text { homes were } \\
\text { damaged in the Churuguara area. }\end{array}$ \\
\hline Aug. 14 & 193913.6 & $1.795 \mathrm{~N}$. & $126.519 \mathrm{E}$ & 33 & $\begin{array}{l}6.6 \mathrm{~m}_{b}(\mathrm{GS}) \\
7.2 \mathrm{M}_{\mathrm{S}}(\mathrm{GS}) \\
7.4 \mathrm{M}_{\mathrm{S}}(\mathrm{BK})\end{array}$ & $\begin{array}{l}\text { Molucca } \\
\text { Passage. }\end{array}$ & $\begin{array}{l}\text { Felt in northern Sulawesi and on Mindanao, Phillippine } \\
\text { Islands. }\end{array}$ \\
\hline Aug. 30 & 212835.4 & $45.547 \mathrm{~N}$ & $26.316 \mathrm{E}$ & 132 & $\begin{array}{l}6.4 \mathrm{~m}_{b}(\mathrm{GS}) \\
6.9 \mathrm{M}_{S}(\mathrm{GS}) \\
5.8 \mathrm{~m}_{\mathrm{b}}(\mathrm{BK})\end{array}$ & $\begin{array}{l}\text { Eastern } \\
\text { Romania. }\end{array}$ & $\begin{array}{l}\text { Two killed, } 558 \text { injured, and about } 55,000 \text { homes damaged } \\
\text { in the Kishinev and Kagul areas, USSR. Substantial } \\
\text { damage in the Focsani-Birlad and Bucharest areas, Ro- } \\
\text { mania. Felt in Bulgaria, Greece, Hungary, Italy, Poland, } \\
\text { and Turkey. }\end{array}$ \\
\hline Sept. 13 & 172431.4 & $37.014 \mathrm{~N}$. & $22.176 \mathrm{E}$ & 11 & $\begin{array}{l}6.0 \mathrm{~m}_{\mathrm{b}}(\mathrm{GS}) \\
5.8 \mathrm{M}_{\mathrm{S}}(\mathrm{GS}) \\
5.7 \mathrm{M}_{\mathrm{S}}(\mathrm{BK}) \\
5.7 \mathrm{M}_{\mathrm{L}}(\mathrm{AT})\end{array}$ & $\begin{array}{l}\text { Peloponnesus, } \\
\text { Greece. }\end{array}$ & $\begin{array}{l}\text { Twenty killed, about } 300 \text { injured, and 1,500 buildings were } \\
\text { damaged or destroyed in the Kalamai area. }\end{array}$ \\
\hline Sept. 16 & 182017.7 & $19.376 \mathrm{~N}$ & 146.301E. & 48 & $\begin{array}{l}6.5 \mathrm{~m}_{\mathrm{b}}(\mathrm{GS}) \\
6.7 \mathrm{Ms}(\mathrm{GS}) \\
6.8 \mathrm{M}_{\mathrm{S}}(\mathrm{BK})\end{array}$ & $\begin{array}{l}\text { Mariana } \\
\text { Islands } \\
\text { region. }\end{array}$ & None. \\
\hline Oct. 10 & 174924.1 & $13.827 \mathrm{~N}$ & $89.118 W$. & 7 & $\begin{array}{l}5.0 \mathrm{~m}_{\mathrm{b}}(\mathrm{GS}) \\
5.4 \mathrm{M}_{\mathrm{s}}(\mathrm{GS}) \\
5.5 \mathrm{M}_{\mathrm{S}}(\mathrm{BK})\end{array}$ & El Salvador. & $\begin{array}{l}\text { At least } 1,000 \text { killed, } 10,000 \text { injured, } 200,000 \text { homeless, and } \\
\text { severe damage in the San Salvador area. Some damage } \\
\text { occurred at Tegucigalpa, Honduras. Felt strongly in } \\
\text { Guatemala. }\end{array}$ \\
\hline Oct. 20 & 064609.9 & $28.117 S$. & $176.367 \mathrm{~W}$. & 29 & $\begin{array}{l}6.6 \mathrm{~m}_{b}(\mathrm{GS}) \\
8.1 \mathrm{M}_{S}(\mathrm{GS}) \\
8.3 \mathrm{M}_{\mathrm{S}}(\mathrm{BK})\end{array}$ & $\begin{array}{l}\text { Northern } \\
\text { Kermadec } \\
\text { Islands. }\end{array}$ & $\begin{array}{l}\text { Felt on Raoul Island and North Island, New Zealand. A } \\
\text { small tsunami was recorded in the Hawaiian Islands, } \\
\text { Papeete, Tahiti, and Samoa Islands. }\end{array}$ \\
\hline Oct. 30 & 012854.5 & $21.702 S$ & 176.616W. & 188 & $\begin{array}{l}6.4 \mathrm{~m}_{b}(\mathrm{GS}) \\
6.4 \mathrm{~m}_{\mathrm{b}}(\mathrm{BK}) \\
6.8 \mathrm{~m}_{\mathrm{b}}(\mathrm{PS})\end{array}$ & $\begin{array}{l}\text { Tonga } \\
\text { Islands. }\end{array}$ & May be two events 5 seconds apart. \\
\hline Nov. 14 & 212010.5 & $23.901 \mathrm{~N}$ & $121.574 \mathrm{E}$. & 34 & $\begin{array}{l}6.3 \mathrm{~m}_{\mathrm{b}}(\mathrm{GS}) \\
7.8 \mathrm{M}(\mathrm{GS}) \\
7.5 \mathrm{M} \text { (BK) }\end{array}$ & $\begin{array}{l}\text { Off the east } \\
\text { coast of } \\
\text { Taiwan. }\end{array}$ & $\begin{array}{l}\text { Fifteen killed, } 44 \text { injured, and damage in the Taipei area. } \\
\text { Undersea cables from Taiwan to Guam and Okinawa } \\
\text { were damaged. Felt in the Ryukyu Islands and on Luzon, } \\
\text { Phillippine Islands. }\end{array}$ \\
\hline Dec. 7 & 141709.5 & $43.274 \mathrm{~N}$ & $25.912 \mathrm{E}$ & 21 & $\begin{array}{l}5.2 \mathrm{~m}_{\mathrm{b}}(\mathrm{GS}) \\
5.6 \mathrm{M}_{\mathrm{S}}(\mathrm{GS}) \\
5.5 \mathrm{M}_{\mathrm{L}}(\mathrm{TG})\end{array}$ & $\begin{array}{l}\text { Northern } \\
\text { Bulgaria. }\end{array}$ & $\begin{array}{l}\text { Three killed, } 60 \text { injured, and damage in the Turyovishte- } \\
\text { Veliko Turnovo area. Felt in Turkey and Yugoslovia. }\end{array}$ \\
\hline
\end{tabular}




\section{STRONG-MOTION ACCELEROGRAPH DATA}

By Ronald L. Porcella and Josephine C. Switzer

U.S. Geological Survey

345 Middlefield Road

Menlo Park, CA 94025

\section{Introduction}

The current United States strong-motion instrumentation program is administered by the U.S. Geological Survey (USGS) in cooperation with both private industry and educational institutions, as well as numerous Federal, State, and local agencies and organizations including: the Army Corps of Engineers, the Veterans Administration, the Metropolitan Water District of Southern California, and others. The objectives of the program are to record strong ground motions and the response of representative types of engineered structures during potentially damaging earthquakes, and to disseminate processed data and information about the records, sites, and structures to external users in earthquake engineering research and design practice and engineering seismology. The dissemination of this information and data is achieved in various ways.

Preliminary earthquake reports are published after all earthquakes that produce significant strong-motion recordings, and a summary of recent accelerograph records is presented on an annual basis in the Catalogue of U.S. Geological Survey Strong Motion Records, a USGS Circular. These preliminary reports include a brief description of the earthquake and strong-motion recording station, the results of routine scalings of those records that contain peak accelerations greater than $0.05 \mathrm{~g}$ (gravity), and photographic reproductions of the more significant accelerograms. The catalogue contains a table of all USGS strong-motion recordings recovered during the calendar year, and includes earthquake, station, and record information.

Strong-motion event and strong-motion data reports are periodically published as USGS open-file reports and include the results of digitization and routine analyses of strong-motion accelerograms that contain peak accelerations of significant amplitude or that are related to a specific event, particular strong-motion station, or geographic group of stations. Although maximum acceleration is not directly related to frequency content or duration of strong motion, the peak acceleration can be readily obtained from an accelerogram, and thus the value is commonly used as a general indicator of the potential significance of the record.

The "Strong-Motion Accelerograph Station List" is periodically published as a USGS open-file report and includes information on all the accelerograph stations in the Western Hemisphere known to the USGS. Because of the ever-changing nature of this information, it is impossible to have a complete list of all of the stations in existence at any one time. Rather, the list is intended to provide that community of persons interested in strong-motion programs with a reasonably complete indication of the current status of the various strong-motion networks. Information presented in this list includes the station name and geographic coordinates, site characteristics, type and size of structure, location of instruments, and the primary sources of data. The current list contains information on about 1,350 stations in the United States, Canada, the Caribbean, and throughout Central and South America (Switzer and others, 1981).

Table 7 is a summary of United States accelerograph records recovered during 1986. 
Table 7. Summary of United States accelerograph records recovered during 1986

[Station owners: ACOE, U.S. Army Corps of Engineers; BECH, Bechtel Power Corporation; CDOT, California Department of Transportation; CDWR, California Department of Water Resources; CLA, City of Los Angeles; MWD, Metropolitan Water District of Southern California; SDGE, San Diego Gas and Electric Company; UCB, University of California at Berkeley; USBR, U.S. Bureau of Reclamation; USGS, U.S. Geological Survey; VA, Veterans Administration. Instrument trigger time in seconds after the minute (or the following minute) listed in earthquake column. S-minus trigger denotes $S$-wave-arrival-minus-trigger-time $(S-t)$ or $S$-wave-minus- $P$-wave-arrival time interval. Direction is of case acceleration for upward trace deflection on accelerogram; horizontal components are listed as azimuth and vertical components as "up" or "down." Maximum amplitude is peak acceleration recorded at ground level on one vertical and two horizontal (orthogonal) components unless otherwise noted. Duration is interval between first and last peaks of acceleration greater than 0.10 g. G.m.t., Greenwich Mean time, is equivalent to UTC, Coordinated Universal time; $\left({ }^{\circ}\right)$, degrees; $N$, north; $W$, west; (s), seconds; (az), azimuth; $(\mathrm{g})$, local acceleration of gravity; Dashes (---), indicate acceleration is less than $0.10 \mathrm{~g}$. Footnotes are at end of table.]

\begin{tabular}{|c|c|c|c|c|c|c|c|}
\hline Earthquake & $\begin{array}{l}\text { Station name } \\
\text { (Owner) }\end{array}$ & $\begin{array}{l}\text { Station } \\
\text { location } \\
\left(^{*}\right)\end{array}$ & $\begin{array}{l}\text { Trigger } \\
\text { time }\end{array}$ & $\begin{array}{l}S \text {-minus } \\
\text { trigger } \\
(\mathrm{s})\end{array}$ & $\begin{array}{l}\text { Direction } \\
\quad(a z)\end{array}$ & $\begin{array}{l}\text { Maximum } \\
\text { amplitude } \\
\quad(g)\end{array}$ & $\begin{array}{l}\text { Duration } \\
\text { (s) }\end{array}$ \\
\hline $\begin{array}{l}4 \text { December } 1985- \\
14 \text { January } 1986 \\
\text { Central Calif. } \\
\text { Epicenter and } \\
\text { magnitude unknown }\end{array}$ & $\begin{array}{l}\text { Bear Valley Station } 10 \\
\text { Webb Residence } \\
\text { (USGS) }\end{array}$ & $\begin{array}{r}36.532 \mathrm{~N} \\
121.143 \mathrm{~W}\end{array}$ & (2) & 0.9 & & (1) & \\
\hline $\begin{array}{l}14 \text { January } 1986 \\
0307: 54.9 \text { G.m.t. } \\
\text { Central Calif. } \\
36.563 \mathrm{~N}, 121.203 \mathrm{~W} \\
\text { Magnitude } 3.3 \mathrm{ML}\end{array}$ & $\begin{array}{l}\text { Bear Valley Station } 10 \\
\text { Webb Residence } \\
\text { (USGS) }\end{array}$ & $\begin{array}{r}36.532 \mathrm{~N} \\
121.143 \mathrm{~W}\end{array}$ & 56.7 & 1.4 & & (1) & \\
\hline \multirow{8}{*}{$\begin{array}{l}14 \text { January } 1986 \\
0309: 36.3 \text { G.m.t. } \\
\text { Central Cal if. } \\
36.572 \mathrm{~N}, 121.205 \mathrm{~W} \\
\text { Magnitude } 4.7 \mathrm{ML}\end{array}$} & $\begin{array}{l}\text { Bear Valley Station } 1 \\
\text { CDF Fire Station } \\
\text { (USGS) }\end{array}$ & $\begin{array}{r}36.573 \mathrm{~N} \\
121.184 \mathrm{~W}\end{array}$ & 38.1 & 0.8 & $\begin{array}{l}310 \\
\text { Up } \\
220\end{array}$ & $\begin{array}{l}.27 \\
.05 \\
.19\end{array}$ & $\begin{array}{l}0.1 \\
--- \\
0.2\end{array}$ \\
\hline & $\begin{array}{l}\text { Bear Valley Station } 2 \\
\text { Stone Canyon West } \\
\text { (USGS) }\end{array}$ & $\begin{array}{r}36.636 \mathrm{~N} \\
121.234 \mathrm{~W}\end{array}$ & 39.3 & (2) & & (1) & \\
\hline & $\begin{array}{l}\text { Bear Valley Station } 5 \\
\text { Callens Ranch } \\
\text { (USGS) }\end{array}$ & $\begin{array}{r}36.673 \mathrm{~N} \\
121.195 \mathrm{~W}\end{array}$ & 39.7 & 2.7 & $\begin{array}{l}310 \\
\text { Up } \\
220\end{array}$ & $\begin{array}{l}.06 \\
.04 \\
.10\end{array}$ & $\begin{array}{l}--- \\
1 \text { peak }\end{array}$ \\
\hline & $\begin{array}{l}\text { Bear Valley Station } 6 \\
\text { James Ranch } \\
\text { (USGS) }\end{array}$ & $\begin{array}{r}36.504 \mathrm{~N} \\
121.101 \mathrm{~W}\end{array}$ & 41.3 & (2) & & (1) & \\
\hline & $\begin{array}{l}\text { Bear Valley Station } 7 \\
\text { Pinnacles } \\
\text { (USGS) }\end{array}$ & $\begin{array}{r}36.483 \mathrm{~N} \\
121.184 \mathrm{~W}\end{array}$ & 38.8 & 1.6 & $\begin{array}{l}310 \\
\text { Up } \\
220\end{array}$ & $\begin{array}{l}.05 \\
.05 \\
.08\end{array}$ & $\begin{array}{l}--- \\
---\end{array}$ \\
\hline & $\begin{array}{l}\text { Bear Valley Station } 10 \\
\text { Webb Residence } \\
\text { (USGS) }\end{array}$ & $\begin{array}{r}36.532 \mathrm{~N} \\
121.143 \mathrm{~W}\end{array}$ & 38.2 & 2.0 & $\begin{array}{l}310 \\
\text { Up } \\
220\end{array}$ & $\begin{array}{l}.22 \\
.12 \\
.22\end{array}$ & $\begin{array}{l}1 \text { peak } \\
0.4 \\
0.5\end{array}$ \\
\hline & $\begin{array}{l}\text { Bear Valley Station } 12 \\
\text { Williams Ranch } \\
\text { (USGS) }\end{array}$ & $\begin{array}{r}36.658 \mathrm{~N} \\
121.249 \mathrm{~W}\end{array}$ & 39.7 & 2.1 & $\begin{array}{l}310 \\
\text { Up } \\
220\end{array}$ & $\begin{array}{l}.09 \\
.05 \\
.14\end{array}$ & $\begin{array}{l}--- \\
--- \\
\cdots\end{array}$ \\
\hline & $\begin{array}{l}\text { Bear Valley Station } 14 \\
\text { Upper Butts Ranch } \\
\text { (USGS) }\end{array}$ & $\begin{array}{r}36.569 \mathrm{~N} \\
121.043 \mathrm{~W}\end{array}$ & 40.1 & (2) & & (1) & \\
\hline
\end{tabular}


Table 7. Summary of United States accelerograph records recovered during 1986-Continued

\begin{tabular}{|c|c|c|c|c|c|c|c|}
\hline Earthquake & $\begin{array}{l}\text { Station name } \\
\text { (Owner) }\end{array}$ & $\begin{array}{l}\text { Station } \\
\text { location } \\
\quad(*)\end{array}$ & $\begin{array}{l}\text { Trigger } \\
\text { time }\end{array}$ & $\begin{array}{l}S \text {-minus } \\
\text { trigger } \\
(\mathrm{s})\end{array}$ & $\begin{array}{l}\text { Direction } \\
\quad(a z)\end{array}$ & $\begin{array}{l}\text { Maximum } \\
\text { amplitude } \\
\quad(g)\end{array}$ & $\begin{array}{l}\text { Duration } \\
\text { (s) }\end{array}$ \\
\hline \multirow{3}{*}{$\begin{array}{l}14 \text { January } 1986 \\
0535: 47.9 \text { G.m.t. } \\
\text { Central Cal if. } \\
36.568 \mathrm{~N}, 121.202 \mathrm{~W} \\
\text { Magnitude } 2.9 \mathrm{ML}\end{array}$} & $\begin{array}{l}\text { Bear Valley Station } 1 \\
\text { CDF Fire Station } \\
\text { (USGS) }\end{array}$ & $\begin{array}{r}.36 .573 \mathrm{~N} \\
121.184 \mathrm{~W}\end{array}$ & 50.3 & (2) & & (1) & \\
\hline & $\begin{array}{l}\text { Bear Valley Station } 7 \\
\text { Pinnacles } \\
\text { (USGS) }\end{array}$ & $\begin{array}{r}36.483 \mathrm{~N} \\
121.184 \mathrm{~W}\end{array}$ & 49.9 & (2) & & (1) & \\
\hline & $\begin{array}{l}\text { Bear Valley Station } 10 \\
\text { Webb Residence } \\
\text { (USGS) }\end{array}$ & $\begin{array}{r}36.532 \mathrm{~N} \\
121.143 \mathrm{~W}\end{array}$ & 49.7 & (2) & & (1) & \\
\hline $\begin{array}{l}14 \text { January } 1986 \\
0550 \text { G.m.t. } \\
\text { Central Cal if. } \\
\text { Epicenter and } \\
\text { magnitude unknown }\end{array}$ & $\begin{array}{l}\text { Bear Valley Station } 10 \\
\text { Webb Residence } \\
\text { (USGS) }\end{array}$ & $\begin{array}{r}36.532 \mathrm{~N} \\
121.143 \mathrm{~W}\end{array}$ & 24.4 & (2) & & & \\
\hline $\begin{array}{l}15 \text { January } 1986- \\
26 \text { January } 1986 \\
\text { Central Cal if. } \\
\text { Epicenters and } \\
\text { magnitudes unknown }\end{array}$ & $\begin{array}{l}\text { Bear Valley Station } 10 \\
\text { Webb Residence } \\
\text { (USGS) } \\
\quad \text { Note: One additional }\end{array}$ & $\begin{array}{r}36.532 \mathrm{~N} \\
121.143 \mathrm{~W}\end{array}$ & recovered & d at Webb & Residence. & (1) & \\
\hline \multirow{10}{*}{$\begin{array}{l}26 \text { January } 1986 \\
1920: 51.2 \mathrm{G.m.t} \text {. } \\
\text { Central Calif. } \\
36.810 \mathrm{~N}, 121.275 \mathrm{~W} \\
\text { Magnitude } 5.5 \mathrm{ML}\end{array}$} & $\begin{array}{l}\text { Bear Valley Station } 1 \\
\text { Fire Station } \\
\text { (USGS) }\end{array}$ & $\begin{array}{r}36.573 \mathrm{~N} \\
121.184 \mathrm{~W}\end{array}$ & 57.8 & (2) & & (1) & \\
\hline & $\begin{array}{l}\text { Bear Valley Station } 2 \\
\text { Stone Canyon West } \\
\text { (USGS) }\end{array}$ & $\begin{array}{r}36.636 \mathrm{~N} \\
121.234 \mathrm{~W}\end{array}$ & 56.4 & (2) & & (1) & \\
\hline & $\begin{array}{l}\text { Bear Valley Station } 5 \\
\text { Callens Ranch }\end{array}$ & $\begin{array}{r}36.673 \mathrm{~N} \\
121.195 \mathrm{~W}\end{array}$ & 54.7 & (2) & & (1) & \\
\hline & (USGS) & & & & & & \\
\hline & $\begin{array}{l}\text { Bear Valley Station } 6 \\
\text { James Ranch } \\
\text { (USGS) }\end{array}$ & $\begin{array}{r}36.504 \mathrm{~N} \\
121.101 \mathrm{~W}\end{array}$ & 03.1 & (2) & & (1) & \\
\hline & $\begin{array}{l}\text { Bear Valley Station } 10 \\
\text { Webb Residence } \\
\text { (USGS) }\end{array}$ & $\begin{array}{r}36.532 \mathrm{~N} \\
121.143 \mathrm{~W}\end{array}$ & (3) & (2) & & (1) & \\
\hline & $\begin{array}{l}\text { Bear Valley Station } 12 \\
\text { Williams Ranch } \\
\text { (USGS) }\end{array}$ & $\begin{array}{r}36.658 \mathrm{~N} \\
121.249 \mathrm{~W}\end{array}$ & (3) & 3.4 & $\begin{array}{l}310 \\
\text { Up } \\
220\end{array}$ & $\begin{array}{l}.12 \\
.01 \\
.12\end{array}$ & $\begin{array}{c}0.6 \\
--- \\
1 \text { peak }\end{array}$ \\
\hline & $\begin{array}{l}\text { Bear Valley Station } 14 \\
\text { Upper Butts Ranch } \\
\text { (USGS) }\end{array}$ & $\begin{array}{r}36.569 \mathrm{~N} \\
121.043 \mathrm{~W}\end{array}$ & 58.3 & (2) & $\begin{array}{l}310 \\
\text { Up } \\
220\end{array}$ & $\begin{array}{l}.03 \\
.02 \\
.06\end{array}$ & --- \\
\hline & $\begin{array}{l}\text { Hollister City Hall } \\
\text { Basement Annex } \\
\text { (USGS) }\end{array}$ & $\begin{array}{r}36.851 \mathrm{~N} \\
121.402 \mathrm{~W}\end{array}$ & 54.8 & 2.8 & $\begin{array}{l}180 \\
\text { Up } \\
090\end{array}$ & $\begin{array}{l}.10 \\
.29 \\
.12\end{array}$ & $\begin{array}{l}1 \text { peak } \\
1.5 \\
0.5\end{array}$ \\
\hline & $\begin{array}{l}\text { Hollister } \\
\text { Damler Residence } \\
\text { (USGS) }\end{array}$ & $\begin{array}{r}36.807 N \\
121.408 W\end{array}$ & $54.5^{4}$ & 2.4 & $\begin{array}{l}360 \\
\text { Up } \\
270\end{array}$ & $\begin{array}{l}.17 \\
.09 \\
.14\end{array}$ & $\begin{array}{l}3.3 \\
--- \\
0.8\end{array}$ \\
\hline
\end{tabular}

See footnotes at end of table 
Table 7. Summary of United States accelerograph records recovered during 1986-Continued

\begin{tabular}{|c|c|c|c|c|c|c|c|}
\hline Earthquake & $\begin{array}{l}\text { Station name } \\
\text { (Owner) }\end{array}$ & $\begin{array}{l}\text { Station } \\
\text { location } \\
(*)\end{array}$ & $\begin{array}{l}\text { Trigger } \\
\text { time }\end{array}$ & $\begin{array}{l}S \text {-minus } \\
\text { trigger } \\
(s)\end{array}$ & $\begin{array}{l}\text { Direction } \\
\quad(\mathrm{az})\end{array}$ & $\begin{array}{l}\text { Maximum } \\
\text { amplitude } \\
\text { (g) }\end{array}$ & $\begin{array}{l}\text { Duration } \\
\text { (s) }\end{array}$ \\
\hline & $\begin{array}{l}\text { Holl ister } \\
\text { SAGO Vault } \\
\text { (USGS) }\end{array}$ & $\begin{array}{r}36.765 \mathrm{~N} \\
121.446 \mathrm{~W}\end{array}$ & $57.7^{4}$ & (2) & & (1) & \\
\hline & $\begin{array}{l}\text { Hollister Diff. } \\
\text { Array (SMA) } \\
\text { (USGS) }\end{array}$ & $\begin{array}{r}36.888 N \\
121.413 W\end{array}$ & 54.7 & (2) & $\begin{array}{l}255 \\
\text { Up } \\
165\end{array}$ & $\begin{array}{l}.09 \\
.15 \\
.10\end{array}$ & 2 peaks \\
\hline & $\begin{array}{l}\text { San Justo Damsite } \\
\text { (USBR) }\end{array}$ & & & & & & \\
\hline & Left Abutment & $\begin{array}{r}36.815 \mathrm{~N} \\
121.447 \mathrm{~W}\end{array}$ & 55.4 & 3.1 & $\begin{array}{l}360 \\
\text { Up } \\
270\end{array}$ & $\begin{array}{l}.16 \\
.07 \\
.14\end{array}$ & $\begin{array}{l}0.5 \\
--- \\
0.4\end{array}$ \\
\hline & $\begin{array}{l}\text { Right Abutment } \\
\text { (Dike) }\end{array}$ & $\begin{array}{r}36.827 N \\
121.445 W\end{array}$ & 55.2 & 2.5 & $\begin{array}{l}360 \\
\text { Up } \\
270\end{array}$ & $\begin{array}{l}.09 \\
.04 \\
.08\end{array}$ & $\begin{array}{l}--- \\
---\end{array}$ \\
\hline $\begin{array}{l}26 \text { January } 1986 \\
2346: 54.9 \mathrm{G.m} . \mathrm{t} . \\
\text { Central Calif. } \\
36.828 \mathrm{~N}, 121.290 \mathrm{~W} \\
\text { Magnitude } 3.8 \mathrm{ML}\end{array}$ & $\begin{array}{l}\text { Hollister City Hall } \\
\text { Basement Annex } \\
\text { (USGS) }\end{array}$ & $\begin{array}{r}36.851 \mathrm{~N} \\
121.402 \mathrm{~W}\end{array}$ & 57.4 & 2.4 & & (1) & \\
\hline
\end{tabular}

Note: One additional record ${ }^{1}$ recovered at Hollister City Hall Annex.

10 February 1986

0340 G.m.t.

Central Calif.

Epicenter and

magnitude unknown

9 March 1986

2241:42.5 G.m.t.

Southern Calif.

$34.110 \mathrm{~N}, 117.770 \mathrm{~W}$

Magnitude $3.5 \mathrm{ML}$
Bear Valley Station 10

Webb Residence

(USGS)

Live Oak Reservoir

(MWD)

Abutment

Structure Array

Ch. 1-Center crest

Ch. 2-Center crest

Ch. 3-Center crest

Ch. 4-Left crest

Ch. 5-Left crest

Ch. 6-Left slope

Ch. 7-Center slope

Ch. 8-Center slope

Ch. 9-Center slope

Ch. 10-Center toe

Ch. 11-Center toe

Ch. 12-Center toe

San Antonio Dam (ACOE)

$34.166 \mathrm{~N}$

$117.680 \mathrm{~W}$
$48.5 \quad 1.4$
$34.134 \mathrm{~N}$

$117.753 \mathrm{~W}$

(3)

180
Up
090

.10

.03

1 peak

.05

$0.8 \quad 155$

(2) Up

$0.7 \quad 245$

$0.7 \quad 155$

$0.6 \quad 245$

$0.7 \quad 245$

$0.6 \quad 155$

(2) Up

$0.7 \quad 245$

$0.6 \quad 155$

(2) Up

$0.6 \quad 245$

(3)

(2)

Crest 
Table 7. Summary of United States accelerograph records recovered during 1986-Continued

\begin{tabular}{|c|c|c|c|c|c|c|c|}
\hline Earthquake & $\begin{array}{l}\text { Station name } \\
\text { (Owner) }\end{array}$ & $\begin{array}{c}\text { Station } \\
\text { location } \\
(*)\end{array}$ & $\begin{array}{c}\text { Trigger } \\
\text { time }\end{array}$ & $\begin{array}{c}S \text {-minus } \\
\text { trigger } \\
\text { (s) }\end{array}$ & $\begin{array}{l}\text { Direction } \\
\text { (az) }\end{array}$ & $\begin{array}{l}\text { Maximum } \\
\text { amplitude } \\
\text { (g) }\end{array}$ & $\begin{array}{c}\text { Duration } \\
\text { (s) }\end{array}$ \\
\hline
\end{tabular}

\begin{tabular}{|c|c|c|c|c|c|c|c|}
\hline & $\begin{array}{l}\text { Weymouth Filter Plant } \\
\text { (MWD) }\end{array}$ & $\begin{array}{r}34.114 \mathrm{~N} \\
117.778 \mathrm{~W}\end{array}$ & (3) & & & & \\
\hline & Ground & & & 0.7 & & (1) & \\
\hline & Tank top & & & & & (1) & \\
\hline $\begin{array}{l}10 \text { March } 1986 \\
1533: 16.2 \text { G.m.t. } \\
\text { Southern Cal if. } \\
34.400 \mathrm{~N}, 119.800 \mathrm{~W} \\
\text { Magnitude } 4.0 \mathrm{ML}\end{array}$ & $\begin{array}{l}\text { Santa Barbara } \\
\text { Courthouse } \\
\text { (USGS) }\end{array}$ & $\begin{array}{r}34.42 \mathrm{~N} \\
119.70 \mathrm{~W}\end{array}$ & (3). & 2.3 & & (1) & \\
\hline $\begin{array}{l}13 \text { March } 1986 \\
0836: 59.4 \mathrm{G} . \mathrm{m} . \mathrm{t} . \\
\text { Central Calif. } \\
36.309 \mathrm{~N}, 120.312 \mathrm{~W} \\
\text { Magnitude } 2.7 \mathrm{ML}\end{array}$ & $\begin{array}{l}\text { Coalinga } \\
\text { oil City } \\
\text { (USGS) }\end{array}$ & $\begin{array}{r}36.229 \mathrm{~N} \\
120.360 \mathrm{~W}\end{array}$ & 03.4 & 0.6 & $\begin{array}{l}360 \\
\text { Up } \\
270\end{array}$ & $\begin{array}{l}.05 \\
.01 \\
.03\end{array}$ & $\begin{array}{l}--- \\
--- \\
--\end{array}$ \\
\hline $\begin{array}{l}24 \text { March } 1986 \\
2255: 34.0 \mathrm{G} . \mathrm{m} . \mathrm{t} \text {. } \\
\text { Central Calif. } \\
36.557 \mathrm{~N}, 121.183 \mathrm{~W} \\
\text { Magnitude } 3.0 \mathrm{ML}\end{array}$ & $\begin{array}{l}\text { Bear Valley Station } 1 \\
\text { CDF Fire Station } \\
\text { (USGS) }\end{array}$ & $\begin{array}{r}36.573 \mathrm{~N} \\
121.184 \mathrm{~W}\end{array}$ & 34.8 & 0.7 & & (1) & \\
\hline \multirow{2}{*}{$\begin{array}{l}29 \text { March } 1986 \\
1624: 04.2 \text { G.m.t. } \\
\text { Central Cal iff. } \\
37.877 \mathrm{~N}, 122.203 \mathrm{~W} \\
\text { Magnitude } 4.0 \mathrm{ML}\end{array}$} & $\begin{array}{l}\text { Emeryville } \\
6363 \text { Christie Ave. } \\
\text { (USGS) }\end{array}$ & $\begin{array}{r}37.844 \mathrm{~N} \\
122.295 \mathrm{~W}\end{array}$ & (3) & (2) & & \multicolumn{2}{|l|}{ 1) } \\
\hline & $\begin{array}{l}\text { U.C. Berkeley } \\
\text { Strawberry Canyon } \\
\text { (UCB) }\end{array}$ & $\begin{array}{r}37.87 \mathrm{~N} \\
122.24 \mathrm{~W}\end{array}$ & 07.5 & (2) & & (1) & \\
\hline \multirow{8}{*}{$\begin{array}{l}31 \text { March } 1986 \\
1155: 40.0 \mathrm{G} . \mathrm{m} . \mathrm{t} \text {. } \\
\text { Central Cal if. } \\
37.483 \mathrm{~N}, 121.690 \mathrm{~W} \\
\text { Magnitude } 5.7 \mathrm{ML}\end{array}$} & $\begin{array}{l}\text { Anderson Dam } \\
\text { (USGS) (SMA) }\end{array}$ & $\begin{array}{r}37.166 \mathrm{~N} \\
121.628 \mathrm{~W}\end{array}$ & & & & & \\
\hline & Crest & & 49.2 & 3.8 & & (1) & \\
\hline & CR-1 (12-channe1) & & 49.2 & (2) & & (1) & \\
\hline & $\begin{array}{l}\text { Del Valle Dam } \\
\text { (CDWR) }\end{array}$ & $\begin{array}{r}37.615 \mathrm{~N} \\
121.745 \mathrm{~W}\end{array}$ & (3) & 2.5 & & & \\
\hline & Crest & & & & $\begin{array}{l}065 \\
\text { Up } \\
335\end{array}$ & $\begin{array}{l}.15 \\
.08 \\
.10\end{array}$ & $\begin{array}{c}1.4 \\
-1 \text { peak }\end{array}$ \\
\hline & $\begin{array}{l}\text { Livermore } \\
\text { VA Hospital, Bldg. } 62 \\
\text { (VA) }\end{array}$ & $\begin{array}{r}37.625 \mathrm{~N} \\
121.762 \mathrm{~W}\end{array}$ & (3) & 2.8 & & & \\
\hline & Basement & & & & $\begin{array}{l}125 \\
\text { Up } \\
035\end{array}$ & $\begin{array}{l}.07 \\
.05 \\
.09\end{array}$ & $\begin{array}{l}--- \\
--- \\
--\end{array}$ \\
\hline & Roof (7th level) & & & & $\begin{array}{l}125 \\
\text { Up } \\
035\end{array}$ & $\begin{array}{l}.15 \\
.10 \\
.39\end{array}$ & $\begin{array}{l}0.7 \\
3 \text { peaks } \\
1.4\end{array}$ \\
\hline
\end{tabular}


Table 7. Summary of United States accelenograph records recovered during 1986-Continued

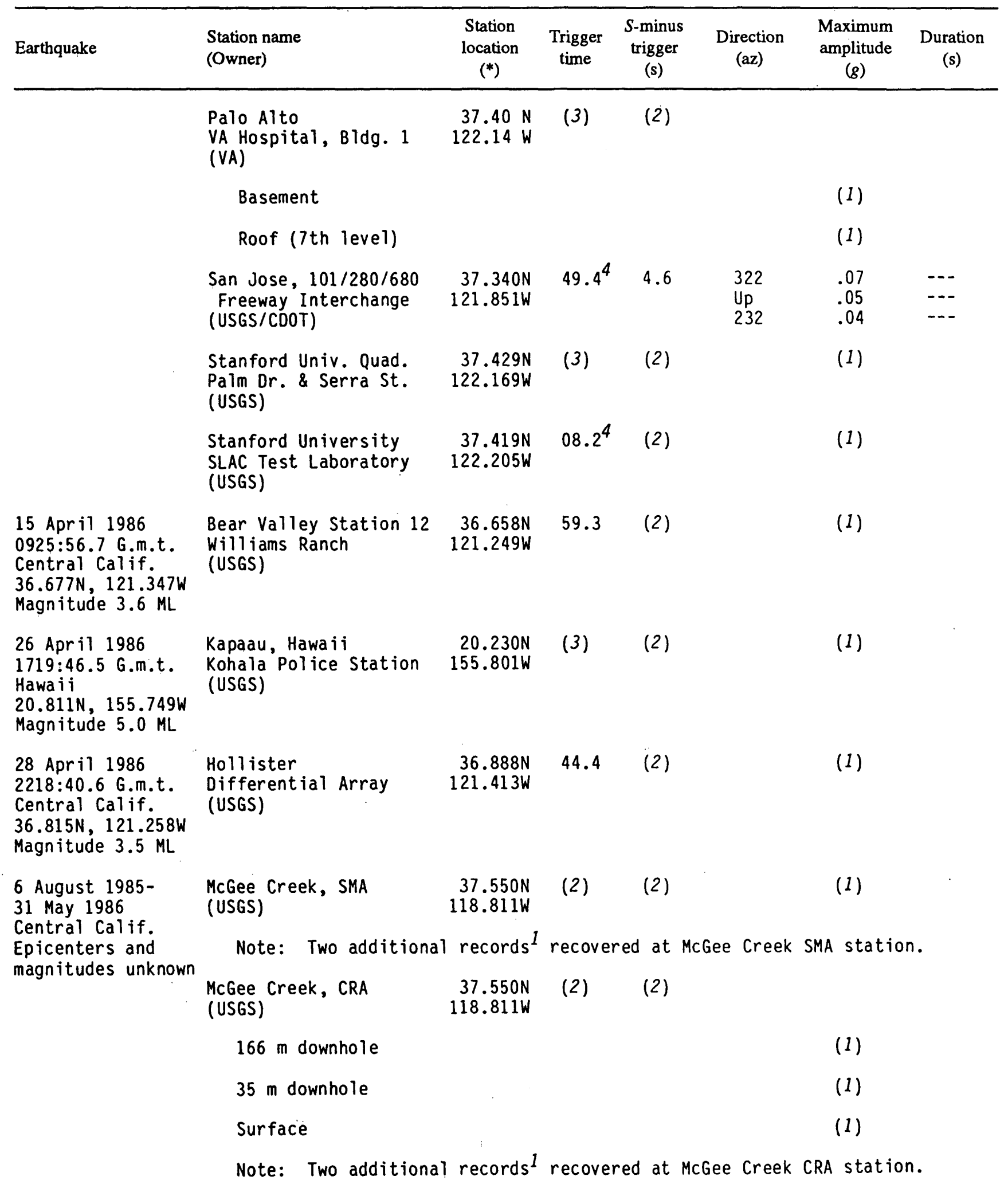


Table 7. Summary of United States accelerograph records recovered during 1986 -Continued

\begin{tabular}{|c|c|c|c|c|c|c|c|}
\hline Earthquake & $\begin{array}{l}\text { Station name } \\
\text { (Owner) }\end{array}$ & $\begin{array}{l}\text { Station } \\
\text { location } \\
\left({ }^{*}\right)\end{array}$ & $\begin{array}{l}\text { Trigger } \\
\text { time }\end{array}$ & $\begin{array}{l}S \text {-minus } \\
\text { trigger } \\
\quad(\mathrm{s})\end{array}$ & $\begin{array}{l}\text { Direction } \\
\quad(a z)\end{array}$ & $\begin{array}{l}\text { Maximum } \\
\text { amplitude } \\
\text { (g) }\end{array}$ & $\begin{array}{c}\text { Duration } \\
\text { (s) }\end{array}$ \\
\hline \multirow{7}{*}{$\begin{array}{l}31 \text { May } 1986 \\
0847: 56.1 \text { G.m.t. } \\
\text { Central Cal if. } \\
36.570 \mathrm{~N}, 121.327 \mathrm{~W} \\
\text { Magnitude } 4.8 \mathrm{ML}\end{array}$} & $\begin{array}{l}\text { Bear Valley Station } 1 \\
\text { CDF Fire Station } \\
\text { (USGS) }\end{array}$ & $\begin{array}{r}36.573 \mathrm{~N} \\
121.184 \mathrm{~W}\end{array}$ & 58.4 & 1.0 & $\begin{array}{l}310 \\
\text { Up } \\
220\end{array}$ & $\begin{array}{l}.05 \\
.03 \\
.08\end{array}$ & --- \\
\hline & $\begin{array}{l}\text { Bear Valley Station } 2 \\
\text { Stone Canyon West } \\
\text { (USGS) }\end{array}$ & $\begin{array}{r}36.636 \mathrm{~N} \\
121.234 \mathrm{~W}\end{array}$ & 58.9 & (2) & & (1) & \\
\hline & $\begin{array}{l}\text { Bear Valley Station } 5 \\
\text { Callens Ranch } \\
\text { (USGS) }\end{array}$ & $\begin{array}{r}36.673 \mathrm{~N} \\
121.195 \mathrm{~W}\end{array}$ & 59.3 & 1.7 & $\begin{array}{l}310 \\
\text { Up } \\
220\end{array}$ & $\begin{array}{l}.05 \\
.03 \\
.04\end{array}$ & --- \\
\hline & $\begin{array}{l}\text { Bear Valley Station } 10 \\
\text { Webb Residence } \\
\text { (USGS) }\end{array}$ & $\begin{array}{r}36.532 \mathrm{~N} \\
121.143 \mathrm{~W}\end{array}$ & 00.3 & 1.2 & & (1) & \\
\hline & $\begin{array}{l}\text { Bear Valley Station } 12 \\
\text { Will iams Ranch } \\
\text { (USGS) }\end{array}$ & $\begin{array}{r}36.658 \mathrm{~N} \\
121.249 \mathrm{~W}\end{array}$ & 58.2 & 2.4 & $\begin{array}{l}310 \\
\text { Up } \\
220\end{array}$ & $\begin{array}{l}.32 \\
.13 \\
.25\end{array}$ & $\begin{array}{l}1.8 \\
0.3 \\
1.3\end{array}$ \\
\hline & $\begin{array}{l}\text { Bear Valley Station } 14 \\
\text { Upper Butts Ranch } \\
\text { (USGS) }\end{array}$ & $\begin{array}{r}36.569 N \\
121.043 W\end{array}$ & 04.7 & (2) & $\begin{array}{l}310 \\
\text { Up } \\
220\end{array}$ & $\begin{array}{l}.02 \\
.02 \\
.05\end{array}$ & --- \\
\hline & $\begin{array}{l}\text { Hollister } \\
\text { Damler Residence } \\
\text { (USGS) }\end{array}$ & $\begin{array}{r}36.807 \mathrm{~N} \\
121.408 \mathrm{~W}\end{array}$ & $07.8^{4}$ & (2) & & (1) & \\
\hline $\begin{array}{l}31 \text { May } 1986 \\
1451: 27.9 \text { G.m.t. } \\
\text { Central Calif. } \\
36.635 \mathrm{~N}, 121.261 \mathrm{~W} \\
\text { Magnitude } 2.6 \mathrm{ML}\end{array}$ & $\begin{array}{l}\text { Bear Valley Station } 12 \\
\text { Will iams Ranch } \\
\text { (USGS) }\end{array}$ & $\begin{array}{r}36.658 \mathrm{~N} \\
121.249 \mathrm{~W}\end{array}$ & 30.3 & 1.9 & . & (1) & \\
\hline \multirow{4}{*}{$\begin{array}{l}1 \text { June } 1986 \\
0649: 34.1 \text { G.m.t. } \\
\text { Central Calif. } \\
36.612 \mathrm{~N}, 121.267 \mathrm{~W} \\
\text { Magnitude } 3.6 \mathrm{ML}\end{array}$} & $\begin{array}{l}\text { Bear Valley Station } 1 \\
\text { CDF Fire Station } \\
\text { (USGS) }\end{array}$ & $\begin{array}{r}36.573 \mathrm{~N} \\
121.184 \mathrm{~W}\end{array}$ & 36.8 & 1.3 & $\begin{array}{l}310 \\
\text { Up } \\
220\end{array}$ & $\begin{array}{l}.07 \\
.02 \\
.10\end{array}$ & 1 peak \\
\hline & $\begin{array}{l}\text { Bear Valley Station } 5 \\
\text { Callens Ranch } \\
\text { (USGS) }\end{array}$ & $\begin{array}{r}36.673 \mathrm{~N} \\
121.195 \mathrm{~W}\end{array}$ & 38.0 & (2) & & (1) & \\
\hline & $\begin{array}{l}\text { Bear Valley Station } 12 \\
\text { Williams Ranch } \\
\text { (USGS) }\end{array}$ & $\begin{array}{r}36.658 \mathrm{~N} \\
121.249 \mathrm{~W}\end{array}$ & 37.0 & 1.9 & $\begin{array}{l}310 \\
\text { Up } \\
220\end{array}$ & $\begin{array}{l}.05 \\
.05 \\
.05\end{array}$ & --- \\
\hline & $\begin{array}{l}\text { Bear Valley Station } 14 \\
\text { Upper Butts Ranch } \\
\text { (USGS) }\end{array}$ & $\begin{array}{r}36.569 \mathrm{~N} \\
121.043 \mathrm{~W}\end{array}$ & 43.0 & (2) & & $(1)$ & \\
\hline $\begin{array}{l}1 \text { June } 1986 \\
1934: 44.6 \mathrm{G.m.t} . \\
\text { Central Cal if. } \\
36.619 \mathrm{~N}, 121.252 \mathrm{~W} \\
\text { Magnitude } 2.5 \mathrm{ML}\end{array}$ & $\begin{array}{l}\text { Bear Valley Station } 12 \\
\text { Williams Ranch } \\
\text { (USGS) }\end{array}$ & $\begin{array}{r}36.658 \mathrm{~N} \\
121.249 \mathrm{~W}\end{array}$ & 47.25 & 1.9 & & (1) & \\
\hline
\end{tabular}


Table 7. Summary of United States accelerograph records recovered during 1986_Continued

\begin{tabular}{|c|c|c|c|c|c|c|c|}
\hline Earthquake & $\begin{array}{l}\text { Station name } \\
\text { (Owner) }\end{array}$ & $\begin{array}{l}\text { Station } \\
\text { location } \\
(*)\end{array}$ & $\begin{array}{l}\text { Trigger } \\
\text { time }\end{array}$ & $\begin{array}{l}S \text {-minus } \\
\text { trigger } \\
\text { (s) }\end{array}$ & $\begin{array}{l}\text { Direction } \\
\quad(a z)\end{array}$ & $\begin{array}{l}\text { Maximum } \\
\text { amplitude } \\
(g)\end{array}$ & $\begin{array}{l}\text { Duration } \\
\text { (s) }\end{array}$ \\
\hline \multirow{3}{*}{$\begin{array}{l}3 \text { June } 1986 \\
1414: 49.2 \text { G.m.t. } \\
\text { Southern Calif. } \\
33.790 \mathrm{~N}, 116.340 \mathrm{~W} \\
\text { Magnitude } 3.7 \mathrm{ML}\end{array}$} & $\begin{array}{l}\text { Fun Valley } \\
\text { Reservoir } 261 \\
\text { (USGS) }\end{array}$ & $\begin{array}{r}33.925 \mathrm{~N} \\
116.389 \mathrm{~W}\end{array}$ & 52.6 & (2) & & (1) & \\
\hline & $\begin{array}{l}\text { Indio, Southern } \\
\text { Calif. Gas Company } \\
\text { (USGS) }\end{array}$ & $\begin{array}{r}33.747 \mathrm{~N} \\
116.214 \mathrm{~W}\end{array}$ & 52.6 & 3.1 & $\begin{array}{l}315 \\
\text { Up } \\
225\end{array}$ & $\begin{array}{l}.05 \\
.01 \\
.02\end{array}$ & --- \\
\hline & $\begin{array}{l}\text { Thousand Paims } \\
\text { Post office } \\
\text { (USGS) }\end{array}$ & $\begin{array}{r}33.82 \mathrm{~N} \\
116.40 \mathrm{~W}\end{array}$ & (3) & 0.5 & $\begin{array}{l}135 \\
\text { Up } \\
045\end{array}$ & $\begin{array}{l}.05 \\
.02 \\
.05\end{array}$ & --- \\
\hline $\begin{array}{l}11 \text { June } 1986 \\
1508: 59.6 \mathrm{G.m} . \mathrm{t} . \\
\text { Central Calif. } \\
\text { 36.622N, } 121.282 \mathrm{~W} \\
\text { Magnitude } 3.1 \mathrm{ML}\end{array}$ & $\begin{array}{l}\text { Bear Valley Station } 12 \\
\text { Williams Ranch } \\
\text { (USGS) }\end{array}$ & $\begin{array}{r}36.658 \mathrm{~N} \\
121.249 \mathrm{~W}\end{array}$ & 01.7 & 2.0 & $\begin{array}{l}310 \\
\text { Up } \\
220\end{array}$ & $\begin{array}{l}.10 \\
.04 \\
.06\end{array}$ & 1 peak \\
\hline \multirow[t]{5}{*}{$\begin{array}{l}9 \text { December 1985- } \\
13 \text { June } 1986 \\
\text { Southern Calif. } \\
\text { Epicenter and } \\
\text { magnitude unknown }\end{array}$} & $\begin{array}{l}\text { Brea Dam } \\
(A C O E) \\
\quad \text { Left abutment }\end{array}$ & $\begin{array}{r}33.890 \mathrm{~N} \\
117.930 \mathrm{~W}\end{array}$ & (3) & 3.6 & & (1) & \\
\hline & Downstream & & & & & (1) & \\
\hline & $\begin{array}{l}\text { Long Beach } \\
\text { VA Hospital } \\
\text { (VA) }\end{array}$ & $\begin{array}{r}33.78 \mathrm{~N} \\
118.12 \mathrm{~W}\end{array}$ & (3) & 2.1 & & & \\
\hline & Basement & & & & & (1) & \\
\hline & 6th floor & & & & & (1) & \\
\hline & 11th floor & & & & & (1) & \\
\hline $\begin{array}{l}30 \text { June } 1986 \\
\text { Time unknown } \\
\text { Central Calif. } \\
\text { Epicenter and } \\
\text { magnitude unknown }\end{array}$ & $\begin{array}{l}\text { Bear Valley Station } 10 \\
\text { Webb Residence } \\
\text { (USGS) }\end{array}$ & $\begin{array}{r}36.532 \mathrm{~N} \\
121.143 \mathrm{~W}\end{array}$ & 19.5 & 0.8 & & (1) & \\
\hline \multirow{3}{*}{$\begin{array}{l}8 \text { July } 1986 \\
0920: 44.5 \text { G.m.t. } \\
\text { N. Palm Springs } \\
34.000 \mathrm{~N}, 116.61 \mathrm{WW} \\
\text { Magnitude } 6.0 \mathrm{ML}\end{array}$} & $\begin{array}{l}\text { Anza Fire Station } \\
\text { ANZA Array } \\
\text { (USGS) }\end{array}$ & $\begin{array}{r}33.556 \mathrm{~N} \\
116.673 \mathrm{~W}\end{array}$ & 54.36 & 5.1 & $\begin{array}{l}315 \\
U p \\
225\end{array}$ & $\begin{array}{l}.07 \\
.06 \\
.11\end{array}$ & .02 \\
\hline & $\begin{array}{l}\text { Big Pines Station } \\
\text { (USGS) }\end{array}$ & $\begin{array}{r}34.38 \mathrm{~N} \\
117.69 \mathrm{~W}\end{array}$ & 16.73 & (2) & & (1) & \\
\hline & $\begin{array}{l}\text { Borrego Springs } \\
\text { Scripps Clinic } \\
\text { (USGS) }\end{array}$ & $\begin{array}{r}33.210 \mathrm{~N} \\
116.330 \mathrm{~W}\end{array}$ & 02.5 & 9.3 & & (1) & \\
\hline
\end{tabular}


Table 7. Summary of United States accelerograph records recovered during 1986-Continued

\begin{tabular}{|c|c|c|c|c|c|c|c|}
\hline Earthquake & $\begin{array}{l}\text { Station name } \\
\text { (Owner) }\end{array}$ & $\begin{array}{c}\text { Station } \\
\text { location } \\
\left({ }^{*}\right)\end{array}$ & $\begin{array}{l}\text { Trigger } \\
\text { time }\end{array}$ & $\begin{array}{c}S \text {-minus } \\
\text { trigger } \\
(\mathrm{s})\end{array}$ & $\begin{array}{l}\text { Direction } \\
\quad(\mathrm{az})\end{array}$ & $\begin{array}{l}\text { Maximum } \\
\text { amplitude } \\
(g)\end{array}$ & $\begin{array}{l}\text { Duration } \\
\quad(\mathrm{s})\end{array}$ \\
\hline & $\begin{array}{l}\text { Brea Dam: } \\
(A C O E)\end{array}$ & $\begin{array}{r}33.889 \mathrm{~N} \\
117.926 \mathrm{~W}\end{array}$ & & & & & \\
\hline & Left abutment & & (3) & (2) & & (1) & \\
\hline & Downstream & & (3) & 14.1 & & (1). & \\
\hline & Crest & & (3) & 14.0 & $\begin{array}{l}130 \\
\text { Up } \\
040\end{array}$ & $\begin{array}{l}.04 \\
.03 \\
.07\end{array}$ & $\begin{array}{l}-- \\
-- \\
--\end{array}$ \\
\hline & Note: One additic & record & recovere & d at Brea & Dam crest. & & \\
\hline & $\begin{array}{l}\text { Cabazon Post office } \\
\text { (USGS) }\end{array}$ & $\begin{array}{r}33.918 \mathrm{~N} \\
116.782 \mathrm{~W}\end{array}$ & 49.50 & 2.4 & $\begin{array}{l}270 \\
\text { Up } \\
180\end{array}$ & $\begin{array}{l}.21 \\
.38 \\
.22\end{array}$ & $\begin{array}{l}3.3 \\
3.2 \\
2.7\end{array}$ \\
\hline
\end{tabular}

Note: One additional record ${ }^{1}$ recovered at Cabazon.

\begin{tabular}{|c|c|c|c|c|c|c|}
\hline $\begin{array}{l}\text { Carbon Canyon Dam } \\
(\mathrm{ACOE})\end{array}$ & $\begin{array}{r}33.92 \mathrm{~N} \\
117.84 \mathrm{~W}\end{array}$ & (3) & (2) & & & \\
\hline Crest & & & & \multicolumn{3}{|c|}{ (1) } \\
\hline \multicolumn{7}{|c|}{ Note: One additional record ${ }^{1} r$} \\
\hline $\begin{array}{l}\text { Cherry Valley } \\
\text { (USGS) }\end{array}$ & $\begin{array}{r}33.98 \mathrm{~N} \\
116.99 \mathrm{~W}\end{array}$ & 51.48 & 5.2 & $\begin{array}{l}295 \\
\text { Up } \\
205\end{array}$ & $\begin{array}{l}.10 \\
.06 \\
.10\end{array}$ & $\begin{array}{l}1 \text { peak } \\
-\ldots \\
1 \text { peak }\end{array}$ \\
\hline $\begin{array}{l}\text { Chihuahua Valley } \\
\text { ANZA Array } \\
\text { (USGS) }\end{array}$ & $\begin{array}{r}33.38 \mathrm{~N} \\
116.68 \mathrm{~W}\end{array}$ & (3) & 7.9 & $\begin{array}{l}270 \\
\text { Up } \\
180\end{array}$ & $\begin{array}{l}.05 \\
.04 \\
.07\end{array}$ & --- \\
\hline \multicolumn{7}{|l|}{ Note: } \\
\hline $\begin{array}{l}\text { Coachella Canal } \\
\text { Station } 1 \\
\text { (USGS) }\end{array}$ & $\begin{array}{r}33.64 \mathrm{~N} \\
116.08 \mathrm{~W}\end{array}$ & 56.8 & 9.8 & $\begin{array}{l}315 \\
\text { Up } \\
225\end{array}$ & $\begin{array}{l}.09 \\
.05 \\
.14\end{array}$ & 2 peaks \\
\hline $\begin{array}{l}\text { Coachella Canal } \\
\text { Station } 2 \\
\text { (USGS) }\end{array}$ & $\begin{array}{r}33.56 \mathrm{~N} \\
115.95 \mathrm{~W}\end{array}$ & (3) & 9.0 & & (1) & \\
\hline $\begin{array}{l}\text { Collins Valley } \\
\text { (USGS). }\end{array}$ & $\begin{array}{r}33.405 \mathrm{~N} \\
116.467 \mathrm{~W}\end{array}$ & 56.3 & (2) & & (1) & \\
\hline $\begin{array}{l}\text { Colton Interchange } \\
\text { (CDOT) }\end{array}$ & $\begin{array}{r}34.06 \mathrm{~N} \\
117.30 \mathrm{~W}\end{array}$ & (3) & 6.4 & & & \\
\hline Bridge cell & & & & $\begin{array}{l}082 \\
\text { Up } \\
352\end{array}$ & $\begin{array}{l}.12 \\
.05 \\
.10\end{array}$ & $\begin{array}{c}1.7 \\
1 \text { peak }\end{array}$ \\
\hline Vault & & (3) & 5.9 & $\begin{array}{l}082 \\
\text { Up } \\
352\end{array}$ & $\begin{array}{l}.06 \\
.02 \\
.06\end{array}$ & $\begin{array}{l}--- \\
---\end{array}$ \\
\hline
\end{tabular}

Note: One each additional record ${ }^{l}$ recovered at Colton Interchange bridge cell and vault. 
Table 7. Summary of United States accelerograph records recovered during 1986—Continued

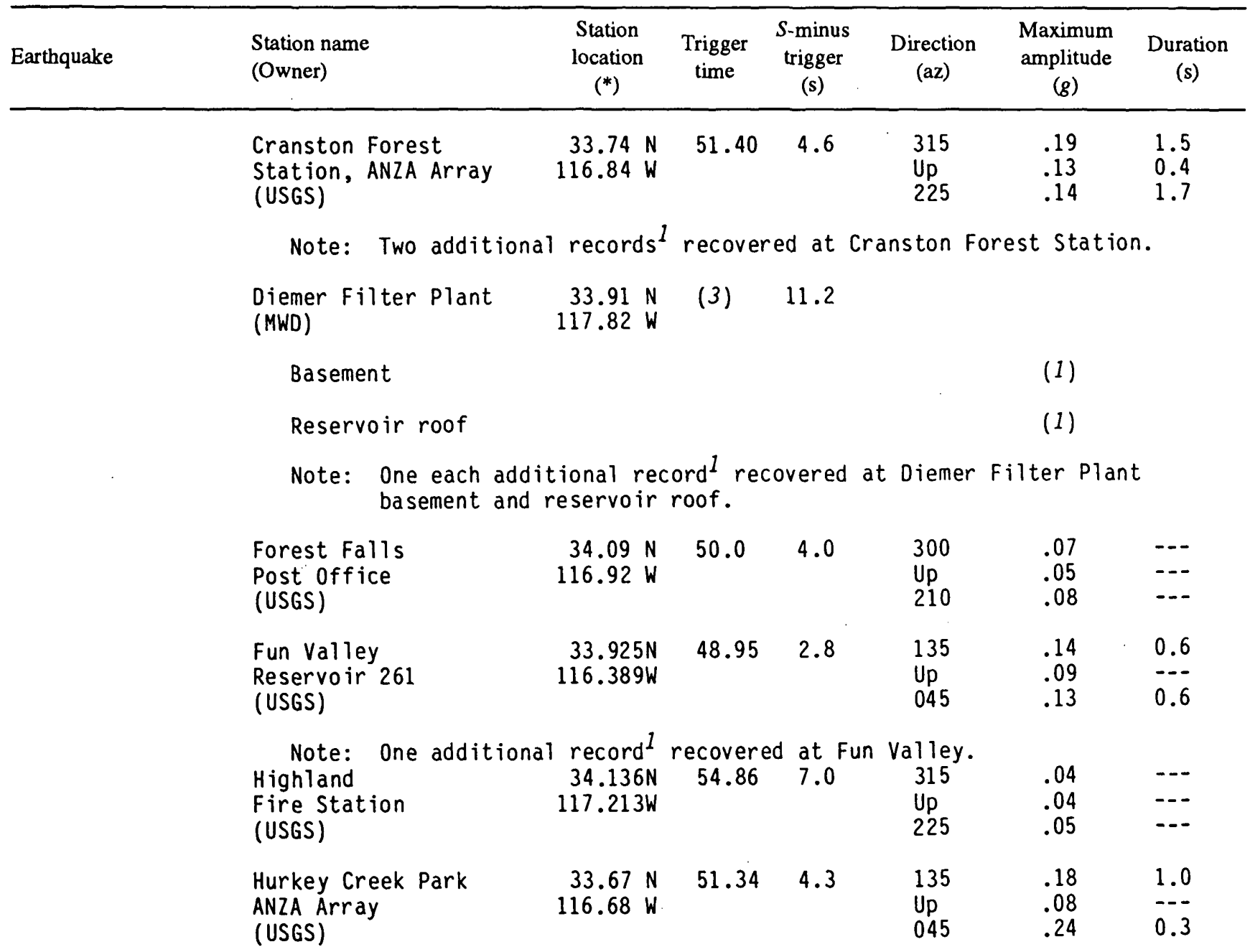

Note: Two additional records ${ }^{1}$ recovered at Hurkey Creek Park.

$\begin{array}{lrccccc}\text { Indio, Southern } & 33.747 \mathrm{~N} & 53.20 & 6.2 & 315 & .12 & 0.4 \\ \text { Calif. Gas Company } & 116.214 \mathrm{~W} & & & \text { Up } & .09 & -.- \\ \text { (USGS) } & & & 225 & .06 & ---\end{array}$

Note: One additional record ${ }^{l}$ recovered at Indio.

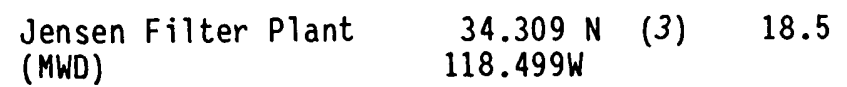

Basement

Admin. bldg.

Generator room

Basement

Reservoir roof

Note: One each additional records ${ }^{1}$ recovered at Jensen Filter Plant administration building basement and generator room basement.

\begin{tabular}{|c|c|}
\hline $\begin{array}{l}\text { Keenwild Forest Station } \\
\text { Anza Array } \\
\text { (USGS) }\end{array}$ & $\begin{array}{r}33.71 \\
116.71\end{array}$ \\
\hline
\end{tabular}


Table 7. Summary of United States accelerograph records recovered during 1986—Continued

\begin{tabular}{|c|c|c|c|c|c|c|c|}
\hline Earthquake & $\begin{array}{l}\text { Station name } \\
\text { (Owner) }\end{array}$ & $\begin{array}{l}\text { Station } \\
\text { location } \\
\left(^{*}\right)\end{array}$ & $\begin{array}{l}\text { Trigger } \\
\text { time }\end{array}$ & $\begin{array}{l}S \text {-minus } \\
\text { trigger } \\
(\mathrm{s})\end{array}$ & $\begin{array}{l}\text { Direction } \\
\quad(a z)\end{array}$ & $\begin{array}{l}\text { Maximum } \\
\text { amplitude } \\
\text { (8) }\end{array}$ & $\begin{array}{l}\text { Duration } \\
\text { (s) }\end{array}$ \\
\hline & $\begin{array}{l}\text { Mathews Dam } \\
\text { Dike Toe } \\
\text { (USGS) }\end{array}$ & $\begin{array}{r}33.852 \mathrm{~N} \\
117.451 \mathrm{~W}\end{array}$ & (3) & 6.8 & $\begin{array}{l}252 \\
\text { Up } \\
162\end{array}$ & $\begin{array}{l}.05 \\
.04 \\
.07\end{array}$ & --- \\
\hline & $\begin{array}{l}\text { Loma Linda University } \\
\text { Medical Center } \\
\text { (USGS) }\end{array}$ & $\begin{array}{r}34.05 \mathrm{~N} \\
117.26 \mathrm{~W}\end{array}$ & (3) & 6.0 & & & \\
\hline & Basement & & & & & (1) & \\
\hline & $\begin{array}{l}\text { Loma Linda } \\
\text { VA Hospital } \\
\text { (VA) }\end{array}$ & & & & & & \\
\hline & South FF & $\begin{array}{r}34.049 \mathrm{~N} \\
117.250 \mathrm{~W}\end{array}$ & (3) & 6.0 & & (1) & \\
\hline & North FF & $\begin{array}{r}34.051 \mathrm{~N} \\
117.248 \mathrm{~W}\end{array}$ & 56.6 & (2) & $\begin{array}{l}360 \\
\text { Up } \\
270\end{array}$ & $\begin{array}{l}.05 \\
.03 \\
.04\end{array}$ & --- \\
\hline & $\begin{array}{l}\text { Structure Array, } \\
9 \text { Channel CRA-1: } \\
\text { 1-1st floor center } \\
\text { 2-1st floor center } \\
\text { 3-1st floor center } \\
\text { 4-4th floor center } \\
\text { 5-1st floor north } \\
\text { 6-4th floor center } \\
\text { 7-4th floor north } \\
\text { 8-1st floor south } \\
\text { 9-4th floor south }\end{array}$ & $\begin{array}{r}34.049 \mathrm{~N} \\
117.248 \mathrm{~W}\end{array}$ & (3) & 6.3 & $\begin{array}{l}\text { Down } \\
180 \\
270 \\
270 \\
270 \\
180 \\
270 \\
180 \\
270\end{array}$ & $\begin{array}{l}.02 \\
.04 \\
.04 \\
.10 \\
.04 \\
.08 \\
.09 \\
.04 \\
.11\end{array}$ & $\begin{array}{l}-- \\
1 \text { peak } \\
-- \\
1 \text { peak }\end{array}$ \\
\hline & $\begin{array}{l}\text { Lone Pine Canyon } \\
\text { (USGS) }\end{array}$ & $\begin{array}{r}34.32 \mathrm{~N} \\
117.57 \mathrm{~W}\end{array}$ & 03.65 & 8.7 & & (1) & \\
\hline & $\begin{array}{l}\text { Los Angeles } \\
\text { Bulk Mail Center } \\
\text { (USGS) }\end{array}$ & $\begin{array}{r}33.99 \mathrm{~N} \\
118.16 \mathrm{~W}\end{array}$ & (3) & 16.5 & & $(1)$ & \\
\hline & $\begin{array}{l}\text { Lyt le Creek } \\
\text { Mann Residence } \\
\text { (USGS) }\end{array}$ & $\begin{array}{r}34.26 \mathrm{~N} \\
117.50 \mathrm{~W}\end{array}$ & 11.98 & (2) & & (1) & \\
\hline & $\begin{array}{l}\text { Mentone Fire Station } \\
\text { (USGS) }\end{array}$ & $\begin{array}{r}34.067 N \\
117.117 W\end{array}$ & 53.02 & 6.2 & $\begin{array}{l}315 \\
\text { Up } \\
225\end{array}$ & $\begin{array}{l}.06 \\
.04 \\
.04\end{array}$ & --- \\
\hline & $\begin{array}{l}\text { Morongo Valley } \\
\text { Fire Station } \\
\text { (USGS). }\end{array}$ & $\begin{array}{r}34.048 \mathrm{~N} \\
116.577 \mathrm{~W}\end{array}$ & 47.0 & 1.9 & $\begin{array}{l}135 \\
\cup p \\
045\end{array}$ & $\begin{array}{l}.22 \\
.35 \\
.23\end{array}$ & $\begin{array}{l}4.7 \\
4.2 \\
4.8\end{array}$ \\
\hline & Note: Two additional & records 1 & \multicolumn{3}{|c|}{ recovered at Morongo Valley } & \multicolumn{2}{|c|}{ Fire Station. } \\
\hline & $\begin{array}{l}\text { North Palm Springs } \\
\text { Post office } \\
\text { (USGS) }\end{array}$ & $\begin{array}{r}33.924 \mathrm{~N} \\
116.543 \mathrm{~W}\end{array}$ & 47.55 & 2.0 & $\begin{array}{l}300 \\
\text { Up } \\
210\end{array}$ & $\begin{array}{l}.68 \\
.78 \\
.70\end{array}$ & $\begin{array}{l}6.0 \\
5.6 \\
5.3\end{array}$ \\
\hline
\end{tabular}

Note: One additional record ${ }^{1}$ recovered at $N$. Palm Springs Post office. 
Table 7. Summary of United States accelerograph records recovered during 1986-Continued

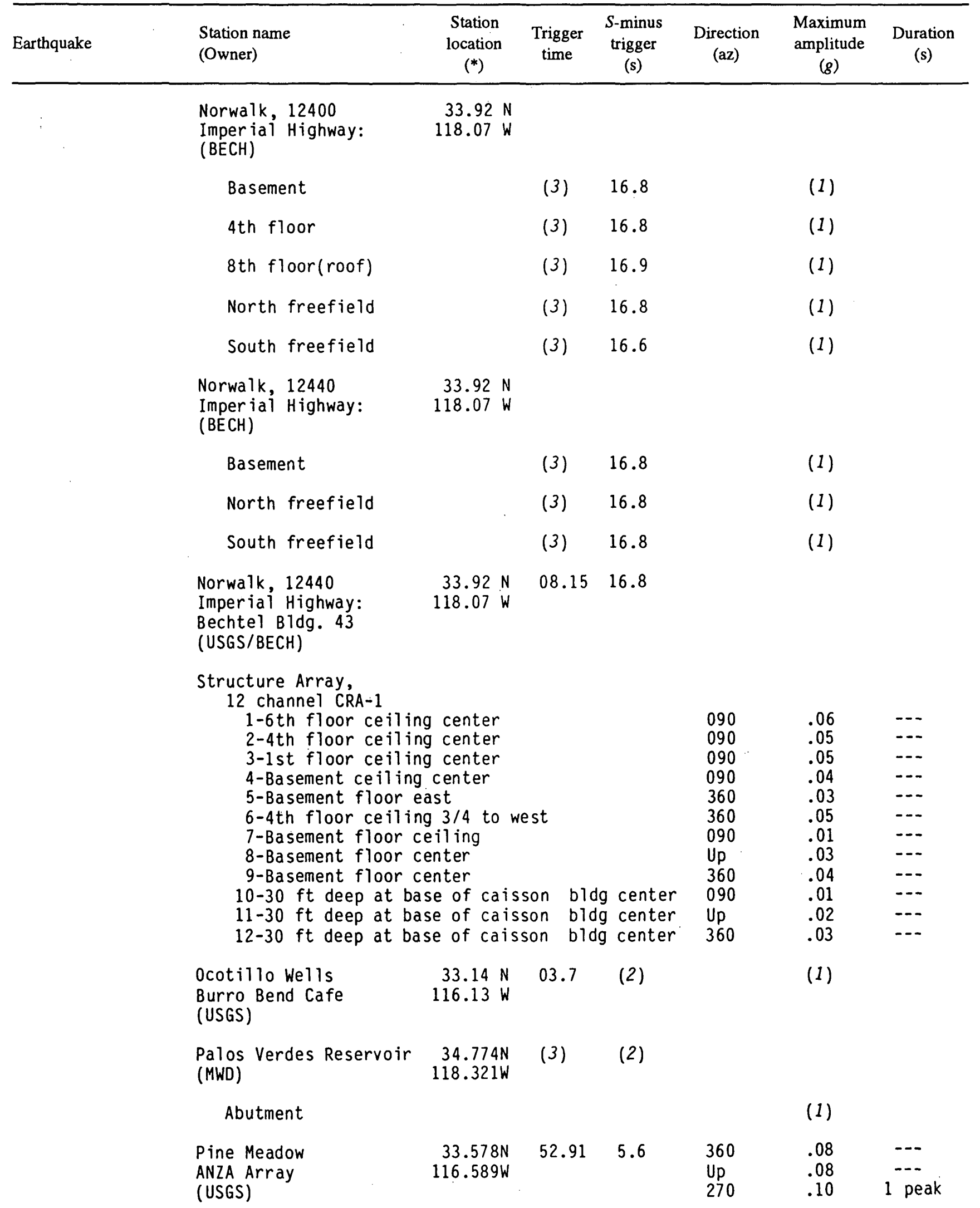


Table 7. Summary of United States accelerograph records recovered during 1986-Continued

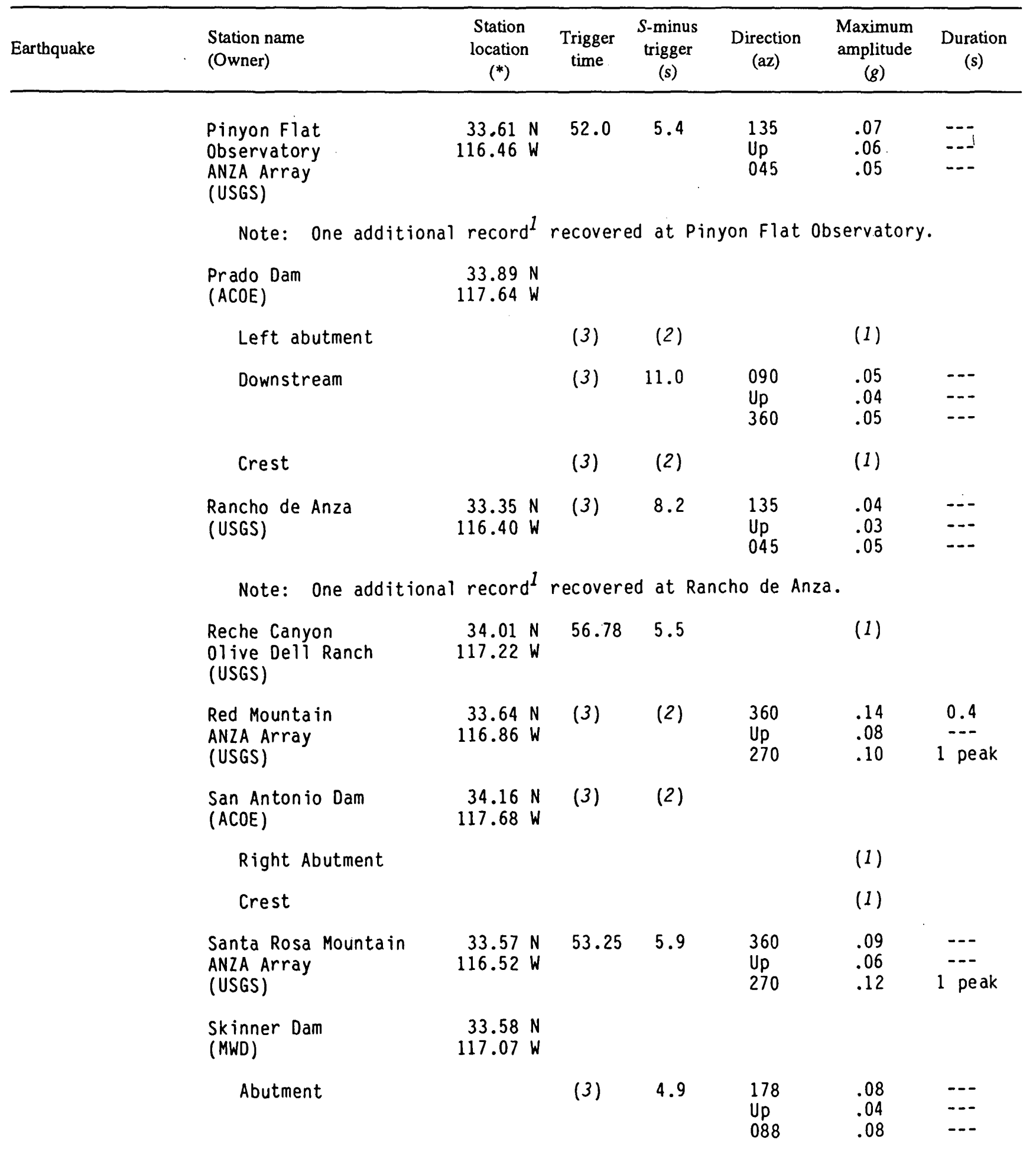


Table 7. Summary of United States accelerograph records recovered during 1986 - Continued

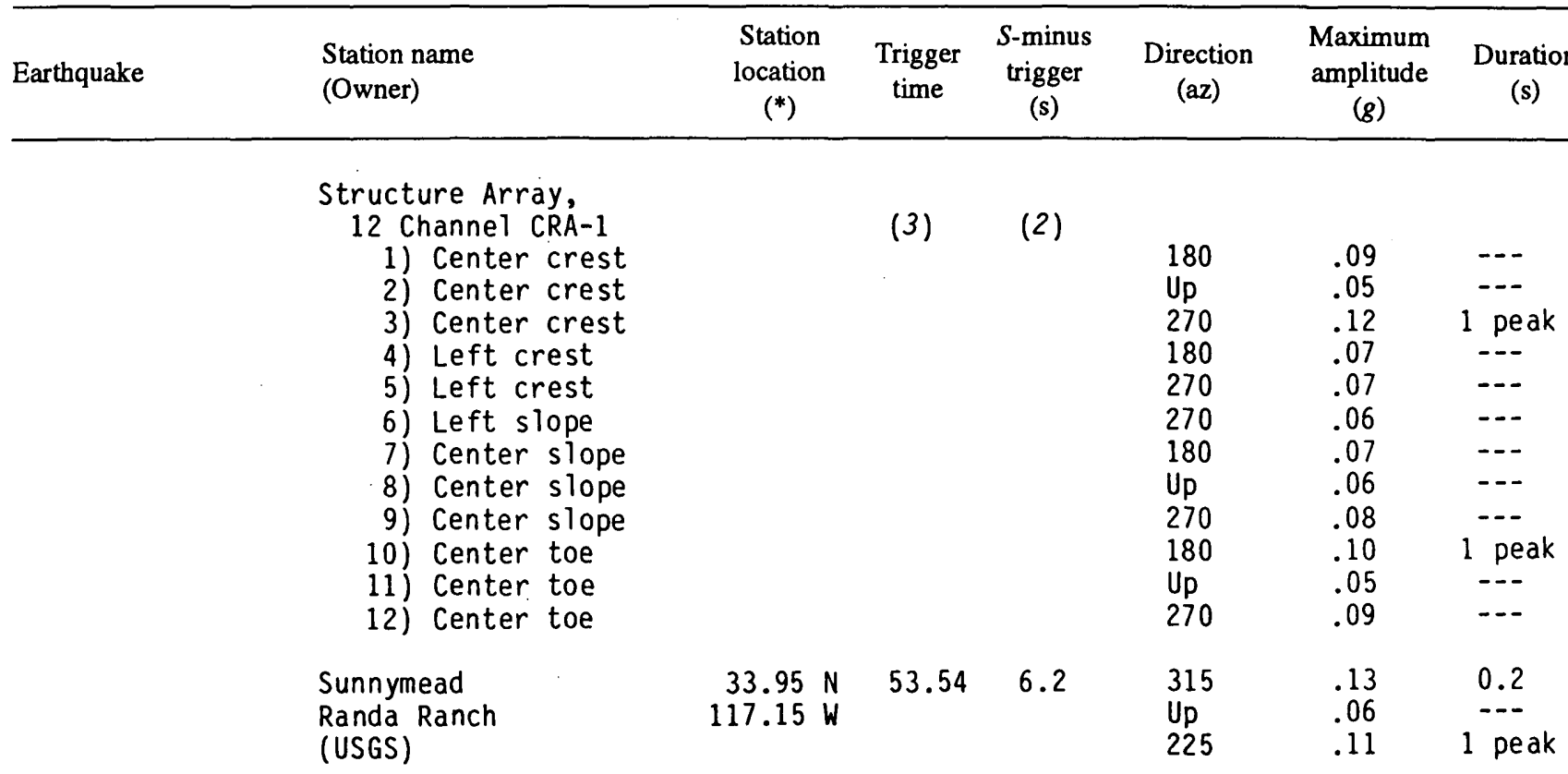

Note: Two additional records ${ }^{1}$ recovered at Sunnymead.

\begin{tabular}{|c|c|c|c|c|c|c|}
\hline $\begin{array}{l}\text { Terwilliger Valley } \\
\text { ANZA Array } \\
\text { (USGS) }\end{array}$ & $\begin{array}{r}33.48 \mathrm{~N} \\
116.59 \mathrm{~W}\end{array}$ & (3) & 6.5 & $\begin{array}{l}135 \\
\text { Up } \\
045\end{array}$ & $\begin{array}{l}.03 \\
.04 \\
.07\end{array}$ & --- \\
\hline $\begin{array}{l}\text { Tripp Flats } \\
\text { ANZA Array } \\
\text { (USGS) }\end{array}$ & $\begin{array}{r}33.60 \mathrm{~N} \\
116.74 \mathrm{~W}\end{array}$ & 53.98 & 4.5 & $\begin{array}{l}360 \\
\text { Up } \\
270\end{array}$ & $\begin{array}{l}.05 \\
.05 \\
.08\end{array}$ & $\cdots$ \\
\hline $\begin{array}{l}\text { Tule Canyon } \\
\text { ANZA Array } \\
\text { (USGS) }\end{array}$ & $\begin{array}{r}33.47 \mathrm{~N} \\
116.64 \mathrm{~W}\end{array}$ & (3) & 6.9 & $\begin{array}{l}360 \\
\text { Up } \\
270\end{array}$ & $\begin{array}{l}.10 \\
.04 \\
.11\end{array}$ & $\begin{array}{l}1 \text { peak } \\
1 \text { peak }\end{array}$ \\
\hline
\end{tabular}

Note: One additional record ${ }^{1}$ recovered at Tule Canyon.

Weymouth Filter Plant $\quad 34.506 \mathrm{~N}$

(MWD)

$117.778 \mathrm{~W}$

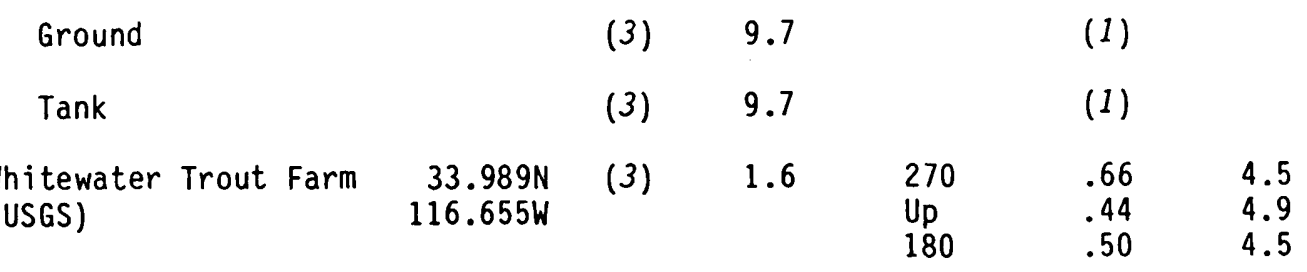

Note: 20 additional records ${ }^{1}$ recovered at Whitewater Trout Farm.

Whittier
7215 Bright Ave.
(USGS)

Basement

5 th floor

10th floor 
Table 7. Summary of United States accelerograph records recovered during 1986-Continued

\begin{tabular}{|c|c|c|c|c|c|c|c|}
\hline Earthquake & $\begin{array}{l}\text { Station name } \\
\text { (Owner) }\end{array}$ & $\begin{array}{l}\text { Station } \\
\text { location } \\
\left(^{*}\right)\end{array}$ & $\begin{array}{l}\text { Trigger } \\
\text { time }\end{array}$ & $\begin{array}{l}S \text {-minus } \\
\text { trigger } \\
(s)\end{array}$ & $\begin{array}{l}\text { Direction } \\
\quad(a z)\end{array}$ & $\begin{array}{l}\text { Maximum } \\
\text { amplitude } \\
\text { (g) }\end{array}$ & $\begin{array}{c}\text { Duration } \\
\text { (s) }\end{array}$ \\
\hline \multirow{3}{*}{$\begin{array}{l}8 \text { July } 1986 \\
\text { 0924 G.m.t. } \\
\text { Southern Calif. } \\
\text { Epicenters and } \\
\text { magnitudes unknown }\end{array}$} & $\begin{array}{l}\text { Morongo Valley } \\
\text { Fire Station } \\
\text { (USGS) }\end{array}$ & $\begin{array}{r}34.048 \mathrm{~N} \\
116.577 \mathrm{~W}\end{array}$ & 15.2 & 2.2 & $\begin{array}{l}135 \\
\text { Up. } \\
045\end{array}$ & $\begin{array}{l}.03 \\
.08 \\
.05\end{array}$ & $\begin{array}{l}--- \\
--- \\
---\end{array}$ \\
\hline & Note: One additional & record 1 & recovered & d at Mo & ingo Valley & Fire stat & ion. \\
\hline & $\begin{array}{l}\text { North Palm Springs } \\
\text { Post office } \\
\text { (USGS) }\end{array}$ & $\begin{array}{r}33.924 \mathrm{~N} \\
116.543 \mathrm{~W}\end{array}$ & 16.5 & 2.8 & $\begin{array}{l}300 \\
\text { Up } \\
210\end{array}$ & $\begin{array}{l}.05 \\
.03 \\
.06\end{array}$ & $\begin{array}{l}--- \\
--- \\
---\end{array}$ \\
\hline
\end{tabular}

\begin{abstract}
8 July 1986
0928 G.m.t.

Southern Calif.

Epicenters and

magnitudes unknown

8 July 1986

0930:23.6 G.m.t.

Southern Calif.

$33.980 \mathrm{~N}, 116.620 \mathrm{~W}$

Magnitude $3.6 \mathrm{ML}$
\end{abstract}

Note: One additional record ${ }^{l}$ recovered at North Palm Springs Post Office.

$\begin{array}{lrrr}\text { North Palm Springs } & 33.924 \mathrm{~N} & 17.0 & 2.1 \\ \text { Post office } & 116.543 \mathrm{~W} & & \\ \text { (USGS) } & & \end{array}$

Note: Two additional records ${ }^{l}$ recovered at North Palm Springs Post Office.

$$
\begin{array}{lrrr}
\text { Cabazon. Post office } & 33.918 \mathrm{~N} & 27.7 & 0.2 \\
\text { (USGS) } & 116.782 \mathrm{~W} & &
\end{array}
$$

\begin{tabular}{|c|c|c|c|c|c|}
\hline $\begin{array}{l}\text { Fun Valley } \\
\text { Reservoir } 261 \\
\text { (USGS) }\end{array}$ & $\begin{array}{r}33.925 \mathrm{~N} \\
116.389 \mathrm{~W}\end{array}$ & 29.4 & (2) & $\begin{array}{l}135 \\
\text { Up } \\
045\end{array}$ & $\begin{array}{l}.04 \\
.02 \\
.05\end{array}$ \\
\hline
\end{tabular}

Note: One additional record ${ }^{l}$ recovered at Cabazon.

Note: One additional record ${ }^{l}$ recovered at Fun Valley.

\section{July 1986}

0932:20.8 G.m.t.

Southern Calif.

$33.980 \mathrm{~N}, 116.620 \mathrm{~W}$

Magnitude $3.1 \mathrm{ML}$

8 July 1986

0949:49.7 G.m.t.

Southern Calif.

$33.990 \mathrm{~N}, 116.560 \mathrm{~W}$

Magnitude $3.5 \mathrm{ML}$

\section{July 1986}

$1004: 52.9$ G.m.t.

Southern Calif.

$33.960 \mathrm{~N}, 116.580 \mathrm{~W}$

Magnitude $3.4 \mathrm{ML}$

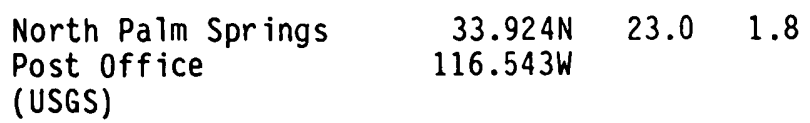

Note: One additional record ${ }^{1}$ recovered at North Palm Springs Post Office.

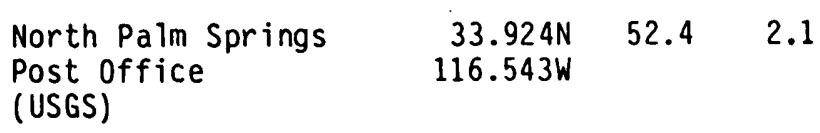

\begin{tabular}{|c|c|c|}
\hline $\begin{array}{l}\text { North Palm Springs } \\
\text { Post office } \\
\text { (USGS) }\end{array}$ & $\begin{array}{r}33.924 \mathrm{~N} \\
116.543 \mathrm{~W}\end{array}$ & 55.0 \\
\hline
\end{tabular}

Note: One additional record ${ }^{1}$ recovered at North Palm Springs Post Office.

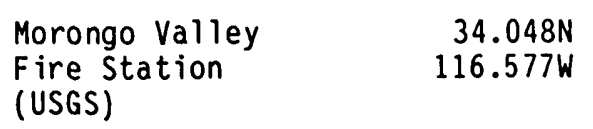

$$
116.577 \mathrm{~W}
$$

$\begin{array}{rlllll}33.924 \mathrm{~N} & 05.1 & 1.7 & 300 & .17 & 0.1 \\ 116.543 \mathrm{~W} & & & \text { Up } & .06 & -1 .- \\ & & & 210 & .14 & 0.3\end{array}$

$\begin{array}{lll}33.918 N & 09.3 & 0.3\end{array}$ 
Table 7. Summary of United States accelerograph records recovered during 1986-Continued

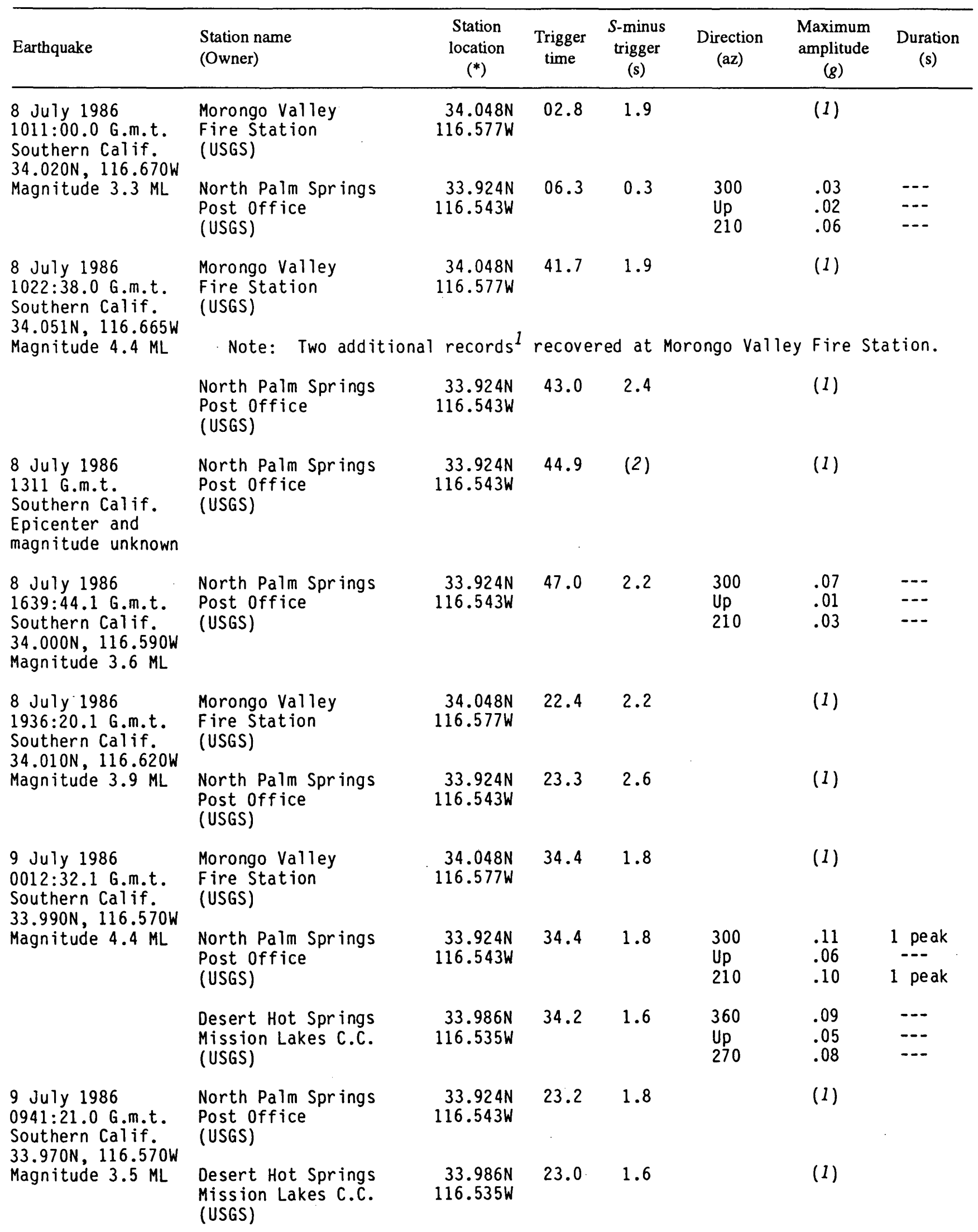


Table 7. Summary of United States accelerograph records recovered during 1986—Continued

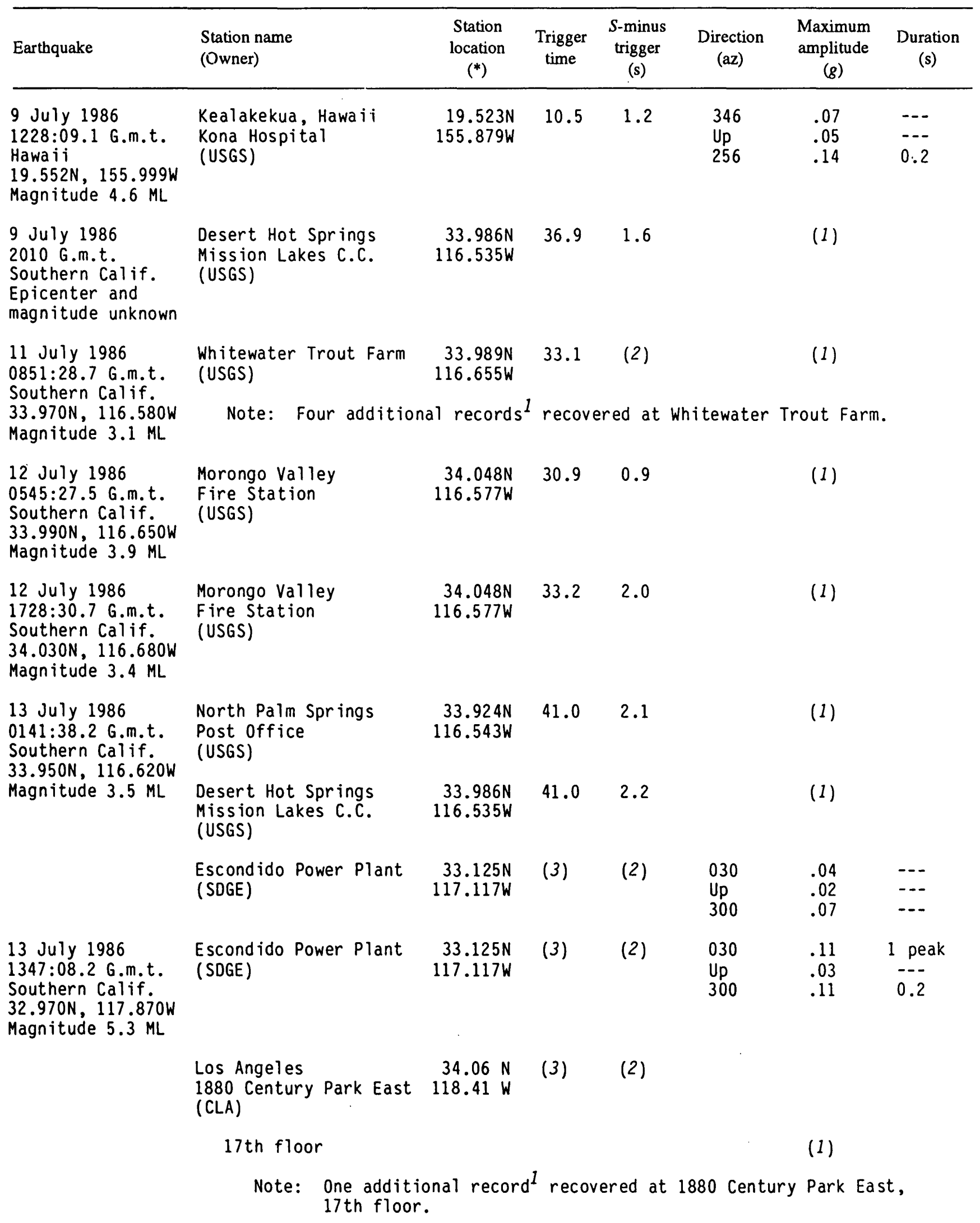


Table 7. Summary of United States accelerograph records recovered during 1986_Continued

\begin{tabular}{|c|c|c|c|c|c|c|c|}
\hline Earthquake & $\begin{array}{l}\text { Station name } \\
\text { (Owner) }\end{array}$ & $\begin{array}{l}\text { Station } \\
\text { location } \\
\left(^{*}\right)\end{array}$ & $\begin{array}{l}\text { Trigger } \\
\text { time }\end{array}$ & $\begin{array}{l}S \text {-minus } \\
\text { trigger } \\
\quad(s)\end{array}$ & $\begin{array}{l}\text { Direction } \\
\quad(a z)\end{array}$ & $\begin{array}{l}\text { Maximum } \\
\text { amplitude } \\
(g)\end{array}$ & $\begin{array}{l}\text { Duration } \\
\text { (s) }\end{array}$ \\
\hline & $\begin{array}{l}\text { Los Angeles } \\
2029 \text { Century Park East } \\
\text { (CLA) }\end{array}$ & $\begin{array}{r}34.060 \mathrm{~N} \\
118.413 \mathrm{~W}\end{array}$ & (3) & (2) & & & \\
\hline & 30 th floor & & & & & (1) & \\
\hline & 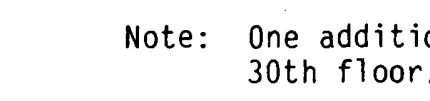 & onal recorc & $\mathrm{d}^{1}$ recov & ered at & 2029 Centur & ry Park E & East, \\
\hline & $\begin{array}{l}\text { Los Angeles } \\
2049 \text { Century Park East } \\
\text { (CLA) }\end{array}$ & $\begin{array}{r}34.06 \mathrm{~N} \\
118.41 \mathrm{~W}\end{array}$ & (3) & (2) & & & \\
\hline & 30 th floor & & & & & (1) & \\
\hline & 43 th floor & & & & & (1) & \\
\hline & $\begin{aligned} \text { Note: } & \text { Two each ac } \\
& \text { East, } 30 \text { th }\end{aligned}$ & $\begin{array}{l}\text { dditional } r \\
\text { floor and }\end{array}$ & $\begin{array}{l}\text { records } \\
43 \mathrm{th} \mathrm{fl}\end{array}$ & $\begin{array}{l}\text { recover } \\
\text { oor. }\end{array}$ & ed at 2049 & Century & Park \\
\hline & $\begin{array}{l}\text { Mission Power station } \\
\text { (SDGE) }\end{array}$ & $\begin{array}{r}32.788 \mathrm{~N} \\
117.138 \mathrm{~W}\end{array}$ & (3) & (2) & $\begin{array}{l}150 \\
\text { Up } \\
060\end{array}$ & $\begin{array}{l}.05 \\
.06 \\
.07\end{array}$ & --- \\
\hline & $\begin{array}{l}\text { San Diego VA Hospital } \\
\text { La Jolla, Bldg } 1 \\
\text { (VA) }\end{array}$ & $\begin{array}{r}32.87 \mathrm{~N} \\
117.23 \mathrm{~W}\end{array}$ & (3) & 6.4 & & & \\
\hline & Basement & & & & $\begin{array}{l}180 \\
\text { Up } \\
090\end{array}$ & $\begin{array}{l}.05 \\
.05 \\
.07\end{array}$ & --- \\
\hline \multirow{7}{*}{$\begin{array}{l}17 \text { July } 1986 \\
2035: 15.0 \text { G.m.t. } \\
\text { Southern Calif. } \\
33.990 \mathrm{~N}, 116.650 \mathrm{~W} \\
\text { Magnitude } 4.0 \mathrm{ML}\end{array}$} & $\begin{array}{l}\text { Morongo Valley } \\
\text { Fire Station } \\
\text { (USGS) }\end{array}$ & $\begin{array}{r}34.048 N \\
116.577 W\end{array}$ & 17.3 & 1.5 & $\begin{array}{l}135 \\
\text { Up } \\
045\end{array}$ & $\begin{array}{l}.04 \\
.04 \\
.07\end{array}$ & $\begin{array}{l}--- \\
--- \\
---\end{array}$ \\
\hline & $\begin{array}{l}\text { North Palm Springs } \\
\text { Post office } \\
\text { (USGS) }\end{array}$ & $\begin{array}{r}33.924 \mathrm{~N} \\
116.543 \mathrm{~W}\end{array}$ & 17.8 & 2.2 & $\begin{array}{l}300 \\
\text { Up } \\
210\end{array}$ & $\begin{array}{l}.04 \\
.03 \\
.08\end{array}$ & $\begin{array}{l}--- \\
---\end{array}$ \\
\hline & $\begin{array}{l}\text { Whitewater Trout Farm } \\
\text { (USGS) }\end{array}$ & $\begin{array}{l}33.989 \mathrm{~N} \\
116.655\end{array}$ & 16.3 & 1.2 & $\begin{array}{l}270 \\
\text { Up } \\
180\end{array}$ & $\begin{array}{l}.14 \\
.08 \\
.11\end{array}$ & $\begin{array}{l}1 \text { peak } \\
--- \\
.05\end{array}$ \\
\hline & $\begin{array}{l}\text { Keenwild Forest Station } \\
\text { ANZA Array } \\
\text { (USGS) }\end{array}$ & $\begin{array}{r}33.71 \mathrm{~N} \\
116.71 \mathrm{~W}\end{array}$ & 20.6 & 4.0 & & (1) & \\
\hline & $\begin{array}{l}\text { Desert Hot Springs } \\
\text { Mission Lakes C.C. } \\
\text { (USGS) }\end{array}$ & $\begin{array}{r}33.986 N \\
116.535 W\end{array}$ & 35.2 & 1.9 & $\begin{array}{l}360 \\
\text { Up } \\
270\end{array}$ & $\begin{array}{l}.05 \\
.04 \\
.13\end{array}$ & 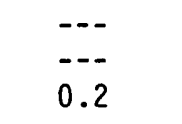 \\
\hline & $\begin{array}{l}\text { W. Palm Springs village } \\
\text { St. John's School } \\
\text { (USGS) }\end{array}$ & $\begin{array}{r}33.925 \mathrm{~N} \\
116.680 \mathrm{~W}\end{array}$ & 16.6 & 1.3 & $\begin{array}{l}360 \\
\text { Up } \\
270\end{array}$ & $\begin{array}{l}.08 \\
.04 \\
.05\end{array}$ & $\begin{array}{l}--- \\
--- \\
---\end{array}$ \\
\hline & $\begin{array}{l}\text { Morongo Valley } \\
\text { Canyon House } \\
\text { (USGS) }\end{array}$ & $\begin{array}{r}34.347 N \\
116.604 W\end{array}$ & 17.3 & (2) & $\begin{array}{l}360 \\
\text { Up } \\
270\end{array}$ & $\begin{array}{l}.08 \\
.07 \\
.08\end{array}$ & --- \\
\hline
\end{tabular}


Table 7. Summary of United States accelerograph records recovered during 1986-Continued

\begin{tabular}{|c|c|c|c|c|c|c|c|}
\hline Earthquake & $\begin{array}{l}\text { Station name } \\
\text { (Owner) }\end{array}$ & $\begin{array}{l}\text { Station } \\
\text { location } \\
(*)\end{array}$ & $\begin{array}{l}\text { Trigger } \\
\text { time }\end{array}$ & $\begin{array}{l}S \text {-minus } \\
\text { trigger } \\
(\mathrm{s})\end{array}$ & $\begin{array}{l}\text { Direction } \\
\quad(a z)\end{array}$ & $\begin{array}{l}\text { Maximum } \\
\text { amplitude } \\
\qquad(g)\end{array}$ & $\begin{array}{l}\text { Duration } \\
\text { (s) }\end{array}$ \\
\hline \multirow{8}{*}{$\begin{array}{l}17 \text { July } 1986 \\
2154: 45.1 \text { G.m.t. } \\
\text { Southern Calif. } \\
33.990 \mathrm{~N}, 116.650 \mathrm{~W} \\
\text { Magnitude } 4.4 \mathrm{ML}\end{array}$} & $\begin{array}{l}\text { Cabazon Post office } \\
\text { (USGS) }\end{array}$ & $\begin{array}{r}33.918 \mathrm{~N} \\
116.782 \mathrm{~W}\end{array}$ & 50.3 & 0.2 & & $(1)$ & \\
\hline & $\begin{array}{l}\text { Desert Hot Springs } \\
\text { Mission Lakes C.C. } \\
\text { (USGS) }\end{array}$ & $\begin{array}{r}33.986 \mathrm{~N} \\
116.535 \mathrm{~W}\end{array}$ & 48.0 & 1.8 & $\begin{array}{l}360 \\
\text { Up } \\
270\end{array}$ & $\begin{array}{l}.06 \\
.02 \\
.07\end{array}$ & --- \\
\hline & $\begin{array}{l}\text { Millard Canyon } \\
\text { (USGS) }\end{array}$ & $\begin{array}{r}33.98 \mathrm{~N} \\
116.78 \mathrm{~W}\end{array}$ & 50.6 & (2) & & (1) & \\
\hline & Note: One additiona & 1 record 1 & recover & ed at $M i$ & lard Cany & & \\
\hline & $\begin{array}{l}\text { Morongo Valley } \\
\text { Fire Station } \\
\text { (USGS) }\end{array}$ & $\begin{array}{r}34.048 \mathrm{~N} \\
116.577 \mathrm{~W}\end{array}$ & 47.4 & 1.7 & & (1) & \\
\hline & $\begin{array}{l}\text { North Palm Springs } \\
\text { Post office } \\
\text { (USGS) }\end{array}$ & $\begin{array}{r}33.924 N \\
116.543 W\end{array}$ & 48.8 & 2.2 & $\begin{array}{l}300 \\
\text { Up } \\
210\end{array}$ & $\begin{array}{l}.03 \\
.03 \\
.05\end{array}$ & --- \\
\hline & $\begin{array}{l}\text { W. Palm Springs village } \\
\text { St. John's School } \\
\text { (USGS) }\end{array}$ & $\begin{array}{r}33.925 \mathrm{~N} \\
116.680 \mathrm{~W}\end{array}$ & 46.9 & 1.3 & $\begin{array}{l}360 \\
\text { Up } \\
270\end{array}$ & $\begin{array}{l}.08 \\
.04 \\
.07\end{array}$ & --- \\
\hline & $\begin{array}{l}\text { Whitewater Trout Farm } \\
\text { (USGS) }\end{array}$ & $\begin{array}{r}33.989 \mathrm{~N} \\
116.655 \mathrm{~W}\end{array}$ & 46.6 & 1.1 & $\begin{array}{l}270 \\
\text { Up } \\
180\end{array}$ & $\begin{array}{l}.16 \\
.08 \\
.16\end{array}$ & $\begin{array}{l}.55 \\
--- \\
.35\end{array}$ \\
\hline \multirow{6}{*}{$\begin{array}{l}18 \text { July } 1986 \\
0718: 05.4 \mathrm{G.m.t.} \\
\text { Eastern Calif. } \\
37.575 \mathrm{~N}, 118.827 \mathrm{~W} \\
\text { Magnitude } 3.0 \mathrm{ML}\end{array}$} & $\begin{array}{l}\text { McGee Creek, SMA } \\
\text { (USGS) }\end{array}$ & $\begin{array}{r}37.550 \mathrm{~N} \\
118.811 \mathrm{~W}\end{array}$ & 07.4 & (2) & & (1) & \\
\hline & $\begin{array}{l}\text { MCGee Creek, CRA } \\
\text { (USGS) }\end{array}$ & $\begin{array}{r}37.550 \mathrm{~N} \\
118.811 \mathrm{~W}\end{array}$ & 07.5 & (2) & & & \\
\hline & $166 \mathrm{~m}$ downhole & & & & & (1) & \\
\hline & $35 \mathrm{~m}$ downhole & & & & & (1) & \\
\hline & Surface & & & & & (1) & \\
\hline & $1 \mathrm{~m}$ downhole & & & & & (1) & \\
\hline $\begin{array}{l}18 \text { July } 1986 \\
1958: 01.8 \mathrm{G} . \mathrm{m} . \mathrm{t} . \\
\text { Southern Calif. } \\
33.970 \mathrm{~N}, 116.570 \mathrm{~W} \\
\text { Magnitude } 3.2 \mathrm{ML}\end{array}$ & $\begin{array}{l}\text { Desert Hot Springs } \\
\text { Mission Lakes C.C. } \\
\text { (USGS) }\end{array}$ & $\begin{array}{r}33.986 \mathrm{~N} \\
116.535 \mathrm{~W}\end{array}$ & 04.0 & 2.2 & & (1) & \\
\hline $\begin{array}{l}20 \text { July } 1986 \\
1429: 45.5 \text { G.m.t. } \\
\text { Eastern Calif. } \\
37.580 \mathrm{~N}, 118.450 \mathrm{~W} \\
\text { Magnitude } 5.9 \mathrm{ML}\end{array}$ & $\begin{array}{l}\text { Long Valley Dam } \\
\text { Lake Crowley } \\
\text { (USGS) } \\
\quad \text { Left abutment }\end{array}$ & $\begin{array}{r}37.588 \mathrm{~N} \\
118.705 \mathrm{~W}\end{array}$ & (3) & 3.3 & $\begin{array}{l}275 \\
\text { Up } \\
185\end{array}$ & $\begin{array}{l}.07 \\
.07 \\
.15\end{array}$ & 1.0 \\
\hline
\end{tabular}


Table 7. Summary of United States accelerograph records recovered during 1986-Continued

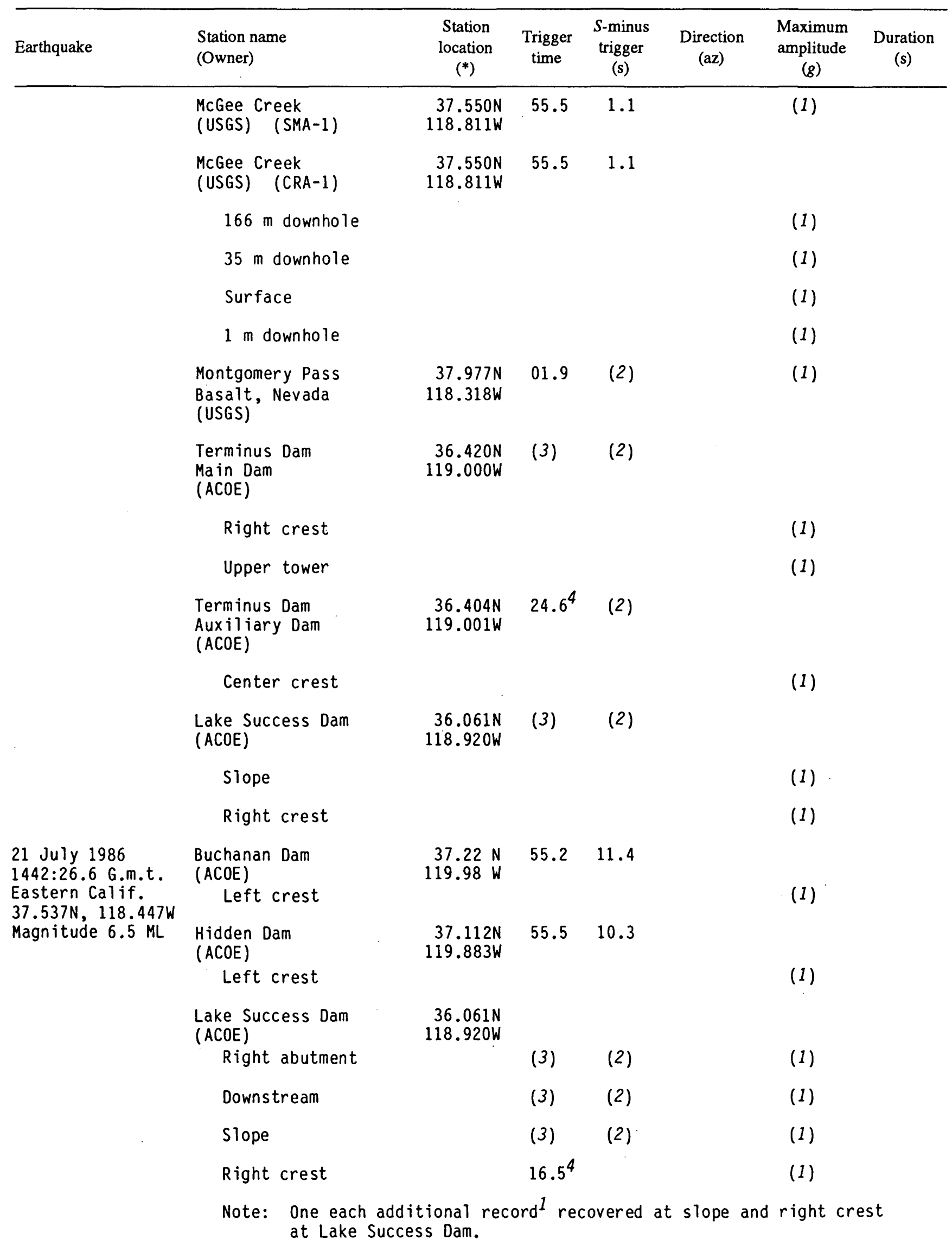


Table 7. Summary of United States accelerograph records recovered during 1986-Continued

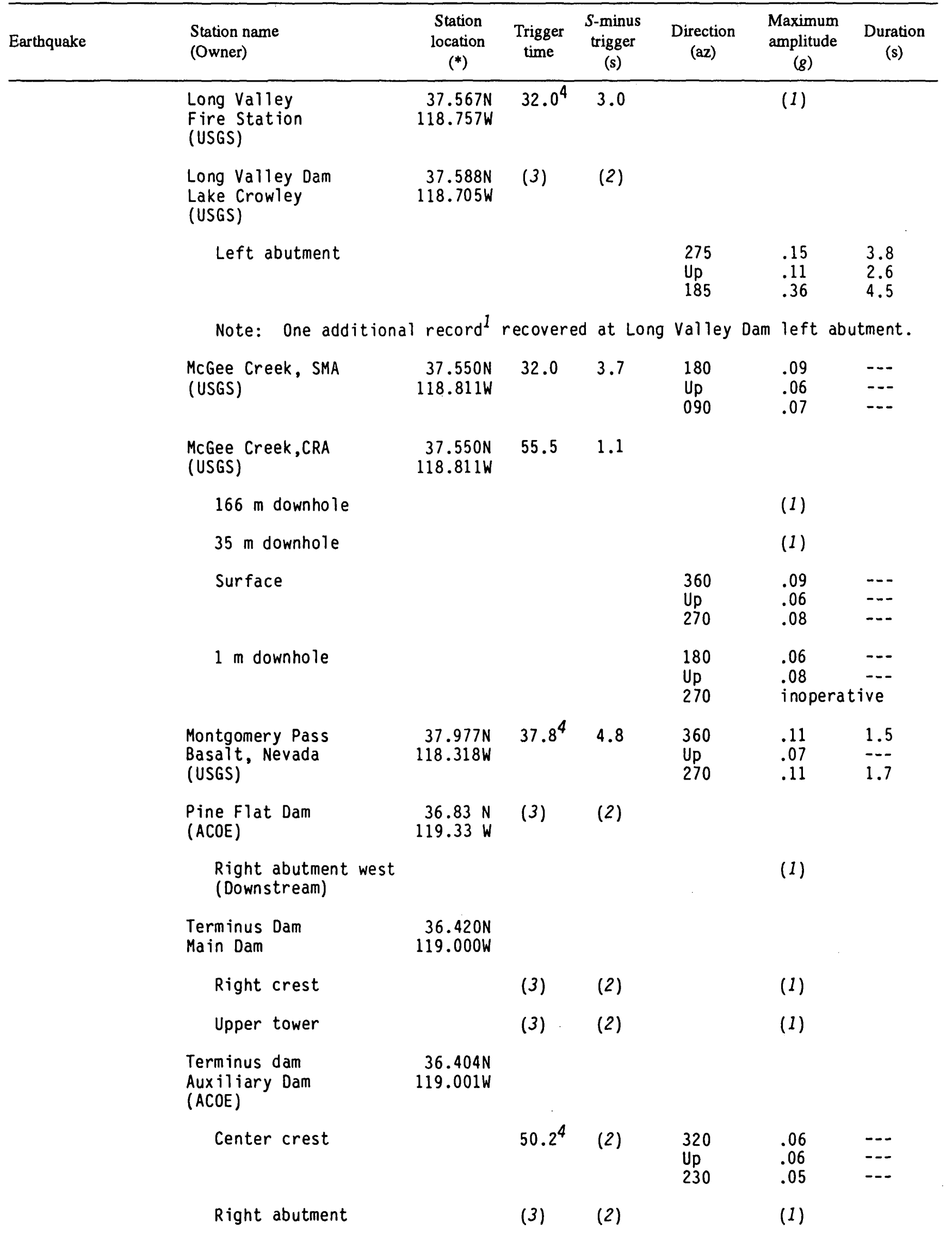

See footnotes at end of table 
Table 7. Summary of United States accelerograph records recovered during 1986 Continued

\begin{tabular}{|c|c|c|c|c|c|c|c|}
\hline Earthquake & $\begin{array}{l}\text { Station name } \\
\text { (Owner) }\end{array}$ & $\begin{array}{l}\text { Station } \\
\text { location } \\
\left({ }^{*}\right)\end{array}$ & $\begin{array}{l}\text { Trigger } \\
\text { time }\end{array}$ & $\begin{array}{l}S \text {-minus } \\
\text { trigger } \\
(\mathrm{s})\end{array}$ & $\begin{array}{l}\text { Direction } \\
\quad(\mathrm{az})\end{array}$ & $\begin{array}{l}\text { Maximum } \\
\text { amplitude } \\
\quad(g)\end{array}$ & $\begin{array}{l}\text { Duration } \\
\quad \text { (s) }\end{array}$ \\
\hline \multirow{9}{*}{$\begin{array}{l}21 \text { July } 1986 \\
1451: 11.0 \mathrm{G} . \mathrm{m} . \mathrm{t} . \\
\text { Eastern Calif. } \\
37.520 \mathrm{~N}, 118.412 \mathrm{~W} \\
\text { Magnitude } 5.7 \mathrm{ML}\end{array}$} & $\begin{array}{l}\text { McGee Creek, SMA } \\
\text { (USGS) }\end{array}$ & $\begin{array}{r}37.550 \mathrm{~N} \\
118.811 \mathrm{~W}\end{array}$ & (3) & (2) & & (1) & \\
\hline & $\begin{array}{l}\text { McGee Creek, CRA } \\
\text { (USGS) }\end{array}$ & $\begin{array}{r}37.550 \mathrm{~N} \\
118.811 \mathrm{~W}\end{array}$ & (3) & (2) & & & \\
\hline & $166 \mathrm{~m}$ downhole & & & & & (1) & \\
\hline & $35 \mathrm{~m}$ downhole & & & & & (1) & \\
\hline & Surface & & & & & (1) & \\
\hline & $1 \mathrm{~m}$ downhole & & & & & (1) & \\
\hline & $\begin{array}{l}\text { Montgomery Pass } \\
\text { Basalt, Nevada } \\
\text { (USGS) }\end{array}$ & $\begin{array}{r}37.977 \mathrm{~N} \\
118.318 \mathrm{~W}\end{array}$ & $29.3^{4}$ & (2) & & (1) & \\
\hline & $\begin{array}{l}\text { Terminus Dam } \\
\text { Auxiliary Dam } \\
\text { (ACOE) }\end{array}$ & $\begin{array}{r}36.404 \mathrm{~N} \\
119.001 \mathrm{~W}\end{array}$ & $46.5^{4}$ & (2) & & & \\
\hline & Center crest & & & & & (1) & \\
\hline \multirow{2}{*}{$\begin{array}{l}21 \text { July } 1986 \\
2207: 18.0 \mathrm{G} . \mathrm{m.t} . \\
\text { Eastern Calif. } \\
37.498 \mathrm{~N}, 118.397 \mathrm{~W} \\
\text { Magnitude } 5.6 \mathrm{ML}\end{array}$} & $\begin{array}{l}\text { Long Valley Dam } \\
\text { Lake Crowley } \\
\text { (USGS) }\end{array}$ & $\begin{array}{r}37.588 \mathrm{~N} \\
118.705 \mathrm{~W}\end{array}$ & & & & & \\
\hline & Left abutment & & (3) & 3.7 & $\begin{array}{l}275 \\
\text { Up } \\
185\end{array}$ & $\begin{array}{l}.09 \\
.04 \\
.19\end{array}$ & $\begin{array}{l}--. \\
--. \\
0.7\end{array}$ \\
\hline
\end{tabular}

Note: Six additional records ${ }^{1}$ recovered at Long Valley Dam left abutment.

\begin{tabular}{|c|c|c|}
\hline $\begin{array}{l}\text { McGee Creek, SMA } \\
\text { (USGS) }\end{array}$ & $\begin{array}{r}37.550 \mathrm{~N} \\
118.811 \mathrm{~W}\end{array}$ & (3) \\
\hline $\begin{array}{l}\text { McGee Creek, CRA } \\
\text { (USGS) }\end{array}$ & $\begin{array}{r}37.550 \mathrm{~N} \\
118.811 \mathrm{~W}\end{array}$ & (3) \\
\hline \multicolumn{3}{|l|}{$166 \mathrm{~m}$ downhole } \\
\hline \multicolumn{3}{|l|}{$35 \mathrm{~m}$ downhole } \\
\hline \multicolumn{3}{|l|}{ Surface } \\
\hline \multicolumn{3}{|l|}{$1 \mathrm{~m}$ downhole } \\
\hline $\begin{array}{l}\text { Montgomery Pass } \\
\text { Basalt, Nevada } \\
\text { (USGS) }\end{array}$ & $\begin{array}{r}37.977 N \\
118.318 W\end{array}$ & $31.5^{4}$ \\
\hline $\begin{array}{l}\text { TerminusDam } \\
\text { Auxiliary Dam } \\
\text { (ACOE) }\end{array}$ & $\begin{array}{r}36.404 \mathrm{~N} \\
119.001 \mathrm{~W}\end{array}$ & $16.1^{4}$ \\
\hline
\end{tabular}


Table 7. Summary of United States accelerograph records recovered during 1986-Continued

\begin{tabular}{|c|c|c|c|c|c|c|c|}
\hline Earthquake & $\begin{array}{l}\text { Station name } \\
\text { (Owner) }\end{array}$ & $\begin{array}{l}\text { Station } \\
\text { location } \\
(*)\end{array}$ & $\begin{array}{l}\text { Trigger } \\
\text { time }\end{array}$ & $\begin{array}{l}S \text {-minus } \\
\text { trigger } \\
\text { (s) }\end{array}$ & $\begin{array}{l}\text { Direction } \\
\quad(a z)\end{array}$ & $\begin{array}{l}\text { Maximum } \\
\text { amplitude } \\
\text { (g) }\end{array}$ & $\begin{array}{c}\text { Duration } \\
\text { (s) }\end{array}$ \\
\hline $\begin{array}{l}22 \text { July } 1986 \\
2017: 00.1 \text { G.m.t. } \\
\text { Eastern Calif. } \\
37.554 \mathrm{~N}, 118.359 \mathrm{~W} \\
\text { Magnitude } 4.2 \mathrm{ML}\end{array}$ & $\begin{array}{l}\text { Laws, Calif. } \\
\text { Northeast Bishop } \\
\text { (USGS) }\end{array}$ & $\begin{array}{r}37.402 \mathrm{~N} \\
118.346 \mathrm{~W}\end{array}$ & (3) & (2) & & (1) & \\
\hline $\begin{array}{l}22 \text { July } 1986 \\
2206: 41.8 \mathrm{G.m.t} . \\
\text { Eastern Cal if. } \\
37.513 \mathrm{~N}, 118.294 \mathrm{~W} \\
\text { Magnitude } 4.2 \mathrm{ML}\end{array}$ & $\begin{array}{l}\text { Chalfant Valley } \\
\text { Fire Station } \\
\text { (USGS) } \\
\quad \text { Note: } 12 \text { additional }\end{array}$ & $\begin{array}{r}37.53 \mathrm{~N} \\
118.37 \mathrm{~W}\end{array}$ & recovered & d at $\mathrm{Ch}$ & $\begin{array}{l}360 \\
\text { Up } \\
270 \\
\text { alfant Valley }\end{array}$ & $\begin{array}{l}.06 \\
.05 \\
.08 \\
\text { y Fire S }\end{array}$ & station. \\
\hline $\begin{array}{l}23 \mathrm{July} 1986 \\
0508 \mathrm{G.m.t.} \\
\text { Eastern Calif. }\end{array}$ & $\begin{array}{l}\text { Chalfant Valley } \\
\text { Fire Station } \\
\text { (USGS) }\end{array}$ & $\begin{array}{r}37.53 \mathrm{~N} \\
118.37 \mathrm{~W}\end{array}$ & $06.1^{4}$ & 1.3 & $\begin{array}{l}360 \\
\text { Up } \\
270\end{array}$ & $\begin{array}{l}.07 \\
.04 \\
.05\end{array}$ & $\begin{array}{l}--- \\
--- \\
---\end{array}$ \\
\hline
\end{tabular}

Epicenters and magnitudes unknown

Note: 3 additional records ${ }^{1}$ recovered at Chalfant Valley Fire Station.

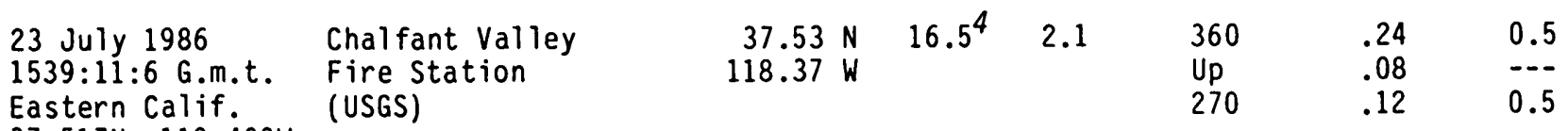

$37.517 \mathrm{~N}, 118.409 \mathrm{~W}$

Magnitude $4.7 \mathrm{MB}$

Note: 11 additional records ${ }^{1}$ recovered at Chalfant Valley Fire Station.

\begin{tabular}{|c|c|c|c|c|c|}
\hline $\begin{array}{l}\text { Hammil, Calif. } \\
\text { Cinnamon Ranch } \\
\text { (USGS) }\end{array}$ & $\begin{array}{r}37.68 \mathrm{~N} \\
118.39 \mathrm{~W}\end{array}$ & $17.0^{4}$ & 2.4 & & (1) \\
\hline $\begin{array}{l}\text { Chalfant Valley } \\
\text { Fire Station } \\
\text { (USGS) }\end{array}$ & $\begin{array}{r}37.53 \mathrm{~N} \\
118.37 \mathrm{~W}\end{array}$ & $57.4^{4}$ & 0.6 & $\begin{array}{l}360 \\
\text { Up } \\
270\end{array}$ & $\begin{array}{l}.07 \\
.01 \\
.03\end{array}$ \\
\hline
\end{tabular}

24 July 1986

$1134: 51.5$ G.m.t.

Eastern Calif.

$37.530 \mathrm{~N}, 118.367 \mathrm{~W}$

Magnitude $3.3 \mathrm{ML}$

Note: 2 additional records ${ }^{1}$ recovered at Chalfant Valley Fire Station.

24 July 1986
$1458: 45.2$ G.m.t.
Eastern Calif.
$37.514 \mathrm{~N}, 118.289 \mathrm{~W}$
Magnitude $3.7 \mathrm{ML}$

24 July 1986

1644:40.7 G.m.t.

Eastern Calif.

$37.529 \mathrm{~N}, 118.398 \mathrm{~W}$

Chalfant Valley

Fire Station

(USGS)

$37.53 \mathrm{~N}$

$118.37 \mathrm{~W}$

$48.6^{4}$

1.7

360
Up
270

.09
.07

.05

Chalfant Valley

Fire Station

$37.53 N \quad 44.9^{4}$

2.0

360
Up
270

.18

.07

0.3

(USGS)

$118.37 \mathrm{~W}$

Chalfant Valley

Fire Station

(USGS)

$\begin{array}{rlll}37.53 \mathrm{~N} & 30.0^{4} & 1.9 & \\ 118.37 \mathrm{~W} & & & 360 \\ & & & \text { Up } \\ & & & 270\end{array}$

.19
.06
.16

0.2

$270 \quad .06 \quad-16$

Eastern Calif. $37.467 \mathrm{~N}, 118.297 \mathrm{~W}$ Magnitude 4.3 ML
28 July 1986
2113 G.m.t.
Southern California
Epicenter and
magnitude unknown

Note: 11 additional records ${ }^{1}$ recovered at Chalfant Valley fire Station.

$$
\begin{array}{lrlll}
\text { Salton Sea } & 33.18 \mathrm{~N} & 24.8 & \text { (2) } \\
\text { Wildlife Refuge } & 115.62 & \mathrm{~W} & &
\end{array}
$$


Table 7. Summary of United States accelerograph records recovered during 1986—Continued

\begin{tabular}{|c|c|c|c|c|c|c|c|}
\hline Earthquake & $\begin{array}{l}\text { Station name } \\
\text { (Owner) }\end{array}$ & $\begin{array}{l}\text { Station } \\
\text { location } \\
\left.\quad{ }^{*}\right)\end{array}$ & $\begin{array}{l}\text { Trigger } \\
\text { time }\end{array}$ & $\begin{array}{l}S \text {-minus } \\
\text { trigger } \\
\quad(s)\end{array}$ & $\begin{array}{l}\text { Direction } \\
\quad \text { (az) }\end{array}$ & $\begin{array}{l}\text { Maximum } \\
\text { amplitude } \\
\quad(g)\end{array}$ & $\begin{array}{l}\text { Duration } \\
\text { (s) }\end{array}$ \\
\hline \multirow{3}{*}{$\begin{array}{l}29 \text { July } 1986 \\
0643: 50.2 \text { G.m.t. } \\
\text { Southern Calif. } \\
33.970 \mathrm{~N}, 116.590 \mathrm{~W} \\
\text { Magnitude } 3.2 \mathrm{ML}\end{array}$} & $\begin{array}{l}\text { Desert Hot Springs } \\
\text { Mission Lakes C.C. } \\
\text { (USGS) }\end{array}$ & $\begin{array}{r}33.986 \mathrm{~N} \\
116.535 \mathrm{~W}\end{array}$ & 52.3 & 1.5 & & (1) & \\
\hline & $\begin{array}{l}\text { North Palm Springs } \\
\text { Post office } \\
\text { (USGS) }\end{array}$ & $\begin{array}{r}33.924 \mathrm{~N} \\
116.543 \mathrm{~W}\end{array}$ & 52.5 & 2.0 & & (1) & \\
\hline & $\begin{array}{l}\text { Whitewater Canyon } \\
\text { Trout Farm } \\
\text { (USGS) }\end{array}$ & $\begin{array}{r}33.989 \mathrm{~N} \\
116.655 \mathrm{~W}\end{array}$ & 53.7 & (2) & & (1) & \\
\hline $\begin{array}{l}29 \text { July } 1986 \\
0957: 57.0 \text { G.m.t. } \\
\text { Eastern Cal if. } \\
37.593 \mathrm{~N}, 118.447 \mathrm{~W} \\
\text { Magnitude } 4.6 \mathrm{ML}\end{array}$ & $\begin{array}{l}\text { Hammil, Calif. } \\
\text { Cinnamon Ranch } \\
\text { (USGS) }\end{array}$ & $\begin{array}{r}37.68 \mathrm{~N} \\
118.39 \mathrm{~W}\end{array}$ & $01.2^{4}$ & 2.1 & & (1) & \\
\hline $\begin{array}{l}30 \text { July } 1986 \\
0603: 32.1 \text { G.m.t. } \\
\text { Eastern Cal if. } \\
37.633 \mathrm{~N}, 118.403 \mathrm{~W} \\
\text { Magnitude } 4.0 \mathrm{ML}\end{array}$ & $\begin{array}{l}\text { Hammil, Calif. } \\
\text { Cinnamon Ranch } \\
\text { (USGS) }\end{array}$ & $\begin{array}{r}37.68 \mathrm{~N} \\
118.39 \mathrm{~W}\end{array}$ & $36.2^{4}$ & 2.2 & & (1) & \\
\hline $\begin{array}{l}30 \text { July } 1986 \\
0641: 52.7 \text { G.m.t. } \\
\text { Eastern Cal if. } \\
37.562 \mathrm{~N}, 118.424 \mathrm{~W} \\
\text { Magnitude } 4.8 \mathrm{ML}\end{array}$ & $\begin{array}{l}\text { Hammil, Calif: } \\
\text { Cinnamon Ranch } \\
\text { (USGS) }\end{array}$ & $\begin{array}{r}37.68 \mathrm{~N} \\
118.39 \mathrm{~W}\end{array}$ & $58.8^{4}$ & 0.5 & & (1) & \\
\hline $\begin{array}{l}22 \text { July } 1986- \\
31 \text { July } 1986 \\
\text { Eastern Calif. }\end{array}$ & $\begin{array}{l}\text { Moran Spring } \\
\text { (USGS) }\end{array}$ & $\begin{array}{r}37.654 \mathrm{~N} \\
118.594 \mathrm{~W}\end{array}$ & (3) & (2) & $\begin{array}{l}360 \\
\text { Up } \\
270\end{array}$ & $\begin{array}{l}.06 \\
.04 \\
.03\end{array}$ & $\begin{array}{ll}--- \\
\cdots-\end{array}$ \\
\hline
\end{tabular}

Epicenters and magnitudes unknown

Note: Five additional records ${ }^{l}$ recovered at Moran Spring.

\begin{tabular}{|c|c|c|c|c|c|}
\hline \multirow[t]{3}{*}{$\begin{array}{l}\text { South Hammil Valley } \\
\text { White Mountain Ranch } \\
\text { (USGS) }\end{array}$} & $\begin{array}{r}37.62 \mathrm{~N} \\
118.39 \mathrm{~W}\end{array}$ & (3) & 2.1 & $\begin{array}{l}360 \\
\text { Up } \\
270\end{array}$ & $\begin{array}{l}.09 \\
.04 \\
.05\end{array}$ \\
\hline & & (3) & 1.6 & $\begin{array}{l}360 \\
\text { Up } \\
270\end{array}$ & $\begin{array}{l}.04 \\
.07 \\
.05\end{array}$ \\
\hline & & (3) & 0.7 & $\begin{array}{l}360 \\
\text { Up } \\
270\end{array}$ & $\begin{array}{l}.07 \\
.06 \\
.06\end{array}$ \\
\hline
\end{tabular}

Note: Four additional records ${ }^{1}$ recovered at White Mounta in Ranch. 
Table 7. Summary of United States accelerograph records recovered during $1986-$ Continued

\begin{tabular}{|c|c|c|c|c|c|c|c|}
\hline Earthquake & $\begin{array}{l}\text { Station name } \\
\text { (Owner) }\end{array}$ & $\begin{array}{l}\text { Station } \\
\text { location } \\
(*)\end{array}$ & $\begin{array}{l}\text { Trigger } \\
\text { time }\end{array}$ & $\begin{array}{c}S \text {-minus } \\
\text { trigger } \\
(s)\end{array}$ & $\begin{array}{l}\text { Direction } \\
\quad(a z)\end{array}$ & $\begin{array}{l}\text { Maximum } \\
\text { amplitude } \\
\quad(g)\end{array}$ & $\begin{array}{l}\text { Duration } \\
\quad(\mathrm{s})\end{array}$ \\
\hline \multirow{14}{*}{$\begin{array}{l}31 \text { July } 1986 \\
0722: 40.2 \text { G.m.t. } \\
\text { Eastern Calif. } \\
37.463 \mathrm{~N}, 118.374 \mathrm{~W} \\
\text { Magnitude } 5.8 \mathrm{ML}\end{array}$} & $\begin{array}{l}\text { Hammil, Calif. } \\
\text { Cinnamon Ranch } \\
\text { (USGS) }\end{array}$ & $\begin{array}{r}37.68 \mathrm{~N} \\
118.39 \mathrm{~W}\end{array}$ & $47.6^{4}$ & 1.3 & & (1) & \\
\hline & $\begin{array}{l}\text { McGee Creek, SMA } \\
\text { (USGS) }\end{array}$ & $\begin{array}{r}37.550 \mathrm{~N} \\
118.811 \mathrm{~W}\end{array}$ & $47.3^{4}$ & (2) & & (1) & \\
\hline & $\begin{array}{l}\text { McGee Creek, CRA } \\
\text { (USGS) }\end{array}$ & $\begin{array}{r}37.550 \mathrm{~N} \\
118.811 \mathrm{~W}\end{array}$ & $47.3^{4}$ & (2) & & & \\
\hline & $166 \mathrm{~m}$ downhole & & & & & (1) & \\
\hline & $35 \mathrm{~m}$ downhole & & & & & (1) & \\
\hline & Surface & & & & & (1) & \\
\hline & $1 \mathrm{~m}$ downhole & & & & & (1) & \\
\hline & $\begin{array}{l}\text { Moran Spring, Calif. } \\
\text { (USGS) }\end{array}$ & $\begin{array}{r}37.654 \mathrm{~N} \\
118.594 \mathrm{~W}\end{array}$ & $46.2^{4}$ & (2) & & (1) & \\
\hline & $\begin{array}{l}\text { Montgomery Pass } \\
\text { Basalt, Nevada } \\
\text { (USGS) }\end{array}$ & $\begin{array}{r}37.977 \mathrm{~N} \\
118.318 \mathrm{~W}\end{array}$ & $01.5^{4}$ & (2) & & (1) & \\
\hline & $\begin{array}{l}\text { Terminus Dam } \\
\text { Ma in Dam } \\
\text { (ACOE) }\end{array}$ & $\begin{array}{r}36.420 \mathrm{~N} \\
119.000 \mathrm{~W}\end{array}$ & (3) & (2) & & & \\
\hline & Right crest & & & & & (1) & \\
\hline & Upper tower & & & & & (1) & \\
\hline & $\begin{array}{l}\text { Terminus Dam } \\
\text { Auxiliary Dam } \\
\text { (ACOE) }\end{array}$ & $\begin{array}{r}36.404 \mathrm{~N} \\
119.001 \mathrm{~W}\end{array}$ & $16.1^{4}$ & (2) & & & \\
\hline & Center crest & & & & & (1) & \\
\hline \multirow{2}{*}{$\begin{array}{l}31 \text { July } 1986 \\
0751: 42.9 \text { G.m.t. } \\
\text { Southern Calif. } \\
33.970 \mathrm{~N}, 116.570 \mathrm{~W} \\
\text { Magnitude } 3.3 \mathrm{ML}\end{array}$} & $\begin{array}{l}\text { Desert Hot Springs } \\
\text { Mission Lakes C.C. } \\
\text { (USGS) }\end{array}$ & $\begin{array}{r}33.986 \mathrm{~N} \\
116.535 \mathrm{~W}\end{array}$ & 44.8 & (2) & & (1) & \\
\hline & $\begin{array}{l}\text { Whitewater Canyon } \\
\text { Trout Farm } \\
\text { (USGS) }\end{array}$ & $\begin{array}{r}33.989 \mathrm{~N} \\
116.655 \mathrm{~W}\end{array}$ & 47.2 & (2) & & (1) & \\
\hline $\begin{array}{l}1 \text { August } 1986 \\
0634: 42.9 \text { G.m.t. } \\
\text { Eastern Cal if. }\end{array}$ & $\begin{array}{l}\text { Chalfant Valley } \\
\text { Fire Station } \\
\text { (USGS) }\end{array}$ & $\begin{array}{r}37.53 \mathrm{~N} \\
118.37 \mathrm{~W}\end{array}$ & 45.6 & 2.1 & $\begin{array}{l}360 \\
U p \\
270\end{array}$ & $\begin{array}{l}.06 \\
.01 \\
.04\end{array}$ & $\begin{array}{l}--\cdot \\
-- \\
--\end{array}$ \\
\hline
\end{tabular}
$37.561 \mathrm{~N}, 118.394 \mathrm{~W}$ Magnitude $3.2 \mathrm{ML}$

Note: Four additional records ${ }^{1}$ recovered at Chalfant Valley Fire Station.
Laws, Calif.
Northeast Bishop
$37.402 \mathrm{~N} \quad 50.5^{4}$
(2) (USGS)

(1) 
Table 7. Summary of United States accelerograph records recovered during 1986-Continued

\begin{tabular}{|c|c|c|c|c|c|c|c|}
\hline Earthquake & $\begin{array}{l}\text { Station name } \\
\text { (Owner) }\end{array}$ & $\begin{array}{l}\text { Station } \\
\text { location } \\
(*)\end{array}$ & $\begin{array}{l}\text { Trigger } \\
\text { time }\end{array}$ & $\begin{array}{l}S \text {-minus } \\
\text { trigger } \\
(\mathrm{s})\end{array}$ & $\begin{array}{l}\text { Direction } \\
\quad(a z)\end{array}$ & $\begin{array}{l}\text { Maximum } \\
\text { amplitude } \\
\quad(g)\end{array}$ & $\begin{array}{c}\text { Duration } \\
\text { (s) }\end{array}$ \\
\hline \multirow{4}{*}{$\begin{array}{l}1 \text { August } 1986 \\
1427: 16.0 \mathrm{G} . \mathrm{m} . \mathrm{t} . \\
\text { Eastern Cal if. } \\
37.501 \mathrm{~N}, 118.352 \mathrm{~W} \\
\text { Magnitude } 4.3 \mathrm{ML}\end{array}$} & $\begin{array}{l}\text { Chalfant Valley } \\
\text { Fire Station } \\
\text { (USGS) }\end{array}$ & $\begin{array}{r}37.53 \mathrm{~N} \\
118.37 \mathrm{~W}\end{array}$ & 17.8 & 0.5 & $\begin{array}{l}360 \\
\text { Up } \\
270\end{array}$ & $\begin{array}{l}.25 \\
.25 \\
.23\end{array}$ & $\begin{array}{l}0.9 \\
0.4 \\
0.8\end{array}$ \\
\hline & $\begin{array}{l}\text { Hammil, Calif. } \\
\text { Cinnamon Ranch } \\
\text { (USGS) }\end{array}$ & $\begin{array}{r}37.68 \mathrm{~N} \\
118.39 \mathrm{~W}\end{array}$ & $20.3^{4}$ & 3.2 & & (1) & \\
\hline & $\begin{array}{l}\text { Laws, Calif. } \\
\text { Northeast Bishop } \\
\text { (USGS) }\end{array}$ & $\begin{array}{r}37.402 \mathrm{~N} \\
118.346 \mathrm{~W}\end{array}$ & $20.55^{4}$ & (2) & & (1) & \\
\hline & $\begin{array}{l}\text { South Hammil Valley } \\
\text { White Mountain Ranch } \\
\text { (USGS) }\end{array}$ & $\begin{array}{r}37.62 \mathrm{~N} \\
118.39 \mathrm{~W}\end{array}$ & $18.9^{4}$ & 1.9 & & (1) & \\
\hline $\begin{array}{l}1 \text { August } 1986 \\
1428: 18.0 \mathrm{G} . \mathrm{m} . \mathrm{t} . \\
\text { Eastern Calif. }\end{array}$ & $\begin{array}{l}\text { Chalfant Valley } \\
\text { Fire Station } \\
\text { (USGS) }\end{array}$ & $\begin{array}{r}37.53 \mathrm{~N} \\
118.37 \mathrm{~W}\end{array}$ & 20.0 & 0.5 & $\begin{array}{l}360 \\
\text { Up } \\
270\end{array}$ & $\begin{array}{l}.40 \\
.30 \\
.29\end{array}$ & $\begin{array}{l}1.4 \\
3.0 \\
1.5\end{array}$ \\
\hline
\end{tabular}

$37.375 \mathrm{~N}, 118.442 \mathrm{~W}$ Magnitude $4.7 \mathrm{ML}$

\section{August 1986 \\ 0505 G.m.t. \\ Southern Calif. \\ Epicenter and \\ magnitude unknown \\ 2 August 1986 \\ 1451:36.2 G.m.t. \\ Eastern Calif. \\ $37.594 \mathrm{~N}, 118.368 \mathrm{~W}$ \\ Magnitude $3.7 \mathrm{ML}$}

3 August 1986

0137 G.m.t.

Eastern Calif.

Epicenters and

magnitudes unknown

3 August 1986

0900:13.6 G.m.t.

Central Calif.

$36.592 \mathrm{~N}, 121.233 \mathrm{~W}$

Magnitude $2.9 \mathrm{ML}$
Note: Six additional records ${ }^{l}$ recovered at Chalfant Valley Fire Station.

$\begin{array}{lrrr}\text { Laws, Calif. } & 37.402 \mathrm{~N} & 21.1^{4} & 0.8 \\ \begin{array}{l}\text { Northeast Bishop } \\ \text { (USGS) }\end{array} & 118.346 \mathrm{~W} & & \\ & & & \\ \text { South Hammil Valley } & 37.62 \mathrm{~N} & 21.4^{4} & 1.7 \\ \begin{array}{l}\text { White Mountain Ranch } \\ \text { (USGS) }\end{array} & 118.39 \mathrm{~W} & & \\ & & & \\ \text { Whitewater Canyon } & 33.989 \mathrm{~N} & 05.3 & \text { (2) } \\ \text { Trout Farm } & 116.655 \mathrm{~W} & & \\ \text { (USGS) } & & & \end{array}$

$\begin{array}{lll}360 & .11 & 0.2 \\ \text { Up } & .09 & -- \\ 270 & .07 & \ldots-\end{array}$

(1)

South Hammil Valley

White Mountain Ranch

$37.62 \mathrm{~N} \quad 38.5^{4} \quad 1.4$ $118.39 \mathrm{~W}$

(USGS)

Hammil, Calif.

Cinnamon Ranch

(USGS)

Chalfant Valley

Fire Station

(USGS)

118.37

$37.68 \mathrm{~N}$

$118.39 \mathrm{~W}$

$40.4^{4}$

(2)

360

Up

270

.04

.05

.05

(1)

Note: 15 additional records ${ }^{1}$ recovered at Chalfant Valley Fire Station.

$$
\begin{array}{lrrr}
\text { Bear Valley Station } 1 & 36.573 \mathrm{~N} & 16.9 & \text { (2) }
\end{array}
$$

CDF Fire Station 121.184W (USGS)

Bear Valley Station $10 \quad 36.532 \mathrm{~N}$

Webb Residence

(USGS)
$16.6 \quad 1.7$

310
Up
220

0.2 
Table 7. Summary of United States accelerograph records recovered during 1986-Continued

\begin{tabular}{|c|c|c|c|c|c|c|c|}
\hline Earthquake & $\begin{array}{l}\text { Station name } \\
\text { (Owner) }\end{array}$ & $\begin{array}{l}\text { Station } \\
\text { location } \\
(*)\end{array}$ & $\begin{array}{l}\text { Trigger } \\
\text { time }\end{array}$ & $\begin{array}{l}S \text {-minus } \\
\text { trigger } \\
(\mathrm{s})\end{array}$ & $\begin{array}{l}\text { Direction } \\
\quad(a z)\end{array}$ & $\begin{array}{l}\text { Maximum } \\
\text { amplitude } \\
\text { (g) }\end{array}$ & $\begin{array}{c}\text { Duration } \\
\text { (s) }\end{array}$ \\
\hline \multirow{3}{*}{$\begin{array}{l}3 \text { August } 1986 \\
1033: 04.5 \mathrm{G} . \mathrm{m} . \mathrm{t} . \\
\text { Eastern Calif. } \\
37.615 \mathrm{~N}, 118.410 \mathrm{~W} \\
\text { Magnitude } 4.0 \mathrm{ML}\end{array}$} & $\begin{array}{l}\text { Laws, Calif. } \\
\text { Northeast Bishop } \\
\text { (USGS) }\end{array}$ & $\begin{array}{r}37.402 \mathrm{~N} \\
118.346 \mathrm{~W}\end{array}$ & $12.9^{4}$ & \multirow[t]{2}{*}{0.6} & \multicolumn{3}{|c|}{ (1) } \\
\hline & $\begin{array}{l}\text { South Hammil Valley } \\
\text { White Mountain Ranch } \\
\text { (USGS) }\end{array}$ & $\begin{array}{r}37.62 \mathrm{~N} \\
118.39 \mathrm{~W}\end{array}$ & 06.8 & & $\begin{array}{l}360 \\
\text { Up } \\
270\end{array}$ & $\begin{array}{l}.04 \\
.06 \\
.04\end{array}$ & \multirow[t]{2}{*}{---} \\
\hline & $\begin{array}{l}\text { Hammil, Calif. } \\
\text { Cinnamon Ranch } \\
\text { (USGS) }\end{array}$ & $\begin{array}{r}37.68 \mathrm{~N} \\
118.39 \mathrm{~W}\end{array}$ & $08.65^{4}$ & 0.5 & & (1) & \\
\hline $\begin{array}{l}4 \text { August } 1986 \\
1231: 06.4 \mathrm{G} . \mathrm{m} . \mathrm{t} . \\
\text { Eastern Calif. } \\
37.521 \mathrm{~N}, 118.415 \mathrm{~W} \\
\text { Magnitude } 3.3 \mathrm{ML}\end{array}$ & $\begin{array}{l}\text { Laws, Calif. } \\
\text { Northeast Bishop } \\
\text { (USGS) }\end{array}$ & $\begin{array}{r}37.402 \mathrm{~N} \\
118.346 \mathrm{~W}\end{array}$ & $12.35^{4}$ & 0.5 & & \multicolumn{2}{|l|}{ (1) } \\
\hline $\begin{array}{l}6 \text { August } 1986 \\
0452 \text { G.m.t. } \\
\text { Central Calif. } \\
\text { Epicenter and } \\
\text { magnitude unknown }\end{array}$ & $\begin{array}{l}\text { Bear Valley Station } 10 \\
\text { Webb Residence } \\
\text { (USGS) }\end{array}$ & $\begin{array}{r}36.532 \mathrm{~N} \\
121.143 \mathrm{~W}\end{array}$ & 50.0 & 1.8 & & \multicolumn{2}{|l|}{ (1) } \\
\hline $\begin{array}{l}6 \text { August } 1986 \\
1116 \text { G.m.t. } \\
\text { Southern Cal if. } \\
\text { Epicenter and } \\
\text { magnitude unknown }\end{array}$ & $\begin{array}{l}\text { Whitewater Canyon } \\
\text { Trout Farm } \\
\text { (USGS) }\end{array}$ & $\begin{array}{r}33.989 \mathrm{~N} \\
116.655 \mathrm{~W}\end{array}$ & 32.7 & (2) & & \multicolumn{2}{|l|}{ (1) } \\
\hline $\begin{array}{l}10 \text { August } 1986 \\
2014 \text { G.m.t. } \\
\text { Eastern Calif. } \\
\text { Epicenter and } \\
\text { magnitude unknown }\end{array}$ & $\begin{array}{l}\text { Laws, Cal if } \\
\text { Northeast Bishop } \\
\text { (USGS) }\end{array}$ & $\begin{array}{r}37.402 \mathrm{~N} \\
118.346 \mathrm{~W}\end{array}$ & $43.45^{4}$ & 0.6 & & \multicolumn{2}{|l|}{ (1) } \\
\hline $\begin{array}{l}11 \text { August } 1986 \\
0426 \text { G.m.t. } \\
\text { Eastern Cal if. } \\
\text { Epicenter and } \\
\text { magnitude unknown }\end{array}$ & $\begin{array}{l}\text { Laws, Calif. } \\
\text { Northeast Bishop } \\
\text { (USGS) }\end{array}$ & $\begin{array}{r}37.402 \mathrm{~N} \\
118.346 \mathrm{~W}\end{array}$ & $52.78^{4}$ & (2) & & \multicolumn{2}{|l|}{ (1) } \\
\hline $\begin{array}{l}12 \text { August } 1986 \\
0929: 48.0 \mathrm{G.m.t} . \\
\text { Eastern Calif. } \\
37.487 \mathrm{~N}, 118.377 \mathrm{~W} \\
\text { Magnitude } 3.5 \mathrm{ML}\end{array}$ & $\begin{array}{l}\text { Chalfant Valley } \\
\text { Fire Station } \\
\text { (USGS) } \\
\text { Note: One additional }\end{array}$ & $\begin{array}{r}37.53 \mathrm{~N} \\
118.37 \mathrm{~W}\end{array}$ & recovered & d at Cha & $\begin{array}{l}360 \\
\text { Up } \\
270 \\
\text { lfant Valley }\end{array}$ & $\begin{array}{l}.11 \\
.06 \\
.07 \\
\text { y Fire St }\end{array}$ & $\begin{array}{l}1 \text { peak } \\
\text { ation. }\end{array}$ \\
\hline $\begin{array}{l}12 \text { August } 1986 \\
1537: 27.9 \text { G.m.t. } \\
\text { Eastern Calif. } \\
37.503 \mathrm{~N}, 118.477 \mathrm{~W} \\
\text { Magnitude } 3.5 \mathrm{ML}\end{array}$ & $\begin{array}{l}\text { Laws, Calif } \\
\text { Northeast Bishop } \\
\text { (USGS) }\end{array}$ & $\begin{array}{r}37.402 \mathrm{~N} \\
118.346 \mathrm{~W}\end{array}$ & $33.0^{4}$ & 0.6 & & \multicolumn{2}{|l|}{ (1) } \\
\hline $\begin{array}{l}14 \text { August } 1986 \\
0836 \text { G.m.t. } \\
\text { Eastern Calif. } \\
\text { Epicenter and } \\
\text { magnitude unknown }\end{array}$ & $\begin{array}{l}\text { Laws, Calif. } \\
\text { Northeast Bishop } \\
\text { (USGS) }\end{array}$ & $\begin{array}{r}37.402 \mathrm{~N} \\
118.346 \mathrm{~W}\end{array}$ & $07.4^{4}$ & (2) & & \multicolumn{2}{|l|}{ (1) } \\
\hline
\end{tabular}

See footnotes at end of table 
Table 7. Summary of United States accelerograph records recovered during 1986-Continued

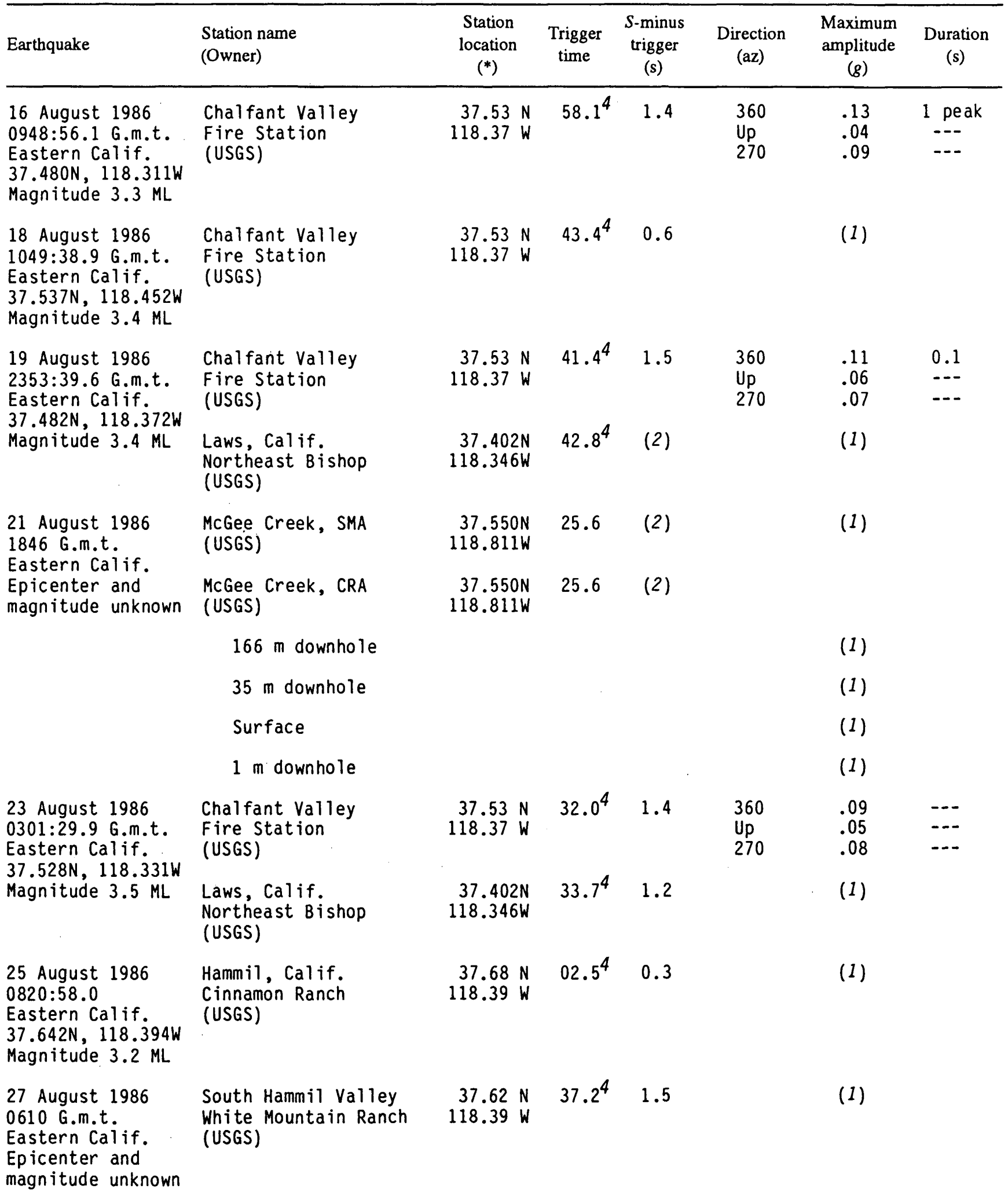


Table 7. Summary of United States accelerograph records recovered during 1986-Continued

\begin{tabular}{|c|c|c|c|c|c|c|c|}
\hline Earthquake & $\begin{array}{l}\text { Station name } \\
\text { (Owner) }\end{array}$ & $\begin{array}{l}\text { Station } \\
\text { location } \\
\quad(*)\end{array}$ & $\begin{array}{l}\text { Trigger } \\
\text { time }\end{array}$ & $\begin{array}{l}S \text {-minus } \\
\text { trigger } \\
(\mathrm{s})\end{array}$ & $\begin{array}{l}\text { Direction } \\
\quad(a z)\end{array}$ & $\begin{array}{l}\text { Maximum } \\
\text { amplitude } \\
\text { (g) }\end{array}$ & $\begin{array}{c}\text { Duration } \\
\text { (s) }\end{array}$ \\
\hline \multirow{4}{*}{$\begin{array}{l}29 \text { August } 1986 \\
0746: 53.3 \text { G.m.t. } \\
\text { Southern Calif. } \\
33.953 \mathrm{~N}, 116.623 \mathrm{~W} \\
\text { Magnitude } 4.0 \mathrm{ML}\end{array}$} & $\begin{array}{l}\text { Desert Hot Springs } \\
\text { Mission Lakes C.C. } \\
\text { (USGS) }\end{array}$ & $\begin{array}{r}33.986 \mathrm{~N} \\
116.535 \mathrm{~W}\end{array}$ & 56.6 & 1.5 & $\begin{array}{l}360 \\
\text { Up } \\
270\end{array}$ & $\begin{array}{l}.13 \\
.08 \\
.12\end{array}$ & $\begin{array}{l}1 \text { peak } \\
1 \text { peak }\end{array}$ \\
\hline & $\begin{array}{l}\text { North Palm Springs } \\
\text { Post office } \\
\text { (USGS) }\end{array}$ & $\begin{array}{r}33.924 \mathrm{~N} \\
116.543 \mathrm{~W}\end{array}$ & 56.5 & 1.7 & $\begin{array}{l}300 \\
\text { Up } \\
210\end{array}$ & $\begin{array}{l}.14 \\
.04 \\
.10\end{array}$ & $\begin{array}{c}0.1 \\
-- \\
1 \text { peak }\end{array}$ \\
\hline & $\begin{array}{l}\text { Whitewater Canyon } \\
\text { Trout Farm } \\
\text { (USGS) }\end{array}$ & $\begin{array}{r}33.989 \mathrm{~N} \\
116.655 \mathrm{~W}\end{array}$ & 57.9 & (2) & & (1) & \\
\hline & Note: One additional & record 1 & recovered & d at Wh & tewater & Canyon Trout & Farm. \\
\hline \multirow{2}{*}{$\begin{array}{l}9 \text { September } 1986 \\
1622: 50.6 \mathrm{G} . \mathrm{m} . \mathrm{t} \text {. } \\
\text { Southern Calif. } \\
33.970 \mathrm{~N}, 116.570 \mathrm{~W} \\
\text { Magnitude } 3.5 \mathrm{ML}\end{array}$} & $\begin{array}{l}\text { Desert Hot Springs } \\
\text { Mission Lakes C.C. } \\
\text { (USGS) }\end{array}$ & $\begin{array}{r}33.986 \mathrm{~N} \\
116.535 \mathrm{~W}\end{array}$ & 52.7 & 1.5 & & (1) & \\
\hline & $\begin{array}{l}\text { North Palm Springs } \\
\text { Post office } \\
\text { (USGS) }\end{array}$ & $\begin{array}{r}33.924 \mathrm{~N} \\
116.543 \mathrm{~W}\end{array}$ & 52.7 & 1.5 & $\begin{array}{l}300 \\
\text { Up } \\
210\end{array}$ & $\begin{array}{l}.05 \\
.02 \\
.05\end{array}$ & 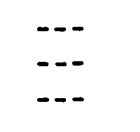 \\
\hline $\begin{array}{l}16 \text { September } 1986 \\
0007: 41.2 \mathrm{G} . \mathrm{m} . \mathrm{t} \text {. } \\
\text { Eastern Calif. } \\
37.625 \mathrm{~N}, 118.455 \mathrm{~W} \\
\text { Magnitude } 3.3 \mathrm{ML}\end{array}$ & $\begin{array}{l}\text { South Hammil Valley } \\
\text { White Mountain Ranch } \\
\text { (USGS) }\end{array}$ & $\begin{array}{r}37.62 \mathrm{~N} \\
118.39 \mathrm{~W}\end{array}$ & $45.1^{4}$ & (2) & & (1) & \\
\hline \multirow{2}{*}{$\begin{array}{l}16 \text { September } 1986 \\
0501: 43.5 \mathrm{G} . \mathrm{m} . \mathrm{t} . \\
\text { Eastern Cal if. } \\
37.642 \mathrm{~N}, 118.398 \mathrm{~W} \\
\text { Magnitude } 3.3 \mathrm{ML}\end{array}$} & $\begin{array}{l}\text { South Hammil Valley } \\
\text { White Mountain Ranch } \\
\text { (USGS) }\end{array}$ & $\begin{array}{r}37.62 \mathrm{~N} \\
118.39 \mathrm{~W}\end{array}$ & $47.2^{4}$ & 0.5 & & (1) & \\
\hline & $\begin{array}{l}\text { Hammil, Cal if. } \\
\text { Cinnamon Ranch } \\
\text { (USGS) }\end{array}$ & $\begin{array}{r}37.68 \mathrm{~N} \\
118.39 \mathrm{~W}\end{array}$ & $48.1^{4}$ & 0.5 & & (1) & \\
\hline $\begin{array}{l}16 \text { September } 1986 \\
0636: 57.8 \mathrm{G} . \mathrm{m} . \mathrm{t} . \\
\text { Eastern Calif. } \\
37.610 \mathrm{~N}, 118.445 \mathrm{~W} \\
\text { Magnitude } 3.3 \mathrm{ML} \text {. }\end{array}$ & $\begin{array}{l}\text { Hammil, Calif. } \\
\text { Cinnamon Ranch } \\
\text { (USGS) }\end{array}$ & $\begin{array}{r}37.68 \mathrm{~N} \\
118.39 \mathrm{~W}\end{array}$ & $01.5^{4}$ & 0.4 & & (1) & \\
\hline $\begin{array}{l}16 \text { September } 1986 \\
1314: 25.9 \mathrm{G} . \mathrm{m} . \mathrm{t} . \\
\text { Eastern Cal if. } \\
37.595 \mathrm{~N}, 118.413 \mathrm{~W} \\
\text { Magnitude } 3.5 \mathrm{ML}\end{array}$ & $\begin{array}{l}\text { South Hammil Valley } \\
\text { White Mountain Ranch } \\
\text { (USGS) }\end{array}$ & $\begin{array}{r}37.62 \mathrm{~N} \\
118.39 \mathrm{~W}\end{array}$ & $29.2^{4}$ & (2) & & (1) & \\
\hline
\end{tabular}


Table 7. Summary of United States accelerograph records recovered during 1986-Continued

\begin{tabular}{|c|c|c|c|c|c|c|c|}
\hline Earthquake & $\begin{array}{l}\text { Station name } \\
\text { (Owner) }\end{array}$ & $\begin{array}{l}\text { Station } \\
\text { location } \\
(*)\end{array}$ & $\begin{array}{l}\text { Trigger } \\
\text { time }\end{array}$ & $\begin{array}{l}S \text {-minus } \\
\text { trigger } \\
\quad(\mathrm{s})\end{array}$ & $\begin{array}{l}\text { Direction } \\
\quad(a z)\end{array}$ & $\begin{array}{l}\text { Maximum } \\
\text { amplitude } \\
(g)\end{array}$ & $\begin{array}{l}\text { Duration } \\
\text { (s) }\end{array}$ \\
\hline \multirow{4}{*}{$\begin{array}{l}\text { 18 September } 1986 \\
0759: 47.5 \mathrm{G} . \mathrm{m} . \mathrm{t} . \\
\text { Eastern Cal if. } \\
37.632 \mathrm{~N}, 118.392 \mathrm{~W} \\
\text { Magnitude } 4.1 \mathrm{ML}\end{array}$} & $\begin{array}{l}\text { Chalfant Valley } \\
\text { Fire Station } \\
\text { (USGS) }\end{array}$ & $\begin{array}{r}37.53 \mathrm{~N} \\
118.37 \mathrm{~W}\end{array}$ & $52.7^{4}$ & 0.8 & $\begin{array}{l}360 \\
\text { Up } \\
270\end{array}$ & $\begin{array}{l}.05 \\
.03 \\
.06\end{array}$ & --- \\
\hline & $\begin{array}{l}\text { Hammil, Cal if. } \\
\text { Cinnamon Ranch } \\
\text { (USGS) }\end{array}$ & $\begin{array}{r}37.68 \mathrm{~N} \\
118.39 \mathrm{~W}\end{array}$ & $51.7^{4}$ & 0.5 & & (1) & \\
\hline & $\begin{array}{l}\text { Laws, Calif. } \\
\text { Northeast Bishop } \\
\text { (USGS) }\end{array}$ & $\begin{array}{r}37.402 \mathrm{~N} \\
118.346 \mathrm{~W}\end{array}$ & $55.7^{4}$ & (2) & & (1) & \\
\hline & $\begin{array}{l}\text { South Hammil Valley } \\
\text { White Mountain Rarich } \\
\text { (USGS) }\end{array}$ & $\begin{array}{r}37.62 \mathrm{~N} \\
118.39 \mathrm{~W}\end{array}$ & $50.8^{4}$ & 0.6 & $\begin{array}{l}360 \\
\text { Up } \\
270\end{array}$ & $\begin{array}{l}.06 \\
.05 \\
.06\end{array}$ & --- \\
\hline $\begin{array}{l}23 \text { September } 1986 \\
0619: 46.2 \mathrm{G} . \mathrm{m} . \mathrm{t} . \\
\text { Central Calif. } \\
36.635 \mathrm{~N}, 121.292 \mathrm{~W} \\
\text { Magnitude } 2.5 \mathrm{ML}\end{array}$ & $\begin{array}{l}\text { Bear Valley Station } 12 \\
\text { Williams Ranch } \\
\text { (USGS) }\end{array}$ & $\begin{array}{r}36.658 \mathrm{~N} \\
121.249 \mathrm{~W}\end{array}$ & 47.7 & 1.5 & $\begin{array}{l}310 \\
\text { Up } \\
220\end{array}$ & $\begin{array}{l}.05 \\
.01 \\
.02\end{array}$ & $\begin{array}{l}--- \\
---\end{array}$ \\
\hline $\begin{array}{l}28 \text { September } 1986 \\
0706: 26.8 \mathrm{G} . \mathrm{m} . \mathrm{t} \text {. } \\
\text { Southern Calif. } \\
34.010 \mathrm{~N}, 116.580 \mathrm{~W} \\
\text { Magnitude } 3.2 \mathrm{ML}\end{array}$ & $\begin{array}{l}\text { Desert Hot Springs } \\
\text { Mission Lakes C.C. } \\
\text { (USGS) }\end{array}$ & $\begin{array}{r}33.986 \mathrm{~N} \\
116.535 \mathrm{~W}\end{array}$ & 31.7 & (2) & & (1) & \\
\hline $\begin{array}{l}29 \text { September } 1986 \\
0617: 32.0 \mathrm{G} . \mathrm{m} . \mathrm{t} . \\
\text { Eastern Cal if. } \\
37.514 \mathrm{~N}, 118.398 \mathrm{~W} \\
\text { Magnitude } 3.4 \mathrm{ML}\end{array}$ & $\begin{array}{l}\text { Laws, Calif. } \\
\text { Northeast Bishop } \\
\text { (USGS) }\end{array}$ & $\begin{array}{r}37.402 \mathrm{~N} \\
118.346 \mathrm{~W}\end{array}$ & $37.2^{4}$ & 0.4 & & (1) & \\
\hline $\begin{array}{l}1 \text { 0ctober } 1986 \\
0802 \text { G.m.t. } \\
\text { Hawaii } \\
\text { Epicenter and }\end{array}$ & $\begin{array}{l}\text { Honokaa, Hawaii } \\
\text { Pol ice Station } \\
\text { (USGS) }\end{array}$ & $\begin{array}{r}20.080 \mathrm{~N} \\
155.465 \mathrm{~W}\end{array}$ & $30.7^{4}$ & $(2)$ & & $(1)$ & \\
\hline magnitude unknown & $\begin{array}{l}\text { Hilo, Hawaii } \\
\text { U.S. Fish \& Wildlife } \\
\text { (USGS) }\end{array}$ & $\begin{array}{r}19.731 \mathrm{~N} \\
155.100 \mathrm{~W}\end{array}$ & $17.9^{4}$ & 4.4 & & (1) & \\
\hline $\begin{array}{l}11 \text { July } 1986- \\
7 \text { october } 1986 \\
\text { Southern Cal if. } \\
\text { Epicenter and }\end{array}$ & $\begin{array}{l}\text { Loma Linda } \\
\text { VA Hospital } \\
\text { (VA/USGS) }\end{array}$ & $\begin{array}{r}34.05 \mathrm{~N} \\
117.26 \mathrm{~W}\end{array}$ & (3) & (2) & & & \\
\hline magnitude unknown & Structure Array & & & & & (1) & \\
\hline $\begin{array}{l}9 \text { october } 1986 \\
0537: 25.2 \mathrm{G.m} . \mathrm{t} . \\
\text { Eastern Calif. } \\
37.358 \mathrm{~N}, 118.335 \mathrm{~W} \\
\text { Magnitude } 4.2 \mathrm{ML}\end{array}$ & $\begin{array}{l}\text { Laws, Calif } \\
\text { Northeast Bishop } \\
\text { (USGS) }\end{array}$ & $\begin{array}{r}37.402 \mathrm{~N} \\
118.346 \mathrm{~W}\end{array}$ & $27.4^{4}$ & 1.9 & & (1) & \\
\hline
\end{tabular}


Table 7. Summary of United States accelerograph records recovered during 1986-Continued

\begin{tabular}{|c|c|c|c|c|c|c|c|}
\hline Earthquake & $\begin{array}{l}\text { Station name } \\
\text { (Owner) }\end{array}$ & $\begin{array}{l}\text { Station } \\
\text { location } \\
(*)\end{array}$ & $\begin{array}{l}\text { Trigger } \\
\text { time }\end{array}$ & $\begin{array}{l}S \text {-minus } \\
\text { trigger } \\
\quad(s)\end{array}$ & $\begin{array}{l}\text { Direction } \\
\quad(a z)\end{array}$ & $\begin{array}{l}\text { Maximum } \\
\text { amplitude } \\
\quad(g)\end{array}$ & $\begin{array}{c}\text { Duration } \\
\text { (s) }\end{array}$ \\
\hline \multirow{3}{*}{$\begin{array}{l}15 \text { October } 1986 \\
0228: 47.7 \text { G.m.t. } \\
\text { Southern Calif. } \\
33.950 \mathrm{~N}, 116.570 \mathrm{~W} \\
\text { Magnitude } 4.7 \mathrm{ML}\end{array}$} & $\begin{array}{l}\text { Desert Hot Springs } \\
\text { Mission Lakes C.C. } \\
\text { (USGS) }\end{array}$ & $\begin{array}{r}33.986 \mathrm{~N} \\
116.535 \mathrm{~W}\end{array}$ & 50.0 & 1.5 & $\begin{array}{l}360 \\
\text { Up } \\
270\end{array}$ & $\begin{array}{l}.11 \\
.05 \\
.09\end{array}$ & 0.2 \\
\hline & $\begin{array}{l}\text { Morongo Valley } \\
\text { Fire Station } \\
\text { (USGS) }\end{array}$ & $\begin{array}{r}34.048 \mathrm{~N} \\
116.577 \mathrm{~W}\end{array}$ & 50.1 & 2.1 & $\begin{array}{l}135 \\
\text { Up } \\
045\end{array}$ & $\begin{array}{l}.03 \\
.08 \\
.03\end{array}$ & $\begin{array}{l}--- \\
--- \\
---\end{array}$ \\
\hline & $\begin{array}{l}\text { North Palm Springs } \\
\text { Post office } \\
\text { (USGS) }\end{array}$ & $\begin{array}{r}33.924 \mathrm{~N} \\
116.543 \mathrm{~W}\end{array}$ & 49.7 & 1.3 & $\begin{array}{l}300 \\
\text { Up } \\
210\end{array}$ & $\begin{array}{l}.15 \\
.09 \\
.07\end{array}$ & $\begin{array}{l}0.3 \\
--- \\
---\end{array}$ \\
\hline $\begin{array}{l}21 \text { 0ctober } 1986 \\
0836: 25.1 \mathrm{G.m.t.} \\
\text { Eastern Calif. } \\
37.510 \mathrm{~N}, 118.338 \mathrm{~W} \\
\text { Magnitude } 3.1 \mathrm{ML}\end{array}$ & $\begin{array}{l}\text { Chalfant Valley } \\
\text { Fire Station } \\
\text { (USGS) }\end{array}$ & $\begin{array}{r}37.53 \mathrm{~N} \\
118.37 \mathrm{~W}\end{array}$ & $27.6^{4}$ & 1.6 & & (1) & \\
\hline $\begin{array}{l}29 \text { August 1986- } \\
23 \text { October } 1986 \\
\text { Southern Cal if. } \\
\text { Epicenter and } \\
\text { magnitude unknown }\end{array}$ & $\begin{array}{l}\text { Whitewater Canyon } \\
\text { Trout Farm } \\
\text { (USGS) }\end{array}$ & $\begin{array}{r}33.989 \mathrm{~N} \\
116.655 \mathrm{~W}\end{array}$ & (3) & 0.5 & & (1) & \\
\hline $\begin{array}{l}26 \text { October } 1986 \\
1020: 13.8 \mathrm{G} . \mathrm{m} . \mathrm{t} . \\
\text { Eastern Calif. } \\
37.473 \mathrm{~N}, 118.371 \mathrm{~W} \\
\text { Magnitude } 3.2 \mathrm{ML}\end{array}$ & $\begin{array}{l}\text { Laws, Calif. } \\
\text { Northeast Bishop } \\
\text { (USGS) }\end{array}$ & $\begin{array}{r}37.402 \mathrm{~N} \\
118.346 \mathrm{~W}\end{array}$ & $17.0^{4}$ & (2) & & (1) & \\
\hline $\begin{array}{l}12 \text { July } 1986- \\
30 \text { October } 1986 \\
\text { Southern Cal if. } \\
\text { Epicenter and } \\
\text { magnitude unknown }\end{array}$ & $\begin{array}{l}\text { Colton, Calif. } \\
\text { I-10/15 Interchange } \\
\text { (CDOT) } \\
\quad \text { Vault }\end{array}$ & $\begin{array}{r}34.06 \mathrm{~N} \\
117.30 \mathrm{~W}\end{array}$ & (3) & (2) & & (1) & \\
\hline $\begin{array}{l}17 \text { November } 1985 \text { or } \\
\text { (1) } \\
1986 ; 0247 \text { G.m.t. } \\
\text { Hawa i } \\
\text { Epicenter and } \\
\text { magnitude unknown }\end{array}$ & $\begin{array}{l}\text { Wahaula Maint. Center } \\
\text { (USGS) }\end{array}$ & $\begin{array}{l}\text { Hawa i } \mathrm{Na} \\
155.031 \mathrm{~W}\end{array}$ & tional $\mathrm{F}$ & Park & $19.329 \mathrm{~N}$ & $25.6^{4}$ & 1.3 \\
\hline $\begin{array}{l}17 \text { November } 1986 \\
1240: 22.4 \mathrm{G} . \mathrm{m} . \mathrm{t} . \\
\text { Eastern Cal if. } \\
37.571 \mathrm{~N}, 118.415 \mathrm{~W} \\
\text { Magnitude } 3.5 \mathrm{ML}\end{array}$ & $\begin{array}{l}\text { South Hammil Valley } \\
\text { White Mountain Ranch } \\
\text { (USGS) }\end{array}$ & $\begin{array}{r}37.62 \mathrm{~N} \\
118.39 \mathrm{~W}\end{array}$ & $25.9^{4}$ & 1.4 & & (1) & \\
\hline
\end{tabular}


Table 7. Summary of United States accelerograph records recovered during 1986-Continued

\begin{tabular}{|c|c|c|c|c|c|c|c|}
\hline Earthquake & $\begin{array}{l}\text { Station name } \\
\text { (Owner) }\end{array}$ & $\begin{array}{l}\text { Station } \\
\text { location } \\
(*)\end{array}$ & $\begin{array}{c}\text { Trigger } \\
\text { time }\end{array}$ & $\begin{array}{l}S \text {-minus } \\
\text { trigger } \\
(\mathrm{s})\end{array}$ & $\begin{array}{l}\text { Direction } \\
\quad(a z)\end{array}$ & $\begin{array}{l}\text { Maximum } \\
\text { amplitude } \\
\quad(g)\end{array}$ & $\begin{array}{c}\text { Duration } \\
\text { (s) }\end{array}$ \\
\hline \multirow{7}{*}{$\begin{array}{l}21 \text { November } 1986 \\
2333: 01.7 \mathrm{G} . \mathrm{m.t} \text {. } \\
\text { Northern Calif. } \\
40.372 \mathrm{~N}, 124.443 \mathrm{~W} \\
\text { Magnitude } 5.1 \mathrm{ML}\end{array}$} & $\begin{array}{l}\text { Eel River Valley Array } \\
\text { Bunker Hill } \\
\text { (USGS) }\end{array}$ & $\begin{array}{r}40.498 \mathrm{~N} \\
124.294 W\end{array}$ & 11.6 & $(2)$ & $\begin{array}{l}360 \\
\text { Up } \\
270\end{array}$ & $\begin{array}{l}.05 \\
.01 \\
.05\end{array}$ & --- \\
\hline & $\begin{array}{l}\text { Eel River Valley Array } \\
\text { Centerville Beach } \\
\text { (USGS) }\end{array}$ & $\begin{array}{r}40.563 \mathrm{~N} \\
124.348 \mathrm{~W}\end{array}$ & 08.9 & 4.1 & $\begin{array}{l}360 \\
\text { Up } \\
270\end{array}$ & $\begin{array}{l}.14 \\
.03 \\
.16\end{array}$ & $\begin{array}{l}0.3 \\
1 \text { peak }\end{array}$ \\
\hline & $\begin{array}{l}\text { Eel River Valley Array } \\
\text { College of the Redwoods } \\
\text { (USGS) }\end{array}$ & $\begin{array}{r}40.699 N \\
124.200 W\end{array}$ & (3) & (2) & & (1) & \\
\hline & $\begin{array}{l}\text { Eel River Valley Array } \\
\text { Ferndale Fire Station } \\
\text { (USGS) }\end{array}$ & $\begin{array}{r}40.576 \mathrm{~N} \\
124.262 \mathrm{~W}\end{array}$ & (3) & 5.0 & $\begin{array}{l}360 \\
\text { Up } \\
270\end{array}$ & $\begin{array}{l}.19 \\
.03 \\
.19\end{array}$ & $\begin{array}{l}1 \text { peak } \\
0.6\end{array}$ \\
\hline & $\begin{array}{l}\text { Eel River Valley Array } \\
\text { Fortuna Fire Station } \\
\text { (USGS) }\end{array}$ & $\begin{array}{r}40.599 \mathrm{~N} \\
124.154 \mathrm{~W}\end{array}$ & (3) & 1.4 & $\begin{array}{l}360 \\
\text { Up } \\
270\end{array}$ & $\begin{array}{l}.13 \\
.03 \\
.28\end{array}$ & $\begin{array}{l}0.3 \\
-.- \\
0.5\end{array}$ \\
\hline & $\begin{array}{l}\text { Eel River Valley Array } \\
\text { Loleta Fire Station } \\
\text { (USGS) }\end{array}$ & $\begin{array}{r}40.644 \mathrm{~N} \\
124.219 \mathrm{~W}\end{array}$ & (3) & 5.9 & $\begin{array}{l}360 \\
\text { Up } \\
270\end{array}$ & $\begin{array}{l}.06 \\
.03 \\
.08\end{array}$ & --- \\
\hline & $\begin{array}{l}\text { Eel River Valley Array } \\
\text { South Bay Union School } \\
\text { (USGS) }\end{array}$ & $\begin{array}{r}40.735 \mathrm{~N} \\
124.207 \mathrm{~W}\end{array}$ & (3) & (2) & & (1) & \\
\hline \multirow{7}{*}{$\begin{array}{l}21 \text { November } 1986 \\
2334: 18.0 \mathrm{G} . \mathrm{m} . \mathrm{t} \text {. } \\
\text { Northern Calif. } \\
40.367 \mathrm{~N}, 124.450 \mathrm{~W} \\
\text { Magnitude } 5.1 \mathrm{ML}\end{array}$} & $\begin{array}{l}\text { Eel River Valley Array } \\
\text { Bunker Hill } \\
\text { (USGS) }\end{array}$ & $\begin{array}{r}40.498 \mathrm{~N} \\
124.294 \mathrm{~W}\end{array}$ & 28.3 & (2) & & (1) & \\
\hline & $\begin{array}{l}\text { Eel River Valley Array } \\
\text { Centerville Beach } \\
\text { (USGS) }\end{array}$ & $\begin{array}{r}40.563 \mathrm{~N} \\
124.348 \mathrm{~W}\end{array}$ & 24.6 & 4.8 & $\begin{array}{l}360 \\
\text { Up } \\
270\end{array}$ & $\begin{array}{l}.21 \\
.05 \\
.10\end{array}$ & $\begin{array}{l}0.3 \\
-.- \\
0.1\end{array}$ \\
\hline & $\begin{array}{l}\text { Eel River Valley Array } \\
\text { College of the Redwoods } \\
\text { (USGS) }\end{array}$ & $\begin{array}{r}40.699 \mathrm{~N} \\
124.200 \mathrm{~W}\end{array}$ & (3) & 6.8 & & (1) & . \\
\hline & $\begin{array}{l}\text { Eel River Valley Array } \\
\text { Ferndale Fire Station } \\
\text { (USGS) }\end{array}$ & $\begin{array}{r}40.576 \mathrm{~N} \\
124.262 \mathrm{~W}\end{array}$ & (3) & 5.5 & $\begin{array}{l}360 \\
\text { Up } \\
270\end{array}$ & $\begin{array}{l}.17 \\
.04 \\
.11\end{array}$ & $\begin{array}{l}1 \text { peak } \\
0.1\end{array}$ \\
\hline & $\begin{array}{l}\text { Eel River Valley Array } \\
\text { Fortuna Fire Station } \\
\text { (USGS) }\end{array}$ & $\begin{array}{r}40.599 \mathrm{~N} \\
124.154 \mathrm{~W}\end{array}$ & (3) & 6.2 & $\begin{array}{l}360 \\
\text { Up } \\
270\end{array}$ & $\begin{array}{l}.16 \\
.03 \\
.17\end{array}$ & $\begin{array}{l}0.3 \\
--. \\
0.3\end{array}$ \\
\hline & $\begin{array}{l}\text { Eel River Valley Array } \\
\text { Loleta Fire Station } \\
\text { (USGS) }\end{array}$ & $\begin{array}{r}40.644 \mathrm{~N} \\
124.219 \mathrm{~W}\end{array}$ & (3) & 6.5 & $\begin{array}{l}360 \\
\text { Up } \\
270\end{array}$ & $\begin{array}{l}.04 \\
.05 \\
.04\end{array}$ & 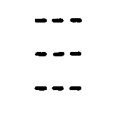 \\
\hline & $\begin{array}{l}\text { Eel River Valley Array } \\
\text { South Bay Union School } \\
\text { (USGS) }\end{array}$ & $\begin{array}{r}40.735 N \\
124.207 W\end{array}$ & (3) & (2) & & (1) & \\
\hline
\end{tabular}


Table 7. Summary of United States accelerograph records recovered during $1986-$ Continued

\begin{tabular}{|c|c|c|c|c|c|c|}
\hline Earthquake & $\begin{array}{l}\text { Station name } \\
\text { (Owner) }\end{array}$ & $\begin{array}{c}\text { Station } \\
\text { location } \\
\left(^{*}\right)\end{array}$ & $\begin{array}{l}\text { Trigger } \\
\text { time }\end{array}$ & $\begin{array}{l}S \text {-minus } \\
\text { trigger } \\
\quad(s)\end{array}$ & $\begin{array}{l}\text { Maximum } \\
\text { amplitude } \\
\text { (g) }\end{array}$ & $\begin{array}{l}\text { Duration } \\
\quad(s)\end{array}$ \\
\hline $\begin{array}{l}24 \text { November } 1986 \\
1508: 01.3 \mathrm{G} . \mathrm{m} . \mathrm{t} . \\
\text { Central Cal if. } \\
36.597 \mathrm{~N}, 121.242 \mathrm{~W} \\
\text { Magnitude } 3.1 \mathrm{ML}\end{array}$ & $\begin{array}{l}\text { Bear Valley Station } 1 \\
\text { CDF Fire Station } \\
\text { (USGS) }\end{array}$ & $\begin{array}{r}36.573 \mathrm{~N} \\
121.184 \mathrm{~W}\end{array}$ & 04.5 & (2) & (1) & \\
\hline $\begin{array}{l}8 \text { December } 1986 \\
1727 \text { G.m.t. } \\
\text { Nevada } \\
\text { Epicenter and } \\
\text { magnitude unknown }\end{array}$ & $\begin{array}{l}\text { Stilliwater, Nevada } \\
\text { Wildlife Refuge } \\
\text { (USGS) }\end{array}$ & $\begin{array}{r}39.518 \mathrm{~N} \\
118.510 \mathrm{~W}\end{array}$ & $08.4^{4}$ & (2) & (1) & \\
\hline $\begin{array}{l}17 \text { November } 1985- \\
11 \text { December } 1986 \\
\text { Hawai } \\
\text { Epicenters and } \\
\text { magnitudes unknown }\end{array}$ & $\begin{array}{l}\text { Wa imea, Hawaij } \\
\text { Fire Station } \\
\text { (USGS) } \\
\text { Note: Two additional }\end{array}$ & $\begin{array}{r}20.03 \mathrm{~N} \\
155.66 \mathrm{~W}\end{array}$ & recovere & ed at Waimea Fire & Station. & \\
\hline $\begin{array}{l}25 \text { December } 1986 \\
0608: 54.4 \mathrm{G} . \mathrm{m} . \mathrm{t} . \\
\text { Eastern Cal if. } \\
37.570 \mathrm{~N}, 118.407 \mathrm{~W} \\
\text { Magnitude } 3.5 \mathrm{ML}\end{array}$ & $\begin{array}{l}\text { South Hammil Valley } \\
\text { White Mounta in Ranch } \\
\text { (USGS) }\end{array}$ & $\begin{array}{r}37.62 \mathrm{~N} \\
118.39 \mathrm{~W}\end{array}$ & $58.0^{4}$ & 1.4 & (1) & \\
\hline \multirow{2}{*}{$\begin{array}{l}26 \text { December } 1986 \\
0956: 27.4 \mathrm{G} . \mathrm{m} . \mathrm{t} . \\
\text { Eastern Calif. } \\
37: 557 \mathrm{~N}, 118.371 \mathrm{~W} \\
\text { Magnitude } 3.9 \mathrm{ML}\end{array}$} & $\begin{array}{l}\text { Chalfant Valley } \\
\text { Fire Station } \\
\text { (USGS) }\end{array}$ & $\begin{array}{r}37.53 \mathrm{~N} \\
118.37 \mathrm{~W}\end{array}$ & $30.9^{4}$ & 1.4 & (1) & \\
\hline & $\begin{array}{l}\text { South Hammil Valley } \\
\text { White Mountain Ranch } \\
\text { (USGS) }\end{array}$ & $\begin{array}{r}37.62 \mathrm{~N} \\
118.39 \mathrm{~W}\end{array}$ & $31.1^{4}$ & (2) & (1) & \\
\hline $\begin{array}{l}29 \text { December } 1986 \\
1528: 04.9 \text { G.m.t. } \\
\text { Central. Cal if. } \\
37.458 \mathrm{~N}, 121.800 \mathrm{~W} \\
\text { Magnitude } 4.5 \mathrm{ML}\end{array}$ & $\begin{array}{l}\text { San Jose, } 101 / 280 / 680 \\
\text { Freeway Interchange } \\
\text { (USGS/CDOT) }\end{array}$ & $\begin{array}{r}37.340 \mathrm{~N} \\
121.851 \mathrm{~W}\end{array}$ & 08.8 & 2.3 & (1) & \\
\hline
\end{tabular}

${ }^{1}$ Less than $0.05 \mathrm{~g}$ at ground level or less than $0.10 \mathrm{~g}$ at nonground level stations.

${ }^{2}$ Questionable or undeterminable.

3 WWVB radio time code illegible or instrument not equipped with a radio receiver; correlation of accelerogram with earthquake may be questionable.

4 Internal clock time; accuracy is variable. 


\section{REFERENCES CITED}

Ake, J.P., 1984, An analysis of the May and July, 1983, Soccorro Mountain microearthquake swarms: New Mexico Institute of Mining and Technology, Geophysics OpenFile Report 49, 107 p.

Ake, J.P., Sanford, A.R., and Jarpe, S., 1983, A magnitude scale for central New Mexico based on signal duration: New Mexico Institute of Mining and Technology, Geophysics Open-File Report 45, 26 p.

Bath, Markus, 1966, Earthquake energy and magnitude, in Physics and chemistry of the Earth, v. 7: New York, Pergamon Press, p. 115-165.

Bolt, B.A., and Herraiz, M., 1983, Simplified estimation of seismic moment from seismograms: Seismological Society of America Bulletin, v. 73, p. 735-748.

Bolt, B.A., and Uhrhammer, R.A., 1986, Report on the March 31, 1986 Mt. Lewis, California earthquake: Earthquake Engineering Research Institute Newsletter, Special Earthquake Report, v. 20, no. 6, p. 1-3.

Bolt, B.A., Friday, J., and Uhrhammer, R.A., 1988, A PCbased broadband digital seismograph network: Geophysical Journal, v. 93, p. 565-573.

Borchardt, Glenn, and Manson, M.W., 1986, North Palm Springs earthquake: California Geology, v. 39, no. 11, p. 248-252.

Brewer, Lindie, 1989 , The intensity of the July 21,1986 , Chalfant Valley, California, earthquake: U.S. Geological Survey Open-File Report 89-135, 24 p.

Cockerham, R.S., and Corbett, E.J., 1987, The July 1986 Chalfant Valley, California, earthquake sequence: Preliminary Results: Seismological Society of America Bulletin, v. 77, no. 1, p. 280-289.

Davison, F. C., and Bode', M.J., 1987, A note on the December 1986-January 1987 Richmond, Virgnia, felt earthquake sequence: Seismological Society of America, Eastern Section, Seismological Research Letters, v. 58, no. 3, p. 73-80.

DePolo, C.M., and Ramelli, A.R., 1987, Preliminary report on surface fractures along the White Mountain faultzone associated with the July 1986 Chalfant Valley earthquake sequence: Seismological Society of America Bulletin, v. 77, no. 1, p. 290-296.

Dziewonski, A.M., Chou, T.A., and Woodhouse, J.H., 1981, Determination of earthquake source parameters from waveform data for studies of global and regional seismicity: Journal of Geophysical Research, v. 86, p. 2825-2852.

Earthquake Engineering Research Institute, 1986a, Reporton the Chalfant Valley, California, earthquake-July 21, 1986: Earthquake Engineering Research Institute Newsletter, Special Earthquake Report, v. 20, no. 11, 8p. 1986b, Report on the North Palm Springs, California, earthquake - July 8, 1986: Earthquake Engineering Research Institute Newsletter, Special Earthquake Report, v. 20, no. 9,12 p.

Engdahl, E.R., Billington, S., and Kisslinger, C., 1989, Teleseismically recorded seismicity before and after the May 7, 1986 Andreanof Islands, Alaska, earthquake: Journal of Geophysical Research, v. 94, no. B11, p. 15,481-15,498.

Glick,D.M., McCormick,D.M., Horstman,N.M., Bass, Neil, andUng, Gary, 1986, Adak, Alaska, earthquake-May 7, 1986: Earthquake Engineering Research Institute Newsletter, SpecialEarthquake Report, v.20, no.5,4p.

Gutenberg, Beno, and Richter, C.F., 1956, Magnitude and energy of earthquakes: Annali di Geofisica, v. 9, no. 1, p. 1-15.

Hanks, T.C., and Kanamori, Hiroo, 1979, A moment magnitude scale: Journal of Geophysical Research, v. 84, no. B5, p. 2348-2350.

Hauksson, Egill, and Jones, L.M., 1988, The July 1986 Oceanside $\left(M_{L}=5.3\right)$ earthquake sequence in the continental borderland, Southern California: Seismological Society of America Bulletin, v. 78, no. 6, p. 1885-1906.

Hildebrand, G.M., Steeples, D.W., Knapp, R. W., Miller, R. D., and Bennett, B.C., 1988, Microearthquakes in Kansas and Nebraska 1977-1987: Seismological Research Letters, v. 59, no. 4, p. 159-163.

Irby, W.L., Presgrave, B.W., and Schmieder, W. S., 1982, Preliminary determination of epicenters, Monthly listing, January 1982: U.S. Geological Survey, 16 p.

Jones, L.M., Hutton, L.K., Given, D.D., and Allen, C.R., 1986, The North Palm Springs, California, earthquake sequence of July 1986: Seismological Society of America Bulletin, v. 76, no. 6, p. 1830-1837.

Lee, W.H.K., and Lahr, J.C., 1975, HYPO71 (revised) - A computer program for determining hypocenter, magnitude, and first motion pattern of local earthquakes: U.S. Geological Survey Open-File Report 75-0311, 113 p.

Lienkaemper, J.J., Pezzopane, S.K., Clark, M.M., and Rymer, M.J., 1987, Fault fractures formed in association with the 1986 Chalfant Valley, California, earthquake sequence-Preliminary report: Seismological Society of America Bulletin, v. 77, no. 1, p. 297-305.

McKenzie, M.R., and Uhrhammer, R.A., 1988, Bulletin of the seismographic stations of the University of California: v. 56, nos. 1 and 2, p. 1-105.

Monroe, N.J., and Stevenson, J.D., 1986, Preliminary report on the Ohio earthquake of January 31, 1986: Earthquake Engineering Research Institute Newsletter, Special Earthquake Report, v. 20, no. 4, p. 5-8. 
Newton, C.A., Cash, D.J., Olsen, K.H., and Homuth, E.F., 1976, LASL seismic programs in the vicinity of Los Alamos, New Mexico: Los Alamos Scientific Laboratory Informal Report LA-6406-MS, 42 p.

Nicholson, C., Roeloffs, E., and Wesson, R.L., 1988, The northeastern Ohio earthquake of 31 January, 1986-Was it induced?: Seismological Society of America Bulletin, v. 78 , no. 1 , p. 188-217.

Nuttli, O.W., 1973, Seismic wave attenuation and magnitude relations for eastern North America: Journal of Geophysical Research, v. 78, no. 5, p. 876-885.

Petrillo, C.C., 1987, Analysis of recent swarm activity along the Hot Springs-Montosa fault east of Socorro, New Mexico: New Mexico Institute of Mining and Technology, Geophysics Open-File Report 58, 60.p.

Qamar, Anthony, Ludwin, Ruth, Crosson, R. S., and Malone, S. D., 1987, Earthquake hypocenters in Washington and Northern Oregon-1982-1986: Washington Division of Geology and Earth Resources, Information Circular 84, $78 \mathrm{p}$.

Richter, C.F., 1958, Elementary seismology: San Francisco, W. H. Freeman and Co., 768 p.

Sanford, A.R., 1965, An instrumental study of New Mexico earthquakes: New Mexico Bureau of Mines and Mineral Resources, Circular 78, 12 p.

Sanford, A.R., and Cash, D.J., 1969, An instrumental study of New Mexico earthquakes, July 1, 1964, through December 31, 1967: New Mexico Bureau of Mines and Mineral Resources, Circular 102, 7 p.

Sanford, A.R., Olsen, K.H., and Jaksha, L.H., 1981, EarthquakesinNewMexico, 1849-1977: NewMexicoBureau of Mines and Mineral Resources, Circular 171, $20 \mathrm{p}$.

Schiff, Anshel, 1986, Lifelines: Earthquake Engineering Research Institute Newsletter, Special Earthquake Report, v. 20 , no. 6, p. $7-8$.

Schwartz, S.Y., and Christensen, D.H., 1988, The 12 July 1986 St. Mary's, Ohio earthquake and recent seismicity in the Anna, Ohio seismogenic zone: Eastern Section, Seismological Society of America, Seismological Research Letters, v. 59, no. 2, p. 57-62.

Sharp, R.V., Rymer, M.J., and Morton, D.M., 1986, Tracefractures on the Banning fault created in association with the North Palm Springs earthquake: Seismological Society of America Bulletin, v. 76, no. 6, p. 1838-1843.

Sibol, M.S., Bollinger, G.A., Mathena, E.C., 1987a, Seismicity of the southeastern United States: Virginia Polytechnic Institute and State University Bulletin 18, 82 p. 1987b, Seismicity of the southeastern United States: Virginia Polytechnic Institute and State University Bulletin 19, $82 \mathrm{p}$.

Sipkin, S.A., 1982, Estimation of earthquake source parameters by the inversion of waveform data-Synthetic seismograms: Physics of the Earth and Planetary Interiors, v. 30, p. 242-259.

Smith, K.D., 1987, Personal experience of the 21 July 1986 $M_{L}$ 6.4 Chalfant Valley earthquake: Seismological Society of America Bulletin, v. 77, no. 1, p. 311-313.

Stickney, M. C., 1988, Montana seismicity: Montana College of Science and Technology, Montana Bureau of Mines and Geology, Open-File Report 204, 39 p.

Stickney, M. C., and Bartholomew, M. J., 1987, Seismicity and late Quaternary faulting of the northern basin and range province, Montana and Idaho: Seismological Society of America Bulletin, v. 77, no. 5, p. 1602-1625.

Stover, C.W., 1986, Preliminary isoseismal map for the northeastern Ohio earthquake of January 31, 1986: U.S. Geological Survey Open-File Report 86-136, 7 p.

Stover, C.W., and Brewer, L.R., 1991, United States earthquakes, 1985: U.S. Geological Survey Bulletin 1954, $170 \mathrm{p}$.

Switzer, J., Johnson, D., Maley, R., and Matthiesen, R., 1981, Western hemisphere strong-motion station list-1980: U.S. Geological Survey Open-File Report $81-664,162$ p.

Thiel, Charles, and Arnold, Christopher, 1986, Observations on the Santa Clara County Government Center building and strong-motion data: Earthquake Engineering Research Institute Newsletter, Special Earthquake Report, v. 20, no. 6, p. 4-6.

Toppozada, T.R. and Sanford, A.R., 1972, Instrumental study of New Mexico earthquakes, January 1968 through June 1971: New Mexico Bureau of Mines and Mineral Resources, Circular 126, $6 \mathrm{p}$.

Virginia Polytechnic Institute and State University, 1986, Blacksburg, Virginia, earthquake of March, 1986: Virginia Polytechnic and State University Seismological Observatory, VTSO Special Study Series 86-2,36 p.

Ward, R.M., 1980, Determination of three-dimensional velocity anomalies within the upper crust in the vicinity of Socorro, New Mexico, using first P-arrival times from local earthquakes: New Mexico Institute of Mining and Technology $\mathrm{Ph}$.D. dissertation, $236 \mathrm{p}$.

Ward, R.M., Schlue, J.W., and Sanford, A.R., 1981, Three-dimensional velocity anomalies in the upper crustnear Socorro, New Mexico: New Mexico Institute of Mining and Technology, Geophysical Research Letters, v. 8, p. 553-556. 
Wesson, R.L., and Nicholson, C., 1986, Studies of the January 31,1986 , northeastern Ohio earthquake- $A$ report to the U.S. Nuclear Regulatory Commission: U.S. Geological Survey Open-File Report 86-331, 131 p.

Wood, H.O., and Newmann, Frank, 1931, Modified Mercalli
Intensity Scale of 1931: Seismological Society of America Bulletin, v. 21, no. 4, p. 277-283.

Zollweg, J.E., and Johnson P.A., 1989, The Darrington seismic zone in northwestern Washington: Seismological Society of America Bulletin, v. 79, no. 6, p. 1833-1845.

Published in the Central Region, Denver, Colorado

Manuscript approved for publication March 15, 1994 


\section{SELECTED SERIES OF U.S. GEOLOGICAL SURVEY PUBLICATIONS}

\section{Periodicals}

Earthquakes \& Volcanoes (issued bimonthly).

Preliminary Determination of Epicenters (issued monthly).

\section{Technical Books and Reports}

Professional Papers are mainly comprehensive scientific reports of wide and lasting interest and importance to professional scientists and engineers. Included are reports on the results of resource studies and of topographic, hydrologic, and geologic investigations. They also include collections of related papers addressing different aspects of a single scientific topic.

Bulletins contain significant data and interpretations that are of lasting scientific interest but are generally more limited in scope or geographic coverage than Professional Papers. They include the results of resource studies and of geologic and topographic investigations; as well as collections of short papers related to a specific topic.

Water-Supply Papers are comprehensive reports that present significant interpretive results of hydrologic investigations of wide interest to professional geologists, hydrologists, and engineers. The series covers investigations in all phases of bydrology, including bydrology, availability of water, quality of water, and use of water.

Circulars present administrative information or important scientific information of wide popular interest in a format designed for distribution at no cost to the public. Information is usually of short-term interest.

Water-Resources Investigations Reports are papers of an interpretive nature made available to the public outside the formal USGS publications series. Copies are reproduced on request unlike formal USGS publications, and they are also available for public inspection at depositories indicated in USGS catalogs.

Open-File Reports include unpublished manuscript reports, maps, and other material that are made available for public consultation at depositories. They are a nonpermanent form of publication that may be cited in other publications as sources of information.

\section{Maps}

Gcologic Quadrangle Maps are multicolor geologic maps on topographic bases in $71 / 2$ - or 15 -minute quadrangle formats (scales mainly $1: 24,000$ or $1: 62,500$ ) showing bedrock, surficial, or engineering geology. Maps generally include brief texts; some maps include structure and columnar sections only.

Geophysical Investigations Maps are on topographic or planimetric bases at various scales, they show results of surveys using geophysical techniques, such as gravity, magnetic, seismic, or radioactivity, which reflect subsurface structures that are of economic or geologic significance. Many maps include correlations with the geology.

Miscellaneous Investigations Series Maps are on planimetric or topographic bases of regular and irregular areas at various scales; they present a wide variety of format and subject matter. The series also includes 7 1/2-minute quadrangle photogeologic maps on planimetric bases which show geology as interpreted from aerial photographs. The series also includes maps of Mars and the Moon.
Coal Investigations Maps are geologic maps on topographic or planimetric bases at various scales showing bedrock or surficial geology, stratigraphy, and structural relations in certain coal-resource areas.

Oil and Gas Investigations Charts show stratigraphic information for certain oil and gas fields and other areas having petroleum potential.

Miscellaneous Field Studies Maps are multicolor or black-andwhite maps on topographic or planimetric bases on quadrangle or irregular areas at various scales. Pre-1971 maps show bedrock geology in relation to specific mining or mineral-deposit problems; post-1971 maps are primarily black-and-white maps on various subjects such as environmental studies or wilderness mineral investigations.

Hydrologic Investigations Atlases are multicolored or black-andwhite maps on topographic or planimetric bases presenting a wide range of geohydrologic data of both regular and irregular areas; the principal scale is 1:24,000, and regional studies are at 1:250,000 scale or smaller.

\section{Catalogs}

Permanent catalogs, as well as some others, giving comprehensive listings of U.S. Geological Survey publications are available under the conditions indicated below from USGS Map Distribution, Box 25286, Building 810, Denver Federal Center, Denver, CO 80225. (See latest Price and Availability List.)

"Publications of the Geological Survey, 1879-1961" may be purchased by mail and over the counter in paperback book form and as a set microfiche.

"Publications of the Geological Survey, 1962-1970" may be purchased by mail and over the counter in paperback book form and as a set of microfiche.

"Publications of the U.S. Geological Survey, 1971-1981" may be purchased by mail and over the counter in paperback book form (two volumes, publications listing and index) and as a set of microfiche.

Supplements for 1982,1983,1984, 1985, 1986, and for subsequent years since the last permanent catalog may be purchased by mail and over the counter in paperback book form.

State catalogs, "List of U.S. Geological Survey Geologic and Water-Supply Reports and Maps For (State)," may be purchased by mail and over the counter in paperback booklet form only.

"Price and Availability List of U.S. Geological Survey Publications," issued annually, is available free of charge in paperback booklet form only.

Selected copies of a monthly catalog "New Publications of the U.S. Geological Survey" is available free of charge by mail or may be obtained over the counter in paperback booklet form only. Those wishing a free subscription to the monthly catalog "New Publications of the U.S. Geological Survey" should write to the U.S. Geological Survey, $582 \mathrm{Na}$ tional Center, Reston, VA 22092.

Note.-Prices of Government publications listed in older catalogs, announcements, and publications may be incorrect. Therefore, the prices charged may differ from the prices in catalogs, announcements, and publications. 